Semi-Annual Report of The Department of Energy, Office of Environmental Management, Quality Assessment Program RECEIVED

FEB 221999

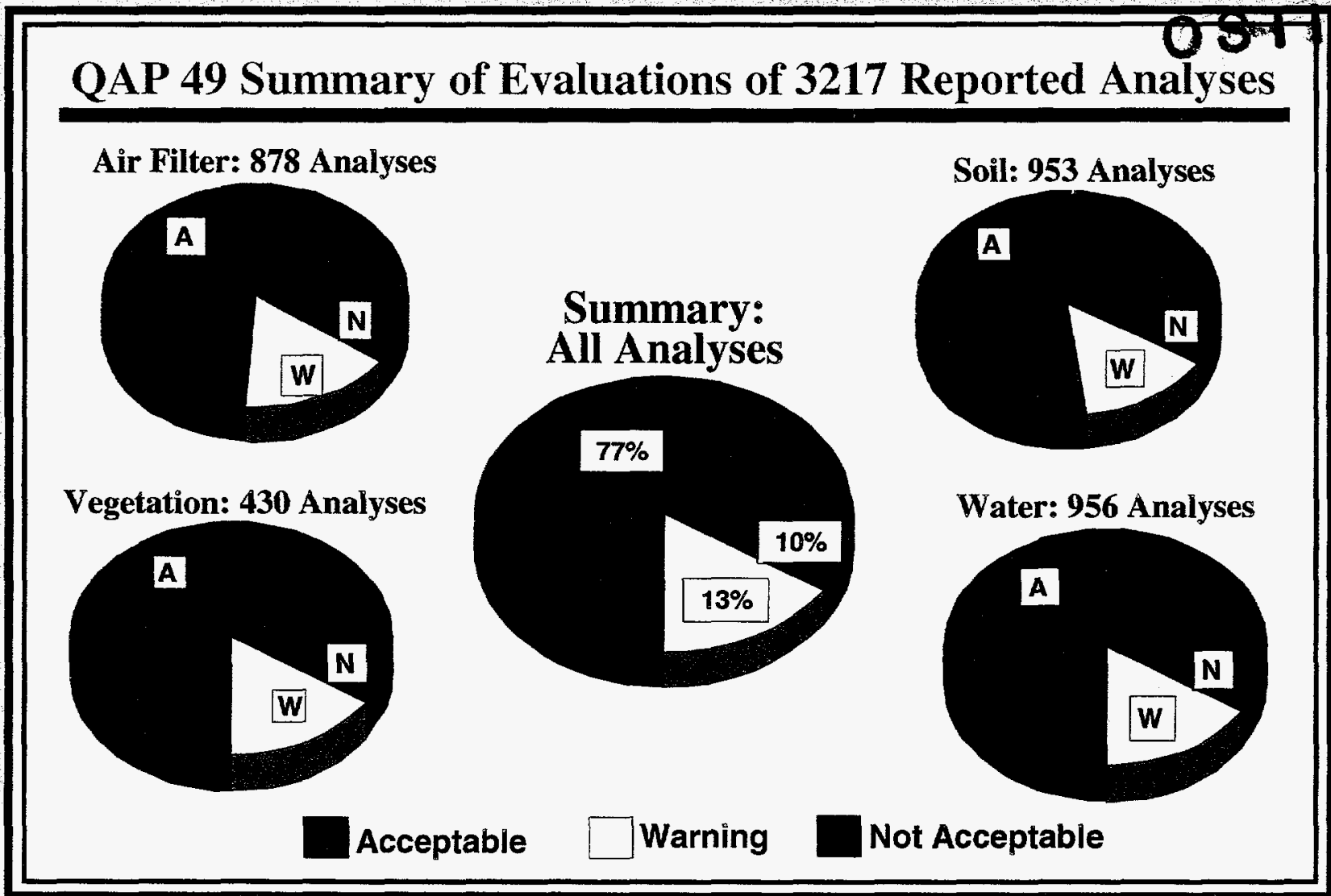

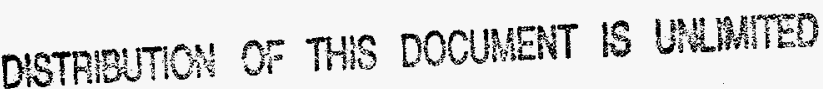

Pamela D. Greenlaw MASTER

December, 1998 
EML-600

\title{
SEMI-ANNUAL REPORT OF THE DEPARTMENT OF ENERGY, OFFICE OF ENVIRONMENTAL MANAGEMENT, QUALITY ASSESSMENT PROGRAM
}

\author{
Pamela D. Greenlaw \\ Environmental Measurements Laboratory \\ U. S. Department of Energy \\ New York, NY 10014-4811
}

December 1998

\section{DISCLAIMER}

"This report was prepared as an account of work sponsored by an agency of the United States Government. Neither the United States Government nor any agency thereof, nor any of their employees, makes any warranty, express or implied, or assumes any legal liability or responsibility for the accuracy, completeness, or usefulness of any information, apparatus, product, or process disclosed, or represents that its use would not infringe privately owned rights. Reference herein to any specific commercial product, process, or service by trade name, trademark, manufacturer, or otherwise, does not necessarily constitute or imply its endorsement, recommendation, or favoring by the United States Government or any agency thereof. The views and opinions of authors expressed herein do not necessarily state or reflect those of the United States Government or any agency thereof."

This report has been reproduced directly from the best available copy.

Available from the National Technical Information Service, U.S. Department of Commerce, 5285 Port Royal Road, Springfield, Virginia 22161. 


\section{DISCLAIMER}

Portions of this document may be illegible in electronic image products. Images are produced from the best available original document. 
EML-600 QAP 9809

December, 1998

\section{Acknowledgement}

This report represents the efforts of the following EML staff: Anna Berne, Karin Decker, Michele

DeGennaro, Isabel M. Fisenne, Richard Godwin, Christina Jaw, John Kada, Ada Kong, Pamela M. Perry, William Rivera, Arnold Boyd, Nancy Chieco, Kevin Clancy, Francis DiPasqua, Brenda Jones, Sylvia Kendall and Camille Marinetti. 
A

This report presents the results from the analysis of the 49th set of environmental quality assessment samples (QAP XLIX) that were received on or before December 1, 1998. 
EML-600 QAP 9809

December, 1998

\section{INTRODUCTION}

This Quality Assessment Program (QAP) is designed to test the quality of the environmental measurements being reported to the Department of Energy by its contractors. Since 1976, real or synthetic environmental samples that have been prepared and thoroughly analyzed at the Environmental Measurements Laboratory (EML) have been distributed at first quarterly and then semi-annually to these contractors. Their results, which are returned to EML within 90 days, are compiled with EML's results and are reported back to the participating contractors 30 days later. A summary of the reported results is available to the participants 4 days after the reporting deadline via the Internet at www.eml.doe.gov.

This is the 55th report of this program. Preceding reports in this series are:

$\begin{array}{ll}\text { HASL-317 } & \text { (February 1, 1977) } \\ \text { HASL-319 } & \text { (May 2, 1977) } \\ \text { HASL-323 } & \text { (August 1, 1977) } \\ \text { HASL-331 } & \text { (November 1, 1977) } \\ \text { EML-336 } & \text { (January 1, 1978) } \\ \text { EML-337 } & \text { (February 1, 1978) } \\ \text { EML-340 } & \text { (May 1, 1978) } \\ \text { EML-343 } & \text { (August 1, 1978) } \\ \text { EML-346 } & \text { (November 1, 1978) } \\ \text { EML-350 } & \text { (February 1, 1979) } \\ \text { EML-351 } & \text { (February 1,1979) } \\ \text { EML-354 } & \text { (May 1, 1979) } \\ \text { EML-358 } & \text { (August 1, 1979) } \\ \text { EML-364 } & \text { (November 1, 1979) } \\ \text { EML-368 } & \text { (February 1, 1980) } \\ \text { EML-377 } & \text { (August 1, 1980) } \\ \text { EML-387 } & \text { (February 1, 1981) } \\ \text { EML-388 } & \text { (February 1, 1981) } \\ \text { EML-393 } & \text { (August 3, 1981) } \\ \text { EML-402 } & \text { (February 1, 1982) } \\ \text { EML-414 } & \text { (April 1, 1983) } \\ \text { EML-417 } & \text { (September 1,1983) } \\ \text { EML-426 } & \text { (March 1, 1984) } \\ \text { PNL-5079 } & \text { (April 1, 1984) } \\ \text { EML-431 } & \text { (September 1, 1984) } \\ \text { EML-432 } & \text { (November 1, 1984) } \\ \text { EML-438 } & \text { (March 1, 1985) } \\ & \end{array}$

HASL-317

HASL-319

HASL-331

EML-336

EML-337

EML-340

EML-343

EML-346

EML-350

EML-351

EML-354

EML-358

EML-364

EML-368

EML-377

EML-387

EML-388

EML-393

EML-402

EML-414

EML-417

EML-426

PNL-5079

EML-431

EML-438
(February 1, 1977)

(May 2, 1977)

(August 1, 1977)

(November 1, 1977)

(January 1, 1978)

(February 1, 1978)

(May 1, 1978)

(August 1, 1978)

(November 1, 1978)

(February 1, 1979)

(February 1, 1979)

(May 1, 1979)

(August 1, 1979)

(November 1, 1979)

(February 1, 1980)

(August 1, 1980)

(February 1, 1981)

(February 1, 1981)

(August 3, 1981)

(February 1, 1982)

(April 1, 1983)

(September 1, 1983)

(March 1, 1984)

(April 1, 1984)

(September 1, 1984)

(March 1, 1985)
EML-439

EML-448

EML-453

EML-454

EML-477

EML-478

EML-498

EML-518

EML-525*

EML-526

EML-530

EML-535

EML-539

EML-543

EML-546

EML-551

EML-556

EML-559

EML-561

EML-565

EML-569

EML-576

EML-581

EML-587

EML-591

EML-594

EML-596
(March 1, 1985)

(October 1, 1985)

(March 1, 1986)

(March 1, 1986)

(October 1, 1986)

(March 1, 1987)

(September 1, 1987)

(January 2, 1989)

(August 1, 1989)

(January 2, 1990)

(July 2, 1990)

(January 1, 1991)

(July 1, 1991)

(January 2, 1992)

(July 1, 1992)

(January 4, 1993)

(July 1, 1993)

(January 5, 1994)

(July 1, 1994)

(January 5, 1995)

(July 3, 1995)

(February 1, 1996, Revised)

(July 1, 1996)

(January 1997)

(July 1997)

(January 1998)

(July 1998)

"Please note this is a corrected report number. 
EML-600 QAP 9809

December, 1998

\section{Results}

The results from the analysis of QAP-XLIX samples received on or before December 1, 1998 are listed according to the TABLE OF CONTENTS. The data for the different kinds of samples are given in the following units:

$\begin{array}{ll}\text { Air Filters } & \mathrm{Bq} \text { filter } \\ \text { Soil } & \mathrm{Bq} \mathrm{kg}^{-1} \\ \text { Tissue } & \mathrm{Bq} \mathrm{kg}^{-1} \\ \text { Vegetation } & \mathrm{Bq} \mathrm{kg}^{-1} \\ \text { Water } & \mathrm{Bq} \mathrm{L}^{-1}\end{array}$

The values for elemental uranium are reported in $\mu \mathrm{g}$ filter $^{-1}, \mathrm{~g}$, or $\mathrm{mL}$. Some programs require the use of $\mathrm{pCi}$ as reporting units, the conversion can be found on page 2 .

The 'EML value' listed in the tables to which the contractors' results are compared is the mean of replicate determinations for each nuclide. The EML uncertainty is the standard deviation of the mean. All other uncertainties are as reported by the participants.

The control limit concept was established from percentiles of historic data distributions (1982-1992). The evaluation of this historic data and the development of the control limits are presented in DOE report EML-564. The control limits for QAP-XLIX were developed from percentiles of data distributions for the years 1993-1998.

Participants' analytical performance is evaluated based on the historical analytical capabilities for individual analyte/matrix pairs. The criteria for acceptable performance, "A", has been chosen to be between the $15^{\text {th }}$ and $85^{\text {th }}$ percentile of the cumulative normalized distribution, which can be viewed as the middle $70 \%$ of all historic measurements. The acceptable with warning criteria, "W", is between the $5^{\text {th }}$ and $15^{\text {th }}$ percentile and between the $85^{\text {th }}$ and $95^{\text {th }}$ percentile. In other words, the middle $90 \%$ of all reported values are acceptable, while the outer $5^{\text {th }}-15^{\text {th }}(10 \%)$ and $85^{\text {th }}-95^{\text {th }}$ percentiles $(10 \%)$ are in the warning area. The not acceptable criteria, " $\mathrm{N}$ ", is established at less than the $5^{\text {th }}$ percentile and greater than the $95^{\text {th }}$ percentile, that is, the outer $10 \%$ of the historical data. These control limits for all $48 \mathrm{i} / \mathrm{j}$ pairs are listed in the Table of Control Limits (p. 3).

QAP is an external assessment of environmental radiological analyses. If your laboratory is performing other types of analyses (screening, high-level radiological), this evaluation system may not be appropriate, and you should continue to use an evaluation system appropriate to your data quality objectives. 


\section{TabLe OF CONTENTS}

Summary Figure of QAP-XLIX Evaluations $\ldots \ldots \ldots \ldots \ldots \ldots \ldots \ldots \ldots \ldots \ldots \ldots \ldots \ldots \ldots$

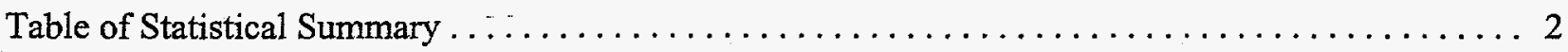

Table of Control Limits and Performance Criteria $\ldots \ldots \ldots \ldots \ldots \ldots \ldots \ldots \ldots \ldots \ldots$

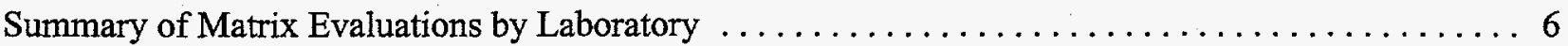

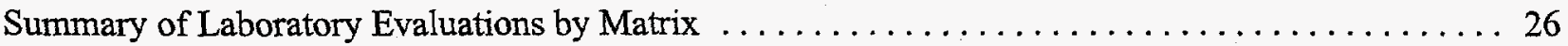

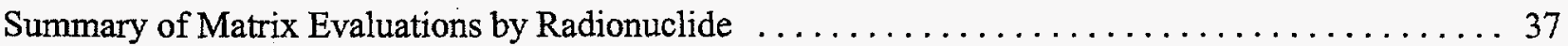

Results Ordered by Laboratory

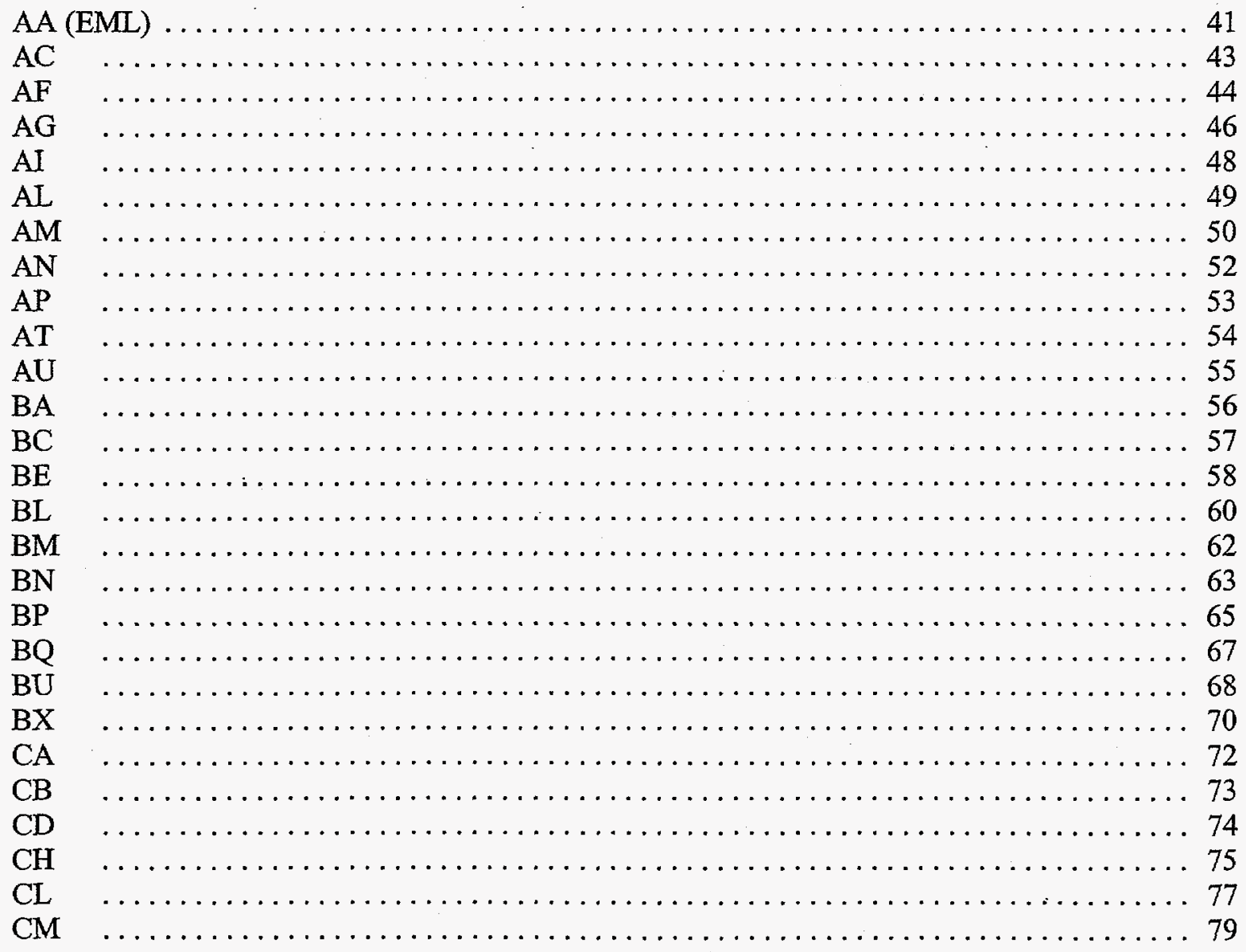




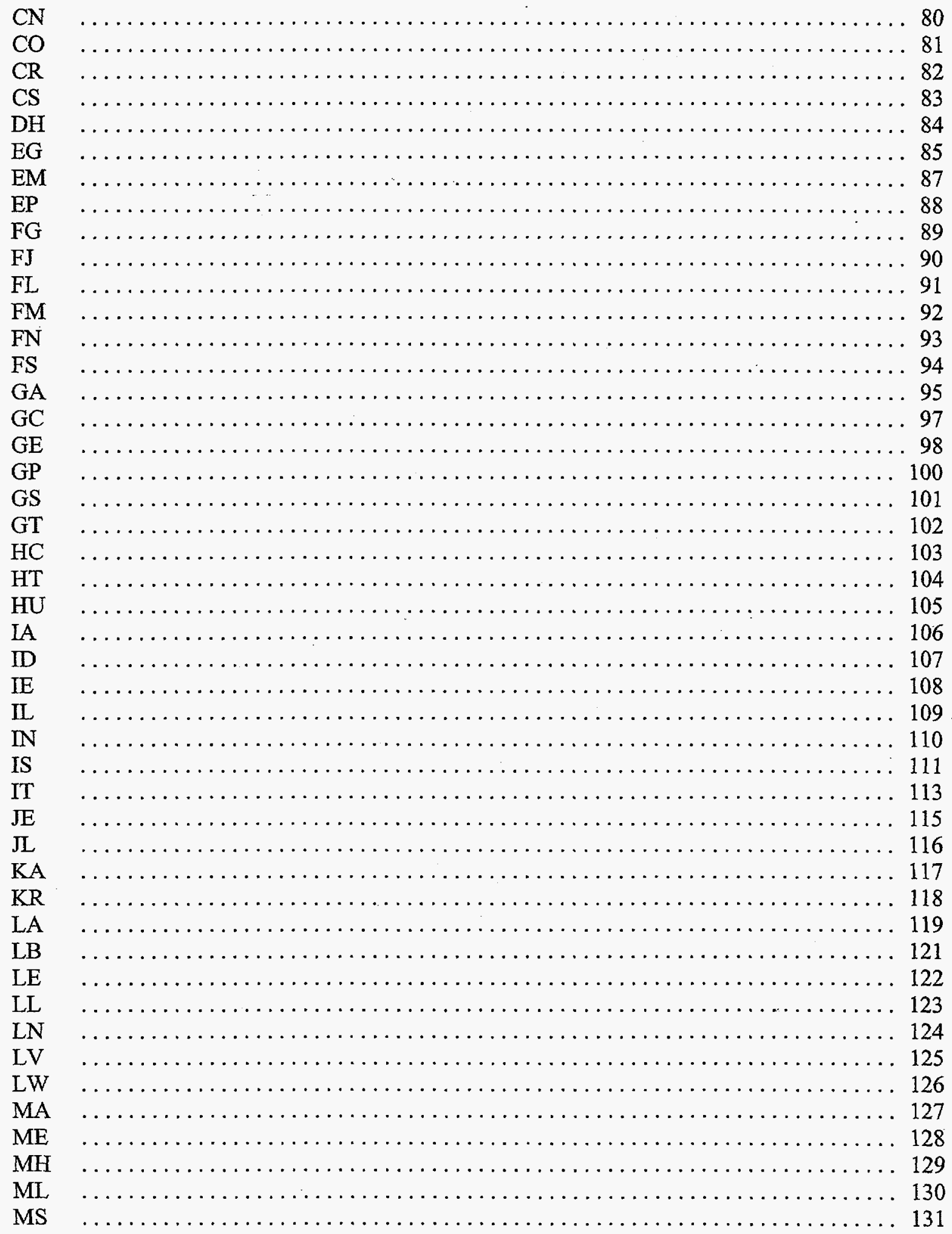


NA

ND

NL

NM

135

NP

136

NQ

NR

NS

NZ

$\mathrm{OB}$

OC

OD

OL

OT

$\mathrm{OU}$

PA

PK

PO

PR

RA

$\mathrm{RC}$

$\mathrm{RE}$

$\mathrm{RG}$

RI

RK

SA

SB

SK

SL

SN

SR

ST

SW

TE

TI

TM

TN

TO

TP

TT

TW

TX

TY

UC

UP

UY 


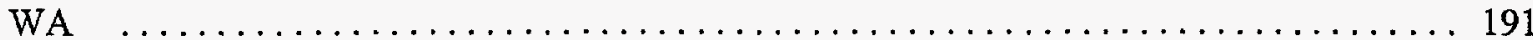

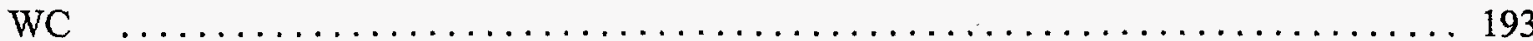

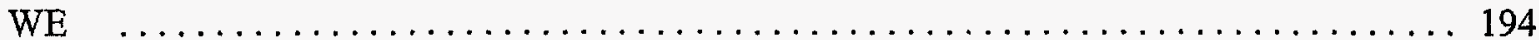

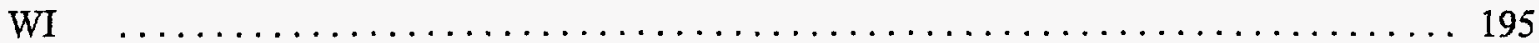

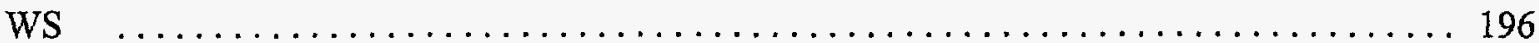

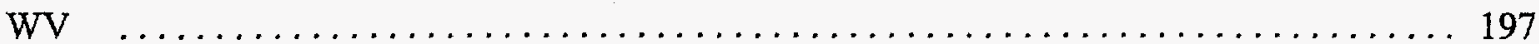

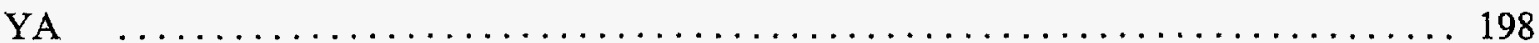

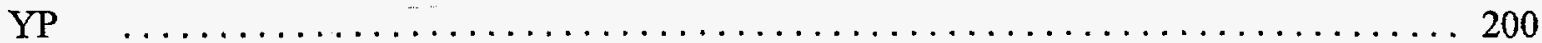

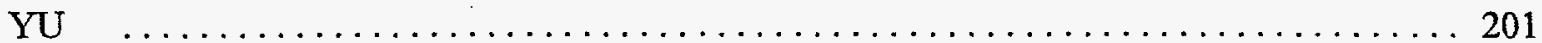

Results Ordered by Matrix/Nuclide

Air

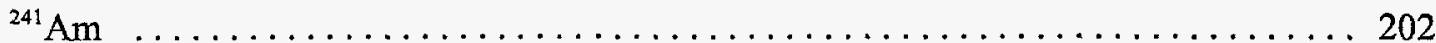

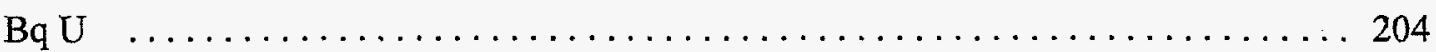

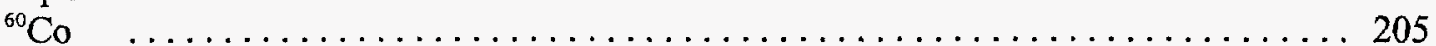

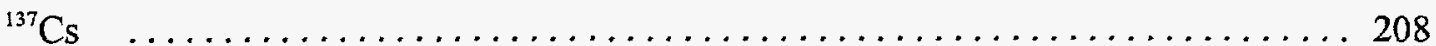

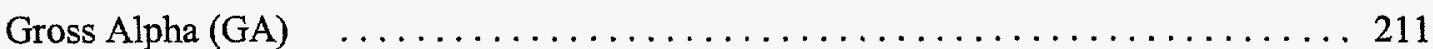

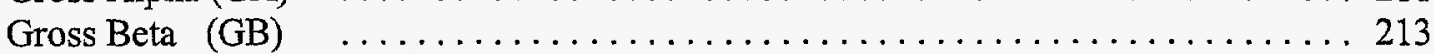

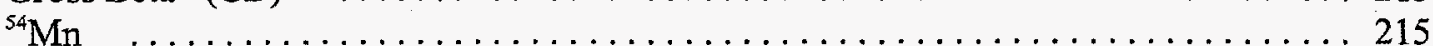

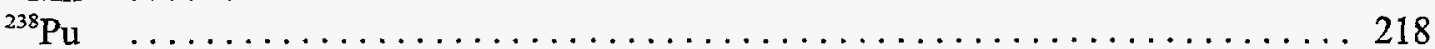

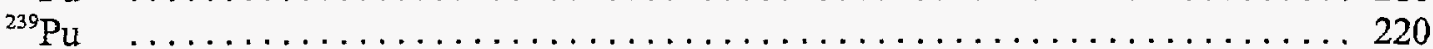

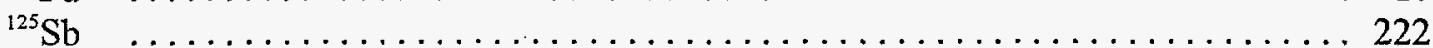

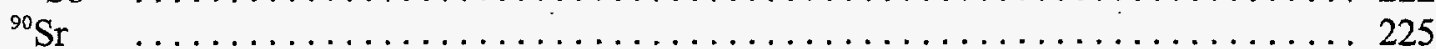

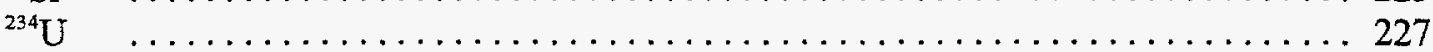

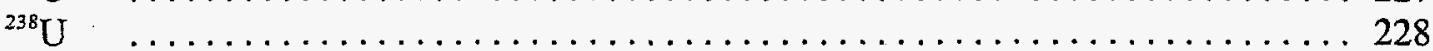

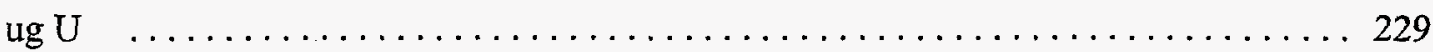

Soil

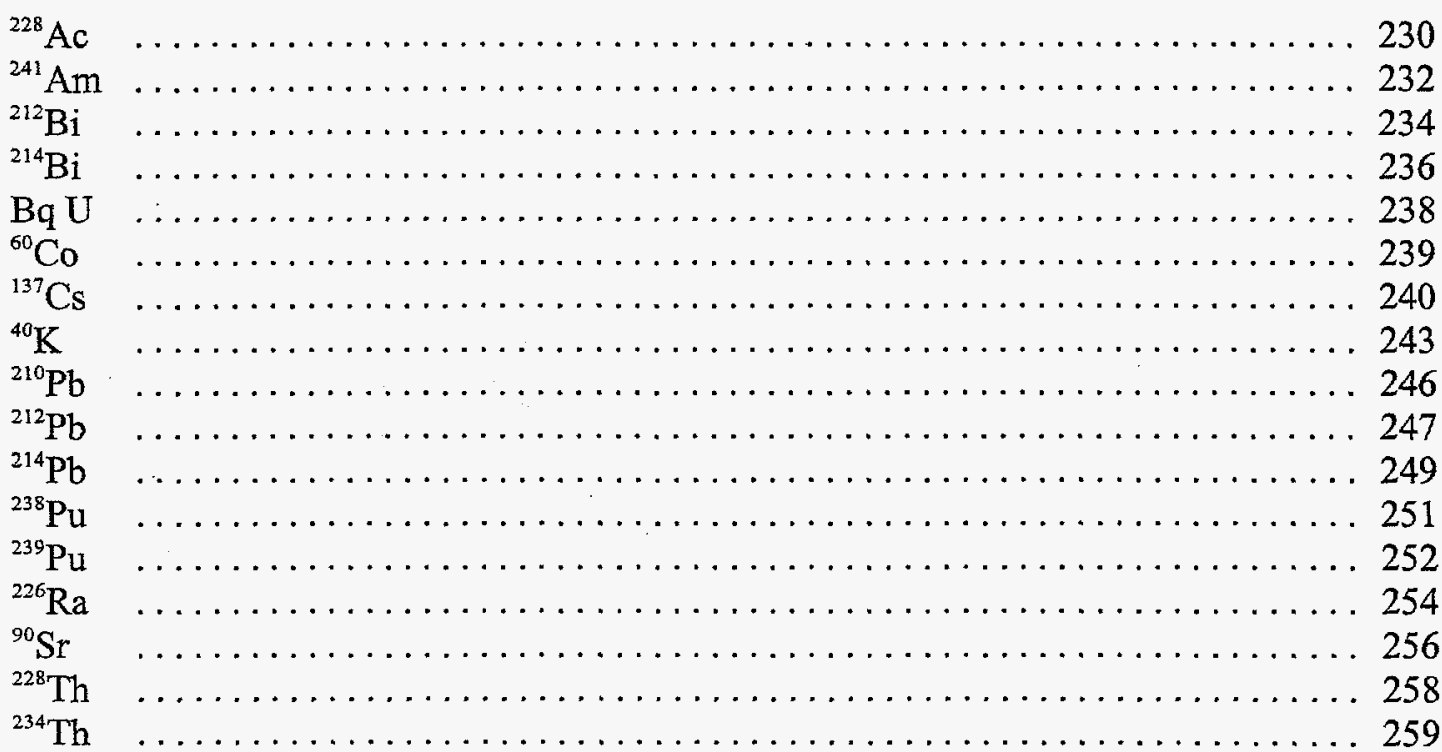


${ }^{208} \mathrm{Tl}$

\section{Vegetation}

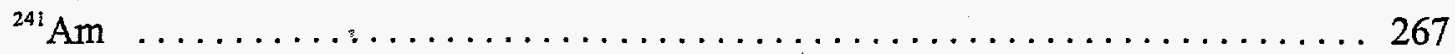

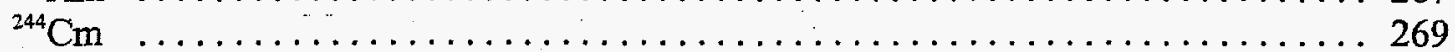

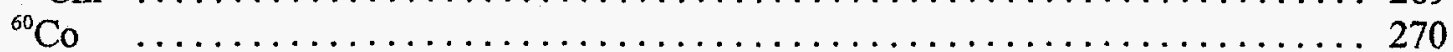

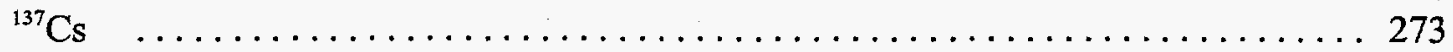

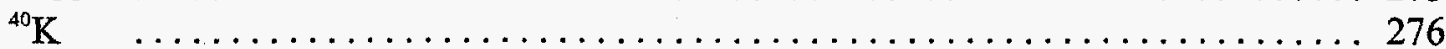

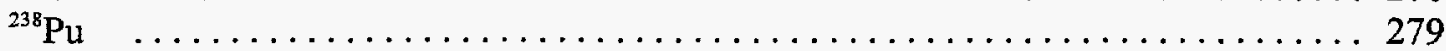

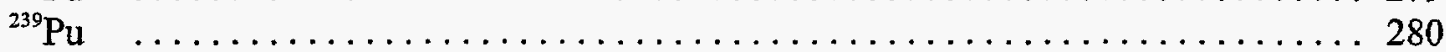

${ }^{90} \mathrm{Sr}$

Water

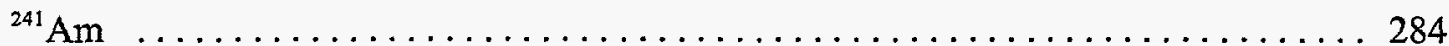

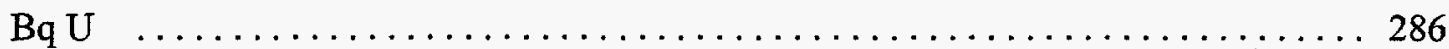

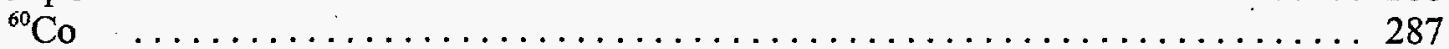

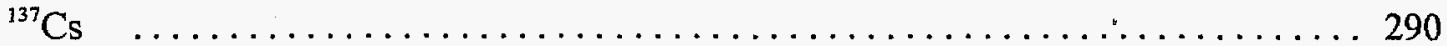

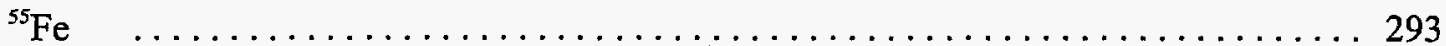

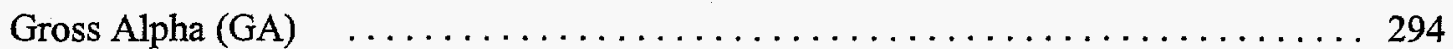

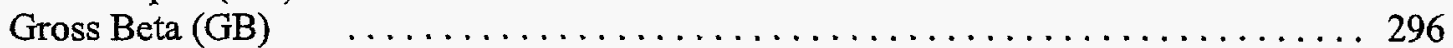

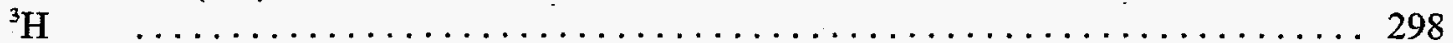

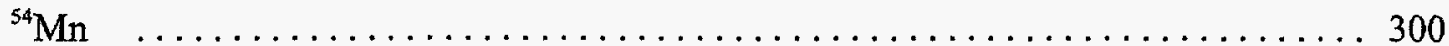

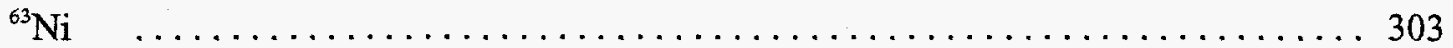

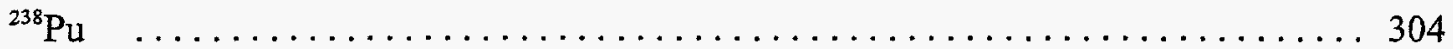

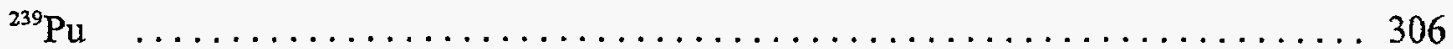

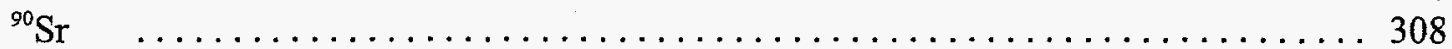

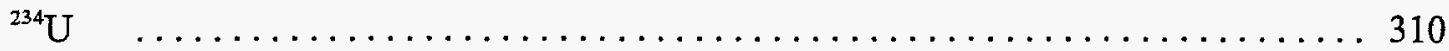

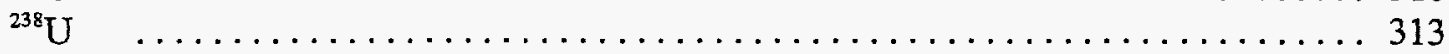

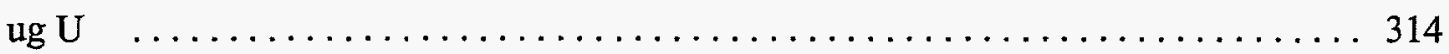

List of Labcodes of Participating Laboratories for EML QAP XLVII 


\section{QAP 49 Summary of Evaluations of 3217 Reported Analyses}

Air Filter:

878 Analyses

Vegetation:

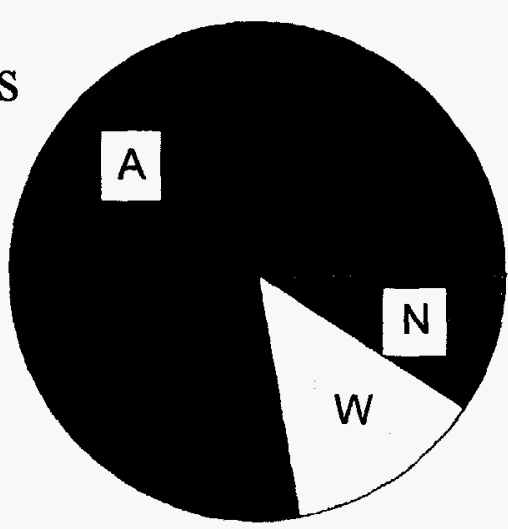

430 Analyses

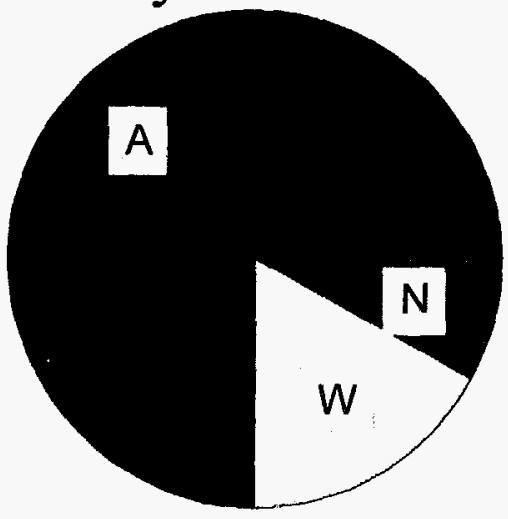

Summary: All Analyses
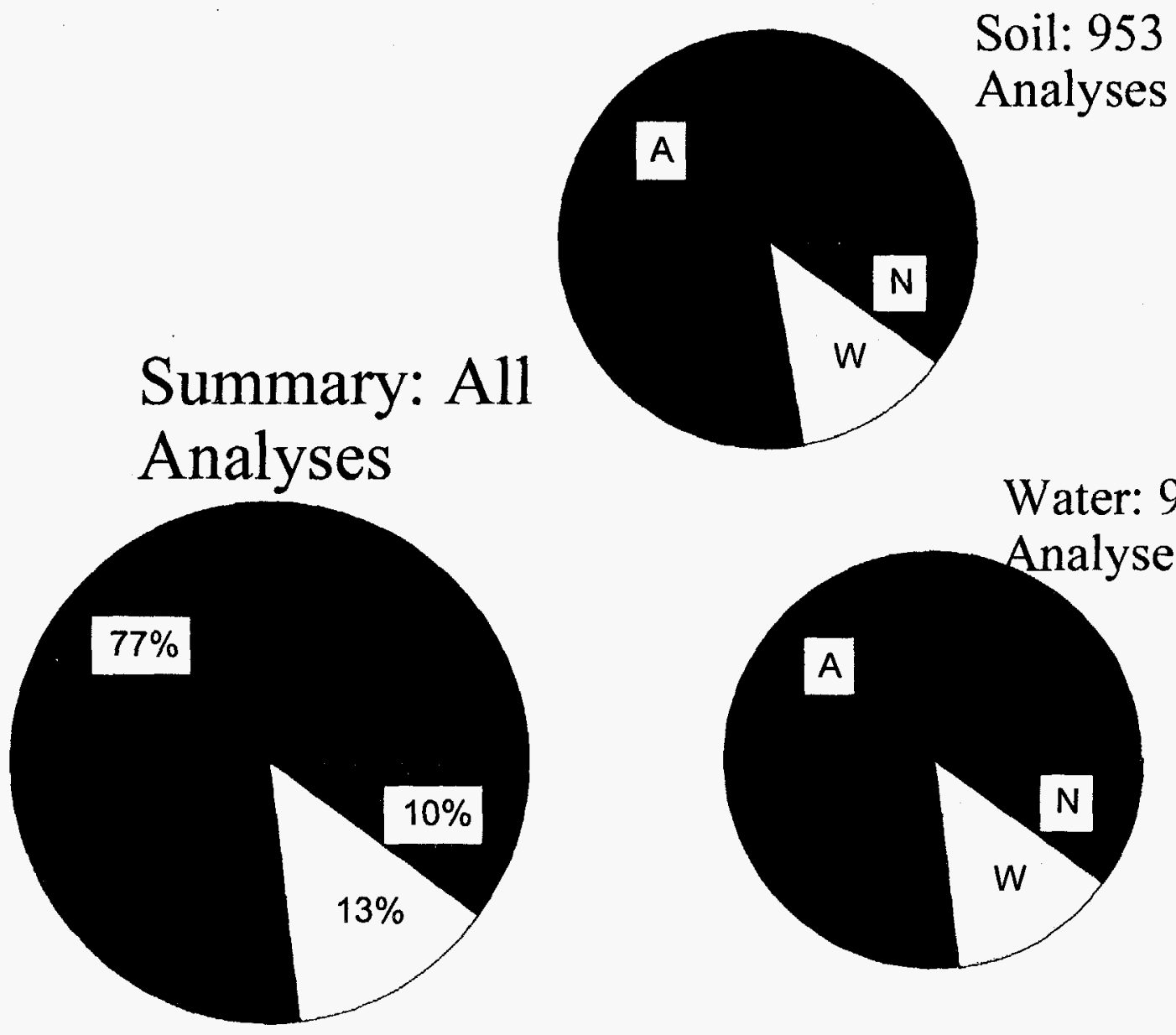

Water: 956

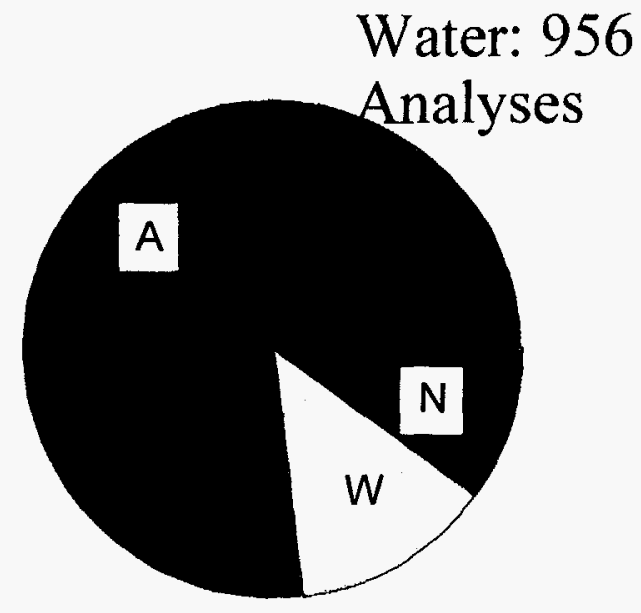

Acceptable

Warning

Not Acceptable 


\section{QAP 49 Statistical Summary}

Reported Values

Nuclide EML Value EML Error
EML Value

$\frac{\text { RML Value }}{\text { EML }}$

Mean Median Std. Dev.
No.* of

Reported

Values

\section{Matrix: AI}

AM241

$\mathrm{Bq} \mathrm{U}$

C060

CS137

0.530

9.160

GROSS ALPHA

GROSS BETA

MN54

PU238

PU239

SB125

SR90

$\mathrm{U} 234$

U238

ug $\mathrm{U}$

22.470
1.650

2.160

4.920

0.460

0.420

8.890

1.120

0.260

0.260

20.960

Matrix: $\quad$ SO

$$
\begin{array}{r}
52.600 \\
7.470 \\
58.300 \\
28.800 \\
237.000 \\
1.240 \\
954.000 \\
314.000 \\
32.000 \\
52.800 \\
29.100 \\
0.530 \\
13.090 \\
29.000 \\
39.630 \\
52.700 \\
114.000 \\
18.300 \\
113.000 \\
120.000 \\
9.700
\end{array}
$$

.700

0.008
0.020
0.580
1.030
0.160
0.070
0.400
0.005
0.006
0.550
0.050
0.010
0.010
0.100

0.008
0.020

0.580

1.030

0.070

0.400

0.005

0.006

0.050

0.010

0.010

0.100

2.900

0.410

5.900

0.500

16.000

0.110

38.000

13.000

3.300

3.700

1.200

0.270

0.570

1.000

0.003

4.000

6.000

1.100

6.000

9.000

0.700

1.102
1.053
0.993
1.005
1.028
0.958
1.042
1.065
1.081
1.004
0.979
1.039
1.062
1.025

1.102
1.053

0.993

1.005

1.028

0.958

1.065

1.081

1.004

0.979

1.062

1.025

1.062
1.015
0.993
1.001
0.982
0.926
1.043
1.080
1.096
1.016
1.005
1.000
1.031
1.001

1.062
1.015

0.993

1.001

0.982

0.926

1.043

1.080

1.016

1.005

1.031

1.001
0.219

0.220
0.074

0.090

0.166

0.160

0.092

0.093

0.095

0.131

0.125

0.175

0.174

0.129
54

15

101

99

73

75

95

43

44

88

34

29

30

16

U238

ug U

$\begin{array}{ll}1.051 & 1.027 \\ 1.092 & 1.051 \\ 0.842 & 0.919 \\ 1.097 & 1.084 \\ 0.869 & 0.920 \\ 1.200 & 1.339 \\ 1.078 & 1.090 \\ 1.114 & 1.122 \\ 1.240 & 1.013 \\ 1.057 & 1.036 \\ 1.101 & 1.076 \\ 1.113 & 1.453 \\ 0.999 & 1.005 \\ 2.442 & 1.393 \\ 1.096 & 1.089 \\ 1.157 & 1.044 \\ 1.069 & 0.984 \\ 1.441 & 1.140 \\ 0.940 & 0.949 \\ 0.888 & 0.902 \\ 0.943 & 0.924\end{array}$

0.219

49

$0.209 \quad 57$

0.278

0.225

0.144

0.319

0.095

0.141

0.908

0.226

0.236

0.309

0.112

2.086

0.278

0.602

0.365

0.713

0.100

0.088

0.077 
Reported Values

EML Value

No. * of

Nuclide

EML Value

EML Error

Mean Median

Std. Dev.

Reported

Values

\section{Matrix: VE}

$\begin{array}{lrrrrrr}\text { AM241 } & 2.330 & 0.060 & 1.207 & 1.138 & 0.344 & 44 \\ \text { CM244 } & 1.760 & 0.070 & 1.051 & 1.071 & 0.228 & 26 \\ \text { CO60 } & 20.000 & 1.000 & 1.051 & 1.050 & 0.136 & 80 \\ \text { CS137 } & 390.000 & 20.000 & 1.087 & 1.108 & 0.100 & 83 \\ \text { K40 } & 460.000 & 20.000 & 1.113 & 1.122 & 0.139 & 77 \\ \text { PU238 } & 0.310 & 0.070 & 1.105 & 1.306 & 0.365 & 4 \\ \text { PU239 } & 3.720 & 0.270 & 1.066 & 1.074 & 0.120 & 38 \\ \text { SR90 } & 606.000 & 40.000 & 0.952 & 1.012 & 0.178 & 43\end{array}$

\begin{tabular}{lr}
\hline Matrix: & WA \\
\hline AM241 & 1.250 \\
Bq U & 1.050 \\
CO60 & 49.400 \\
CS137 & 50.000 \\
FE55 & 139.000 \\
GROSS ALPHA & 1080.000 \\
GROSS BETA & 1420.000 \\
H3 & 76.200 \\
MN54 & 32.400 \\
NI63** & 95.700 \\
PU238 & 1.100 \\
PU239 & 1.410 \\
SR90 & 2.110 \\
U234 & 0.510 \\
U238 & 0.520 \\
ug U & 0.040
\end{tabular}

$\begin{array}{rrr}0.080 & 1.051 & 1.040 \\ 0.080 & 1.014 & 1.029 \\ 1.200 & 1.039 & 1.032 \\ 1.700 & 1.051 & 1.036 \\ 2.000 & 0.986 & 1.018 \\ 60.000 & 0.939 & 0.971 \\ 60.000 & 0.905 & 0.880 \\ 2.900 & 1.116 & 1.062 \\ 1.400 & 1.093 & 1.090 \\ 0.900 & 1.094 & 1.190 \\ 0.010 & 1.049 & 1.071 \\ 0.040 & 1.085 & 1.085 \\ 0.180 & 1.102 & 1.090 \\ 0.030 & 1.045 & 1.039 \\ 0.050 & 1.026 & 1.019 \\ 0.003 & 1.039 & 1.050\end{array}$

0.112

0.136

0.049

0.078

0.098

0.130

0.122

0.187

0.068

0.226

0.092

0.095

0.132

0.093

0.058

0.073

58
21
105
107
16
65
70
59
93
14
51
55
52
37
37
21

Units for matrices:

Air filter: $\mathrm{AI}=\mathrm{Bq} /$ filter

Soil: $\mathrm{SO}=\mathrm{Bq} / \mathrm{kg}$

Vegetation: $\mathrm{VE}=\mathrm{Bq} / \mathrm{kg}$

Water: $\mathrm{WA}=\mathrm{Bq} / \mathrm{L}$.

Values for elemental uranium in $\mu \mathrm{g} /$ filter, $\mathrm{g}$ or $\mathrm{mL}$.

Conversion from $\mathrm{Bq} / \mathrm{kg}$ or $\mathrm{L}$ to $\mathrm{pCi} / \mathrm{g}$ or $\mathrm{mL}$ :

$1 \mathrm{~Bq} / \mathrm{kg}$ or $\mathrm{L}=0.027 \mathrm{pCi} / \mathrm{g}$ or $\mathrm{mL}$

Example: Convert $3 \mathrm{~Bq} / \mathrm{kg}$ to $\mathrm{pCi} / \mathrm{g}$

$3 \mathrm{~Bq} / \mathrm{kg} \times 27 \mathrm{pCi} / \mathrm{Bq} / 1000 \mathrm{~g} / \mathrm{kg}=0.081 \mathrm{pCi} / \mathrm{g}$

*Statistical summary of " $A$ " and "W" reported values 


\section{QAP 49 Control Limits* by Matrix}

Nuclide Lower Limit $\quad$ Lower Middle Limit $\quad$ Upper Middle Limit $\quad$ Upper Limit

\begin{tabular}{|c|c|c|c|c|}
\hline \multicolumn{2}{|l|}{ Matrix: $\mathbf{A I}$} & \multirow[b]{2}{*}{0.88} & \multirow[b]{2}{*}{1.46} & \multirow[b]{2}{*}{2.58} \\
\hline AM241 & 0.73 & & & \\
\hline $\mathrm{BqU}$ & 0.80 & 0.90 & 1.53 & 3.35 \\
\hline CO60 & 0.75 & 0.83 & 1.10 & 1.32 \\
\hline CS137 & 0.73 & 0.82 & 1.14 & 1.37 \\
\hline GROSS ALPHA & 0.50 & 0.81 & 1.32 & 1.55 \\
\hline GROSS BETA & 0.72 & 0.89 & 1.39 & 1.67 \\
\hline MN54 & 0.76 & 0.84 & 1.18 & 1.42 \\
\hline PU238 & 0.74 & 0.89 & 1.15 & 1.40 \\
\hline PU239 & 0.76 & 0.90 & 1.19 & 1.44 \\
\hline SB125 & 0.61 & 0.83 & 1.19 & 1.43 \\
\hline SR90 & 0.61 & 0.83 & 1.33 & 1.93 \\
\hline U234 & 0.83 & 0.90 & 1.40 & 1.92 \\
\hline U238 & 0.84 & 0.90 & 1.31 & 2.61 \\
\hline ug U & 0.72 & 0.90 & 1.29 & 1.93 \\
\hline \multicolumn{2}{|l|}{ Matrix: $\quad$ SO } & & & \\
\hline $\mathrm{AC} 228^{* *}$ & 0.00 & 0.00 & 0.00 & 0.00 \\
\hline AM241 & 0.63 & 0.79 & 1.48 & 2.31 \\
\hline $\mathrm{BI} 212 * *$ & 0.00 & 0.00 & 0.00 & 0.00 \\
\hline $\mathrm{BI} 214^{* *}$ & 0.00 & 0.00 & 0.00 & 0.00 \\
\hline $\mathrm{Bq} \mathrm{U}$ & 0.42 & 0.61 & 1.16 & 1.39 \\
\hline CO60 & 0.67 & 0.83 & 1.74 & 2.54 \\
\hline CS137 & 0.83 & 0.90 & 1.21 & 1.32 \\
\hline $\mathrm{K} 40$ & 0.78 & 0.90 & 1.25 & 1.53 \\
\hline PB210** & 0.00 & 0.00 & 0.00 & 0.00 \\
\hline PB212** & 0.00 & 0.00 & 0.00 & 0.00 \\
\hline PB214** & 0.00 & 0.00 & 0.00 & 0.00 \\
\hline PU238 & 0.52 & 0.74 & 1.37 & 2.84 \\
\hline PU239 & 0.69 & 0.89 & 1.24 & 1.74 \\
\hline RA226** & 0.00 & 0.00 & 0.00 & 0.00 \\
\hline SR90 & 0.60 & 0.77 & 1.64 & 3.66 \\
\hline TH228** & 0.00 & 0.00 & 0.00 & 0.00 \\
\hline $\mathrm{TH} 234 * *$ & 0.00 & 0.00 & 0.00 & 0.00 \\
\hline TL208** & 0.00 & 0.00 & 0.00 & 0.00 \\
\hline U234 & 0.47 & 0.70 & 1.11 & 1.30 \\
\hline U238 & 0.44 & 0.69 & 1.10 & 1.42 \\
\hline ug U & 0.46 & 0.67 & 1.10 & 1.22 \\
\hline
\end{tabular}

${ }^{*}$ Control limits are established from historical QAP data and reported as: the ratio of Reported Value vs. EML Value **Where historical data are insufficient, limits were not applied 


\section{QAP 49 Control Limits* by Matrix}

\begin{tabular}{llll} 
Nuclide $\quad$ Lower Limit $\quad$ Lower Middle Limit $\quad$ Upper Middle Limit & Upper Limit \\
\hline
\end{tabular}

$\begin{array}{lllll}\text { Matrix: } & \text { VE } & & & \\ \text { AM241 } & 0.68 & 0.89 & 1.60 & 2.70 \\ \text { CM244 } & 0.44 & 0.81 & 1.30 & 1.62 \\ \text { CO60 } & 0.69 & 0.86 & 1.24 & 1.46 \\ \text { CS137 } & 0.80 & 0.90 & 1.25 & 1.40 \\ \text { K40 } & 0.79 & 0.90 & 1.24 & 1.42 \\ \text { PU238 } & 0.66 & 0.81 & 2.89 & 7.94 \\ \text { PU239 } & 0.68 & 0.86 & 1.23 & 1.59 \\ \text { SR90 } & 0.50 & 0.73 & 1.13 & 1.33\end{array}$

\section{Matrix: WA}

$\begin{array}{lllll}\text { AM241 } & 0.75 & 0.90 & 1.24 & 1.49 \\ \text { Bq U } & 0.67 & 0.90 & 1.26 & 1.42 \\ \text { CO60 } & 0.80 & 0.90 & 1.14 & 1.20 \\ \text { CS137 } & 0.80 & 0.90 & 1.18 & 1.26 \\ \text { FE55 } & 0.44 & 0.60 & 1.34 & 1.53 \\ \text { GROSS ALPHA } & 0.61 & 0.83 & 1.17 & 1.32 \\ \text { GROSS BETA } & 0.55 & 0.71 & 1.32 & 1.54 \\ \text { H3 } & 0.71 & 0.82 & 1.22 & 1.79 \\ \text { MN54 } & 0.80 & 0.90 & 1.17 & 1.25 \\ \text { NI63** } & 0.00 & 0.00 & 0.00 & 0.00 \\ \text { PU238 } & 0.78 & 0.90 & 1.11 & 1.25 \\ \text { PU239 } & 0.80 & 0.90 & 1.15 & 1.39 \\ \text { SR90 } & 0.75 & 0.89 & 1.21 & 1.50 \\ \text { U234 } & 0.80 & 0.90 & 1.22 & 1.20 \\ \text { U238 } & 0.80 & 0.90 & 1.17 & 1.34 \\ \text { ug U } & 0.80 & 0.90 & 1.18 & \end{array}$

The following are recommended performance criteria for analysis of environmental levels of analytes: Acceptable: $\quad$ Lower Middle Limit $\leq \mathrm{A} \leq$ Upper Middle Limit

Acceptable with Warning: Lower Limit $\leq \mathrm{W}<$ Lower Middle Limit or Upper Middle Limit $<\mathrm{W} \leq \mathrm{Upper}$ Limit

Not Acceptable: $\mathbf{N}<$ Lower Limit or $\mathbf{N}>$ Upper Limit

* Control limits are established from historical QAP data and

reported as: the ratio of Reported Value vs. EML Value

** Where historical data are insufficient, limits were not applied 


\section{QAP 49 Summary of Matrix Evaluations by Laboratory}

Evaluation Summary

\begin{tabular}{|c|c|c|c|c|}
\hline \multirow{2}{*}{ Matrix } & \multicolumn{4}{|c|}{ 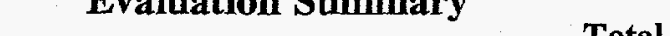 } \\
\hline & A & $\mathbf{w}$ & $\mathbf{N}$ & Analyse \\
\hline \multicolumn{5}{|c|}{ Lab: AC Analytical Chemistry Laboratory, Argonne N } \\
\hline $\begin{array}{l}\text { WA } \\
\text { SO }\end{array}$ & 2 & 0 & 0 & 2 \\
\hline SO & 2 & 0 & 0 & \\
\hline Totals: & 4 & 0 & 0 & \\
\hline
\end{tabular}

Lab: AF Air Force Analytical Lab, Brooks AFB

\begin{tabular}{|c|c|c|c|c|c|c|c|}
\hline $\begin{array}{l}\text { VE } \\
\text { SO } \\
\text { WA } \\
\text { AI }\end{array}$ & $\begin{array}{r}3 \\
12 \\
5 \\
6\end{array}$ & $\begin{array}{l}1 \\
2 \\
2 \\
0\end{array}$ & $\begin{array}{l}2 \\
2 \\
4 \\
3\end{array}$ & $\begin{array}{r}6 \\
16 \\
11 \\
9 \\
\end{array}$ & $\begin{array}{l}50 \\
75 \\
45 \\
67\end{array}$ & $\begin{array}{r}17 \\
13 \\
18 \\
0\end{array}$ & $\begin{array}{l}33 \\
13 \\
36 \\
33\end{array}$ \\
\hline Totals: & 26 & 5 & 11 & 42 & $62 \%$ & $12 \%$ & $26 \%$ \\
\hline
\end{tabular}

Lab: AG Paragon Analytics, Inc, Fort Collins, CO

\begin{tabular}{lrrrrrrr} 
& & & & & & & \\
WA & 12 & 0 & 0 & 12 & 100 & 0 & 0 \\
AI & 9 & 0 & 3 & 12 & 75 & 0 & 25 \\
SO & 15 & 2 & 0 & 17 & 88 & 12 & 0 \\
VE & 7 & 0 & 0 & 7 & 100 & 0 & 0 \\
\cline { 2 - 8 } Totals: & 43 & 2 & 3 & 48 & $90 \%$ & $4 \%$ & $\mathbf{6 \%}$
\end{tabular}

Lab: AI Nuclear Technology Services, Inc, Roswell, GA

\begin{tabular}{|c|c|c|c|c|c|c|c|}
\hline $\mathrm{AI}$ & 0 & 2 & 0 & 2 & 0 & 100 & 0 \\
\hline SO & 11 & 1 & 0 & 12 & 92 & 8 & 0 \\
\hline WA & 5 & 4 & 2 & 11 & 45 & 36 & 18 \\
\hline VE & 3 & 3 & 1 & 7 & 43 & 43 & 14 \\
\hline Totals: & 19 & 10 & 3 & 32 & $59 \%$ & $31 \%$ & \\
\hline
\end{tabular}

Lab: AL Ames Laboratory, Ames, IA

\begin{tabular}{|c|c|c|c|c|c|c|c|}
\hline WA & 0 & 0 & 3 & 3 & 0 & 0 & 100 \\
\hline $\mathrm{AI}$ & 4 & 2 & 0 & 6 & 67 & 33 & 0 \\
\hline VE & 0 & 0 & 3 & 3 & 0 & 0 & 100 \\
\hline SO & 1 & 0 & 2 & 3 & 33 & 0 & 67 \\
\hline Totals: & 5 & 2 & 8 & 15 & $33 \%$ & $13 \%$ & $53 \%$ \\
\hline
\end{tabular}

Lab: AM American Radiation Services, Inc., Baton Rouge

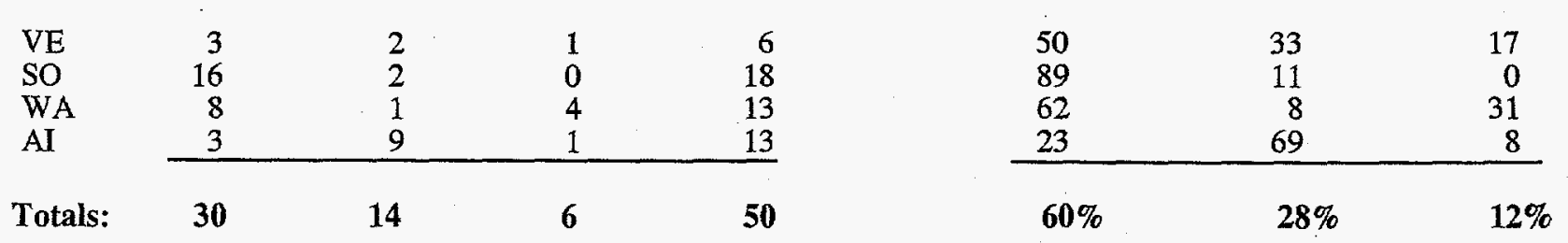




\section{QAP 49 Summary of Matrix Evaluations by Laboratory}

Evaluation Summary

\begin{tabular}{lcccr} 
Matrix & A & W & N & $\begin{array}{r}\text { Total } \\
\text { Analy }\end{array}$ \\
Lab: AN & Argonne National Laboratory & \\
\hline \multirow{2}{*}{ SO } & 6 & 0 & 2 & 8 \\
WA & 10 & 0 & 0 & 10 \\
AI & 10 & 0 & 0 & 10 \\
\cline { 2 - 5 } Totals: & 26 & 0 & 2 & 28
\end{tabular}

Evaluation Percentages \%A $\% \mathrm{~W}$ $\% \mathrm{~N}$

Lab: AP Aberdeen Proving Ground, Aberdeen, MD

AI

Totals:

\begin{tabular}{llll}
2 & 0 & 0 & 2 \\
\hline 2 & 0 & 0 & 2
\end{tabular}
2

2

\begin{tabular}{rlr}
75 & 0 & 25 \\
100 & 0 & 0 \\
100 & 0 & 0 \\
\hline $93 \%$ & $0 \%$ & $7 \%$
\end{tabular}

Lab: AT ATL International inc., Germantown, MD

\begin{tabular}{lrllllll} 
WA & 4 & 0 & 0 & 4 & 100 & 0 & 0 \\
VE & 4 & 0 & 0 & 4 & 100 & 0 & 0 \\
SO & 3 & 0 & 0 & 3 & 100 & 0 & 0 \\
\cline { 2 - 8 } & & & 0 & 0 & 11 & $100 \%$ & $0 \%$
\end{tabular}

Lab: AU ORISE EESD/ESSAP, Oak Ridge

\begin{tabular}{lrrrrrrr} 
VE & 6 & 0 & 0 & 6 & 100 & 0 & 0 \\
SO & 7 & 0 & 0 & 7 & 100 & 0 & 0 \\
WA & 11 & 1 & 0 & 12 & 92 & 8 & 0 \\
AT & 6 & 1 & 4 & 11 & 55 & 9 & 36 \\
\cline { 2 - 8 } Totals: & $\mathbf{3 0}$ & $\mathbf{2}$ & $\mathbf{4}$ & $\mathbf{3 6}$ & $\mathbf{8 3 \%}$ & $\mathbf{6 \%}$ & $\mathbf{1 1 \%}$
\end{tabular}

Lab: BA Bettis Atomic Power Lab, West Mifflin, PA

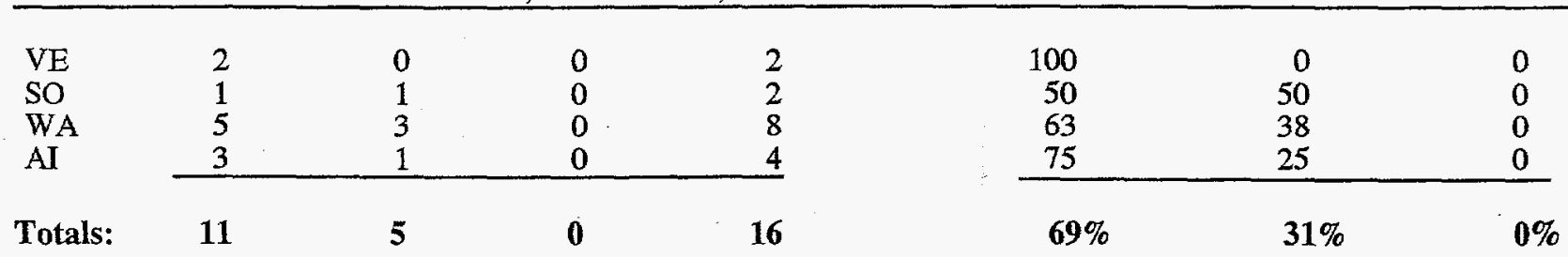

Lab: BC Babcock \& Wilcox MC \#42, Lynchburg, VA

\begin{tabular}{|c|c|c|c|c|c|c|c|}
\hline WA & 6 & 1 & 1 & 8 & 75 & 13 & 13 \\
\hline AI & 4 & 1 & 4 & 9 & 44 & 11 & 44 \\
\hline SO & 2 & 2 & 1 & 5 & 40 & 40 & 20 \\
\hline VE & 3 & 1 & 0 & 4 & 75 & 25 & 0 \\
\hline Totals: & 15 & 5 & 6 & 26 & 58 & $19 \%$ & 23 \\
\hline
\end{tabular}

Lab: BE RUST Geotech, Grand Junction, CO

\begin{tabular}{lrrrrrrr}
\hline & & & & & & & \\
SO & 8 & 0 & 0 & 8 & 100 & 0 & 0 \\
WA & 14 & 1 & 0 & 15 & 93 & 7 & 0 \\
\hline
\end{tabular}




\section{QAP 49 Summary of Matrix Evaluations by Laboratory}

\begin{tabular}{|c|c|c|c|c|c|c|c|}
\hline \multirow[b]{2}{*}{ Matrix } & \multicolumn{4}{|c|}{ Evaluation Summary } & \multicolumn{3}{|c|}{ Evaluation Percentages } \\
\hline & A & $\mathbf{W}$ & $\mathbf{N}$ & Analyses & $\% A$ & $\% W$ & $\% \mathbf{N}$ \\
\hline $\begin{array}{l}\text { AI } \\
\text { VE }\end{array}$ & $\begin{array}{r}12 \\
6 \\
\end{array}$ & $\begin{array}{l}1 \\
1 \\
\end{array}$ & $\begin{array}{l}0 \\
0 \\
\end{array}$ & $\begin{array}{r}13 \\
7 \\
\end{array}$ & $\begin{array}{l}92 \\
86 \\
\end{array}$ & $\begin{array}{r}8 \\
14 \\
\end{array}$ & $\begin{array}{l}0 \\
0 \\
\end{array}$ \\
\hline $\begin{array}{l}\text { Totals: } \\
\text { Lab: B }\end{array}$ & $\begin{array}{r}40 \\
\text { Barr } \\
\end{array}$ & 3 & $\mathbf{0}$ & 43 & $93 \%$ & $7 \%$ & $0 \%$ \\
\hline $\begin{array}{l}\text { VE } \\
\text { SO } \\
\text { WA } \\
\text { AI }\end{array}$ & $\begin{array}{r}6 \\
24 \\
15 \\
15 \\
\end{array}$ & $\begin{array}{l}2 \\
0 \\
3 \\
3 \\
\end{array}$ & $\begin{array}{l}0 \\
1 \\
2 \\
0 \\
\end{array}$ & $\begin{array}{r}8 \\
25 \\
20 \\
18 \\
\end{array}$ & $\begin{array}{l}75 \\
96 \\
75 \\
83 \\
\end{array}$ & $\begin{array}{r}25 \\
0 \\
15 \\
17 \\
\end{array}$ & $\begin{array}{r}0 \\
4 \\
10 \\
0 \\
\end{array}$ \\
\hline Totals: & 60 & 8 & 3 & 71 & $85 \%$ & $11 \%$ & $4 \%$ \\
\hline
\end{tabular}

Lab: BM Battelle Memorial Institute, Columbus, $\mathrm{OH}$

\begin{tabular}{llllllll} 
AI & 8 & 0 & 0 & 8 & 100 & 0 & 0 \\
WA & 8 & 0 & 0 & 8 & 100 & 0 & 0 \\
SO & 6 & 0 & 0 & 6 & 100 & 0 & 0 \\
VE & 5 & 0 & 0 & 5 & 100 & 0 & 0 \\
\cline { 2 - 8 } & & & 0 & 0 & 27 & $100 \%$ & $0 \%$
\end{tabular}

Lab: BN Brookhaven National Laboratory, Upton, NY

\begin{tabular}{|c|c|c|c|c|c|c|c|}
\hline $\begin{array}{l}\text { SO } \\
\text { WA } \\
\mathrm{AI} \\
\mathrm{VE}\end{array}$ & $\begin{array}{r}24 \\
3 \\
4 \\
9 \\
\end{array}$ & $\begin{array}{l}0 \\
7 \\
4 \\
0 \\
\end{array}$ & $\begin{array}{r}0 \\
10 \\
10 \\
0\end{array}$ & $\begin{array}{r}24 \\
20 \\
18 \\
9 \\
\end{array}$ & $\begin{array}{r}100 \\
15 \\
22 \\
100 \\
\end{array}$ & $\begin{array}{r}0 \\
35 \\
22 \\
0\end{array}$ & $\begin{array}{r}0 \\
50 \\
56 \\
0\end{array}$ \\
\hline Totals: & 40 & 11 & 20 & 71 & $56 \%$ & $15 \%$ & $28 \%$ \\
\hline
\end{tabular}

Lab: BP Battelle Pacific Northwest National Laboratory

\begin{tabular}{lrrrrrrr} 
WA & 12 & 2 & 0 & 14 & 86 & 14 & 0 \\
AI & 13 & 0 & 0 & 13 & 100 & 0 & 0 \\
SO & 12 & 1 & 0 & 13 & 92 & 8 & 0 \\
VE & 7 & 0 & 0 & 7 & 100 & 0 & 0 \\
\cline { 2 - 8 } & Totals: & 44 & 3 & 0 & 47 & $94 \%$ & $6 \%$
\end{tabular}

Lab: BQ Becquerel Laboratories Inc., Mississauga, Ontario, Canada

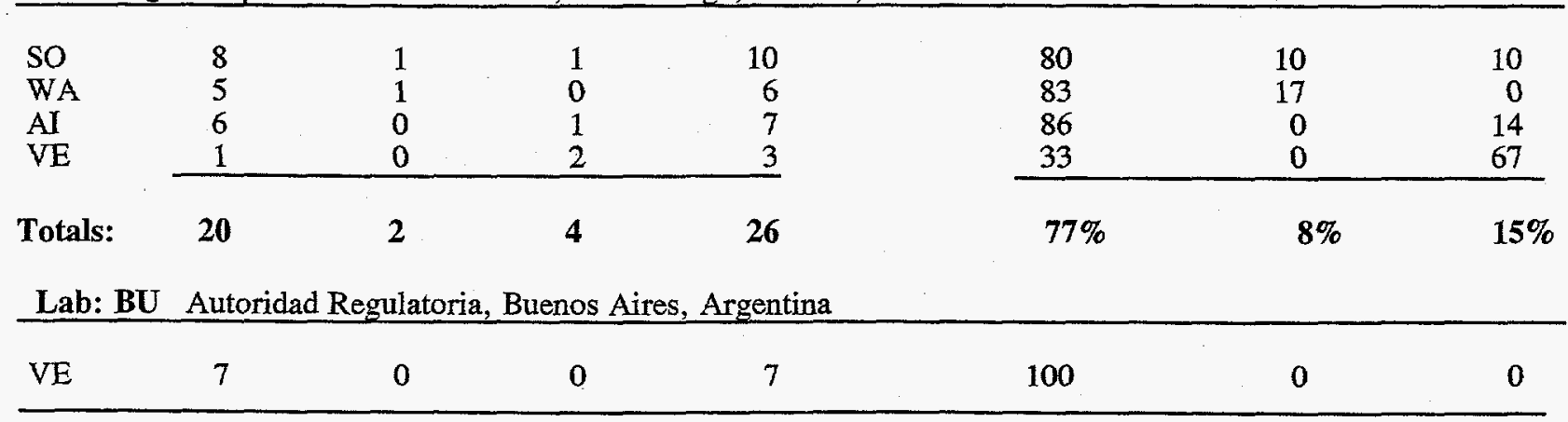




\section{QAP 49 Summary of Matrix Evaluations by Laboratory}

\section{Evaluation Summary}

\begin{tabular}{|c|c|c|c|c|}
\hline \multirow[b]{2}{*}{ Matrix } & \\
\hline & $\mathbf{A}$ & W & $\mathbf{N}$ & Analys \\
\hline AI & 11 & 2 & 0 & 13 \\
\hline SO & 8 & 1 & 0 & 9 \\
\hline WA & 10 & 2 & 0 & 12 \\
\hline Totals: & 36 & 5 & 0 & 41 \\
\hline
\end{tabular}

\section{Evaluation Percentages}

\begin{tabular}{lll} 
\%A & $\% \mathbf{W}$ & \%N \\
\hline 85 & 15 & 0 \\
89 & 11 & 0 \\
83 & 17 & 0 \\
\hline $88 \%$ & $12 \%$ & $0 \%$
\end{tabular}

Lab: BX B\&W Nuclear Envir. Services, Lynchburg, VA

\begin{tabular}{lrrrrrrrr}
\hline & & & & & 7 & 57 & 29 & 14 \\
VE & 4 & 2 & 1 & 7 & 63 & 38 & 0 \\
SO & 5 & 3 & 0 & 8 & 71 & 21 & 7 \\
WA & 10 & 3 & 1 & 14 & 5 & 9 & 36 \\
AI & 6 & 1 & 4 & 11 & $\mathbf{5 5}$ & $\mathbf{6 3}$ & $\mathbf{2 3 \%}$ & $\mathbf{1 5 \%}$
\end{tabular}

Lab: CA Atomic Energy Control Board, Ottawa, Canada

\begin{tabular}{|c|c|c|c|c|c|c|c|}
\hline $\begin{array}{l}\text { SO } \\
\text { WA } \\
\text { AI }\end{array}$ & $\begin{array}{l}1 \\
4 \\
5\end{array}$ & $\begin{array}{l}0 \\
1 \\
1\end{array}$ & $\begin{array}{l}0 \\
1 \\
0\end{array}$ & $\begin{array}{l}1 \\
6 \\
6 \\
\end{array}$ & $\begin{array}{r}100 \\
67 \\
83\end{array}$ & $\begin{array}{r}0 \\
17 \\
17\end{array}$ & $\begin{array}{r}0 \\
17 \\
0\end{array}$ \\
\hline Totals: & 10 & 2 & 1 & 13 & $77 \%$ & $15 \%$ & $8 \%$ \\
\hline
\end{tabular}

Lab: CB Radiation Protection Bureau, Ontario, Canada

\begin{tabular}{|c|c|c|c|c|c|c|c|}
\hline $\mathrm{Al}$ & 14 & 1 & 0 & 15 & 93 & 7 & 0 \\
\hline WA & 2 & 0 & 0 & 2 & 100 & 0 & 0 \\
\hline Totals: & 16 & 1 & 0 & 17 & $94 \%$ & $6 \%$ & $0 \%$ \\
\hline
\end{tabular}

Lab: CD Gentilly-2 Nuclear Power Plant, Quebec Canada

\begin{tabular}{lrrrrrrr} 
AI & 6 & 0 & 0 & 6 & 100 & 0 & 0 \\
WA & 4 & 1 & 0 & 5 & 80 & 20 & 0 \\
VE & 3 & 0 & 0 & 3 & 100 & 0 & 0 \\
SO & 8 & 0 & 0 & 8 & 100 & 0 & 0 \\
\cline { 2 - 8 } & & 1 & 0 & 22 & $95 \%$ & $\mathbf{5 \%}$ & $\mathbf{0 \%}$
\end{tabular}

Lab: CH California State Dept. Health Serv.,Sanitation \& Radiation Laboratory

\begin{tabular}{|c|c|c|c|c|c|c|c|}
\hline $\begin{array}{l}\text { VE } \\
\text { SO } \\
\text { WA } \\
\text { AI }\end{array}$ & $\begin{array}{r}6 \\
17 \\
13 \\
14 \\
\end{array}$ & $\begin{array}{l}1 \\
0 \\
2 \\
0 \\
\end{array}$ & $\begin{array}{l}0 \\
0 \\
0 \\
0 \\
\end{array}$ & $\begin{array}{r}7 \\
17 \\
15 \\
14 \\
\end{array}$ & $\begin{array}{r}86 \\
100 \\
87 \\
100 \\
\end{array}$ & $\begin{array}{r}14 \\
0 \\
13 \\
0 \\
\end{array}$ & $\begin{array}{l}0 \\
0 \\
0 \\
0 \\
\end{array}$ \\
\hline $\begin{array}{l}\text { Totals: } \\
\text { Lab: }\end{array}$ & $\begin{array}{r}50 \\
\text { Core } \\
\end{array}$ & 3 & 0 & 53 & $94 \%$ & $6 \%$ & $0 \%$ \\
\hline $\begin{array}{l}\text { VE } \\
\text { SO } \\
\text { WA }\end{array}$ & $\begin{array}{r}5 \\
12 \\
10\end{array}$ & $\begin{array}{l}2 \\
3 \\
2\end{array}$ & $\begin{array}{l}0 \\
0 \\
1\end{array}$ & $\begin{array}{r}7 \\
15 \\
13\end{array}$ & $\begin{array}{l}71 \\
80 \\
77\end{array}$ & $\begin{array}{l}29 \\
20 \\
15\end{array}$ & $\begin{array}{l}0 \\
0 \\
8\end{array}$ \\
\hline
\end{tabular}




\section{QAP 49 Summary of Matrix Evaluations by Laboratory}

\section{Evaluation Summary}

\begin{tabular}{lcccr} 
Matrix & A & W & N & Analys \\
AI & 10 & 0 & 1 & 11 \\
\cline { 2 - 5 } Totals: & 37 & 7 & 2 & 46
\end{tabular}

Totals: $\quad 37$

46

Total

Evaluation Percentages

Lab: CM Metropolitan Water Reclamation District of Greater Chicago, IL

\begin{tabular}{|c|c|c|c|c|c|c|c|}
\hline WA & 20 & 0 & 0 & 20 & 100 & 0 & 0 \\
\hline SO & 9 & 0 & 0 & 9 & 100 & 0 & 0 \\
\hline Totals: & 29 & 0 & 0 & 29 & $100 \%$ & $0 \%$ & \\
\hline
\end{tabular}

Lab: CN China Institute for Radiation Protection

\begin{tabular}{|c|c|c|c|c|c|c|c|}
\hline $\mathrm{AI}$ & 4 & 1 & 0 & 5 & 80 & 20 & 0 \\
\hline VE & 4 & 0 & 0 & 4 & 100 & 0 & 0 \\
\hline SO & 3 & 0 & 0 & 3 & 100 & 0 & 0 \\
\hline & 11 & 1 & 0 & 12 & $92 \%$ & $8 \%$ & $0 \%$ \\
\hline
\end{tabular}

Lab: CO Bedford Institute of Oceanography, Dartmouth. Nova Scotia, Canada

\begin{tabular}{|c|c|c|c|c|c|c|c|}
\hline VE & 2 & 0 & 0 & 2 & 100 & 0 & 0 \\
\hline SO & 1 & 0 & 2 & 3 & 33 & 0 & 67 \\
\hline AI & 4 & 0 & 0 & 4 & 100 & 0 & 0 \\
\hline Totals: & 7 & 0 & 2 & 9 & $78 \%$ & $0 \%$ & $22 \%$ \\
\hline
\end{tabular}

Lab: CR Laboratorio de Fisica Nuclear Aplicada, Costa Rica

\begin{tabular}{|c|c|c|c|c|c|c|c|}
\hline VE & 2 & 1 & 0 & 3 & 67 & 33 & 0 \\
\hline so & 8 & 1 & 0 & 9 & 89 & 11 & 0 \\
\hline $\mathrm{AI}$ & 0 & 0 & 4 & 4 & 0 & 0 & 100 \\
\hline Totals: & 10 & 2 & 4 & 16 & $63 \%$ & $13 \%$ & $25 \%$ \\
\hline
\end{tabular}

Lab: CS Boeing North American, Canoga Park, CA

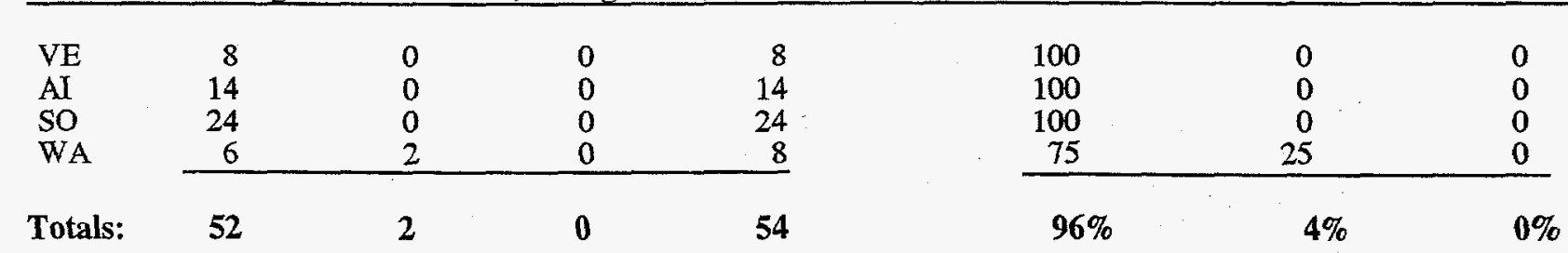

Lab: DH Duke Engineering Services Hanford

\begin{tabular}{|c|c|c|c|c|c|c|c|}
\hline SO & 3 & 1 & 0 & 4 & 75 & 25 & 0 \\
\hline WA & 4 & 1 & 0 & 5 & 80 & 20 & 0 \\
\hline AI & 1 & 1 & 0 & 2 & 50 & 50 & 0 \\
\hline Totals: & 8 & 3 & 0 & 11 & $73 \%$ & $27 \%$ & $0 \%$ \\
\hline
\end{tabular}




\section{QAP 49 Summary of Matrix Evaluations by Laboratory}

\section{Evaluation Summary}

Matrix A
Total Analyses

\section{Evaluation Percentages} \%A \%W $\% \mathrm{~N}$

Lab: EG LMITCO/NEL, Scoville

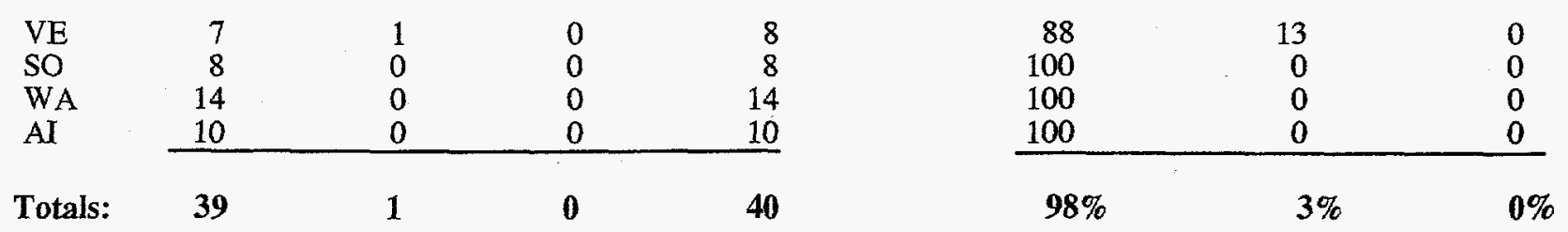

Lab: EM 3M, Empore Disks, St. Paul, MN

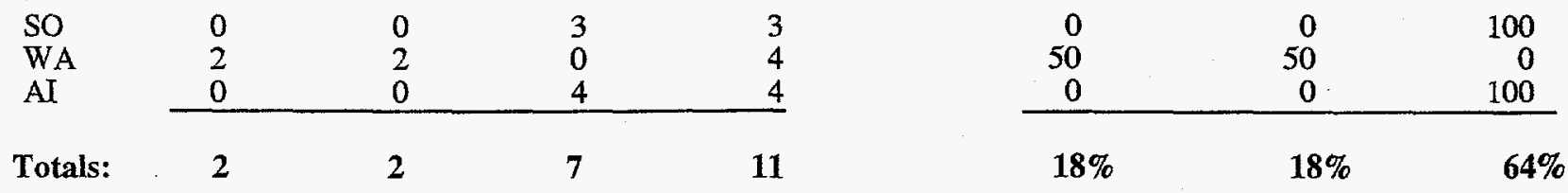

Lab: EP US EPA, Las Vegas

\begin{tabular}{lllll} 
WA & 8 & 0 & 0 & 8 \\
AI & 7 & 0 & 0 & 7 \\
SO & 2 & 0 & 0 & 2 \\
VE & 3 & 0 & 0 & 3 \\
\hline
\end{tabular}

Totals: $\quad 20$

$\mathbf{0}$

20

\begin{tabular}{lll}
100 & 0 & 0 \\
100 & 0 & 0 \\
100 & 0 & 0 \\
100 & 0 & 0 \\
\hline $\mathbf{1 0 0 \%}$ & $\mathbf{0 \%}$ & $\mathbf{0 \%}$
\end{tabular}

Lab: FG FGL Environmental, Santa Paula, CA

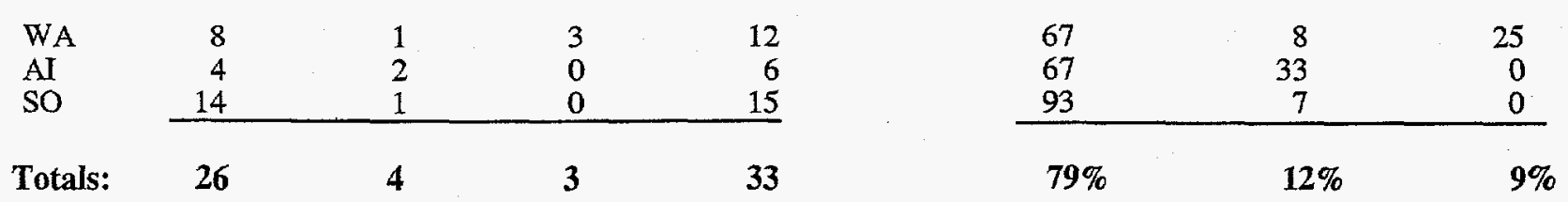

Lab: FJ The University of the South Pacific, Fiji Islands

\begin{tabular}{lllllllll} 
AI & 3 & 1 & 1 & 5 & & 60 & 20 & 20 \\
\cline { 2 - 8 } Totals: & 3 & 1 & 1 & 5 & & $60 \%$ & $20 \%$ & $20 \%$
\end{tabular}

Lab: FL Florida Dept of Health \& Rehab. Serv., Orlando

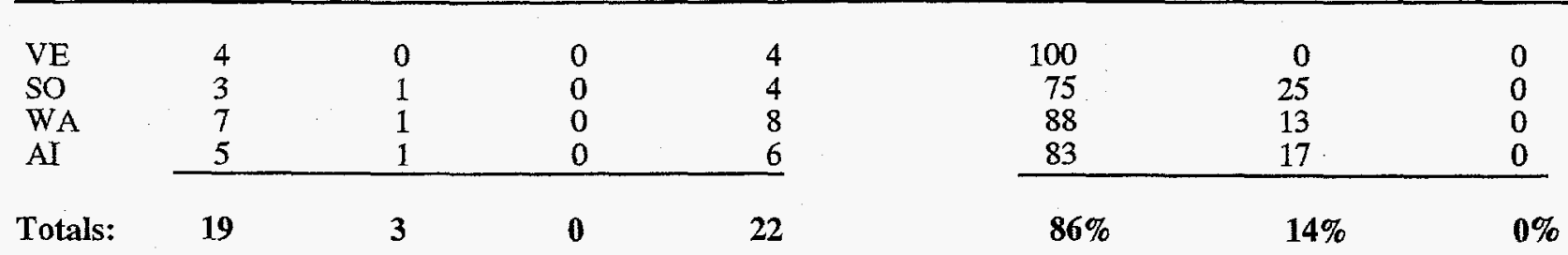

Lab: FM Florida Mobile Emergency Radiological Laboratory, Orlando

\begin{tabular}{|c|c|c|c|c|c|c|c|}
\hline AI & 5 & 0 & 0 & 5 & 100 & 0 & 0 \\
\hline WA & 4 & 0 & 0 & 4 & 100 & 0 & 0 \\
\hline
\end{tabular}




\section{QAP 49 Summary of Matrix Evaluations by Laboratory}

Evaluation Summary

\begin{tabular}{lccccccc} 
& \multicolumn{3}{c}{ Evaluation Summary } & \multicolumn{3}{c}{ Total } & \multicolumn{3}{c}{ Evaluation Percentages } \\
Matrix & A & W & N & Analyses & \%A & \%W & $\% \mathbf{N}$ \\
\cline { 2 - 8 } Totals: & 9 & 0 & 0 & 9 & $100 \%$ & $0 \%$ & $0 \%$
\end{tabular}

Lab: FN Fermi Lab, Batavia, IL

\begin{tabular}{|c|c|c|c|c|c|c|c|}
\hline WA & 4 & 0 & 0 & 4 & 100 & 0 & 0 \\
\hline $\mathrm{AI}$ & 4 & 0 & 0 & 4 & 100 & 0 & 0 \\
\hline VE & 3 & 0 & 0 & 3 & 100 & 0 & 0 \\
\hline So & 2 & 0 & 0 & 2 & 100 & 0 & 0 \\
\hline
\end{tabular}

Lab: FS Florida State University, Tallahassee

\begin{tabular}{|c|c|c|c|c|c|c|c|}
\hline SO & 8 & 0 & 1 & 9 & 89 & 0 & 11 \\
\hline Totals: & 8 & 0 & 1 & 9 & $89 \%$ & $0 \%$ & $11 \%$ \\
\hline
\end{tabular}

Lab: GA Lockheed Martin, Pikton, $\mathrm{OH}$

\begin{tabular}{|c|c|c|c|c|c|c|c|}
\hline VE & 4 & 2 & 0 & 6 & 67 & 33 & 0 \\
\hline SO & 12 & 1 & 0 & 13 & 92 & 8 & 0 \\
\hline WA & 5 & 2 & 3 & 10 & 50 & 20 & 30 \\
\hline $\mathrm{AI}$ & 9 & 2 & 0 & 11 & 82 & 18 & 0 \\
\hline Totals: & 30 & 7 & 3 & 40 & $75 \%$ & $18 \%$ & $8 \%$ \\
\hline
\end{tabular}

Lab: GC Georgia Power Company Environmental Lab

\begin{tabular}{|c|c|c|c|c|c|c|c|}
\hline VE & 3 & 0 & 0 & 3 & 100 & 0 & 0 \\
\hline so & 6 & 0 & 0 & 6 & 100 & 0 & 0 \\
\hline WA & 6 & 1 & 1 & 8 & 75 & 13 & 13 \\
\hline $\mathrm{AI}$ & 1 & 1 & 0 & 2 & 50 & 50 & 0 \\
\hline Totals: & 16 & 2 & 1 & 19 & $84 \%$ & $11 \%$ & $5 \%$ \\
\hline
\end{tabular}

Lab: GE General Engineering Labs, Charleston, SC

\begin{tabular}{|c|c|c|c|c|c|c|c|}
\hline $\begin{array}{l}\text { VE } \\
\text { AI } \\
\text { SO } \\
\text { WA }\end{array}$ & $\begin{array}{r}6 \\
10 \\
14 \\
14 \\
\end{array}$ & $\begin{array}{l}1 \\
2 \\
0 \\
0\end{array}$ & $\begin{array}{l}0 \\
1 \\
0 \\
1 \\
\end{array}$ & $\begin{array}{r}7 \\
13 \\
14 \\
15 \\
\end{array}$ & $\begin{array}{r}86 \\
77 \\
100 \\
93 \\
\end{array}$ & $\begin{array}{r}14 \\
15 \\
0 \\
0\end{array}$ & $\begin{array}{l}0 \\
8 \\
0 \\
7\end{array}$ \\
\hline $\begin{array}{l}\text { Totals: } \\
\text { Lab: }\end{array}$ & $\begin{array}{r}44 \\
\text { GPL }\end{array}$ & 3 & ourg & 49 & $90 \%$ & $6 \%$ & $4 \%$ \\
\hline $\begin{array}{l}\text { VE } \\
\text { SO } \\
\text { WA } \\
\text { AI }\end{array}$ & $\begin{array}{r}8 \\
2 \\
12 \\
9\end{array}$ & $\begin{array}{l}0 \\
0 \\
0 \\
0\end{array}$ & $\begin{array}{l}0 \\
1 \\
1 \\
4\end{array}$ & $\begin{array}{r}8 \\
3 \\
13 \\
13\end{array}$ & $\begin{array}{r}100 \\
67 \\
92 \\
69\end{array}$ & $\begin{array}{l}0 \\
0 \\
0 \\
0\end{array}$ & $\begin{array}{r}0 \\
33 \\
8 \\
31\end{array}$ \\
\hline
\end{tabular}




\section{QAP 49 Summary of Matrix Evaluations by Laboratory}

\begin{tabular}{|c|c|c|c|c|c|c|c|}
\hline \multirow[b]{2}{*}{ Matrix } & \multicolumn{3}{|c|}{ Evaluation Summary } & \multirow[b]{2}{*}{$\begin{array}{c}\text { Total } \\
\text { Analyses }\end{array}$} & \multicolumn{3}{|c|}{ Evaluation Percentages } \\
\hline & A & $\mathbf{W}$ & $\mathbf{N}$ & & $\% \mathbf{A}$ & $\% \mathbf{W}$ & $\% \mathrm{~N}$ \\
\hline Totals: & 31 & $\mathbf{0}$ & 6 & 37 & $84^{\circ}$ & $0 \%$ & \\
\hline
\end{tabular}

Lab: GS USGS/NWQL, Arvada, CO

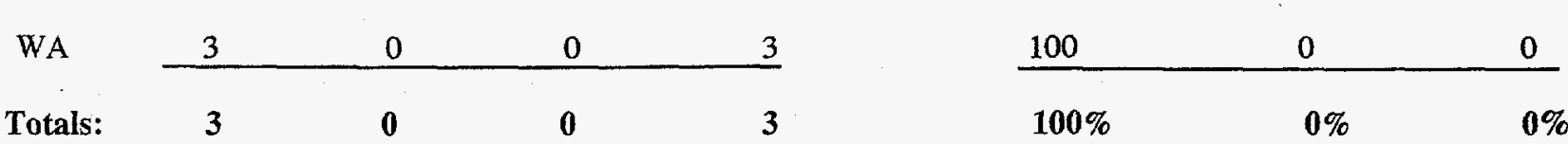

Lab: GT Georgia Institute of Technology

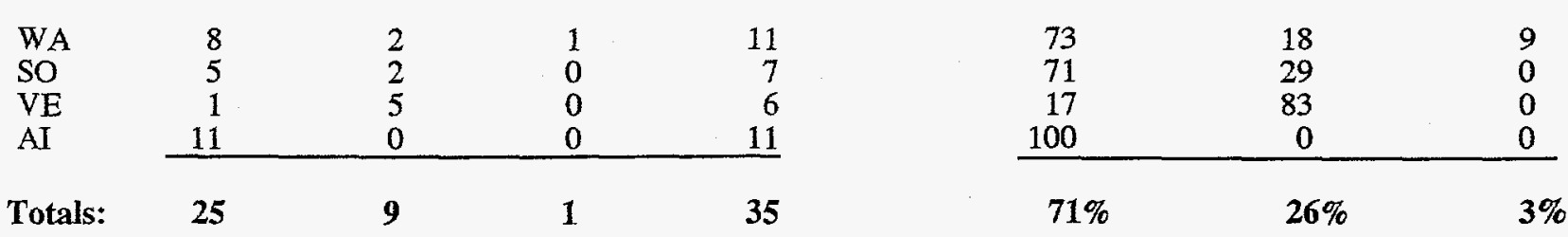

Lab: HC Lawrence Livermore Laboratory, California

\begin{tabular}{|c|c|c|c|c|c|c|}
\hline $\begin{array}{l}\text { WA } \\
\text { AI }\end{array}$ & $\begin{array}{l}3 \\
1 \\
\end{array}$ & $\begin{array}{l}0 \\
1\end{array}$ & $\begin{array}{l}0 \\
0 \\
\end{array}$ & $\begin{array}{l}3 \\
2 \\
\end{array}$ & $\begin{array}{r}100 \\
50\end{array}$ & $\begin{array}{r}0 \\
50\end{array}$ \\
\hline Totals: & 4 & 1 & 0 & 5 & $80 \%$ & $20 \%$ \\
\hline
\end{tabular}

Lab: HT Technical University, Budapest, Hungary

\begin{tabular}{|c|c|c|c|c|c|c|c|}
\hline $\begin{array}{l}\text { SO } \\
\text { WA }\end{array}$ & $\begin{array}{l}0 \\
0\end{array}$ & $\begin{array}{l}0 \\
1\end{array}$ & $\begin{array}{l}6 \\
6\end{array}$ & $\begin{array}{l}6 \\
7 \\
\end{array}$ & $\begin{array}{l}0 \\
0\end{array}$ & $\begin{array}{r}0 \\
14\end{array}$ & $\begin{array}{r}100 \\
86\end{array}$ \\
\hline & 0 & 1 & 12 & 13 & $0 \%$ & $8 \%$ & $92 \%$ \\
\hline
\end{tabular}

Lab: HU Water Resources Research Centre (VITUKI), Hungary

\begin{tabular}{|c|c|c|c|c|c|c|c|}
\hline VE & 3 & 1 & 0 & 4 & 75 & 25 & 0 \\
\hline SO & 8 & 0 & 2 & 10 & 80 & 0 & 20 \\
\hline AI & 3 & 1 & 1 & 5 & 60 & 20 & 20 \\
\hline Totals: & 14 & 2 & 3 & 19 & 74 & $11 \%$ & $16 \%$ \\
\hline
\end{tabular}

Lab: IA Bhabha Atomic Research Centre, India

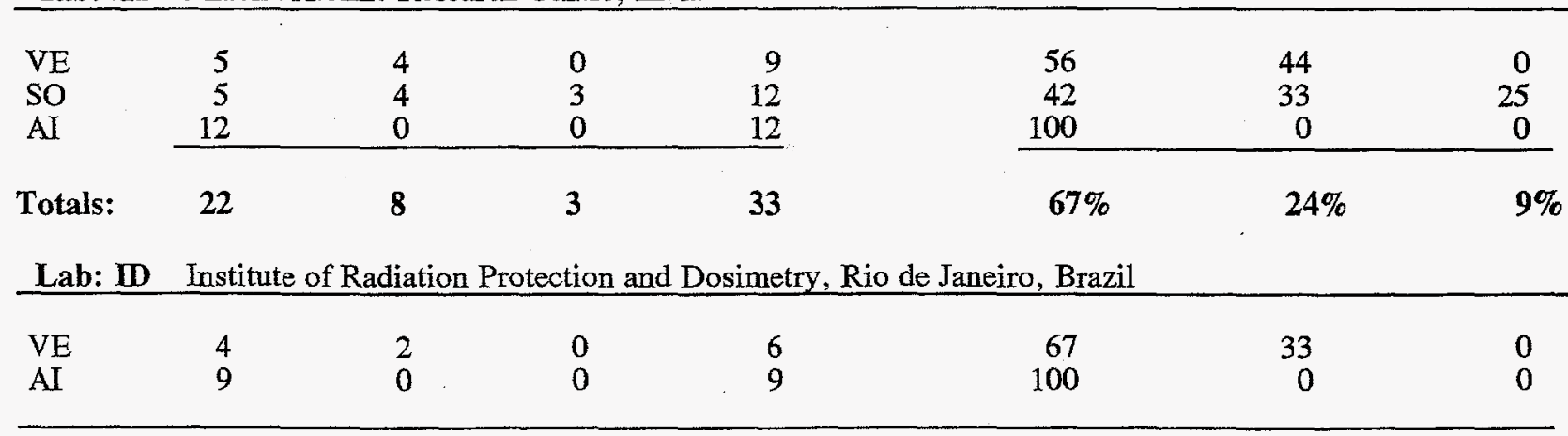




\section{QAP 49 Summary of Matrix Evaluations by Laboratory}

Evaluation Summary

Matrix A

SO

WA

12

6

W

W

$\mathbf{N}$

Totals: $\quad 31$

0
1

0
1

3

1
Total Analyses

12

8

35
Evaluation Percentages

\begin{tabular}{rrr} 
\%A & \%W & \%N \\
100 & 0 & 0 \\
75 & 13 & 13 \\
\hline
\end{tabular}

$\begin{array}{lll}89 \% & 9 \% & 3 \%\end{array}$

Lab: IE IEA, Inc., Morrisville, NC

\begin{tabular}{|c|c|c|c|c|c|c|c|}
\hline $\begin{array}{l}\text { VE } \\
\text { SO } \\
\text { WA }\end{array}$ & $\begin{array}{l}0 \\
7 \\
5\end{array}$ & $\begin{array}{l}1 \\
1 \\
1\end{array}$ & $\begin{array}{l}2 \\
1 \\
1\end{array}$ & $\begin{array}{l}3 \\
9 \\
7 \\
\end{array}$ & $\begin{array}{r}0 \\
78 \\
71\end{array}$ & $\begin{array}{l}33 \\
11 \\
14\end{array}$ & $\begin{array}{l}67 \\
11 \\
14\end{array}$ \\
\hline Totals: & 12 & 3 & 4 & 19 & $63 \%$ & $16 \%$ & $21 \%$ \\
\hline
\end{tabular}

Lab: IL ISU Environmental Monitoring Program, Pocatello, ID

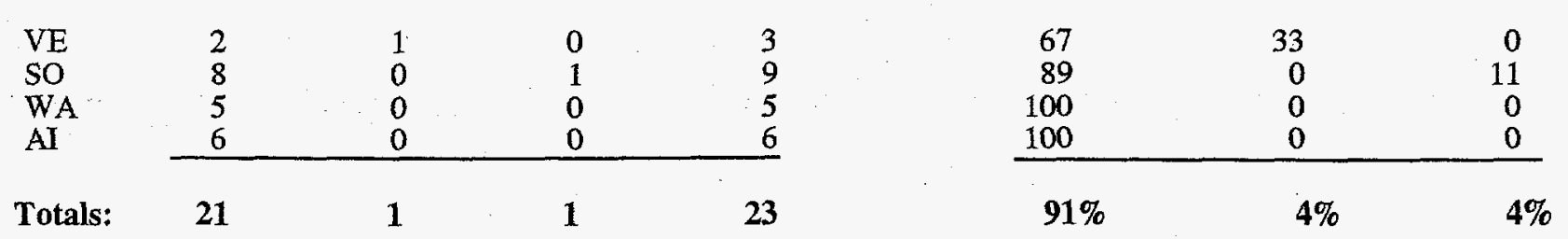

Lab: IN Lockheed Martin Idaho Technical Corp., Analytical Laboratory

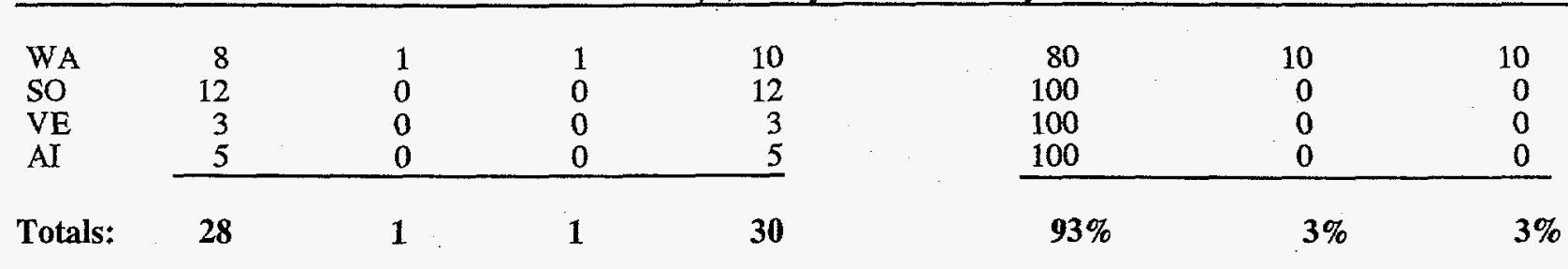

Lab: IS Quanterra- St. Louis

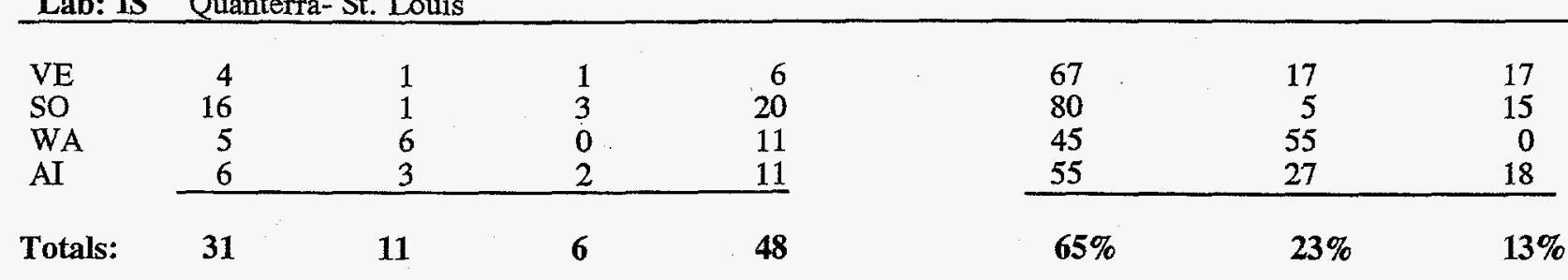

Lab: IT Quanterra-Richland Laboratory

\begin{tabular}{|c|c|c|c|c|c|c|c|}
\hline VE & 6 & 0 & 0 & 6 & 100 & 0 & 0 \\
\hline So & 18 & 2 & 0 & 20 & 90 & 10 & 0 \\
\hline WA & 13 & 1 & 0 & 14 & 93 & 7 & 0 \\
\hline $\mathrm{AI}$ & 13 & 1 & 0 & 14 & 93 & 7 & 0 \\
\hline Totals: & 50 & 4 & 0 & 54 & $93 \%$ & $7 \%$ & $0 \%$ \\
\hline
\end{tabular}

Lab: JE Jacobs Engineering, Oak Ridge, TN

\begin{tabular}{|c|c|c|c|c|c|c|c|}
\hline AI & 2 & 0 & 0 & 2 & 100 & 0 & 0 \\
\hline SO & 6 & 0 & 0 & 6 & 100 & 0 & 0 \\
\hline
\end{tabular}




\section{QAP 49 Summary of Matrix Evaluations by Laboratory}

\section{Evaluation Summary}

\begin{tabular}{llllr} 
Matrix & A & W & N & Analyse \\
WA & 2 & 0 & 3 & 5 \\
\cline { 2 - 5 } & & 0 & 3
\end{tabular}

Totals: $\quad 10$

13

Total

nalyses

5

3

Evaluation Percentages

\%A $\quad \% W \quad \% N$

\begin{tabular}{lll}
40 & 0 & 60 \\
\hline $77 \%$ & $0 \%$ & $23 \%$
\end{tabular}

Lab: JL Jefferson Lab, Newport News, VA

\begin{tabular}{lllll} 
WA & 3 & 0 & 1 & 4 \\
AI & 0 & 0 & 2 & 2 \\
\cline { 2 - 5 } Totals: & 3 & 0 & 3 & 6
\end{tabular}

Lab: KA Knolls Atomic Power Lab, Schenectady

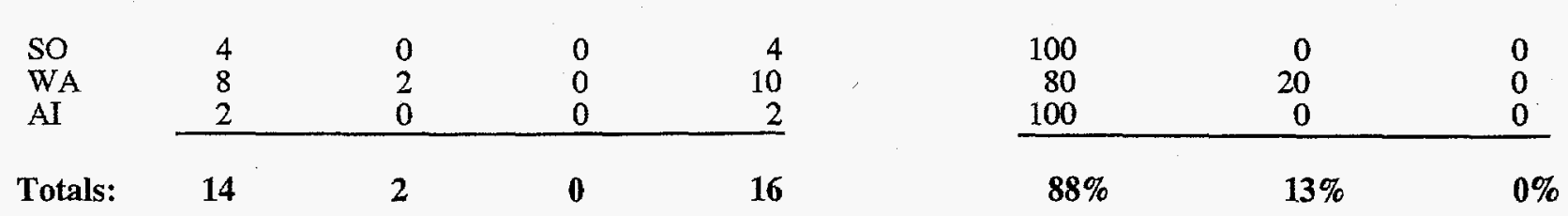

Lab: KR Korea Atomic Energy Research Institute

\begin{tabular}{|c|c|c|c|c|c|c|c|}
\hline VE & 3 & 1 & 0 & 4 & 75 & 25 & 0 \\
\hline SO & 4 & 0 & 0 & 4 & 100 & 0 & 0 \\
\hline AI & 6 & 1 & 0 & 7 & 86 & 14 & 0 \\
\hline & 13 & 2 & 0 & 15 & $87^{\circ}$ & $13 \%$ & $0 \%$ \\
\hline
\end{tabular}

Lab: LA Los Alamos National Laboratory, NM

\begin{tabular}{lrrrrrrr}
\hline WA & 21 & 9 & 1 & 31 & 68 & 29 & 3 \\
VE & 8 & 4 & 0 & 12 & 67 & 33 & 0 \\
AI & 21 & 0 & 0 & 21 & 100 & 0 & 0 \\
SO & 8 & 1 & 3 & 12 & 67 & 8 & 25 \\
\cline { 2 - 8 } Totals: & $\mathbf{5 8}$ & $\mathbf{1 4}$ & $\mathbf{4}$ & $\mathbf{7 6}$ & $\mathbf{7 6 \%}$ & $\mathbf{1 8 \%}$ & $\mathbf{5 \%}$
\end{tabular}

Lab: LB Lawrence Berkeley Lab UCB

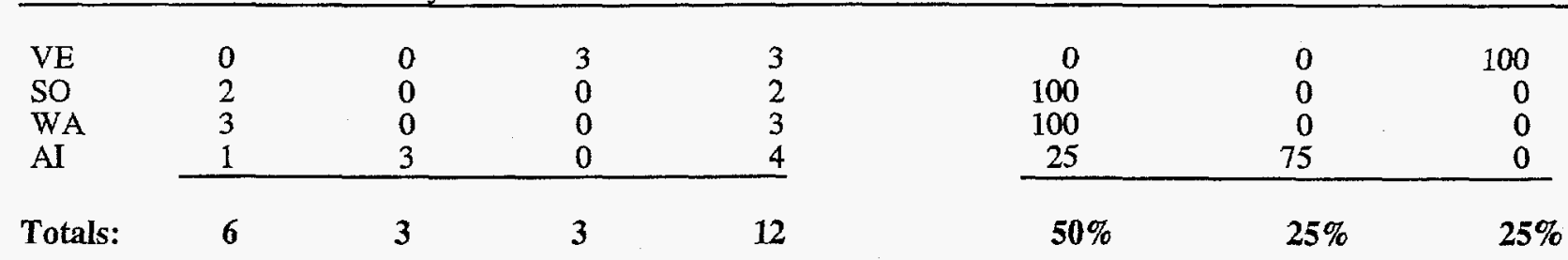

Lab: LE Lyle Environmental Management, Columbus, Ohio

\begin{tabular}{lllllllll} 
WA & 1 & 0 & 1 & 2 & & 50 & 0 & 50 \\
\cline { 2 - 9 } Totals: & 1 & 0 & 1 & 2 & & $50 \%$ & $0 \%$ & $50 \%$
\end{tabular}




\section{QAP 49 Summary of Matrix Evaluations by Laboratory}

Evaluation Summary

Matrix A

$\mathbf{W}$

$\mathbf{N}$

Total Analyses

Lab: LL LLNL Chemistry and Material Science/Environmental

\begin{tabular}{lrrrr} 
VE & 5 & 0 & 1 & 6 \\
SO & 6 & 0 & 2 & 8 \\
WA & 9 & 2 & 0 & 11 \\
AI & 11 & 0 & 0 & 11 \\
\cline { 2 - 5 } Totals: & 31 & 2 & 3 & 36
\end{tabular}

Totals:

2

3

36

1

Lab: LN Los Alamos National Lab, ES\&H

\begin{tabular}{lllll} 
WA & 1 & 1 & 2 & 4 \\
AI & 3 & 3 & 1 & 7 \\
\cline { 2 - 4 } Totals: & 4 & 4 & 3 & 11
\end{tabular}

Totals:

4

3

11

Lab: LV UNLV, Dept of Health Physics

\begin{tabular}{lrrrrrrr}
\hline & & & & & 82 & 18 & 0 \\
SO & 9 & 2 & 0 & 11 & 78 & 0 & 22 \\
WA & 7 & 0 & 2 & 9 & 75 & 25 & 0 \\
VE & 3 & 1 & 0 & 4 & 44 & 22 & 33 \\
\cline { 2 - 7 } & 4 & 2 & 3 & 9 & $70 \%$ & $15 \%$ & $15 \%$
\end{tabular}

Lab: LW Lawrence Livermore National Lab, Waste

\begin{tabular}{lllllllll} 
SO & 4 & 0 & 0 & 4 & & 100 & 0 & 0 \\
WA & 3 & 0 & 0 & 3 & & 100 & 0 & 0 \\
\cline { 2 - 9 } Totals: & 7 & $\mathbf{0}$ & $\mathbf{0}$ & $\mathbf{7}$ & & $\mathbf{1 0 0 \%}$ & $\mathbf{0 \%}$ & $\mathbf{0 \%}$
\end{tabular}

Lab: MA ORNL Health Sciences Research Div.

\begin{tabular}{|c|c|c|c|c|c|c|c|}
\hline VE & 3 & 0 & 1 & 4 & 75 & 0 & 25 \\
\hline So & 3 & 0 & 0 & 3 & 100 & 0 & 0 \\
\hline AI & 0 & 0 & 4 & 4 & 0 & 0 & 100 \\
\hline Totals: & 6 & 0 & 5 & 11 & $55 \%$ & $0 \%$ & $45 \%$ \\
\hline
\end{tabular}

Lab: ME Radiation Control Program, Jamaica Plain, MA

\begin{tabular}{|c|c|c|c|c|c|c|c|}
\hline $\begin{array}{l}\text { VE } \\
\text { SO } \\
\text { WA } \\
\text { AI }\end{array}$ & $\begin{array}{l}3 \\
4 \\
4 \\
2 \\
\end{array}$ & $\begin{array}{l}1 \\
2 \\
1 \\
5 \\
\end{array}$ & $\begin{array}{l}0 \\
1 \\
0 \\
0\end{array}$ & $\begin{array}{l}4 \\
7 \\
5 \\
7 \\
\end{array}$ & $\begin{array}{l}75 \\
57 \\
80 \\
29\end{array}$ & $\begin{array}{l}25 \\
29 \\
20 \\
71\end{array}$ & $\begin{array}{r}0 \\
14 \\
0 \\
0\end{array}$ \\
\hline $\begin{array}{l}\text { Totals: } \\
\text { Lab: } \\
\end{array}$ & $\begin{array}{l}13 \\
\text { Main }\end{array}$ & 9 & 1 & 23 & $\mathbf{5 7 \%}$ & $39 \%$ & $4 \%$ \\
\hline $\begin{array}{l}\text { SO } \\
\text { WA }\end{array}$ & $\begin{array}{l}9 \\
8\end{array}$ & $\begin{array}{l}0 \\
0\end{array}$ & $\begin{array}{l}0 \\
0\end{array}$ & $\begin{array}{l}9 \\
8\end{array}$ & $\begin{array}{l}100 \\
100\end{array}$ & $\begin{array}{l}0 \\
0\end{array}$ & $\begin{array}{l}0 \\
0\end{array}$ \\
\hline
\end{tabular}




\section{QAP 49 Summary of Matrix Evaluations by Laboratory}

\begin{tabular}{|c|c|c|c|c|c|c|c|}
\hline \multirow[b]{2}{*}{ Matrix } & \multicolumn{3}{|c|}{ Evaluation Summary } & \multirow{2}{*}{$\begin{array}{c}\text { Total } \\
\text { Analyses }\end{array}$} & \multicolumn{3}{|c|}{ Evaluation Percentages } \\
\hline & $\mathbf{A}$ & $\mathbf{W}$ & $\mathbf{N}$ & & $\% A$ & $\% \mathrm{~W}$ & $\% \mathrm{~N}$ \\
\hline $\begin{array}{l}\text { VE } \\
\text { AI }\end{array}$ & $\begin{array}{l}3 \\
2\end{array}$ & $\begin{array}{l}1 \\
4\end{array}$ & $\begin{array}{l}0 \\
0\end{array}$ & $\begin{array}{l}4 \\
6\end{array}$ & $\begin{array}{l}75 \\
33\end{array}$ & $\begin{array}{l}25 \\
67\end{array}$ & $\begin{array}{l}0 \\
0\end{array}$ \\
\hline Totals: & 22 & 5 & 0 & 27 & 81 & 19 & \\
\hline
\end{tabular}

Lab: ML Babcock \& Wilcox of Ohio, Mound, Miamisburg, OH

\begin{tabular}{|c|c|c|c|c|c|c|}
\hline $\begin{array}{l}\text { VE } \\
\text { SO } \\
\text { WA }\end{array}$ & $\begin{array}{l}1 \\
3 \\
5 \\
\end{array}$ & $\begin{array}{l}0 \\
0 \\
0 \\
\end{array}$ & $\begin{array}{l}0 \\
0 \\
0 \\
\end{array}$ & $\begin{array}{l}1 \\
3 \\
5 \\
\end{array}$ & $\begin{array}{l}100 \\
100 \\
100 \\
\end{array}$ & $\begin{array}{l}0 \\
0 \\
0 \\
\end{array}$ \\
\hline Totals: & 9 & 0 & 0 & 9 & $100 \%$ & $0 \%$ \\
\hline
\end{tabular}

Lab: NA US EPA NAREL, Montgomery, AL

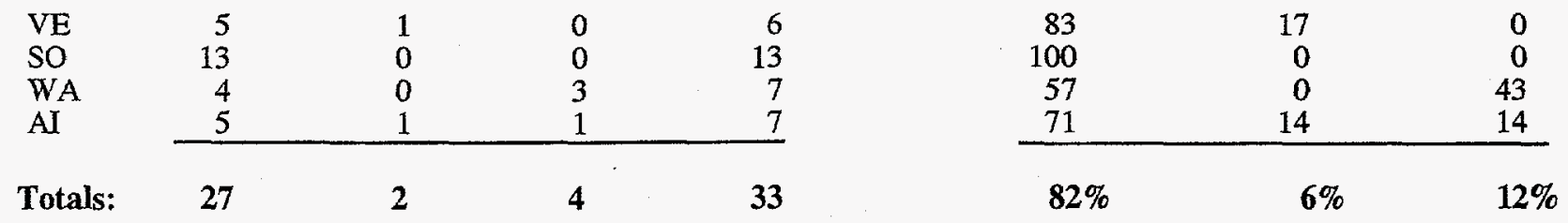

Lab: ND Dept. of Environmental Health and Safety, NC State University

\begin{tabular}{|c|c|c|c|c|c|c|c|}
\hline $\begin{array}{l}\text { WA } \\
\text { AI }\end{array}$ & $\begin{array}{l}3 \\
5\end{array}$ & $\begin{array}{l}0 \\
1\end{array}$ & $\begin{array}{l}0 \\
0\end{array}$ & $\begin{array}{l}3 \\
6 \\
\end{array}$ & $\begin{array}{r}100 \\
83\end{array}$ & $\begin{array}{r}0 \\
17\end{array}$ & $\begin{array}{l}0 \\
0\end{array}$ \\
\hline Totals: & 8 & 1 & 0 & 9 & 89 & $11 \%$ & $0 \%$ \\
\hline
\end{tabular}

Lab: NL Fluor Daniel Fernald, Inc., Ohio

\begin{tabular}{|c|c|c|c|c|c|c|c|}
\hline WA & 8 & 0 & 0 & 8 & 100 & 0 & 0 \\
\hline AI & 8 & 1 & 0 & 9 & 89 & 11 & 0 \\
\hline SO & 14 & 0 & 0 & 14 & 100 & 0 & 0 \\
\hline Totals: & 30 & 1 & 0 & 31 & 97 & 3 & $0 \%$ \\
\hline
\end{tabular}

Lab: NM Environmental Evaluation Group, Carlsbad, NM

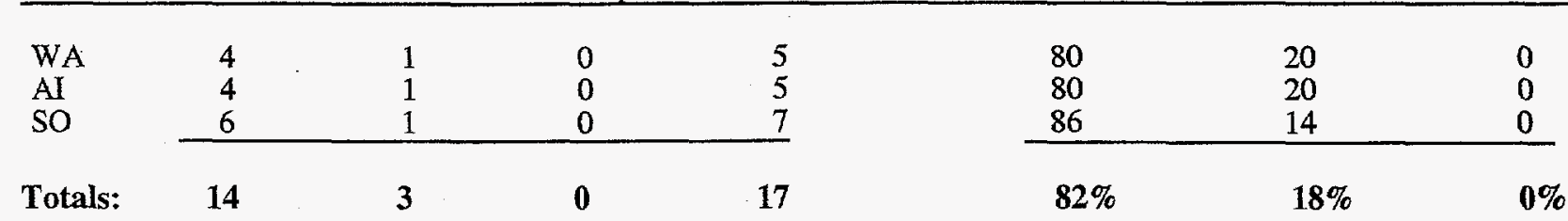

Lab: NP JAF Environmental Laboratory, New York Power Authority

\begin{tabular}{llllrrrr}
\hline VE & 2 & 1 & 0 & 3 & 67 & 33 & 0 \\
SO & 0 & 1 & 1 & 2 & 0 & 50 & 50 \\
WA & 4 & 0 & 1 & 5 & 80 & 0 & 20 \\
AI & 5 & 0 & 0 & 5 & 100 & 0 & 0
\end{tabular}




\section{QAP 49 Summary of Matrix Evaluations by Laboratory}

\section{Evaluation Summary}

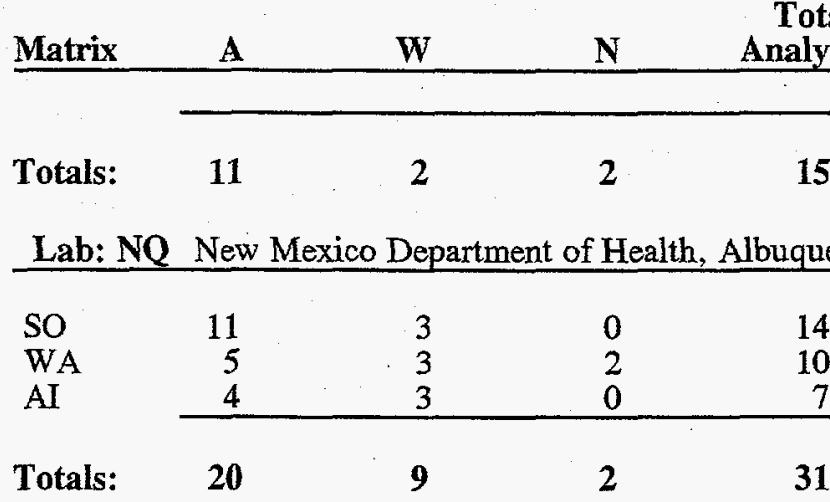

Evaluation Percentages

Total

$\% \mathbf{A}$

$\mathbf{7 3 \%}$
$\% \mathrm{~W}$

$13 \%$
15 $\% \mathrm{~N}$

\begin{tabular}{rrr}
79 & 21 & 0 \\
50 & 30 & 20 \\
57 & 43 & 0 \\
\hline
\end{tabular}

$65 \% \quad 29 \% \quad 6 \%$

Lab: NR Naval Reactors Facility Chemistry, Scoville, ID

\begin{tabular}{lllll} 
VE & 3 & 0 & 0 & 3 \\
SO & 2 & 0 & 0 & 2 \\
\cline { 2 - 5 } Totals: & 5 & 0 & 0 & 5
\end{tabular}

Lab: NS State Lab of Public Health, North Carolina

\begin{tabular}{lrlllllll}
\hline AI & 5 & 0 & 1 & 6 & 83 & 0 & 17 \\
WA & 5 & 1 & 1 & 7 & & 71 & 14 & 14 \\
\cline { 2 - 9 } & Totals: & $\mathbf{1 0}$ & 1 & 2 & 13 & $77 \%$ & $8 \%$ & $15 \%$
\end{tabular}

Lab: NZ National Radiation Laboratory, New Zealand

\begin{tabular}{|c|c|c|c|c|c|c|c|}
\hline VE & 6 & 0 & 0 & 6 & 100 & 0 & 0 \\
\hline SO & 10 & 0 & 0 & 10 & 100 & 0 & 0 \\
\hline WA & 14 & 2 & 4 & 20 & 70 & 10 & 20 \\
\hline AI & 14 & 6 & 2 & 22 & 64 & 27 & 9 \\
\hline tals: & 44 & 8 & 6 & 58 & $76 \%$ & $14 \%$ & $10 \%$ \\
\hline
\end{tabular}

Lab: OB OBG Laboratories, East Syracuse, NY

\begin{tabular}{lrrrrrrr}
\hline & & & & & 0 & 0 & 100 \\
VE & 0 & 0 & 3 & 3 & 80 & 0 & 20 \\
SO & 8 & 0 & 2 & 10 & 5 & 0 & 20 \\
WA & 4 & 0 & 1 & 5 & 17 & 17 & 67 \\
AI & 1 & 1 & 4 & 6 & $\mathbf{5 4 \%}$ & $\mathbf{4 \%}$ & $\mathbf{4 2 \%}$
\end{tabular}

Lab: OC Radiation Protection Service Laboratory, Ontario, Canada

\begin{tabular}{|c|c|c|c|c|c|c|c|}
\hline $\begin{array}{l}\text { WA } \\
\text { AI } \\
\text { SO } \\
\text { VE }\end{array}$ & $\begin{array}{r}15 \\
7 \\
28 \\
5 \\
\end{array}$ & $\begin{array}{r}0 \\
11 \\
5 \\
7 \\
\end{array}$ & $\begin{array}{l}4 \\
0 \\
0 \\
0 \\
\end{array}$ & $\begin{array}{l}19 \\
18 \\
33 \\
12 \\
\end{array}$ & $\begin{array}{l}79 \\
39 \\
85 \\
42 \\
\end{array}$ & $\begin{array}{r}0 \\
61 \\
15 \\
58 \\
\end{array}$ & $\begin{array}{r}21 \\
0 \\
0 \\
0 \\
\end{array}$ \\
\hline Totals: & 55 & 23 & 4 & 82 & $67 \%$ & $28 \%$ & $5 \%$ \\
\hline
\end{tabular}




\section{QAP 49 Summary of Matrix Evaluations by Laboratory}

\section{Evaluation Summary}

Matrix A W $\mathbf{N}$

Total Analyses

20

26

46

\section{Evaluation Percentages} \%A $\% \mathrm{~W}$ $\% \mathrm{~N}$

Lab: OD ORNL, Radiobioassay Lab

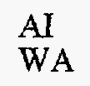

Totals:

\begin{tabular}{llll}
20 & 0 & 0 & 20 \\
24 & 2 & 0 & 26 \\
\hline 44 & 2 & 0 & 46
\end{tabular}

\begin{tabular}{lll}
100 & 0 & 0 \\
92 & 8 & 0 \\
\hline $96 \%$ & $4 \%$ & $0 \%$
\end{tabular}

Lab: OL ORNL Environmental Sciences Div.

\begin{tabular}{lrlllrrr} 
VE & 3 & 0 & 0 & 3 & 100 & 0 & 0 \\
SO & 3 & 0 & 0 & 3 & 100 & 0 & 0 \\
WA & 3 & 0 & 0 & 3 & 100 & 0 & 0 \\
AI & 3 & 1 & 0 & 4 & 75 & 25 & 0 \\
\cline { 2 - 8 } & & 1 & 0 & $\mathbf{1 3}$ & $\mathbf{9 2 \%}$ & $\mathbf{8 \%}$ & $\mathbf{0 \%}$
\end{tabular}

Lab: OT ORNL Radioactive Material Analysis Lab

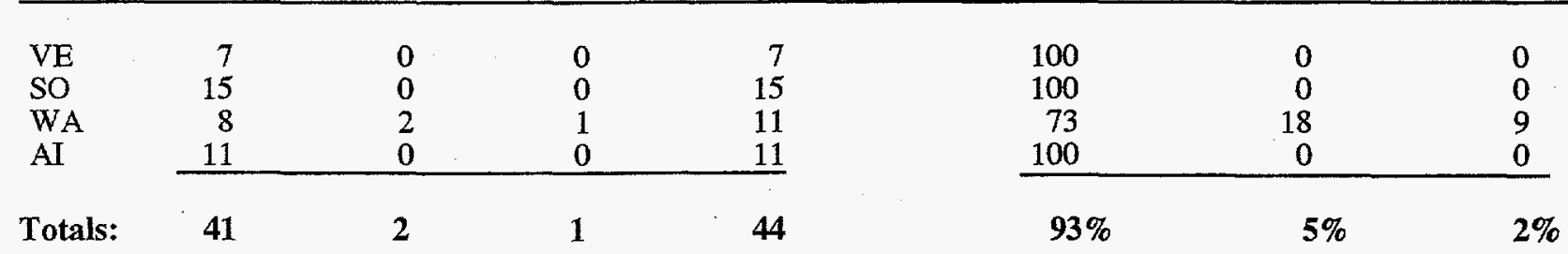

Lab: OU Outreach Laboratory, Broken Arrow, OK

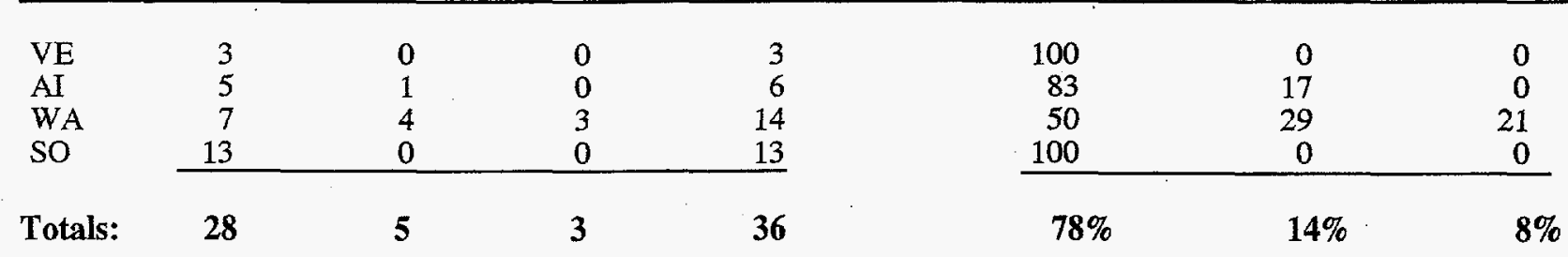

Lab: PA Mason \& Hanger-Silas Mason Co., Inc., Battelle Pantex, Amarillo, TX

AI

Totals:

2

2

0

$$
0
$$

0

0

$\mathbf{0}$

Lab: PK Pakistan Institute of Nuclear Science \& Technology

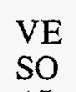

AI

Totals:

2
1
4

7
$0 \quad 0$

$0 \quad 0$

0

o

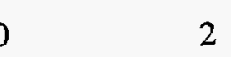

1
7
100

$100 \%$
0

0\%
0

$0 \%$

Lab: PO Institute of Oceanology PAN, Poland

\begin{tabular}{|c|c|c|c|c|c|c|c|}
\hline VE & 3 & 1 & 0 & 4 & 75 & 25 & 0 \\
\hline SO & 4 & 0 & 0 & 4 & 100 & 0 & 0 \\
\hline $\mathrm{AI}$ & 2 & 3 & 0 & 5 & 40 & 60 & 0 \\
\hline
\end{tabular}




\section{QAP 49 Summary of Matrix Evaluations by Laboratory}

\begin{tabular}{|c|c|c|c|c|c|c|c|}
\hline \multirow[b]{2}{*}{ Matrix } & \multicolumn{3}{|c|}{ Evaluation Summary } & & \multicolumn{3}{|c|}{ Evaluation Percentages } \\
\hline & $\mathbf{A}$ & $\mathbf{W}$ & $\mathbf{N}$ & $\begin{array}{c}\text { Total } \\
\text { Analyses } \\
\end{array}$ & $\% \mathbf{A}$ & $\% \mathrm{~W}$ & $\% \mathrm{~N}$ \\
\hline
\end{tabular}

Lab: PR Princeton Plasma Physics Lab

\begin{tabular}{lllllllll} 
WA & 5 & 0 & 0 & 5 & 100 & 0 & 0 \\
AI & 5 & 0 & 0 & 5 & & 100 & 0 & 0 \\
\cline { 2 - 8 } & & 10 & 0 & 0 & 10 & $100 \%$ & $0 \%$
\end{tabular}

Lab: RA V. G. Khlopin Radium Institute, St. Petersburg, Russia

\begin{tabular}{|c|c|c|c|c|c|c|c|}
\hline $\begin{array}{l}\text { SO } \\
\text { AI } \\
\text { VE }\end{array}$ & $\begin{array}{r}22 \\
9 \\
9 \\
\end{array}$ & $\begin{array}{l}3 \\
0 \\
0 \\
\end{array}$ & $\begin{array}{l}0 \\
2 \\
0\end{array}$ & $\begin{array}{r}25 \\
11 \\
9 \\
\end{array}$ & $\begin{array}{r}88 \\
82 \\
100\end{array}$ & $\begin{array}{r}12 \\
0 \\
0\end{array}$ & $\begin{array}{r}0 \\
18 \\
0\end{array}$ \\
\hline Totals: & 40 & 3 & 2 & 45 & $89 \%$ & $7 \%$ & \\
\hline
\end{tabular}

Lab: RC US NRC Region I Laboratory, PA

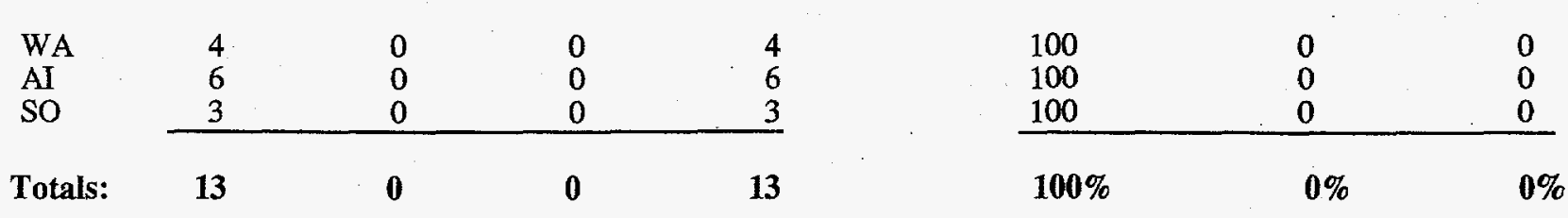

Lab: RE Bechtel Nevada, Mercury, NV

\begin{tabular}{|c|c|c|c|c|c|c|c|}
\hline VE & 6 & 1 & 0 & 7 & 86 & 14 & 0 \\
\hline SO & 9 & 0 & 0 & 9 & 100 & 0 & 0 \\
\hline WA & 12 & 0 & 0 & 12 & 100 & 0 & 0 \\
\hline AI & 9 & 1 & 0 & 10 & 90 & 10 & 0 \\
\hline Totals: & 36 & 2 & 0 & 38 & 95 & & \\
\hline
\end{tabular}

Lab: RG Thermo Nutech Rocky Flats Plant, Golden

\begin{tabular}{lllllllll}
\hline WA & 2 & 0 & 0 & 2 & & 100 & 0 & 0 \\
\cline { 2 - 8 } Totals: & 2 & 0 & 0 & 2 & & $100 \%$ & $0 \%$ & $0 \%$
\end{tabular}

Lab: RI Waste Management Services of Hanford, Inc., 222S Lab

\begin{tabular}{|c|c|c|c|c|c|c|c|}
\hline VE & 1 & 0 & 2 & 3 & 33 & 0 & 67 \\
\hline $\mathrm{AI}$ & 6 & 1 & 1 & 8 & 75 & 13 & 13 \\
\hline WA & 4 & 2 & 1 & 7 & 57 & 29 & 14 \\
\hline so & 1 & 0 & 0 & 1 & 100 & 0 & 0 \\
\hline Totals: & 12 & 3 & 4 & 19 & $63 \%$ & $16 \%$ & $21 \%$ \\
\hline
\end{tabular}




\section{QAP 49 Summary of Matrix Evaluations by Laboratory}

\begin{tabular}{|c|c|c|c|c|c|c|c|}
\hline \multirow[b]{2}{*}{ Matrix } & \multicolumn{3}{|c|}{ Evaluation Summary } & & \multicolumn{3}{|c|}{ Evaluation Percentages } \\
\hline & A & W & $\mathbf{N}$ & $\begin{array}{l}\text { Total } \\
\text { Analyses }\end{array}$ & $\% \mathrm{~A}$ & $\% W$ & $\% \mathbf{N}$ \\
\hline
\end{tabular}

Lab: RK Rock Island Arsenal, Illinois

\begin{tabular}{lllllllll} 
AI & 1 & 1 & 0 & 2 & & 50 & 50 & 0 \\
\cline { 2 - 8 } Totals: & 1 & 1 & 0 & 2 & & $50 \%$ & $50 \%$ & $0 \%$
\end{tabular}

Lab: SA Sandia Labs Radioactive Sample Diag. Prog., NM

\begin{tabular}{|c|c|c|c|c|c|c|c|}
\hline $\begin{array}{l}\text { SO } \\
\text { WA } \\
\text { AI }\end{array}$ & $\begin{array}{l}2 \\
2 \\
7\end{array}$ & $\begin{array}{l}0 \\
1 \\
0\end{array}$ & $\begin{array}{l}0 \\
0 \\
0\end{array}$ & $\begin{array}{l}2 \\
3 \\
7\end{array}$ & $\begin{array}{r}100 \\
67 \\
100\end{array}$ & $\begin{array}{r}0 \\
33 \\
0\end{array}$ & $\begin{array}{l}0 \\
0 \\
0\end{array}$ \\
\hline Totals: & 11 & 1 & 0 & 12 & $92 \%$ & $8 \%$ & $0 \%$ \\
\hline
\end{tabular}

Lab: SB SC Dept. of Health and Environment Control Radiological Lab

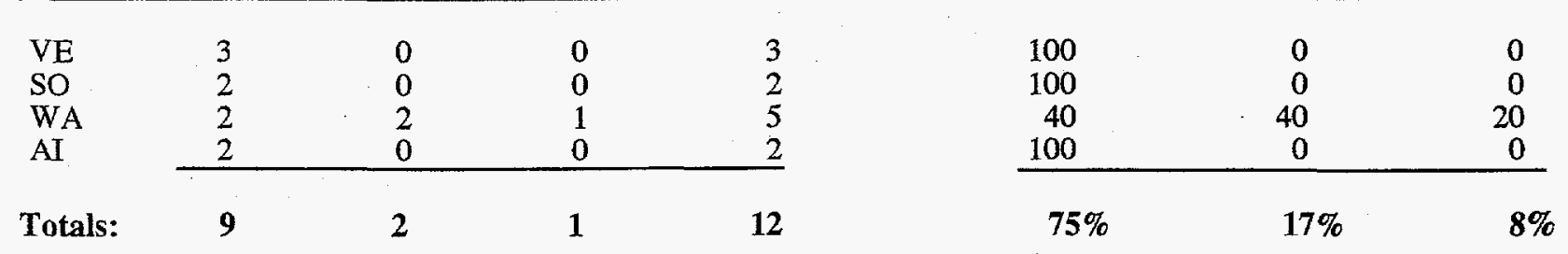

Lab: SK Savannah River Plant

\begin{tabular}{|c|c|c|c|c|c|c|c|}
\hline $\mathrm{AI}$ & 4 & 1 & 0 & 5 & 80 & 20 & 0 \\
\hline SO & 12 & 0 & 0 & 12 & 100 & 0 & 0 \\
\hline WA & 4 & 0 & 2 & 6 & 67 & 0 & 33 \\
\hline Totals: & 20 & 1 & 2 & 23 & $87 \%$ & $4 \%$ & $9 \%$ \\
\hline
\end{tabular}

Lab: SL Stanford Linear Accelerator Center

\begin{tabular}{|c|c|c|c|c|c|c|c|}
\hline WA & 3 & 0 & 0 & 3 & 100 & 0 & 0 \\
\hline Totals: & 3 & 0 & 0 & 3 & $100 \%$ & $0 \%$ & $0 \%$ \\
\hline
\end{tabular}

Lab: SN Sanford Cohen Associates, Inc., Montgomery, AL

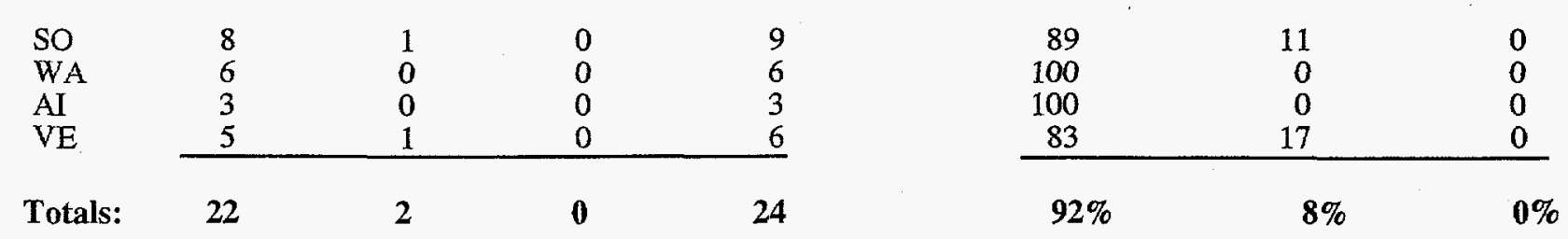

Lab: SR Savannah River Environmental Laboratory

$\begin{array}{lrrrrrrr}\text { VE } & 6 & 1 & 0 & 7 & 86 & 14 & 0 \\ \text { SO } & 8 & 1 & 0 & 9 & 89 & 11 & 0 \\ \text { WA } & 7 & 5 & 0 & 12 & 58 & 42 & 0 \\ \text { AI } & 11 & 0 & 1 & 12 & 92 & 0 & 8\end{array}$




\section{QAP 49 Summary of Matrix Evaluations by Laboratory}

Evaluation Summary

\begin{tabular}{lcccr} 
Matrix & A & W & N & $\begin{array}{r}\text { Total } \\
\text { Analys }\end{array}$ \\
\cline { 2 - 5 } Totals: & $\mathbf{3 2}$ & $\mathbf{7}$ & $\mathbf{1}$ & $\mathbf{4 0}$ \\
Lab: ST & SC DHEC, & Aiken, & South Carolina & \\
\hline WA & 1 & 0 & 0 & 1 \\
Totals: & $\mathbf{1}$ & $\mathbf{0}$ & $\mathbf{0}$ & $\mathbf{1}$
\end{tabular}

Evaluation Percentages $\%$ A

$\begin{array}{lll}80 \% & 18 \% & 3 \%\end{array}$

Lab: SW Southwest Research Institute, San Antonio, TX

\begin{tabular}{lrrrrrrr} 
AI & 5 & 2 & 4 & 11 & 45 & 18 & 36 \\
WA & 4 & 3 & 4 & 11 & 36 & 27 & 36 \\
SO & 12 & 2 & 2 & 16 & 75 & 13 & 13 \\
VE & 0 & 5 & 2 & 7 & 0 & 71 & 29 \\
\cline { 2 - 8 } Totals: & $\mathbf{2 1}$ & $\mathbf{1 2}$ & $\mathbf{1 2}$ & $\mathbf{4 5}$ & $\mathbf{4 7 \%}$ & $\mathbf{2 7 \%}$ & $\mathbf{2 7 \%}$
\end{tabular}

Lab: TE Teledyne Isotopes Midwest Lab, Northbrook, $\mathrm{IL}$

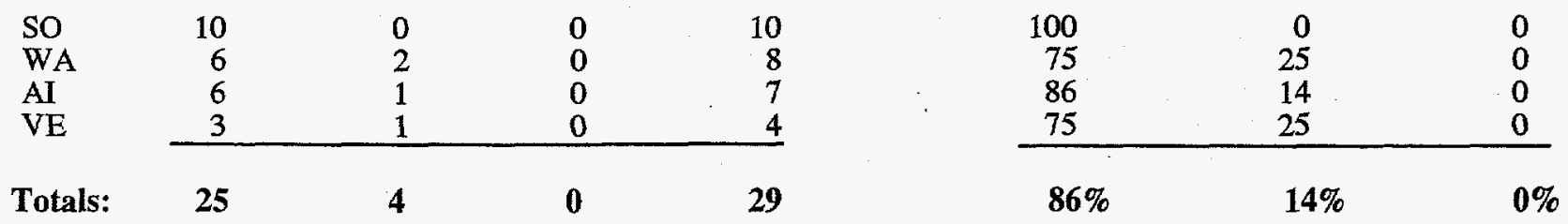

Lab: TI Teledyne Brown Engineering Environmental Services, Westwood, NJ

\begin{tabular}{|c|c|c|c|c|c|c|c|}
\hline WA & 12 & 0 & 1 & 13 & 92 & 0 & 8 \\
\hline $\mathrm{AI}$ & 9 & 2 & 0 & 11 & 82 & 18 & 0 \\
\hline SO & 5 & 1 & 1 & 7 & 71 & 14 & 14 \\
\hline VE & 6 & 1 & 0 & 7 & 86 & 14 & 0 \\
\hline Totals: & 32 & 4 & 2 & 38 & $84^{\circ}$ & $11 \%$ & \\
\hline
\end{tabular}

Lab: TM Thermo NUtech Albuquerque Lab, NM

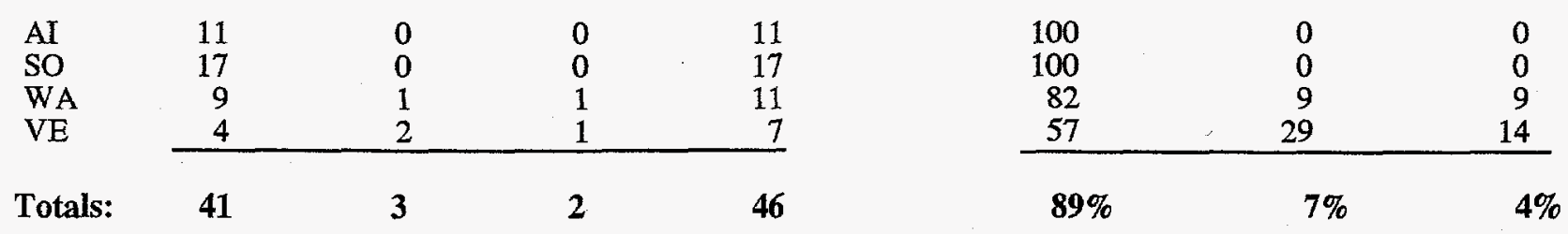

Lab: TN Thermo NUTech, Richmond, CA

$\begin{array}{lrrrrrrr}\text { AI } & 11 & 1 & 1 & 13 & 85 & 8 & 8 \\ \text { WA } & 11 & 1 & 3 & 15 & 73 & 7 & 20 \\ \text { VE } & 4 & 0 & 3 & 7 & 57 & 0 & 43 \\ \text { SO } & 8 & 0 & 2 & 10 & 80 & 0 & 20\end{array}$




\section{QAP 49 Summary of Matrix Evaluations by Laboratory}

\begin{tabular}{|c|c|c|c|c|c|c|c|}
\hline \multirow[b]{2}{*}{ Matrix } & \multicolumn{3}{|c|}{ Evaluation Summary } & & \multicolumn{3}{|c|}{ Evaluation Percentages } \\
\hline & $\mathbf{A}$ & W & $\mathbf{N}$ & Analyses & $\% \mathrm{~A}$ & $\% W$ & $\% \mathrm{~N}$ \\
\hline
\end{tabular}

Lab: TO Thermo NUtech Oak Ridge Laboratory

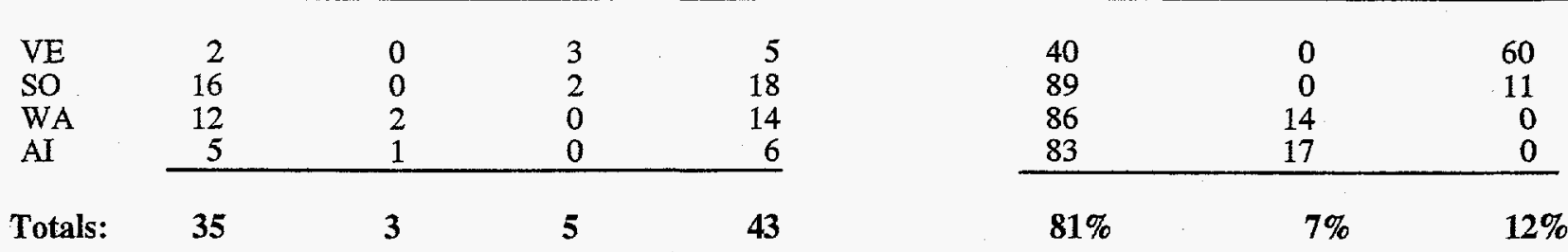

Lab: TP Taiwan Power Company, Taipei, Taiwan

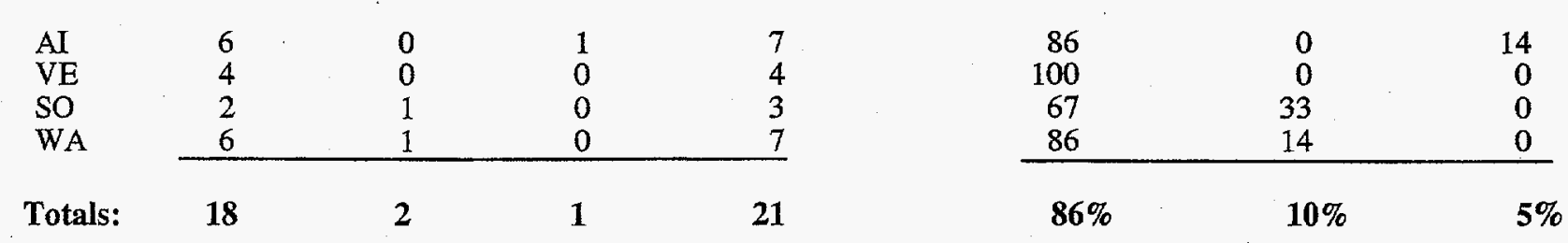

Lab: TT Tracer Technologies International, Inc., Cleveland

\begin{tabular}{llllllll}
\hline SO & 0 & 1 & 1 & 2 & 0 & 50 & 50 \\
WA & 3 & 1 & 0 & 4 & 75 & 25 & 0 \\
\cline { 2 - 9 } Totals: & 3 & 2 & 1 & 6 & $50 \%$ & $\mathbf{3 3 \%}$ & $\mathbf{1 7 \%}$
\end{tabular}

Lab: TW Taiwan Radiation Monitoring Center

\begin{tabular}{|c|c|c|c|c|c|c|c|}
\hline VE & 3 & 0 & 0 & 3 & 100 & 0 & 0 \\
\hline SO & 7 & 0 & 0 & 7 & 100 & 0 & 0 \\
\hline WA & 4 & 1 & 0 & 5 & 80 & 20 & 0 \\
\hline AI & 5 & 1 & 0 & 6 & 83 & 17 & 0 \\
\hline & 19 & 2 & 0 & 21 & $90 \%$ & $10 \%$ & \\
\hline
\end{tabular}

Lab: TX Texas Dept. of Health/Laboratories, Austin

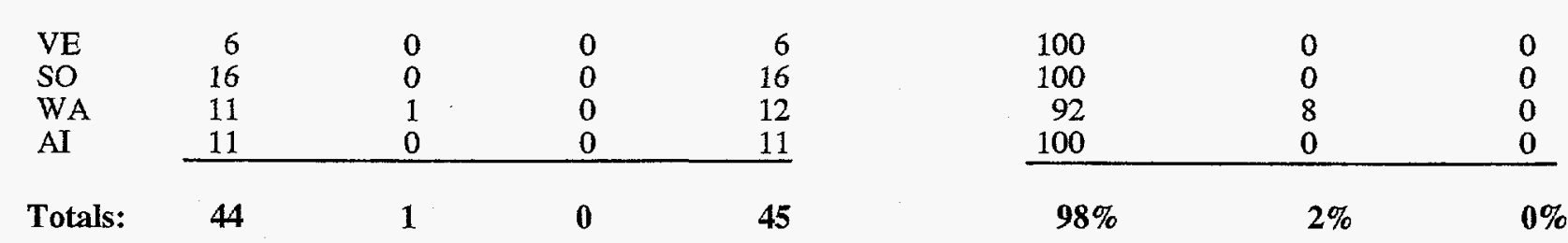

Lab: TY Scientific Production Association, Russia

\begin{tabular}{llllllll} 
So & 3 & 0 & 1 & 4 & 75 & 0 & 25 \\
\cline { 2 - 8 } Totals: & 3 & 0 & 1 & 4 & $75 \%$ & $0 \%$ & $\mathbf{2 5 \%}$
\end{tabular}




\section{QAP 49 Summary of Matrix Evaluations by Laboratory}

Evaluation Summary

Matrix A

W

N

Total Analyses

Lab: UC Lockheed Martin, Paducah, KY

\begin{tabular}{lllll} 
WA & 5 & 0 & 0 & 5 \\
AI & 3 & 0 & 3 & 6 \\
SO & 1 & 1 & 0 & 2 \\
VE & 0 & 0 & 3 & 3 \\
\cline { 2 - 4 } Totals: & 9 & 1 & 6 & 16
\end{tabular}

Lab: UP Lockheed Martin Energy Systerns, Y-12 Plant, Oak Ridge

\begin{tabular}{lrrrr} 
WA & 11 & 1 & 0 & $\mathbf{1 2}$ \\
AI & 10 & 1 & 1 & 12 \\
SO & 6 & 0 & 0 & 6 \\
\cline { 2 - 6 } Totals: & $\mathbf{2 7}$ & $\mathbf{2}$ & $\mathbf{1}$ & $\mathbf{3 0}$
\end{tabular}

Totals:

27

30

12

12

Lab: UY Lockheed Martin Energy Systems, Y-12 Plant, Oak Ridge

\begin{tabular}{lrrrrrrr} 
SO & 13 & 1 & 0 & 14 & 93 & 7 & 0 \\
WA & 11 & 0 & 0 & 11 & 100 & 0 & 0 \\
AI & 9 & 2 & 0 & 11 & 82 & 18 & 0 \\
\cline { 2 - 9 } Totals: & $\mathbf{3 3}$ & $\mathbf{3}$ & $\mathbf{0}$ & $\mathbf{3 6}$ & $\mathbf{9 2 \%}$ & $\mathbf{8 \%}$ & $\mathbf{0 \%}$
\end{tabular}

Lab: WA Environmental Radiation Lab, Off. of Public Health Labs. Seattle

\begin{tabular}{lrrrrrrr} 
AI & 9 & 4 & 0 & 13 & 69 & 31 & 0 \\
VE & 4 & 3 & 0 & 7 & 57 & 43 & 0 \\
SO & 17 & 1 & 0 & 18 & 94 & 6 & 0 \\
WA & 12 & 2 & 0 & 14 & 86 & 14 & 0 \\
\cline { 2 - 8 } Totals: & $\mathbf{4 2}$ & $\mathbf{1 0}$ & $\mathbf{0}$ & $\mathbf{5 2}$ & $\mathbf{8 1 \%}$ & $\mathbf{1 9 \%}$ & $\mathbf{0 \%}$
\end{tabular}

Lab: WC Waste Management Federal Services of Hanford

\begin{tabular}{lrrrrrrr}
\hline & & & & & 12 & 0 & 0 \\
AI & 12 & 0 & 0 & 12 & 75 & 17 & 8 \\
WA & 9 & 2 & 1 & 12 & 71 & 29 & 0 \\
VE & 5 & 2 & 0 & 7 & 80 & 20 & 0 \\
SO & 4 & 1 & 0 & 5 & $83 \%$ & $14 \%$ & $3 \%$ \\
\cline { 2 - 8 } Totals: & $\mathbf{3 0}$ & 5 & 1 & 36 &
\end{tabular}

Lab: WE Westinghouse Electric Corp., Madison, PA

$\begin{array}{lrrrrrrr} & & & & & 75 & 25 & 0 \\ \text { VE } & 3 & 1 & 0 & 4 & 100 & 0 & 0 \\ \text { SO } & 12 & 0 & 0 & 12 & 50 & 0 & 50 \\ \text { WA } & 2 & 0 & 2 & 4 & 20 & 60 & 20 \\ \text { AI } & 1 & 3 & 1 & 5 & 72 \% & 16 \% & 12 \% \\ \text { Tratals: } & 18 & 4 & 3 & 25 & & 72 \%\end{array}$




\section{QAP 49 Summary of Matrix Evaluations by Laboratory}

\section{Evaluation Summary}

Matrix
Total

N Analyses
Evaluation Percentages

$\% \mathrm{~A} \quad \% \mathrm{~W} \quad \% \mathrm{~N}$

Lab: WI WIPP Site, Westinghouse Electric Corp.

\begin{tabular}{|c|c|c|c|c|c|c|c|}
\hline WA & 6 & 3 & 0 & 9 & 67 & 33 & 0 \\
\hline Totals: & 6 & 3 & 0 & 9 & $67 \%$ & $33 \%$ & \\
\hline
\end{tabular}

Lab: WS Weldon Springs Site, St Charles, MO

\begin{tabular}{|c|c|c|c|c|c|c|c|}
\hline $\begin{array}{l}\text { SO } \\
\mathrm{AI}\end{array}$ & $\begin{array}{l}3 \\
1 \\
\end{array}$ & $\begin{array}{l}1 \\
0 \\
\end{array}$ & $\begin{array}{l}0 \\
0 \\
\end{array}$ & $\begin{array}{l}4 \\
1 \\
\end{array}$ & $\begin{array}{r}75 \\
100 \\
\end{array}$ & $\begin{array}{r}25 \\
0 \\
\end{array}$ & $\begin{array}{l}0 \\
0 \\
\end{array}$ \\
\hline Totals: & 4 & 1 & 0 & 5 & $80 \%$ & $20 \%$ & \\
\hline
\end{tabular}

Lab: WV West Valley Nuclear Services Co, Inc, NY

\begin{tabular}{|c|c|c|c|c|c|c|c|}
\hline $\begin{array}{l}\text { WA } \\
\text { AI }\end{array}$ & $\begin{array}{l}6 \\
2 \\
\end{array}$ & $\begin{array}{l}1 \\
0\end{array}$ & $\begin{array}{l}0 \\
0\end{array}$ & $\begin{array}{l}7 \\
2 \\
\end{array}$ & $\begin{array}{r}86 \\
100 \\
\end{array}$ & $\begin{array}{r}14 \\
0\end{array}$ & $\begin{array}{l}0 \\
0\end{array}$ \\
\hline & 8 & 1 & 0 & 9 & $89 \%$ & $11 \%$ & $0 \%$ \\
\hline
\end{tabular}

Lab: YA Duke Engineering Environmental Lab, Westboro, MA

\begin{tabular}{lrrrrrrr} 
VE & 6 & 1 & 0 & 7 & 86 & 14 & 0 \\
AI & 9 & 1 & 0 & 10 & 90 & 10 & 0 \\
SO & 7 & 1 & 0 & 8 & 88 & 13 & 8 \\
WA & 11 & 1 & 1 & 13 & 85 & 8 & 8 \\
\cline { 2 - 8 } Totals: & $\mathbf{3 3}$ & $\mathbf{4}$ & $\mathbf{1}$ & $\mathbf{3 8}$ & $\mathbf{8 7 \%}$ & $\mathbf{1 1 \%}$ & $\mathbf{3 \%}$
\end{tabular}

Lab: YP US Army Proving Ground, Yuma, AZ

\begin{tabular}{llllllll} 
SO & 1 & 0 & 0 & 1 & 100 & 0 & 0 \\
WA & 1 & 0 & 0 & 1 & 100 & 0 & 0 \\
AI & 1 & 0 & 0 & 1 & 100 & 0 & 0 \\
\cline { 2 - 9 } Totals: & $\mathbf{3}$ & $\mathbf{0}$ & $\mathbf{0}$ & $\mathbf{3}$ & $\mathbf{1 0 0 \%}$ & $\mathbf{0 \%}$ & $\mathbf{0 \%}$
\end{tabular}

Lab: YU Institute of Occupational and Radiological Health, Yugoslovia

\begin{tabular}{lrllllll} 
AI & 3 & 1 & 0 & 4 & 75 & 25 & 0 \\
VE & 3 & 0 & 0 & 3 & 100 & 0 & 0 \\
SO & 2 & 0 & 0 & 2 & 100 & 0 & 0 \\
WA & 3 & 0 & 0 & 3 & 100 & 0 & 0 \\
\cline { 2 - 8 } Totals: & $\mathbf{1 1}$ & $\mathbf{1}$ & $\mathbf{0}$ & $\mathbf{1 2}$ & $\mathbf{9 2 \%}$ & $\mathbf{8 \%}$ & $\mathbf{0 \%}$
\end{tabular}




\section{QAP 49 Summary of Laboratory Evaluations by Matrix}

\section{Matrix: AI Air Filter}

\section{Evaluation Summary}

Labcode

\begin{tabular}{|c|c|c|c|c|}
\hline AF & 6 & 0 & 3 & 9 \\
\hline AG & 9 & 0 & 3 & 12 \\
\hline $\mathrm{AI}$ & 0 & 2 & 0 & 2 \\
\hline AL & 4 & 2 & 0 & 6 \\
\hline $\mathrm{AM}$ & 3 & 9 & 1 & 13 \\
\hline AN & 10 & 0 & 0 & 10 \\
\hline AP & 2 & 0 & 0 & 2 \\
\hline $\mathrm{AU}$ & 6 & 1 & 4 & 11 \\
\hline $\mathrm{BA}$ & 3 & 1 & 0 & 4 \\
\hline$B C$ & 4 & 1 & 4 & \\
\hline $\mathrm{BE}$ & 12 & $\overline{1}$ & 0 & 13 \\
\hline BL & 15 & 3 & 0 & 18 \\
\hline $\mathrm{BM}$ & 8 & 0 & 0 & 8 \\
\hline BN & 4 & 4 & 10 & 18 \\
\hline $\mathrm{BP}$ & 13 & 0 & 0 & 13 \\
\hline $\mathrm{BQ}$ & 6 & 0 & 1 & \\
\hline BU & 11 & 2 & 0 & 13 \\
\hline BX & 6 & 1 & 4 & 11 \\
\hline $\mathrm{CA}$ & 5 & 1 & 0 & 6 \\
\hline $\mathrm{CB}$ & 14 & 1 & 0 & 15 \\
\hline$C D$ & 6 & 0 & 0 & 6 \\
\hline $\mathrm{CH}$ & 14 & 0 & 0 & 14 \\
\hline $\mathrm{CL}$ & 10 & 0 & 1 & 11 \\
\hline $\mathrm{CN}$ & 4 & 1 & 0 & 5 \\
\hline $\mathrm{CO}$ & 4 & 0 & 0 & 4 \\
\hline CR & 0 & 0 & 4 & 4 \\
\hline CS & 14 & 0 & 0 & 14 \\
\hline $\mathrm{DH}$ & 1 & 1 & 0 & 2 \\
\hline $\mathrm{EG}$ & 10 & 0 & 0 & 10 \\
\hline $\mathrm{EM}$ & 0 & 0 & 4 & 4 \\
\hline $\mathrm{EP}$ & 7 & 0 & 0 & 7 \\
\hline $\mathrm{FG}$ & 4 & 2 & 0 & 6 \\
\hline FJ & 3 & $\overrightarrow{1}$ & 1 & 5 \\
\hline FL & 5 & 1 & 0 & 6 \\
\hline $\mathrm{FM}$ & 5 & 0 & 0 & 5 \\
\hline FN & 4 & 0 & 0 & 4 \\
\hline $\mathrm{GA}$ & 9 & 2 & 0 & 11 \\
\hline GC & 1 & 1 & 0 & 2 \\
\hline GE & 10 & 2 & 1 & 13 \\
\hline GP & 9 & 0 & 4 & 13 \\
\hline GT & 11 & 0 & 0 & 11 \\
\hline $\mathrm{HC}$ & 1 & 1 & 0 & 2 \\
\hline HU & 3 & 1 & 1 & 5 \\
\hline IA & 12 & 0 & 0 & 12 \\
\hline ID & 9 & 0 & 0 & \\
\hline IL & 6 & 0 & 0 & 6 \\
\hline IN & 5 & 0 & 0 & \\
\hline IS & 6 & 3 & 2 & 11 \\
\hline IT & 13 & 1 & 0 & 14 \\
\hline JE & 2 & 0 & 0 & \\
\hline ЛL & 0 & 0 & 2 & \\
\hline $\mathrm{KA}$ & 2 & 0 & 0 & 2 \\
\hline $\mathrm{KR}$ & 6 & 1 & 0 & 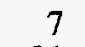 \\
\hline LA & 21 & 0 & 0 & 21 \\
\hline LB & 1 & 3 & 0 & \\
\hline
\end{tabular}

Evaluation Percentages

Total Analyses $\%$ A \%W \%N

$\begin{array}{rr}9 & 67 \\ 12 & 75 \\ 2 & 0 \\ 6 & 67 \\ 13 & 23 \\ 10 & 100 \\ 2 & 100 \\ 11 & 55 \\ 4 & 75 \\ 9 & 44 \\ 13 & 92 \\ 18 & 83 \\ 8 & 100 \\ 18 & 22 \\ 13 & 100 \\ 7 & 86 \\ 13 & 85 \\ 11 & 55 \\ 6 & 83 \\ 15 & 93 \\ 6 & 100 \\ 14 & 100 \\ 11 & 91 \\ 5 & 80 \\ 4 & 100 \\ 4 & \end{array}$

67
75
0
67
23
100
100
55
75
44
92
83
100
22
100
86
85
55
83
93
100
100
91
80
100

0
100

50

100

100

67

60

83

100

100

82

50

77

69

100

50

60

100

100

100

100

55

93
100

100
0

100

86

100

25

$\begin{array}{rr}0 & 33 \\ 0 & 25 \\ 100 & 0\end{array}$

33

69

0
0

9
25

25

11
8

17

0

22
0

0
15

15
9

17

7

0
0

0
20

20

0
0

0
50

50
0

0

0
33

20

17

0

0
18

50

15

0

50

20

0
0

0

0
27

7

0
0

0
0

14

0
75
33

0

0

8

0

36

44

0

0

0

56

14

0

36

0

0

0

9

0
0

100

0

0

100

0

20

0

0

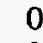

0

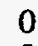

8

31

0

20

0

18

100 


\section{QAP 49 Summary of Laboratory Evaluations by Matrix}

Matrix: AI Air Filter

Evaluation Summary

Labcode

LL

LN

MA

ME

MH

NA

ND

NL

NM

NO

NS

$\mathrm{NZ}$

$\mathrm{OB}$

$\mathrm{OC}$

OD

OL

OU

PA

PK

PO

RA

$\mathrm{RC}$

RE

RI

RK

SA

SB

SK

$\mathrm{SN}$

SR

SW

TE

TI

TM

TN

TO

TP

TX

UC

UP

UY

WA

WC

WE

WS

WV

YA

YP

YU

W

0
3
2
0

0

5

4

$$
1
$$$$
1
$$$$
1
$$$$
1
$$$$
\begin{aligned}
& 0 \\
& 3
\end{aligned}
$$$$
0
$$$$
6
$$$$
1
$$$$
11
$$$$
0
$$$$
1
$$$$
\begin{aligned}
& 0 \\
& 1
\end{aligned}
$$$$
0
$$$$
3
$$$$
0
$$$$
\begin{aligned}
& 0 \\
& 0
\end{aligned}
$$$$
1
$$$$
1
$$$$
\begin{aligned}
& 1 \\
& 1 \\
& 0
\end{aligned}
$$$$
\begin{aligned}
& 0 \\
& 0
\end{aligned}
$$$$
\begin{aligned}
& 0 \\
& 1 \\
& 0
\end{aligned}
$$$$
\begin{aligned}
& 0 \\
& 0 \\
& 2
\end{aligned}
$$$$
\begin{aligned}
& 2 \\
& 2
\end{aligned}
$$$$
\begin{aligned}
& 2 \\
& 0
\end{aligned}
$$$$
1
$$$$
1
$$$$
\begin{aligned}
& 0 \\
& 1 \\
& 0
\end{aligned}
$$$$
\begin{aligned}
& 0 \\
& 1
\end{aligned}
$$$$
1
$$$$
\begin{aligned}
& 2 \\
& 4
\end{aligned}
$$$$
\begin{aligned}
& 4 \\
& 0 \\
& 3
\end{aligned}
$$$$
\begin{aligned}
& 3 \\
& 0
\end{aligned}
$$$$
0
$$$$
\begin{aligned}
& 0 \\
& 1 \\
& 0 \\
& 1
\end{aligned}
$$

Total Analyses

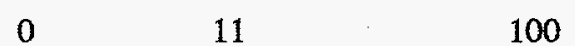

0
1
3
4
0
0
1
0

0

0

0
0

1

$$
\begin{aligned}
& 2 \\
& 4 \\
& \hline
\end{aligned}
$$$$
0
$$$$
0
$$$$
0
$$$$
\begin{aligned}
& 0 \\
& 0
\end{aligned}
$$$$
\begin{aligned}
& 0 \\
& 0
\end{aligned}
$$$$
\text { o }
$$$$
0
$$$$
\begin{aligned}
& 0 \\
& 2
\end{aligned}
$$$$
\begin{aligned}
& 2 \\
& 0
\end{aligned}
$$$$
\text { 0 }
$$$$
0
$$$$
1
$$$$
\begin{aligned}
& 0 \\
& 0
\end{aligned}
$$$$
\begin{aligned}
& 0 \\
& 0
\end{aligned}
$$$$
\begin{aligned}
& 0 \\
& 0
\end{aligned}
$$$$
0
$$$$
\begin{aligned}
& 0 \\
& 1 \\
& 4
\end{aligned}
$$$$
\begin{aligned}
& 4 \\
& 0
\end{aligned}
$$$$
\begin{aligned}
& 0 \\
& 0 \\
& 0
\end{aligned}
$$$$
\begin{array}{ll}
0 & 11 \\
1 & 11
\end{array}
$$$$
\begin{array}{lr}
1 & 13 \\
0 & 6
\end{array}
$$$$
\begin{array}{ll}
0 & 6 \\
1 & 7
\end{array}
$$$$
\begin{array}{ll}
1 & 7 \\
0 & 6
\end{array}
$$$$
0 \quad 11
$$$$
\begin{array}{rr}
3 & 6 \\
1 & 12
\end{array}
$$$$
\begin{array}{ll}
1 & 12 \\
0 & 11
\end{array}
$$$$
\begin{array}{ll}
0 & 11 \\
0 & 13
\end{array}
$$$$
\begin{array}{ll}
0 & 13 \\
0 & 12
\end{array}
$$$$
1 \quad 5
$$$$
\begin{array}{ll}
0 & 1 \\
0 & 2
\end{array}
$$$$
\begin{array}{lr}
0 & 2 \\
0 & 10
\end{array}
$$$$
\begin{array}{rr}
0 & 10 \\
0 & 1 \\
0 & 4
\end{array}
$$
$\% \mathrm{~A}$

\begin{tabular}{|c|c|c|}
\hline 100 & 0 & 0 \\
\hline 43 & 43 & 14 \\
\hline 44 & 22 & 33 \\
\hline 0 & 0 & 100 \\
\hline 29 & 71 & 0 \\
\hline 33 & 67 & 0 \\
\hline 71 & 14 & 14 \\
\hline 83 & 17 & 0 \\
\hline 89 & 11 & 0 \\
\hline 80 & 20 & 0 \\
\hline 100 & 0 & 0 \\
\hline 57 & 43 & 0 \\
\hline 83 & 0 & 17 \\
\hline 64 & 27 & 9 \\
\hline 17 & 17 & 67 \\
\hline 39 & 61 & 0 \\
\hline 100 & 0 & 0 \\
\hline 75 & 25 & 0 \\
\hline 100 & 0 & 0 \\
\hline 83 & 17 & 0 \\
\hline 100 & 0 & 0 \\
\hline 100 & 0 & 0 \\
\hline 40 & 60 & 0 \\
\hline 100 & 0 & 0 \\
\hline 82 & 0 & 18 \\
\hline 100 & 0 & 0 \\
\hline 90 & 10 & 0 \\
\hline 75 & 13 & 13 \\
\hline 50 & 50 & 0 \\
\hline 100 & 0 & 0 \\
\hline 100 & 0 & 0 \\
\hline 80 & 20 & 0 \\
\hline 100 & 0 & 0 \\
\hline 92 & 0 & 8 \\
\hline 45 & 18 & 36 \\
\hline 86 & 14 & 0 \\
\hline 82 & 18 & 0 \\
\hline 100 & 0 & 0 \\
\hline 85 & 8 & 8 \\
\hline 83 & 17 & 0 \\
\hline 86 & 0 & 14 \\
\hline 83 & 17 & 0 \\
\hline 100 & 0 & 0 \\
\hline 50 & 0 & 50 \\
\hline 83 & 8 & 8 \\
\hline 82 & 18 & 0 \\
\hline 69 & 31 & 0 \\
\hline 100 & 0 & 0 \\
\hline 20 & 60 & 20 \\
\hline 100 & 0 & 0 \\
\hline 100 & 0 & 0 \\
\hline 90 & 10 & 0 \\
\hline 100 & 0 & 0 \\
\hline 75 & 25 & 0 \\
\hline
\end{tabular}

Evaluation Percentages 
QAP 49 Summary of Laboratory Evaluations by Matrix

Matrix: AI Air Filter

Evaluation Summary

Labcode A W

Total Analyses

81

Totals

109 Labs: 678

877
Evaluation Percentages $\% A$ $\% \mathrm{~W}$ $\% \mathrm{~N}$

$77 \%$

$13 \%$

9\% 
QAP 49 Summary of Laboratory Evaluations by Matrix

Matrix: SO Soil

Evaluation Summary

Labcode

\begin{tabular}{|c|c|c|c|c|}
\hline $\mathrm{AC}$ & 2 & 0 & 0 & 2 \\
\hline $\mathrm{AF}$ & 12 & 2 & 2 & 16 \\
\hline$A G$ & 15 & 2 & 0 & 17 \\
\hline AI & 11 & 1 & 0 & 12 \\
\hline $\mathrm{AL}$ & 1 & 0 & 2 & 3 \\
\hline $\mathrm{AM}$ & 16 & 2 & 0 & 18 \\
\hline AN & 6 & 0 & 2 & 8 \\
\hline $\mathrm{AT}$ & 3 & 0 & 0 & 3 \\
\hline $\mathrm{AU}$ & 7 & 0 & 0 & 7 \\
\hline $\mathrm{BA}$ & 1 & 1 & 0 & 2 \\
\hline $\mathrm{BC}$ & 2 & 2 & 1 & 5 \\
\hline $\mathrm{BE}$ & 8 & 0 & 0 & 8 \\
\hline $\mathrm{BL}$ & 24 & 0 & 1 & 25 \\
\hline BM & 6 & 0 & 0 & 6 \\
\hline $\mathrm{BN}$ & 24 & 0 & 0 & 24 \\
\hline BP & 12 & 1 & 0 & 13 \\
\hline $\mathrm{BQ}$ & 8 & 1 & 1 & 10 \\
\hline BÙ & 8 & 1 & 0 & 9 \\
\hline $\mathrm{BX}$ & 5 & 3 & 0 & 8 \\
\hline $\mathrm{CA}$ & 1 & 0 & 0 & 1 \\
\hline $\mathrm{CD}$ & 8 & 0 & 0 & 8 \\
\hline $\mathrm{CH}$ & 17 & 0 & 0 & 17 \\
\hline $\mathrm{CL}$ & 12 & 3 & 0 & 15 \\
\hline $\mathrm{CM}$ & 9 & 0 & 0 & 9 \\
\hline $\mathrm{CN}$ & 3 & 0 & 0 & 3 \\
\hline $\mathrm{CO}$ & 1 & 0 & 2 & 3 \\
\hline $\mathrm{CR}$ & 8 & 1 & 0 & 9 \\
\hline $\mathrm{CS}$ & 24 & 0 & 0 & 24 \\
\hline $\mathrm{DH}$ & 3 & 1 & 0 & 4 \\
\hline $\mathrm{EG}$ & 8 & 0 & 0 & 8 \\
\hline EM & 0 & 0 & 3 & 3 \\
\hline EP & 2 & 0 & 0 & \\
\hline FG & 14 & 1 & 0 & 15 \\
\hline $\mathrm{FL}$ & 3 & 1 & 0 & 4 \\
\hline FN & 2 & 0 & 0 & 2 \\
\hline FS & 8 & 0 & 1 & 9 \\
\hline GA & 12 & 1 & 0 & 13 \\
\hline$G C$ & 6 & 0 & 0 & 6 \\
\hline GE & 14 & 0 & 0 & 14 \\
\hline GP & 2 & 0 & 1 & 3 \\
\hline GT & 5 & 2 & 0 & 7 \\
\hline HT & 0 & 0 & 6 & 6 \\
\hline HU & 8 & 0 & 2 & 10 \\
\hline IA & 5 & 4 & 3 & 12 \\
\hline ID & 12 & 0 & 0 & 12 \\
\hline IE & 7 & 1 & 1 & 9 \\
\hline IL & 8 & 0 & 1 & 9 \\
\hline IN & 12 & 0 & 0 & 12 \\
\hline IS & 16 & 1 & 3 & 20 \\
\hline IT & 18 & 2 & 0 & 20 \\
\hline $\mathrm{JE}$ & 6 & $\overline{0}$ & 0 & \\
\hline $\mathrm{KA}$ & 4 & 0 & 0 & 4 \\
\hline $\mathrm{KR}$ & 4 & 0 & 0 & 4 \\
\hline LA & 8 & 1 & 3 & 12 \\
\hline $\mathrm{LB}$ & 2 & 0 & 0 & 2 \\
\hline
\end{tabular}

Evaluation Percentages

Total $\% \mathrm{~A} \quad \% \mathrm{~W} \quad \% \mathrm{~N}$

2.100

75

88

92

33

89

75

100

100

50

40

100

96

100

100

92

80

89

63

100

100

100

80

100

100

33

89

100

75

100

100

100
93

75

100

89

92

100

100

67

71

0

80

42

100

78

89

100

80

90

100

100

100

67

100
0

13

12

8

11

0

0

50

40

0

0

0
0
8

8

10

11

38

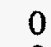

0

20

0
0

0
0

11

25

0

0

0
7

25

0

0

8

0

0

29

0

0

33

11

0

0

5
10

10

0

0

0

8
0
0 
QAP 49 Summary of Laboratory Evaluations by Matrix

Matrix: SO Soil

Evaluation Summary

Labcode

LL

LV

MA

ME

MH

ML

NA

NL

NM

NP

NQ

NR

$\mathrm{NZ}$

$\mathrm{OB}$

OC

OL

OT

PK

PO

RA

$\mathrm{RC}$

RE

RI

SA

SB

SK

SN

SR

SW

TE

TI

TM

TN

TO

TP

TT

TW

TX

TY

$\mathrm{UC}$

UP

UY

WA

WC

WE

WS

YA

YP

YU
A W

6
9
4

3

4

9

3

13

14$$
6
$$$$
11
$$$$
2
$$$$
10
$$$$
8
$$$$
28
$$$$
3
$$$$
15
$$$$
13
$$$$
1
$$$$
22
$$$$
3
$$$$
\begin{aligned}
& 9 \\
& 1
\end{aligned}
$$$$
1
$$$$
2
$$$$
12
$$$$
8
$$

12

10

5
17

17
8

8
16

2
0
7

16

$$
3
$$$$
\begin{aligned}
& 1 \\
& 6
\end{aligned}
$$$$
13
$$$$
17
$$$$
4
$$$$
12
$$$$
\begin{array}{r}
12 \\
3
\end{array}
$$$$
\begin{aligned}
& 7 \\
& 1 \\
& 2
\end{aligned}
$$

Total Analyses

$\begin{array}{rr}8 & 75 \\ 11 & 82 \\ 4 & 100 \\ 3 & 100 \\ 7 & 57 \\ 9 & 100 \\ 3 & 100 \\ 13 & 100 \\ 14 & 100 \\ 7 & 86\end{array}$

2

14

2

10

10

33

3

15

13

1
4

25 .

3

9
1

1

2

12

9
9

16

10

7

17

18

3

2

16

4

2
6

14

18

5

12

4

8

1
Evaluation Percentages \%A \%W $\% N$

\section{5}

82

100

57

100

100

100

100
86

0
79

100

100

80

85

100

100

100

100

100

88

100

100

100

100

100

100

89

89

75

100

71

100

80

89

67

0

100

75

50

100

93

94

80

100

75

88

100

100

$0 \quad 25$

$18 \quad 0$

$\begin{array}{ll}0 & 0 \\ 0 & 0\end{array}$

$29 \quad 14$

$0 \quad 0$

0

0 . 0

$14 \quad 0$

$50 \quad 50$

21

$\begin{array}{ll}0 & 0 \\ 0 & 0\end{array}$

$0 \quad 20$

15

0 0

$0 \quad 0$

$\begin{array}{ll}0 & 0 \\ 0 & 0\end{array}$

$\begin{array}{rl}0 & 0 \\ 12 & 0\end{array}$

0 . 0

$\begin{array}{ll}0 & 0 \\ 0 & 0\end{array}$

$\begin{array}{ll}0 & 0 \\ 0 & 0\end{array}$

$0 \quad 0$

$\begin{array}{ll}11 & 0 \\ 11 & 0\end{array}$

$13 \quad 13$

$\begin{array}{rr}0 & 0 \\ 14 & 14 \\ 0 & 0\end{array}$

$\begin{array}{rr}0 & 0 \\ 0 & 20\end{array}$

$0 \quad 11$

33

50

$0 \quad 0$

$0 \quad 0$

$\begin{array}{rr}0 & 25 \\ 50 & 0\end{array}$

$\begin{array}{rl}50 & 0 \\ 0 & 0\end{array}$

$7 \quad 0$

$20 \quad 0$

$\begin{array}{rl}0 & 0 \\ 13 & 0\end{array}$

130

$\begin{array}{ll}0 & 0 \\ 0 & 0\end{array}$ 
QAP 49 Summary of Laboratory Evaluations by Matrix

Matrix: SO Soil

Evaluation Summary

Labcode A W N Analyses \%A $\% \mathrm{~N}$ 
QAP 49 Summary of Laboratory Evaluations by Matrix

Matrix: VE Vegetation

Evaluation Summary

\section{Labcode}

AF

AG

AL

AM

AT

AU

$\mathrm{BA}$

$\mathrm{BC}$

BE

$\mathrm{BL}$

BM

BN

$\mathrm{BP}$

BQ

BX

$\mathrm{CD}$

$\mathrm{CH}$

CL

$\mathrm{CN}$

$\mathrm{CO}$

CS

EG

EP

FN

GA

GC

GE

GP

GT

HU

IA

ID

IE

IL

IN

IS

IT

KR

LA

LB

LL

MA

ME

MH

ML

NA

NP

NR

$\mathrm{NZ}$

$\mathrm{OB}$
Total Analvses

650

7

7

3

6
4

4
6

6

4

7

8

5

9

3

7

7

3

7

7

4

2

3
8

8

3

4

3

6

3

7
8

6

4

9

6

3

3

3
6

6
4

12

3

6

4

4

4

4

1

6

3

3

3

50
100

43
Evaluation Percentages

0
50

100

100

100

75

86
75

100

100

100

33

100

57

100

86

71

100

100

67

100

88

100

100

100

67

100

86

100

17

75

56

67

0

67
100

67

100

100
75

67

0
83

75

75

75

75

100

83

67

100

100
$\% W$

17

0
43

.0

33

0

0

25

14

25

0

0
0

0
0

0

29

14

29

0

0

33
0

13

0

0
0

33

0

14
0

83

25

44

33

33

33

0

17

0
25

33

0

25

0

25

25

0

17

33

0

0
$\% N$

33

0

100

17

0

0
0

0

0

0

0

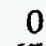

67

14

0

0

0

0

0

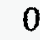

0

0

0

0

0

0

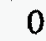

0

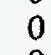

67

0

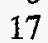

0

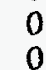

100

17

0
25

0

0

0

0 


\section{QAP 49 Summary of Laboratory Evaluations by Matrix}

Matrix: VE Vegetation

Evaluation Summary

\begin{tabular}{l} 
Labcode \\
\hline OC \\
OL \\
OT \\
OU \\
PK \\
PO \\
RA \\
RE \\
RI \\
SB \\
SN \\
SR \\
SW \\
TE \\
TI \\
TM \\
TN \\
TO \\
TP \\
TW \\
TX \\
UC \\
WA \\
WC \\
WE \\
YA \\
YU
\end{tabular}

W N

$\begin{array}{ll}5 & 7 \\ 3 & 0 \\ 7 & 0 \\ 3 & 0 \\ 2 & 0 \\ 3 & 1 \\ 9 & 0 \\ 6 & 1 \\ 1 & 0 \\ 3 & 0 \\ 5 & 1 \\ 6 & 1 \\ 0 & 5 \\ 3 & 1 \\ 6 & 1 \\ 4 & 2 \\ 4 & 0 \\ 2 & 0 \\ 4 & 0 \\ 3 & 0 \\ 6 & 0 \\ 0 & 0 \\ 4 & 3 \\ 5 & 2 \\ 3 & 1 \\ 6 & 1 \\ 3 & 0\end{array}$

Total

N Analyses

Totals

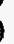

$\begin{array}{ll}0 & 0 \\ 0 & 0\end{array}$

$1 \quad 0$

0

$1 \quad 0$

2
0

$1 \quad 0$

0

(2)

2
0

0

1

\begin{tabular}{l}
1 \\
3 \\
\hline
\end{tabular}

3
0

0

0

0
3
0

0

0

0
0
0

0 Analyses

3

2

4

9

7
3

3

6

6
7

7

4

7

7

7

5
4
3

4
3
6

3
6
3
7

3
7

7

4
7
3

12
3
7

Evaluation Percentages $\%$ A \%W $\% \mathrm{~N}$

$\begin{array}{rrr}42 & 58 & 0 \\ 100 & 0 & 0 \\ 100 & 0 & 0 \\ 100 & 0 & 0 \\ 100 & 0 & 0 \\ 75 & 25 & 0 \\ 100 & 0 & 0 \\ 86 & 14 & 0 \\ 33 & 0 & 67 \\ 100 & 0 & 0 \\ 83 & 17 & 0 \\ 86 & 14 & 0 \\ 0 & 71 & 29 \\ 75 & 25 & 0 \\ 86 & 14 & 0 \\ 57 & 29 & 14 \\ 57 & 0 & 43 \\ 40 & 0 & 60 \\ 100 & 0 & 0 \\ 100 & 0 & 0 \\ 100 & 0 & 0 \\ 0 & 0 & 100 \\ 57 & 43 & 0 \\ 71 & 29 & 0 \\ 75 & 25 & 0 \\ 86 & 14 & 0 \\ 100 & 0 & 0\end{array}$

82 Labs:

323

72

35

430

$\mathbf{7 5} \%$

$17 \%$

$8 \%$ 


\section{QAP 49 Summary of Laboratory Evaluations by Matrix}

Matrix: WAWater

Evaluation Summary

\begin{tabular}{|c|c|c|c|c|c|c|c|}
\hline Labcode & A & W & $\mathbf{N}$ & Analyses & \%A & $\% \mathrm{~W}$ & $\% \mathbf{N}$ \\
\hline $\mathrm{AC}$ & 2 & 0 & 0 & 2 & 100 & 0 & 0 \\
\hline $\mathrm{AF}$ & 5 & 2 & 4 & 11 & 45 & 18 & 36 \\
\hline$A G$ & 12 & 0 & 0 & 12 & 100 & 0 & 0 \\
\hline $\mathrm{AI}$ & 5 & 4 & 2 & 11 & 45 & 36 & 18 \\
\hline AL & 0 & 0 & 3 & 3 & 0 & 0 & 100 \\
\hline $\mathrm{AM}$ & 8 & 1 & 4 & 13 & 62 & 8 & 31 \\
\hline AN & 10 & 0 & 0 & 10 & 100 & 0 & 0 \\
\hline AT & 4 & 0 & 0 & 4 & 100 & 0 & 0 \\
\hline $\mathrm{AU}$ & 11 & 1 & 0 & 12 & 92 & 8 & 0 \\
\hline BA & 5 & 3 & 0 & 8 & 63 & 38 & 0 \\
\hline $\mathrm{BC}$ & 6 & 1 & 1 & 8 & 75 & 13 & 13 \\
\hline $\mathrm{BE}$ & 14 & 1 & 0 & 15 & 93 & 7 & 0 \\
\hline BL & 15 & 3 & 2 & 20 & 75 & 15 & 10 \\
\hline BM & 8 & 0 & 0 & 8 & 100 & 0 & 0 \\
\hline BN & 3 & 7 & 10 & 20 & 15 & 35 & 50 \\
\hline BP & 12 & 2 & 0 & 14 & 86 & 14 & 0 \\
\hline $\mathrm{BQ}$ & 5 & 1 & 0 & 6 & 83 & 17 & 0 \\
\hline $\mathrm{BU}$ & 10 & 2 & 0 & 12 & 83 & 17 & 0 \\
\hline BX & 10 & 3 & 1 & 14 & 71 & 21 & 7 \\
\hline $\mathrm{CA}$ & 4 & 1 & 1 & 6 & 67 & 17 & 17 \\
\hline $\mathrm{CB}$ & 2 & 0 & 0 & 2 & 100 & 0 & 0 \\
\hline$C D$ & 4 & 1 & 0 & 5 & 80 & 20 & 0 \\
\hline $\mathrm{CH}$ & 13 & 2 & 0 & 15 & 87 & 13 & 0 \\
\hline CL & 10 & 2 & 1 & 13 & 77 & 15 & 8 \\
\hline $\mathrm{CM}$ & 20 & 0 & 0 & 20 & 100 & 0 & 0 \\
\hline $\mathrm{CS}$ & 6 & 2 & 0 & 8 & 75 & 25 & 0 \\
\hline DH & 4 & 1 & 0 & 5 & 80 & 20 & 0 \\
\hline EG & 14 & 0 & 0 & 14 & 100 & 0 & 0 \\
\hline EM & 2 & 2 & 0 & 4 & 50 & 50 & 0 \\
\hline EP & 8 & 0 & 0 & 8 & 100 & 0 & 0 \\
\hline $\mathrm{FG}$ & 8 & 1 & 3 & 12 & 67 & 8 & 25 \\
\hline FL & 7 & 1 & 0 & 8 & 88 & 13 & 0 \\
\hline FM & 4 & 0 & 0 & 4 & 100 & 0 & 0 \\
\hline FN & 4 & 0 & 0 & 4 & 100 & 0 & 0 \\
\hline GA & 5 & 2 & 3 & 10 & 50 & 20 & 30 \\
\hline $\mathrm{GC}$ & 6 & 1 & 1 & 8 & 75 & 13 & 13 \\
\hline GE & 14 & 0 & 1 & 15 & 93 & 0 & 7 \\
\hline GP & 12 & 0 & 1 & 13 & 92 & 0 & 8 \\
\hline GS & 3 & 0 & 0 & 3 & 100 & 0 & 0 \\
\hline GT & 8 & 2 & 1 & 11 & 73 & 18 & 9 \\
\hline $\mathrm{HC}$ & 3 & 0 & 0 & 3 & 100 & 0 & 0 \\
\hline $\mathrm{HT}$ & 0 & 1 & 6 & 7 & 0 & 14 & 86 \\
\hline ID & 6 & 1 & 1 & 8 & 75 & 13 & 13 \\
\hline IE & 5 & 1 & 1 & 7 & 71 & 14 & 14 \\
\hline IL & 5 & 0 & 0 & 5 & 100 & 0 & 0 \\
\hline IN & 8 & 1 & 1 & 10 & 80 & 10 & 10 \\
\hline IS & 5 & 6 & 0 & 11 & 45 & 55 & 0 \\
\hline IT & 13 & 1 & 0 & 14 & 93 & 7 & 0 \\
\hline JE & 2 & 0 & 3 & 5 & 40 & 0 & 60 \\
\hline JL & 3 & 0 & 1 & 4 & 75 & 0 & 25 \\
\hline $\mathrm{KA}$ & 8 & 2 & 0 & 10 & 80 & 20 & 0 \\
\hline LA & 21 & 9 & 1 & 31 & 68 & 29 & 3 \\
\hline LB & 3 & 0 & 0 & 3 & 100 & 0 & 0 \\
\hline LE & 1 & 0 & 1 & 2 & 50 & 0 & 50 \\
\hline LL & 9 & 2 & 0 & 11 & 82 & 18 & 0 \\
\hline
\end{tabular}

Evaluation Percentages

Total 
Evaluation Summary

Labcode A $\quad$ W $\quad$ N

$\begin{array}{lrl}\text { LN } & 1 & 1 \\ \text { LV } & 7 & 0 \\ \text { LW } & 3 & 0 \\ \text { ME } & 4 & 1 \\ \text { MH } & 8 & 0 \\ \text { ML } & 5 & 0 \\ \text { NA } & 4 & 0 \\ \text { ND } & 3 & 0 \\ \text { NL } & 8 & 0 \\ \text { NM } & 4 & 1 \\ \text { NP } & 4 & 0 \\ \text { NQ } & 5 & 3 \\ \text { NS } & 5 & 1 \\ \text { NZ } & 14 & 2 \\ \text { OB } & 4 & 0 \\ \text { OC } & 15 & 0 \\ \text { OD } & 24 & 2 \\ \text { OL } & 3 & 0 \\ \text { OT } & 8 & 2 \\ \text { OU } & 7 & 4 \\ \text { PR } & 5 & 0 \\ \text { RC } & 4 & 0 \\ \text { RE } & 12 & 0 \\ \text { RG } & 2 & 0 \\ \text { RI } & 4 & 2 \\ \text { SA } & 2 & 1 \\ \text { SB } & 2 & 2 \\ \text { SK } & 4 & 0 \\ \text { SL } & 3 & 0 \\ \text { SN } & 6 & 0 \\ \text { SR } & 7 & 5 \\ \text { ST } & 1 & 0 \\ \text { SW } & 4 & 3 \\ \text { TE } & 6 & 2 \\ \text { TI } & 12 & 0 \\ \text { TM } & 9 & 1 \\ \text { TN } & 11 & 1 \\ \text { TO } & 12 & 2 \\ \text { TP } & 6 & 1 \\ \text { TT } & 3 & 1 \\ \text { TW } & 4 & 1 \\ \text { TX } & 11 & 1 \\ \text { UC } & 5 & 0 \\ \text { UP } & 11 & 1 \\ \text { UY } & 11 & 0 \\ \text { WA } & 12 & 2 \\ \text { WC } & 9 & 2 \\ \text { WE } & 2 & 0 \\ \text { WI } & 6 & 3 \\ \text { WV } & 6 & 1 \\ \text { YA } & 11 & 1 \\ \text { YP } & 1 & 0 \\ \text { YU } & 3 & 0\end{array}$

Total Analuses

$\begin{array}{ll}4 & \\ 9 & \\ 3 & 1 \\ 5 & \end{array}$

8

5

3

8

5

10

7

20

5
19

26

3

11

14
5

5
4
4

$i^{4}$

2

3

5

6

3

12

1

11

8
13

11

15

14

7

4

5
12

5

12

11

14

12

4

9

7

13
Evaluation Percentages $\% A$ $\% \mathrm{~W}$

$\% \mathrm{~N}$

25
78
100
80
100
100
57

100

100

80

80

50

71

70

80
79

92

100

73

50
100

100

100

100

57

67

40
67

67
100

100

58

100

36
75

92

82

73

86

86

75

80

92

100

92

100

86

75

50

67

86

85

100

100
25. 50

$0 \quad 22$

0

20

0
0

0
0

0
20

20
0

30

14

10

$\begin{array}{ll}14 & 20 \\ 0 & 20\end{array}$

$0 \quad 21$

8

0

18

29
.0

.0
0

0

0

29

33

40

0

0

0
42

42
0

27

25

0

9
7

14

14

25

20

8

0
8

8
0

14

17

0

33

14

8

0
0 


\section{QAP 49 Summary of Laboratory Evaluations by Matrix}

Matrix: WAWater

Evaluation Summary

Labcode

A W N

Total Analyses
Evaluation Percentages $\% A$ $\% \mathrm{~W}$ $\% \mathbf{N}$

Totals

108 Labs: 738

123

95

956

$77 \%$

$13 \%$

$10 \%$ 


\section{QAP 49 Summary of Matrix Evaluations by Radionuclide}

Matrix: $\quad$ Air Filter

Evaluation Summary

\begin{tabular}{|c|c|c|c|c|c|c|c|}
\hline \multirow[b]{2}{*}{$\begin{array}{l}\text { Radio- } \\
\text { Nuclide } \\
\end{array}$} & \multicolumn{3}{|c|}{ Evaluation Summary } & \multirow[b]{2}{*}{$\begin{array}{l}\text { Total } \\
\text { Analyses }\end{array}$} & \multicolumn{3}{|c|}{ Evaluation Percentages } \\
\hline & $\mathbf{A}$ & W & $\mathbf{N}$ & & $\% \mathbf{A}$ & $\% W$ & $\% \mathbf{N}$ \\
\hline SB125 & 74 & 14 & 15 & 103 & 72 & 14 & 15 \\
\hline GROSS BETA & 44 & 31 & 1 & 76 & 58 & 41 & 1 \\
\hline $\mathrm{CO60}$ & 95 & 6 & 6 & 107 & 89 & 6 & 6 \\
\hline CS137 & 85 & 14 & 9 & 108 & 79 & 13 & 8 \\
\hline SR90 & 29 & 5 & 7 & 41 & 71 & 12 & 17 \\
\hline PU238 & 37 & 6 & 9 & 52 & 71 & 12 & 17 \\
\hline PU239 & 39 & 5 & 8 & 52 & 75 & 10 & 15 \\
\hline GROSS ALPHA & 63 & 10 & 3 & 76 & 83 & 13 & 4 \\
\hline U234 & 28 & 1 & 4 & 33 & 85 & 3 & 12 \\
\hline U238 & 27 & 3 & 5 & 35 & 77 & 9 & 14 \\
\hline $\mathrm{Bq} \mathrm{U}$ & 13 & 2 & 1 & 16 & 81 & 13 & 6 \\
\hline ug U & 14 & 2 & 1 & 17 & 82 & 12 & 6 \\
\hline AM241 & 45 & 9 & 4 & 58 & 78 & 16 & 7 \\
\hline MN54 & 85 & 10 & 9 & 104 & 82 & 10 & 9 \\
\hline
\end{tabular}




\title{
QAP 49 Summary of Matrix Evaluations by Radionuclide
}

\author{
Matrix: Soil
}

\begin{tabular}{|c|c|c|c|c|c|c|c|}
\hline \multirow[b]{2}{*}{$\begin{array}{l}\text { Radio- } \\
\text { Nuclide } \\
\end{array}$} & \multicolumn{3}{|c|}{ Evaluation Summary } & \multirow{2}{*}{$\begin{array}{l}\text { Total } \\
\text { Analyses }\end{array}$} & \multicolumn{3}{|c|}{ Evaluation Percentages } \\
\hline & $\mathbf{A}$ & $\mathbf{W}$ & $\mathbf{N}$ & & $\% \mathbf{A}$ & $\% \mathrm{~W}$ & $\% \mathbf{N}$ \\
\hline TL208 & 50 & 0 & 0 & 50 & 100 & 0 & 0 \\
\hline PB210 & 14 & 0 & 0 & 14 & 100 & 0 & 0 \\
\hline BI214 & 52 & 0 & 0 & 52 & 100 & 0 & 0 \\
\hline PB214 & 53 & 0 & 0 & 53 & 100 & 0 & 0 \\
\hline BI212 & 42 & 0 & 0 & 42 & 100 & 0 & 0 \\
\hline $\mathrm{TH} 228$ & 34 & 0 & 0 & 34 & 100 & 0 & 0 \\
\hline ug $U$ & 19 & 1 & 4 & 24 & 79 & 4 & 17 \\
\hline SR90 & 38 & 6 & 2 & 46 & 83 & 13 & 4 \\
\hline PB212 & 52 & 0 & 0 & 52 & 100 & 0 & 0 \\
\hline $\mathrm{Bq} \mathrm{U}$ & 16 & 1 & 2 & 19 & 84 & 5 & 11 \\
\hline U238 & 40 & 2 & 4 & 46 & 87 & 4 & 9 \\
\hline $\mathrm{U} 234$ & 33 & 2 & 5 & 40 & 83 & 5 & 13 \\
\hline AM241 & 52 & 5 & 4 & 61 & 85 & 8 & 7 \\
\hline RA226 & 45 & 0 & 0 & 45 & 100 & 0 & 0 \\
\hline PU238 & 2 & 1 & 0 & 3 & 67 & 33 & 0 \\
\hline K40 & 70 & 24 & 8 & 102 & 69 & 24 & 8 \\
\hline $\mathrm{CS} 137$ & 85 & 11 & 16 & 112 & 76 & 10 & 14 \\
\hline CO60 & 10 & 2 & 2 & 14 & 71 & 14 & 14 \\
\hline PU239 & 46 & 10 & 3 & 59 & 78 & 17 & 5 \\
\hline TH234 & 36 & 0 & 0 & 36 & 100 & 0 & 0 \\
\hline AC228 & 49 & 0 & 0 & 49 & 100 & 0 & 0 \\
\hline Totals: & 838 & 65 & 50 & 953 & $88 \%$ & $7 \%$ & $5 \%$ \\
\hline
\end{tabular}


EML-600 QAP 9809

December, 1998

QAP 49 Summary of Matrix Evaluations by Radionuclide

Matrix: Vegetation

\begin{tabular}{|c|c|c|c|c|c|c|c|}
\hline \multirow[b]{2}{*}{$\begin{array}{l}\text { Radio- } \\
\text { Nuclide } \\
\end{array}$} & \multicolumn{3}{|c|}{ Evaluation Summary } & \multirow[b]{2}{*}{$\begin{array}{l}\text { Total } \\
\text { Analyses }\end{array}$} & \multicolumn{3}{|c|}{ Evaluation Percentages } \\
\hline & $\mathbf{A}$ & $\mathbf{W}$ & $\mathbf{N}$ & & $\% \mathbf{A}$ & $\% W$ & $\% \mathbf{N}$ \\
\hline $\mathrm{CO} 60$ & 66 & 14 & 9 & 89 & 74 & 16 & 10 \\
\hline CS137 & 78 & 5 & 7 & 90 & 87 & 6 & 8 \\
\hline SR90 & 31 & 12 & 4 & 47 & 66 & 26 & 9 \\
\hline PU238 & 4 & 0 & 0 & 4 & 100 & 0 & 0 \\
\hline PU239 & 35 & 3 & 3 & 41 & 85 & 7 & 7 \\
\hline AM241 & 33 & 11 & 2 & 46 & 72 & 24 & 4 \\
\hline CM244 & 19 & 7 & 1 & 27 & 70 & 26 & 4 \\
\hline $\mathrm{K} 40$ & 57 & 20 & 9 & 86 & 66 & 23 & 10 \\
\hline Totals: & 323 & 72 & 35 & 430 & $75 \%$ & $17 \%$ & $8 \%$ \\
\hline
\end{tabular}




\section{QAP 49 Summary of Matrix Evaluations by Radionuclide}

Matrix: Water

\begin{tabular}{|c|c|c|c|c|c|c|c|}
\hline \multirow[b]{2}{*}{$\begin{array}{l}\text { Radio- } \\
\text { Nuclide } \\
\end{array}$} & \multicolumn{3}{|c|}{ Evaluation Summary } & \multirow[b]{2}{*}{$\begin{array}{l}\text { Total } \\
\text { Analyses }\end{array}$} & \multicolumn{3}{|c|}{ Evaluation Percentages } \\
\hline & $\mathbf{A}$ & W & $\mathbf{N}$ & & \%A & $\% W$ & $\% \mathrm{~N}$ \\
\hline GROSS ALPHA & 50 & 15 & 7 & 72 & 69 & 21 & 10 \\
\hline FE55 & 16 & 0 & 0 & 16 & 100 & 0 & 0 \\
\hline $\mathrm{H} 3$ & 45 & 14 & 15 & 74 & 61 & 19 & 20 \\
\hline MN54 & 76 & 17 & 14 & 107 & 71 & 16 & 13 \\
\hline GROSS BETA & 68 & 2 & 3 & 73 & 93 & 3 & 4 \\
\hline ug U & 19 & 2 & 3 & 24 & 79 & 8 & 13 \\
\hline $\mathrm{BqU}$ & 17 & $\overline{4}$ & 1 & 22 & 77 & 18 & 5 \\
\hline $\mathrm{U}^{1} 38$ & 36 & $i$ & 8 & 45 & 80 & 2 & 18 \\
\hline U234 & 35 & 2 & 5 & 42 & 83 & 5 & 12 \\
\hline AM241 & 49 & 9 & 4 & 62 & 79 & 15 & 6 \\
\hline PU239 & 41 & 14 & 6 & 61 & 67 & 23 & 10 \\
\hline PU238 & 35 & 16 & 8 & 59 & 59 & 27 & 14 \\
\hline SR90 & 39 & 13 & 12 & 64 & 61 & 20 & 19 \\
\hline $\mathrm{CS} 137$ & 97 & 10 & 4 & 111 & 87 & 9 & 4 \\
\hline CO60 & 101 & 4 & 5 & 110 & 92 & 4 & 5 \\
\hline NI63 & 14 & 0 & 0 & 14 & 100 & 0 & 0 \\
\hline Totals: & 738 & 123 & 95 & 956 & $77 \%$ & $13 \%$ & $10 \%$ \\
\hline
\end{tabular}


QAP 49 EML Results

Environmental Measurements Laboratory, New York, NY

\begin{tabular}{|c|c|c|c|}
\hline Matrix & Radionuclide & EML Value & EML Error \\
\hline Air Filter & Gross Alpha & 1.650 & 0.160 \\
\hline Air Filter & Gross Beta & 2.160 & 0.070 \\
\hline Air Filter & $54 \mathrm{Mn}$ & 4.920 & 0.400 \\
\hline Air Filter & $60 \mathrm{Co}$ & 9.160 & 0.580 \\
\hline Air Filter & $90 \mathrm{Sr}$ & 1.120 & 0.050 \\
\hline Air Filter & $125 \mathrm{Sb}$ & 8.890 & 0.550 \\
\hline Air Filter & $137 \mathrm{Cs}$ & 22.470 & 1.030 \\
\hline Air Filter & $234 \mathrm{U}$ & 0.260 & 0.010 \\
\hline Air Filter & $238 \mathrm{U}$ & 0.260 & 0.010 \\
\hline Air Filter & $\mathrm{Bq} \mathrm{U}$ & 0.530 & 0.020 \\
\hline Air Filter & ug U & 20.960 & 0.100 \\
\hline Air Filter & $238 \mathrm{Pu}$ & 0.460 & 0.005 \\
\hline Air Filter & $239 \mathrm{Pu}$ & 0.420 & 0.006 \\
\hline Air Filter & $241 \mathrm{Am}$ & 0.510 & 0.008 \\
\hline Soil & $40 \mathrm{~K}$ & 314.000 & 13.000 \\
\hline Soil & $60 \mathrm{Co}$ & 1.240 & 0.110 \\
\hline Soil & $90 \mathrm{Sr}$ & 39.630 & 0.003 \\
\hline Soil & $137 \mathrm{Cs}$ & 954.000 & 38.000 \\
\hline Soil & $234 \mathrm{U}$ & 113.000 & 6.000 \\
\hline Soil & $238 \mathrm{U}$ & 120.000 & 9.000 \\
\hline Soil & $\mathrm{BqU}$ & 237.000 & 16.000 \\
\hline Soil & ug U & 9.700 & 0.700 \\
\hline Soil & $238 \mathrm{Pu}$ & 0.530 & 0.270 \\
\hline Soil & $239 \mathrm{Pu}$ & 13.090 & 0.570 \\
\hline Soil & $241 \mathrm{Am}$ & 7.470 & 0.410 \\
\hline Soil & $208 \mathrm{Tl}$ & 18.300 & 1.100 \\
\hline Soil & $210 \mathrm{~Pb}$ & 32.000 & 3.300 \\
\hline Soil & $212 \mathrm{Bi}$ & 58.300 & 5.900 \\
\hline Soil & $212 \mathrm{~Pb}$ & 52.800 & 3.700 \\
\hline Soil & $214 \mathrm{Bi}$ & 28.800 & 0.500 \\
\hline Soil & $214 \mathrm{~Pb}$ & 29.100 & 1.200 \\
\hline Soil & $226 \mathrm{Ra}$ & 29.000 & 1.000 \\
\hline Soil & $228 \mathrm{Ac}$ & 52.600 & 2.900 \\
\hline Soil & $228 \mathrm{Th}$ & 52.700 & 4.000 \\
\hline Soil & $234 \mathrm{Th}$ & 114.000 & 6.000 \\
\hline Water & $3 \mathrm{H}$ & 76.200 & 2.900 \\
\hline Water & $54 \mathrm{Mn}$ & 32.400 & 1.400 \\
\hline Water & $55 \mathrm{Fe}$ & 139.000 & 2.000 \\
\hline Water & $60 \mathrm{Co}$ & 49.400 & 1.200 \\
\hline Water & $90 \mathrm{Sr}$ & 2.110 & 0.180 \\
\hline Water & $137 \mathrm{Cs}$ & 50.000 & 1.700 \\
\hline Water & $234 U$ & 0.510 & 0.030 \\
\hline Water & $238 \mathrm{U}$ & 0.520 & 0.050 \\
\hline Water & $\mathrm{Bq} \mathrm{U}$ & 1.050 & 0.080 \\
\hline Water & ug U & 0.040 & 0.003 \\
\hline Water & $238 \mathrm{Pu}$ & 1.100 & 0.010 \\
\hline Water & $239 \mathrm{Pu}$ & 1.410 & 0.040 \\
\hline Water & $241 \mathrm{Am}$ & 1.250 & 0.080 \\
\hline Water & $63 \mathrm{Ni}$ & 95.700 & 0.900 \\
\hline
\end{tabular}

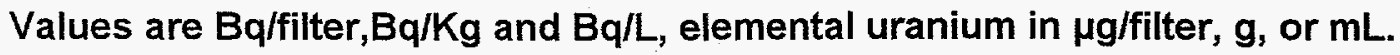
$\mathrm{pCi} / \mathrm{g}$ or $\mathrm{mL}=\mathrm{Bq} \times 0.027$ 
QAP 49 EML Results

Environmental Measurements Laboratory, New York, NY

Matrix

Water

Water

Vegetation

Vegetation

Vegetation

Vegetation

Vegetation

Vegetation

Vegetation

Vegetation
Radionuclide

Gross Alpha

Gross Beta

$40 \mathrm{~K}$

$60 \mathrm{Co}$

$90 \mathrm{Sr}$

$137 \mathrm{Cs}$

$238 \mathrm{Pu}$

$239 \mathrm{Pu}$

$241 \mathrm{Am}$

$244 \mathrm{Cm}$
EML Value

1080.000

1420.000

460.000

20.000

606.000

390.000

0.310

3.720

2.330

1.760
EML Error

60.000

60.000

20.000

1.000

40.000

20.000

0.070

0.270

0.060

0.070

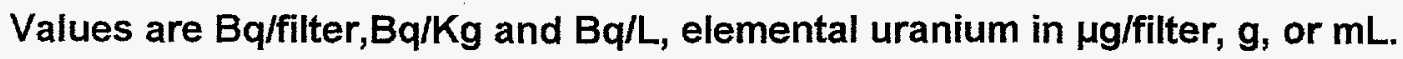
$\mathrm{pCi} / \mathrm{g}$ or $\mathrm{mL}=\mathrm{Bq} \times 0.027$ 
Lab: AC Analytical Chemistry Laboratory, Argonne National Lab

\begin{tabular}{|c|c|c|c|c|c|c|c|}
\hline $\begin{array}{l}\text { No. } \\
\text { Test }\end{array}$ & Radionuclide & $\begin{array}{c}\text { Reported } \\
\text { Value }\end{array}$ & $\begin{array}{c}\text { Reported } \\
\text { Error }\end{array}$ & $\begin{array}{l}\text { EML } \\
\text { Value } \\
\end{array}$ & $\begin{array}{l}\text { EML } \\
\text { Error }\end{array}$ & $\frac{\text { Reported }}{\text { EML }}$ & Evalua \\
\hline \multicolumn{8}{|c|}{ Matrix: SO Soil Bq/kg } \\
\hline $\begin{array}{l}1 \\
1\end{array}$ & $\begin{array}{l}\text { SR90 } \\
\text { Ig U }\end{array}$ & $\begin{array}{r}39.600 \\
8.780\end{array}$ & $\begin{array}{l}2.600 \\
0.880\end{array}$ & $\begin{array}{r}39.630 \\
9.700\end{array}$ & $\begin{array}{l}0.003 \\
0.700\end{array}$ & $\begin{array}{l}0.999 \\
0.905\end{array}$ & \\
\hline
\end{tabular}

Matrix: WA Water $\mathrm{Bq} / \mathrm{L}$

$\begin{array}{llllllll}1 & \text { SR90 } & 2.140 & 0.090 & 2.110 & 0.180 & 1.014 & \mathrm{~A} \\ 1 & \text { ug U } & 0.044 & 0.004 & 0.040 & 0.003 & 1.100 & \mathrm{~A}\end{array}$




\section{QAP 49 Results by Laboratory}

Lab: AF Air Force Analytical Lab, Brooks AFB

\begin{tabular}{lccccc} 
No. & Reported & Reported & EML & EML & Reported \\
Test & Radionuclide & $\begin{array}{l}\text { Value } \\
\text { Error }\end{array}$ & Value & $\begin{array}{c}\text { Error } \\
\text { EML }\end{array}$ Evaluation Evaluation \\
\hline
\end{tabular}

Matrix: AI Air Filter $\mathrm{Bq} /$ filter

$\begin{array}{llrrrrrrr}1 & \text { AM241 } & 0.140 & 0.020 & 0.510 & 0.008 & 0.275 & \mathrm{~N} & \text { W } \\ 1 & \text { Bq U } & 0.510 & 0.060 & 0.530 & 0.020 & 0.962 & \mathrm{~A} & \\ 1 & \text { CO60 } & 7.870 & 0.880 & 9.160 & 0.580 & 0.859 & \mathrm{~A} & \mathrm{~A} \\ 1 & \text { CS137 } & 19.580 & 2.140 & 22.470 & 1.030 & 0.871 & \mathrm{~A} & \mathrm{~A} \\ 1 & \text { MN54 } & 4.500 & 0.600 & 4.920 & 0.400 & 0.915 & \mathrm{~A} & \mathrm{~A} \\ 1 & \text { PU238 } & 0.107 & 0.020 & 0.460 & 0.005 & 0.233 & \mathrm{~N} & \\ 1 & \text { PU239 } & 0.120 & 0.020 & 0.420 & 0.006 & 0.286 & \mathrm{~N} & \mathrm{~W} \\ 1 & \text { SB125 } & 8.120 & 1.220 & 8.890 & 0.550 & 0.913 & \mathrm{~A} & \mathrm{~A} \\ 1 & \text { SR90 } & 1.160 & 0.160 & 1.120 & 0.050 & 1.036 & \mathrm{~A} & \mathrm{~W}\end{array}$

Matrix: SO Soil $\mathrm{Bq} / \mathrm{kg}$

$\begin{array}{ll}1 & \text { AC228 } \\ 1 & \text { AM241 } \\ 1 & \text { BI212 } \\ 1 & \text { BI214 } \\ 1 & \text { Bq U } \\ 1 & \text { CS137 } \\ 1 & \text { K40 } \\ 1 & \text { PB210 } \\ 1 & \text { PB212 } \\ 1 & \text { PB214 } \\ 1 & \text { PU239 } \\ 1 & \text { RA226 } \\ 1 & \text { SR90 } \\ 1 & \text { TH228 } \\ 1 & \text { TH234 } \\ 1 & \text { TL208 }\end{array}$

46.620
7.400
37.370
22.200
121.730
723.350
231.990
35.150
39.590
29.600
9.990
113.220
48.100
103.230
67.340
16.650

$\begin{array}{rr}8.510 & 52.600 \\ 1.480 & 7.470 \\ 17.760 & 58.300 \\ 9.620 & 28.800 \\ 11.870 & 237.000 \\ 83.990 & 954.000 \\ 44.770 & 314.000 \\ 6.230 & 32.000 \\ 7.400 & 52.800 \\ 6.290 & 29.100 \\ 2.590 & 13.090 \\ 96.200 & 29.000 \\ 18.500 & 39.630 \\ 49.580 & 52.700 \\ 54.020 & 114.000 \\ 3.330 & 18.300\end{array}$

2.900
0.410
5.900
0.500
16.000
38.000
13.000
3.300
3.700
1.200
0.570
1.000
0.003
4.000
6.000
1.100

0.886

0.991

0.641

0.771

0.514

0.758

0.739

1.098

0.750

1.017

0.763

3.904

1.214

1.959

0.591

0.910

$\begin{array}{ll}\text { A } & \\ \text { A } & \text { N } \\ \text { A } & \\ \text { A } & \\ \text { W } & \\ \text { N } & \text { W } \\ \text { N } & \text { A } \\ \text { A } & \\ \text { A } & \\ \text { A } & \\ \text { W } & \text { A } \\ \text { A } & \\ \text { A } & \text { N } \\ \text { A } & \\ \text { A } & \\ \text { A } & \end{array}$

Matrix: VE Vegetation $\mathrm{Bq} / \mathrm{kg}$

$\begin{array}{ll}1 & \text { AM241 } \\ 1 & \text { CO60 } \\ 1 & \text { CS137 } \\ 1 & \text { K40 } \\ 1 & \text { PU239 } \\ 1 & \text { SR90 }\end{array}$

6.290
31.450
444.000
1400.450
4.070
551.300

1.110

27.010

58.830

407.370

0.740

22.200

$\begin{array}{rr}2.330 & 0.060 \\ 20.000 & 1.000 \\ 390.000 & 20.000 \\ 460.000 & 20.000 \\ 3.720 & 0.270 \\ 606.000 & 40.000\end{array}$

0.060

20.000

0.000

40.000
2.700
1.572
1.138
3.044
1.094
0.910

W
$\mathrm{N}$
$\mathrm{A}$
$\mathrm{N}$
$\mathrm{A}$
$\mathrm{A}$

Matrix: WA Water $\mathrm{Bq} / \mathrm{L}$

$\begin{array}{llr}1 & \text { AM241 } & 1.050 \\ 1 & \text { Bq U } & 1.170 \\ 1 & \text { CO60 } & 0.210 \\ 1 & \text { CS137 } & 0.200 \\ 1 & \text { GROSS ALPHA } & 1110.000 \\ 1 & \text { GROSS BETA } & 1235.800\end{array}$

$$
\begin{array}{rr}
0.170 & 1.250 \\
0.110 & 1.050 \\
0.020 & 49.400 \\
0.030 & 50.000 \\
59.200 & 1080.000 \\
44.400 & 1420.000
\end{array}
$$

0.840
1.114
0.004
0.004
1.028
0.870

W
A
N
$\mathrm{N}$
$\mathrm{A}$
$\mathrm{A}$

A

$\mathrm{N}$

A

A

A

$\mathrm{N}$

Values for elemental uranium are reported in $\mu \mathrm{g} /$ filter, $\mathrm{g}$, or $\mathrm{mL}$.

$\mathrm{pCi} / \mathrm{g}$ or $\mathrm{mL}=\mathrm{Bq} \times 0.027$

Evaluation: $A=$ Acceptable, $W=$ Acceptable with Warning, $N=$ Not Acceptable

If the evaluation system is not appropriate for the types of analyses performed in your lab, apply a site specific evaluation. 


\section{QAP 49 Results by Laboratory}

Lab: AF Air Force Analytical Lab, Brooks AFB

No.

Reported Reported EML

EML

Reported

QAP 48

Test Radionuclide

Value

Error

Value

Error EML

Evaluation

Evaluation

Matrix: WA Water $\mathrm{Bq} / \mathrm{L}$

$\begin{array}{llrrrrrrr}1 & \text { H3 } & 1498.500 & 3.700 & 76.200 & 2.900 & 19.665 & \mathrm{~N} & \mathrm{~N} \\ 1 & \text { MN54 } & 0.140 & 0.020 & 32.400 & 1.400 & 0.004 & \mathrm{~N} & \mathrm{~A} \\ 1 & \text { PU238 } & 0.890 & 0.140 & 1.100 & 0.010 & 0.809 & \mathrm{~W} & \mathrm{~W} \\ 1 & \text { PU239 } & 1.440 & 0.200 & 1.410 & 0.040 & 1.021 & \mathrm{~A} & \mathrm{~A} \\ 1 & \text { SR90 } & 2.250 & 0.210 & 2.110 & 0.180 & 1.066 & \mathrm{~A} & \mathrm{~A}\end{array}$

Values for elemental uranium are reported in $\mu \mathrm{g} /$ filter, $\mathrm{g}$, or $\mathrm{mL}$. $\mathrm{pCi} / \mathrm{g}$ or $\mathrm{mL}=\mathrm{Bq} \times 0.027$

Evaluation: $A=$ Acceptable, $W=$ Acceptable with Warning, $N=$ Not Acceptable

If the evaluation system is not appropriate for the types of analyses performed in your lab, apply a site specific evaluation. 


\section{QAP 49 Results by Laboratory}

Lab: AG Paragon Analytics, Inc, Fort Collins, CO

\begin{tabular}{|c|c|c|c|c|c|c|c|}
\hline Test & Radionuclide & $\begin{array}{l}\text { Reported } \\
\text { Value }\end{array}$ & $\begin{array}{l}\text { Reported } \\
\text { Error }\end{array}$ & $\begin{array}{l}\text { EML } \\
\text { Value }\end{array}$ & $\begin{array}{l}\text { EML } \\
\text { Error }\end{array}$ & $\frac{\text { Reported }}{\text { EML }}$ & $\begin{array}{c}\text { QAP 48 } \\
\text { Evaluation }\end{array}$ \\
\hline
\end{tabular}

Matrix: AI Air Filter $\mathrm{Bq} /$ filter

$\begin{array}{llrlrllll}1 & \text { AM241 } & 0.226 & 0.030 & 0.510 & 0.008 & 0.443 & \mathrm{~N} & \text { W } \\ 1 & \text { Bq U } & 0.526 & 0.045 & 0.530 & 0.020 & 0.992 & \mathrm{~A} & \mathrm{~A} \\ 1 & \text { CO60 } & 8.500 & 1.700 & 9.160 & 0.580 & 0.928 & \mathrm{~A} & \mathrm{~A} \\ 1 & \text { CS137 } & 22.300 & 4.100 & 22.470 & 1.030 & 0.992 & \mathrm{~A} & \mathrm{~A} \\ 1 & \text { MN54 } & 5.000 & 1.300 & 4.920 & 0.400 & 1.016 & \mathrm{~A} & \mathrm{~A} \\ 1 & \text { PU238 } & 0.310 & 0.040 & 0.460 & 0.005 & 0.674 & \mathrm{~N} & \mathrm{~W} \\ 1 & \text { PU239 } & 0.303 & 0.040 & 0.420 & 0.006 & 0.721 & \mathrm{~N} & \mathrm{~A} \\ 1 & \text { SB125 } & 9.600 & 2.200 & 8.890 & 0.550 & 1.080 & \mathrm{~A} & \mathrm{~A} \\ 1 & \text { SR90 } & 1.000 & 0.180 & 1.120 & 0.050 & 0.893 & \mathrm{~A} & \mathrm{~A} \\ 1 & \text { U234 } & 0.250 & 0.032 & 0.260 & 0.010 & 0.962 & \mathrm{~A} & \mathrm{~W} \\ 1 & \text { U238 } & 0.262 & 0.033 & 0.260 & 0.010 & 1.008 & \mathrm{~A} & \mathrm{~A} \\ 1 & \text { ug U } & 20.600 & 3.400 & 20.960 & 0.100 & 0.983 & \mathrm{~A} & \mathrm{~A}\end{array}$

Matrix: SO Soil $\mathrm{Bq} / \mathrm{kg}$

$\begin{array}{ll}1 & \text { AC228 } \\ 1 & \text { AM241 } \\ 1 & \text { BI212 } \\ 1 & \text { BI214 } \\ 1 & \text { Bq U } \\ 1 & \text { CS137 } \\ 1 & \text { K40 } \\ 1 & \text { PB212 } \\ 1 & \text { PB214 } \\ 1 & \text { PU239 } \\ 1 & \text { SR90 } \\ 1 & \text { TH228 } \\ 1 & \text { TH234 } \\ 1 & \text { TL208 } \\ 1 & \text { U234 } \\ 1 & \text { U238 } \\ 1 & \text { ug U }\end{array}$

$\begin{array}{rrr}58.000 & 11.000 & 52.600 \\ 8.600 & 2.000 & 7.470 \\ 57.000 & 28.000 & 58.300 \\ 32.600 & 7.800 & 28.800 \\ 212.000 & 18.000 & 237.000 \\ 1173.000 & 200.000 & 954.000 \\ 396.000 & 74.000 & 314.000 \\ 60.000 & 11.000 & 52.800 \\ 35.000 & 7.400 & 29.100 \\ 13.400 & 2.100 & 13.090 \\ 45.300 & 9.300 & 39.630 \\ 59.000 & 32.000 & 52.700 \\ 110.000 & 31.000 & 114.000 \\ 21.500 & 4.400 & 18.300 \\ 102.000 & 13.000 & 113.000 \\ 105.000 & 13.000 & 120.000 \\ 8.900 & 1.500 & 9.700\end{array}$

2.900
0.410
5.900
0.500
16.000
38.000
13.000
3.700
1.200
0.570
0.003
4.000
6.000
1.100
6.000
9.000
0.700

1.103
1.151
0.978
1.132
0.895
1.230
1.261
1.136
1.203
1.024
1.143
1.120
0.965
1.175
0.903
0.875
0.918

A

A $\quad$ A

A

A

A $\quad$ A

$\mathrm{W} \quad \mathrm{A}$

A

A

A $\quad$ A

A $\quad$ A

A

A

A

A $\quad$ A

A A

A A

Matrix: VE Vegetation $\mathrm{Bq} / \mathrm{kg}$

$\begin{array}{ll}1 & \text { AM241 } \\ 1 & \text { CM244 } \\ 1 & \text { CO60 } \\ 1 & \text { CS137 } \\ 1 & \text { K40 } \\ 1 & \text { PU239 } \\ 1 & \text { SR90 }\end{array}$

Matrix: WA Water $\mathrm{Bq} / \mathrm{L}$

2.230
2.090
21.600
480.000
516.000
3.930
644.000

0.460

0.440

4.100

81.000

93.000

0.560

115.000

2.330
1.760
20.000
390.000
460.000
3.720
606.000

0.060
0.070
1.000
20.000
20.000
0.270
40.000

$$
\begin{aligned}
& 0.957 \\
& 1.188 \\
& 1.080 \\
& 1.231 \\
& 1.122 \\
& 1.056 \\
& 1.063
\end{aligned}
$$

$\begin{array}{ll}\mathrm{A} & \mathrm{A} \\ \mathrm{A} & \mathrm{A} \\ \mathrm{A} & \mathrm{A} \\ \mathrm{A} & \mathrm{A} \\ \mathrm{A} & \mathrm{A} \\ \mathrm{A} & \mathrm{A} \\ \mathrm{A} & \mathrm{A}\end{array}$

$1 \quad$ AM241

1.280

0.160

1.250

0.080

1.024

A

A

Values for elemental uranium are reported in $\mu \mathrm{g} /$ filter, $\mathrm{g}$, or $\mathrm{mL}$.

$\mathrm{pCi} / \mathrm{g}$ or $\mathrm{mL}=\mathrm{Bq} \times 0.027$

Evaluation: $A=$ Acceptable, $W=$ Acceptable with $W$ arning, $N=$ Not Acceptable

If the evaluation system is not appropriate for the types of analyses performed in your lab, apply a site specific evaluation. 


\section{QAP 49 Results by Laboratory}

Lab: AG Paragon Analytics, Inc, Fort Collins, CO

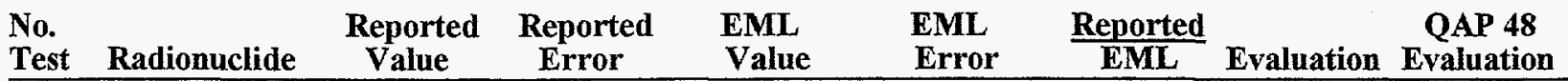

Matrix: WA Water $\mathrm{Bq} / \mathrm{L}$

$\begin{array}{llrrrrrrr}1 & \text { Bq U } & 1.080 & 0.100 & 1.050 & 0.080 & 1.029 & \mathrm{~A} & \mathrm{~A} \\ 1 & \text { CO60 } & 53.400 & 8.500 & 49.400 & 1.200 & 1.081 & \mathrm{~A} & \mathrm{~A} \\ 1 & \text { CS137 } & 51.600 & 8.500 & 50.000 & 1.700 & 1.032 & \mathrm{~A} & \mathrm{~A} \\ 1 & \text { H3 } & 79.000 & 21.000 & 76.200 & 2.900 & 1.037 & \mathrm{~A} & \mathrm{~A} \\ 1 & \text { MN54 } & 35.200 & 5.900 & 32.400 & 1.400 & 1.086 & \mathrm{~A} & \mathrm{~A} \\ 1 & \text { PU238 } & 1.180 & 0.150 & 1.100 & 0.010 & 1.073 & \mathrm{~A} & \mathrm{~W} \\ 1 & \text { PU239 } & 1.430 & 0.180 & 1.410 & 0.040 & 1.014 & \mathrm{~A} & \mathrm{~A} \\ 1 & \text { SR90 } & 1.890 & 0.340 & 2.110 & 0.180 & 0.896 & \mathrm{~A} & \mathrm{~A} \\ 1 & \text { U234 } & 0.531 & 0.073 & 0.510 & 0.030 & 1.041 & \mathrm{~A} & \mathrm{~A} \\ 1 & \text { U238 } & 0.523 & 0.072 & 0.520 & 0.050 & 1.006 & \mathrm{~A} & \mathrm{~A} \\ 1 & \text { ugU } & 0.043 & 0.007 & 0.040 & 0.003 & 1.077 & \mathrm{~A} & \mathrm{~A}\end{array}$

Values for elemental uranium are reported in $\mu \mathrm{g} /$ filter, $\mathrm{g}$, or $\mathrm{mL}$.

$\mathrm{pCi} / \mathrm{g}$ or $\mathrm{mL}=\mathrm{Bq} \times 0.027$

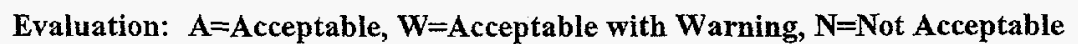

If the evaluation system is not appropriate for the types of analyses performed in your lab, apply a site specific evaluation. 


\section{QAP 49 Results by Laboratory}

Lab: AI Nuclear Technology Services, Inc., Roswell, GA

\begin{tabular}{|c|c|c|c|c|}
\hline Radionuclide & $\begin{array}{l}\text { Reported } \\
\text { Value }\end{array}$ & $\begin{array}{l}\text { Reported } \\
\text { Error }\end{array}$ & $\begin{array}{l}\text { EML } \\
\text { Value }\end{array}$ & $\begin{array}{cc}\text { QAP 48 } \\
\text { Evaluation } \\
\text { Evaluation }\end{array}$ \\
\hline
\end{tabular}

Matrix: $\mathrm{AI}$ Air Filter $\mathrm{Bq} /$ filter

$\begin{array}{llllllll}1 & \text { GROSS ALPHA } & 1.280 & 0.051 & 1.650 & 0.160 & 0.776 & \text { W } \\ 1 & \text { GROSS BETA } & 1.700 & 0.050 & 2.160 & 0.070 & 0.787 & \text { W }\end{array}$

Matrix: SO Soil $\mathrm{Bq} / \mathrm{kg}$

$\begin{array}{llrrrrrrr}1 & \text { AC228 } & 51.500 & 3.450 & 52.600 & 2.900 & 0.979 & \mathrm{~A} & \\ 1 & \text { AM241 } & 11.000 & 2.000 & 7.470 & 0.410 & 1.473 & \mathrm{~A} & \mathrm{~N} \\ 1 & \text { B214 } & 38.900 & 2.190 & 28.800 & 0.500 & 1.351 & \mathrm{~A} & \mathrm{~N} \\ 1 & \text { CS137 } & 1010.000 & 6.160 & 954.000 & 38.000 & 1.059 & \mathrm{~A} & \mathrm{~W} \\ 1 & \text { K40 } & 389.000 & 17.700 & 314.000 & 13.000 & 1.239 & \mathrm{~A} & \mathrm{~W} \\ 1 & \text { PB212 } & 12.600 & 2.260 & 52.800 & 3.700 & 0.239 & \mathrm{~A} & \\ 1 & \text { PB214 } & 37.000 & 2.630 & 29.100 & 1.200 & 1.271 & \mathrm{~A} & \\ 1 & \text { PU239 } & 16.400 & 1.860 & 13.090 & 0.570 & 1.253 & \mathrm{~W} & \mathrm{~A} \\ 1 & \text { RA226 } & 174.000 & 33.600 & 29.000 & 1.000 & 6.000 & \mathrm{~A} & \\ 1 & \text { TH228 } & 49.700 & 1.830 & 52.700 & 4.000 & 0.943 & \mathrm{~A} & \\ 1 & \text { TH234 } & 103.000 & 17.700 & 114.000 & 6.000 & 0.904 & \mathrm{~A} & \\ 1 & \text { TL208 } & 19.500 & 1.260 & 18.300 & 1.100 & 1.066 & \mathrm{~A} & \end{array}$

Matrix: VE Vegetation $\mathrm{Bq} / \mathrm{kg}$

$\begin{array}{ll}1 & \text { AM241 } \\ 1 & \text { CM244 } \\ 1 & \text { CO60 } \\ 1 & \text { CS137 } \\ 1 & \text { K40 } \\ 1 & \text { PU239 } \\ 1 & \text { SR90 }\end{array}$

$\begin{array}{rr}2.010 & 0.440 \\ 2.620 & 0.730 \\ 21.300 & 1.470 \\ 459.000 & 5.290 \\ 646.000 & 28.300 \\ 3.450 & 0.270 \\ 4.610 & 0.034\end{array}$

$$
\begin{array}{r}
2.330 \\
1.760 \\
20.000 \\
390.000 \\
460.000 \\
3.720 \\
606.000
\end{array}
$$

$$
\begin{array}{r}
0.060 \\
0.070 \\
1.000 \\
20.000 \\
20.000 \\
0.270 \\
40.000
\end{array}
$$

\begin{tabular}{|c|c|c|c|c|c|c|}
\hline AM241 & 0.960 & 0.013 & 1.250 & 0.080 & 0.768 & W \\
\hline $\mathrm{BqU}$ & 0.860 & 0.043 & 1.050 & 0.080 & 0.819 & W \\
\hline CÓ60 & 54.500 & 0.443 & 49.400 & 1.200 & 1.103 & A \\
\hline $\operatorname{CS} 137$ & 50.200 & 4.740 & 50.000 & 1.700 & 1.004 & A \\
\hline GROSS ALPHA & 915.000 & 70.100 & 1080.000 & 60.000 & 0.847 & A \\
\hline GROSS BETA & 1230.000 & 56.900 & 1420.000 & 60.000 & 0.866 & A \\
\hline $\mathrm{H} 3$ & 634.000 & 86.000 & 76.200 & 2.900 & 8.320 & $\mathrm{~N}$ \\
\hline MN54 & 35.000 & 0.473 & 32.400 & 1.400 & 1.080 & A \\
\hline PU238 & 0.920 & 0.023 & 1.100 & 0.010 & 0.836 & W \\
\hline PU239 & 1.210 & 0.029 & 1.410 & 0.040 & 0.858 & W \\
\hline SR90 & 1.430 & 0.322 & 2.110 & 0.180 & 0.678 & $\mathrm{~N}$ \\
\hline
\end{tabular}

$\begin{array}{ll}0.863 & \mathrm{~W} \\ 1.489 & \mathrm{~W} \\ 1.065 & \mathrm{~A} \\ 1.177 & \mathrm{~A} \\ 1.404 & \mathrm{~W} \\ 0.927 & \mathrm{~A} \\ 0.008 & \mathrm{~N}\end{array}$
A
A
A
A
A

Matrix: WA Water $\mathrm{Bq} / \mathrm{L}$

\section{Values for elemental uranium are reported in $\mu \mathrm{g} /$ filter, $\mathrm{g}$, or $\mathrm{mL} . \quad \mathrm{pCi} / \mathrm{g}$ or $\mathrm{mL}=\mathrm{Bq} \times 0.027$}

Evaluation: $A=A c c e p t a b l e, W=A c c e p t a b l e$ with Warning, $N=$ Not Acceptable

If the evaluation system is not appropriate for the types of analyses performed in your lab, apply a site specific evaluation. 


\section{QAP 49 Results by Laboratory}

Lab: AL Ames Laboratory, Ames, IA

No.

Reported Reported

EML

EML

Reported

QAP 48

Test Radionuclide Value Error

Value EML

Evaluation Evaluation

Matrix: AI Air Filter $\mathrm{Bq} /$ filter

$\begin{array}{llrrrrrrr}1 & \text { CO60 } & 9.905 & 0.300 & 9.160 & 0.580 & 1.081 & \mathrm{~A} & \mathrm{~A} \\ 1 & \text { CS137 } & 22.556 & 0.100 & 22.470 & 1.030 & 1.004 & \mathrm{~A} & \mathrm{~W} \\ 1 & \text { GROSS ALPHA } & 1.683 & 0.400 & 1.650 & 0.160 & 1.020 & \mathrm{~A} & \mathrm{~A} \\ 1 & \text { GROSS BETA } & 1.866 & 0.300 & 2.160 & 0.070 & 0.864 & \mathrm{~W} & \mathrm{~W} \\ 1 & \text { MN54 } & 5.053 & 0.500 & 4.920 & 0.400 & 1.027 & \mathrm{~A} & \mathrm{~W} \\ 1 & \text { SB125 } & 6.185 & 0.600 & 8.890 & 0.550 & 0.696 & \mathrm{~W} & \mathrm{~N}\end{array}$

Matrix: $\mathrm{SO}$ Soil $\mathrm{Bq} / \mathrm{kg}$

$1 \quad \operatorname{CS} 137$

$1 \mathrm{~K} 40$

1 RA226

0.070

0.500

3.400

954.000

314.000

29.000

38.000

13.000

1.000

Matrix: VE Vegetation $\mathrm{Bq} / \mathrm{kg}$

$1 \quad \mathrm{CO} 60$

$1 \mathrm{CS} 137$

$1 \mathrm{~K} 40$
36.373

763.800

870.140
0.800

0.100

0.700

20.000

390.000

460.000
1.875

1.963

10.632

$\begin{array}{ll}\mathrm{N} & \mathrm{N} \\ \mathrm{N} & \mathrm{N}\end{array}$

Matrix: WA Water $\mathrm{Bq} / \mathrm{L}$

$\begin{array}{ll}1 & \mathrm{CO} 60 \\ 1 & \mathrm{CS} 137\end{array}$

1 MN54
71.470
79.025
45.536

0.200

0.200

0.300
49.400
50.000
32.400
1.819

1.958

1.892

$\mathrm{N}$
$\mathrm{N}$

$\mathrm{N}$

$\mathrm{N}$

N




\begin{tabular}{|c|c|c|c|c|c|c|c|}
\hline $\begin{array}{l}\text { No. } \\
\text { Test }\end{array}$ & Radionuclide & $\begin{array}{c}\text { Reported } \\
\text { Value }\end{array}$ & $\begin{array}{c}\text { Reported } \\
\text { Error }\end{array}$ & $\begin{array}{l}\text { EML } \\
\text { Value }\end{array}$ & $\begin{array}{l}\text { EML } \\
\text { Error }\end{array}$ & $\frac{\text { Reported }}{\text { EML }}$ & $\begin{array}{c}\text { QAP 48 } \\
\text { Evaluation }\end{array}$ \\
\hline
\end{tabular}

Matrix: AI Air Filter Bq/filter

$\begin{array}{llrrrrrrr}1 & \text { AM241 } & 0.480 & 0.050 & 0.510 & 0.008 & 0.941 & \mathrm{~A} & \mathrm{~N} \\ 1 & \text { Bq U } & 0.940 & 0.020 & 0.530 & 0.020 & 1.774 & \mathrm{~W} & \mathrm{~W} \\ 1 & \text { CO60 } & 7.250 & 0.110 & 9.160 & 0.580 & 0.791 & \mathrm{~W} & \mathrm{~A} \\ 1 & \text { CS137 } & 17.940 & 0.180 & 22.470 & 1.030 & 0.798 & \mathrm{~W} & \mathrm{~A} \\ 1 & \text { GROSS ALPHA } & 2.470 & 0.020 & 1.650 & 0.160 & 1.497 & \mathrm{~W} & \mathrm{~W} \\ 1 & \text { GROSS BETA } & 2.980 & 0.030 & 2.160 & 0.070 & 1.380 & \mathrm{~A} & \mathrm{~W} \\ 1 & \text { MN54 } & 4.150 & 0.110 & 4.920 & 0.400 & 0.843 & \mathrm{~A} & \mathrm{~A} \\ 1 & \text { PU238 } & 0.270 & 0.010 & 0.460 & 0.005 & 0.587 & \mathrm{~N} & \\ 1 & \text { PU239 } & 0.340 & 0.010 & 0.420 & 0.006 & 0.810 & \mathrm{~W} & \\ 1 & \text { SB125 } & 5.720 & 0.280 & 8.890 & 0.550 & 0.643 & \mathrm{~W} & \mathrm{~W} \\ 1 & \text { SR90 } & 0.830 & 0.080 & 1.120 & 0.050 & 0.741 & \mathrm{~W} & \\ 1 & \text { U234 } & 0.480 & 0.020 & 0.260 & 0.010 & 1.846 & \mathrm{~W} & \mathrm{~N} \\ 1 & \text { U238 } & 0.470 & 0.020 & 0.260 & 0.010 & 1.808 & \mathrm{~W} & \mathrm{~N}\end{array}$

Matrix: SO Soil $\mathrm{Bq} / \mathrm{kg}$

$\begin{array}{ll}1 & \text { AC228 } \\ 1 & \text { AM241 } \\ 1 & \text { BI212 } \\ 1 & \text { BI214 } \\ 1 & \text { Bq U } \\ 1 & \text { CS137 } \\ \text { I } & \text { K40 } \\ 1 & \text { PB210 } \\ 1 & \text { PB212 } \\ 1 & \text { PB214 } \\ 1 & \text { PU239 } \\ 1 & \text { RA226 } \\ 1 & \text { SR90 } \\ 1 & \text { TH228 } \\ 1 & \text { TH234 } \\ 1 & \text { TL208 } \\ 1 & \text { U234 } \\ 1 & \text { U238 }\end{array}$

Matrix: VE Vegetation $\mathrm{Bq} / \mathrm{kg}$

$\begin{array}{rrr}51.050 & 5.180 & 52.600 \\ 10.320 & 1.850 & 7.470 \\ 30.330 & 7.030 & 58.300 \\ 40.320 & 2.590 & 28.800 \\ 146.010 & 13.310 & 237.000 \\ 1049.940 & 9.990 & 954.000 \\ 372.180 & 38.850 & 314.000 \\ 72.880 & 31.440 & 32.000 \\ 60.300 & 1.470 & 52.800 \\ 36.260 & 3.700 & 29.100 \\ 12.570 & 2.220 & 13.090 \\ 54.750 & 12.210 & 29.000 \\ 54.750 & 8.510 & 39.630 \\ 29.510 & 2.600 & 52.700 \\ 139.110 & 31.450 & 114.000 \\ 16.270 & 0.730 & 18.300 \\ 68.470 & 18.200 & 113.000 \\ 72.540 & 18.600 & 120.000\end{array}$

3.840

23.310

432.850

547.540

2.180

406.210

$\begin{array}{rr}1.850 & 2.330 \\ 2.960 & 20.000 \\ 6.280 & 390.000 \\ 35.520 & 460.000 \\ 0.630 & 3.720 \\ 73.990 & 606.000\end{array}$

2.900
0.410
5.900
0.500
16.000
38.000
13.000
3.300
3.700
1.200
0.570
1.000
0.003
4.000
6.000
1.100
6.000
9.000

0.971
1.382
0.520
1.400
0.616
1.101
1.185
2.277
1.142
1.246
0.960
1.888
1.382
0.560
1.220
0.889
0.606
0.604

$\begin{array}{ll}\text { A } & \\ \text { A } & \text { W } \\ \text { A } & \\ \text { A } & \\ \text { A } & \text { W } \\ \text { A } & \text { A } \\ \text { A } & \text { A } \\ \text { A } & \\ \text { A } & \\ \text { A } & \\ \text { A } & \\ \text { A } & \\ \text { A } & \\ \text { A } & \\ \text { A } & \\ \text { A } & \\ \text { W } & \text { W } \\ \text { W } & \text { W }\end{array}$

Values for elemental uranium are reported in $\mu \mathrm{g} /$ filter, $\mathrm{g}$, or $\mathrm{mL}$.

$\mathrm{pCi} / \mathrm{g}$ or $\mathrm{mL}=\mathrm{Bq} \times 0.027$

Evaluation: $A=A c c e p t a b l e, W=A c c e p t a b l e$ with Warning, $N=$ Not Acceptable

If the evaluation system is not appropriate for the types of analyses performed in your lab, apply a site specific evaluation. 


\section{QAP 49 Results by Laboratory}

Lab: AM American Radiation Services, Inc., Baton Rouge

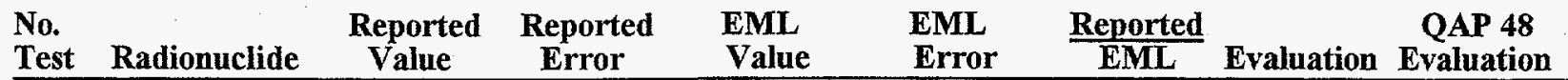

Matrix: WA Water $\mathrm{Bq} / \mathrm{L}$

$\begin{array}{llrrrrrrr}1 & \text { AM241 } & 2.180 & 0.530 & 1.250 & 0.080 & 1.744 & \mathrm{~N} & \mathrm{~A} \\ 1 & \text { Bq U } & 1.060 & 0.220 & 1.050 & 0.080 & 1.010 & \mathrm{~A} & \mathrm{~A} \\ 1 & \text { CO60 } & 49.570 & 0.630 & 49.400 & 1.200 & 1.003 & \mathrm{~A} & \mathrm{~W} \\ 1 & \text { CS137 } & 53.390 & 0.750 & 50.000 & 1.700 & 1.068 & \mathrm{~A} & \mathrm{~W} \\ 1 & \text { GROSS ALPHA } & 1139.370 & 7.740 & 1080.000 & 60.000 & 1.055 & \mathrm{~A} & \mathrm{~W} \\ 1 & \text { GROSS BETA } & 1801.150 & 8.170 & 1420.000 & 60.000 & 1.268 & \mathrm{~A} & \mathrm{~A} \\ 1 & \text { H3 } & 323.100 & 15.000 & 76.200 & 2.900 & 4.240 & \mathrm{~N} & \\ 1 & \text { MN54 } & 30.240 & 3.030 & 32.400 & 1.400 & 0.933 & \mathrm{~A} & \mathrm{~A} \\ 1 & \text { PU238 } & 0.580 & 0.020 & 1.100 & 0.010 & 0.527 & \mathrm{~N} & \\ 1 & \text { PU239 } & 0.820 & 0.020 & 1.410 & 0.040 & 0.582 & \mathrm{~N} & \\ 1 & \text { SR90 } & 1.860 & 0.020 & 2.110 & 0.180 & 0.882 & \mathrm{~W} & \\ 1 & \text { U234 } & 0.590 & 0.220 & 0.510 & 0.030 & 1.157 & \mathrm{~A} & \mathrm{~W} \\ 1 & \text { U238 } & 0.490 & 0.220 & 0.520 & 0.050 & 0.942 & \mathrm{~A} & \mathrm{~A}\end{array}$

Values for elemental uranium are reported in $\mu \mathrm{g} /$ filter, $\mathrm{g}$, or $\mathrm{mL}$.

$\mathrm{pCi} / \mathrm{g}$ or $\mathrm{mL}=\mathrm{Bq} \times 0.027$

Evaluation: A=Acceptable, $W=$ Acceptable with Warning, $N=$ Not Acceptable

If the evaluation system is not appropriate for the types of analyses performed in your lab, apply a site specific evaluation. 


\title{
QAP 49 Results by Laboratory
}

Lab: AN Argonne National Laboratory

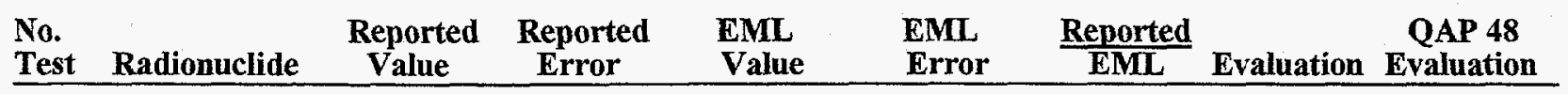

Matrix: AI Air Filter $\mathrm{Bq} /$ filter

$\begin{array}{llrrrrrrr}1 & \text { AM241 } & 0.530 & 0.020 & 0.510 & 0.008 & 1.039 & \text { A } & \text { A } \\ 1 & \text { CO60 } & 9.720 & 0.530 & 9.160 & 0.580 & 1.061 & \text { A } & \text { W } \\ 1 & \text { CS137 } & 22.600 & 0.700 & 22.470 & 1.030 & 1.006 & \text { A } & \text { A } \\ 1 & \text { MN54 } & 5.300 & 0.350 & 4.920 & 0.400 & 1.077 & \text { A } & \text { A } \\ 1 & \text { PU238 } & 0.520 & 0.020 & 0.460 & 0.005 & 1.130 & \text { A } & \text { A } \\ 1 & \text { PU239 } & 0.470 & 0.020 & 0.420 & 0.006 & 1.119 & \text { A } & \text { A } \\ 1 & \text { SB125 } & 10.590 & 0.930 & 8.890 & 0.550 & 1.191 & \text { A } & \text { A } \\ 1 & \text { SR90 } & 1.220 & 0.020 & 1.120 & 0.050 & 1.089 & \text { A } & \text { A } \\ 1 & \text { U234 } & 0.290 & 0.010 & 0.260 & 0.010 & 1.115 & \text { A } & \text { A } \\ 1 & \text { U238 } & 0.280 & 0.010 & 0.260 & 0.010 & 1.077 & \text { A } & \end{array}$

Matrix: SO Soil $\mathrm{Bq} / \mathrm{kg}$

$\begin{array}{ll}1 & \text { AM241 } \\ 1 & \text { CS137 } \\ 1 & \text { K40 } \\ 1 & \text { PU239 } \\ 1 & \text { SR90 } \\ 1 & \text { TH228 } \\ 1 & \text { U234 } \\ 1 & \text { U238 }\end{array}$

Matrix: WA Water $\mathrm{Bq} / \mathrm{L}$

7.400
1092.000
363.000
13.640
44.800
55.800
174.000
186.000

$\begin{array}{rr}0.450 & 7.470 \\ 4.000 & 954.000 \\ 11.000 & 314.000 \\ 0.600 & 13.090 \\ 0.900 & 39.630 \\ 4.000 & 52.700 \\ 19.000 & 113.000 \\ 22.000 & 120.000\end{array}$

1.250

50.600

50.800

79.400

34.800

1.170

1.570

2.160

0.520

0.510

$\begin{array}{lr}0.030 & 1.250 \\ 2.100 & 49.400 \\ 0.800 & 50.000 \\ 3.000 & 76.200 \\ 0.700 & 32.400 \\ 0.060 & 1.100 \\ 0.080 & 1.410 \\ 0.050 & 2.110 \\ 0.010 & 0.510 \\ 0.010 & 0.520\end{array}$

$$
\begin{array}{r}
0.410 \\
38.000 \\
13.000 \\
0.570 \\
0.003 \\
4.000 \\
6.000 \\
9.000
\end{array}
$$

0.991
1.145
1.156
1.042
1.130
1.059
1.540
1.550

$\begin{array}{ll}\mathrm{A} & \mathrm{A} \\ \mathrm{A} & \mathrm{A} \\ \mathrm{A} & \mathrm{A} \\ \mathrm{A} & \mathrm{A} \\ \mathrm{A} & \mathrm{A} \\ \mathrm{A} & \\ \mathrm{N} & \mathrm{A} \\ \mathrm{N} & \mathrm{A}\end{array}$
A
A

A

W
A
A
A
A
A
A
A
A

$1 \mathrm{U} 238$

\begin{abstract}
0.080
1.200

1.700

2.900

1.400

0.010

0.040

0.180

0.030

0.050
\end{abstract}

1.000
1.024
1.016
1.042
1.074
1.064
1.113
1.024
1.020
0.981

$\begin{array}{ll}\mathrm{A} & \mathrm{A} \\ \mathrm{A} & \mathrm{A} \\ \mathrm{A} & \mathrm{A} \\ \mathrm{A} & \mathrm{A} \\ \mathrm{A} & \mathrm{A} \\ \mathrm{A} & \mathrm{A} \\ \mathrm{A} & \mathrm{A} \\ \mathrm{A} & \mathrm{A} \\ \mathrm{A} & \mathrm{A} \\ \mathrm{A} & \mathrm{A}\end{array}$

A

A




\section{QAP 49 Results by Laboratory}

Lab: AP Aberdeen Proving Ground, Aberdeen, MD

No.

Reported Reported

EML

EML

Reported

QAP 48

Test Radionuclide Value Error

Value

Error

EML

Evaluation Evaluation

Matrix: AI Air Filter $\mathrm{Bq} /$ filter

1 GROSS ALPHA 2.060

1 GROSS BETA : $\quad 2.930$

0.240

1.650

0.160

0.070

1.248

1.356

A

2.160

A

Values for elemental uranium are reported in $\mu \mathrm{g} /$ filter, $\mathrm{g}$, or $\mathrm{mL}$.

$\mathrm{pCi} / \mathrm{g}$ or $\mathrm{mL}=\mathrm{Bq} \times 0.027$

Evaluation: $A=$ Acceptable, $W=$ Acceptable with Warning, $N=$ Not Acceptable

If the evaluation system is not appropriate for the types of analyses performed in your lab, apply a site specific evaluation. 


\section{QAP 49 Results by Laboratory}

Lab: AT ATL International inc., Germantown, MD

$\begin{array}{llclll}\text { No. } & \text { Reported } \\ \text { Test } & \text { Radionuclide } & \begin{array}{c}\text { Reported } \\ \text { Value }\end{array} & \begin{array}{c}\text { EMror } \\ \text { Value }\end{array} & \begin{array}{c}\text { EML } \\ \text { Error }\end{array} & \frac{\text { Reported }}{\text { EML }} \\ \text { Evaluation Evaluation }\end{array}$

Matrix: SO Soil $\mathrm{Bq} / \mathrm{kg}$

$1 \quad$ AM241

1 CS137

$1 \mathrm{~K} 40$

7.818

970.500

380.800

1.441

81.000

7.470

954.000

314.000

41.100

Matrix: $\mathrm{VE}$ Vegetation $\mathrm{Bq} / \mathrm{kg}$

1 AM241

$1 \mathrm{CO60}$

$1 \quad \mathrm{CS} 137$

$1 \mathrm{~K} 40$

2.768

19.550

396.600

514.500

0.963

2.680

33.200

56.300

2.330
20.000
390.000

460.000

0.221

4.460

4.220

48.980

47.820

32.480

3.600
1.250

49.400

50.000

32.400
1 MN54
0.410
38.000
13.000
1.047

1.017

1.213

A

A

W
W
W

32.480

1.040

0.991

0.956

1.002

$\begin{array}{ll}\text { A } & \text { A } \\ \text { A } & \text { W } \\ \text { A } & \text { A } \\ \text { A } & \text { A }\end{array}$

Values for elemental uranium are reported in $\mu \mathrm{g} /$ filter, $\mathrm{g}$, or $\mathrm{mL}$.

$\mathrm{pCi} / \mathrm{g}$ or $\mathrm{mL}=\mathrm{Bq} \times 0.027$

Evaluation: $A=$ Acceptable,$W=$ Acceptable with Warning, $N=$ Not Acceptable

If the evaluation system is not appropriate for the types of analyses performed in your lab, apply a site specific evaluation. 


\section{QAP 49 Results by Laboratory}

Lab: AU ORISE EESD/ESSAP, Oak Ridge

\begin{tabular}{llcllll} 
No. & Reported & Reported & EML & EML & Reported & QP 48 \\
Test & Radionuclide & $\begin{array}{c}\text { Value } \\
\text { Error }\end{array}$ & $\begin{array}{c}\text { Value } \\
\text { Error }\end{array}$ & $\begin{array}{c}\text { EML } \\
\text { Evaluation Evaluation }\end{array}$ \\
\hline
\end{tabular}

Matrix: AI Air Filter $\mathrm{Bq} /$ filter

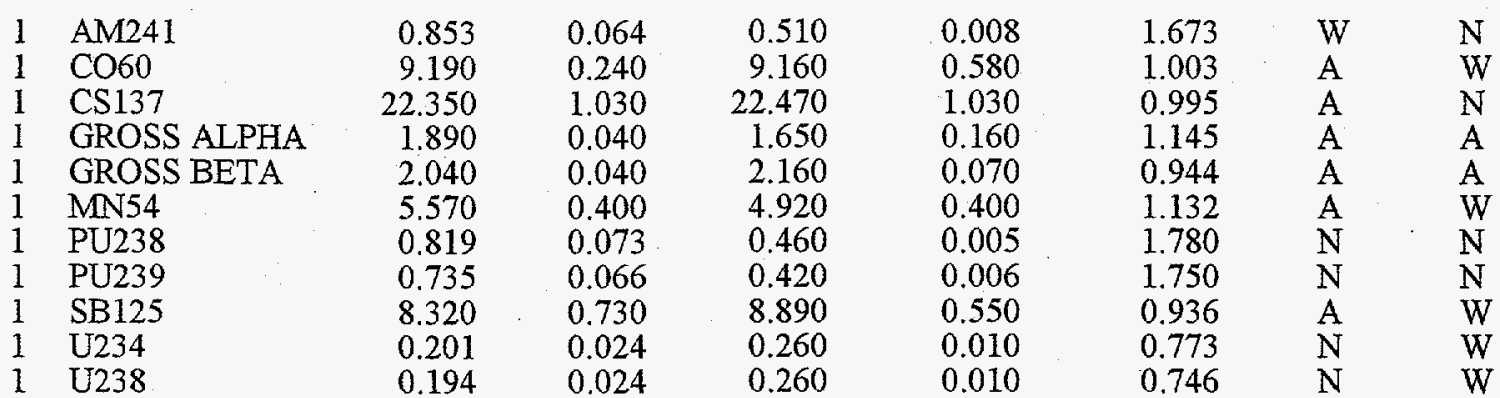

Matrix: SO Soil $\mathrm{Bq} / \mathrm{kg}$

$\begin{array}{ll}1 & \text { AM241 } \\ 1 & \text { CS137 } \\ 1 & \text { K40 } \\ 1 & \text { PU239 } \\ 1 & \text { SR90 } \\ 1 & \text { U234 } \\ 1 & \text { U238 }\end{array}$

$\begin{array}{rr}8.300 & 1.400 \\ 1113.000 & 58.000 \\ 388.000 & 24.000 \\ 12.400 & 1.900 \\ 49.000 & 7.000 \\ 102.000 & 12.000 \\ 105.000 & 14.000\end{array}$

7.470
954.000
314.000
13.090
39.630
113.000
120.000

0.410
38.000
13.000
0.570
0.003
6.000
9.000

1.111

1.167

1.236

0.947

1.236

0.903

0.875

$\begin{array}{ll}\mathrm{A} & \mathrm{A} \\ \mathrm{A} & \mathrm{A} \\ \mathrm{A} & \mathrm{A} \\ \mathrm{A} & \mathrm{A} \\ \mathrm{A} & \mathrm{A} \\ \mathrm{A} & \mathrm{A} \\ \mathrm{A} & \mathrm{A}\end{array}$

Matrix: VE Vegetation $\mathrm{Bq} / \mathrm{kg}$

$\begin{array}{ll}1 & \text { AM241 } \\ 1 & \text { CO60 } \\ 1 & \text { CS137 } \\ 1 & \text { K40 } \\ 1 & \text { PU239 } \\ 1 & \text { SR90 }\end{array}$

2.160
23.000
446.000
559.000
3.720
624.000

0.300

3.000

25.000

48.000

0.460

16.000

0.060
1.000
20.000
20.000
0.270
40.000
0.927
1.150
1.144
1.215
1.000
1.030

$\begin{array}{ll}\mathrm{A} & \mathrm{A} \\ \mathrm{A} & \mathrm{A} \\ \mathrm{A} & \mathrm{A} \\ \mathrm{A} & \mathrm{A} \\ \mathrm{A} & \mathrm{A} \\ \mathrm{A} & \mathrm{A}\end{array}$

Matrix: WA Water $\mathrm{Bq} / \mathrm{L}$

$\begin{array}{ll}1 & \text { AM241 } \\ 1 & \text { CO60 } \\ 1 & \text { CS137 } \\ 1 & \text { GROSS A } \\ 1 & \text { GROSS B } \\ 1 & \text { H3 } \\ 1 & \text { MN54 } \\ 1 & \text { PU238 } \\ 1 & \text { PU239 } \\ 1 & \text { SR90 } \\ 1 & \text { U234 } \\ 1 & \text { U238 }\end{array}$

1.350
52.000
52.000
991.000
1303.000
61.000
36.800
1.200
1.500
2.070
0.509
0.514

0.120
1.500
2.600
40.000
37.000
15.000
2.300
0.110
0.130
0.400
0.089
0.089

1.250
49.400
50.000
1080.000
1420.000
76.200
32.400
1.100
1.410
2.110
0.510
0.520

0.080
1.200
1.700
60.000
60.000
2.900
1.400
0.010
0.040
0.180
0.030
0.050

1.080
1.053
1.040
0.918
0.918
0.801
1.136
1.091
1.064
0.981
0.998
0.988

$\begin{array}{ll}\text { A } & \text { W } \\ \text { A } & \text { W } \\ \text { A } & \text { A } \\ \text { A } & \text { A } \\ \text { A } & \text { A } \\ \text { W } & \text { A } \\ \text { A } & \text { A } \\ \text { A } & \text { A } \\ \text { A } & \text { W } \\ \text { A } & \text { A } \\ \text { A } & \text { W } \\ \text { A } & \text { N }\end{array}$

\section{Values for elemental uranium are reported in $\mu \mathrm{g} /$ filter, $g$, or $\mathrm{mL}$.}

\section{$\mathrm{pCi} / \mathrm{g}$ or $\mathrm{mL}=\mathrm{Bq} \times 0.027$}

Evaluation: $A=$ Acceptable, $W=$ Acceptable with Warning, $N=N$ ot Acceptable

If the evaluation system is not appropriate for the types of analyses performed in your lab, apply a site specific evaluation. 


\section{QAP 49 Results by Laboratory}

Lab: BA Bettis Atomic Power Lab, West Mifflin, PA

\begin{tabular}{llllll} 
No. & Reported & Reported \\
Test & Radionuclide & $\begin{array}{c}\text { EML } \\
\text { Value }\end{array}$ & $\begin{array}{c}\text { EML } \\
\text { Ealue }\end{array}$ & $\begin{array}{c}\text { Reported } \\
\text { Error }\end{array}$ & $\begin{array}{c}\text { EML } \\
\text { Evaluation Evaluation }\end{array}$ \\
\hline
\end{tabular}

Matrix: AI Air Filter $\mathrm{Bq} /$ filter

$\begin{array}{llrrrrrrr}1 & \text { CO60 } & 8.970 & 0.360 & 9.160 & 0.580 & 0.979 & \mathrm{~A} & \mathrm{~A} \\ 1 & \text { CS137 } & 23.600 & 1.700 & 22.470 & 1.030 & 1.050 & \mathrm{~A} & \mathrm{~A} \\ 1 & \text { MN54 } & 5.630 & 0.440 & 4.920 & 0.400 & 1.144 & \mathrm{~A} & \mathrm{~W} \\ 1 & \text { SB125 } & 6.850 & 0.320 & 8.890 & 0.550 & 0.771 & \text { W } & \mathrm{A}\end{array}$

Matrix: SO Soil Bq/kg

$\begin{array}{llrrrrrrr}1 & \text { CS137 } & 1041.000 & 45.000 & 954.000 & 38.000 & 1.091 & \text { A } & \text { A } \\ 1 & \text { SR90 } & 24.400 & 2.330 & 39.630 & 0.003 & 0.616 & \text { W } & \end{array}$

Matrix: VE Vegetation $\mathrm{Bq} / \mathrm{kg}$

\begin{tabular}{|c|c|c|c|c|c|}
\hline $\begin{array}{ll}1 & C 060 \\
1 & \text { CS137 }\end{array}$ & $\begin{array}{r}24.700 \\
420.000\end{array}$ & $\begin{array}{r}3.900 \\
20.000\end{array}$ & $\begin{array}{r}20.000 \\
390.000\end{array}$ & $\begin{array}{r}1.000 \\
20.000\end{array}$ & $\begin{array}{l}1.235 \\
1.077\end{array}$ \\
\hline
\end{tabular}

Matrix: WA Water $\mathrm{Bq} / \mathrm{L}$

$\begin{array}{llrrrrrrr}1 & \text { CO60 } & 50.300 & 3.300 & 49.400 & 1.200 & 1.018 & \mathrm{~A} & \mathrm{~A} \\ 1 & \text { CS137 } & 53.300 & 7.000 & 50.000 & 1.700 & 1.066 & \mathrm{~A} & \mathrm{~A} \\ 1 & \text { MN54 } & 38.400 & 5.300 & 32.400 & 1.400 & 1.185 & \mathrm{~W} & \mathrm{~A} \\ 1 & \text { PU238 } & 1.260 & 0.290 & 1.100 & 0.010 & 1.145 & \mathrm{~W} & \mathrm{~A} \\ 1 & \text { PU239 } & 1.700 & 0.370 & 1.410 & 0.040 & 1.206 & \mathrm{~W} & \mathrm{~A} \\ 1 & \text { SR90 } & 2.410 & 0.270 & 2.110 & 0.180 & 1.142 & \mathrm{~A} & \mathrm{~A} \\ 1 & \text { U234 } & 0.570 & 0.100 & 0.510 & 0.030 & 1.118 & \mathrm{~A} & \\ 1 & \text { U238 } & 0.540 & 0.100 & 0.520 & 0.050 & 1.038 & \mathrm{~A} & \end{array}$

Values for elemental uranium are reported in $\mu \mathrm{g} /$ filter, $\mathrm{g}$, or $\mathrm{mL} . \quad \mathrm{pCi} / \mathrm{g}$ or $\mathrm{mL}=\mathrm{Bq} \times 0.027$

Evaluation: $A=$ Acceptable, $W=$ Acceptable with Warning, $N=$ Not Acceptable

If the evaluation system is not appropriate for the types of analyses performed in your lab, apply a site specific evaluation. 


\section{QAP 49 Results by Laboratory}

Lab: BC Babcock \& Wilcox MC \#42, Lynchburg, VA

\begin{tabular}{|c|c|c|c|c|c|c|c|}
\hline $\begin{array}{l}\text { No. } \\
\text { Test }\end{array}$ & Radio & $\begin{array}{c}\text { Reported } \\
\text { Value }\end{array}$ & $\begin{array}{c}\text { Reported } \\
\text { Error }\end{array}$ & $\begin{array}{l}\text { EML } \\
\text { Value }\end{array}$ & $\begin{array}{l}\text { EML } \\
\text { Error }\end{array}$ & $\frac{\text { Reported }}{\text { EML }}$ & $\begin{array}{c}\text { QAP 48 } \\
\text { Evaluation }\end{array}$ \\
\hline
\end{tabular}

Matrix: AI Air Filter $\mathrm{Bq} /$ filter

$\begin{array}{llrrrrrrr}1 & \text { CO60 } & 15.100 & 0.770 & 9.160 & 0.580 & 1.648 & \mathrm{~N} & \mathrm{~N} \\ 1 & \text { CS137 } & 44.200 & 3.400 & 22.470 & 1.030 & 1.967 & \mathrm{~N} & \mathrm{~N} \\ 1 & \text { GROSS ALPHA } & 2.400 & 0.050 & 1.650 & 0.160 & 1.455 & \mathrm{~W} & \mathrm{~A} \\ 1 & \text { GROSS BETA } & 2.080 & 0.040 & 2.160 & 0.070 & 0.963 & \mathrm{~A} & \mathrm{~A} \\ 1 & \text { MN54 } & 9.070 & 0.540 & 4.920 & 0.400 & 1.843 & \mathrm{~N} & \mathrm{~N} \\ 1 & \text { SB125 } & 18.600 & 0.970 & 8.890 & 0.550 & 2.092 & \mathrm{~N} & \mathrm{~N} \\ 1 & \text { SR90 } & 1.150 & 0.100 & 1.120 & 0.050 & 1.027 & \mathrm{~A} & \mathrm{~W} \\ 1 & \text { U234 } & 0.300 & 0.020 & 0.260 & 0.010 & 1.154 & \mathrm{~A} & \mathrm{~W} \\ 1 & \text { U238 } & 0.310 & 0.030 & 0.260 & 0.010 & 1.192 & \mathrm{~A} & \mathrm{~W}\end{array}$

Matrix: SO Soil $\mathrm{Bq} / \mathrm{kg}$

$\begin{array}{ll}1 & \text { CS137 } \\ 1 & \text { K40 } \\ 1 & \text { SR90 } \\ 1 & \text { U234 } \\ 1 & \text { U238 }\end{array}$

$$
\begin{array}{r}
1099.000 \\
403.000 \\
27.200 \\
165.000 \\
95.800
\end{array}
$$

$$
\begin{array}{r}
954.000 \\
314.000 \\
39.630 \\
113.000 \\
120.000
\end{array}
$$

1.152
1.283
0.686
1.460
0.798

$\begin{array}{ll}\mathrm{A} & \mathrm{A} \\ \mathrm{W} & \mathrm{W} \\ \mathrm{W} & \\ \mathrm{N} & \mathrm{A} \\ \mathrm{A} & \mathrm{A}\end{array}$

Matrix: VE Vegetation $\mathrm{Bq} / \mathrm{kg}$

$\begin{array}{ll}1 & \text { CO60 } \\ 1 & \text { CS137 } \\ 1 & \text { K40 } \\ 1 & \text { SR90 }\end{array}$

Matrix: WA Water $\mathrm{Bq} / \mathrm{L}$

$\begin{array}{ll}1 & \text { CO60 } \\ 1 & \text { CS137 } \\ 1 & \text { GROSS ALPHA } \\ 1 & \text { GROSS BETA } \\ 1 & \text { MN54 } \\ 1 & \text { SR90 } \\ 1 & \text { U234 } \\ 1 & \text { U238 }\end{array}$

21.100
447.000
559.000
337.800

1.500

29.600

31.500

22.700

1.000
20.000
20.000
40.000

606.000

$\begin{array}{rr}2.600 & 49.400 \\ 4.200 & 50.000 \\ 25.600 & 1080.000 \\ 20.000 & 1420.000 \\ 2.400 & 32.400 \\ 0.370 & 2.110 \\ 0.060 & 0.510 \\ 0.070 & 0.520\end{array}$

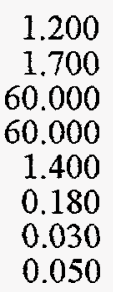

1.043
1.106
1.031
0.844
1.148
2.280
1.255
1.058
1.055

1.146

1.215

0.557 


\section{QAP 49 Results by Laboratory}

Lab: BE RUST Geotech, Grand Junction, CO

No.

Reported Reported EML EML Reported

QAP 48

Test Radionuclide

Value Error

Value

Error EML

Evaluation Evaluation

Matrix: AI Air Filter $\mathrm{Bq} /$ filter

\begin{tabular}{|c|c|c|c|c|c|c|}
\hline $\mathrm{AM} 241$ & 0.560 & 0.050 & 0.510 & 0.008 & 1.098 & A \\
\hline $\mathrm{CO} 60$ & 9.100 & 0.500 & 9.160 & 0.580 & 0.993 & A \\
\hline CS137 & 23.100 & 2.000 & 22.470 & 1.030 & 1.028 & A \\
\hline GROSS ALPHA & 1.580 & 0.090 & 1.650 & 0.160 & 0.958 & A \\
\hline GROSS BETA & 1.750 & 0.070 & 2.160 & 0.070 & 0.810 & W \\
\hline MN54 & 5.600 & 0.700 & 4.920 & 0.400 & 1.138 & A \\
\hline PU238 & 0.500 & 0.040 & 0.460 & 0.005 & 1.087 & A \\
\hline PU239 & 0.450 & 0.040 & 0.420 & 0.006 & 1.071 & A \\
\hline SB125 & 8.300 & 0.500 & 8.890 & 0.550 & 0.934 & A \\
\hline SR90 & 1.110 & 0.080 & 1.120 & 0.050 & 0.991 & A \\
\hline $\mathrm{U} 234$ & 0.250 & 0.020 & 0.260 & 0.010 & 0.962 & A \\
\hline U238 & 0.250 & 0.020 & 0.260 & 0.010 & 0.962 & A \\
\hline ug U & 20.600 & & 20.960 & 0.100 & 0.983 & A \\
\hline
\end{tabular}

Matrix: SO Soil $\mathrm{Bq} / \mathrm{kg}$

$\begin{array}{ll}1 & \text { AM241 } \\ 1 & \text { CS137 } \\ 1 & \text { K40 } \\ 1 & \text { PU239 } \\ 1 & \text { SR90 } \\ 1 & \text { U234 } \\ 1 & \text { U238 } \\ 1 & \text { ugU }\end{array}$

7.850
1069.000
390.000
13.300
41.800
105.100
108.300
8.900

0.770
150.000
80.000
1.300
4.500
7.900
8.000

7.470

954.000

314.000

13.090

39.630

113.000

120.000

9.700

Matrix: VE Vegetation $\mathrm{Bq} / \mathrm{kg}$

$\begin{array}{ll}1 & \text { AM241 } \\ 1 & \text { CM244 } \\ 1 & \text { CO60 } \\ 1 & \text { CS137 } \\ 1 & \text { K40 } \\ 1 & \text { PU239 } \\ 1 & \text { SR90 }\end{array}$

$$
\begin{array}{r}
2.540 \\
2.070 \\
22.000 \\
447.000 \\
601.000 \\
4.500 \\
635.000
\end{array}
$$

0.360

0.290

3.000

50.000

140.000

0.460

39.000

$$
\begin{array}{r}
2.330 \\
1.760 \\
20.000 \\
390.000 \\
460.000 \\
3.720 \\
606.000
\end{array}
$$

$\begin{array}{rr}1.400 & 0.140 \\ 52.200 & 4.000 \\ 53.100 & 4.000 \\ 142.200 & 6.800 \\ 1045.000 & 71.000 \\ 1122.000 & 53.000 \\ 93.000 & 7.400 \\ 38.500 & 3.000 \\ 122.200 & 1.900\end{array}$

$$
\begin{array}{r}
0.410 \\
38.000 \\
13.000 \\
0.570 \\
0.003 \\
6.000 \\
9.000 \\
0.700
\end{array}
$$

$$
\begin{array}{r}
0.060 \\
0.070 \\
1.000 \\
20.000 \\
20.000 \\
0.270 \\
40.000
\end{array}
$$

$\begin{array}{rr}0.080 & 1.120 \\ 1.200 & 1.057 \\ 1.700 & 1.062 \\ 2.000 & 1.023 \\ 60.000 & 0.968 \\ 60.000 & 0.790 \\ 2.900 & 1.220 \\ 1.400 & 1.188 \\ 0.900 & 1.277\end{array}$

1.051
1.121
1.242
1.016
1.055
0.930
0.903
0.918

$\begin{array}{ll}\mathrm{A} & \mathrm{A} \\ \mathrm{A} & \mathrm{A} \\ \mathrm{A} & \mathrm{A} \\ \mathrm{A} & \mathrm{A} \\ \mathrm{A} & \mathrm{A} \\ \mathrm{A} & \mathrm{A} \\ \mathrm{A} & \mathrm{A} \\ \mathrm{A} & \mathrm{A}\end{array}$

Matrix: WA Water $\mathrm{Bq} / \mathrm{L}$

$\begin{array}{ll}1 & \text { AM241 } \\ 1 & \text { CO60 } \\ 1 & \text { CS137 } \\ 1 & \text { FE55 } \\ 1 & \text { GROSS ALPHA } \\ 1 & \text { GROSS BETA } \\ 1 & \text { H3 } \\ 1 & \text { MN54 } \\ 1 & \text { N163 }\end{array}$

1.250
49.400
50.000
139.000
1080.000
1420.000
76.200
32.400
95.700
1.090
1.176
1.100
1.146
1.307
1.210
1.048

$\begin{array}{ll}\text { A } & \text { A } \\ \text { A } & \text { A } \\ \text { A } & \text { A } \\ \text { A } & \text { A } \\ \text { W } & \text { W } \\ \text { A } & \text { A } \\ \text { A } & \text { A }\end{array}$

\section{Values for elemental uranium are reported in $\mu \mathrm{g} /$ filter, $g$, or $\mathrm{mL}$.}

\section{$\mathrm{pCi} / \mathrm{g}$ or $\mathrm{mL}=\mathrm{Bq} \times 0.027$}

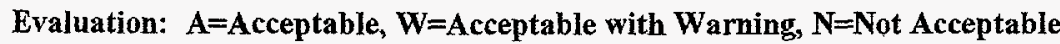

If the evaluation system is not appropriate for the types of analyses performed in your lab, apply a site specific evaluation. 


\section{QAP 49 Results by Laboratory}

Lab: BE RUST Geotech, Grand Junction, CO

\begin{tabular}{|c|c|c|c|c|c|c|c|}
\hline $\begin{array}{l}\text { No. } \\
\text { Test }\end{array}$ & adionuclide & $\begin{array}{l}\text { Reported } \\
\text { Value }\end{array}$ & $\begin{array}{l}\text { Reported } \\
\text { Error }\end{array}$ & $\begin{array}{l}\text { EML } \\
\text { Value }\end{array}$ & $\begin{array}{l}\text { EML } \\
\text { Error }\end{array}$ & $\frac{\text { Reported }}{\text { EML }}$ & $\begin{array}{c}\text { QAP 48 } \\
\text { Evaluation } \\
\text { Evaluation }\end{array}$ \\
\hline
\end{tabular}

Matrix: WA Water $\mathrm{Bq} / \mathrm{L}$

$\begin{array}{lllllllll}1 & \text { PU238 } & 1.170 & 0.110 & 1.100 & 0.010 & 1.064 & \text { A } & \text { A } \\ 1 & \text { PU239 } & 1.590 & 0.150 & 1.410 & 0.040 & 1.128 & \text { A } & \text { A } \\ 1 & \text { SR90 } & 2.200 & 0.200 & 2.110 & 0.180 & 1.043 & \text { A } & \text { A } \\ 1 & \text { U234 } & 0.530 & 0.060 & 0.510 & 0.030 & 1.039 & \text { A } & \text { A } \\ 1 & \text { U238 } & 0.530 & 0.060 & 0.520 & 0.050 & 1.019 & \text { A } & \text { A } \\ 1 & \text { ug U } & 0.042 & & 0.040 & 0.003 & 1.060 & \text { A } & \text { A }\end{array}$

Values for elemental uranium are reported in $\mu \mathrm{g} /$ filter, $\mathrm{g}$, or $\mathrm{mL}$.

$\mathrm{pCi} / \mathrm{g}$ or $\mathrm{mL}=\mathrm{Bq} \times 0.027$

Evaluation: $A=A c c e p t a b l e, W=A c c e p t a b l e$ with Warning, $N=$ Not Acceptable

If the evaluation system is not appropriate for the types of analyses performed in your lab, apply a site specific evaluation. 


\section{QAP 49 Results by Laboratory}

Lab: BL Barringer Laboratories Inc., Golden, CO

\begin{tabular}{|c|c|c|c|c|c|c|c|}
\hline st & Radionuclide & $\begin{array}{c}\text { Reported } \\
\text { Value }\end{array}$ & $\begin{array}{c}\text { Reported } \\
\text { Error }\end{array}$ & $\begin{array}{l}\text { EML } \\
\text { Value }\end{array}$ & $\begin{array}{l}\text { EML } \\
\text { Error }\end{array}$ & $\frac{\text { Reported }}{\text { EML }}$ & $\begin{array}{cc}\text { QAP 48 } \\
\text { Evaluation } \\
\text { Evaluation }\end{array}$ \\
\hline
\end{tabular}

Matrix: AI Air Filter $\mathrm{Bq} /$ filter

$\begin{array}{llrlrllll}\text { I } & \text { AM241 } & 0.599 & 0.041 & 0.510 & 0.008 & 1.175 & \mathrm{~A} & \mathrm{~W} \\ 2 & \text { AM241 } & 0.552 & 0.035 & 0.510 & 0.008 & 1.082 & \mathrm{~A} & \mathrm{~W} \\ 1 & \text { Bq U } & 0.528 & 0.008 & 0.530 & 0.020 & 0.996 & \mathrm{~A} & \mathrm{~A} \\ \mathrm{I} & \text { CO60 } & 10.000 & 0.300 & 9.160 & 0.580 & 1.092 & \mathrm{~A} & \mathrm{~A} \\ 1 & \text { CS137 } & 26.700 & 0.800 & 22.470 & 1.030 & 1.188 & \mathrm{~W} & \mathrm{~A} \\ 1 & \text { GROSS ALPHA } & 1.560 & 0.030 & 1.650 & 0.160 & 0.945 & \mathrm{~A} & \mathrm{~A} \\ 1 & \text { GROSS BETA } & 1.700 & 0.030 & 2.160 & 0.070 & 0.787 & \mathrm{~W} & \mathrm{~A} \\ 1 & \text { MN54 } & 5.980 & 0.220 & 4.920 & 0.400 & 1.215 & \mathrm{~W} & \mathrm{~A} \\ 1 & \text { PU238 } & 0.522 & 0.039 & 0.460 & 0.005 & 1.135 & \mathrm{~A} & \mathrm{~A} \\ 2 & \text { PU238 } & 0.518 & 0.041 & 0.460 & 0.005 & 1.126 & \mathrm{~A} & \mathrm{~A} \\ 1 & \text { PU239 } & 0.476 & 0.037 & 0.420 & 0.006 & 1.133 & \mathrm{~A} & \mathrm{~A} \\ 2 & \text { PU239 } & 0.444 & 0.037 & 0.420 & 0.006 & 1.057 & \mathrm{~A} & \mathrm{~A} \\ 1 & \text { SB125 } & 10.200 & 0.500 & 8.890 & 0.550 & 1.147 & \mathrm{~A} & \mathrm{~A} \\ 1 & \text { SR90 } & 1.220 & 0.240 & 1.120 & 0.050 & 1.089 & \mathrm{~A} & \mathrm{~A} \\ 2 & \text { SR90 } & 1.090 & 0.310 & 1.120 & 0.050 & 0.973 & \mathrm{~A} & \mathrm{~A} \\ 1 & \text { U234 } & 0.261 & 0.004 & 0.260 & 0.010 & 1.004 & \mathrm{~A} & \mathrm{~A} \\ 1 & \text { U238 } & 0.254 & 0.004 & 0.260 & 0.010 & 0.977 & \mathrm{~A} & \mathrm{~A} \\ 1 & \text { ug U } & 21.070 & 0.330 & 20.960 & 0.100 & 1.005 & \mathrm{~A} & \end{array}$

Matrix: SO Soil $\mathrm{Bq} / \mathrm{kg}$

$\begin{array}{ll}1 & \text { AC228 } \\ 1 & \text { AM241 } \\ 1 & \text { BI212 } \\ 1 & \text { BI214 } \\ 1 & \text { Bq U } \\ 2 & \text { Bq U } \\ 1 & \text { CS137 } \\ 1 & \text { K40 } \\ 1 & \text { PB210 } \\ 1 & \text { PB212 } \\ 1 & \text { PB214 } \\ 1 & \text { PU239 } \\ 2 & \text { PU239 } \\ 1 & \text { RA226 } \\ 1 & \text { SR90 } \\ 2 & \text { SR90 } \\ 1 & \text { TH228 } \\ 1 & \text { TH234 } \\ 1 & \text { TL208 } \\ 1 & \text { U234 } \\ 2 & \text { U234 } \\ 2 & \text { U238 } \\ 1 & \text { U238 } \\ 1 & \text { ug U } \\ 2 & \text { ug U }\end{array}$

58.000
3.680
58.000
31.000
223.000
213.000
1097.000
355.000
27.700
62.100
35.500
15.500
13.100
44.400
34.000
39.700
60.300
141.000
19.100
110.000
105.000
103.000
108.000
8.910
8.510

$\begin{array}{rr}5.200 & 52.600 \\ 1.270 & 7.470 \\ 2.100 & 58.300 \\ 2.100 & 28.800 \\ & 237.000 \\ 3.000 & 237.000 \\ 40.000 & 954.000 \\ 16.000 & 314.000 \\ 5.800 & 32.000 \\ 3.700 & 52.800 \\ 2.300 & 29.100 \\ 0.500 & 13.090 \\ 0.600 & 13.090 \\ 3.300 & 29.000 \\ 4.400 & 39.630 \\ 4.900 & 39.630 \\ 2.200 & 52.700 \\ 10.000 & 114.000 \\ 1.400 & 18.300 \\ & 113.000 \\ 2.000 & 113.000 \\ 2.000 & 120.000 \\ & 120.000 \\ & 9.700 \\ 0.140 & 9.700\end{array}$

2.900
0.410
5.900
0.500
16.000
16.000
38.000
13.000
3.300
3.700
1.200
0.570
0.570
1.000
0.003
0.003
4.000
6.000
1.100
6.000
6.000
9.000
9.000
0.700
0.700

1.103

0.493

0.995

1.076

0.941

0.899

1.150

1.131

0.866

1.176

1.220

1.184

1.001

1.531

0.858

1.002

1.144

1.237

1.044

0.973

0.929

0.858

0.900

0.919

0.877
A
A
A
A

A

A

A

A $\quad$ A

A A

A A

A A

Values for elemental uranium are reported in $\mu \mathrm{g} /$ filter, $\mathrm{g}$, or $\mathrm{mL}$.

$\mathrm{pCi} / \mathrm{g}$ or $\mathrm{mL}=\mathrm{Bq} \times \mathbf{0 . 0 2 7}$

Evaluation: $A=A c c e p t a b l e, W=A c c e p t a b l e$ with Warning, $N=$ Not Acceptable

If the evaluation system is not appropriate for the types of analyses performed in your lab, apply a site specific evaluation. 


\section{QAP 49 Results by Laboratory}

Lab: BL Barringer Laboratories Inc., Golden, CO

\begin{tabular}{|c|c|c|c|c|c|c|c|}
\hline $\begin{array}{l}\text { No. } \\
\text { Test }\end{array}$ & Radionuclide & $\begin{array}{l}\text { Reported } \\
\text { Value }\end{array}$ & $\begin{array}{c}\text { Reported } \\
\text { Error }\end{array}$ & $\begin{array}{l}\text { EML } \\
\text { Value }\end{array}$ & $\begin{array}{l}\text { EML } \\
\text { Error }\end{array}$ & $\frac{\text { Reported }}{\text { EML }}$ & $\begin{array}{cc}\text { QAP 48 } \\
\text { Evaluation } \\
\text { Evaluation }\end{array}$ \\
\hline
\end{tabular}

Matrix: VE Vegetation $\mathrm{Bq} / \mathrm{kg}$

$\begin{array}{ll}1 & \text { AM241 } \\ 1 & \text { CM244 } \\ 1 & \text { CO60 } \\ 1 & \text { CS137 } \\ 1 & \text { K40 } \\ 2 & \text { PU239 } \\ 1 & \text { PU239 } \\ 1 & \text { SR90 }\end{array}$

$\begin{array}{rr}2.930 & 0.510 \\ 1.330 & 0.250 \\ 20.000 & 1.400 \\ 438.000 & 16.000 \\ 483.000 & 25.000 \\ 3.950 & 0.370 \\ 4.370 & 0.240 \\ 417.000 & 18.000\end{array}$

2.330
1.760
20.000
390.000
460.000
3.720
3.720
606.000

0.060
0.070
1.000
20.000
20.000
0.270
0.270
40.000

1.258
0.756
1.000
1.123
1.050
1.062
1.175
0.688

$\begin{array}{ll}\text { A } & \text { N } \\ \text { W } & \text { N } \\ \text { A } & \text { A } \\ \text { A } & \text { A } \\ \text { A } & \text { A } \\ \text { A } & \text { A } \\ \text { A } & \text { A } \\ \text { W } & \text { A }\end{array}$

Matrix: WA Water $\mathrm{Bq} / \mathrm{L}$

$\begin{array}{llrrrrrrr}2 & \text { AM241 } & 1.270 & 0.220 & 1.250 & 0.080 & 1.016 & \mathrm{~A} & \mathrm{~A} \\ 1 & \text { AM241 } & 1.510 & 0.230 & 1.250 & 0.080 & 1.208 & \mathrm{~A} & \mathrm{~A} \\ 1 & \text { CO60 } & 51.000 & 2.100 & 49.400 & 1.200 & 1.032 & \mathrm{~A} & \mathrm{~A} \\ 1 & \text { CS137 } & 52.000 & 2.100 & 50.000 & 1.700 & 1.040 & \mathrm{~A} & \mathrm{~A} \\ 1 & \text { FE55 } & 148.000 & 5.000 & 139.000 & 2.000 & 1.065 & \mathrm{~A} & \mathrm{~W} \\ 1 & \text { GROSS ALPHA } & 956.000 & 14.000 & 1080.000 & 60.000 & 0.885 & \mathrm{~A} & \mathrm{~W} \\ 1 & \text { GROSS BETA } & 1055.000 & 14.000 & 1420.000 & 60.000 & 0.743 & \mathrm{~A} & \mathrm{~A} \\ 1 & \text { H3 } & 81.800 & 4.100 & 76.200 & 2.900 & 1.073 & \mathrm{~A} & \mathrm{~W} \\ 1 & \text { MN54 } & 35.100 & 1.500 & 32.400 & 1.400 & 1.083 & \mathrm{~A} & \mathrm{~A} \\ 1 & \text { NI63 } & 103.000 & 3.000 & 995.700 & 0.900 & 1.076 & \mathrm{~A} & \\ 1 & \text { PU238 } & 1.470 & 0.120 & 1.100 & 0.010 & 1.336 & \mathrm{~N} & \mathrm{~W} \\ 2 & \text { PU238 } & 1.340 & 0.130 & 1.100 & 0.010 & 1.218 & \mathrm{~W} & \mathrm{~W} \\ 1 & \text { PU239 } & 1.680 & 0.130 & 1.410 & 0.040 & 1.191 & \mathrm{~W} & \mathrm{~A} \\ 2 & \text { PU239 } & 1.790 & 0.140 & 1.410 & 0.040 & 1.270 & \mathrm{~W} & \mathrm{~A} \\ 2 & \text { SR90 } & 2.280 & 0.630 & 2.110 & 0.180 & 1.081 & \mathrm{~A} & \mathrm{~A} \\ 1 & \text { SR90 } & 2.170 & 0.500 & 2.110 & 0.180 & 1.028 & \mathrm{~A} & \mathrm{~A} \\ 1 & \text { U234 } & 0.510 & 0.010 & 0.510 & 0.030 & 1.000 & \mathrm{~A} & \mathrm{~A} \\ 1 & \text { U238 } & 0.495 & 0.010 & 0.520 & 0.050 & 0.952 & \mathrm{~A} & \mathrm{~W} \\ 1 & \text { ug U } & 1.030 & 0.020 & 0.040 & 0.003 & 25.750 & \mathrm{~N} & \\ 2 & \text { ug U } & 0.041 & 0.001 & 0.040 & 0.003 & 1.025 & \mathrm{~A} & \end{array}$

\footnotetext{
Values for elemental uranium are reported in $\mu \mathrm{g} /$ filter, $\mathrm{g}$, or $\mathrm{mL}$.
}

$\mathrm{pCi} / \mathrm{g}$ or $\mathrm{mL}=\mathrm{Bq} \times 0.027$

Evaluation: $A=$ Acceptable, $W=$ Acceptable with Warning, $N=$ Not Acceptable

If the evaluation system is not appropriate for the types of analyses performed in your lab, apply a site specific evaluation. 


\section{QAP 49 Results by Laboratory}

Lab: BM Battelle Memorial Institute, Columbus, $\mathrm{OH}$

No.

Reported Reported

EML

EMIL

Reported

QAP 48

Test Radionuclide Value

Error

Error EML

Evaluation Evaluation

Matrix: AI Air Filter $\mathrm{Bq} /$ filter

$\begin{array}{ll}1 & \text { AM241 } \\ 1 & \text { CO60 } \\ 1 & \text { CS137 } \\ 1 & \text { PU238 } \\ 1 & \text { PU239 } \\ 1 & \text { SR90 } \\ 1 & \text { U234 } \\ 1 & \text { U238 }\end{array}$

0.460
9.260
22.200
0.497
0.472
1.140
0.260
0.270

0.060

0.280

0.330

0.070

0.064

0.040

0.040

0.040

0.510
9.160
22.470
0.460
0.420
1.120
0.260
0.260

1.180

7.000

2.500

2.700

14.600

40.600

110.000

114.400

15.200

7.470

954.000

13.090

39.630

113.000

120.000

0.008
0.580
1.030
0.005
0.006
0.050
0.010
0.010
0.902
1.011
0.988
1.080
1.124
1.018
1.000
1.038

1.031

1.091

0.963

1.024

0.973

0.953

$\begin{array}{ll}\text { A } & \text { A } \\ \text { A } & \text { A } \\ \text { A } & \text { A } \\ \text { A } & \text { A } \\ \text { A } & \text { A } \\ \text { A } & \text { A } \\ \text { A } & \text { A } \\ \text { A } & \text { A }\end{array}$

Matrix: VE Vegetation $\mathrm{Bq} / \mathrm{kg}$

$\begin{array}{ll}1 & \text { AM241 } \\ 1 & \text { CO60 } \\ 1 & \text { CS137 } \\ 1 & \text { PU239 } \\ 1 & \text { SR90 }\end{array}$

$$
\begin{array}{r}
2.360 \\
20.500 \\
431.000 \\
3.670 \\
663.000
\end{array}
$$

$$
\begin{array}{r}
0.600 \\
1.600 \\
4.000 \\
0.730 \\
12.000
\end{array}
$$

$\begin{array}{rr}2.330 & 0.060 \\ 20.000 & 1.000 \\ 390.000 & 20.000 \\ 3.720 & 0.270 \\ 606.000 & 40.000\end{array}$
1.013
1.025
1.105
0.987
1.094

$\begin{array}{ll}\mathrm{A} & \mathrm{A} \\ \mathrm{A} & \mathrm{A} \\ \mathrm{A} & \mathrm{W} \\ \mathrm{A} & \mathrm{A} \\ \mathrm{A} & \mathrm{A} \\ \mathrm{A} & \mathrm{A}\end{array}$

Matrix: WA Water $\mathrm{Bq} / \mathrm{L}$

$\begin{array}{ll}1 & \text { AM241 } \\ 1 & \text { CO60 } \\ 1 & \text { CS137 } \\ 1 & \text { PU238 } \\ 1 & \text { PU239 } \\ 1 & \text { SR90 } \\ 1 & \text { U234 } \\ 1 & \text { U238 }\end{array}$

1.220
46.500
49.300
1.200
1.580
2.230
0.540
0.540

0.180
3.500
3.300
0.190
0.240
0.260
0.080
0.080

1.250

49.400

50.000

0.080
1.200
1.700
0.010
0.040
0.180
0.030
0.050
0.976
0.941
0.986
1.091
1.121
1.057
1.059

1.100

2.110

0.510

0.520

$\begin{array}{ll}\mathrm{A} & \mathrm{A} \\ \mathrm{A} & \mathrm{A} \\ \mathrm{A} & \mathrm{A} \\ \mathrm{A} & \mathrm{A} \\ \mathrm{A} & \mathrm{A}\end{array}$

$\mathrm{A}$
$\mathrm{A}$
$\mathrm{A}$
$\mathrm{A}$
$\mathrm{A}$
$\mathrm{A}$
$\mathrm{A}$
$\mathrm{A}$




\section{QAP 49 Results by Laboratory}

Lab: BN Brookhaven National Laboratory, Upton, NY

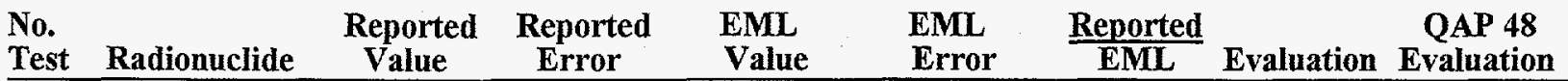

Matrix: AI Air Filter $\mathrm{Bq} /$ filter

$\begin{array}{llrrrrrrr}1 & \text { CO60 } & 12.210 & 1.010 & 9.160 & 0.580 & 1.333 & \mathrm{~N} & \mathrm{~A} \\ 2 & \text { CO60 } & 9.990 & 1.010 & 9.160 & 0.580 & 1.091 & \mathrm{~A} & \mathrm{~A} \\ 3 & \text { CO60 } & 12.030 & 1.010 & 9.160 & 0.580 & 1.313 & \mathrm{~W} & \mathrm{~A} \\ 3 & \text { CS137 } & 38.480 & 1.990 & 22.470 & 1.030 & 1.713 & \mathrm{~N} & \mathrm{~W} \\ 2 & \text { CS137 } & 33.670 & 1.990 & 22.470 & 1.030 & 1.498 & \mathrm{~N} & \mathrm{~W} \\ 1 & \text { CS137 } & 36.700 & 1.990 & 22.470 & 1.030 & 1.633 & \mathrm{~N} & \mathrm{~W} \\ 2 & \text { GROSS ALPHA } & 1.490 & 0.020 & 1.650 & 0.160 & 0.903 & \mathrm{~A} & \mathrm{~W} \\ 3 & \text { GROSS ALPHA } & 1.450 & 0.020 & 1.650 & 0.160 & 0.879 & \mathrm{~A} & \mathrm{~W} \\ 1 & \text { GROSS ALPHA } & 1.460 & 0.020 & 1.650 & 0.160 & 0.885 & \mathrm{~A} & \mathrm{~W} \\ 2 & \text { GROSS BETA } & 1.770 & 0.040 & 2.160 & 0.070 & 0.819 & \mathrm{~W} & \mathrm{~A} \\ 3 & \text { GROSS BETA } & 1.760 & 0.040 & 2.160 & 0.070 & 0.815 & \mathrm{~W} & \mathrm{~A} \\ 1 & \text { GROSS BETA } & 1.690 & 0.040 & 2.160 & 0.070 & 0.782 & \mathrm{~W} & \mathrm{~A} \\ 3 & \text { MN54 } & 8.880 & 0.660 & 4.920 & 0.400 & 1.805 & \mathrm{~N} & \mathrm{~W} \\ 2 & \text { MN54 } & 7.290 & 0.660 & 4.920 & 0.400 & 1.482 & \mathrm{~N} & \mathrm{~W} \\ 1 & \text { MN54 } & 8.360 & 0.660 & 4.920 & 0.400 & 1.699 & \mathrm{~N} & \mathrm{~W} \\ 2 & \text { SB125 } & 13.990 & 0.360 & 8.890 & 0.550 & 1.574 & \mathrm{~N} & \mathrm{~W} \\ 1 & \text { SB125 } & 14.210 & 0.360 & 8.890 & 0.550 & 1.598 & \mathrm{~N} & \mathrm{~W} \\ 3 & \text { SB125 } & 14.840 & 0.360 & 8.890 & 0.550 & 1.669 & \mathrm{~N} & \mathrm{~W}\end{array}$

Matrix: SO Soil $\mathrm{Bq} / \mathrm{kg}$

$\begin{array}{llrrrrrr}1 & \text { AC228 } & 45.030 & 0.750 & 52.600 & 2.900 & 0.856 & \mathrm{~A} \\ 2 & \text { AC228 } & 43.360 & 0.750 & 52.600 & 2.900 & 0.824 & \mathrm{~A} \\ 3 & \text { AC228 } & 43.510 & 0.750 & 52.600 & 2.900 & 0.827 & \mathrm{~A} \\ 1 & \text { BL212 } & 31.570 & 1.520 & 58.300 & 5.900 & 0.542 & \mathrm{~A} \\ 3 & \text { BI212 } & 35.280 & 1.520 & 58.300 & 5.900 & 0.605 & \mathrm{~A} \\ 2 & \text { BI212 } & 33.150 & 1.520 & 58.300 & 5.900 & 0.569 & \mathrm{~A} \\ 1 & \text { BI214 } & 25.810 & 0.580 & 28.800 & 0.500 & 0.896 & \mathrm{~A} \\ 2 & \text { BI214 } & 27.200 & 0.580 & 28.800 & 0.500 & 0.944 & \mathrm{~A} \\ 3 & \text { BI214 } & 26.260 & 0.580 & 28.800 & 0.500 & 0.912 & \mathrm{~A} \\ 1 & \text { CS137 } & 959.410 & 2.190 & 954.000 & 38.000 & 1.006 & \mathrm{~A} \\ 3 & \text { CS137 } & 959.780 & 2.190 & 954.000 & 38.000 & 1.006 & \mathrm{~A} \\ 2 & \text { CS137 } & 954.970 & 2.190 & 954.000 & 38.000 & 1.001 & \mathrm{~A} \\ 3 & \text { K40 } & 284.460 & 3.950 & 314.000 & 13.000 & 0.906 & \mathrm{~A} \\ 2 & \text { K40 } & 282.500 & 3.950 & 314.000 & 13.000 & 0.900 & \mathrm{~A} \\ 1 & \text { K40 } & 291.670 & 3.950 & 314.000 & 13.000 & 0.929 & \mathrm{~A} \\ 1 & \text { PB212 } & 51.280 & 0.690 & 52.800 & 3.700 & 0.971 & \mathrm{~A} \\ 2 & \text { PB212 } & 49.950 & 0.690 & 52.800 & 3.700 & 0.946 & \mathrm{~A} \\ 3 & \text { PB212 } & 49.730 & 0.690 & 52.800 & 3.700 & 0.942 & \mathrm{~A} \\ 1 & \text { PB214 } & 26.850 & 1.790 & 29.100 & 1.200 & 0.923 & \mathrm{~A} \\ 2 & \text { PB214 } & 27.380 & 1.790 & 29.100 & 1.200 & 0.941 & \mathrm{~A} \\ 3 & \text { PB214 } & 30.900 & 1.790 & 29.100 & 1.200 & 1.062 & \mathrm{~A} \\ 1 & \text { TL208 } & 16.420 & 0.990 & 18.300 & 1.100 & 0.897 & \mathrm{~A} \\ 2 & \text { TL208 } & 18.710 & 0.990 & 18.300 & 1.100 & 1.022 & \mathrm{~A} \\ 3 & \text { TL208 } & 16.920 & 0.990 & 18.300 & 1.100 & 0.925 & \mathrm{~A}\end{array}$
A
A
A
A
A
A

Values for elemental uranium are reported in $\mu \mathrm{g} /$ filter, $\mathrm{g}$, or $\mathrm{mL}$.

$\mathrm{pCi} / \mathrm{g}$ or $\mathrm{mL}=\mathrm{Bq} \times 0.027$

Evaluation: A=Acceptable, W=Acceptable with Warning, $N=$ Not Acceptable

If the evaluation system is not appropriate for the types of analyses performed in your lab, apply a site specific evaluation. 


\section{QAP 49 Results by Laboratory}

Lab: BN Brookhaven National Laboratory, Upton, NY

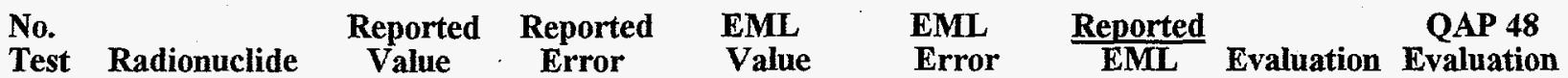

Matrix: VE Vegetation $\mathrm{Bq} / \mathrm{kg}$

$\begin{array}{llrrrrrrr}1 & \text { CO60 } & 18.630 & 0.380 & 20.000 & 1.000 & 0.931 & \mathrm{~A} & \mathrm{~A} \\ 2 & \text { CO60 } & 18.160 & 0.380 & 20.000 & 1.000 & 0.908 & \mathrm{~A} & \mathrm{~A} \\ 3 & \text { CO60 } & 17.700 & 0.380 & 20.000 & 1.000 & 0.885 & \mathrm{~A} & \mathrm{~A} \\ 2 & \text { CS137 } & 435.860 & 1.490 & 390.000 & 20.000 & 1.118 & \mathrm{~A} & \mathrm{~A} \\ 1 & \text { CS137 } & 432.530 & 1.490 & 390.000 & 20.000 & 1.109 & \mathrm{~A} & \mathrm{~A} \\ 3 & \text { CS137 } & 432.900 & 1.490 & 390.000 & 20.000 & 1.110 & \mathrm{~A} & \mathrm{~A} \\ 3 & \text { K40 } & 444.370 & 8.970 & 460.000 & 20.000 & 0.966 & \mathrm{~A} & \mathrm{~A} \\ 1 & \text { K40 } & 451.030 & 8.970 & 460.000 & 20.000 & 0.980 & \mathrm{~A} & \mathrm{~A} \\ 2 & \mathrm{~K} 40 & 429.570 & 8.970 & 460.000 & 20.000 & 0.934 & \mathrm{~A} & \mathrm{~A}\end{array}$

Matrix: WA Water $\mathrm{Bq} / \mathrm{L}$

\begin{tabular}{|c|c|c|c|c|c|c|}
\hline $\mathrm{CO} 60$ & 54.170 & 1.090 & 49.400 & 1.200 & 1.097 & A \\
\hline CO60 & 52.840 & 1.090 & 49.400 & 1.200 & 1.070 & A \\
\hline $\mathrm{CO} 60$ & 51.500 & 1.090 & 49.400 & 1.200 & 1.043 & A \\
\hline CS137 & 61.680 & 0.190 & 50.000 & 1.700 & 1.234 & W \\
\hline CS137 & 61.240 & 0.190 & 50.000 & 1.700 & 1.225 & W \\
\hline $\mathrm{CS} 137$ & 61.310 & 0.190 & 50.000 & 1.700 & 1.226 & W \\
\hline GROSS ALPHA & 8.410 & 0.600 & 1080.000 & 60.000 & 0.008 & $\mathrm{~N}$ \\
\hline GROSS ALPHA & 8.720 & 0.600 & 1080.000 & 60.000 & 0.008 & $\mathrm{~N}$ \\
\hline GROSS ALPHA & 9.820 & 0.600 & 1080.000 & 60.000 & 0.009 & $\mathrm{~N}$ \\
\hline GROSS BETA & 12.770 & 0.270 & 1420.000 & 60.000 & 0.009 & $\mathrm{~N}$ \\
\hline GROSS BETA & 12.430 & 0.270 & 1420.000 & 60.000 & 0.009 & $\mathrm{~N}$ \\
\hline GROSS BETA & 12.090 & 0.270 & 1420.000 & 60.000 & 0.009 & $\mathrm{~N}$ \\
\hline $\mathrm{H} 3$ & 57.310 & 3.020 & 76.200 & 2.900 & 0.752 & W \\
\hline $\mathrm{H} 3$ & 50.410 & 3.020 & 76.200 & 2.900 & 0.662 & $\mathrm{~N}$ \\
\hline $\mathrm{H} 3$ & 56.180 & 3.020 & 76.200 & 2.900 & 0.737 & W \\
\hline MN54 & 39.410 & 1.360 & 32.400 & 1.400 & 1.216 & W \\
\hline MN54 & 39.180 & 1.360 & 32.400 & 1.400 & 1.209 & W \\
\hline MN54 & 42.180 & 1.360 & 32.400 & 1.400 & 1.302 & $N$ \\
\hline SR90 & 7.070 & 0.300 & 2.110 & 0.180 & 3.351 & $N$ \\
\hline SR90 & 7.660 & 0.300 & 2.110 & 0.180 & 3.630 & $\mathrm{~N}$ \\
\hline
\end{tabular}

\section{Values for elemental uranium are reported in $\mu \mathrm{g} /$ filter, $\mathrm{g}$, or $\mathrm{mL} . \quad \mathrm{pCi} / \mathrm{g}$ or $\mathrm{mL}=\mathrm{Bq} \times 0.027$}

Evaluation: $A=$ Acceptable, $W=$ Acceptable with Warning, $N=$ Not Acceptable

If the evaluation system is not appropriate for the types of analyses performed in your lab, apply a site specific evaluation. 


\section{QAP 49 Results by Laboratory}

Lab: BP Battelle Pacific Northwest National Laboratory

\begin{tabular}{|c|c|c|c|c|c|c|c|}
\hline $\begin{array}{l}\text { No. } \\
\text { Test }\end{array}$ & Radionuclide & $\begin{array}{l}\text { Reported } \\
\text { Value }\end{array}$ & $\begin{array}{l}\text { Reported } \\
\text { Error }\end{array}$ & $\begin{array}{l}\text { EML } \\
\text { Value }\end{array}$ & $\begin{array}{l}\text { EML } \\
\text { Error }\end{array}$ & $\frac{\text { Reported }}{\text { EML }}$ & $\begin{array}{cc}\text { QAP 48 } \\
\text { Evaluation } \\
\text { Evaluation }\end{array}$ \\
\hline
\end{tabular}

Matrix: AI Air Filter Bq / filter

$\begin{array}{llrlrllll}1 & \text { AM241 } & 0.575 & 0.019 & 0.510 & 0.008 & 1.127 & \mathrm{~A} & \mathrm{~A} \\ \mathrm{I} & \text { Bq } & 0.542 & 0.014 & 0.530 & 0.020 & 1.023 & \mathrm{~A} & \\ 1 & \text { CO60 } & 9.500 & 0.200 & 9.160 & 0.580 & 1.037 & \mathrm{~A} & \mathrm{~A} \\ 1 & \text { CS137 } & 22.900 & 0.500 & 22.470 & 1.030 & 1.019 & \mathrm{~A} & \mathrm{~A} \\ 1 & \text { GROSS ALPHA } & 1.650 & 0.110 & 1.650 & 0.160 & 1.000 & \mathrm{~A} & \mathrm{~A} \\ 1 & \text { GROSS BETA } & 1.950 & 0.060 & 2.160 & 0.070 & 0.903 & \mathrm{~A} & \mathrm{~A} \\ 1 & \text { MN54 } & 5.200 & 0.100 & 4.920 & 0.400 & 1.057 & \mathrm{~A} & \mathrm{~A} \\ 1 & \text { PU238 } & 0.519 & 0.010 & 0.460 & 0.005 & 1.128 & \mathrm{~A} & \mathrm{~A} \\ 1 & \text { PU239 } & 0.489 & 0.010 & 0.420 & 0.006 & 1.164 & \mathrm{~A} & \mathrm{~A} \\ 1 & \text { SB125 } & 7.900 & 0.200 & 8.890 & 0.550 & 0.889 & \mathrm{~A} & \mathrm{~A} \\ 1 & \text { SR90 } & 1.160 & 0.090 & 1.120 & 0.050 & 1.036 & \mathrm{~A} & \mathrm{~W} \\ 1 & \text { U234 } & 0.264 & 0.010 & 0.260 & 0.010 & 1.015 & \mathrm{~A} & \\ 1 & \text { U238 } & 0.267 & 0.010 & 0.260 & 0.010 & 1.027 & \mathrm{~A} & \mathrm{~A}\end{array}$

Matrix: SO Soil $\mathrm{Bq} / \mathrm{kg}$

$\begin{array}{ll}1 & \text { AC228 } \\ 1 & \text { BI212 } \\ 1 & \text { BI214 } \\ 1 & \text { BqU } \\ 1 & \text { CS137 } \\ 1 & \text { K40 } \\ 1 & \text { PB212 } \\ 1 & \text { PB214 } \\ 1 & \text { PU239 } \\ 1 & \text { SR90 } \\ 1 & \text { TL208 } \\ 1 & U 234 \\ 1 & \text { U238 }\end{array}$

44.000
64.000
27.000
226.000
902.000
305.000
50.000
29.000
14.000
88.000
41.000
109.000
112.000

$\begin{array}{rr}3.000 & 52.600 \\ 10.000 & 58.300 \\ 3.000 & 28.800 \\ 6.000 & 237.000 \\ 18.000 & 954.000 \\ 7.000 & 314.000 \\ 8.000 & 52.800 \\ 3.000 & 29.100 \\ 0.300 & 13.090 \\ 16.000 & 39.630 \\ 7.000 & 18.300 \\ 4.000 & 113.000 \\ 4.000 & 120.000\end{array}$

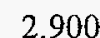

$$
5.900
$$

0.500

16.000

$\begin{array}{ll}0.837 & \mathrm{~A} \\ 1.098 & \mathrm{~A} \\ 0.938 & \mathrm{~A} \\ 0.954 & \mathrm{~A} \\ 0.945 & \mathrm{~A} \\ 0.971 & \mathrm{~A} \\ 0.947 & \mathrm{~A} \\ 0.997 & \mathrm{~A} \\ 1.070 & \mathrm{~A} \\ 2.221 & \mathrm{~W} \\ 2.240 & \mathrm{~A} \\ 0.965 & \mathrm{~A} \\ 0.933 & \mathrm{~A}\end{array}$

A

A

A

A

A

A

A

Matrix: VE Vegetation $\mathrm{Bq} / \mathrm{kg}$

$\begin{array}{ll}1 & \text { AM241 } \\ 1 & \text { CM244 } \\ 1 & \text { CO60 } \\ 1 & \text { CS137 } \\ 1 & \text { K40 } \\ 1 & \text { PU239 } \\ 1 & \text { SR90 }\end{array}$

2.670
1.620
18.000
374.000
451.000
4.100
651.000

0.170

0.160

0.400

8.000

9.000

0.400

88.000
38.000

13.000

3.700

1.200

0.570

0.003

1.100

6.000

$$
\begin{array}{r}
0.060 \\
0.070 \\
1.000 \\
20.000 \\
20.000 \\
0.270 \\
40.000
\end{array}
$$
1.146
0.920
0.900
0.959
0.980
1.102
1.074

$\begin{array}{ll}\text { A } & \text { A } \\ \text { A } & \text { A } \\ \text { A } & \text { A } \\ \text { A } & \text { A } \\ \text { A } & \text { A } \\ \text { A } & \text { A } \\ \text { A } & \text { A }\end{array}$

A

A

$\stackrel{\mathrm{A}}{\mathrm{W}}$

$\begin{array}{lll}1.144 & \mathrm{~A} & \mathrm{~A} \\ 1.076 & \mathrm{~A} & \\ 1.018 & \mathrm{~A} & \mathrm{~A} \\ 1.042 & \mathrm{~A} & \mathrm{~A}\end{array}$

0.080
0.080
1.200
1.700

Matrix: WA Water $\mathrm{Bq} / \mathrm{L}$

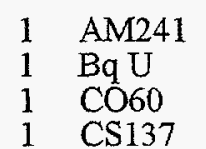

1 CS137

\begin{abstract}
1.430
1.130

50.300

52.100
\end{abstract}

$$
\begin{array}{r}
1.250 \\
1.050 \\
49.400 \\
50.000
\end{array}
$$

$\mathrm{pCi} / \mathrm{g}$ or $\mathrm{mL}=\mathrm{Bq} \times 0.027$

Values for elemental uranium are reported in $\mu \mathrm{g} /$ filter, $\mathrm{g}$, or $\mathrm{mL}$.

Evaluation: $A=A c c e p t a b l e, W=A c c e p t a b l e$ with Warning, $N=$ Not Acceptable

If the evaluation system is not appropriate for the types of analyses performed in your lab, apply a site specific evaluation. 


\section{QAP 49 Results by Laboratory}

Lab: BP Battelle Pacific Northwest National Laboratory

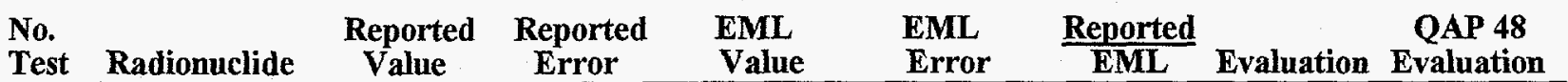

Matrix: WA Water $\mathrm{Bq} / \mathrm{L}$

$\begin{array}{llrrrrrrr}1 & \text { FE55 } & 145.000 & 10.000 & 139.000 & 2.000 & 1.043 & \mathrm{~A} & \mathrm{~A} \\ 1 & \text { GROSS ALPHA } & 1066.000 & 26.000 & 1080.000 & 60.000 & 0.987 & \mathrm{~A} & \mathrm{~A} \\ 1 & \text { GROSS BETA } & 1376.000 & 30.000 & 1420.000 & 60.000 & 0.969 & \mathrm{~A} & \mathrm{~A} \\ 1 & \text { H3 } & 80.800 & 4.300 & 76.200 & 2.900 & 1.060 & \mathrm{~A} & \mathrm{~A} \\ 1 & \text { MN54 } & 35.100 & 0.700 & 32.400 & 1.400 & 1.083 & \mathrm{~A} & \mathrm{~A} \\ 1 & \text { NI63 } & 86.000 & 10.000 & 95.700 & 0.900 & 0.899 & \mathrm{~A} & \\ 1 & \text { PU238 } & 1.230 & 0.040 & 1.100 & 0.010 & 1.118 & \mathrm{~W} & \mathrm{~A} \\ 1 & \text { PU239 } & 1.640 & 0.030 & 1.410 & 0.040 & 1.163 & \text { W } & \mathrm{A} \\ 1 & \text { U234 } & 0.560 & 0.030 & 0.510 & 0.030 & 1.098 & \mathrm{~A} & \\ 1 & \text { U238 } & 0.550 & 0.010 & 0.520 & 0.050 & 1.058 & \mathrm{~A} & \mathrm{~A}\end{array}$

\section{Values for elemental uranium are reported in $\mu \mathrm{g} /$ filter, $\mathrm{g}$, or $\mathrm{mL} . \quad \mathrm{pCi} / \mathrm{g}$ or $\mathrm{mL}=\mathrm{Bq} \times 0.027$}

Evaluation: $A=$ Acceptable, $W=$ Acceptable with $W$ arning, $N=$ Not Acceptable

If the evaluation system is not appropriate for the types of analyses performed in your lab, apply a site specific evaluation. 


\section{QAP 49 Results by Laboratory}

Lab: BQ Becquerel Laboratories Inc., Mississauga, Ontario, Canada

\begin{tabular}{|c|c|c|c|c|c|c|c|}
\hline $\begin{array}{l}\text { Jo. } \\
\text { Cest }\end{array}$ & Radionuclide & $\begin{array}{l}\text { Reported } \\
\text { Value }\end{array}$ & $\begin{array}{l}\text { Reported } \\
\text { Error }\end{array}$ & $\begin{array}{l}\text { EML } \\
\text { Value }\end{array}$ & $\begin{array}{l}\text { EML } \\
\text { Error }\end{array}$ & $\frac{\text { Reported }}{\text { EML }}$ & $\begin{array}{cc}\text { QAP 48 } \\
\text { Evaluation } \\
\text { Evaluation }\end{array}$ \\
\hline
\end{tabular}

Matrix: AI Air Filter $\mathrm{Bq} /$ filter

$\begin{array}{llrrrrrrr}1 & \text { CO60 } & 8.600 & 0.200 & 9.160 & 0.580 & 0.939 & \mathrm{~A} & \mathrm{~A} \\ 1 & \text { CS137 } & 24.400 & 0.200 & 22.470 & 1.030 & 1.086 & \mathrm{~A} & \mathrm{~A} \\ 1 & \text { GROSS ALPHA } & 3.090 & 0.080 & 1.650 & 0.160 & 1.873 & \mathrm{~N} & \\ 1 & \text { GROSS BETA } & 2.160 & 0.040 & 2.160 & 0.070 & 1.000 & \mathrm{~A} & \\ 1 & \text { MN54 } & 4.600 & 0.100 & 4.920 & 0.400 & 0.935 & \mathrm{~A} & \mathrm{~A} \\ 1 & \text { SB125 } & 9.000 & 0.300 & 8.890 & 0.550 & 1.012 & \mathrm{~A} & \mathrm{~N} \\ 1 & \text { ug U } & 26.000 & 1.000 & 20.960 & 0.100 & 1.240 & \mathrm{~A} & \mathrm{~A}\end{array}$

Matrix: SO Soil $\mathrm{Bq} / \mathrm{kg}$

$\begin{array}{llrrrrrrr}1 & \text { AC228 } & 87.000 & 30.000 & 52.600 & 2.900 & 1.654 & \mathrm{~A} & \\ 1 & \text { CS137 } & 1070.000 & 30.000 & 954.000 & 38.000 & 1.122 & \mathrm{~A} & \mathrm{~N} \\ 1 & \text { K40 } & 490.000 & 5.000 & 314.000 & 13.000 & 1.561 & \mathrm{~N} & \mathrm{~A} \\ 1 & \text { PB210 } & 33.000 & 10.000 & 32.000 & 3.300 & 1.031 & \mathrm{~A} & \\ 1 & \text { PB212 } & 52.000 & 7.000 & 52.800 & 3.700 & 0.985 & \mathrm{~A} & \\ 1 & \text { RA226 } & 39.000 & 2.000 & 29.000 & 1.000 & 1.345 & \mathrm{~A} & \\ 1 & \text { TH228 } & 53.000 & 3.000 & 52.700 & 4.000 & 1.006 & \mathrm{~A} & \\ 1 & \text { TH234 } & 120.000 & 40.000 & 114.000 & 6.000 & 1.053 & \mathrm{~A} & \\ 1 & \text { TL208 } & 30.000 & 9.000 & 18.300 & 1.100 & 1.639 & \mathrm{~A} & \\ 1 & \text { ug U } & 11.300 & 0.300 & 9.700 & 0.700 & 1.165 & \text { W } & \mathrm{A}\end{array}$

Matrix: VE Vegetation $\mathrm{Bq} / \mathrm{kg}$

$\begin{array}{llrrrrrrr}1 & \text { CO60 } & 68.000 & 20.000 & 20.000 & 1.000 & 3.400 & \text { N } & \\ 1 & \text { CS137 } & 434.000 & 17.000 & 390.000 & 20.000 & 1.113 & \text { A } & \text { A } \\ 1 & \text { K40 } & 721.000 & 7.000 & 460.000 & 20.000 & 1.567 & \text { N } & \text { W }\end{array}$

Matrix: WA Water $\mathrm{Bq} / \mathrm{L}$

$\begin{array}{llrrrrrrr}1 & \text { CO60 } & 49.000 & 1.000 & 49.400 & 1.200 & 0.992 & \mathrm{~A} & \mathrm{~A} \\ 1 & \text { CS137 } & 45.000 & 1.000 & 50.000 & 1.700 & 0.900 & \mathrm{~A} & \mathrm{~A} \\ 1 & \text { GROSS ALPHA } & 1140.000 & 40.000 & 1080.000 & 60.000 & 1.056 & \mathrm{~A} & \\ 1 & \text { GROSS BETA } & 1130.000 & 20.000 & 1420.000 & 60.000 & 0.796 & \mathrm{~A} & \\ 1 & \text { MN54 } & 30.600 & 0.900 & 32.400 & 1.400 & 0.944 & \mathrm{~A} & \mathrm{~A} \\ 1 & \text { ug U } & 0.048 & 0.002 & 0.040 & 0.003 & 1.200 & \text { W } & \mathrm{A}\end{array}$

\section{Values for elemental uranium are reported in $\mu \mathrm{g} /$ filter, $\mathrm{g}$, or $\mathrm{mL} . \quad \mathrm{pCi} / \mathrm{g}$ or $\mathrm{mL}=\mathrm{Bq} \times 0.027$}

Evaluation: $A=$ Acceptable, $W=$ Acceptable with Warning, $N=$ Not Acceptable

If the evaluation system is not appropriate for the types of analyses performed in your lab, apply a site specific evaluation. 


\section{QAP 49 Results by Laboratory}

Lab: BU Autoridad Regulatoria, Buenos Aires, Argentina

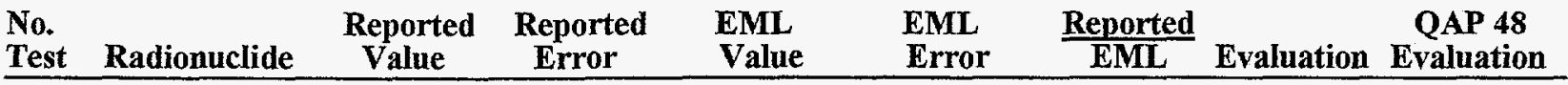

Matrix: $\mathrm{AI}$ Air Filter $\mathrm{Bq} /$ filter

$\begin{array}{llrrrrrrr}1 & \text { AM241 } & 0.600 & 0.030 & 0.510 & 0.008 & 1.176 & \mathrm{~A} & \mathrm{~A} \\ 1 & \text { Bq U } & 0.490 & 0.030 & 0.530 & 0.020 & 0.925 & \mathrm{~A} & \mathrm{~A} \\ 1 & \text { CO60 } & 9.100 & 0.500 & 9.160 & 0.580 & 0.993 & \mathrm{~A} & \mathrm{~A} \\ 1 & \text { CS137 } & 23.000 & 1.200 & 22.470 & 1.030 & 1.024 & \mathrm{~A} & \mathrm{~A} \\ 1 & \text { GROSS ALPHA } & 1.500 & 0.100 & 1.650 & 0.160 & 0.909 & \mathrm{~A} & \mathrm{~A} \\ 1 & \text { GROSS BETA } & 1.800 & 0.100 & 2.160 & 0.070 & 0.833 & \mathrm{~W} & \mathrm{~A} \\ 1 & \text { MN54 } & 4.700 & 0.800 & 4.920 & 0.400 & 0.955 & \mathrm{~A} & \mathrm{~A} \\ 1 & \text { PU238 } & 0.510 & 0.020 & 0.460 & 0.005 & 1.109 & \mathrm{~A} & \mathrm{~A} \\ 1 & \text { PU239 } & 0.460 & 0.020 & 0.420 & 0.006 & 1.095 & \mathrm{~A} & \mathrm{~A} \\ 1 & \text { SB125 } & 8.500 & 0.800 & 8.890 & 0.550 & 0.956 & \mathrm{~A} & \mathrm{~A} \\ 1 & \text { U234 } & 0.240 & 0.010 & 0.260 & 0.010 & 0.923 & \mathrm{~A} & \mathrm{~A} \\ 1 & \text { U238 } & 0.240 & 0.010 & 0.260 & 0.010 & 0.923 & \mathrm{~A} & \mathrm{~A} \\ 1 & \text { ug U } & 18.000 & 1.300 & 20.960 & 0.100 & 0.859 & \mathrm{~W} & \mathrm{~A}\end{array}$

Matrix: SO Soil $\mathrm{Bq} / \mathrm{kg}$

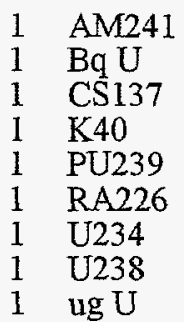

Matrix: VE Vegetation $\mathrm{Bq} / \mathrm{kg}$

$\begin{array}{ll}1 & \text { AM241 } \\ 1 & \text { CM244 } \\ 1 & \text { CO60 } \\ 1 & \text { CS137 } \\ 1 & \text { K40 } \\ 1 & \text { PU239 } \\ 1 & \text { SR90 }\end{array}$

2.900
1.800
21.000
430.000
530.000
4.300
678.000

$\begin{array}{rr}7.600 & 0.400 \\ 218.000 & 10.000 \\ 860.000 & 90.000 \\ 290.000 & 30.000 \\ 11.000 & 0.800 \\ 34.000 & 2.000 \\ 105.000 & 8.000 \\ 108.000 & 6.000 \\ 8.400 & 0.600\end{array}$

0.410

16.000

38.000

13.000

0.570

1.000

6.000

9.000

0.700
1.017

0.920

0.901

0.924

0.840

1.172

0.929

0.900

0.866

$\begin{array}{ll}\text { A } & \text { A } \\ \text { A } & \text { A } \\ \text { A } & \text { W } \\ \text { A } & \text { W } \\ \text { W } & \text { A } \\ \text { A } & \\ \text { A } & \text { A } \\ \text { A } & \text { A } \\ \text { A } & \text { A }\end{array}$

Matrix: WA Water $\mathrm{Bq} / \mathrm{L}$

$\begin{array}{llr}1 & \text { AM241 } & 1.390 \\ 1 & \text { Bq U } & 1.160 \\ 1 & \text { CO60 } & 57.000 \\ 1 & \text { CS137 } & 47.000 \\ 1 & \text { GROSS ALPHA } & 1140.000 \\ 1 & \text { GROSS BETA } & 1090.000 \\ 1 & \text { H3 } & 76.900 \\ 1 & \text { PU238 } & 1.230\end{array}$

$$
\begin{array}{r}
0.090 \\
0.060 \\
3.000 \\
3.000 \\
75.000 \\
50.000 \\
1.500
\end{array}
$$

2330

1.760

20.000

390.000

460.000

3.720

606.000

$$
\begin{array}{r}
0.060 \\
0.070 \\
1.000 \\
20.000 \\
20.000 \\
0.270 \\
40.000
\end{array}
$$

1.245
1.023
1.050
1.103
1.152
1.156
1.119

A
A
A
A
A
A
A

A
A
A
W
A
A
A

Values for elemental uranium are reported in $\mu \mathrm{g} /$ filter, $\mathrm{g}$, or $\mathrm{mL}$.

$\begin{array}{rlll}0.080 & 1.112 & \mathrm{~A} & \mathrm{~A} \\ 0.080 & 1.105 & \mathrm{~A} & \mathrm{~A} \\ 1.200 & 1.154 & \mathrm{~W} & \mathrm{~A} \\ 1.700 & 0.940 & \mathrm{~A} & \mathrm{~A} \\ 60.000 & 1.056 & \mathrm{~A} & \mathrm{~W} \\ 60.000 & 0.768 & \mathrm{~A} & \\ 2.900 & 1.009 & \mathrm{~A} & \mathrm{~A} \\ 0.010 & 1.118 & \mathrm{~W} & \mathrm{~A}\end{array}$

Evaluation: $A=A c c e p t a b l e, W=A c c e p t a b l e$ with Warning, $N=$ Not Acceptable

If the evaluation system is not appropriate for the types of analyses performed in your lab, apply a site specific evaluation. 


\section{QAP 49 Results by Laboratory}

Lab: BU Autoridad Regulatoria, Buenos Aires, Argentina

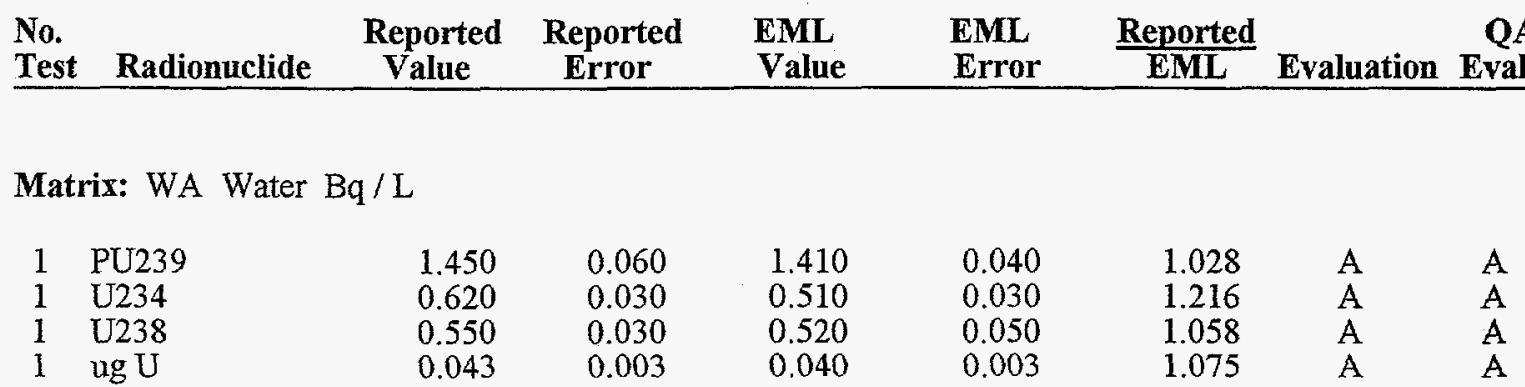

Values for elemental uranium are reported in $\mu \mathrm{g} /$ filter, $\mathrm{g}$, or $\mathrm{mL}$.

$\mathrm{pCi} / \mathrm{g}$ or $\mathrm{mL}=\mathrm{Bq} \times 0.027$

Evaluation: $A=$ Acceptable, $W=$ Acceptable with Warning, $N=$ Not Acceptable

If the evaluation system is not appropriate for the types of analyses performed in your lab, apply a site specific evaluation. 


\section{QAP 49 Results by Laboratory}

Lab: BX B\&W Nuclear Envir. Services, Lynchburg, VA

\begin{tabular}{llllll} 
No. & Reported & Reported & EML & EML & Reported \\
Test & Radionuclide & $\begin{array}{c}\text { Value } \\
\text { Error }\end{array}$ & $\begin{array}{c}\text { Value } \\
\text { Error }\end{array}$ & $\begin{array}{c}\text { EML } \\
\text { Evaluation Evaluation }\end{array}$ \\
\hline
\end{tabular}

Matrix: $\mathrm{AI}$ Air Filter $\mathrm{Bq} /$ filter

$\begin{array}{llrrrrrrr}1 & \text { AM241 } & 0.500 & 0.040 & 0.510 & 0.008 & 0.980 & \mathrm{~A} & \mathrm{~A} \\ 1 & \text { CO60 } & 16.500 & 0.010 & 9.160 & 0.580 & 1.801 & \mathrm{~N} & \mathrm{~N} \\ 1 & \text { CS137 } & 46.900 & 3.110 & 22.470 & 1.030 & 2.087 & \mathrm{~N} & \mathrm{~N} \\ 1 & \text { GROSS ALPHA } & 2.360 & 0.050 & 1.650 & 0.160 & 1.430 & \mathrm{~W} & \mathrm{~A} \\ 1 & \text { GROSS BETA } & 2.180 & 0.040 & 2.160 & 0.070 & 1.009 & \mathrm{~A} & \mathrm{~A} \\ 1 & \text { MN54 } & 10.300 & 0.010 & 4.920 & 0.400 & 2.093 & \mathrm{~N} & \mathrm{~N} \\ 1 & \text { PU238 } & 0.500 & 0.020 & 0.460 & 0.005 & 1.087 & \mathrm{~A} & \mathrm{~W} \\ 1 & \text { PU239 } & 0.420 & 0.020 & 0.420 & 0.006 & 1.000 & \mathrm{~A} & \mathrm{~A} \\ 1 & \text { SB125 } & 19.600 & 1.080 & 8.890 & 0.550 & 2.205 & \mathrm{~N} & \mathrm{~N} \\ 1 & \text { U234 } & 0.250 & 0.020 & 0.260 & 0.010 & 0.962 & \mathrm{~A} & \mathrm{~W} \\ 1 & \text { U238 } & 0.320 & 0.040 & 0.260 & 0.010 & 1.231 & \mathrm{~A} & \mathrm{~A}\end{array}$

Matrix: SO Soil $\mathrm{Bq} / \mathrm{kg}$

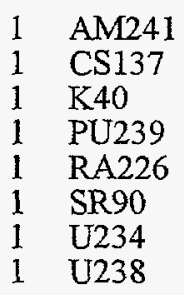
6.500
1094.000
418.000
13.200
222.000
26.500
146.200
105.500

Matrix: VE Vegetation $\mathrm{Bq} / \mathrm{kg}$

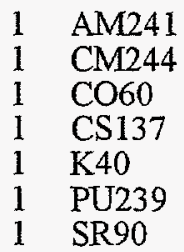

$$
\begin{array}{r}
3.000 \\
1.600 \\
24.600 \\
480.000 \\
596.000 \\
0.500 \\
370.000
\end{array}
$$

$\begin{array}{rr}7.470 & 0.410 \\ 954.000 & 38.000 \\ 314.000 & 13.000 \\ 13.090 & 0.570 \\ 29.000 & 1.000 \\ 39.630 & 0.003 \\ 113.000 & 6.000 \\ 120.000 & 9.000\end{array}$

0.870
1.147
1.331
1.008
7.655
0.669
1.294
0.879

A
A
W
A
A
W
W
A

$$
\begin{aligned}
& \text { A } \\
& \text { A } \\
& \text { W } \\
& \text { A } \\
& \\
& \text { A } \\
& \text { A }
\end{aligned}
$$

Matrix: WA Water $\mathrm{Bq} / \mathrm{L}$

$\begin{array}{lll}1 & \text { AM241 } \\ 1 & \text { CO60 } \\ 1 & \text { CS137 } \\ 1 & \text { FE55 } & \\ 1 & \text { GROSS ALPHA } & 116 \\ 1 & \text { GROSS BETA } & 1206 \\ 1 & \text { H3 } & \\ 1 & \text { MN54 } & \text { N163 } \\ 1 & \text { PU238 } & \\ 1 & \text { PU239 }\end{array}$

1.270
50.500
54.200
116.200
980.500
1206.000
116.500
35.700
93.600
1.230
1.530

0.140

2.800

3.900

9.770

23.830

20.000

10.700

2.100

7.800

0.060

0.080

1.250
49.400
50.000
139.000
080.000
420.000
76.200
32.400
95.700
1.100
1.410

0.080
1.200
1.700
2.000
60.000
60.000
2.900
1.400
0.900
0.010
0.040

1.288
0.909
1.230
1.231
1.296
0.134
0.611

$\begin{array}{ll}\text { A } & \text { W } \\ \text { A } & \text { W } \\ \text { A } & \text { A } \\ \text { A } & \text { A } \\ \text { W } & \text { W } \\ \text { N } & \text { W } \\ \text { W } & \text { W }\end{array}$

\section{Values for elemental uranium are reported in $\mu \mathrm{g} /$ filter, $\mathrm{g}$, or $\mathrm{mL}$.}

\section{$\mathrm{pCi} / \mathrm{g}$ or $\mathrm{mL}=\mathrm{Bq} \times 0.027$}

Evaluation: $A=$ Acceptable, $W=$ Acceptable with Warning, $N=$ Not Acceptable

If the evaluation system is not appropriate for the types of analyses performed in your lab, apply a site specific evaluation. 


\section{QAP 49 Results by Laboratory}

Lab: BX B\&W Nuclear Envir. Services, Lynchburg, VA

\begin{tabular}{|c|c|c|c|c|c|c|c|}
\hline $\begin{array}{l}\text { No. } \\
\text { Test }\end{array}$ & Radionuclide & $\begin{array}{c}\text { Reported } \\
\text { Value }\end{array}$ & $\begin{array}{c}\text { Reported } \\
\text { Error }\end{array}$ & $\begin{array}{l}\text { EML } \\
\text { Value }\end{array}$ & $\begin{array}{l}\text { EML } \\
\text { Error }\end{array}$ & $\frac{\text { Reported }}{\text { EML }}$ & Evaluation \\
\hline \multicolumn{8}{|c|}{ Matrix: WA Water Bq/L } \\
\hline $\begin{array}{l}1 \\
1 \\
1\end{array}$ & $\begin{array}{l}\text { SR90 } \\
\text { U234 } \\
\text { U238 }\end{array}$ & $\begin{array}{l}2.260 \\
0.670 \\
0.680\end{array}$ & $\begin{array}{l}0.500 \\
0.070 \\
0.090\end{array}$ & $\begin{array}{l}2.110 \\
0.510 \\
0.520\end{array}$ & $\begin{array}{l}0.180 \\
0.030 \\
0.050\end{array}$ & $\begin{array}{l}1.071 \\
1.314 \\
1.308\end{array}$ & $\begin{array}{l}\mathrm{A} \\
\mathrm{W} \\
\mathrm{N}\end{array}$ \\
\hline
\end{tabular}

Values for elemental uranium are reported in $\mu \mathrm{g} /$ filter, $\mathrm{g}$, or $\mathrm{mL}$.

$\mathrm{pCi} / \mathrm{g}$ or $\mathrm{mL}=\mathrm{Bq} \times \mathbf{0 . 0 2 7}$

Evaluation: $A=$ Acceptable,$W=A c c e p t a b l e$ with Warning, $N=$ Not Acceptable

If the evaluation system is not appropriate for the types of analyses performed in your lab, apply a site specific evaluation. 


\section{QAP 49 Results by Laboratory}

Lab: CA Atomic Energy Control Board, Ottawa, Canada

\begin{tabular}{|c|c|c|c|c|c|c|c|}
\hline $\begin{array}{l}\text { No. } \\
\text { Test }\end{array}$ & Radionuclide & $\begin{array}{l}\text { Reported } \\
\text { Value }\end{array}$ & $\begin{array}{l}\text { Reported } \\
\text { Error }\end{array}$ & $\begin{array}{l}\text { EML } \\
\text { Value }\end{array}$ & $\begin{array}{l}\text { EML } \\
\text { Error }\end{array}$ & $\frac{\text { Reported }}{\text { EML }}$ & $\begin{array}{cc}\text { QAP 48 } \\
\text { Evaluation } \\
\text { Evaluation }\end{array}$ \\
\hline
\end{tabular}

Matrix: AI Air Filter Bq/filter

$\begin{array}{llrrrrrrr}1 & \text { CO60 } & 8.300 & 0.200 & 9.160 & 0.580 & 0.906 & \mathrm{~A} & \mathrm{~A} \\ 1 & \text { CS137 } & 22.500 & 2.300 & 22.470 & 1.030 & 1.001 & \mathrm{~A} & \mathrm{~A} \\ 1 & \text { GROSS ALPHA } & 1.800 & 0.200 & 1.650 & 0.160 & 1.091 & \mathrm{~A} & \mathrm{~A} \\ 1 & \text { GROSS BETA } & 1.700 & 0.200 & 2.160 & 0.070 & 0.787 & \mathrm{~W} & \mathrm{~A} \\ 1 & \text { MN54 } & 4.500 & 0.300 & 4.920 & 0.400 & 0.915 & \mathrm{~A} & \mathrm{~A} \\ 1 & \text { SB125 } & 8.200 & 0.300 & 8.890 & 0.550 & 0.922 & \mathrm{~A} & \mathrm{~A}\end{array}$

Matrix: SO Soil $\mathrm{Bq} / \mathrm{kg}$

$1 \mathrm{ug} U$

$9.400 \quad 0.900$

9.700

0.700

0.969

A

A

Matrix: WA Water $\mathrm{Bq} / \mathrm{L}$

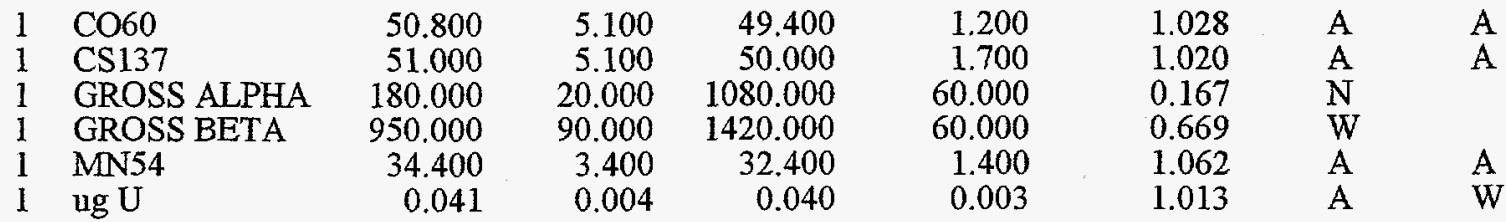

Values for elemental uranium are reported in $\mu \mathrm{g} /$ filter, $g$, or $\mathbf{m L}$.

$\mathrm{pCi} / \mathrm{g}$ or $\mathrm{mL}=\mathrm{Bq} \times 0.027$

Evaluation: $A=A c c e p t a b l e, W=A c c e p t a b l e$ with Warning, $N=$ Not Acceptable

If the evaluation system is not appropriate for the types of analyses performed in your lab, apply a site specific evaluation. 


\section{QAP 49 Results by Laboratory}

Lab: CB Radiation Protection Bureau, Ontario, Canada

\begin{tabular}{lccccc} 
No. & Reported & Reported & EML & EML & Reported \\
Test & Radionuclide & $\begin{array}{c}\text { Value } \\
\text { Error }\end{array}$ & $\begin{array}{l}\text { Value } \\
\text { Error }\end{array}$ & $\begin{array}{l}\text { QAP } 48 \\
\text { Evaluation }\end{array}$ Evaluation \\
\hline
\end{tabular}

Matrix: AI Air Filter $\mathrm{Bq} /$ filter

$\begin{array}{llrlrlll}3 & \text { AM241 } & 0.500 & 0.030 & 0.510 & 0.008 & 0.980 & \mathrm{~A} \\ 2 & \text { AM241 } & 0.420 & 0.090 & 0.510 & 0.008 & 0.824 & \mathrm{~W} \\ 1 & \text { AM241 } & 0.600 & 0.070 & 0.510 & 0.008 & 1.176 & \mathrm{~A} \\ 3 & \text { CO60 } & 9.200 & 0.120 & 9.160 & 0.580 & 1.004 & \mathrm{~A} \\ 2 & \text { CO60 } & 8.500 & 0.110 & 9.160 & 0.580 & 0.928 & \mathrm{~A} \\ 1 & \text { CO60 } & 9.100 & 0.120 & 9.160 & 0.580 & 0.993 & \mathrm{~A} \\ 1 & \text { CS137 } & 22.800 & 0.670 & 22.470 & 1.030 & 1.015 & \mathrm{~A} \\ 3 & \text { CS137 } & 23.300 & 0.540 & 22.470 & 1.030 & 1.037 & \mathrm{~A} \\ 2 & \text { CS137 } & 23.100 & 0.640 & 22.470 & 1.030 & 1.028 & \mathrm{~A} \\ 2 & \text { MN54 } & 5.200 & 0.130 & 4.920 & 0.400 & 1.057 & \mathrm{~A} \\ 1 & \text { MN54 } & 5.100 & 0.140 & 4.920 & 0.400 & 1.037 & \mathrm{~A} \\ 3 & \text { MN54 } & 5.200 & 0.130 & 4.920 & 0.400 & 1.057 & \mathrm{~A} \\ 3 & \text { SB125 } & 9.000 & 0.160 & 8.890 & 0.550 & 1.012 & \mathrm{~A} \\ 1 & \text { SB125 } & 9.700 & 0.190 & 8.890 & 0.550 & 1.091 & \mathrm{~A} \\ 2 & \text { SB125 } & 9.400 & 0.170 & 8.890 & 0.550 & 1.057 & \mathrm{~A}\end{array}$

Matrix: WA Water $\mathrm{Bq} / \mathrm{L}$

$\begin{array}{lllllllll}1 & \text { SR90 } & 2.460 & 0.250 & 2.110 & 0.180 & 1.166 & \text { A } & \text { A } \\ 2 & \text { SR90 } & 2.270 & 0.250 & 2.110 & 0.180 & 1.076 & \text { A } & \text { A }\end{array}$

Values for elemental uranium are reported in $\mu \mathrm{g} /$ filter, $\mathrm{g}$, or $\mathrm{mL}$.

$\mathrm{pCi} / \mathrm{g}$ or $\mathrm{mL}=\mathrm{Bq} \times 0.027$

Evaluation: $A=$ Acceptable,$W=$ Acceptable with Warning, $N=$ Not Acceptable

If the evaluation system is not appropriate for the types of analyses performed in your lab, apply a site specific evaluation. 


\section{QAP 49 Results by Laboratory}

Lab: CD Gentilly-2 Nuclear Power Plant, Quebec Canada

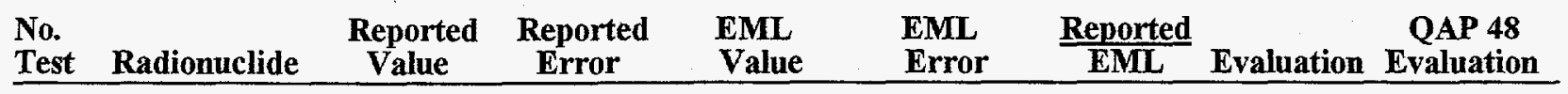

Matrix: AI Air Filter $\mathrm{Bq} /$ filter

$\begin{array}{llrrrrrrr}1 & \text { CO60 } & 8.900 & 0.100 & 9.160 & 0.580 & 0.972 & \mathrm{~A} & \mathrm{~A} \\ 1 & \text { CS137 } & 20.700 & 0.200 & 22.470 & 1.030 & 0.921 & \mathrm{~A} & \text { W } \\ 1 & \text { GROSS ALPHA } & 1.500 & 0.100 & 1.650 & 0.160 & 0.909 & \mathrm{~A} & \text { W } \\ 1 & \text { GROSS BETA } & 3.000 & 0.200 & 2.160 & 0.070 & 1.389 & \mathrm{~A} & \mathrm{~N} \\ 1 & \text { MN54 } & 5.200 & 0.100 & 4.920 & 0.400 & 1.057 & \mathrm{~A} & \mathrm{~A} \\ 1 & \text { SB125 } & 8.600 & 0.200 & 8.890 & 0.550 & 0.967 & \mathrm{~A} & \mathrm{~A}\end{array}$

Matrix: SO Soil Bq/kg

$\begin{array}{ll}1 & \mathrm{AC} 228 \\ 1 & \mathrm{BI212} \\ 1 & \mathrm{BI} 14 \\ 1 & \mathrm{CS} 137 \\ 1 & \mathrm{~K} 40 \\ 1 & \mathrm{~PB} 212 \\ 1 & \mathrm{~PB} 214 \\ 1 & \mathrm{TL} 208\end{array}$

$\begin{array}{rrr}55.000 & 2.000 & 52.600 \\ 55.000 & 1.000 & 58.300 \\ 32.000 & 2.000 & 28.800 \\ 1051.000 & 4.000 & 954.000 \\ 338.000 & 10.000 & 314.000 \\ 58.000 & 1.000 & 52.800 \\ 30.000 & 2.000 & 29.100 \\ 61.000 & 3.000 & 18.300\end{array}$

2.900
5.900
0.500
38.000
13.000
3.700
1.200
1.100

\subsection{6}

0.943

1.111

1.102

1.076

1.098

1.031

3.333

A
A
A
A
A
A
A
A

Matrix: VE Vegetation $\mathrm{Bq} / \mathrm{kg}$

$\begin{array}{llrrrrrrr}1 & \text { CO60 } & 21.000 & 1.000 & 20.000 & 1.000 & 1.050 & \mathrm{~A} & \mathrm{~A} \\ 1 & \text { CS137 } & 419.000 & 3.000 & 390.000 & 20.000 & 1.074 & \mathrm{~A} & \mathrm{~A} \\ 1 & \mathrm{~K} 40 & 481.000 & 1.000 & 460.000 & 20.000 & 1.046 & \mathrm{~A} & \mathrm{~A}\end{array}$

Matrix: WA Water $\mathrm{Bq} / \mathrm{L}$

$\begin{array}{llrrrrrrr}1 & \text { CO60 } & 51.000 & 0.500 & 49.400 & 1.200 & 1.032 & \mathrm{~A} & \mathrm{~A} \\ 1 & \text { CS137 } & 51.000 & 0.500 & 50.000 & 1.700 & 1.020 & \mathrm{~A} & \mathrm{~A} \\ 1 & \text { GROSS ALPHA } & 770.000 & 40.000 & 1080.000 & 60.000 & 0.713 & \text { W } & \text { W } \\ 1 & \text { GROSS BETA } & 1170.000 & 60.000 & 1420.000 & 60.000 & 0.824 & \mathrm{~A} & \mathrm{~A} \\ 1 & \text { MN54 } & 35.000 & 0.500 & 32.400 & 1.400 & 1.080 & \mathrm{~A} & \mathrm{~A}\end{array}$




\section{QAP 49 Results by Laboratory}

Lab: CH California State Dept. Health Serv.,Sanitation \& Radiation Laboratory

\begin{tabular}{|c|c|c|c|c|c|c|}
\hline Radionu & $\begin{array}{l}\text { Reported } \\
\text { Value }\end{array}$ & $\begin{array}{c}\text { Reported } \\
\text { Error }\end{array}$ & $\begin{array}{l}\text { EML } \\
\text { Value }\end{array}$ & $\begin{array}{l}\text { EML } \\
\text { Error }\end{array}$ & $\frac{\text { Reported }}{\text { EML }}$ & $\begin{array}{cc}\text { QAP 48 } \\
\text { Evaluation } \\
\text { Evaluation }\end{array}$ \\
\hline
\end{tabular}

Matrix: AI Air Filter Bq / filter

$\begin{array}{llrlrllll}1 & \text { AM241 } & 0.535 & 0.027 & 0.510 & 0.008 & 1.049 & \mathrm{~A} & \mathrm{~A} \\ 1 & \text { BqU } & 0.510 & 0.025 & 0.530 & 0.020 & 0.962 & \mathrm{~A} & \mathrm{~A} \\ 1 & \text { CO60 } & 9.080 & 0.170 & 9.160 & 0.580 & 0.991 & \mathrm{~A} & \mathrm{~A} \\ 1 & \text { CS137 } & 21.400 & 0.180 & 22.470 & 1.030 & 0.952 & \mathrm{~A} & \mathrm{~A} \\ 1 & \text { GROSS ALPHA } & 1.800 & 0.046 & 1.650 & 0.160 & 1.091 & \mathrm{~A} & \mathrm{~A} \\ 1 & \text { GROSS BETA } & 2.060 & 0.039 & 2.160 & 0.070 & 0.954 & \mathrm{~A} & \mathrm{~A} \\ 1 & \text { MN54 } & 4.800 & 0.130 & 4.920 & 0.400 & 0.976 & \mathrm{~A} & \mathrm{~A} \\ 1 & \text { PU238 } & 0.484 & 0.026 & 0.460 & 0.005 & 1.052 & \mathrm{~A} & \mathrm{~A} \\ 1 & \text { PU239 } & 0.441 & 0.024 & 0.420 & 0.006 & 1.050 & \mathrm{~A} & \mathrm{~A} \\ 1 & \text { SB125 } & 9.610 & 0.310 & 8.890 & 0.550 & 1.081 & \mathrm{~A} & \mathrm{~A} \\ 1 & \text { SR90 } & 1.140 & 0.077 & 1.120 & 0.050 & 1.018 & \mathrm{~A} & \mathrm{~A} \\ 1 & \text { U234 } & 0.239 & 0.011 & 0.260 & 0.010 & 0.919 & \mathrm{~A} & \mathrm{~A} \\ 1 & \text { U238 } & 0.249 & 0.110 & 0.260 & 0.010 & 0.958 & \mathrm{~A} & \mathrm{~A} \\ 1 & \text { ug U } & 20.700 & 2.100 & 20.960 & 0.100 & 0.988 & \mathrm{~A} & \mathrm{~A}\end{array}$

Matrix: SO Soil $\mathrm{Bq} / \mathrm{kg}$

$\begin{array}{ll}1 & \text { AC228 } \\ 1 & \text { AM241 } \\ 1 & \text { BL212 } \\ 1 & \text { BI214 } \\ 1 & \text { Bq U } \\ 1 & \text { CS137 } \\ 1 & \text { K40 } \\ 1 & \text { PB212 } \\ 1 & \text { PB214 } \\ 1 & \text { PU239 } \\ 1 & \text { RA226 } \\ 1 & \text { SR90 } \\ 1 & \text { TH228 } \\ 1 & \text { TL208 } \\ 1 & \text { U234 } \\ 1 & \text { U238 } \\ 1 & \text { ug U }\end{array}$

Matrix: VE Vegetation $\mathrm{Bq} / \mathrm{kg}$

$\begin{array}{ll}1 & \mathrm{AM} 241 \\ 1 & \mathrm{CM} 244 \\ 1 & \mathrm{CO} 60 \\ 1 & \mathrm{CS} 137 \\ 1 & \text { K40 } \\ 1 & \text { PU239 } \\ 1 & \text { SR90 }\end{array}$

$\begin{array}{rr}2.530 & 0.130 \\ 1.810 & 0.120 \\ 23.200 & 0.900 \\ 457.000 & 2.300 \\ 594.000 & 13.000 \\ 3.870 & 0.190 \\ 613.000 & 7.900\end{array}$

$$
\begin{array}{r}
52.600 \\
7.470 \\
58.300 \\
28.800 \\
237.000 \\
954.000 \\
314.000 \\
52.800 \\
29.100 \\
13.090 \\
29.000 \\
39.630 \\
52.700 \\
18.300 \\
113.000 \\
120.000 \\
9.700
\end{array}
$$

$$
\begin{array}{r}
2.900 \\
0.410 \\
5.900 \\
0.500 \\
16.000 \\
38.000 \\
13.000 \\
3.700 \\
1.200 \\
0.570 \\
1.000 \\
0.003 \\
4.000 \\
1.100 \\
6.000 \\
9.000 \\
0.700
\end{array}
$$

$$
\begin{array}{r}
0.060 \\
0.070 \\
1.000 \\
20.000 \\
20.000 \\
0.270 \\
40.000
\end{array}
$$

1.099

1.122

1.187

0.899

0.945

1.140

1.175

1.051

0.990

1.039

0.893

1.166

1.028

2.814

0.965

0.917

1.030
$\mathrm{A}$
$\mathrm{A}$
$\mathrm{A}$
$\mathrm{A}$
$\mathrm{A}$
$\mathrm{A}$
$\mathrm{A}$
$\mathrm{A}$
$\mathrm{A}$
$\mathrm{A}$
$\mathrm{A}$
$\mathrm{A}$
$\mathrm{A}$
$\mathrm{A}$
$\mathrm{A}$
$\mathrm{A}$
$\mathrm{A}$

$\begin{array}{lll}1.086 & \mathrm{~A} & \mathrm{~A} \\ 1.028 & \mathrm{~A} & \mathrm{~A} \\ 1.160 & \mathrm{~A} & \mathrm{~A} \\ 1.172 & \mathrm{~A} & \mathrm{~A} \\ 1.291 & \mathrm{~W} & \mathrm{~A} \\ 1.040 & \mathrm{~A} & \mathrm{~A} \\ 1.012 & \mathrm{~A} & \mathrm{~A}\end{array}$

Values for elemental uranium are reported in $\mu \mathrm{g} /$ filter, $\mathrm{g}$, or $\mathrm{mL}$.

$\mathrm{pCi} / \mathrm{g}$ or $\mathrm{mL}=\mathrm{Bq} \times 0.027$

Evaluation: A=Acceptable, $W=A c c e p t a b l e$ with Warning, $\mathbf{N}=$ Not Acceptable

If the evaluation system is not appropriate for the types of analyses performed in your lab, apply a site specific evaluation. 


\section{QAP 49 Results by Laboratory}

Lab: $\mathrm{CH}$ California State Dept. Health Serv.,Sanitation \& Radiation Laboratory

\begin{tabular}{|c|c|c|c|c|c|c|c|}
\hline ast & Radi & $\begin{array}{l}\text { Reported } \\
\text { Value }\end{array}$ & $\begin{array}{l}\text { Reported } \\
\text { Error }\end{array}$ & $\begin{array}{l}\text { EML } \\
\text { Value }\end{array}$ & $\begin{array}{l}\text { EML } \\
\text { Error }\end{array}$ & $\frac{\text { Reported }}{\text { EML }}$ & 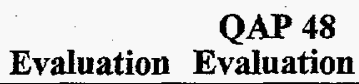 \\
\hline
\end{tabular}

Matrix: WA Water $\mathrm{Bq} / \mathrm{L}$

$\begin{array}{llrrrrrr}1 & \text { AM241 } & 1.310 & 0.025 & 1.250 & 0.080 & 1.048 & \mathrm{~A} \\ 1 & \text { Bq U } & 1.090 & 0.039 & 1.050 & 0.080 & 1.038 & \mathrm{~A} \\ 1 & \text { CO60 } & 52.700 & 0.740 & 49.400 & 1.200 & 1.067 & \mathrm{~A} \\ 1 & \text { CS137 } & 53.400 & 0.640 & 50.000 & 1.700 & 1.068 & \mathrm{~A} \\ 1 & \text { FE55 } & 142.000 & 1.900 & 139.000 & 2.000 & 1.022 & \mathrm{~A} \\ 1 & \text { GROSS ALPHA } & 885.000 & 117.000 & 1080.000 & 60.000 & 0.819 & \text { W } \\ 1 & \text { GROSS BETA } & 1176.000 & 86.000 & 1420.000 & 60.000 & 0.828 & \mathrm{~A} \\ 1 & \text { H3 } & 80.400 & 4.400 & 76.200 & 2.900 & 1.055 & \mathrm{~A} \\ 1 & \text { MN54 } & 35.900 & 0.580 & 32.400 & 1.400 & 1.108 & \mathrm{~A} \\ 1 & \text { PU238 } & 1.200 & 0.082 & 1.100 & 0.010 & 1.091 & \mathrm{~A} \\ 1 & \text { PU239 } & 1.530 & 0.095 & 1.410 & 0.040 & 1.085 & \mathrm{~A} \\ 1 & \text { SR90 } & 2.750 & 0.220 & 2.110 & 0.180 & 1.303 & \text { W } \\ 1 & \text { U234 } & 0.518 & 0.027 & 0.510 & 0.030 & 1.016 & \mathrm{~A} \\ 1 & \text { U238 } & 0.551 & 0.028 & 0.520 & 0.050 & 1.060 & \mathrm{~A} \\ 1 & \text { ug U } & 0.041 & 0.004 & 0.040 & 0.003 & 1.015 & \mathrm{~A}\end{array}$

Values for elemental uranium are reported in $\mu \mathrm{g} /$ filter, $\mathrm{g}$, or $\mathrm{mL} . \quad \mathrm{pCi} / \mathrm{g}$ or $\mathrm{mL}=\mathrm{Bq} \times \mathbf{0 . 0 2 7}$

Evaluation: $A=A c c e p t a b l e, W=A c c e p t a b l e$ with Warning, $N=$ Not Acceptable

If the evaluation system is not appropriate for the types of analyses performed in your lab, apply a site specific evaluation. 


\section{QAP 49 Results by Laboratory}

Lab: CL Core Laboratories, Casper, WY

\begin{tabular}{llllll} 
No. & Reported & Reported & EML & EML & Reported \\
Test & Radionuclide & $\begin{array}{c}\text { Value } \\
\text { Error }\end{array}$ & $\begin{array}{c}\text { Value } \\
\text { Error }\end{array}$ & $\begin{array}{c}\text { QAP 48 } \\
\text { EML }\end{array}$ \\
\hline
\end{tabular}

Matrix: AI Air Filter $\mathrm{Bq} /$ filter

$\begin{array}{llrrrrrrr}1 & \text { AM241 } & 0.510 & 0.100 & 0.510 & 0.008 & 1.000 & \mathrm{~A} & \mathrm{~A} \\ 1 & \text { Bq U } & 0.550 & 0.100 & 0.530 & 0.020 & 1.038 & \mathrm{~A} & \mathrm{~A} \\ 1 & \text { CO60 } & 9.400 & 0.500 & 9.160 & 0.580 & 1.026 & \mathrm{~A} & \mathrm{~A} \\ 1 & \text { CS137 } & 22.000 & 0.900 & 22.470 & 1.030 & 0.979 & \mathrm{~A} & \mathrm{~A} \\ 1 & \text { MN54 } & 5.300 & 0.600 & 4.920 & 0.400 & 1.077 & \mathrm{~A} & \mathrm{~A} \\ 1 & \text { PU238 } & 0.030 & 0.010 & 0.460 & 0.005 & 0.065 & \mathrm{~N} & \mathrm{~W} \\ 1 & \text { PU239 } & 0.480 & 0.100 & 0.420 & 0.006 & 1.143 & \mathrm{~A} & \mathrm{~A} \\ 1 & \text { SB125 } & 8.500 & 0.600 & 8.890 & 0.550 & 0.956 & \mathrm{~A} & \mathrm{~A} \\ 1 & \text { SR90 } & 1.200 & 0.300 & 1.120 & 0.050 & 1.071 & \mathrm{~A} & \mathrm{~N} \\ 1 & \text { U234 } & 0.280 & 0.050 & 0.260 & 0.010 & 1.077 & \mathrm{~A} & \mathrm{~A} \\ 1 & \text { U238 } & 0.280 & 0.050 & 0.260 & 0.010 & 1.077 & \mathrm{~A} & \mathrm{~A}\end{array}$

Matrix: SO Soil $\mathrm{Bq} / \mathrm{kg}$

$\begin{array}{ll}1 & \text { AC228 } \\ 1 & \text { AM241 } \\ 1 & \text { BI214 } \\ 1 & \text { Bq U } \\ 1 & \text { CS137 } \\ 1 & \text { K40 } \\ 1 & \text { PB212 } \\ 1 & \text { PB214 } \\ 1 & \text { PU239 } \\ 1 & \text { RA226 } \\ 1 & \text { SR90 } \\ 1 & \text { TH228 } \\ 1 & \text { TL208 } \\ 1 & \text { U234 } \\ 1 & \text { U238 }\end{array}$

65.300
5.800
41.000
186.000
950.000
400.000
61.500
42.900
13.100
331.000
26.300
58.200
21.700
101.000
86.100

$\begin{array}{rr}11.700 & 52.600 \\ 1.500 & 7.470 \\ 7.400 & 28.800 \\ 36.000 & 237.000 \\ 28.500 & 954.000 \\ 36.000 & 314.000 \\ 3.100 & 52.800 \\ 7.300 & 29.100 \\ 2.700 & 13.090 \\ 66.300 & 29.000 \\ 12.500 & 39.630 \\ 5.800 & 52.700 \\ 4.600 & 18.300 \\ 15.700 & 113.000 \\ 14.900 & 120.000\end{array}$

2.900
0.410
0.500
16.000
38.000
13.000
3.700
1.200
0.570
1.000
0.003
4.000
1.100
6.000
9.000

1.241

0.776

1.424

0.785

0.996

1.274

1.165

1.474

1.001

11.414

0.664

1.104

1.186

0.894

0.717

$\begin{array}{rrrr}3.800 & 1.500 & 2.330 & 0.060 \\ 1.200 & 1.100 & 1.760 & 0.070 \\ 23.300 & 2.600 & 20.000 & 1.000 \\ 366.000 & 14.600 & 390.000 & 20.000 \\ 500.000 & 45.000 & 460.000 & 20.000 \\ 4.100 & 1.900 & 3.720 & 0.270 \\ 610.000 & 30.000 & 606.000 & 40.000\end{array}$

610.000
1.631

0.682

1.165

0.938

1.087

1.102

1.007

$\begin{array}{ll}\text { A } & \\ \text { W } & \text { A } \\ \text { A } & \\ \text { A } & \text { A } \\ \text { A } & \text { A } \\ \text { W } & \text { A } \\ \text { A } & \\ \text { A } & \\ \text { A } & \text { A } \\ \text { A } & \\ \text { W } & \text { W } \\ \text { A } & \\ \text { A } & \\ \text { A } & \text { A } \\ \text { A } & \text { A }\end{array}$

Matrix: WA Water $\mathrm{Bq} / \mathrm{L}$

$\begin{array}{ll}1 & \mathrm{AM} 241 \\ 1 & \mathrm{~Bq} \mathrm{U} \\ 1 & \mathrm{CO60} \\ 1 & \mathrm{CS} 137\end{array}$

\begin{tabular}{llrrrr}
1 & AM241 & 1.300 & 0.200 & 1.250 & 0.080 \\
1 & Bq U & 1.000 & 0.200 & 1.050 & 0.080 \\
1 & CO60 & 49.700 & 2.000 & 49.400 & 1.200 \\
1 & CS137 & 50.700 & 1.500 & 50.000 & 1.700 \\
\\
\multicolumn{7}{l}{ Values for elemental uranium are reported in $\boldsymbol{\mu g}$ /filter, $\mathbf{g}$, or $\mathbf{m L}$. } \\
Evaluation: A=Acceptable, W=Acceptable with Warning, $\mathbf{N = N o t}$ Acceptable
\end{tabular}

If the evaluation system is not appropriate for the types of analyses performed in your lab, apply a site specific evaluation. 


\section{QAP 49 Results by Laboratory}

Lab: CL Core Laboratories, Casper, WY

\begin{tabular}{|c|c|c|c|c|c|c|c|}
\hline $\begin{array}{l}\text { No. } \\
\text { Test }\end{array}$ & adi & $\begin{array}{c}\text { Reported } \\
\text { Value }\end{array}$ & $\begin{array}{c}\text { Reported } \\
\text { Error }\end{array}$ & $\begin{array}{l}\text { EML } \\
\text { Value }\end{array}$ & $\begin{array}{l}\text { EML } \\
\text { Error }\end{array}$ & $\frac{\text { Reporte }}{\text { EML }}$ & Evaluation Evaluation \\
\hline
\end{tabular}

Matrix: WA Water $\mathrm{Bq} / \mathrm{L}$

$\begin{array}{llrrrrrrr}1 & \text { FE55 } & 136.000 & 8.000 & 139.000 & 2.000 & 0.978 & \mathrm{~A} & \mathrm{~A} \\ 1 & \text { H3 } & 108.000 & 7.600 & 76.200 & 2.900 & 1.417 & \mathrm{~W} & \mathrm{~A} \\ 1 & \text { MN54 } & 35.300 & 1.400 & 32.400 & 1.400 & 1.090 & \mathrm{~A} & \mathrm{~A} \\ 1 & \text { NI63 } & 136.000 & 5.000 & 95.700 & 0.900 & 1.421 & \mathrm{~A} & \\ 1 & \text { PU238 } & 0.100 & 0.050 & 1.100 & 0.010 & 0.091 & \mathrm{~N} & \mathrm{~N} \\ 1 & \text { PU239 } & 1.400 & 0.200 & 1.410 & 0.040 & 0.993 & \mathrm{~A} & \mathrm{~N} \\ 1 & \text { SR90 } & 2.700 & 0.300 & 2.110 & 0.180 & 1.280 & \mathrm{~W} & \mathrm{~A} \\ 1 & \text { U234 } & 0.500 & 0.100 & 0.510 & 0.030 & 0.980 & \mathrm{~A} & \mathrm{~W} \\ 1 & \text { U238 } & 0.600 & 0.200 & 0.520 & 0.050 & 1.154 & \mathrm{~A} & \mathrm{~A}\end{array}$

Values for elemental uranium are reported in $\mu \mathrm{g} /$ filter, $\mathrm{g}$, or $\mathrm{mL}$.

$\mathrm{pCi} / \mathrm{g}$ or $\mathrm{mL}=\mathrm{Bq} \times 0.027$

Evaluation: $A=$ Acceptable, $W=$ Acceptable with Warning, $N=$ Not Acceptable

If the evaluation system is not appropriate for the types of analyses performed in your lab, apply a site specific evaluation. 


\section{QAP 49 Results by Laboratory}

Lab: CM Metropolitan Water Reclamation District of Greater Chicago, IL

\begin{tabular}{|c|c|c|c|c|c|c|c|}
\hline $\begin{array}{l}\text { No. } \\
\text { Test }\end{array}$ & Radionuclide & $\begin{array}{c}\text { Reporte } \\
\text { Value }\end{array}$ & $\begin{array}{l}\text { Reported } \\
\text { Error }\end{array}$ & $\begin{array}{l}\text { EML } \\
\text { Value }\end{array}$ & $\begin{array}{l}\text { EML } \\
\text { Error }\end{array}$ & $\frac{\text { Reported }}{\text { EML }}$ & $\begin{array}{c}\text { QAP 48 } \\
\text { Evaluation } \\
\text { Evaluation }\end{array}$ \\
\hline
\end{tabular}

Matrix: SO Soil $\mathrm{Bq} / \mathrm{kg}$

$\begin{array}{llrrrrrr}2 & \text { CS137 } & 1010.000 & 48.000 & 954.000 & 38.000 & 1.059 & \mathrm{~A} \\ 3 & \text { CS137 } & 1050.000 & 50.000 & 954.000 & 38.000 & 1.101 & \mathrm{~A} \\ 1 & \text { CS137 } & 987.000 & 47.000 & 954.000 & 38.000 & 1.035 & \mathrm{~A} \\ 1 & \text { K40 } & 379.000 & 23.000 & 314.000 & 13.000 & 1.207 & \mathrm{~A} \\ 2 & \text { K40 } & 379.000 & 23.000 & 314.000 & 13.000 & 1.207 & \mathrm{~A} \\ 3 & \text { K40 } & 391.000 & 24.000 & 314.000 & 13.000 & 1.245 & \mathrm{~A} \\ 3 & \text { RA226 } & 154.000 & 13.600 & 29.000 & 1.000 & 5.310 & \mathrm{~A} \\ 2 & \text { RA226 } & 156.000 & 13.700 & 29.000 & 1.000 & 5.379 & \mathrm{~A} \\ 1 & \text { RA226 } & 155.000 & 13.500 & 29.000 & 1.000 & 5.345 & \mathrm{~A}\end{array}$

Matrix: WA Water $\mathrm{Bq} / \mathrm{L}$

$\begin{array}{llrrrrrr}3 & \text { CO60 } & 48.500 & 1.600 & 49.400 & 1.200 & 0.982 & \mathrm{~A} \\ 2 & \text { CO60 } & 48.800 & 1.600 & 49.400 & 1.200 & 0.988 & \mathrm{~A} \\ 1 & \text { CO60 } & 47.900 & 1.500 & 49.400 & 1.200 & 0.970 & \mathrm{~A} \\ 2 & \text { CS137 } & 49.400 & 1.700 & 50.000 & 1.700 & 0.988 & \mathrm{~A} \\ 3 & \text { CS137 } & 49.600 & 1.600 & 50.000 & 1.700 & 0.992 & \mathrm{~A} \\ 1 & \text { CS137 } & 48.300 & 1.700 & 50.000 & 1.700 & 0.966 & \mathrm{~A} \\ 1 & \text { GROSS ALPHA } & 977.000 & 26.000 & 1080.000 & 60.000 & 0.905 & \mathrm{~A} \\ 4 & \text { GROSS ALPHA } & 1073.000 & 40.000 & 1080.000 & 60.000 & 0.994 & \mathrm{~A} \\ 2 & \text { GROSS ALPHA } & 1121.000 & 42.000 & 1080.000 & 60.000 & 1.038 & \mathrm{~A} \\ 3 & \text { GROSS ALPHA } & 1069.000 & 40.000 & 1080.000 & 60.000 & 0.990 & \mathrm{~A} \\ 3 & \text { GROSS BETA } & 1439.000 & 33.000 & 1420.000 & 60.000 & 1.013 & \mathrm{~A} \\ 2 & \text { GROSS BETA } & 1439.000 & 33.000 & 1420.000 & 60.000 & 1.013 & \mathrm{~A} \\ 4 & \text { GROSS BETA } & 1398.000 & 32.000 & 1420.000 & 60.000 & 0.985 & \mathrm{~A} \\ 1 & \text { GROSS BETA } & 1413.000 & 33.000 & 1420.000 & 60.000 & 0.995 & \mathrm{~A} \\ 3 & \text { H3 } & 80.800 & 2.300 & 76.200 & 2.900 & 1.060 & \mathrm{~A} \\ 1 & \text { H3 } & 83.900 & 2.300 & 76.200 & 2.900 & 1.101 & \mathrm{~A} \\ 2 & \text { H3 } & 79.700 & 2.300 & 76.200 & 2.900 & 1.046 & \mathrm{~A} \\ 1 & \text { MN54 } & 32.600 & 1.100 & 32.400 & 1.400 & 1.006 & \mathrm{~A} \\ 2 & \text { MN54 } & 33.100 & 1.100 & 32.400 & 1.400 & 1.022 & \mathrm{~A} \\ 3 & \text { MN54 } & 33.100 & 1.100 & 32.400 & 1.400 & 1.022 & \mathrm{~A}\end{array}$

Values for elemental uranium are reported in $\mu \mathrm{g} /$ filter, $\mathrm{g}$, or $\mathrm{mL} . \quad$ pCi/g or $\mathrm{mL}=\mathrm{Bq} \times \mathbf{0 . 0 2 7}$

Evaluation: $A=A$ cceptable, $W=$ Acceptable with Warning, $N=$ Not Acceptable

If the evaluation system is not appropriate for the types of analyses performed in your lab, apply a site specific evaluation. 


\section{QAP 49 Results by Laboratory}

Lab: CN China Institute for Radiation Protection

\begin{tabular}{|c|c|c|c|c|c|c|c|}
\hline $\begin{array}{l}\text { No. } \\
\text { Test }\end{array}$ & Radionuclide & $\begin{array}{l}\text { Reported } \\
\text { Value }\end{array}$ & $\begin{array}{c}\text { Reported } \\
\text { Error }\end{array}$ & $\begin{array}{l}\text { EML } \\
\text { Value }\end{array}$ & $\begin{array}{l}\text { EML } \\
\text { Error }\end{array}$ & $\frac{\text { Reported }}{\text { EML }}$ & $\begin{array}{c}\text { QAP 48 } \\
\text { Evaluation }\end{array}$ \\
\hline
\end{tabular}

Matrix: $\mathrm{AI}$ Air Filter Bq / filter

$\begin{array}{ll}1 & \text { AM241 } \\ 1 & \text { CO60 } \\ 1 & \text { CS137 } \\ 1 & \text { MN54 } \\ 1 & \text { SB125 }\end{array}$

$\begin{array}{rrrr}0.910 & 0.130 & 0.510 & 0.008 \\ 9.860 & 0.580 & 9.160 & 0.580 \\ 23.020 & 1.190 & 22.470 & 1.030 \\ 5.030 & 0.290 & 4.920 & 0.400 \\ 9.350 & 0.520 & 8.890 & 0.550\end{array}$

1.784
1.076
1.024
1.022
1.052

W
A
A
A

A

A

A

Matrix: SO Soil $\mathrm{Bq} / \mathrm{kg}$

$\begin{array}{ll}1 & \operatorname{AM} 241 \\ 1 & \operatorname{CS} 137 \\ 1 & \mathrm{~K} 40\end{array}$

8.340

1046.700

331.600

1.100
63.770

7.470

954.000

31.640

314.000

Matrix: VE Vegetation $\mathrm{Bq} / \mathrm{kg}$

$\begin{array}{ll}1 & \text { AM241 } \\ 1 & \text { CO60 } \\ 1 & \text { CS137 } \\ 1 & \text { K40 }\end{array}$

3.700

19.940

437.500

469.900
0.230

1.200

27.850

40.120
2.330

20.000

460.000
0.410

38.000

13.000

0.060
1.000
20.000
20.000

1.588
0.997
1.122
1.022

1.116

1.097

1.056

A
A
A

A

$\begin{array}{ll}\text { A } & \\ \text { A } & \text { A } \\ \text { A } & \text { A } \\ \text { A } & \text { A }\end{array}$




\section{QAP 49 Results by Laboratory}

Lab: CO Bedford Institute of Oceanography, Dartmouth. Nova Scotia, Canada

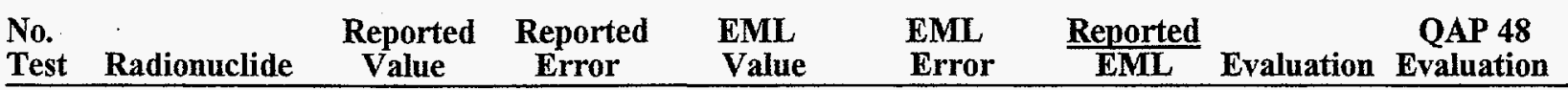

Matrix: AI Air Filter $\mathrm{Bq} /$ filter

$\begin{array}{llrrrrrrr}1 & \text { CO60 } & 9.500 & 0.300 & 9.160 & 0.580 & 1.037 & \mathrm{~A} & \mathrm{~A} \\ 1 & \text { CS137 } & 22.800 & 0.600 & 22.470 & 1.030 & 1.015 & \mathrm{~A} & \mathrm{~A} \\ 1 & \text { MN54 } & 5.300 & 0.200 & 4.920 & 0.400 & 1.077 & \mathrm{~A} & \mathrm{~A} \\ 1 & \text { SB125 } & 8.800 & 0.600 & 8.890 & 0.550 & 0.990 & \mathrm{~A} & \mathrm{~A}\end{array}$

Matrix: SO Soil $\mathrm{Bq} / \mathrm{kg}$

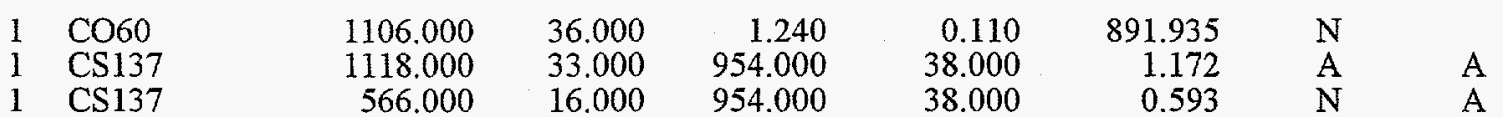

Matrix: $\mathrm{VE}$ Vegetation $\mathrm{Bq} / \mathrm{kg}$

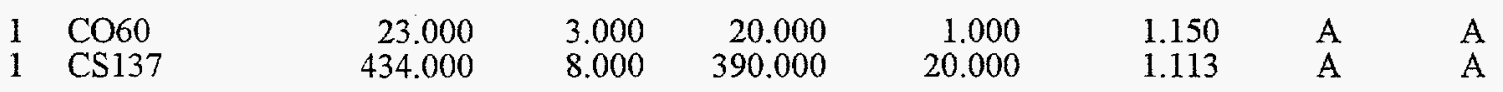

Values for elemental uranium are reported in $\mu \mathrm{g} /$ filter, $\mathrm{g}$, or $\mathbf{~} \mathbf{m L}$.

$\mathrm{pCi} / \mathrm{g}$ or $\mathrm{mL}=\mathrm{Bq} \times 0.027$

Evaluation: A=Acceptable, W=Acceptable with Warning, $N=$ Not Acceptable

If the evaluation system is not appropriate for the types of analyses performed in your lab, apply a site specific evaluation. 


\section{QAP 49 Results by Laboratory}

Lab: CR Laboratorio de Fisica Nuclear Aplicada, Costa Rica

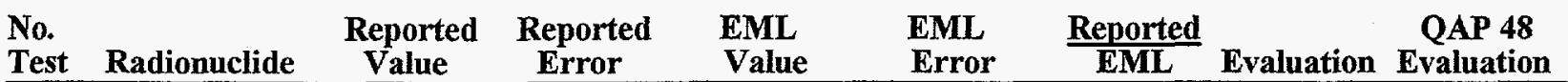

Matrix: AI Air Filter Bq / filter

$\begin{array}{llrrrrrrr}1 & \text { CO60 } & 16.200 & 0.800 & 9.160 & 0.580 & 1.769 & \mathrm{~N} & \mathrm{~A} \\ 1 & \text { CS137 } & 38.500 & 1.500 & 22.470 & 1.030 & 1.713 & \mathrm{~N} & \mathrm{~W} \\ 1 & \text { MN54 } & 8.300 & 0.400 & 4.920 & 0.400 & 1.687 & \mathrm{~N} & \mathrm{~A} \\ 1 & \text { SB125 } & 16.400 & 0.200 & 8.890 & 0.550 & 1.845 & \mathrm{~N} & \mathrm{~W}\end{array}$

Matrix: SO Soil Bq/kg

$\begin{array}{llrrrrrrl}1 & \text { AC228 } & 70.000 & 13.000 & 52.600 & 2.900 & 1.331 & \text { A } & \\ 1 & \text { BI212 } & 69.000 & 31.000 & 58.300 & 5.900 & 1.184 & \text { A } & \\ 1 & \text { BI214 } & 40.000 & 6.000 & 28.800 & 0.500 & 1.389 & \text { A } & \\ 1 & \text { CS137 } & 1180.000 & 47.000 & 954.000 & 38.000 & 1.237 & \text { W } & \text { W } \\ 1 & \text { K40 } & 328.000 & 58.000 & 314.000 & 13.000 & 1.045 & \text { A } & \text { W } \\ 1 & \text { PB212 } & 63.000 & 6.000 & 52.800 & 3.700 & 1.193 & \text { A } & \\ 1 & \text { PB214 } & 41.000 & 7.000 & 29.100 & 1.200 & 1.409 & \text { A } & \\ 1 & \text { RA226 } & 152.000 & 37.000 & 29.000 & 1.000 & 5.241 & \text { A } & \\ 1 & \text { TL208 } & 48.000 & 9.000 & 18.300 & 1.100 & 2.623 & \text { A }\end{array}$

Matrix: VE Vegetation $\mathrm{Bq} / \mathrm{kg}$

$\begin{array}{llrrrrrrr}1 & \text { CO60 } & 25.000 & 3.000 & 20.000 & 1.000 & 1.250 & \text { W } & \text { W } \\ 1 & \text { CS137 } & 464.000 & 19.000 & 390.000 & 20.000 & 1.190 & \text { A } & \text { A } \\ 1 & \text { K40 } & 546.000 & 74.000 & 460.000 & 20.000 & 1.187 & \text { A } & \text { W }\end{array}$

Values for elemental uranium are reported in $\mu \mathrm{g} /$ filter, $\mathrm{g}$, or $\mathrm{mL} . \quad \mathrm{pCi} / \mathrm{g}$ or $\mathrm{mL}=\mathrm{Bq} \times \mathbf{0 . 0 2 7}$

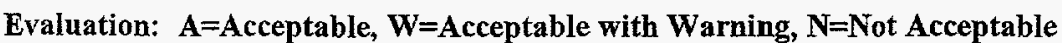

If the evaluation system is not appropriate for the types of analyses performed in your lab, apply a site specific evaluation. 


\section{QAP 49 Results by Laboratory}

Lab: CS Boeing North American, Canoga Park, CA

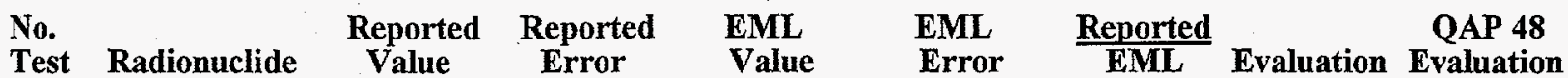

Matrix: AI Air Filter $\mathrm{Bq} /$ filter

$\begin{array}{llrrrrrrr}1 & \text { AM241 } & 0.520 & 0.039 & 0.510 & 0.008 & 1.019 & \mathrm{~A} & \mathrm{~W} \\ 1 & \text { CO60 } & 8.351 & 0.276 & 9.160 & 0.580 & 0.912 & \mathrm{~A} & \mathrm{~N} \\ 1 & \text { CS137 } & 19.960 & 0.880 & 22.470 & 1.030 & 0.888 & \mathrm{~A} & \mathrm{~N} \\ 1 & \text { GROSS ALPHA } & 1.780 & 0.080 & 1.650 & 0.160 & 1.079 & \mathrm{~A} & \mathrm{~A} \\ 1 & \text { GROSS BETA } & 2.940 & 0.230 & 2.160 & 0.070 & 1.361 & \mathrm{~A} & \mathrm{~W} \\ 1 & \text { MN54 } & 4.442 & 0.222 & 4.920 & 0.400 & 0.903 & \mathrm{~A} & \mathrm{~N} \\ 1 & \text { SB125 } & 8.586 & 0.317 & 8.890 & 0.550 & 0.966 & \mathrm{~A} & \mathrm{~N}\end{array}$

Matrix: SO Soil Bq/ kg

$\begin{array}{ll}1 & \text { AC228 } \\ 1 & \text { AM241 } \\ 1 & \text { B214 } \\ 1 & \text { CS137 } \\ 1 & \text { K40 } \\ 1 & \text { PB210 } \\ 1 & \text { PB212 } \\ 1 & \text { PB214 } \\ 1 & \text { RA226 } \\ 1 & \text { TH234 } \\ 1 & \text { TL208 } \\ 1 & \text { U238 }\end{array}$

Matrix: VE Vegetation $\mathrm{Bq} / \mathrm{kg}$

46.780
7.684
27.950
950.900
322.600
21.970
54.170
28.950
69.850
97.660
17.230
100.500

0.546

0.727

40.980

15.100

4.622

2.784

1.031

4.477

6.690

0.650

21.940

$$
\begin{array}{r}
52.600 \\
7.470 \\
28.800 \\
954.000 \\
314.000 \\
32.000 \\
52.800 \\
29.100 \\
29.000 \\
114.000 \\
18.300 \\
120.000
\end{array}
$$

\subsection{0}

0.410

0.500

38.000

13.000

3.300

3.700

1.200

1.000

6.000

1.100

9.000
0.889

1.029

0.970

0.997

1.027

0.687

1.026

0.995

2.409

0.857

0.942

0.837

$\begin{array}{ll}\text { A } & \\ \text { A } & \text { A } \\ \text { A } & \\ \text { A } & \text { A } \\ \text { A } & \text { A } \\ \text { A } & \\ \text { A } & \\ \text { A } & \\ \text { A } & \\ \text { A } & \\ \text { A } & \\ \text { A } & \text { N }\end{array}$

$\mathrm{N}$

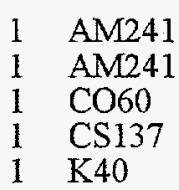

2.476

2.476

18.240

355.800

433.800

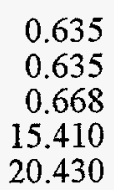

2.330

2.330

20.000

390.000

460.000
0.060

0.060

1.000

20.000

20.000
1.063

1.063

0.912

0.912

0.943

$\begin{array}{ll}\text { A } & \text { N } \\ \text { A } & \text { N } \\ \text { A } & \text { A } \\ \text { A } & \text { A } \\ \text { A } & \text { A }\end{array}$

N
A
A
A

$\begin{array}{ll}\text { A } & \text { A } \\ \text { A } & \text { A } \\ \text { A } & \text { A } \\ \text { A } & \text { A } \\ \text { W } & \text { A } \\ \text { W } & \text { A }\end{array}$

1.275
50.660
50.660
50.750
38.820
38.820

0.108
1.606
1.606
2.197
1.898
1.898

1.250
49.400
49.400
50.000
32.400
32.400

0.080
1.200
1.200
1.700
1.400
1.400

1.020
1.026
1.026
1.015
1.198
1.198
1.020
1.026
1.015
1.198

Values for elemental uranium are reported in $\mu \mathrm{g} /$ filter, $\mathrm{g}$, or $\mathrm{mL}$.

$\mathrm{pCi} / \mathrm{g}$ or $\mathrm{mL}=\mathrm{Bq} \times \mathbf{0 . 0 2 7}$

Evaluation: $A=A c c e p t a b l e, W=A c c e p t a b l e$ with Warning, $N=$ Not Acceptable

If the evaluation system is not appropriate for the types of analyses performed in your lab, apply a site specific evaluation. 


\section{QAP 49 Results by Laboratory}

Lab: DH Duke Engineering Services Hanford

\begin{tabular}{|c|c|c|c|c|c|c|c|c|}
\hline To. & adi & $\begin{array}{c}\text { Reported } \\
\text { Value }\end{array}$ & $\begin{array}{c}\text { Reported } \\
\text { Error }\end{array}$ & $\begin{array}{l}\text { EML } \\
\text { Value }\end{array}$ & $\begin{array}{l}\text { EML } \\
\text { Error }\end{array}$ & $\frac{\text { Reported }}{\text { EML }}$ & Evaluation & $\begin{array}{c}\text { QAP 48 } \\
\text { Evaluation }\end{array}$ \\
\hline
\end{tabular}

Matrix: $\mathrm{AI}$ Air Filter $\mathrm{Bq} /$ filter

$\begin{array}{lllllllll}1 & \text { GROSS ALPHA } & 1.500 & 0.220 & 1.650 & 0.160 & 0.909 & \mathrm{~A} & \mathrm{~A} \\ 1 & \text { GROSS BETA } & 1.750 & 0.200 & 2.160 & 0.070 & 0.810 & \mathrm{~W} & \mathrm{~A}\end{array}$

Matrix: SO Soil $\mathrm{Bq} / \mathrm{kg}$

$1 \quad$ AM241

$1 \mathrm{CO} 60$

$1 \quad \operatorname{CS} 137$

$1 \mathrm{~K} 40$

Matrix: WA Water $\mathrm{Bq} / \mathrm{L}$

$1 \quad \mathrm{CO} 60$

$1 \quad \operatorname{CS} 137$

1 GROSS ALPHA

1 GROSS BETA

1 MN54

$\begin{array}{rr}5.900 & 1.800 \\ 1.370 & 0.630 \\ 969.000 & 8.000 \\ 331.000 & 27.000\end{array}$

1.800

8.000

27.000
0.790

1.105

1.016

1.054
W
A
A
A

A

A
A

A 


\section{QAP 49 Results by Laboratory}

Lab: EG LMITCO/INEL, Scoville

\begin{tabular}{|c|c|c|c|c|c|c|c|}
\hline est & Radionuclide & $\begin{array}{l}\text { Reported } \\
\text { Value }\end{array}$ & $\begin{array}{l}\text { Reported } \\
\text { Error }\end{array}$ & $\begin{array}{l}\text { EML } \\
\text { Value }\end{array}$ & $\begin{array}{l}\text { EML } \\
\text { Error }\end{array}$ & $\frac{\text { Reported }}{\text { EML }}$ & $\begin{array}{cc}\text { QAP 48 } \\
\text { Evaluation } \\
\text { Evaluation }\end{array}$ \\
\hline
\end{tabular}

Matrix: AI Air Filter $\mathrm{Bq} /$ filter

$\begin{array}{llrrrrrrr}1 & \text { AM241 } & 0.568 & 0.044 & 0.510 & 0.008 & 1.114 & \text { A } & \text { A } \\ 1 & \text { CO60 } & 8.800 & 0.100 & 9.160 & 0.580 & 0.961 & \text { A } & \text { A } \\ 1 & \text { CS137 } & 21.800 & 0.200 & 22.470 & 1.030 & 0.970 & \text { A } & \text { A } \\ 1 & \text { MN54 } & 5.000 & 0.100 & 4.920 & 0.400 & 1.016 & \text { A } & \text { A } \\ 1 & \text { PU238 } & 0.502 & 0.035 & 0.460 & 0.005 & 1.091 & \text { A } & \text { A } \\ 1 & \text { PU239 } & 0.462 & 0.033 & 0.420 & 0.006 & 1.100 & \text { A } & \text { A } \\ 1 & \text { SB125 } & 8.830 & 0.130 & 8.890 & 0.550 & 0.993 & \text { A } & \text { A } \\ 1 & \text { SR90 } & 1.230 & 0.050 & 1.120 & 0.050 & 1.098 & \text { A } & \text { A } \\ 1 & \text { U234 } & 0.271 & 0.033 & 0.260 & 0.010 & 1.042 & \text { A } & \text { A } \\ 1 & \text { U238 } & 0.283 & 0.039 & 0.260 & 0.010 & 1.088 & \text { A } & \text { A }\end{array}$

Matrix: SO Soil Bq/kg

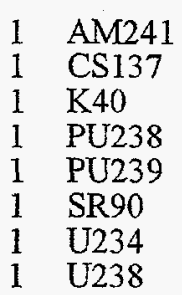

Matrix: VE Vegetation $\mathrm{Bq} / \mathrm{kg}$

$\begin{array}{ll}1 & \text { AM241 } \\ 1 & \text { CM244 } \\ 1 & \text { CO60 } \\ 1 & \text { CS137 } \\ 1 & \text { K40 } \\ 1 & \text { PU238 } \\ 1 & \text { PU239 } \\ 1 & \text { SR90 }\end{array}$

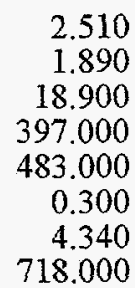

0.230

0.180

2.300

9.000

68.000

0.040

0.420

21.000
7.470

954.000

314.000

0.530

13.090

39.630

113.000

120.000

$$
\begin{array}{r}
0.410 \\
38.000 \\
13.000 \\
0.270 \\
0.570 \\
0.003 \\
6.000 \\
9.000
\end{array}
$$

0.993

1.164

1.115

0.849

1.047

1.226

0.938

0.925

$\begin{array}{ll}\text { A } & \text { A } \\ \text { A } & \text { A } \\ \text { A } & \text { A } \\ \text { A } & \\ \text { A } & \text { A } \\ \text { A } & \text { A } \\ \text { A } & \text { A } \\ \text { A } & \text { A }\end{array}$

1.077

1.074

0.945

1.018

1.050

0.968

1.167

1.185

A
A
A
A
A
A
A
W

A
A
A
A
A
A
A
A

Matrix: WA Water $\mathrm{Bq} / \mathrm{L}$

$\begin{array}{ll}1 & \text { AM241 } \\ 1 & \text { CO60 } \\ 1 & \text { CS137 } \\ 1 & \text { FE55 } \\ 1 & \text { GROSS ALPHA } \\ 1 & \text { GROSS BETA } \\ 1 & \text { H3 } \\ 1 & \text { MN54 } \\ 1 & \text { NI63 } \\ 1 & \text { PU238 } \\ 1 & \text { PU239 }\end{array}$

1.240
50.500
51.300
114.000
1160.000
1630.000
75.000
34.700
78.000
1.180
1.540

0.100
1.100
1.100
48.000
70.000
50.000
6.000
1.000
7.000
0.100
0.130

1.250
49.400
50.000
139.000
1080.000
142.000
76.200
32.400
95.700
1.100
1.410
0.080
1.200
1.700
2.000
60.000
60.000
2.900
1.400
0.900
0.010
0.040

$\begin{array}{ll}0.992 & \mathrm{~A} \\ 1.022 & \mathrm{~A} \\ 1.026 & \mathrm{~A} \\ 0.820 & \mathrm{~A} \\ 1.074 & \mathrm{~A} \\ 1.148 & \mathrm{~A} \\ 0.984 & \mathrm{~A} \\ 1.071 & \mathrm{~A} \\ 0.815 & \mathrm{~A} \\ 1.073 & \mathrm{~A} \\ 1.092 & \mathrm{~A}\end{array}$

A

A

A

A

W

A

A

\footnotetext{
Values for elemental uranium are reported in $\mu \mathrm{g} /$ filter, $\mathrm{g}$, or $\mathrm{mL}$.

$\mathrm{pCi} / \mathrm{g}$ or $\mathrm{mL}=\mathrm{Bq} \times 0.027$

Evaluation: $A=A c c e p t a b l e, W=A c c e p t a b l e$ with Warning, $N=$ Not Acceptable

If the evaluation system is not appropriate for the types of analyses performed in your lab, apply a site specific evaluation.
} 


\section{QAP 49 Results by Laboratory}

Lab: EG LMITCO/INEL, Scoville

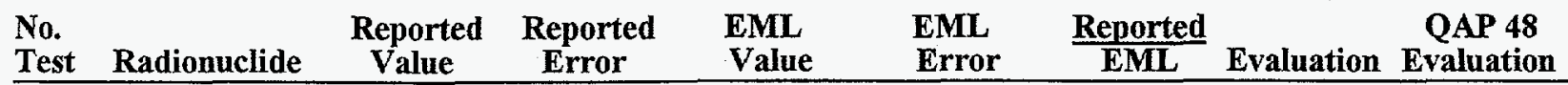

Matrix: WA Water $\mathrm{Bq} / \mathrm{L}$

$\begin{array}{lllllllll}\mathrm{l} & \mathrm{SR90} & 2.200 & 0.200 & 2.110 & 0.180 & 1.043 & \mathrm{~A} & \mathrm{~A} \\ \mathrm{l} & \mathrm{U} 234 & 0.510 & 0.070 & 0.510 & 0.030 & 1.000 & \mathrm{~A} & \mathrm{~A} \\ \mathrm{l} & \mathrm{U} 238 & 0.490 & 0.080 & 0.520 & 0.050 & 0.942 & \mathrm{~A} & \mathrm{~A}\end{array}$

Values for elemental uranium are reported in $\mu \mathrm{g} /$ filter, $\mathrm{g}$, or $\mathrm{mL}$.

$\mathrm{pCi} / \mathrm{g}$ or $\mathrm{mL}=\mathrm{Bq} \times 0.027$

Evaluation: $A=$ Acceptable, $W=$ Acceptable with Warning, $N=$ Not Acceptable

If the evaluation system is not appropriate for the types of analyses performed in your lab, apply a site specific evaluation. 


\section{QAP 49 Results by Laboratory}

Lab: EM 3M, Empore Disks, St. Paul, MN

\begin{tabular}{|c|c|c|c|c|c|c|c|}
\hline $\begin{array}{l}\text { No. } \\
\text { Test }\end{array}$ & adionuclide & $\begin{array}{c}\text { Reported } \\
\text { Value }\end{array}$ & $\begin{array}{c}\text { Reported } \\
\text { Error }\end{array}$ & $\begin{array}{l}\text { EML } \\
\text { Value }\end{array}$ & $\begin{array}{l}\text { EML } \\
\text { Error }\end{array}$ & $\frac{\text { Reporter }}{\text { EML }}$ & $\begin{array}{c}\text { QAP 48 } \\
\text { Evaluation }\end{array}$ \\
\hline
\end{tabular}

Matrix: AI Air Filter $\mathrm{Bq} /$ filter

$\begin{array}{llllllll}1 & \text { PU238 } & 0.075 & 0.003 & 0.460 & 0.005 & 0.163 & \mathrm{~N} \\ 1 & \text { PU239 } & 0.072 & 0.003 & 0.420 & 0.006 & 0.171 & \mathrm{~N} \\ 1 & \text { U234 } & 0.101 & 0.004 & 0.260 & 0.010 & 0.388 & \mathrm{~N} \\ 1 & \text { U238 } & 0.095 & 0.004 & 0.260 & 0.010 & 0.365 & \mathrm{~N}\end{array}$

Matrix: SO Soil $\mathrm{Bq} / \mathrm{kg}$

$\begin{array}{llllllll}1 & \text { PU239 } & 0.009 & 0.001 & 13.090 & 0.570 & 0.001 & \mathrm{~N} \\ 1 & \mathrm{U} 234 & 0.118 & 0.007 & 113.000 & 6.000 & 0.001 & \mathrm{~N} \\ 1 & \mathrm{U} 238 & 0.108 & 0.006 & 120.000 & 9.000 & 0.001 & \mathrm{~N}\end{array}$

Matrix: WA Water $\mathrm{Bq} / \mathrm{L}$

$\begin{array}{llllllll}1 & \text { PU238 } & 0.862 & 0.027 & 1.100 & 0.010 & 0.784 & \text { W } \\ 1 & \text { PU239 } & 1.149 & 0.032 & 1.410 & 0.040 & 0.815 & \text { W } \\ 1 & \text { U234 } & 0.460 & 0.022 & 0.510 & 0.030 & 0.902 & \text { A } \\ 1 & \text { U238 } & 0.489 & 0.023 & 0.520 & 0.050 & 0.940 & \text { A }\end{array}$

Values for elemental uranium are reported in $\mu \mathrm{g} /$ filter, $\mathrm{g}$, or $\mathrm{mL} . \quad \mathrm{pCi} / \mathrm{g}$ or $\mathrm{mL}=\mathrm{Bq} \times 0.027$

Evaluation: $A=$ Acceptable, $W=$ Acceptable with Warning, $N=$ Not Acceptable

If the evaluation system is not appropriate for the types of analyses performed in your lab, apply a site specific evaluation. 


\section{QAP 49 Results by Laboratory}

\begin{tabular}{|c|c|c|c|c|c|c|c|}
\hline $\begin{array}{l}\text { No. } \\
\text { Test }\end{array}$ & Radion & $\begin{array}{l}\text { Reported } \\
\text { Value }\end{array}$ & $\begin{array}{l}\text { Reported } \\
\text { Error }\end{array}$ & $\begin{array}{l}\text { EML } \\
\text { Value }\end{array}$ & $\begin{array}{l}\text { EML } \\
\text { Error }\end{array}$ & $\frac{\text { Reported }}{\text { EML }}$ & $\begin{array}{cc}\text { QAP 48 } \\
\text { Evaluation } \\
\text { Evaluation }\end{array}$ \\
\hline
\end{tabular}

Matrix: AI Air Filter Bq/filter

$\begin{array}{llrrrrrrr}1 & \text { AM241 } & 0.547 & 0.050 & 0.510 & 0.008 & 1.073 & \text { A } & \text { A } \\ 1 & \text { CO60 } & 9.420 & 1.270 & 9.160 & 0.580 & 1.028 & \text { A } & \text { A } \\ 1 & \text { CS137 } & 22.310 & 2.870 & 22.470 & 1.030 & 0.993 & \text { A } & \text { A } \\ 1 & \text { MN54 } & 5.130 & 0.790 & 4.920 & 0.400 & 1.043 & \text { A } & \text { A } \\ 1 & \text { PU238 } & 0.476 & 0.036 & 0.460 & 0.005 & 1.035 & \text { A } & \text { A } \\ 1 & \text { PU239 } & 0.446 & 0.034 & 0.420 & 0.006 & 1.062 & \text { A } & \text { A } \\ 1 & \text { SB125 } & 8.770 & 1.530 & 8.890 & 0.550 & 0.987 & \text { A } & \text { A }\end{array}$

Matrix: SO Soil $\mathrm{Bq} / \mathrm{kg}$

$\begin{array}{llrrrrrrr}1 & \text { AM241 } & 7.620 & 0.803 & 7.470 & 0.410 & 1.020 & \text { A } & \text { A } \\ 1 & \text { PU239 } & 13.000 & 1.030 & 13.090 & 0.570 & 0.993 & \text { A } & \text { A }\end{array}$

Matrix: VE Vegetation $\mathrm{Bq} / \mathrm{kg}$

$\begin{array}{lllllllll}1 & \text { AM241 } & 2.630 & 0.305 & 2.330 & 0.060 & 1.129 & \text { A } & \text { A } \\ 1 & \text { CM244 } & 1.880 & 0.190 & 1.760 & 0.070 & 1.068 & \text { A } & \text { A } \\ 1 & \text { PU239 } & 4.080 & 0.355 & 3.720 & 0.270 & 1.097 & \text { A } & \text { A }\end{array}$

Matrix: WA Water $\mathrm{Bq} / \mathrm{L}$

$\begin{array}{llrrrrrrr}1 & \text { AM241 } & 1.320 & 0.120 & 1.250 & 0.080 & 1.056 & \text { A } & \text { A } \\ 1 & \text { CO60 } & 50.570 & 6.600 & 49.400 & 1.200 & 1.024 & \text { A } & \text { W } \\ 1 & \text { CS137 } & 50.190 & 6.500 & 50.000 & 1.700 & 1.004 & \text { A } & \text { A } \\ 1 & \text { H3 } & 74.660 & 5.890 & 76.200 & 2.900 & 0.980 & \text { A } & \text { A } \\ 1 & \text { MN54 } & 34.940 & 4.770 & 32.400 & 1.400 & 1.078 & \text { A } & \text { A } \\ 1 & \text { PU238 } & 1.120 & 0.091 & 1.100 & 0.010 & 1.018 & \text { A } & \text { A } \\ 1 & \text { PU239 } & 1.470 & 0.116 & 1.410 & 0.040 & 1.043 & \text { A } & \text { A } \\ 1 & \text { SR90 } & 2.320 & 0.402 & 2.110 & 0.180 & 1.100 & \text { A } & \text { A }\end{array}$

Values for elemental uranium are reported in $\mu \mathrm{g} /$ filter, $\mathrm{g}$, or $\mathrm{mL} . \quad$ pCi/g or $\mathrm{mL}=\mathrm{Bq} \times 0.027$

Evaluation: $A=A c c e p t a b l e, W=A c c e p t a b l e$ with Warning, $N=$ Not Acceptable

If the evaluation system is not appropriate for the types of analyses performed in your lab, apply a site specific evaluation. 


\section{QAP 49 Results by Laboratory}

Lab: FG FGL Environmental, Santa Paula, CA

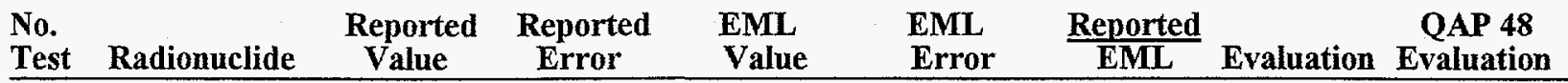

Matrix: AI Air Filter Bq / filter

\begin{tabular}{|c|c|c|c|c|c|c|}
\hline CO60 & 8.910 & 0.140 & 9.160 & 0.580 & 0.973 & A \\
\hline CS137 & 23.180 & 0.140 & 22.470 & 1.030 & 1.032 & A \\
\hline GROSS ALPHA & 1.602 & 0.118 & 1.650 & 0.160 & 0.971 & A \\
\hline GROSS BETA & 1.624 & 0.122 & 2.160 & 0.070 & 0.752 & W \\
\hline MN54 & 5.078 & 0.140 & 4.920 & 0.400 & 1.032 & A \\
\hline SB125 & 10.770 & 0.600 & 8.890 & 0.550 & 1.211 & W \\
\hline
\end{tabular}

Matrix: SO Soil $\mathrm{Bq} / \mathrm{kg}$

$\begin{array}{ll}1 & \mathrm{AC} 228 \\ 1 & \mathrm{BI} 212 \\ 1 & \mathrm{BI214} \\ 1 & \mathrm{CS} 137 \\ 1 & \mathrm{~K} 40 \\ 1 & \mathrm{~PB} 210 \\ 1 & \mathrm{~PB} 212 \\ 1 & \mathrm{~PB} 214 \\ 1 & \mathrm{PU} 239 \\ 1 & \mathrm{RA226} \\ 1 & \mathrm{TH228} \\ 1 & \mathrm{TH234} \\ 1 & \mathrm{TL} 208 \\ 1 & \mathrm{U} 234 \\ 1 & \mathrm{U} 238\end{array}$

$$
\begin{array}{r}
60.110 \\
66.280 \\
47.800 \\
888.300 \\
302.000 \\
130.000 \\
69.040 \\
41.410 \\
9.450 \\
204.100 \\
32.640 \\
66.300 \\
29.110 \\
110.400 \\
97.370
\end{array}
$$

$\begin{array}{rr}1.200 & 52.600 \\ 6.690 & 58.300 \\ 8.000 & 28.800 \\ 1.000 & 954.000 \\ 6.410 & 314.000 \\ 58.700 & 32.000 \\ 1.200 & 52.800 \\ 2.050 & 29.100 \\ 1.300 & 13.090 \\ 15.300 & 29.000 \\ 5.600 & 52.700 \\ 6.100 & 114.000 \\ 1.000 & 18.300 \\ 8.990 & 113.000 \\ 4.660 & 120.000\end{array}$

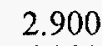

5.900

0.500

38.000

13.000

3.300

3.700

1.200

0.570

1.000

4.000

6.000

1.100

6.000

9.000

$\begin{array}{ll}1.143 & \mathrm{~A} \\ 1.137 & \mathrm{~A} \\ 1.660 & \mathrm{~A} \\ 0.931 & \mathrm{~A} \\ 0.962 & \mathrm{~A} \\ 4.063 & \mathrm{~A} \\ 1.308 & \mathrm{~A} \\ 1.423 & \mathrm{~A} \\ 0.722 & \mathrm{~W} \\ 7.038 & \mathrm{~A} \\ 0.619 & \mathrm{~A} \\ 0.582 & \mathrm{~A} \\ 1.591 & \mathrm{~A} \\ 0.977 & \mathrm{~A} \\ 0.811 & \mathrm{~A}\end{array}$

A
A
A
A
A
A

Matrix: WA Water $\mathrm{Bq} / \mathrm{L}$

$\begin{array}{llr}1 & \text { AM241 } & 1.638 \\ 1 & \text { Bq U } & 1.058 \\ 1 & \text { CO60 } & 50.760 \\ 1 & \text { CS137 } & 46.160 \\ 1 & \text { GROSS ALPHA } & 1153.000 \\ 1 & \text { GROSS BETA } & 1231.000 \\ 1 & \text { H3 } & 3220.000 \\ 1 & \text { MN54 } & 32.600 \\ 1 & \text { PU238 } & 1.118 \\ 1 & \text { PU239 } & 1.414 \\ 1 & \text { U234 } & 0.246 \\ 1 & \text { U238 } & 0.190\end{array}$

$$
\begin{array}{r}
0.063 \\
0.370 \\
0.400 \\
0.850 \\
53.000 \\
63.000 \\
340.000 \\
0.900 \\
0.062 \\
0.071 \\
0.109 \\
0.060
\end{array}
$$

1.250
1.050
49.400
50.000
1080.000
1420.000
76.200
32.400
1.100
1.410
0.510
0.520

0.080
0.080
1.200
1.700
60.000
60.000
2.900
1.400
0.010
0.040
0.030
0.050

1.310
1.008
1.028
0.923
1.068
0.867
42.257
1.006
1.016
1.003
0.482
0.366

A

A

A

A

ran 


\section{QAP 49 Results by Laboratory}

Lab: FJ The University of the South Pacific, Fiji Islands

\begin{tabular}{|c|c|c|c|c|c|c|c|c|}
\hline $\begin{array}{l}\text { No. } \\
\text { Test }\end{array}$ & Radionuclide & $\begin{array}{c}\text { Reported } \\
\text { Value }\end{array}$ & $\begin{array}{c}\text { Reported } \\
\text { Error } \\
\end{array}$ & $\begin{array}{l}\text { EML } \\
\text { Value }\end{array}$ & $\begin{array}{l}\text { EML } \\
\text { Error }\end{array}$ & $\frac{\text { Reported }}{\text { EML }}$ & Evaluation & $\begin{array}{r}\text { QA } \\
\text { Eval } \\
\end{array}$ \\
\hline \multicolumn{9}{|c|}{ Matrix: AI Air Filter $\mathrm{Bq} /$ filter } \\
\hline 1 & $\mathrm{AM} 241$ & 0.460 & 0.090 & 0.510 & 0.008 & 0.902 & A & $\mathrm{A}$ \\
\hline 1 & $\mathrm{CO} 60$ & 10.400 & 0.280 & 9.160 & 0.580 & 1.135 & $\mathrm{~W}$ & $W$ \\
\hline 1 & CS137 & 23.900 & 0.220 & 22.470 & 1.030 & 1.064 & A & $\ddot{A}$ \\
\hline 1 & MN54 & 7.590 & 0.490 & 4.920 & 0.400 & 1.543 & $\mathrm{~N}$ & $N$ \\
\hline 1 & SB125 & 9.510 & 0.210 & 8.890 & 0.550 & 1.070 & A & W \\
\hline
\end{tabular}

Values for elemental uranium are reported in $\mu \mathrm{g} /$ filter, $\mathrm{g}$, or $\mathrm{mL}$.

$\mathrm{pCi} / \mathrm{g}$ or $\mathrm{mL}=\mathrm{Bq} \times 0.027$

Evaluation: $A=A c c e p t a b l e, W=A c c e p t a b l e$ with Warning, $N=$ Not Acceptable

If the evaluation system is not appropriate for the types of analyses performed in your lab, apply a site specific evaluation. 


\section{QAP 49 Results by Laboratory}

Lab: FL Florida Dept of Health \& Rehab. Serv, Orlando

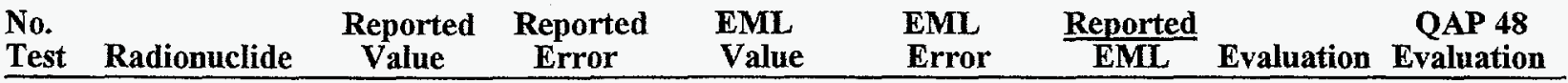

Matrix: AI Air Filter $\mathrm{Bq} /$ filter

$\begin{array}{llrrrrrrr}1 & \text { CO60 } & 8.630 & 0.050 & 9.160 & 0.580 & 0.942 & \mathrm{~A} & \mathrm{~A} \\ 1 & \text { CS137 } & 23.830 & 0.100 & 22.470 & 1.030 & 1.061 & \mathrm{~A} & \mathrm{~A} \\ 1 & \text { GROSS ALPHA } & 1.460 & 0.070 & 1.650 & 0.160 & 0.885 & \mathrm{~A} & \mathrm{~W} \\ 1 & \text { GROSS BETA } & 2.080 & 0.070 & 2.160 & 0.070 & 0.963 & \mathrm{~A} & \mathrm{~A} \\ 1 & \text { MN54 } & 5.250 & 0.050 & 4.920 & 0.400 & 1.067 & \mathrm{~A} & \mathrm{~A} \\ 1 & \text { SB125 } & 7.300 & 0.200 & 8.890 & 0.550 & 0.821 & \text { W } & \mathrm{A}\end{array}$

Matrix: SO Soil $\mathrm{Bq} / \mathrm{kg}$

$\begin{array}{llrrrrrrr}1 & \text { AM241 } & 6.600 & 0.600 & 7.470 & 0.410 & 0.884 & \text { A } & \text { A } \\ 1 & \text { CS137 } & 945.000 & 2.000 & 954.000 & 38.000 & 0.991 & \text { A } & \text { A } \\ 1 & \text { K40 } & 332.000 & 6.000 & 314.000 & 13.000 & 1.057 & \text { A } & \text { A } \\ 1 & \text { U238 } & 82.000 & 4.000 & 120.000 & 9.000 & 0.683 & \text { W } & \text { A }\end{array}$

Matrix: VE Vegetation $\mathrm{Bq} / \mathrm{kg}$

\begin{tabular}{|c|c|c|c|c|c|c|c|}
\hline AM241 & 2.600 & 0.500 & 2.330 & 0.060 & 1.116 & A & W \\
\hline CO60 & 20.200 & 0.500 & 20.000 & 1.000 & 1.010 & A & A \\
\hline CS137 & 402.000 & 2.000 & 390.000 & 20.000 & 1.031 & A & A \\
\hline $\mathrm{K} 40$ & 499.000 & 9.000 & 460.000 & 20.000 & 1.085 & A & A \\
\hline
\end{tabular}

Matrix: WA Water $\mathrm{Bq} / \mathrm{L}$

$\begin{array}{llrrrrrrr}1 & \text { AM241 } & 1.600 & 0.400 & 1.250 & 0.080 & 1.280 & \text { W } & \text { W } \\ 1 & \text { CO60 } & 51.300 & 0.400 & 49.400 & 1.200 & 1.038 & \text { A } & \text { A } \\ 1 & \text { CS137 } & 52.700 & 0.600 & 50.000 & 1.700 & 1.054 & \text { A } & \text { A } \\ 1 & \text { GROSS ALPHA } & 1207.380 & 19.510 & 1080.000 & 60.000 & 1.118 & \text { A } & \text { N } \\ 1 & \text { GROSS BETA } & 1405.500 & 13.940 & 1420.000 & 60.000 & 0.990 & \text { A } & \text { A } \\ 1 & \text { H3 } & 84.080 & 3.100 & 76.200 & 2.900 & 1.103 & \text { A } & \text { A } \\ 1 & \text { MN54 } & 36.400 & 0.500 & 32.400 & 1.400 & 1.123 & \text { A } & \text { A } \\ 1 & \text { NI63 } & 95.230 & 0.670 & 95.700 & 0.900 & 0.995 & \text { A } & \end{array}$

Values for elemental uranium are reported in $\mu \mathrm{g} /$ filter, $\mathrm{g}$, or $\mathrm{mL} . \quad \mathrm{pCi} / \mathrm{g}$ or $\mathrm{mL}=\mathrm{Bq} \times \mathbf{0 . 0 2 7}$

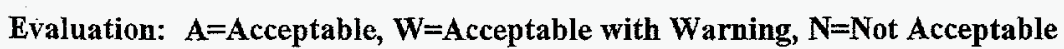

If the evaluation system is not appropriate for the types of analyses performed in your lab, apply a site specific evaluation. 


\section{QAP 49 Results by Laboratory}

Lab: FM Florida Mobile Emergency Radiological Laboratory, Orlando

\begin{tabular}{|c|c|c|c|c|c|c|c|}
\hline $\begin{array}{l}\text { No. } \\
\text { Test }\end{array}$ & Radionuclide & $\begin{array}{l}\text { Reported } \\
\text { Value }\end{array}$ & $\underset{\text { Error }}{\text { Reported }}$ & $\begin{array}{l}\text { EML } \\
\text { Value }\end{array}$ & $\begin{array}{l}\text { EML } \\
\text { Error }\end{array}$ & $\frac{\text { Reported }}{\text { EML }}$ & 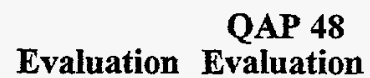 \\
\hline
\end{tabular}

Matrix: AI Air Filter $\mathrm{Bq} /$ filter

$\begin{array}{llrrrrrrr}1 & \text { AM241 } & 0.570 & 0.030 & 0.510 & 0.008 & 1.118 & \mathrm{~A} & \\ 1 & \text { CO60 } & 9.150 & 0.070 & 9.160 & 0.580 & 0.999 & \mathrm{~A} & \mathrm{~A} \\ \mathrm{l} & \text { CS137 } & 24.400 & 0.160 & 22.470 & 1.030 & 1.086 & \mathrm{~A} & \mathrm{~A} \\ 1 & \text { MN54 } & 5.600 & 0.160 & 4.920 & 0.400 & 1.138 & \mathrm{~A} & \mathrm{~A} \\ 1 & \text { SB125 } & 9.780 & 0.090 & 8.890 & 0.550 & 1.100 & \mathrm{~A} & \mathrm{~A}\end{array}$

Matrix: WA Water $\mathrm{Bq} / \mathrm{L}$

$\begin{array}{llrlrllll}1 & \text { AM241 } & 1.300 & 0.110 & 1.250 & 0.080 & 1.040 & \text { A } & \\ \text { I } & \text { CO60 } & 49.700 & 0.320 & 49.400 & 1.200 & 1.006 & \text { A } & \text { A } \\ 1 & \text { CS137 } & 51.200 & 0.360 & 50.000 & 1.700 & 1.024 & \text { A } & \text { A } \\ 1 & \text { MN54 } & 34.400 & 0.320 & 32.400 & 1.400 & 1.062 & \text { A } & \text { A }\end{array}$

Values for elemental uranium are reported in $\mu \mathrm{g} /$ filter, $\mathrm{g}$, or $\mathrm{mL} . \quad \mathrm{pCi} / \mathrm{g}$ or $\mathrm{mL}=\mathrm{Bq} \times 0.027$

Evaluation: $A=$ Acceptable, $W=$ Acceptable with Warning, $N=$ Not Acceptable

If the evaluation system is not appropriate for the types of analyses performed in your lab, apply a site specific evaluation. 


\section{QAP 49 Results by Laboratory}

Lab: FN Fermi Lab, Batavia, IL

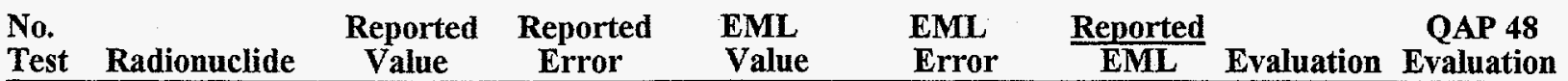

Matrix: AI Air Filter $\mathrm{Bq} /$ filter

$\begin{array}{llrrrrrrr}1 & \text { CO60 } & 8.760 & 0.660 & 9.160 & 0.580 & 0.956 & \text { A } & \text { A } \\ 1 & \text { CS137 } & 21.100 & 2.100 & 22.470 & 1.030 & 0.939 & \text { A } & \text { A } \\ 1 & \text { MN54 } & 4.640 & 0.510 & 4.920 & 0.400 & 0.943 & \text { A } & \text { A } \\ 1 & \text { SB125 } & 10.000 & 0.600 & 8.890 & 0.550 & 1.125 & \text { A } & \text { A }\end{array}$

Matrix: SO Soil $\mathrm{Bq} / \mathrm{kg}$

$\begin{array}{lllllllll}1 & \mathrm{CS} 137 & 954.000 & 96.000 & 954.000 & 38.000 & 1.000 & \mathrm{~A} & \mathrm{~A} \\ \mathrm{l} & \mathrm{K} 40 & 321.000 & 34.000 & 314.000 & 13.000 & 1.022 & \mathrm{~A} & \mathrm{~A}\end{array}$

Matrix: VE Vegetation $\mathrm{Bq} / \mathrm{kg}$

$\begin{array}{llrrrrrrr}1 & \text { CO60 } & 20.700 & 1.700 & 20.000 & 1.000 & 1.035 & \mathrm{~A} & \text { W } \\ 1 & \text { CS137 } & 388.000 & 39.000 & 390.000 & 20.000 & 0.995 & \mathrm{~A} & \text { W } \\ 1 & \text { K40 } & 452.000 & 47.000 & 460.000 & 20.000 & 0.983 & \mathrm{~A} & \mathrm{~A}\end{array}$

Matrix: WA Water $\mathrm{Bq} / \mathrm{L}$

$\begin{array}{lllllllll}1 & \text { CO60 } & 47.500 & 3.500 & 49.400 & 1.200 & 0.962 & \mathrm{~A} & \mathrm{~A} \\ \mathrm{l} & \text { CS137 } & 49.600 & 5.100 & 50.000 & 1.700 & 0.992 & \mathrm{~A} & \mathrm{~A} \\ \mathrm{l} & \mathrm{H} 3 & 79.800 & 8.700 & 76.200 & 2.900 & 1.047 & \mathrm{~A} & \mathrm{~A} \\ 1 & \text { MN54 } & 34.300 & 3.500 & 32.400 & 1.400 & 1.059 & \mathrm{~A} & \mathrm{~A}\end{array}$

Values for elemental uranium are reported in $\mu \mathrm{g} /$ filter, $\mathrm{g}$, or $\mathrm{mL} . \quad \quad \mathrm{pCi} / \mathrm{g}$ or $\mathrm{mL}=\mathrm{Bq} \times \mathbf{0 . 0 2 7}$

Evaluation: A=Acceptable, $W=$ Acceptable with Warning, $N=$ Not Acceptable

If the evaluation system is not appropriate for the types of analyses performed in your lab, apply a site specific evaluation. 


\section{QAP 49 Results by Laboratory}

Lab: FS Florida State University, Tallahassee

\begin{tabular}{|c|c|c|c|c|c|c|c|}
\hline $\begin{array}{l}\text { No. } \\
\text { Test }\end{array}$ & adio & $\begin{array}{l}\text { Reported } \\
\text { Value }\end{array}$ & $\begin{array}{l}\text { Reported } \\
\text { Error }\end{array}$ & $\begin{array}{l}\text { EML } \\
\text { Value }\end{array}$ & $\begin{array}{l}\text { EML } \\
\text { Error }\end{array}$ & $\frac{\text { Reportec }}{\text { EML }}$ & $\begin{array}{c}\text { QAP 48 } \\
\text { Evaluation } \\
\text { Evaluation }\end{array}$ \\
\hline
\end{tabular}

Matrix: SO Soil $\mathrm{Bq} / \mathrm{kg}$

$\begin{array}{llrrrrrrr}1 & \text { AC228 } & 47.500 & 3.600 & 52.600 & 2.900 & 0.903 & \text { A } & \\ 1 & \text { AM241 } & 4.370 & 0.240 & 7.470 & 0.410 & 0.585 & \text { N } & \text { A } \\ 1 & \text { BI214 } & 29.400 & 2.000 & 28.800 & 0.500 & 1.021 & \text { A } & \\ 1 & \text { CS137 } & 954.000 & 36.000 & 954.000 & 38.000 & 1.000 & \text { A } & \text { A } \\ 1 & \text { K40 } & 325.000 & 20.000 & 314.000 & 13.000 & 1.035 & \text { A } & \text { A } \\ 1 & \text { PB210 } & 29.600 & 8.400 & 32.000 & 3.300 & 0.925 & \text { A } & \\ 1 & \text { PB214 } & 25.000 & 4.800 & 29.100 & 1.200 & 0.859 & \text { A } & \\ 1 & \text { TH234 } & 96.200 & 17.800 & 114.000 & 6.000 & 0.844 & \text { A } & \\ 1 & \text { TL208 } & 54.500 & 5.200 & 18.300 & 1.100 & 2.978 & \text { A } & \end{array}$

Values for elemental uranium are reported in $\mu$ g/filter, $\mathrm{g}$, or $\mathrm{mL} . \quad \mathrm{pCi} / \mathrm{g}$ or $\mathrm{mL}=\mathrm{Bq} \times 0.027$

Evaluation: $A=A c c e p t a b l e, ~ W=A c c e p t a b l e$ with Warning, $N=$ Not Acceptable

If the evaluation system is not appropriate for the types of analyses performed in your lab, apply a site specific evaluation. 


\section{QAP 49 Results by Laboratory}

Lab: GA Lockheed Martin, Pikton, $\mathrm{OH}$

\begin{tabular}{lccccc} 
No, & $\begin{array}{c}\text { Reported } \\
\text { Test }\end{array}$ & $\begin{array}{c}\text { Reported } \\
\text { Error }\end{array}$ & $\begin{array}{c}\text { EML } \\
\text { Value }\end{array}$ & $\begin{array}{c}\text { EML } \\
\text { Error }\end{array}$ & $\begin{array}{c}\text { Reported } \\
\text { EML }\end{array}$ Evaluation Evaluation \\
\hline
\end{tabular}

Matrix: AI Air Filter $\mathrm{Bq} /$ filter

$\begin{array}{llrrrrrrr}1 & \text { AM241 } & 0.560 & 0.018 & 0.510 & 0.008 & 1.098 & \mathrm{~A} & \mathrm{~A} \\ 1 & \text { CO60 } & 8.770 & 0.560 & 9.160 & 0.580 & 0.957 & \mathrm{~A} & \mathrm{~A} \\ 1 & \text { CS137 } & 21.500 & 0.900 & 22.470 & 1.030 & 0.957 & \mathrm{~A} & \mathrm{~A} \\ 1 & \text { MN54 } & 5.220 & 0.640 & 4.920 & 0.400 & 1.061 & \mathrm{~A} & \mathrm{~A} \\ 1 & \text { PU238 } & 0.550 & 0.021 & 0.460 & 0.005 & 1.196 & \mathrm{~W} & \mathrm{~A} \\ 1 & \text { PU239 } & 0.480 & 0.020 & 0.420 & 0.006 & 1.143 & \mathrm{~A} & \mathrm{~W} \\ 1 & \text { SB125 } & 9.230 & 1.430 & 8.890 & 0.550 & 1.038 & \mathrm{~A} & \mathrm{~A} \\ 1 & \text { SR90 } & 0.780 & 0.052 & 1.120 & 0.050 & 0.696 & \mathrm{~W} & \\ 1 & \text { U234 } & 0.340 & 0.051 & 0.260 & 0.010 & 1.308 & \mathrm{~A} & \mathrm{~W} \\ 1 & \text { U238 } & 0.340 & 0.050 & 0.260 & 0.010 & 1.308 & \mathrm{~A} & \mathrm{~A} \\ 1 & \text { ug U } & 27.000 & 3.800 & 20.960 & 0.100 & 1.288 & \mathrm{~A} & \end{array}$

Matrix: SO Soil $\mathrm{Bq} / \mathrm{kg}$

$\begin{array}{ll}1 & \text { AM241 } \\ 1 & \text { BI212 } \\ 1 & \text { BI214 } \\ 1 & \text { CS137 } \\ 1 & \text { K40 } \\ 1 & \text { PB212 } \\ 1 & \text { PB214 } \\ 1 & \text { PU239 } \\ 1 & \text { TH234 } \\ 1 & \text { TL208 } \\ 1 & \text { U234 } \\ 1 & \text { U238 } \\ 1 & \text { ug U }\end{array}$

6.900
65.700
31.300
1023.000
351.000
53.700
36.800
11.000
108.000
21.400
110.000
113.000
8.560

1.200

16.600

7.700

30.000

59.000

7.400

10.300

0.790

22.000

4.100

9.400

4.200

8.560

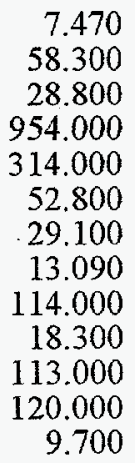
0.410
5.900
0.500
38.000
13.000
3.700
1.200
0.570
6.000
1.100
6.000
9.000
0.700

$$
\begin{aligned}
& 0.924 \\
& 1.127 \\
& 1.087 \\
& 1.072 \\
& 1.118 \\
& 1.017 \\
& 1.265 \\
& 0.840 \\
& 0.947 \\
& 1.169 \\
& 0.973 \\
& 0.942 \\
& 0.882
\end{aligned}
$$

A

A

A

A

W

W

A

Matrix: VE Vegetation $\mathrm{Bq} / \mathrm{kg}$

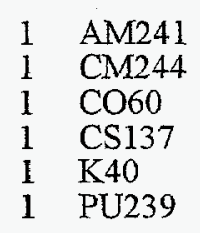

$$
\begin{array}{r}
2.900 \\
1.900 \\
26.600 \\
432.000 \\
581.000 \\
3.800
\end{array}
$$

$$
\begin{array}{r}
2.330 \\
1.760 \\
20.000 \\
390.000 \\
460.000 \\
3.720
\end{array}
$$

$$
\begin{array}{r}
0.060 \\
0.070 \\
1.000 \\
20.000 \\
20.000 \\
0.270
\end{array}
$$
1.245
1.080
1.330
1.108
1.263
1.022

$\begin{array}{ll}\text { A } & \text { A } \\ \text { A } & \text { A } \\ \text { W } & \text { A } \\ \text { A } & \text { A } \\ \text { W } & \text { A } \\ \text { A } & \text { A }\end{array}$

Matrix: WA Water $\mathrm{Bq} / \mathrm{L}$

$\begin{array}{ll}1 & \text { AM241 } \\ 1 & \text { CO60 } \\ 1 & \text { CS137 } \\ 1 & \text { MN54 } \\ 1 & \text { PU238 } \\ 1 & \text { PU239 } \\ 1 & \text { SR90 }\end{array}$

1.400
46.800
51.000
38.900
1.100
1.600
2.600

$\begin{array}{rr}0.210 & 1.250 \\ 4.600 & 49.400 \\ 4.000 & 50.000 \\ 5.300 & 32.400 \\ 0.040 & 1.100 \\ 0.050 & 1.410 \\ 0.030 & 2.110\end{array}$
0.080
1.200
1.700
1.400
0.010
0.040
0.180
1.120
0.947
1.020
1.201
1.000
1.135
1.232

$\begin{array}{ll}\text { A } & \text { A } \\ \text { A } & \text { W } \\ \text { A } & \text { A } \\ \text { W } & \text { A } \\ \text { A } & \text { W } \\ \text { A } & \text { A } \\ \text { W } & \end{array}$

\footnotetext{
Values for elemental uranium are reported in $\mu \mathrm{g} /$ filter, $\mathrm{g}$, or $\mathrm{mL}$.
}

\section{$\mathrm{pCi} / \mathrm{g}$ or $\mathrm{mL}=\mathrm{Bq} \times 0.027$}

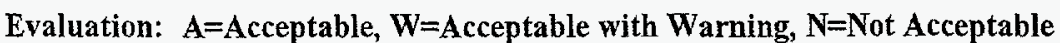

If the evaluation system is not appropriate for the types of analyses performed in your lab, apply a site specific evaluation. 


\section{QAP 49 Results by Laboratory}

Lab: GA Lockheed Martin, Pikton, $\mathrm{OH}$

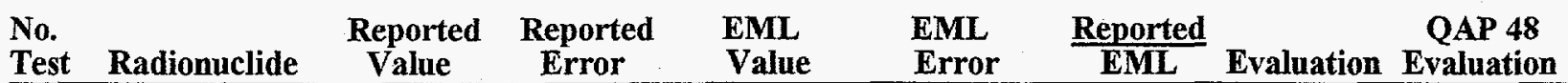

Matrix: WA Water $\mathrm{Bq} / \mathrm{L}$

$\begin{array}{lllllllll}\mathrm{I} & \mathrm{U} 234 & 0.740 & 0.080 & 0.510 & 0.030 & 1.451 & \mathrm{~N} & \mathrm{~A} \\ \mathrm{I} & \mathrm{U} 238 & 0.740 & 0.080 & 0.520 & 0.050 & 1.423 & \mathrm{~N} & \mathrm{~A} \\ \mathrm{I} & \text { ug U } & 0.059 & 0.007 & 0.040 & 0.003 & 1.475 & \mathrm{~N} & \end{array}$

Values for elemental uranium are reported in $\mu \mathrm{g} /$ filter, $g$, or $\mathrm{mL}$.

$\mathrm{pCi} / \mathrm{g}$ or $\mathrm{mL}=\mathrm{Bq} \times 0.027$

Evaluation: $A=A c c e p t a b l e, W=A c c e p t a b l e$ with Warning, $N=$ Not Acceptable

If the evaluation system is not appropriate for the types of analyses performed in your lab, apply a site specific evaluation. 


\section{QAP 49 Results by Laboratory}

Lab: GC Georgia Power Company Environmental Lab

\begin{tabular}{|c|c|c|c|c|c|c|c|}
\hline $\begin{array}{l}\text { No. } \\
\text { Test }\end{array}$ & Rad & $\begin{array}{c}\text { Reported } \\
\text { Value }\end{array}$ & $\begin{array}{c}\text { Reported } \\
\text { Error }\end{array}$ & $\begin{array}{l}\text { EML } \\
\text { Value }\end{array}$ & $\begin{array}{l}\text { EML } \\
\text { Error }\end{array}$ & $\frac{\text { Reported }}{\text { EML }}$ & $\begin{array}{c}\text { QAP 48 } \\
\text { Evaluation }\end{array}$ \\
\hline
\end{tabular}

Matrix: AI Air Filter Bq / filter

$\begin{array}{lllllllll}1 & \text { GROSS ALPHA } & 1.550 & 0.003 & 1.650 & 0.160 & 0.939 & \text { A } & \text { W } \\ 1 & \text { GROSS BETA } & 1.690 & 0.020 & 2.160 & 0.070 & 0.782 & \text { W } & \text { A }\end{array}$

Matrix: SO Soil $\mathrm{Bq} / \mathrm{kg}$

$\begin{array}{ll}1 & \mathrm{AC} 228 \\ 1 & \mathrm{BI} 14 \\ 1 & \mathrm{CS} 137 \\ 1 & \mathrm{~K} 40 \\ 1 & \text { PB212 } \\ 1 & \text { RA226 }\end{array}$

$\begin{array}{rr}54.700 & 9.000 \\ 34.000 & 1.800 \\ 933.100 & 35.100 \\ 369.500 & 21.230 \\ 48.100 & 2.400 \\ 212.600 & 56.100\end{array}$

52.600
28.800
954.000
314.000
52.800
29.000

2.900
0.500
38.000
13.000
3.700
1.000

1.040

1.181

0.978

1.177

0.911

7.331

$\begin{array}{ll}\text { A } & \\ \text { A } & \\ \text { A } & \text { A } \\ \text { A } & \text { A } \\ \text { A } & \\ \text { A } & \end{array}$

Matrix: VE Vegetation $\mathrm{Bq} / \mathrm{kg}$

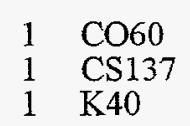

$$
\begin{array}{r}
21.200 \\
399.200 \\
494.000
\end{array}
$$

2.500

5.300

47.400
20.000
390.000
460.000

1.000
20.000
20.000

1.060

1.024

1.074

$\begin{array}{ll}\text { A } & \text { A } \\ \mathrm{A} & \mathrm{A} \\ \mathrm{A} & \mathrm{A}\end{array}$

Matrix: WA Water $\mathrm{Bq} / \mathrm{L}$

$\begin{array}{llr}1 & \text { CO60 } & 51.200 \\ 1 & \text { CS137 } & 54.000 \\ 1 & \text { FE55 } & 141.000 \\ 1 & \text { GROSS ALPHA } & 504.000 \\ 1 & \text { GROSS BETA } & 1218.000 \\ 1 & \text { H3 } & 79.000 \\ 1 & \text { MN54 } & 36.000 \\ 1 & \text { SR90 } & 2.620\end{array}$

2.530
3.140
3.700
53.200
25.200
1.310
2.180
0.160

49.400
50.000
139.000
1080.000
1420.000
76.200
32.400
2.110
1.200
1.700
2.000
60.000
60.000
2.900
1.400
0.180
1.036
1.080
1.014
0.467
0.858
1.037
1.111
1.242

$\begin{array}{ll}\text { A } & \text { A } \\ \text { A } & \text { A } \\ \text { A } & \text { A } \\ \text { N } & \text { A } \\ \text { A } & \text { W } \\ \text { A } & \text { A } \\ \text { A } & \text { A } \\ \text { W } & \text { A }\end{array}$

Values for elemental uranium are reported in $\mu \mathrm{g} /$ filter, $\mathrm{g}$, or $\mathrm{mL}$.

$\mathrm{pCi} / \mathrm{g}$ or $\mathrm{mL}=\mathrm{Bq} \times 0.027$

Evaluation: $A=A c c e p t a b l e, W=A c c e p t a b l e$ with Warning, $N=$ Not Acceptable

If the evaluation system is not appropriate for the types of analyses performed in your lab, apply a site specific evaluation. 


\section{QAP 49 Results by Laboratory}

Lab: GE General Engineering Labs, Charleston, SC

\begin{tabular}{|c|c|c|c|c|c|c|c|}
\hline $\begin{array}{l}\text { No. } \\
\text { Test }\end{array}$ & Radionuclide & $\begin{array}{c}\text { Reported } \\
\text { Value }\end{array}$ & $\begin{array}{l}\text { Reported } \\
\text { Error }\end{array}$ & $\begin{array}{l}\text { EML } \\
\text { Value }\end{array}$ & $\begin{array}{l}\text { EML } \\
\text { Error }\end{array}$ & $\frac{\text { Reported }}{\text { EML }}$ & 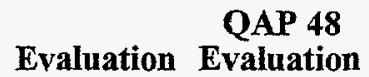 \\
\hline
\end{tabular}

Matrix: AI Air Filter $\mathrm{Bq}$ / filter

$\begin{array}{llrrrrrrr}1 & \text { AM241 } & 0.549 & 0.142 & 0.510 & 0.008 & 1.077 & \mathrm{~A} & \text { W } \\ 1 & \text { CO60 } & 9.183 & 1.553 & 9.160 & 0.580 & 1.003 & \mathrm{~A} & \mathrm{~A} \\ 1 & \text { CS137 } & 22.588 & 3.056 & 22.470 & 1.030 & 1.005 & \mathrm{~A} & \mathrm{~A} \\ 1 & \text { GROSS ALPHA } & 1.632 & 0.007 & 1.650 & 0.160 & 0.989 & \mathrm{~A} & \mathrm{~A} \\ 1 & \text { GROSS BETA } & 1.780 & 0.004 & 2.160 & 0.070 & 0.824 & \mathrm{~W} & \mathrm{~A} \\ 1 & \text { MN54 } & 5.300 & 1.022 & 4.920 & 0.400 & 1.077 & \mathrm{~A} & \mathrm{~A} \\ 1 & \text { PU238 } & 0.509 & 0.117 & 0.460 & 0.005 & 1.106 & \mathrm{~A} & \mathrm{~A} \\ 1 & \text { PU239 } & 0.461 & 0.107 & 0.420 & 0.006 & 1.097 & \mathrm{~A} & \mathrm{~A} \\ 1 & \text { SB125 } & 2.722 & 1.082 & 8.890 & 0.550 & 0.306 & \mathrm{~N} & \mathrm{~A} \\ 1 & \text { SR90 } & 1.184 & 0.097 & 1.120 & 0.050 & 1.057 & \mathrm{~A} & \mathrm{~N} \\ 1 & \text { U234 } & 0.242 & 0.073 & 0.260 & 0.010 & 0.932 & \mathrm{~A} & \mathrm{~A} \\ 1 & \text { U238 } & 0.246 & 0.073 & 0.260 & 0.010 & 0.945 & \mathrm{~A} & \mathrm{~A} \\ 1 & \text { ug U } & 16.940 & 0.662 & 20.960 & 0.100 & 0.808 & \mathrm{~W} & \mathrm{~A}\end{array}$

Matrix: SO Soil $\mathrm{Bq} / \mathrm{kg}$

$\begin{array}{ll}1 & \text { AC228 } \\ 1 & \text { AM241 } \\ 1 & \text { BI212 } \\ 1 & \text { CS137 } \\ 1 & \text { K40 } \\ 1 & \text { PB212 } \\ 1 & \text { PB214 } \\ 1 & \text { PU239 } \\ 1 & \text { RA226 } \\ 1 & \text { SR90 } \\ 1 & \text { TH228 } \\ 1 & \text { TH234 } \\ 1 & \text { U234 } \\ 1 & \text { U238 }\end{array}$

Matrix: VE Vegetation $\mathrm{Bq} / \mathrm{kg}$

$\begin{array}{rr}53.595 & 14.605 \\ 6.956 & 1.541 \\ 31.758 & 14.905 \\ 980.685 & 140.339 \\ 350.316 & 63.867 \\ 56.534 & 9.290 \\ 32.560 & 8.984 \\ 12.173 & 2.554 \\ 29.981 & 11.380 \\ 32.930 & 4.186 \\ 53.600 & 14.600 \\ 109.280 & 84.147 \\ 103.045 & 21.009 \\ 117.290 & 23.785\end{array}$

$\begin{array}{rr}2.690 & 0.824 \\ 2.035 & 0.673 \\ 19.349 & 4.423 \\ 377.955 & 54.560 \\ 468.420 & 81.073 \\ 5.032 & 1.091 \\ 588.239 & 7.950\end{array}$

$$
\begin{array}{r}
52.600 \\
7.470 \\
58.300 \\
954.000 \\
314.000 \\
52.800 \\
29.100 \\
13.090 \\
29.000 \\
39.630 \\
52.700 \\
114.000 \\
113.000 \\
120.000
\end{array}
$$

$\begin{array}{ll}1.019 & \mathrm{~A} \\ 0.931 & \mathrm{~A} \\ 0.545 & \mathrm{~A} \\ 1.028 & \mathrm{~A} \\ 1.116 & \mathrm{~A} \\ 1.071 & \mathrm{~A} \\ 1.119 & \mathrm{~A} \\ 0.930 & \mathrm{~A} \\ 1.034 & \mathrm{~A} \\ 0.831 & \mathrm{~A} \\ 1.017 & \mathrm{~A} \\ 0.959 & \mathrm{~A} \\ 0.912 & \mathrm{~A} \\ 0.977 & \mathrm{~A}\end{array}$

A

A

A

A

A

A

$\begin{array}{rr}2.330 & 0.060 \\ 1.760 & 0.070 \\ 20.000 & 1.000 \\ 390.000 & 20.000 \\ 460.000 & 20.000 \\ 3.720 & 0.270 \\ 606.000 & 40.000\end{array}$

$\begin{array}{ll}\mathrm{A} & \mathrm{A} \\ \mathrm{A} & \mathrm{A} \\ \mathrm{A} & \mathrm{A} \\ \mathrm{A} & \mathrm{A} \\ \mathrm{A} & \mathrm{A} \\ \mathrm{W} & \mathrm{W} \\ \mathrm{A} & \mathrm{A}\end{array}$

Matrix: WA Water $\mathrm{Bq} / \mathrm{L}$

$\begin{array}{ll}1 & \operatorname{AM} 241 \\ 1 & \operatorname{CO} 60 \\ 1 & \operatorname{CS} 137\end{array}$

1.230

53.502

52.558
0.239
7.493
7.238

1.250

49.400

50.000
0.984
1.083
1.051

W
A
A
A
A
A
A
A
A
N
A
A
A

\footnotetext{
Values for elemental uranium are reported in $\mu \mathrm{g} /$ filter, $\mathrm{g}$, or $\mathrm{mL}$.

$\mathrm{pCi} / \mathrm{g}$ or $\mathrm{mL}=\mathrm{Bq} \times 0.027$

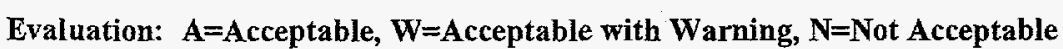

If the evaluation system is not appropriate for the types of analyses performed in your lab, apply a site specific evaluation.
} 


\section{QAP 49 Results by Laboratory}

Lab: GE General Engineering Labs, Charleston, SC

\begin{tabular}{|c|c|c|c|c|c|c|c|c|}
\hline $\begin{array}{l}\text { No. } \\
\text { Test }\end{array}$ & Radionuclide & $\begin{array}{c}\text { Reported } \\
\text { Value }\end{array}$ & $\begin{array}{c}\text { Reported } \\
\text { Error }\end{array}$ & $\begin{array}{l}\text { EML } \\
\text { Value } \\
\end{array}$ & $\begin{array}{l}\text { EML } \\
\text { Error }\end{array}$ & $\frac{\text { Reported }}{\text { EML }}$ & Evaluation & $\begin{array}{r}\text { QA } \\
\text { Eval }\end{array}$ \\
\hline \multicolumn{9}{|c|}{ Matrix: WA Water $\mathrm{Bq} / \mathrm{L}$} \\
\hline 1 & FE55 & 116.971 & 21.328 & 139.000 & 2.000 & 0.842 & A & A \\
\hline 1 & GROSS ALPHA & 1124.800 & 46.990 & 1080.000 & 60.000 & 1.041 & A & W \\
\hline 1 & GROSS BETA & 1228.400 & 39.220 & 1420.000 & 60.000 & 0.865 & A & A \\
\hline 1 & $\mathrm{H} 3$ & 91.865 & 17.109 & 76.200 & 2.900 & 1.206 & A & A \\
\hline 1 & $\mathrm{MN} 54$ & 36.419 & 5.788 & 32.400 & 1.400 & 1.124 & $A$ & $\mathrm{~A}$ \\
\hline 1 & N163 & 55.445 & 6.660 & 95.700 & 0.900 & 0.579 & A & \\
\hline$\hat{1}$ & PU238 & 1.136 & 0.246 & 1.100 & 0.010 & 1.033 & A & A \\
\hline 1 & PU239 & 1.462 & 0.308 & 1.410 & 0.040 & 1.037 & A & A \\
\hline 1 & SR90 & 0.201 & 0.035 & 2.110 & 0.180 & 0.095 & $\mathrm{~N}$ & A \\
\hline 1 & U234 & 0.540 & 0.172 & 0.510 & 0.030 & 1.059 & A & $A$ \\
\hline 1 & U238 & 0.518 & 0.166 & 0.520 & 0.050 & 0.996 & A & A \\
\hline 1 & ug U & 0.041 & 0.001 & 0.040 & 0.003 & 1.013 & $\mathrm{~A}$ & A \\
\hline
\end{tabular}

Values for elemental uranium are reported in $\mu \mathrm{g} /$ filter, $\mathrm{g}$, or $\mathrm{mL}$. $\mathrm{pCi} / \mathrm{g}$ or $\mathrm{mL}=\mathrm{Bq} \times 0.027$

Evaluation: $A=A c c e p t a b l e, W=$ Acceptable with $W$ arning, $N=$ Not Acceptable If the evaluation system is not appropriate for the types of analyses performed in your lab, apply a site specific evaluation. 


\section{QAP 49 Results by Laboratory}

Lab: GP GPU Nuclear, Inc., Harrisburg, PA

\begin{tabular}{|c|c|c|c|c|c|c|c|}
\hline 0 . & Radi & $\begin{array}{l}\text { Reported } \\
\text { Value }\end{array}$ & $\begin{array}{c}\text { Reported } \\
\text { Error }\end{array}$ & $\begin{array}{l}\text { EML } \\
\text { Value }\end{array}$ & $\begin{array}{l}\text { EML } \\
\text { Error }\end{array}$ & $\frac{\text { Reported }}{\text { EML }}$ & $\begin{array}{c}\text { QAP 48 } \\
\text { Evaluation }\end{array}$ \\
\hline
\end{tabular}

Matrix: AI Air Filter Bq / filter

$\begin{array}{llrrrrrrr}1 & \text { AM241 } & 0.500 & 0.050 & 0.510 & 0.008 & 0.980 & \mathrm{~A} & \mathrm{~A} \\ 1 & \text { Bq U } & 0.420 & & 0.530 & 0.020 & 0.792 & \mathrm{~N} & \mathrm{~A} \\ 1 & \text { CO60 } & 9.300 & 1.600 & 9.160 & 0.580 & 1.015 & \mathrm{~A} & \mathrm{~A} \\ 1 & \text { CS137 } & 22.000 & 3.000 & 22.470 & 1.030 & 0.979 & \mathrm{~A} & \mathrm{~N} \\ 1 & \text { GROSS ALPHA } & 1.700 & 0.200 & 1.650 & 0.160 & 1.030 & \mathrm{~A} & \mathrm{~A} \\ 1 & \text { GROSS BETA } & 2.000 & 0.200 & 2.160 & 0.070 & 0.926 & \mathrm{~A} & \mathrm{~A} \\ 1 & \text { MN54 } & 5.000 & 0.900 & 4.920 & 0.400 & 1.016 & \mathrm{~A} & \mathrm{~A} \\ 1 & \text { PU238 } & 0.480 & 0.050 & 0.460 & 0.005 & 1.043 & \mathrm{~A} & \mathrm{~W} \\ 1 & \text { PU239 } & 0.440 & 0.040 & 0.420 & 0.006 & 1.048 & \mathrm{~A} & \mathrm{~A} \\ 1 & \text { SB125 } & 13.000 & 2.000 & 8.890 & 0.550 & 1.462 & \mathrm{~N} & \mathrm{~W} \\ 1 & \text { SR90 } & 1.000 & 0.300 & 1.120 & 0.050 & 0.893 & \mathrm{~A} & \mathrm{~A} \\ 1 & \text { U234 } & 0.200 & 0.020 & 0.260 & 0.010 & 0.769 & \mathrm{~N} & \mathrm{~A} \\ 1 & \text { U238 } & 0.210 & 0.030 & 0.260 & 0.010 & 0.808 & \mathrm{~N} & \mathrm{~A}\end{array}$

Matrix: SO Soil $\mathrm{Bq} / \mathrm{kg}$

$\begin{array}{llrrrrrrr}1 & \text { CS137 } & 1000.000 & 100.000 & 954.000 & 38.000 & 1.048 & \text { A } & \text { A } \\ 1 & \text { K40 } & 350.000 & 30.000 & 314.000 & 13.000 & 1.115 & \text { A } & \text { A } \\ 1 & \text { SR90 } & 19.000 & 7.000 & 39.630 & 0.003 & 0.479 & \text { N } & \end{array}$

Matrix: VE Vegetation $\mathrm{Bq} / \mathrm{kg}$

$\begin{array}{llrrrrrrr}1 & \text { AM241 } & 2.800 & 0.800 & 2.330 & 0.060 & 1.202 & \text { A } & \\ 1 & \text { CM244 } & 1.500 & 0.600 & 1.760 & 0.070 & 0.852 & \text { A } & \\ 1 & \text { CO60 } & 21.000 & 3.000 & 20.000 & 1.000 & 1.050 & \text { A } & \text { A } \\ 1 & \text { CS137 } & 410.000 & 40.000 & 390.000 & 20.000 & 1.051 & \text { A } & \text { A } \\ 1 & \text { K40 } & 520.000 & 50.000 & 460.000 & 20.000 & 1.130 & \text { A } & \text { A } \\ 1 & \text { PU238 } & 0.260 & 0.150 & 0.310 & 0.070 & 0.839 & \text { A } & \\ 1 & \text { PU239 } & 3.400 & 0.600 & 3.720 & 0.270 & 0.914 & \text { A } & \\ 1 & \text { SR90 } & 580.000 & 50.000 & 606.000 & 40.000 & 0.957 & \text { A } & \text { A }\end{array}$

Matrix: WA Water $\mathrm{Bq} / \mathrm{L}$

$\begin{array}{llr}1 & \text { AM241 } & 1.400 \\ 1 & \text { Bq U } & 0.970 \\ 1 & \text { CO60 } & 51.000 \\ 1 & \text { CS137 } & 52.000 \\ 1 & \text { FE55 } & 160.000 \\ 1 & \text { GROSS ALPHA } & 980.000 \\ 1 & \text { GROSS BETA } & 1300.000 \\ 1 & \text { MN54 } & 34.000 \\ 1 & \text { PU238 } & 1.100 \\ 1 & \text { PU239 } & 1.500 \\ 1 & \text { SR90 } & 1.500 \\ 1 & \text { U234 } & 0.470 \\ 1 & \text { U238 } & 0.500\end{array}$

0.300
5.000
5.000
20.000
100.000
100.000
3.000
0.100
0.200
0.600
0.110
0.120

1.250
1.050
49.400
50.000
139.000
1080.000
1420.000
32.400
1.100
1.410
2.110
0.510
0.520

$\begin{array}{rr}0.080 & 1.120 \\ 0.080 & 0.924 \\ 1.200 & 1.032 \\ 1.700 & 1.040 \\ 2.000 & 1.151 \\ 60.000 & 0.907 \\ 60.000 & 0.915 \\ 1.400 & 1.049 \\ 0.010 & 1.000 \\ 0.040 & 1.064 \\ 0.180 & 0.711 \\ 0.030 & 0.922 \\ 0.050 & 0.962\end{array}$

$\begin{array}{ll}\text { A } & \text { A } \\ \text { A } & \text { A } \\ \text { A } & \text { N } \\ \text { A } & \text { A } \\ \text { A } & \text { N } \\ \text { A } & \text { A } \\ \text { A } & \text { A } \\ \text { A } & \text { A } \\ \text { A } & \text { A } \\ \text { A } & \text { A } \\ \text { N } & \text { A } \\ \text { A } & \text { A } \\ \text { A } & \text { A }\end{array}$

Values for elemental uranium are reported in $\mu \mathrm{g} / \mathrm{filter}, \mathrm{g}$, or $\mathrm{mL}$.

$\mathrm{pCi} / \mathrm{g}$ or $\mathrm{mL}=\mathrm{Bq} \times 0.027$

Evaluation: $A=$ Acceptable, $W=$ Acceptable with Warning, $N=$ Not Acceptable

If the evaluation system is not appropriate for the types of analyses performed in your lab, apply a site specific evaluation. 


\section{QAP 49 Results by Laboratory}

Lab: GS USGS/NWQL, Arvada, CO

No.

Reported Reported

EML

EML

Reported Error EML Error Value

$\mathbf{E}$

Matrix: WA Water $\mathrm{Bq} / \mathrm{L}$

$\begin{array}{llrrrrrrr}1 & \text { GROSS ALPHA } & 1208.700 & 81.000 & 1080.000 & 60.000 & 1.119 & \mathrm{~A} & \mathrm{~N} \\ 1 & \text { GROSS BETA } & 1363.500 & 65.500 & 1420.000 & 60.000 & 0.960 & \mathrm{~A} & \mathrm{~A} \\ 1 & \text { ug U } & 0.040 & 0.002 & 0.040 & 0.003 & 1.000 & \mathrm{~A} & \mathrm{~A}\end{array}$

Values for elemental uranium are reported in $\mu \mathrm{g} /$ filter, $\mathrm{g}$, or $\mathrm{mL}$.

$\mathrm{pCi} / \mathrm{g}$ or $\mathrm{mL}=\mathrm{Bq} \times 0.027$

Evaluation: $A=$ Acceptable, $W=$ Acceptable with Warning, $N=$ Not Acceptable

If the evaluation system is not appropriate for the types of analyses performed in your lab, apply a site specific evaluation. 


\section{QAP 49 Results by Laboratory}

Lab: GT Georgia Institute of Technology

\begin{tabular}{|c|c|c|c|c|c|c|c|}
\hline $\begin{array}{l}\text { No. } \\
\text { Test }\end{array}$ & Radic & $\begin{array}{l}\text { Reported } \\
\text { Value }\end{array}$ & $\begin{array}{l}\text { Reported } \\
\text { Error }\end{array}$ & $\begin{array}{l}\text { EML } \\
\text { Value }\end{array}$ & $\begin{array}{l}\text { EML } \\
\text { Error }\end{array}$ & $\frac{\text { Reported }}{\text { EML }}$ & $\begin{array}{cc}\text { QAP 48 } \\
\text { Evaluation } \\
\text { Evaluation }\end{array}$ \\
\hline
\end{tabular}

Matrix: AI Air Filter Bq / filter

$\begin{array}{llrrrrrrr}1 & \text { AM241 } & 0.540 & 0.100 & 0.510 & 0.008 & 1.059 & \mathrm{~A} & \mathrm{~A} \\ 1 & \text { CO60 } & 8.300 & 1.000 & 9.160 & 0.580 & 0.906 & \mathrm{~A} & \mathrm{~A} \\ 1 & \text { CS137 } & 21.000 & 4.000 & 22.470 & 1.030 & 0.935 & \mathrm{~A} & \mathrm{~A} \\ 1 & \text { GROSS ALPHA } & 1.800 & 0.100 & 1.650 & 0.160 & 1.091 & \mathrm{~A} & \mathrm{~A} \\ 1 & \text { GROSS BETA } & 2.000 & 0.100 & 2.160 & 0.070 & 0.926 & \mathrm{~A} & \mathrm{~A} \\ 1 & \text { MN54 } & 4.800 & 1.000 & 4.920 & 0.400 & 0.976 & \mathrm{~A} & \mathrm{~A} \\ 1 & \text { PU238 } & 0.520 & 0.100 & 0.460 & 0.005 & 1.130 & \mathrm{~A} & \mathrm{~A} \\ 1 & \text { PU239 } & 0.490 & 0.100 & 0.420 & 0.006 & 1.167 & \mathrm{~A} & \mathrm{~A} \\ 1 & \text { SB125 } & 9.600 & 1.000 & 8.890 & 0.550 & 1.080 & \mathrm{~A} & \mathrm{~W} \\ 1 & \text { SR90 } & 0.960 & 0.100 & 1.120 & 0.050 & 0.857 & \mathrm{~A} & \mathrm{~A} \\ 1 & \text { U238 } & 0.250 & 0.050 & 0.260 & 0.010 & 0.962 & \mathrm{~A} & \mathrm{~A}\end{array}$

Matrix: SO Soil $\mathrm{Bq} / \mathrm{kg}$

$\begin{array}{ll}1 & \text { AM241 } \\ 1 & \text { CO60 } \\ 1 & \text { CS137 } \\ 1 & \text { K40 } \\ 1 & \text { PU239 } \\ 1 & \text { SR90 } \\ 1 & \text { U238 }\end{array}$

Matrix: $\mathrm{VE}$ Vegetation $\mathrm{Bq} / \mathrm{kg}$

$\begin{array}{ll}1 & \text { AM241 } \\ 1 & \text { CO60 } \\ 1 & \text { CS137 } \\ 1 & \text { K40 } \\ 1 & \text { PU239 } \\ 1 & \text { SR90 }\end{array}$

1.900
25.000
490.000
580.000
4.300
440.000

0.500

5.000

100.000

50.000

0.500

10.000

$$
\begin{array}{r}
2.330 \\
20.000 \\
390.000 \\
460.000 \\
3.720 \\
606.000
\end{array}
$$

0.410
0.110
38.000
13.000
0.570
0.003
9.000

0.410

0.110

13.000

0.570

9.003

$$
\begin{aligned}
& 0.991 \\
& 1.532 \\
& 1.258 \\
& 1.274 \\
& 1.016 \\
& 1.110 \\
& 0.850
\end{aligned}
$$

\subsection{5 \\ 1.250 \\ 1.256 \\ 1.261 \\ 1.156 \\ 0.726}

A

A

A

A

A

Matrix: WA Water Bq/L

$\begin{array}{ll}1 & \text { AM241 } \\ 1 & \text { CO60 } \\ 1 & \text { CS137 } \\ 1 & \text { GROSS ALPHA } \\ 1 & \text { GROSS BETA } \\ 1 & \text { H3 } \\ 1 & \text { MN54 } \\ 1 & \text { PU238 } \\ 1 & \text { PU239 } \\ 1 & \text { SR90 } \\ 1 & \text { U238 }\end{array}$

1.200
50.000
52.000
1000.000
1300.000
82.000
35.000
1.300
1.800
1.900
0.690

$\begin{array}{rr}0.400 & 1.250 \\ 6.000 & 49.400 \\ 8.000 & 50.000 \\ 50.000 & 1080.000 \\ 50.000 & 1420.000 \\ 10.000 & 76.200 \\ 6.000 & 32.400 \\ 0.400 & 1.100 \\ 0.400 & 1.410 \\ 0.500 & 2.110 \\ 0.150 & 0.520\end{array}$

0.080
1.200
1.700
60.000
60.000
2.900
1.400
0.010
0.040
0.180
0.050

0.960
1.012
1.040
0.926
0.915
1.076
1.080
1.182
1.277
0.900
1.327

$\begin{array}{ll}\text { W } & \text { A } \\ \text { W } & \text { A } \\ \text { W } & \text { A } \\ \text { W } & \text { A } \\ \text { A } & \text { A } \\ \text { W } & \text { A }\end{array}$

Values for elemental uranium are reported in $\mu \mathrm{g} /$ filter, $\mathrm{g}$, or $\mathrm{mL}$.

$\mathrm{pCi} / \mathrm{g}$ or $\mathrm{mL}=\mathrm{Bq} \times 0.027$

Evaluation: $A=A c c e p t a b l e, W=A c c e p t a b l e$ with Warning, $N=$ Not Acceptable

If the evaluation system is not appropriate for the types of analyses performed in your lab, apply a site specific evaluation. 


\section{QAP 49 Results by Laboratory}

Lab: HC Lawrence Livermore Laboratory, California

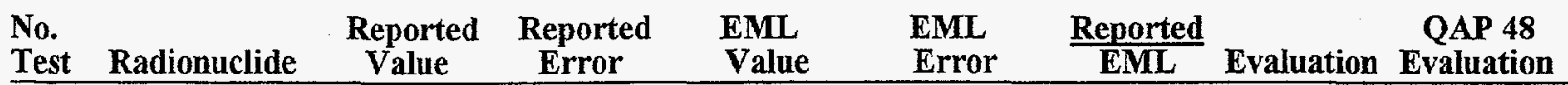

Matrix: AI Air Filter $\mathrm{Bq} /$ filter

$\begin{array}{lllllllll}1 & \text { GROSS ALPHA } & 1.497 & 0.150 & 1.650 & 0.160 & 0.907 & \mathrm{~A} & \mathrm{~W} \\ 1 & \text { GROSS BETA } & 1.603 & 0.160 & 2.160 & 0.070 & 0.742 & \mathrm{~W} & \mathrm{~W}\end{array}$

Matrix: WA Water $\mathrm{Bq} / \mathrm{L}$

$\begin{array}{llrrrrrrr}1 & \text { GROSS ALPHA } & 1012.000 & 58.000 & 1080.000 & 60.000 & 0.937 & \text { A } & \text { A } \\ 1 & \text { GROSS BETA } & 1347.000 & 57.000 & 1420.000 & 60.000 & 0.949 & \text { A } & \text { A } \\ 1 & \text { H3 } & 78.100 & 14.400 & 76.200 & 2.900 & 1.025 & \text { A } & \text { A }\end{array}$

Values for elemental uranium are reported in $\mu \mathrm{g} /$ filter, $\mathrm{g}$, or $\mathrm{mL}$.

$\mathrm{pCi} / \mathrm{g}$ or $\mathrm{mL}=\mathrm{Bq} \times 0.027$

Evaluation: $A=A c c e p t a b l e, W=A c c e p t a b l e$ with Warning, $N=$ Not Acceptable

If the evaluation system is not appropriate for the types of analyses performed in your lab, apply a site specific evaluation. 


\section{QAP 49 Results by Laboratory}

December, 1998

Lab: HT Technical University, Budapest, Hungary

No.

Reported Reported

EML

EML

Reported

QAP 48

Test Radionuclide Value Error Value Error EML

Matrix: $\mathrm{SO}$ Soil $\mathrm{Bq} / \mathrm{kg}$

$\begin{array}{ll}1 & \text { AM241 } \\ 1 & \text { Bq U } \\ 1 & \text { PU239 } \\ 1 & \text { U234 } \\ 1 & \text { U238 } \\ 1 & \text { ug U }\end{array}$

41.000
470.500
5.000
220.000
223.500
18.100

5.000

7.470

45.000

0.600

237.000

0.410

16.000

25.000

13.090

0.570

113.000

6.000

25.000

120.000

9.000

9.700

0.700

5.489
1.985
0.382
1.947
1.862
1.866

$N$
$N$
$N$
$N$
$N$

Matrix: WA Water $\mathrm{Bq} / \mathrm{L}$

$\begin{array}{ll}1 & \text { AM241 } \\ 1 & \text { Bq U } \\ 1 & \text { PU238 } \\ 1 & \text { PU239 } \\ 1 & \text { U234 } \\ 1 & \text { U238 } \\ 1 & \text { ug U }\end{array}$

2.600

1.670

0.200

1.250

1.050

0.080

0.167

1.250

0.200

1.100

1.100

0.150

1.410

0.090

0.510

0.785

0.090

0.520

0.065

0.007

0.040

0.080

0.010

0.040

0.030

0.050

0.003

$\begin{array}{ll}2.080 & \mathrm{~N} \\ 1.590 & \mathrm{~N} \\ 1.136 & \mathrm{~W} \\ 0.780 & \mathrm{~N} \\ 1.539 & \mathrm{~N} \\ 1.538 & \mathrm{~N} \\ 1.625 & \mathrm{~N}\end{array}$
$\mathrm{N}$ 


\section{QAP 49 Results by Laboratory}

Lab: HU Water Resources Research Centre (VITUKI), Hungary

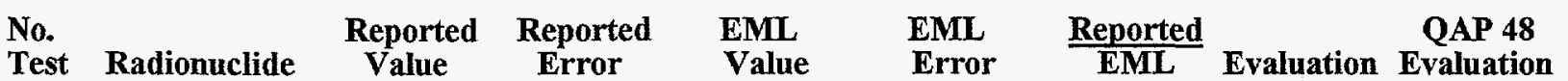

Matrix: AI Air Filter $\mathrm{Bq} /$ filter

$\begin{array}{llrrrrrrr}1 & \text { AM241 } & 0.340 & 0.190 & 0.510 & 0.008 & 0.667 & \mathrm{~N} & \\ 1 & \text { CO60 } & 7.880 & 0.260 & 9.160 & 0.580 & 0.860 & \mathrm{~A} & \mathrm{~A} \\ 1 & \text { CS137 } & 25.900 & 0.770 & 22.470 & 1.030 & 1.153 & \mathrm{~W} & \mathrm{~A} \\ 1 & \text { MN54 } & 5.760 & 0.200 & 4.920 & 0.400 & 1.171 & \mathrm{~A} & \mathrm{~A} \\ 1 & \text { SB125 } & 10.230 & 0.200 & 8.890 & 0.550 & 1.151 & \mathrm{~A} & \mathrm{~A}\end{array}$

Matrix: SO Soil $\mathrm{Bq} / \mathrm{kg}$

$\begin{array}{ll}1 & \text { AC228 } \\ 1 & \text { AM241 } \\ 1 & \text { BI214 } \\ 1 & \text { Bq U } \\ 1 & \text { CO60 } \\ 1 & \text { CS137 } \\ 1 & \text { K40 } \\ 1 & \text { PB212 } \\ 1 & \text { PB214 } \\ 1 & \text { TL208 }\end{array}$

49.100
8.300
25.600
9.400
0.760
896.000
342.000
52.600
31.200
16.600

Matrix: VE Vegetation $\mathrm{Bq} / \mathrm{kg}$

$\begin{array}{ll}1 & \text { AM241 } \\ 1 & \text { CO60 } \\ 1 & \text { CS137 } \\ \text { I } & \text { K40 }\end{array}$

$\begin{array}{rr}1.200 & 2.330 \\ 0.500 & 20.000 \\ 11.000 & 390.000 \\ 40.000 & 460.000\end{array}$

2.330

390.000

588.000

40.000

1.200
3.800
1.000
0.700
0.220
23.000
24.000
1.500
1.000
0.600

52.600

7.470

28.800

237.000

1.240

954.000

314.000

52.800

29.100

18.300

$$
\begin{array}{r}
2.900 \\
0.410 \\
0.500 \\
16.000 \\
0.110 \\
38.000 \\
13.000 \\
3.700 \\
1.200 \\
1.100
\end{array}
$$
0.060
1.000
20.000
20.000

$\begin{array}{ll}0.933 & \mathrm{~A} \\ 1.111 & \mathrm{~A} \\ 0.889 & \mathrm{~A} \\ 0.040 & \mathrm{~N} \\ 0.613 & \mathrm{~N} \\ 0.939 & \mathrm{~A} \\ 1.089 & \mathrm{~A} \\ 0.996 & \mathrm{~A} \\ 1.072 & \mathrm{~A} \\ 0.907 & \mathrm{~A}\end{array}$

W

A

$\begin{array}{ll}1.159 & \mathrm{~A} \\ 1.075 & \mathrm{~A} \\ 1.103 & \mathrm{~A} \\ 1.278 & \mathrm{~W}\end{array}$

Values for elemental uranium are reported in $\mu \mathrm{g} /$ filter, $\mathrm{g}$, or $\mathrm{mL}$.

$\mathrm{pCi} / \mathrm{g}$ or $\mathrm{mL}=\mathrm{Bq} \times 0.027$

Evaluation: $A=$ Acceptable, $W=A c c e p t a b l e$ with Warning, $N=$ Not Acceptable

If the evaluation system is not appropriate for the types of analyses performed in your lab, apply a site specific evaluation. 


\section{QAP 49 Results by Laboratory}

Lab: IA Bhabha Atomic Research Centre, India

\begin{tabular}{|c|c|c|c|c|c|c|c|}
\hline est & Radionuclide & $\begin{array}{c}\text { Reported } \\
\text { Value }\end{array}$ & $\begin{array}{c}\text { Reported } \\
\text { Error }\end{array}$ & $\begin{array}{l}\text { EML } \\
\text { Value }\end{array}$ & $\begin{array}{l}\text { EML } \\
\text { Error }\end{array}$ & $\frac{\text { Reported }}{\text { EML }}$ & $\begin{array}{c}\text { QAP 48 } \\
\text { Evaluation }\end{array}$ \\
\hline
\end{tabular}

Matrix: AI Air Filter Bq / filter

$\begin{array}{llrlrllll}2 & \text { CO60 } & 8.310 & 0.090 & 9.160 & 0.580 & 0.907 & \mathrm{~A} & \text { W } \\ 3 & \text { CO60 } & 8.230 & 0.090 & 9.160 & 0.580 & 0.898 & \mathrm{~A} & \text { W } \\ 1 & \text { CO60 } & 8.530 & 0.090 & 9.160 & 0.580 & 0.931 & \mathrm{~A} & \mathrm{~W} \\ 1 & \text { CS137 } & 21.120 & 0.110 & 22.470 & 1.030 & 0.940 & \mathrm{~A} & \mathrm{~A} \\ 2 & \text { CS137 } & 20.710 & 0.110 & 22.470 & 1.030 & 0.922 & \mathrm{~A} & \mathrm{~A} \\ 3 & \text { CS137 } & 21.190 & 0.110 & 22.470 & 1.030 & 0.943 & \mathrm{~A} & \mathrm{~A} \\ 1 & \text { MN54 } & 4.400 & 0.060 & 4.920 & 0.400 & 0.894 & \mathrm{~A} & \mathrm{~W} \\ 2 & \text { MN54 } & 4.480 & 0.060 & 4.920 & 0.400 & 0.911 & \mathrm{~A} & \mathrm{~W} \\ 3 & \text { MN54 } & 4.320 & 0.060 & 4.920 & 0.400 & 0.878 & \mathrm{~A} & \mathrm{~W} \\ 2 & \text { SB125 } & 8.640 & 0.240 & 8.890 & 0.550 & 0.972 & \mathrm{~A} & \mathrm{~A} \\ 3 & \text { SB125 } & 7.940 & 0.230 & 8.890 & 0.550 & 0.893 & \mathrm{~A} & \mathrm{~A} \\ 1 & \text { SB125 } & 8.330 & 0.230 & 8.890 & 0.550 & 0.937 & \mathrm{~A} & \mathrm{~A}\end{array}$

Matrix: SO Soil $\mathrm{Bq} / \mathrm{kg}$

$\begin{array}{ll}2 & \text { CO60 } \\ 3 & \text { CO60 } \\ 1 & \text { CO60 } \\ 3 & \text { CS137 } \\ 2 & \text { CS137 } \\ 1 & \text { CS137 } \\ 1 & \text { K40 } \\ 3 & \text { K40 } \\ 2 & \text { K40 } \\ 3 & \text { RA226 } \\ 2 & \text { RA226 }\end{array}$

$\begin{array}{rrr}1.210 & 0.200 & 1.240 \\ 0.850 & 0.200 & 1.240 \\ 1.580 & 0.200 & 1.240 \\ 683.000 & 2.000 & 954.000 \\ 687.000 & 2.000 & 954.000 \\ 685.000 & 2.000 & 954.000 \\ 254.000 & 6.000 & 314.000 \\ 246.000 & 6.000 & 314.000 \\ 250.000 & 6.000 & 314.000 \\ 21.300 & 1.500 & 29.000 \\ 23.700 & 1.500 & 29.000 \\ 21.500 & 1.600 & 29.000\end{array}$

0.110
0.110
0.110
38.000
38.000
38.000
13.000
13.000
13.000
1.000
1.000
1.000

0.976

0.685

1.274

0.716

0.720

0.718

0.809

0.783

0.796

0.734

0.817

0.741

$\begin{array}{ll}\text { A } & \\ \text { W } & \\ \text { A } & \\ \text { N } & \text { N } \\ \text { N } & \text { N } \\ \text { N } & \text { N } \\ \text { W } & \text { N } \\ \text { W } & \text { N } \\ \text { W } & \text { N } \\ \text { A } & \\ \text { A } & \\ \text { A } & \end{array}$

Matrix: VE Vegetation $\mathrm{Bq} / \mathrm{kg}$

$\begin{array}{llrrrrrrr}1 & \text { CO60 } & 15.700 & 0.700 & 20.000 & 1.000 & 0.785 & \text { W } & \text { W } \\ 3 & \text { CO60 } & 16.600 & 0.700 & 20.000 & 1.000 & 0.830 & \text { W } & \text { W } \\ 2 & \text { CO60 } & 16.700 & 0.700 & 20.000 & 1.000 & 0.835 & \text { W } & \text { W } \\ 3 & \text { CS137 } & 361.000 & 2.000 & 390.000 & 20.000 & 0.926 & \text { A } & \text { N } \\ 2 & \text { CS137 } & 359.000 & 2.000 & 390.000 & 20.000 & 0.921 & \text { A } & \text { N } \\ 1 & \text { CS137 } & 370.000 & 2.000 & 390.000 & 20.000 & 0.949 & \text { A } & \text { N } \\ 3 & \text { K40 } & 437.000 & 11.000 & 460.000 & 20.000 & 0.950 & \text { A } & \text { W } \\ 1 & \text { K40 } & 397.000 & 11.000 & 460.000 & 20.000 & 0.863 & \text { W } & \text { W } \\ 2 & \text { K40 } & 424.000 & 11.000 & 460.000 & 20.000 & 0.922 & \text { A } & \text { W }\end{array}$

Values for elemental uranium are reported in $\mu \mathrm{g} /$ filter, $g$, or $\mathrm{mL}$.

$\mathrm{pCi} / \mathrm{g}$ or $\mathrm{mL}=\mathrm{Bq} \times 0.027$

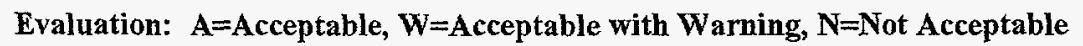

If the evaluation system is not appropriate for the types of analyses performed in your lab, apply a site specific evaluation. 


\section{QAP 49 Results by Laboratory}

Lab: ID Institute of Radiation Protection and Dosimetry, Rio de Janeiro, Brazil

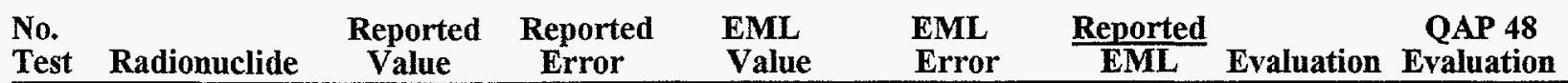

Matrix: AI Air Filter Bq / filter

\begin{tabular}{|c|c|c|c|c|c|c|}
\hline CO60 & 9.700 & 0.592 & 9.160 & 0.580 & 1.059 & A \\
\hline CS137 & 22.820 & 1.230 & 22.470 & 1.030 & 1.016 & A \\
\hline GROSS ALPHA & 1.397 & 0.103 & 1.650 & 0.160 & 0.847 & A \\
\hline GROSS BETA & 2.257 & 0.116 & 2.160 & 0.070 & 1.045 & A \\
\hline MN54 & 5.250 & 0.315 & 4.920 & 0.400 & 1.067 & A \\
\hline PU238 & 0.497 & 0.071 & 0.460 & 0.005 & 1.080 & A \\
\hline PU239 & 0.463 & 0.039 & 0.420 & 0.006 & 1.102 & A \\
\hline $\mathrm{SB} 125$ & 9.600 & 0.657 & 8.890 & 0.550 & 1.080 & A \\
\hline SR90 & 1.080 & 0.088 & 1.120 & 0.050 & 0.964 & A \\
\hline
\end{tabular}

Matrix: SO Soil Bq/ kg

$\begin{array}{ll}1 & \mathrm{AC} 228 \\ 1 & \mathrm{AM} 241 \\ 1 & \mathrm{BI} 12 \\ 1 & \mathrm{BI214} \\ 1 & \mathrm{~Bq} \mathrm{U} \\ 1 & \mathrm{CS} 137 \\ 1 & \mathrm{~K} 40 \\ 1 & \mathrm{~PB} 212 \\ 1 & \mathrm{~PB} 214 \\ 1 & \mathrm{PU} 239 \\ 1 & \mathrm{SR90} \\ 1 & \mathrm{U} 238\end{array}$

60.060
7.900
30.110
29.850
217.770
1056.330
303.700
57.280
35.470
12.530
48.630
112.170

3.690

0.750

2.340

1.650

12.470

52.910

27.160

3.070

2.510

0.650

4.110

6.410

$$
\begin{array}{r}
2.900 \\
0.410 \\
5.900 \\
0.500 \\
16.000 \\
38.000 \\
13.000 \\
3.700 \\
1.200 \\
0.570 \\
0.003 \\
9.000
\end{array}
$$

120.000

0.940

18.540

23.880

23.880

0.505

29.530

$\begin{array}{rr}20.000 & 1.000 \\ 390.000 & 20.000 \\ 460.000 & 20.000 \\ 460.000 & 20.000 \\ 3.720 & 0.270 \\ 606.000 & 40.000\end{array}$

586.330

$\begin{array}{rr}17.960 & 0.940 \\ 367.270 & 18.540 \\ 403.530 & 23.880 \\ 403.530 & 23.880 \\ 3.570 & 0.505 \\ 586.330 & 29.530\end{array}$

Matrix: WA Water $\mathrm{Bq} / \mathrm{L}$

1.130
51.540
51.390
118.330
34.970
1.100
2.480
0.577

0.068
2.750
2.630
11.040
1.920
0.350
0.132
0.036

1.050
49.400
50.000
76.200
32.400
1.410
2.110
0.520

0.080
1.200
1.700
2.900
1.400
0.040
0.180
0.050

1.142
1.058
0.516
1.036
0.919
1.107
0.967
1.085
1.219
0.957
1.227
0.935

A
A
A
A
A
A
A
A
A
A
A
A

0.898

0.942

0.877

0.877

0.960

0.968

$\begin{array}{ll}\text { A } & \text { A } \\ \text { A } & \text { A } \\ \text { W } & \text { A } \\ \text { W } & \text { A } \\ \text { A } & \text { A } \\ \text { A } & \text { A }\end{array}$

A

A

A

W

A

W
W
W
N
W
W




\section{QAP 49 Results by Laboratory}

Lab: IE IEA, Inc., Morrisville, NC

No.

Reported Reported

EML

EML

Reported

QAP 48

Test Radionuclide

Value

Error

Error EML

Evaluation Evaluation

Matrix: SO Soil $\mathrm{Bq} / \mathrm{kg}$

$\begin{array}{ll}1 & \mathrm{AC} 228 \\ 1 & \mathrm{BI} 212 \\ 1 & \mathrm{CS} 137 \\ 1 & \mathrm{~K} 40 \\ 1 & \mathrm{~PB} 212 \\ 1 & \mathrm{~PB} 214 \\ 1 & \text { TH234 } \\ 1 & \text { TL208 } \\ 1 & \mathrm{U} 238\end{array}$

$\begin{array}{rr}50.410 & 7.340 \\ 39.220 & 8.550 \\ 1125.000 & 7.000 \\ 424.600 & 19.200 \\ 46.750 & 1.650 \\ 33.200 & 2.430 \\ 226.700 & 17.700 \\ 21.050 & 1.500 \\ 226.700 & 17.700\end{array}$

$\begin{array}{rr}52.600 & 2.900 \\ 58.300 & 5.900 \\ 954.000 & 38.000 \\ 314.000 & 13.000 \\ 52.800 & 3.700 \\ 29.100 & 1.200 \\ 114.000 & 6.000 \\ 18.300 & 1.100 \\ 120.000 & 9.000\end{array}$

0.958
0.673
1.179
1.352
0.885
1.141
1.989
1.150
1.889

A

A

A

A

A

A

A

A

A

N

A

Matrix: VE Vegetation $\mathrm{Bq} / \mathrm{kg}$

$\begin{array}{ll}1 & \text { CO60 } \\ 1 & \text { CS137 } \\ 1 & \mathrm{~K} 40\end{array}$

27.200

565.700

672.400

1.670

6.400

28.300

1.000
20.000
20.000

460.000

1.380

1.200

1.530

19.900

15.500

8.010

1.280

1.250
49.400
50.000
1080.000
1420.000
76.200
32.400

0.080

1.200

1.700

60.000

60.000

2.900

1.400
1.360

1.451

1.462
A
A

1.528
1.052
1.095
0.864
0.678
0.860
0.996

$\begin{array}{ll}\mathrm{N} & \mathrm{A} \\ \mathrm{A} & \mathrm{A} \\ \mathrm{A} & \mathrm{A} \\ \mathrm{A} & \mathrm{A} \\ \mathrm{W} & \mathrm{A} \\ \mathrm{A} & \mathrm{A} \\ \mathrm{A} & \mathrm{A}\end{array}$

Values for elemental uranium are reported in $\mu \mathrm{g} /$ filter, $\mathrm{g}$, or $\mathrm{mL}$.

$\mathrm{pCi} / \mathrm{g}$ or $\mathrm{mL}=\mathrm{Bq} \times 0.027$

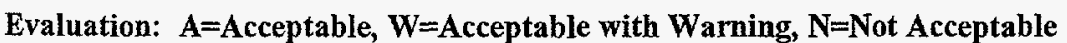

If the evaluation system is not appropriate for the types of analyses performed in your lab, apply a site specific evaluation. 


\section{QAP 49 Results by Laboratory}

Lab: IL ISU Environmental Monitoring Program, Pocatello, ID

\begin{tabular}{llllll} 
No. & Reported & Reported & EML & EML & Reported \\
Test & Radionuclide & $\begin{array}{c}\text { Value } \\
\text { Error }\end{array}$ & $\begin{array}{c}\text { Value } \\
\text { Error }\end{array}$ & $\frac{1}{\text { EML }}$ Evaluation Evaluation \\
\hline
\end{tabular}

Matrix: $\mathrm{AI}$ Air Filter $\mathrm{Bq} /$ filter

$\begin{array}{llrrrrrrr}1 & \text { CO60 } & 8.900 & 0.100 & 9.160 & 0.580 & 0.972 & \mathrm{~A} & \mathrm{~A} \\ 1 & \text { CS137 } & 21.300 & 0.300 & 22.470 & 1.030 & 0.948 & \mathrm{~A} & \mathrm{~A} \\ 1 & \text { GROSS ALPHA } & 1.990 & 0.030 & 1.650 & 0.160 & 1.206 & \mathrm{~A} & \\ 1 & \text { GROSS BETA } & 2.220 & 0.020 & 2.160 & 0.070 & 1.028 & \mathrm{~A} & \\ 1 & \text { MN54 } & 4.900 & 0.100 & 4.920 & 0.400 & 0.996 & \mathrm{~A} & \mathrm{~A} \\ 1 & \text { SB125 } & 9.200 & 0.100 & 8.890 & 0.550 & 1.035 & \mathrm{~A} & \mathrm{~A}\end{array}$

Matrix: SO Soil $\mathrm{Bq} / \mathrm{kg}$

$\begin{array}{llrrrrrrr}1 & \text { AC228 } & 78.700 & 7.400 & 52.600 & 2.900 & 1.496 & \mathrm{~A} & \\ 1 & \text { BI212 } & 63.900 & 3.900 & 58.300 & 5.900 & 1.096 & \mathrm{~A} & \\ 1 & \text { BI214 } & 49.100 & 6.500 & 28.800 & 0.500 & 1.705 & \mathrm{~A} & \\ 1 & \text { CS137 } & 994.900 & 17.500 & 954.000 & 38.000 & 1.043 & \mathrm{~A} & \mathrm{~N} \\ 1 & \text { K40 } & 609.500 & 90.300 & 314.000 & 13.000 & 1.941 & \mathrm{~N} & \mathrm{~N} \\ 1 & \text { PB212 } & 64.000 & 3.900 & 52.800 & 3.700 & 1.212 & \mathrm{~A} & \\ 1 & \text { PB214 } & 49.100 & 6.500 & 29.100 & 1.200 & 1.687 & \mathrm{~A} & \\ 1 & \text { RA226 } & 62.700 & 8.300 & 29.000 & 1.000 & 2.162 & \mathrm{~A} & \\ 1 & \text { TL208 } & 23.000 & 1.400 & 18.300 & 1.100 & 1.257 & \mathrm{~A} & \end{array}$

Matrix: VE Vegetation $\mathrm{Bq} / \mathrm{kg}$

$\begin{array}{llrrrrrrr}1 & \text { CO60 } & 18.800 & 2.500 & 20.000 & 1.000 & 0.940 & \mathrm{~A} & \mathrm{~W} \\ 1 & \text { CS137 } & 437.800 & 10.300 & 390.000 & 20.000 & 1.123 & \mathrm{~A} & \mathrm{~W} \\ 1 & \mathrm{~K} 40 & 580.100 & 99.500 & 460.000 & 20.000 & 1.261 & \mathrm{~W} & \mathrm{~N}\end{array}$

Matrix: WA Water $\mathrm{Bq} / \mathrm{L}$

$\begin{array}{llrrrrrrr}1 & \text { CO60 } & 52.200 & 0.600 & 49.400 & 1.200 & 1.057 & \mathrm{~A} & \mathrm{~A} \\ 1 & \text { CS137 } & 52.300 & 0.800 & 50.000 & 1.700 & 1.046 & \mathrm{~A} & \mathrm{~A} \\ 1 & \text { GROSS ALPHA } & 1034.900 & 29.200 & 1080.000 & 60.000 & 0.958 & \mathrm{~A} & \\ 1 & \text { GROSS BETA } & 1060.000 & 25.500 & 1420.000 & 60.000 & 0.746 & \mathrm{~A} & \\ 1 & \text { MN54 } & 36.000 & 0.500 & 32.400 & 1.400 & 1.111 & \mathrm{~A} & \mathrm{~A}\end{array}$

Values for elemental uranium are reported in $\mu \mathrm{g} /$ filter, $\mathrm{g}$, or $\mathrm{mL} . \quad \mathrm{pCi} / \mathrm{g}$ or $\mathrm{mL}=\mathrm{Bq} \times \mathbf{0 . 0 2 7}$

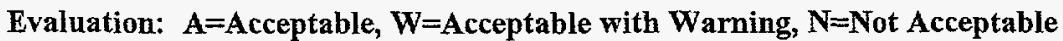

If the evaluation system is not appropriate for the types of analyses performed in your lab, apply a site specific evaluation. 


\section{QAP 49 Results by Laboratory}

Lab: IN Lockheed Martin Idaho Technical Corp., Analytical Laboratory

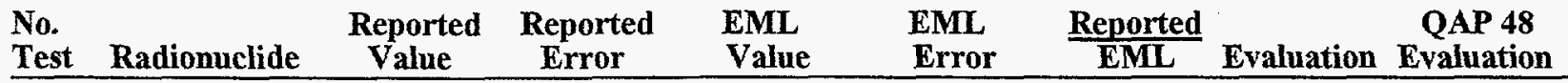

Matrix: AI Air Filter $\mathrm{Bq} /$ filter

$\begin{array}{llrrrrrrr}1 & \text { AM241 } & 0.540 & 0.050 & 0.510 & 0.008 & 1.059 & \text { A } & \\ 1 & \text { CO60 } & 8.500 & 0.300 & 9.160 & 0.580 & 0.928 & \text { A } & \text { A } \\ 1 & \text { CS137 } & 21.600 & 0.800 & 22.470 & 1.030 & 0.961 & \text { A } & \text { A } \\ 1 & \text { MN54 } & 4.890 & 0.500 & 4.920 & 0.400 & 0.994 & \text { A } & \text { A } \\ 1 & \text { SB125 } & 8.900 & 0.900 & 8.890 & 0.550 & 1.001 & \text { A } & \text { A }\end{array}$

Matrix: SO Soil $\mathrm{Bq} / \mathrm{kg}$

$\begin{array}{ll}1 & \text { AM241 } \\ 1 & \text { BI212 } \\ 1 & \text { BI214 } \\ 1 & \text { CS137 } \\ 1 & \text { K40 } \\ 1 & \text { PB212 } \\ 1 & \text { PB214 } \\ 1 & \text { PU239 } \\ 1 & \text { TH234 } \\ 1 & \text { TL208 } \\ 1 & \text { U234 } \\ 1 & \text { U238 }\end{array}$

8.300
47.500
34.000
1136.000
356.000
56.900
33.400
12.980
114.500
19.400
116.000
115.000

1.250

3.000

8.000

175.000

101.000

6.000

9.800

2.700

5.000

2.200

17.000

17.000

0.410
5.900
0.500
38.000
13.000
3.700
1.200
0.570
6.000
1.100
6.000
9.000

1.111

0.815

1.181

1.191

1.134

1.078

1.148

0.992

1.004

1.060

1.027

0.958

$\begin{array}{ll}\text { A } & \text { A } \\ \text { A } & \\ \text { A } & \\ \text { A } & \text { A } \\ \text { A } & \text { A } \\ \text { A } & \\ \text { A } & \\ \text { A } & \text { A } \\ \text { A } & \\ \text { A } & \\ \text { A } & \text { W } \\ \text { A } & \text { W }\end{array}$

Matrix: VE Vegetation $\mathrm{Bq} / \mathrm{kg}$

$\begin{array}{ll}1 & \text { CO60 } \\ 1 & \text { CS137 } \\ 1 & \text { K } 40\end{array}$
21.200
455.800
513.600

4.000

18.400

48.300
20.000

390.000

460.000
1.000
20.000
20.000

1.060

1.169

1.117

$\begin{array}{ll}\text { A } & \text { A } \\ \text { A } & \text { A } \\ \text { A } & \text { A }\end{array}$

1.008

1.036

1.040

0.696

1.105

1.091

1.021

1.275

0.984

0.962

$\begin{array}{ll}\text { A } & \text { A } \\ \text { A } & \text { W } \\ \text { A } & \text { A } \\ \text { N } & \\ \text { A } & \text { W } \\ \text { A } & \text { A } \\ \text { A } & \text { A } \\ \text { W } & \text { A } \\ \text { A } & \text { A } \\ \text { A } & \text { A }\end{array}$

Values for elemental uranium are reported in $\mu \mathrm{g} /$ filter, $\mathrm{g}$, or $\mathrm{mL}$.

$\mathrm{pCi} / \mathrm{g}$ or $\mathrm{mL}=\mathrm{Bq} \times 0.027$

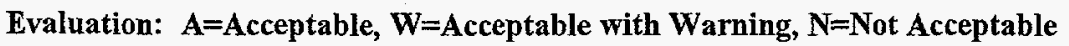

If the evaluation system is not appropriate for the types of analyses performed in your lab, apply a site specific evaluation. 


\section{QAP 49 Results by Laboratory}

Lab: IS Quanterra- St. Louis

\begin{tabular}{llllll} 
No. & Reported & Reported & EML \\
Test & Radionuclide & $\begin{array}{c}\text { Value } \\
\text { Error }\end{array}$ & $\begin{array}{c}\text { VML } \\
\text { Value }\end{array}$ & $\begin{array}{c}\text { Error } \\
\text { Eeported } \\
\text { EML }\end{array}$ Evaluation Evaluation \\
\hline
\end{tabular}

Matrix: AI Air Filter $\mathrm{Bq} /$ filter

$\begin{array}{llrrrrrrr}1 & \text { AM241 } & 0.397 & 0.184 & 0.510 & 0.008 & 0.778 & \text { W } & \text { N } \\ 1 & \text { CO60 } & 9.500 & 1.170 & 9.160 & 0.580 & 1.037 & \text { A } & \text { A } \\ 1 & \text { CS137 } & 22.400 & 2.440 & 22.470 & 1.030 & 0.997 & \text { A } & \text { A } \\ 1 & \text { GROSS ALPHA } & 2.010 & 0.200 & 1.650 & 0.160 & 1.218 & \text { A } & \text { A } \\ 1 & \text { GROSS BETA } & 2.010 & 0.200 & 2.160 & 0.070 & 0.931 & \text { A } & \text { A } \\ 1 & \text { MN54 } & 5.340 & 0.690 & 4.920 & 0.400 & 1.085 & \mathrm{~A} & \mathrm{~A} \\ 1 & \text { PU238 } & 0.392 & 0.112 & 0.460 & 0.005 & 0.851 & \text { W } & \\ 1 & \text { PU239 } & 0.378 & 0.108 & 0.420 & 0.006 & 0.899 & \text { W } & \\ 1 & \text { SB125 } & 9.710 & 1.280 & 8.890 & 0.550 & 1.092 & \text { A } & \text { A } \\ 1 & \text { SR90 } & 0.256 & 0.074 & 1.120 & 0.050 & 0.228 & \text { N } & \text { A } \\ 1 & \text { ug U } & 14.900 & 2.000 & 20.960 & 0.100 & 0.711 & \text { N } & \text { W }\end{array}$

Matrix: SO Soil $\mathrm{Bq} / \mathrm{kg}$

$\begin{array}{ll}1 & \text { AC228 } \\ 1 & \text { AM241 } \\ 1 & \text { BI212 } \\ 1 & \text { BI214 } \\ 1 & \text { CS137 } \\ 1 & \text { K40 } \\ 1 & \text { PB210 } \\ 1 & \text { PB212 } \\ 1 & \text { PB214 } \\ 1 & \text { PU239 } \\ 1 & \text { RA226 } \\ 1 & \text { SR90 } \\ 3 & \text { TH228 } \\ 2 & \text { TH228 } \\ 1 & \text { TH228 } \\ 1 & \text { TH234 } \\ 1 & \text { TL208 } \\ 1 & \text { ug U } \\ 2 & \text { ug U } \\ 3 & \text { ug U }\end{array}$

$\begin{array}{rrr}48.500 & 14.500 & 52.600 \\ 10.340 & 5.810 & 7.470 \\ 62.300 & 29.000 & 58.300 \\ 21.900 & 5.800 & 28.800 \\ 923.000 & 116.000 & 954.000 \\ 326.000 & 46.900 & 314.000 \\ 31.800 & 32.000 & 32.000 \\ 50.100 & 10.000 & 52.800 \\ 28.500 & 7.400 & 29.100 \\ 17.075 & 5.226 & 13.090 \\ 103.600 & 50.400 & 29.000 \\ 51.060 & 17.390 & 39.630 \\ 66.300 & 22.300 & 52.700 \\ 65.400 & 23.400 & 52.700 \\ 57.800 & 20.200 & 52.700 \\ 110.000 & 31.000 & 114.000 \\ 19.900 & 4.100 & 18.300 \\ 2.700 & 0.390 & 9.700 \\ 2.630 & 0.380 & 9.700 \\ 2.560 & 0.370 & 9.700\end{array}$

2.900
0.410
5.900
0.500
38.000
13.000
3.300
3.700
1.200
0.570
1.000
0.003
4.000
4.000
4.000
6.000
1.100
0.700
0.700
0.700

0.922
1.384
1.069
0.760
0.968
1.038
0.994
0.949
0.979
1.304
3.572
1.288
1.258
1.241
1.097
0.965
1.087
0.278
0.271
0.264

$\begin{array}{ll}\text { A } & \\ \text { A } & \text { A } \\ \text { A } & \\ \text { A } & \\ \text { A } & \text { A } \\ \text { A } & \text { W } \\ \text { A } & \\ \text { A } & \\ \text { A } & \\ \text { W } & \\ \text { A } & \\ \text { A } & \text { A } \\ \text { A } & \\ \text { A } & \\ \text { A } & \\ \text { A } & \\ \text { A } & \\ \text { N } & \text { N } \\ \text { N } & \text { N } \\ \text { N } & \text { N }\end{array}$

Matrix: VE Vegetation $\mathrm{Bq} / \mathrm{kg}$

$\begin{array}{ll}1 & \text { AM241 } \\ 1 & \text { CO60 } \\ 1 & \text { CS137 } \\ 1 & \text { K40 } \\ 1 & \text { PU239 } \\ 1 & \text { SR90 }\end{array}$

3.354
23.200
459.200
563.500
6.220
416.250

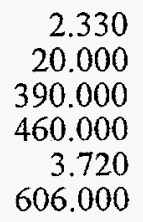
0.060
1.000
20.000
20.000
0.270
40.000

1.439
1.160
1.177
1.225
1.672
0.687

$\begin{array}{ll}\text { A } & \text { N } \\ \text { A } & \text { W } \\ \text { A } & \text { A } \\ \text { A } & \text { W } \\ \text { N } & \text { W } \\ \text { W } & \text { W }\end{array}$

\section{Values for elemental uranium are reported in $\mu \mathrm{g} /$ filter, $\mathrm{g}$, or $\mathrm{mL} . \quad \mathrm{pCi} / \mathrm{g}$ or $\mathrm{mL}=\mathrm{Bq} \times \mathbf{0 . 0 2 7}$}

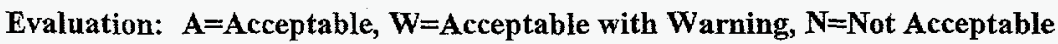

If the evaluation system is not appropriate for the types of analyses performed in your lab, apply a site specific evaluation. 


\begin{tabular}{|c|c|c|c|c|c|c|c|c|}
\hline $\begin{array}{l}\text { No. } \\
\text { Test }\end{array}$ & Radionuclide & $\begin{array}{c}\text { Reported } \\
\text { Value }\end{array}$ & $\begin{array}{c}\text { Reported } \\
\text { Error }\end{array}$ & $\begin{array}{l}\text { EML } \\
\text { Value } \\
\end{array}$ & $\begin{array}{l}\text { EML } \\
\text { Error } \\
\end{array}$ & $\frac{\text { Reported }}{\text { EML }}$ & Evaluation & $\begin{array}{r}\mathbf{Q} \\
\mathbf{E v a} \\
\end{array}$ \\
\hline \multicolumn{9}{|c|}{ Matrix: WA Water $\mathrm{Bq} / \mathrm{L}$} \\
\hline 1 & $\mathrm{AM} 241$ & 1.032 & 0.249 & 1.250 & 0.080 & 0.826 & W & W \\
\hline 1 & $\mathrm{CO} 60$ & 55.100 & 5.400 & 49.400 & 1.200 & 1.115 & $\ddot{A}$ & W \\
\hline 1 & CS137 & 55.900 & 5.700 & 50.000 & 1.700 & 1.118 & A & W \\
\hline 1 & GROSS ALPHA & 1188.000 & 122.000 & 1080.000 & 60.000 & 1.100 & $\mathrm{~A}$ & $\mathrm{~A}$ \\
\hline 1 & GROSS BETA & 1243.000 & 124.000 & 1420.000 & 60.000 & 0.875 & A & A \\
\hline 1 & $\mathrm{H} 3$ & 118.400 & 12.100 & 76.200 & 2.900 & 1.554 & $\mathrm{~W}$ & $\mathrm{~A}$ \\
\hline 1 & MN54 & 39.000 & 3.900 & 32.400 & 1.400 & 1.204 & W & W \\
\hline 1 & PU238 & 1.303 & 0.362 & 1.100 & 0.010 & 1.185 & W & A \\
\hline 1 & PU239 & 1.733 & 0.470 & 1.410 & 0.040 & 1.229 & W & W \\
\hline 1 & SR90 & 1.699 & 0.383 & 2.110 & 0.180 & 0.805 & W & $\mathrm{A}$ \\
\hline 1 & ug U & 0.040 & 0.005 & 0.040 & 0.003 & 1.008 & A & \\
\hline
\end{tabular}

\section{Values for elemental uranium are reported in $\mu \mathrm{g} /$ filter, $\mathrm{g}$, or $\mathrm{mL} . \quad \mathrm{pCi} / \mathrm{g}$ or $\mathrm{mL}=\mathrm{Bq} \times 0.027$}

Evaluation: $A=$ Acceptable, $W=$ Acceptable with Warning, $N=$ Not Acceptable

If the evaluation system is not appropriate for the types of analyses performed in your lab, apply a site specific evaluation. 


\section{QAP 49 Results by Laboratory}

Lab: IT Quanterra-Richland Laboratory

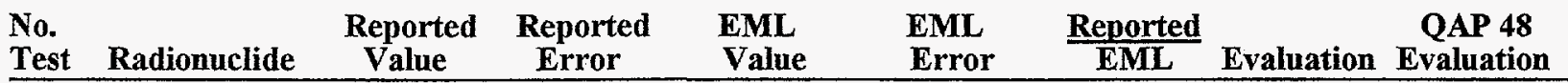

Matrix: AI Air Filter $\mathrm{Bq} /$ filter

\begin{tabular}{|c|c|c|c|c|c|c|c|}
\hline $\mathrm{AM} 241$ & 0.460 & 0.070 & 0.510 & 0.008 & 0.902 & A & A \\
\hline $\mathrm{BqU}$ & 0.490 & 0.020 & 0.530 & 0.020 & 0.925 & A & \\
\hline CO60 & 8.820 & 0.330 & 9.160 & 0.580 & 0.963 & A & A \\
\hline CS137 & 21.800 & 0.300 & 22.470 & 1.030 & 0.970 & A & A \\
\hline GROSS ALPHA & 1.960 & 0.030 & 1.650 & 0.160 & 1.188 & A & A \\
\hline GROSS BETA & 2.200 & 0.070 & 2.160 & 0.070 & 1.019 & A & A \\
\hline MN54 & 5.220 & 0.170 & 4.920 & 0.400 & 1.061 & A & A \\
\hline PU238 & 0.500 & 0.030 & 0.460 & 0.005 & 1.087 & A & A \\
\hline PU239 & 0.480 & 0.020 & 0.420 & 0.006 & 1.143 & A & A \\
\hline SB125 & 9.100 & 0.500 & 8.890 & 0.550 & 1.024 & $A$ & A \\
\hline SR90 & 1.090 & 0.120 & 1.120 & 0.050 & 0.973 & A & \\
\hline $\mathrm{U} 234$ & 0.240 & 0.010 & 0.260 & 0.010 & 0.923 & A & \\
\hline $\mathrm{U} 238$ & 0.230 & 0.010 & 0.260 & 0.010 & 0.885 & W & A \\
\hline ug U & 20.400 & 0.500 & 20.960 & 0.100 & 0.973 & A & A \\
\hline
\end{tabular}

Matrix: SO Soil $\mathrm{Bq} / \mathrm{kg}$

\begin{tabular}{|c|}
\hline $\mathrm{AC} 228$ \\
\hline $\mathrm{AM} 241$ \\
\hline $\mathrm{BI212}$ \\
\hline $\mathrm{BI} 214$ \\
\hline $\mathrm{Bq} \mathrm{U}$ \\
\hline CS137 \\
\hline $\mathrm{K} 40$ \\
\hline PB210 \\
\hline PB212 \\
\hline PB214 \\
\hline PU238 \\
\hline PU239 \\
\hline RA226 \\
\hline SR90 \\
\hline TH228 \\
\hline $\mathrm{TH} 234$ \\
\hline TL208 \\
\hline U234 \\
\hline U238 \\
\hline ug U \\
\hline
\end{tabular}

62.000
4.880
61.000
32.000
193.000
1129.000
373.000
41.000
61.000
35.000
0.770
14.500
32.000
43.000
57.000
236.000
20.300
92.000
98.000
10.200

1.800
0.760
3.000
3.000
13.000
46.000
22.000
5.500
3.000
1.500
0.080
1.200
3.000
2.400
7.000
13.000
2.200
4.000
7.000
0.500

52.600

7.470

58.300

28.800

237.000

954.000

314.000

32.000

52.800

29.100

0.530

13.090

29.000

39.630

52.700

114.000

18.300

113.000

120.000

9.700

\subsection{0}

0.410

5.900

0.500

16.000

38.000

13.000

3.300

3.700

1.200

0.270

0.570

1.000

0.003

4.000

6.000

1.100

6.000

9.000

0.700
1.179

0.653

1.046

1.111

0.814

1.183

1.188

1.281

1.155

1.203

1.453

1.108

1.103

1.085

1.082

2.070

1.109

0.814

0.817

1.052

$\begin{array}{ll}\text { A } & \\ \text { W } & \text { A } \\ \text { A } & \\ \text { A } & \\ \text { A } & \\ \text { A } & \text { A } \\ \text { A } & \text { A } \\ \text { A } & \end{array}$

$\mathrm{A}$
$\mathrm{A}$

A

A

A

A

Matrix: VE Vegetation $\mathrm{Bq} / \mathrm{kg}$

$\begin{array}{ll}1 & \text { AM241 } \\ 1 & \text { CM244 } \\ 1 & \text { CO60 } \\ 1 & \text { CS137 } \\ 1 & \text { K40 } \\ 1 & \text { PU239 }\end{array}$

$$
\begin{array}{r}
2.200 \\
1.940 \\
24.700 \\
486.000 \\
567.000 \\
3.930
\end{array}
$$

$$
\begin{array}{r}
0.240 \\
0.110 \\
2.400 \\
20.000 \\
8.000 \\
0.170
\end{array}
$$

$$
\begin{array}{r}
2.330 \\
1.760 \\
20.000 \\
390.000 \\
460.000 \\
3.720
\end{array}
$$

0.944
1.102
1.235
1.246
1.233
1.056

A

\footnotetext{
Values for elemental uranium are reported in $\mu \mathrm{g} /$ filter, $\mathrm{g}$, or $\mathrm{mL}$.
}

\section{$\mathrm{pCi} / \mathrm{g}$ or $\mathrm{mL}=\mathrm{Bq} \times 0.027$}

Evaluation: $A=$ Acceptable, $W=$ Acceptable with Warning, $N=$ Not Acceptable

If the evaluation system is not appropriate for the types of analyses performed in your lab, apply a site specific evaluation. 


\section{QAP 49 Results by Laboratory}

December, 1998

Lab: IT Quanterra- Richland Laboratory

\begin{tabular}{|c|c|c|c|c|c|c|c|}
\hline est & Radio & $\begin{array}{c}\text { Reported } \\
\text { Value }\end{array}$ & $\begin{array}{l}\text { Reported } \\
\text { Error }\end{array}$ & $\begin{array}{l}\text { EML } \\
\text { Value }\end{array}$ & $\begin{array}{l}\text { EML } \\
\text { Error }\end{array}$ & $\frac{\text { Reported }}{\text { EML }}$ & $\begin{array}{c}\text { QAP 48 } \\
\text { Evaluation }\end{array}$ \\
\hline
\end{tabular}

Matrix: WA Water $\mathrm{Bq} / \mathrm{L}$

$\begin{array}{llrrrrrrr}1 & \text { AM241 } & 1.410 & 0.080 & 1.250 & 0.080 & 1.128 & \mathrm{~A} & \mathrm{~A} \\ 1 & \text { Bq U } & 1.050 & 0.100 & 1.050 & 0.080 & 1.000 & \mathrm{~A} & \\ 1 & \text { CO60 } & 53.400 & 3.000 & 49.400 & 1.200 & 1.081 & \mathrm{~A} & \mathrm{~W} \\ 1 & \text { CS137 } & 51.800 & 2.500 & 50.000 & 1.700 & 1.036 & \mathrm{~A} & \mathrm{~A} \\ 1 & \text { GROSS ALPHA } & 933.000 & 4.000 & 1080.000 & 60.000 & 0.864 & \mathrm{~A} & \mathrm{~A} \\ 1 & \text { GROSS BETA } & 1182.000 & 2.000 & 1420.000 & 60.000 & 0.832 & \mathrm{~A} & \mathrm{~A} \\ 1 & \text { H3 } & 79.000 & 5.000 & 76.200 & 2.900 & 1.037 & \mathrm{~A} & \mathrm{~A} \\ 1 & \text { MN54 } & 35.800 & 1.500 & 32.400 & 1.400 & 1.105 & \mathrm{~A} & \mathrm{~A} \\ 1 & \text { PU238 } & 1.270 & 0.030 & 1.100 & 0.010 & 1.155 & \mathrm{~W} & \mathrm{~A} \\ 1 & \text { PU239 } & 1.580 & 0.120 & 1.410 & 0.040 & 1.121 & \mathrm{~A} & \mathrm{~A} \\ 1 & \text { SR90 } & 2.240 & 0.300 & 2.110 & 0.180 & 1.062 & \mathrm{~A} & \mathrm{~A} \\ 1 & \text { U234 } & 0.500 & 0.010 & 0.510 & 0.030 & 0.980 & \mathrm{~A} & \mathrm{~A} \\ 1 & \text { U238 } & 0.530 & 0.080 & 0.520 & 0.050 & 1.019 & \mathrm{~A} & \mathrm{~A} \\ 1 & \text { ug U } & 0.040 & 0.000 & 0.040 & 0.003 & 0.998 & \mathrm{~A} & \mathrm{~A}\end{array}$

Values for elemental uranium are reported in $\mu \mathrm{g} / \mathrm{filter}, \mathrm{g}$, or $\mathrm{mL} . \quad \mathrm{pCi} / \mathrm{g}$ or $\mathrm{mL}=\mathrm{Bq} \times 0.027$

Evaluation: A=Acceptable, $W=$ Acceptable with Warning, $N=$ Not Acceptable

If the evaluation system is not appropriate for the types of analyses performed in your lab, apply a site specific evaluation. 


\section{QAP 49 Results by Laboratory}

Lab: JE Jacobs Engineering, Oak Ridge, TN

No. $\quad$ Reported Reported EML EML

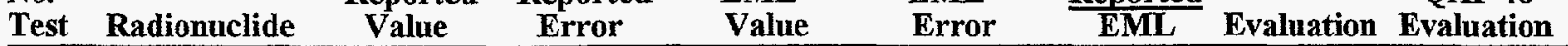

Matrix: AI Air Filter Bq / filter

$\begin{array}{lllllllll}1 & \text { GROSS ALPHA } & 1.811 & 0.200 & 1.650 & 0.160 & 1.098 & \text { A } & \text { A } \\ 1 & \text { GROSS BETA } & 2.250 & 0.200 & 2.160 & 0.070 & 1.042 & \text { A } & \text { A }\end{array}$

Matrix: SO Soil $\mathrm{Bq} / \mathrm{kg}$

$\begin{array}{ll}1 & \text { AC228 } \\ 1 & \text { BI214 } \\ 1 & \text { CS137 } \\ 1 & \text { K40 } \\ 1 & \text { PB212 } \\ 1 & \text { PB214 }\end{array}$

53.280
39.800
1124.960
363.110
42.610
28.390

$\begin{array}{rr}6.590 & 52.600 \\ 5.120 & 28.800 \\ 27.630 & 954.000 \\ 58.290 & 314.000 \\ 3.370 & 52.800 \\ 5.500 & 29.100\end{array}$

2.900
0.500
38.000
13.000
3.700
1.200

1.013

1.382

1.179

1.156

0.807

0.976

$\begin{array}{ll}\text { A } & \\ \text { A } & \\ \text { A } & \text { A } \\ \text { A } & \text { A } \\ \text { A } & \\ \text { A } & \end{array}$

Matrix: WA Water $\mathrm{Bq} / \mathrm{L}$

$\begin{array}{llrrrrrrr}1 & \text { CO60 } & 91.670 & 2.710 & 49.400 & 1.200 & 1.856 & \mathrm{~N} & \mathrm{~N} \\ 1 & \text { CS137 } & 92.450 & 3.510 & 50.000 & 1.700 & 1.849 & \mathrm{~N} & \mathrm{~A} \\ 1 & \text { GROSS ALPHA } & 967.750 & 97.800 & 1080.000 & 60.000 & 0.896 & \mathrm{~A} & \mathrm{~A} \\ 1 & \text { GROSS BETA } & 1477.500 & 95.600 & 1420.000 & 60.000 & 1.040 & \mathrm{~A} & \mathrm{~A} \\ 1 & \text { MN54 } & 68.310 & 3.250 & 32.400 & 1.400 & 2.108 & \mathrm{~N} & \mathrm{~W}\end{array}$

Values for elemental uranium are reported in $\mu \mathrm{g} /$ filter, $\mathrm{g}$, or $\mathrm{mL}$.

$\mathrm{pCi} / \mathrm{g}$ or $\mathrm{mL}=\mathrm{Bq} \times 0.027$

Evaluation: $A=A c c e p t a b l e, W=$ Acceptable with Warning, $N=$ Not Acceptable

If the evaluation system is not appropriate for the types of analyses performed in your lab, apply a site specific evaluation. 


\section{QAP 49 Results by Laboratory}

Lab: JL Jefferson Lab, Newport News, VA

\begin{tabular}{|c|c|c|c|c|c|c|c|}
\hline $\begin{array}{l}\text { to. } \\
\text { est }\end{array}$ & adi & $\begin{array}{l}\text { Reported } \\
\text { Value }\end{array}$ & $\begin{array}{l}\text { Reported } \\
\text { Error }\end{array}$ & $\begin{array}{l}\text { EML } \\
\text { Value }\end{array}$ & $\begin{array}{l}\text { EML } \\
\text { Error }\end{array}$ & $\frac{\text { Reported }}{\text { EML }}$ & $\begin{array}{cc}\text { QAP 48 } \\
\text { Evaluation } \\
\text { Evaluation }\end{array}$ \\
\hline
\end{tabular}

Matrix: AI Air Filter $\mathrm{Bq} /$ filter

$\begin{array}{llllllll}1 & \text { GROSS ALPHA } & 3.010 & 0.070 & 1.650 & 0.160 & 1.824 & \mathrm{~N} \\ 1 & \text { GROSS BETA } & 0.480 & 0.040 & 2.160 & 0.070 & 0.222 & \mathrm{~N}\end{array}$

Matrix: WA Water $\mathrm{Bq} / \mathrm{L}$

$\begin{array}{llrllllll}1 & \text { AM241 } & 0.750 & 0.360 & 1.250 & 0.080 & 0.600 & \mathrm{~N} & \mathrm{~W} \\ 1 & \text { CO60 } & 50.900 & 2.500 & 49.400 & 1.200 & 1.030 & \mathrm{~A} & \mathrm{~N} \\ 1 & \text { CS137 } & 50.600 & 3.500 & 50.000 & 1.700 & 1.012 & \mathrm{~A} & \mathrm{~A} \\ 1 & \text { MN54 } & 35.200 & 2.700 & 32.400 & 1.400 & 1.086 & \mathrm{~A} & \mathrm{~A}\end{array}$




\section{QAP 49 Results by Laboratory}

Lab: KA Knolls Atomic Power Lab, Schenectady

No.

Reported Reported

EML

EML Reported

QAP 48

Test Radionuclide

Value

Error

Value

Error

EML

Evaluation Evaluation

Matrix: $\mathrm{AI}$ Air Filter $\mathrm{Bq} /$ filter

$\begin{array}{lllllllll}1 & \text { GROSS ALPHA } & 1.700 & 0.060 & 1.650 & 0.160 & 1.030 & \mathrm{~A} & \mathrm{~A} \\ 1 & \text { GROSS BETA } & 2.060 & 0.050 & 2.160 & 0.070 & 0.954 & \mathrm{~A} & \mathrm{~A}\end{array}$

Matrix: $\mathrm{SO}$ Soil $\mathrm{Bq} / \mathrm{kg}$

$1 \quad \operatorname{CS} 137$

$1 \mathrm{~K} 40$

1 PU239

1 SR90
967.500

328.100

13.760

43.300

$\begin{array}{rr}108.500 & 954.000 \\ 51.300 & 314.000 \\ 0.080 & 13.090 \\ 1.900 & 39.630\end{array}$

1.690

3.310

6.000

61.000

55.000

9.000

2.270

0.008

0.308

0.000
49.400

50.000

139.000

1080.000

1420.000

76.200

32.400

1.410

2.110

0.040
1.200

1.700

2.000

60.000

60.000

2.900

1.400

0.040

0.180

0.003
0.971

0.968

1.072

0.974

0.978

1.299

0.957

1.193

1.136

1.050

$\begin{array}{ll}\text { A } & \text { A } \\ \text { A } & \text { A } \\ \text { A } & \text { W } \\ \text { A } & \text { A } \\ \text { A } & \text { A } \\ \text { W } & \text { A } \\ \text { A } & \text { A } \\ \text { W } & \text { A } \\ \text { A } & \text { A } \\ \text { A } & \text { A }\end{array}$

$\begin{array}{ll}\mathrm{A} & \mathrm{A} \\ \mathrm{A} & \mathrm{A} \\ \mathrm{A} & \mathrm{A} \\ \mathrm{A} & \mathrm{A}\end{array}$

A
A
A

A

Values for elemental uranium are reported in $\mu \mathrm{g} /$ filter, $\mathrm{g}$, or $\mathrm{mL}$.

$\mathrm{pCi} / \mathrm{g}$ or $\mathrm{mL}=\mathrm{Bq} \times 0.027$

Evaluation: $A=A c c e p t a b l e, W=A c c e p t a b l e$ with Warning, $N=$ Not Acceptable

If the evaluation system is not appropriate for the types of analyses performed in your lab, apply a site specific evaluation. 


\section{QAP 49 Results by Laboratory}

Lab: KR Korea Atomic Energy Research Institute

\begin{tabular}{|c|c|c|}
\hline $\begin{array}{l}\text { No. } \\
\text { Test Radionuclide }\end{array}$ & $\begin{array}{c}\text { Reported } \\
\text { Value }\end{array}$ & $\begin{array}{c}\text { Reported } \\
\text { Error }\end{array}$ \\
\hline
\end{tabular}

Matrix: AI Air Filter Bq / filter

$\begin{array}{llrrrrrrr}1 & \text { CO60 } & 8.800 & 0.900 & 9.160 & 0.580 & 0.961 & \mathrm{~A} & \\ 1 & \text { CS137 } & 21.500 & 2.300 & 22.470 & 1.030 & 0.957 & \mathrm{~A} & \\ 1 & \text { GROSS ALPHA } & 1.600 & 0.030 & 1.650 & 0.160 & 0.970 & \mathrm{~A} & \mathrm{~A} \\ 1 & \text { GROSS BETA } & 1.930 & 0.030 & 2.160 & 0.070 & 0.894 & \mathrm{~A} & \mathrm{~A} \\ 1 & \text { MN54 } & 4.600 & 0.500 & 4.920 & 0.400 & 0.935 & \mathrm{~A} & \\ 1 & \text { SB125 } & 7.700 & 0.900 & 8.890 & 0.550 & 0.866 & \mathrm{~A} & \\ 1 & \text { SR90 } & 0.860 & 0.050 & 1.120 & 0.050 & 0.768 & \text { W } & \end{array}$

Matrix: SO Soil $\mathrm{Bq} / \mathrm{kg}$

$\begin{array}{ll}1 & \text { CS137 } \\ 1 & \text { K40 } \\ 1 & \text { PU239 } \\ 1 & \text { SR90 }\end{array}$
1043.100
381.100
11.700
35.700

44.200
49.600
0.200
0.700

954.000

314.000

13.090

39.630
38.000
13.000
0.570
0.003

1.093
1.214
0.894
0.901

A

A

A $\quad$ A

A $\mathrm{A}$

Matrix: VE Vegetation $\mathrm{Bq} / \mathrm{kg}$

$\begin{array}{ll}1 & \text { CO60 } \\ 1 & \text { CS137 } \\ 1 & \text { K40 } \\ 1 & \text { SR90 }\end{array}$

20.400

397.400

486.800

714.400
20.000
390.000
460.000
606.000

1.000

20.000

20.000

40.000
1.020
1.019
1.058
1.179

A
A
A
W

$\mathrm{A}$
$\mathrm{A}$
$\mathrm{A}$
$\mathrm{A}$
A

Values for elemental uranium are reported in $\mu \mathrm{g} /$ filter, $\mathrm{g}$, or $\mathrm{mL}$.

$\mathrm{pCi} / \mathrm{g}$ or $\mathrm{mL}=\mathrm{Bq} \times 0.027$

Evaluation: $A=$ Acceptable, $W=$ Acceptable with Warning, $N=$ Not Acceptable

If the evaluation system is not appropriate for the types of analyses performed in your lab, apply a site specific evaluation. 


\section{QAP 49 Results by Laboratory}

Lab: LA Los Alamos National Laboratory, NM

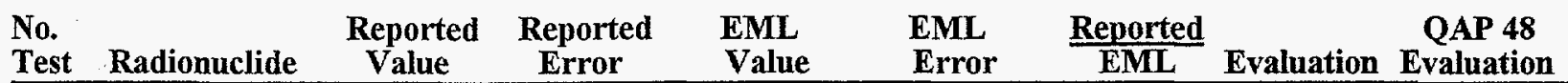

Matrix: AI Air Filter $\mathrm{Bq} /$ filter

\begin{tabular}{|c|c|c|c|c|c|c|}
\hline AM241 & 0.563 & 0.017 & 0.510 & 0.008 & 1.104 & A \\
\hline AM241 & 0.560 & 0.017 & 0.510 & 0.008 & 1.098 & A \\
\hline AM241 & 0.543 & 0.017 & 0.510 & 0.008 & 1.065 & A \\
\hline $\mathrm{CO} 60$ & 9.190 & 0.720 & 9.160 & 0.580 & 1.003 & A \\
\hline CO60 & 9.070 & 0.730 & 9.160 & 0.580 & 0.990 & A \\
\hline $\mathrm{CO} 60$ & 9.120 & 0.740 & 9.160 & 0.580 & 0.996 & A \\
\hline CS137 & 22.000 & 1.700 & 22.470 & 1.030 & 0.979 & A \\
\hline $\mathrm{CS} 137$ & 22.100 & 1.700 & 22.470 & 1.030 & 0.984 & A \\
\hline CS137 & 22.100 & 1.700 & 22.470 & 1.030 & 0.984 & A \\
\hline MN54 & 5.160 & 0.430 & 4.920 & 0.400 & 1.049 & A \\
\hline MN54 & 5.150 & 0.430 & 4.920 & 0.400 & 1.047 & A \\
\hline MN54 & 5.060 & 0.420 & 4.920 & 0.400 & 1.028 & A \\
\hline PU238 & 0.467 & 0.017 & 0.460 & 0.005 & 1.015 & A \\
\hline PU238 & 0.490 & 0.017 & 0.460 & 0.005 & 1.065 & A \\
\hline PU238 & 0.446 & 0.015 & 0.460 & 0.005 & 0.970 & A \\
\hline PU239 & 0.471 & 0.017 & 0.420 & 0.006 & 1.121 & A \\
\hline PU239 & 0.437 & 0.015 & 0.420 & 0.006 & 1.040 & $\mathrm{~A}$ \\
\hline PU239 & 0.466 & 0.016 & 0.420 & 0.006 & 1.110 & A \\
\hline SB125 & 9.150 & 0.770 & 8.890 & 0.550 & 1.029 & A \\
\hline SB125 & 9.110 & 0.770 & 8.890 & 0.550 & 1.025 & A \\
\hline SB125 & 8.910 & 0.750 & 8.890 & 0.550 & 1.002 & A \\
\hline
\end{tabular}

Matrix: SO Soil $\mathrm{Bq} / \mathrm{kg}$

$\begin{array}{ll}1 & \text { AM241 } \\ 2 & \text { AM241 } \\ 3 & \text { AM241 } \\ 3 & \text { CS137 } \\ 2 & \text { CS137 } \\ 1 & \text { CS137 } \\ 3 & \text { K40 } \\ 2 & \text { K40 } \\ 1 & \text { K40 } \\ 3 & \text { PU239 } \\ 2 & \text { PU239 } \\ 1 & \text { PU239 }\end{array}$

$\begin{array}{rr}8.470 & 0.740 \\ 7.660 & 0.740 \\ 7.920 & 0.740 \\ 785.000 & 56.000 \\ 629.000 & 45.000 \\ 628.000 & 45.000 \\ 333.000 & 35.000 \\ 317.000 & 34.000 \\ 253.000 & 29.000 \\ 13.000 & 0.500 \\ 13.100 & 0.500 \\ 12.600 & 0.500\end{array}$

7.470
7.470
7.470
954.000
954.000
954.000
314.000
314.000
314.000
13.090
13.090
13.090

0.410

0.410

0.410

38.000

38.000

38.000

13.000

13.000

13.000

0.570

0.570

0.570

3.200
3.100
2.700
36.000
37.000
37.000
51.000
48.000

20.000
20.000
20.000
390.000
390.000
390.000
460.000
460.000

1.134
1.025
1.060
0.823
0.659
0.658
1.061
1.010
0.806
0.993
1.001
0.963
A
$\mathrm{N}$

A

A

A

A

A

A

A
A
A
W
W
W
A
A
A
A
A
A

Matrix: $\mathrm{VE}$ Vegetation $\mathrm{Bq} / \mathrm{kg}$

$\begin{array}{ll}2 & \text { CO60 } \\ 1 & \text { CO60 } \\ 3 & \text { CO60 } \\ 1 & \text { CS137 } \\ 2 & \text { CS137 } \\ 3 & \text { CS137 } \\ 3 & \text { K40 } \\ 2 & \text { K40 }\end{array}$

26.800
25.800
25.000
443.000
462.000
456.000
470.000
436.000

1.000
1.000
1.000
20.000
20.000
20.000
20.000
20.000
1.340 1.290 1.250 1.136 1.185 1.169 1.022 0.948

Values for elemental uranium are reported in $\mu \mathrm{g} /$ filter, $g$, or $\mathrm{mL}$.

$\mathrm{pCi} / \mathrm{g}$ or $\mathrm{mL}=\mathrm{Bq} \times 0.027$

Evaluation: $A=$ Acceptable, $W=$ Acceptable with Warning, $N=$ Not Acceptable

If the evaluation system is not appropriate for the types of analyses performed in your lab, apply a site specific evaluation. 


\section{QAP 49 Results by Laboratory}

Lab: LA Los Alamos National Laboratory, NM

No. $\quad$ Reported Reported EML EML Reported $\quad$ QAP 48

Test Radionuclide Value Error Value Error $\frac{\text { EML }}{\text { Evaluation Evaluation }}$

Matrix: VE Vegetation $\mathrm{Bq} / \mathrm{kg}$

$\begin{array}{lllllllll}1 & \text { K40 } & 382.000 & 43.000 & 460.000 & 20.000 & 0.830 & \text { W } & \text { W } \\ 2 & \text { SR90 } & 671.000 & 41.700 & 606.000 & 40.000 & 1.107 & \text { A } & \\ 1 & \text { SR90 } & 620.000 & 39.400 & 606.000 & 40.000 & 1.023 & \text { A } & \\ 3 & \text { SR90 } & 622.400 & 39.200 & 606.000 & 40.000 & 1.027 & \text { A } & \end{array}$

Matrix: WA Water $\mathrm{Bq} / \mathrm{L}$

$\begin{array}{llrrrrrrr}2 & \text { AM241 } & 1.180 & 0.040 & 1.250 & 0.080 & 0.944 & \mathrm{~A} & \mathrm{~A} \\ 3 & \text { AM241 } & 1.340 & 0.040 & 1.250 & 0.080 & 1.072 & \mathrm{~A} & \mathrm{~A} \\ 1 & \text { AM241 } & 1.300 & 0.040 & 1.250 & 0.080 & 1.040 & \mathrm{~A} & \mathrm{~A} \\ 3 & \text { BqU } & 1.050 & 0.250 & 1.050 & 0.080 & 1.000 & \mathrm{~A} & \\ 2 & \text { Bq } & 1.025 & 0.250 & 1.050 & 0.080 & 0.976 & \mathrm{~A} & \\ 1 & \text { Bq U } & 1.025 & 0.250 & 1.050 & 0.080 & 0.976 & \mathrm{~A} & \\ 1 & \text { CO60 } & 56.900 & 6.100 & 49.400 & 1.200 & 1.152 & \mathrm{~W} & \mathrm{~W} \\ 3 & \text { CO60 } & 52.100 & 5.700 & 49.400 & 1.200 & 1.055 & \mathrm{~A} & \mathrm{~W} \\ 2 & \text { CO60 } & 54.300 & 5.800 & 49.400 & 1.200 & 1.099 & \mathrm{~A} & \mathrm{~W} \\ 1 & \text { CS137 } & 60.100 & 6.300 & 50.000 & 1.700 & 1.202 & \mathrm{~W} & \mathrm{~W} \\ 2 & \text { CS137 } & 58.800 & 6.200 & 50.000 & 1.700 & 1.176 & \mathrm{~A} & \mathrm{~W} \\ 3 & \text { CS137 } & 58.800 & 6.200 & 50.000 & 1.700 & 1.176 & \mathrm{~A} & \mathrm{~W} \\ 1 & \text { GROSS ALPHA } & 884.300 & 156.880 & 1080.000 & 60.000 & 0.819 & \mathrm{~W} & \mathrm{~A} \\ 3 & \text { GROSS ALPHA } & 1110.000 & 193.510 & 1080.000 & 60.000 & 1.028 & \mathrm{~A} & \mathrm{~A} \\ 1 & \text { GROSS BETA } & 1102.600 & 62.160 & 1420.000 & 60.000 & 0.776 & \mathrm{~A} & \mathrm{~A} \\ 3 & \text { GROSS BETA } & 1158.100 & 65.120 & 1420.000 & 60.000 & 0.816 & \mathrm{~A} & \mathrm{~A} \\ 2 & \text { H3 } & 104.000 & 30.000 & 76.200 & 2.900 & 1.365 & \mathrm{~W} & \mathrm{~A} \\ 3 & \text { H3 } & 106.000 & 30.000 & 76.200 & 2.900 & 1.391 & \mathrm{~W} & \mathrm{~A} \\ 1 & \text { H3 } & 115.000 & 31.000 & 76.200 & 2.900 & 1.509 & \mathrm{~W} & \mathrm{~A} \\ 1 & \text { MN54 } & 40.600 & 4.600 & 32.400 & 1.400 & 1.253 & \mathrm{~N} & \mathrm{~W} \\ 2 & \text { MN54 } & 37.100 & 4.200 & 32.400 & 1.400 & 1.145 & \mathrm{~A} & \mathrm{~W} \\ 3 & \text { MN54 } & 37.200 & 4.300 & 32.400 & 1.400 & 1.148 & \mathrm{~A} & \mathrm{~W} \\ 3 & \text { PU238 } & 1.110 & 0.040 & 1.100 & 0.010 & 1.009 & \mathrm{~A} & \mathrm{~A} \\ 2 & \text { PU238 } & 1.110 & 0.040 & 1.100 & 0.010 & 1.009 & \mathrm{~A} & \mathrm{~A} \\ 1 & \text { PU238 } & 1.060 & 0.040 & 1.100 & 0.010 & 0.964 & \mathrm{~A} & \mathrm{~A} \\ 1 & \text { PU239 } & 1.460 & 0.050 & 1.410 & 0.040 & 1.035 & \mathrm{~A} & \mathrm{~A} \\ 3 & \text { PU239 } & 1.450 & 0.050 & 1.410 & 0.040 & 1.028 & \mathrm{~A} & \mathrm{~A} \\ 2 & \text { PU239 } & 1.480 & 0.050 & 1.410 & 0.040 & 1.050 & \mathrm{~A} & \mathrm{~A} \\ 3 & \text { SR90 } & 2.819 & 0.335 & 2.110 & 0.180 & 1.336 & \mathrm{~W} & \\ 2 & \text { SR90 } & 2.756 & 0.333 & 2.110 & 0.180 & 1.306 & \mathrm{~W} & \\ 1 & \text { SR90 } & 2.851 & 0.336 & 2.110 & 0.180 & 1.351 & \mathrm{~W} & \end{array}$

Values for elemental uranium are reported in $\mu$ g/filter, g, or $\mathrm{mL} . \quad \mathrm{pCi} / \mathrm{g}$ or $\mathrm{mL}=\mathrm{Bq} \times 0.027$

Evaluation: $A=A c c e p t a b l e, W=A c c e p t a b l e$ with Warning, $N=$ Not Acceptable

If the evaluation system is not appropriate for the types of analyses performed in your lab, apply a site specific evaluation. 
QAP 49 Results by Laboratory

Lab: LB Lawrence Berkeley Lab UCB

Reported Reported

EML

EML

Reported

QAP 48

Test Radionuclide Value Error

Value

Error EML

Evaluation Evaluation

Matrix: AI Air Filter $\mathrm{Bq} /$ filter

$\begin{array}{ll}1 & \text { CO60 } \\ 1 & \text { CS137 } \\ 1 & \text { MN54 } \\ 1 & \text { SB125 }\end{array}$

10.400

26.000

5.900

1.100

2.000

9.160

22.470

0.800

4.920

0.700

8.890

0.580

1.030

0.400

0.550

1.135
1.157
1.199
1.080

W

W

W

Matrix: $\mathrm{SO}$ Soil $\mathrm{Bq} / \mathrm{kg}$

$1 \quad \operatorname{CS} 137$

1131.000

124.000

954.000

66.000

314.000

38.000

13.000

1.186

1.220

A

A

Matrix: VE Vegetation $\mathrm{Bq} / \mathrm{kg}$

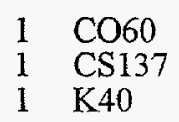
34.000
648.000
753.000
4.000
52.000
90.000

20.000

390.000

460.000

1.000
20.000
20.000

1.700

1.662

1.637

$N$
$N$

Matrix: WA Water $\mathrm{Bq} / \mathrm{L}$

$\begin{array}{llllllll}1 & \text { CO60 } & 51.000 & 9.000 & 49.400 & 1.200 & 1.032 . & \mathrm{A} \\ 1 & \text { CS137 } & 52.000 & 7.000 & 50.000 & 1.700 & 1.040 & \mathrm{~A} \\ 1 & \text { MN54 } & 34.000 & 5.000 & 32.400 & 1.400 & 1.049 & \mathrm{~A}\end{array}$




\section{QAP 49 Results by Laboratory}

Lab: LE Lyle Environmental Management, Columbus, Ohio

No.

Reported Reported

EML

EML

Reported

QAP 48

Test Radionuclide Value Error Value Error EML

Evaluation Evaluation

Matrix: WA Water $\mathrm{Bq} / \mathrm{L}$

1 GROSS ALPHA 545.000

1 GROSS BETA $\quad 1630.000$

1080.000

1420.000
60.000
60.000

60.000
60.000

0.505
1.148

0.505
1.148

N

Values for elemental uranium are reported in $\mu \mathrm{g} /$ filter, $\mathrm{g}$, or $\mathrm{mL}$.

$\mathrm{pCi} / \mathrm{g}$ or $\mathrm{mL}=\mathrm{Bq} \times 0.027$

Evaluation: $A=$ Acceptable, $W=$ Acceptable with Warning, $N=$ Not Acceptable

If the evaluation system is not appropriate for the types of analyses performed in your lab, apply a site specific evaluation. 


\section{QAP 49 Results by Laboratory}

Lab: LL LLNL Chemistry and Material Science/Environmental

\begin{tabular}{lcccccc}
$\begin{array}{l}\text { No. } \\
\text { Test }\end{array}$ & Radionuclide & $\begin{array}{c}\text { Reported } \\
\text { Value }\end{array}$ & $\begin{array}{c}\text { Reported } \\
\text { Error }\end{array}$ & $\begin{array}{c}\text { EML } \\
\text { Value }\end{array}$ & $\begin{array}{c}\text { EML } \\
\text { Error }\end{array}$ & $\begin{array}{c}\text { Reported } \\
\text { EML }\end{array}$ Evaluation Evaluation \\
\hline
\end{tabular}

Matrix: AI Air Filter $\mathrm{Bq} /$ filter

$\begin{array}{llrrrrrrr}1 & \text { AM241 } & 0.567 & 0.031 & 0.510 & 0.008 & 1.112 & \mathrm{~A} & \mathrm{~A} \\ 1 & \text { CO60 } & 9.010 & 0.252 & 9.160 & 0.580 & 0.984 & \mathrm{~A} & \mathrm{~A} \\ 1 & \text { CS137 } & 22.400 & 0.493 & 22.470 & 1.030 & 0.997 & \mathrm{~A} & \mathrm{~N} \\ 2 & \text { GROSS ALPHA } & 1.520 & 0.010 & 1.650 & 0.160 & 0.921 & \mathrm{~A} & \mathrm{~A} \\ 2 & \text { GROSS BETA } & 2.330 & 0.011 & 2.160 & 0.070 & 1.079 & \mathrm{~A} & \mathrm{~A} \\ 1 & \text { MN54 } & 5.160 & 0.269 & 4.920 & 0.400 & 1.049 & \mathrm{~A} & \mathrm{~A} \\ 1 & \text { PU238 } & 0.479 & 0.045 & 0.460 & 0.005 & 1.041 & \mathrm{~A} & \mathrm{~A} \\ 1 & \text { PU239 } & 0.463 & 0.044 & 0.420 & 0.006 & 1.102 & \mathrm{~A} & \mathrm{~A} \\ 1 & \text { SB125 } & 9.670 & 0.774 & 8.890 & 0.550 & 1.088 & \mathrm{~A} & \mathrm{~W} \\ 1 & \text { U234 } & 0.251 & 0.023 & 0.260 & 0.010 & 0.965 & \mathrm{~A} & \mathrm{~N} \\ 1 & \text { U238 } & 0.252 & 0.023 & 0.260 & 0.010 & 0.969 & \mathrm{~A} & \mathrm{~N}\end{array}$

Matrix: SO Soil $\mathrm{Bq} / \mathrm{kg}$

$\begin{array}{ll}1 & \text { AM241 } \\ 1 & \text { CS137 } \\ 1 & \text { K40 } \\ 1 & \text { PU239 } \\ 1 & \text { RA226 } \\ 1 & \text { TH228 } \\ 1 & \text { U234 } \\ 1 & \text { U238 }\end{array}$

$\begin{array}{rr}7.620 & 1.860 \\ 716.000 & 21.500 \\ 242.000 & 68.300 \\ 13.200 & 1.570 \\ 26.300 & 20.500 \\ 39.900 & 6.540 \\ 98.900 & 8.540 \\ 105.000 & 9.010\end{array}$

7.470
954.000
314.000
13.090
29.000
52.700
113.000
120.000
0.410 38.000 13.000 0.570 1.000 4.000 6.000 9.000

1.020
0.751
0.771
1.008
0.907
0.757
0.875
0.875

$\begin{array}{ll}\text { A } & \text { A } \\ \text { N } & \text { A } \\ \text { N } & \text { A } \\ \text { A } & \text { A } \\ \text { A } & \\ \text { A } & \\ \text { A } & \text { A } \\ \text { A } & \text { N }\end{array}$

Matrix: VE Vegetation $\mathrm{Bq} / \mathrm{kg}$

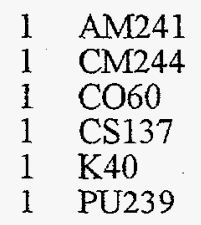

$\begin{array}{rr}2.810 & 0.638 \\ 3.580 & 0.806 \\ 22.300 & 3.520 \\ 454.000 & 14.500 \\ 519.000 & 76.800 \\ 3.420 & 0.620\end{array}$

2.330
1.760
20.000
390.000
460.000
3.720

0.060
0.070
1.000
20.000
20.000
0.270
1.206
2.034
1.115
1.164
1.128
0.919

$\begin{array}{ll}\mathrm{A} & \mathrm{A} \\ \mathrm{N} & \mathrm{A} \\ \mathrm{A} & \mathrm{A} \\ \mathrm{A} & \mathrm{A} \\ \mathrm{A} & \mathrm{A} \\ \mathrm{A} & \mathrm{A}\end{array}$

Matrix: WA Water $\mathrm{Bq} / \mathrm{L}$

$\begin{array}{ll}1 & \text { AM241 } \\ 1 & \text { CO60 } \\ 1 & \text { CS137 } \\ 1 & \text { GROSS ALPHA } \\ 1 & \text { GROSS BETA } \\ 1 & \text { H3 } \\ 1 & \text { MN54 } \\ 1 & \text { PU238 } \\ 1 & \text { PU239 } \\ 1 & \text { U234 } \\ 1 & \text { U238 }\end{array}$

1.350
49.100
49.400
819.000
1330.000
91.500
33.600
1.240
1.530
0.503
0.519
0.119
3.240
3.560
13.400
14.400
4.030
3.430
0.138
0.165
0.047
0.048
1.250
49.400
50.000
1080.000
1420.000
76.200
32.400
1.100
1.410
0.510
0.520
0.080
1.200
1.700
60.000
60.000
2.900
1.400
0.010
0.040
0.030
0.050
1.080
0.994
0.988
0.758
0.937
1.201
1.037
1.127
1.085
0.986
0.998

$\begin{array}{ll}\mathrm{A} & \mathrm{A} \\ \mathrm{A} & \mathrm{A} \\ \mathrm{A} & \mathrm{A} \\ \mathrm{W} & \mathrm{A} \\ \mathrm{A} & \mathrm{A} \\ \mathrm{A} & \mathrm{A} \\ \mathrm{A} & \mathrm{A} \\ \mathrm{W} & \mathrm{A} \\ \mathrm{A} & \mathrm{A} \\ \mathrm{A} & \mathrm{A} \\ \mathrm{A} & \mathrm{A}\end{array}$

Values for elemental uranium are reported in $\mu \mathrm{g} /$ filter, $\mathrm{g}$, or $\mathrm{mL}$.

$\mathrm{pCi} / \mathrm{g}$ or $\mathrm{mL}=\mathrm{Bq} \times 0.027$

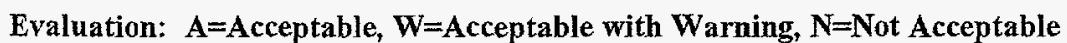

If the evaluation system is not appropriate for the types of analyses performed in your lab, apply a site specific evaluation. 


\section{QAP 49 Results by Laboratory}

Lab: LN Los Alamos National Lab, ES\&H

No.

Reported Reported

EML

EML Reported

QAP 48

Test Radionuclide

Value

Error

Value

Error EML

Evaluation Evaluation

Matrix: AI Air Filter $\mathrm{Bq} /$ filter

$\begin{array}{llrrrrrrr}1 & \text { AM241 } & 0.370 & 0.070 & 0.510 & 0.008 & 0.725 & \text { N } & \\ 1 & \text { CO60 } & 8.100 & 0.280 & 9.160 & 0.580 & 0.884 & \text { A } & \text { A } \\ 1 & \text { CS137 } & 18.100 & 0.290 & 22.470 & 1.030 & 0.806 & \text { W } & \text { A } \\ 1 & \text { GROSS ALPHA } & 1.700 & 0.200 & 1.650 & 0.160 & 1.030 & \text { A } & \text { A } \\ 1 & \text { GROSS BETA } & 1.650 & 0.160 & 2.160 & 0.070 & 0.764 & \text { W } & \text { A } \\ 1 & \text { MN54 } & 4.300 & 0.220 & 4.920 & 0.400 & 0.874 & \text { A } & \text { A } \\ 1 & \text { SB125 } & 5.700 & 0.350 & 8.890 & 0.550 & 0.641 & \text { W } & \text { A }\end{array}$

Matrix: WA Water $\mathrm{Bq} / \mathrm{L}$

$\begin{array}{llrrrrrrr}1 & \text { CO60 } & 50.300 & 2.890 & 49.400 & 1.200 & 1.018 & \mathrm{~A} & \mathrm{~N} \\ 1 & \text { CS137 } & 61.600 & 8.930 & 50.000 & 1.700 & 1.232 & \mathrm{~W} & \mathrm{~N} \\ 1 & \text { H3 } & 370.000 & 13.690 & 76.200 & 2.900 & 4.856 & \mathrm{~N} & \mathrm{~W} \\ 1 & \text { MN54 } & 48.700 & 8.970 & 32.400 & 1.400 & 1.503 & \mathrm{~N} & \mathrm{~A}\end{array}$

Values for elemental uranium are reported in $\mu \mathrm{g} /$ filter, $\mathrm{g}$, or $\mathrm{mL}$.

$\mathrm{pCi} / \mathrm{g}$ or $\mathrm{mL}=\mathrm{Bq} \times 0.027$

Evaluation: A=Acceptable, $W=A c c e p t a b l e$ with Warning, $N=$ Not Acceptable

If the evaluation system is not appropriate for the types of analyses performed in your lab, apply a site specific evaluation. 


\section{QAP 49 Results by Laboratory}

Lab: LV UNLV, Dept of Health Physics

\begin{tabular}{|c|c|c|c|c|c|c|c|}
\hline $\begin{array}{l}\text { No. } \\
\text { Test }\end{array}$ & Radionuclide & $\begin{array}{c}\text { Reported } \\
\text { Value }\end{array}$ & $\begin{array}{l}\text { Reported } \\
\text { Error }\end{array}$ & $\begin{array}{l}\text { EML } \\
\text { Value }\end{array}$ & $\begin{array}{l}\text { EML } \\
\text { Error }\end{array}$ & $\frac{\text { Reported }}{\text { EML }}$ & $\begin{array}{cc}\text { QAP 48 } \\
\text { Evaluation } \\
\text { Evaluation }\end{array}$ \\
\hline
\end{tabular}

Matrix: AI Air Filter Bq / filter

$\begin{array}{llrrrrrrr}1 & \text { AM241 } & 0.894 & 0.051 & 0.510 & 0.008 & 1.753 & \text { W } & \text { N } \\ 1 & \text { CO60 } & 8.400 & 0.160 & 9.160 & 0.580 & 0.917 & \text { A } & \text { A } \\ \text { I } & \text { CSI37 } & 20.500 & 0.200 & 22.470 & 1.030 & 0.912 & \text { A } & \text { A } \\ 1 & \text { GROSS ALPHA } & 1.600 & 0.200 & 1.650 & 0.160 & 0.970 & \text { A } & \text { W } \\ 1 & \text { GROSS BETA } & 1.780 & 0.100 & 2.160 & 0.070 & 0.824 & \text { W } & \text { N } \\ 1 & \text { MN54 } & 4.280 & 0.200 & 4.920 & 0.400 & 0.870 & \text { A } & \text { A } \\ 1 & \text { PU238 } & 0.251 & 0.026 & 0.460 & 0.005 & 0.546 & \mathrm{~N} & \\ 1 & \text { PU239 } & 0.248 & 0.007 & 0.420 & 0.006 & 0.590 & \text { N } & \\ 1 & \text { SB125 } & 4.420 & 0.250 & 8.890 & 0.550 & 0.497 & \text { N } & \text { A }\end{array}$

Matrix: SO Soil $\mathrm{Bq} / \mathrm{kg}$

$\begin{array}{ll}1 & \text { AC228 } \\ 1 & \text { AM241 } \\ 1 & \text { BI212 } \\ 1 & \text { BI214 } \\ 1 & \text { CS137 } \\ 1 & \text { K40 } \\ 1 & \text { PB212 } \\ 1 & \text { PB214 } \\ 1 & \text { PU239 } \\ 1 & \text { TH234 } \\ 1 & \text { TL208 }\end{array}$

68.700
13.100
37.900
36.300
1140.000
391.000
70.300
43.700
9.870
90.100
21.000

$\begin{array}{rr}3.100 & 52.600 \\ 0.700 & 7.470 \\ 2.900 & 58.300 \\ 1.800 & 28.800 \\ 4.000 & 954.000 \\ 10.000 & 314.000 \\ 4.600 & 52.800 \\ 2.500 & 29.100 \\ 0.740 & 13.090 \\ 5.100 & 114.000 \\ 0.700 & 18.300\end{array}$

2.900

0.410

5.900

0.500

38.000

13.000

3.700

1.200

0.570

6.000

1.100

1.306

1.754

0.650

1.260

1.195

1.245

1.331

1.502

0.754

0.790

1.148

A
W
A
A
A
A
A
A
W
A
A

W W

A $\quad$ W

A

$\mathrm{N}$

Matrix: VE Vegetation $\mathrm{Bq} / \mathrm{kg}$

$\begin{array}{ll}1 & \text { AM241 } \\ 1 & \text { CO60 } \\ 1 & \text { CS137 } \\ 1 & \text { K40 }\end{array}$
3.790
20.000
415.000
475.000
1.440
0.700
6.000
27.000

2.330
20.000
390.000
460.000
0.060
1.000
20.000
20.000
1.627
1.000
1.064
1.033

W
A
A
A

A

A

Matrix: WA Water $\mathrm{Bq} / \mathrm{L}$

$\begin{array}{llr}1 & \text { AM241 } & 1.470 \\ 1 & \text { CO60 } & 50.100 \\ 1 & \text { CS137 } & 51.300 \\ 1 & \text { GROSS ALPHA } & 1240.000 \\ 1 & \text { GROSS BETA } & 1030.000 \\ 1 & \text { H3 } & 89.000 \\ 1 & \text { MN54 } & 37.400 \\ 1 & \text { PU238 } & 0.259 \\ 1 & \text { PU239 } & 0.394\end{array}$
0.240
1.100
1.200
25.000
120.000
7.100
3.000
0.249
0.012

0.080
1.200
1.700
60.000
60.000
2.900
1.400
0.010
0.040

1.176

1.014

1.026

1.148

0.725

1.168

1.154

0.235

0.279

$\begin{array}{ll}\text { A } & \text { W } \\ \text { A } & \text { W } \\ \text { A } & \text { A } \\ \text { A } & \text { N } \\ \text { A } & \text { N } \\ \text { A } & \text { A } \\ \text { A } & \text { W } \\ \text { N } & \text { N } \\ \text { N } & \text { N }\end{array}$

\section{Values for elemental uranium are reported in $\mu$ g/filter, $g$, or $\mathrm{mL}$.}

$\mathrm{pCi} / \mathrm{g}$ or $\mathrm{mL}=\mathrm{Bq} \times 0.027$

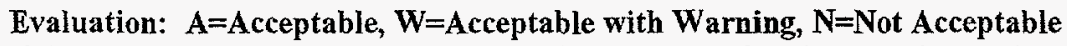

If the evaluation system is not appropriate for the types of analyses performed in your lab, apply a site specific evaluation. 


\section{QAP 49 Results by Laboratory}

Lab: LW Lawrence Livermore National Lab, Waste

\begin{tabular}{|c|c|c|c|c|c|c|c|}
\hline $\begin{array}{l}\text { No. } \\
\text { Test }\end{array}$ & Radi & $\begin{array}{c}\text { Reported } \\
\text { Value }\end{array}$ & $\begin{array}{c}\text { Reported } \\
\text { Error }\end{array}$ & $\begin{array}{l}\text { EML } \\
\text { Value }\end{array}$ & $\begin{array}{l}\text { EML } \\
\text { Error }\end{array}$ & $\frac{\text { Reported }}{\text { EML }}$ & $\begin{array}{c}\text { QAP 48 } \\
\text { Evaluation }\end{array}$ \\
\hline
\end{tabular}

Matrix: $\mathrm{SO}$ Soil $\mathrm{Bq} / \mathrm{kg}$

$\begin{array}{llrrrrrr}1 & \text { AM241 } & 9.630 & 2.530 & 7.470 & 0.410 & 1.289 & \text { A } \\ 1 & \text { PU239 } & 12.800 & 1.540 & 13.090 & 0.570 & 0.978 & \text { A } \\ 1 & \text { U234 } & 88.900 & 20.700 & 113.000 & 6.000 & 0.787 & \text { A } \\ 1 & \text { U238 } & 94.000 & 21.500 & 120.000 & 9.000 & 0.783 & \text { A }\end{array}$

Matrix: WA Water $\mathrm{Bq} / \mathrm{L}$

$\begin{array}{llrrrrrrr}1 & \text { GROSS ALPHA } & 1048.600 & 6.900 & 1080.000 & 60.000 & 0.971 & \text { A } & \\ 1 & \text { GROSS BETA } & 1292.400 & 4.800 & 1420.000 & 60.000 & 0.910 & \text { A } & \\ 1 & \text { H3 } & 78.440 & 20.000 & 76.200 & 2.900 & 1.029 & \text { A } & \text { A }\end{array}$

Values for elemental uranium are reported in $\mu \mathrm{g} /$ filter, $\mathrm{g}$, or $\mathrm{mL}$.

$\mathrm{pCi} / \mathrm{g}$ or $\mathrm{mL}=\mathrm{Bq} \times 0.027$

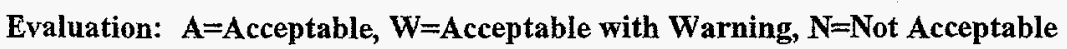

If the evaluation system is not appropriate for the types of analyses performed in your lab, apply a site specific evaluation. 


\section{QAP 49 Results by Laboratory}

Lab: MA ORNL Health Sciences Research Div.

No. Reported Reported EML EML

Test Radionuclide Value Error Value Error $\frac{\text { EML }}{\text { Evaluation Evaluation }}$

Matrix: AI Air Filter Bq / filter

$\begin{array}{llrrrrrr}1 & \text { CO60 } & 9398.000 & 1295.000 & 9.160 & 0.580 & 1,025.983 & \mathrm{~N} \\ 1 & \text { CS137 } & * * * * * * * & 3663.000 & 22.470 & 1.030 & 983.044 & \mathrm{~N} \\ 1 & \text { MN54 } & 4440.000 & 1443.000 & 4.920 & 0.400 & 902.439 & \mathrm{~N} \\ \mathrm{I} & \text { SB125 } & 8695.000 & 3293.000 & 8.890 & 0.550 & 978.065 & \mathrm{~N}\end{array}$

Matrix: SO Soil $\mathrm{Bq} / \mathrm{kg}$

$\begin{array}{llrrrrrrr}1 & \text { AM241 } & 8.900 & 3.000 & 7.470 & 0.410 & 1.191 & \text { A } & \text { A } \\ 1 & \text { CS137 } & 1114.000 & 74.000 & 954.000 & 38.000 & 1.168 & \text { A } & \text { W } \\ 1 & \text { K40 } & 370.000 & 44.000 & 314.000 & 13.000 & 1.178 & \text { A } & \text { A }\end{array}$

Matrix: VE Vegetation $\mathrm{Bq} / \mathrm{kg}$

\begin{tabular}{|c|c|c|c|c|c|c|}
\hline AM241 & 0.370 & 0.370 & 2.330 & 0.060 & 0.159 & $\mathrm{~N}$ \\
\hline CO60 & 24.000 & 3.300 & 20.000 & 1.000 & 1.200 & A \\
\hline $\operatorname{CS} 137$ & 477.000 & 33.000 & 390.000 & 20.000 & 1.223 & A \\
\hline $\mathrm{K} 40$ & 562.000 & 64.000 & 460000 & 20000 & 1200 & A \\
\hline
\end{tabular}

Values for elemental uranium are reported in $\mu \mathrm{g} /$ filter, g, or $\mathrm{mL} . \quad$ pCi/g or $\mathrm{mL}=\mathrm{Bq} \times 0.027$

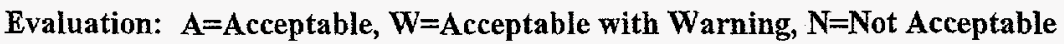

If the evaluation system is not appropriate for the types of analyses performed in your lab, apply a site specific evaluation. 


\section{QAP 49 Results by Laboratory}

Lab: ME Radiation Control Program, Jamaica Plain, MA

$\begin{array}{llclll}\text { No. } & \text { Reported } & \text { Reported } & \text { EML } & \text { EML } & \text { Reported } \\ \text { Test } & \text { Radionuclide } & \begin{array}{c}\text { Value } \\ \text { Error }\end{array} & \text { Value } & \text { Error } & \begin{array}{c}\text { QAP 48 } \\ \text { EML }\end{array} \text { Evaluation Evaluation }\end{array}$

Matrix: AI Air Filter Bq / filter

\begin{tabular}{|c|c|c|c|c|c|c|}
\hline AM241 & 0.760 & 0.060 & 0.510 & 0.008 & 1.490 & W \\
\hline $\mathrm{CO} 60$ & 10.200 & 0.310 & 9.160 & 0.580 & 1.114 & W \\
\hline CS137 & 25.600 & 1.230 & 22.470 & 1.030 & 1.139 & W \\
\hline GROSS ALPHA & 2.220 & 0.080 & 1.650 & 0.160 & 1.345 & W \\
\hline GROSS BETA & 2.400 & 0.070 & 2.160 & 0.070 & 1.111 & A \\
\hline MN54 & 6.100 & 0.350 & 4.920 & 0.400 & 1.240 & W \\
\hline SB125 & 9.700 & 0.390 & 8.890 & 0.550 & 1.091 & A \\
\hline
\end{tabular}

Matrix: SO Soil $\mathrm{Bq} / \mathrm{kg}$

$\begin{array}{ll}1 & \text { AM241 } \\ 1 & \text { CO60 } \\ 1 & \text { CS137 } \\ 1 & \text { K40 } \\ 1 & \text { PB214 } \\ 1 & \text { TH228 } \\ 1 & \text { TH234 }\end{array}$

$\begin{array}{rrr}6.610 & 0.700 & 7.470 \\ 1.010 & 0.200 & 1.240 \\ 703.000 & 33.000 & 954.000 \\ 280.000 & 15.600 & 314.000 \\ 13.700 & 1.080 & 29.100 \\ 115.000 & 18.400 & 52.700 \\ 209.000 & 9.870 & 114.000\end{array}$

$$
\begin{array}{r}
0.410 \\
0.110 \\
38.000 \\
13.000 \\
1.200 \\
4.000 \\
6.000
\end{array}
$$
0.885
0.815
0.737
0.892
0.471
2.182
1.833

$\begin{array}{ll}\text { A } & \text { A } \\ \text { W } & \\ \text { N } & \text { A } \\ \text { W } & \text { A } \\ \text { A } & \\ \text { A } & \\ \text { A } & \end{array}$

Matrix: VE Vegetation $\mathrm{Bq} / \mathrm{kg}$

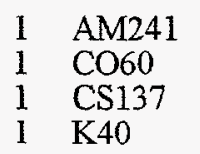

$$
\begin{array}{r}
2.070 \\
21.500 \\
437.000 \\
491.000
\end{array}
$$

0.470
0.790
20.000
27.300

2.330

0.060

1.000

0.888

1.075

1.121

390.000

20.000

460.000

20.000

1.067

$\begin{array}{ll}\text { W } & \\ \text { A } & \text { W } \\ \text { A } & \text { N } \\ \text { A } & \text { N }\end{array}$

Matrix: WA Water $\mathrm{Bq} / \mathrm{L}$

$\begin{array}{ll}1 & \text { AM241 } \\ 1 & \text { CO60 } \\ 1 & \text { CS137 } \\ 1 & \text { H3 } \\ 1 & \text { MN54 }\end{array}$

1.250
53.000
55.200
109.000
34.200

$$
\begin{aligned}
& 0.320 \\
& 1.500 \\
& 2.530 \\
& 4.500 \\
& 1.280
\end{aligned}
$$

1.250

49.400

50.000

76.200

0.080
1.200
1.700
2.900
1.400

1.000
1.073
1.104
1.430
1.056

$\mathrm{A}$
$\mathrm{A}$
$\mathrm{A}$
$\mathrm{W}$
$\mathrm{A}$

A
A
A
W
A 


\section{QAP 49 Results by Laboratory}

Lab: MH Maine Health \& Environmental Testing Laboratory

\begin{tabular}{|c|c|c|c|c|c|c|c|}
\hline $\begin{array}{l}\text { No. } \\
\text { Test }\end{array}$ & Radionuclide & $\begin{array}{c}\text { Reported } \\
\text { Value }\end{array}$ & $\begin{array}{c}\text { Reported } \\
\text { Error }\end{array}$ & $\begin{array}{l}\text { EML } \\
\text { Value }\end{array}$ & $\begin{array}{l}\text { EML } \\
\text { Error }\end{array}$ & $\frac{\text { Reported }}{\text { EML }}$ & $\begin{array}{c}\text { QAP 48 } \\
\text { Evaluation }\end{array}$ \\
\hline
\end{tabular}

Matrix: AI Air Filter $\mathrm{Bq} /$ filter

$\begin{array}{llrrrrrrr}1 & \text { CO60 } & 9.840 & 0.260 & 9.160 & 0.580 & 1.074 & \mathrm{~A} & \mathrm{~W} \\ 1 & \text { CS137 } & 25.860 & 0.970 & 22.470 & 1.030 & 1.151 & \mathrm{~W} & \mathrm{~W} \\ 1 & \text { GROSS ALPHA } & 1.650 & 0.060 & 1.650 & 0.160 & 1.000 & \mathrm{~A} & \\ 1 & \text { GROSS BETA } & 1.850 & 0.020 & 2.160 & 0.070 & 0.856 & \mathrm{~W} & \\ 1 & \text { MN54 } & 5.840 & 0.230 & 4.920 & 0.400 & 1.187 & \text { W } & \text { W } \\ 1 & \text { SB125 } & 6.170 & 0.220 & 8.890 & 0.550 & 0.694 & \text { W } & \text { W }\end{array}$

Matrix: SO Soil $\mathrm{Bq} / \mathrm{kg}$

$\begin{array}{ll}1 & \text { AC228 } \\ 1 & \text { AM241 } \\ 1 & \text { BI212 } \\ 1 & \text { BI214 } \\ 1 & \text { CS137 } \\ 1 & \text { K40 } \\ 1 & \text { PB212 } \\ 1 & \text { PB214 } \\ 1 & \text { TL208 }\end{array}$

51.240
6.390
27.230
29.120
969.500
328.400
50.680
29.040
16.080

$\begin{array}{rr}3.780 & 52.600 \\ 0.640 & 7.470 \\ 3.650 & 58.300 \\ 2.160 & 28.800 \\ 46.850 & 954.000 \\ 17.660 & 314.000 \\ 3.700 & 52.800 \\ 2.170 & 29.100 \\ 1.050 & 18.300\end{array}$

2.900

0.974

0.855

0.467

1.011

5.900

0.500

38.000

13.000

3.700

1.016

1.046

0.960

1.200

0.998

0.879

A
A
A
A
A
A
A
A
A

A

A A

Matrix: VE Vegetation $\mathrm{Bq} / \mathrm{kg}$

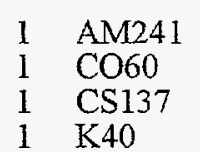

I $\mathrm{CO} 60$

$1 \mathrm{~K} 40$

$$
\begin{array}{r}
0.410 \\
0.810 \\
20.440 \\
26.540
\end{array}
$$

$$
\begin{array}{r}
0.060 \\
1.000 \\
20.000 \\
20.000
\end{array}
$$

0.845
1.035
1.081
1.100

$\begin{array}{ll}\text { W } & \\ \text { A } & \text { A } \\ \text { A } & \text { A } \\ \text { A } & \text { A }\end{array}$

Matrix: WA Water $\mathrm{Bq} / \mathrm{L}$

$\begin{array}{llrrrrrrr}1 & \text { CO60 } & 50.030 & 1.540 & 49.400 & 1.200 & 1.013 & \mathrm{~A} & \mathrm{~W} \\ 1 & \text { CS137 } & 50.730 & 2.530 & 50.000 & 1.700 & 1.015 & \mathrm{~A} & \mathrm{~A} \\ 1 & \text { GROSS ALPHA } & 1229.930 & 6.210 & 1080.000 & 60.000 & 1.139 & \mathrm{~A} & \mathrm{~W} \\ 1 & \text { GROSS BETA } & 1551.570 & 10.170 & 1420.000 & 60.000 & 1.093 & \mathrm{~A} & \mathrm{~A} \\ 1 & \text { H3 } & 78.360 & 5.220 & 76.200 & 2.900 & 1.028 & \mathrm{~A} & \mathrm{~A} \\ 1 & \text { MN54 } & 34.270 & 1.420 & 32.400 & 1.400 & 1.058 & \mathrm{~A} & \mathrm{~A} \\ 1 & \text { U234 } & 0.514 & 0.051 & 0.510 & 0.030 & 1.008 & \mathrm{~A} & \mathrm{~A} \\ 1 & \text { U238 } & 0.529 & 0.051 & 0.520 & 0.050 & 1.017 & \mathrm{~A} & \mathrm{~A}\end{array}$

Values for elemental uranium are reported in $\mu \mathrm{g} /$ filter, $g$, or $\mathrm{mL}$.

$\mathrm{pCi} / \mathrm{g}$ or $\mathrm{mL}=\mathrm{Bq} \times \mathbf{0 . 0 2 7}$

Evaluation: $A=$ Acceptable, $W=$ Acceptable with Warning, $N=$ Not Acceptable

If the evaluation system is not appropriate for the types of analyses performed in your lab, apply a site specific evaluation. 


\section{QAP 49 Results by Laboratory}

December, 1998

Lab: ML Babcock \& Wilcox of Ohio, Mound, Miamisburg, OH

No.

Test Radionuclide

Reported

Reported

EML

EML

Reported

QAP 48

Test Radionuclide

Value

Error EML

Evaluation Evaluation

Matrix: SO Soil $\mathrm{Bq} / \mathrm{kg}$

$\begin{array}{ll}1 & \text { PU239 } \\ 1 & \text { U234 } \\ 1 & \text { U238 }\end{array}$

13.540

99.090

1.030

7.560

13.090

109.800

8.280

113.000

120.000

0.570

6.000

9.000

3.720

0.270

1.169

A

A

Matrix: WA Water $\mathrm{Bq} / \mathrm{L}$
1.034

0.877

0.915

A
A

A

A

A
Matrix: VE Vegetation $\mathrm{Bq} / \mathrm{kg}$

$\begin{array}{rrr}81.030 & 17.390 & 76.200 \\ 1.210 & 0.090 & 1.100 \\ 1.600 & 0.120 & 1.410 \\ 0.490 & 0.040 & 0.510 \\ 0.500 & 0.040 & 0.520\end{array}$

2.900

0.010

0.040

0.030

0.050
1 PU238

$1 \mathrm{U} 234$

$1 \mathrm{U} 238$

0.490

0.040

0.520

1.063

1.100

1.135

0.961

0.962

$\begin{array}{ll}\mathrm{A} & \mathrm{A} \\ \mathrm{A} & \mathrm{A} \\ \mathrm{A} & \mathrm{A} \\ \mathrm{A} & \mathrm{A} \\ \mathrm{A} & \mathrm{A}\end{array}$

Values for elemental uranium are reported in $\mu \mathrm{g} /$ filter, $\mathrm{g}$, or $\mathrm{mL}$.

$\mathrm{pCi} / \mathrm{g}$ or $\mathrm{mL}=\mathrm{Bq} \times 0.027$

Evaluation: $A=A c c e p t a b l e, W=A c c e p t a b l e$ with Warning, $N=$ Not Acceptable

If the evaluation system is not appropriate for the types of analyses performed in your lab, apply a site specific evaluation. 


\section{QAP 49 Results by Laboratory}

Lab: MS Manufacturing Sciences Corporation, Oak Ridge

\begin{tabular}{llllll}
$\begin{array}{l}\text { No. } \\
\text { Test }\end{array}$ Radionuclide & $\begin{array}{c}\text { Reported } \\
\text { Value }\end{array}$ & $\begin{array}{c}\text { Reported } \\
\text { Error }\end{array}$ & $\begin{array}{l}\text { EML } \\
\text { Value }\end{array}$ & $\begin{array}{c}\text { EML } \\
\text { Error }\end{array}$ & $\frac{\text { Reported }}{\text { EML }}$ \\
Evaluation Evaluation \\
\hline
\end{tabular}

Matrix: AI Air Filter $\mathrm{Bq} /$ filter

$\begin{array}{llrrrrrrr}1 & \text { AM241 } & 0.450 & 0.050 & 0.510 & 0.008 & 0.882 & \mathrm{~A} & \mathrm{~A} \\ 1 & \text { CO60 } & 8.960 & 0.900 & 9.160 & 0.580 & 0.978 & \mathrm{~A} & \mathrm{~A} \\ 1 & \text { CS137 } & 21.100 & 1.100 & 22.470 & 1.030 & 0.939 & \mathrm{~A} & \mathrm{~A} \\ 1 & \text { GROSS ALPHA } & 1.980 & 0.200 & 1.650 & 0.160 & 1.200 & \mathrm{~A} & \\ 1 & \text { GROSS BETA } & 2.080 & 0.210 & 2.160 & 0.070 & 0.963 & \mathrm{~A} & \\ 1 & \text { MN54 } & 4.620 & 0.460 & 4.920 & 0.400 & 0.939 & \mathrm{~A} & \mathrm{~A} \\ 1 & \text { SB125 } & 9.050 & 0.450 & 8.890 & 0.550 & 1.018 & \mathrm{~A} & \mathrm{~A}\end{array}$

Matrix: SO Soil $\mathrm{Bq} / \mathrm{kg}$

$\begin{array}{ll}1 & \text { AM241 } \\ 1 & \text { CS137 } \\ 1 & \text { K40 }\end{array}$

Matrix: WA Water $\mathrm{Bq} / \mathrm{L}$

$\begin{array}{llr}1 & \text { AM241 } & 1.590 \\ 1 & \text { CO60 } & 52.500 \\ 1 & \text { CS137 } & 52.100 \\ 1 & \text { GROSS ALPHA } & 1025.000 \\ 1 & \text { GROSS BETA } & 1300.000 \\ 1 & \text { H3 } & 74.000 \\ 1 & \text { MN54 } & 35.600\end{array}$

$$
\begin{array}{r}
1.000 \\
103.000 \\
35.000
\end{array}
$$

0.410
38.000
13.000

1.165

1.074

1.130

0.200
5.300
5.200
100.000
130.000
3.700
3.600

1.250

49.400

50.000

1080.000

1420.000

76.200

32.400

0.080
1.200
1.700
60.000
60.000
2.900
1.400

1.272

1.063

1.042

0.949

0.915

1.015

1.099
A
A
A
$\mathrm{A}$
$\mathrm{A}$
$\mathrm{A}$

Values for elemental uranium are reported in $\mu \mathrm{g} /$ filter, $\mathrm{g}$, or $\mathrm{mL}$.

$\mathrm{pCi} / \mathrm{g}$ or $\mathrm{mL}=\mathrm{Bq} \times 0.027$

Evaluation: $A=$ Acceptable, $W=$ Acceptable with Warning, $N=$ Not Acceptable

If the evaluation system is not appropriate for the types of analyses performed in your lab, apply a site specific evaluation. 


\section{QAP 49 Results by Laboratory}

Lab: NA US EPA NAREL, Montgomery, AL

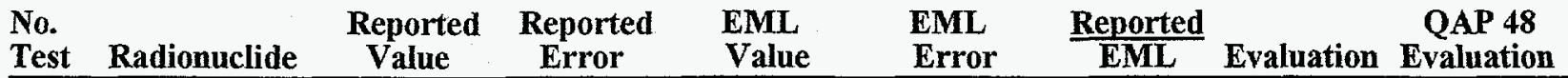

Matrix: AI Air Filter $\mathrm{Bq} /$ filter

$\begin{array}{llrrrrrrr}1 & \text { CO60 } & 8.710 & 0.080 & 9.160 & 0.580 & 0.951 & \mathrm{~A} & \mathrm{~A} \\ 1 & \text { CS137 } & 23.280 & 0.160 & 22.470 & 1.030 & 1.036 & \mathrm{~A} & \mathrm{~A} \\ 1 & \text { MN54 } & 5.010 & 0.080 & 4.920 & 0.400 & 1.018 & \mathrm{~A} & \mathrm{~A} \\ 1 & \text { PU238 } & 0.480 & 0.028 & 0.460 & 0.005 & 1.043 & \mathrm{~A} & \\ 1 & \text { PU239 } & 0.439 & 0.026 & 0.420 & 0.006 & 1.045 & \mathrm{~A} & \\ 1 & \text { SB125 } & 1.400 & 0.110 & 8.890 & 0.550 & 0.157 & \mathrm{~N} & \mathrm{~A} \\ 1 & \text { SR90 } & 0.840 & 0.170 & 1.120 & 0.050 & 0.750 & \mathrm{~W} & \mathrm{~A}\end{array}$

Matrix: SO Soil $\mathrm{Bq} / \mathrm{kg}$

$\begin{array}{ll}1 & \text { BI212 } \\ 1 & \text { BI214 } \\ 1 & \text { CS137 } \\ 1 & \text { K40 } \\ 1 & \text { PB212 } \\ 1 & \text { PB214 } \\ 1 & \text { PU239 } \\ 1 & \text { RA226 } \\ 1 & \text { TH228 } \\ 1 & \text { TH234 } \\ 1 & \text { TL208 } \\ 1 & \text { U234 } \\ 1 & \text { U238 }\end{array}$

45.000

24.000

923.200

304.000

49.800

27.400

12.200

35.000

55.000

98.000

17.000

96.000

100.000

$$
\begin{array}{r}
8.000 \\
1.500 \\
4.400 \\
12.000 \\
1.500 \\
1.700 \\
2.200 \\
1.500 \\
8.000 \\
10.000 \\
0.900 \\
12.000 \\
11.000
\end{array}
$$

$$
\begin{array}{r}
58.300 \\
28.800 \\
954.000 \\
314.000 \\
52.800 \\
29.100 \\
13.090 \\
29.000 \\
52.700 \\
114.000 \\
18.300 \\
113.000 \\
120.000
\end{array}
$$

$$
\begin{array}{r}
5.900 \\
0.500 \\
38.000 \\
13.000 \\
3.700 \\
1.200 \\
0.570 \\
1.000 \\
4.000 \\
6.000 \\
1.100 \\
6.000 \\
9.000
\end{array}
$$

0.772
0.833
0.968
0.968
0.943
0.942
0.932
1.207
1.044
0.860
0.929
0.850
0.833

$\begin{array}{ll}\text { A } & \\ \text { A } & \\ \text { A } & \text { A } \\ \text { A } & \text { A } \\ \text { A } & \\ \text { A } & \\ \text { A } & \text { A } \\ \text { A } & \\ \text { A } & \\ \text { A } & \\ \text { A } & \\ \text { A } & \text { A } \\ \text { A } & \text { A }\end{array}$

Matrix: VE Vegetation $\mathrm{Bq} / \mathrm{kg}$

\section{$1 \quad \mathrm{CO} 60$ \\ $1 \quad$ CS137 \\ $1 \mathrm{~K} 40$ \\ 1 PU238 \\ 1 PU239}

I SR90

\begin{abstract}
22.200
486.600

544.000

0.510

4.610

627.000
\end{abstract}

0.700

3.500

17.000

0.100

0.330

12.000

$\begin{array}{rr}20.000 & 1.000 \\ 390.000 & 20.000 \\ 460.000 & 20.000 \\ 0.310 & 0.070 \\ 3.720 & 0.270 \\ 606.000 & 40.000\end{array}$

1.110
1.248
1.183
1.645
1.239
1.035

$\begin{array}{ll}\text { A } & \text { W } \\ \text { A } & \text { A } \\ \text { A } & \text { A } \\ \text { A } & \\ \text { W } & \text { A } \\ \text { A } & \text { A }\end{array}$

Matrix: WA Water $\mathrm{Bq} / \mathrm{L}$

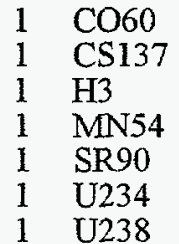

$\begin{array}{ll}1 & \text { CO60 } \\ 1 & \text { CS137 } \\ 1 & \text { H3 } \\ 1 & \text { MN54 } \\ 1 & \text { SR90 } \\ 1 & \text { U234 } \\ 1 & \text { U238 }\end{array}$

$$
\begin{array}{r}
49.800 \\
52.600 \\
79.200 \\
35.300 \\
1.200 \\
0.074 \\
0.060
\end{array}
$$

$\begin{array}{lr}0.600 & 49.400 \\ 0.800 & 50.000 \\ 3.000 & 76.200 \\ 0.700 & 32.400 \\ 0.600 & 2.110 \\ 0.010 & 0.510 \\ 0.009 & 0.520\end{array}$

1.008

1.052

1.039

1.090

0.569

0.145

0.115
$\mathrm{A}$
$\mathrm{A}$
$\mathrm{A}$
$\mathrm{A}$
$\mathrm{N}$
$\mathrm{N}$
$\mathrm{N}$

A
A
W
A
A
A
A
A

$\mathrm{A}$
$\mathrm{A}$
$\mathrm{A}$




\section{QAP 49 Results by Laboratory}

Lab: ND Dept. of Environmental Health and Safety, NC State University

\begin{tabular}{lccccc} 
No. & Reported \\
Test & Radionuclide & $\begin{array}{c}\text { Reported } \\
\text { Value }\end{array}$ & $\begin{array}{c}\text { EML } \\
\text { Value }\end{array}$ & $\begin{array}{c}\text { EML } \\
\text { Error }\end{array}$ & $\begin{array}{c}\text { Reported } \\
\text { EML }\end{array}$ Evaluation Evaluation \\
\hline
\end{tabular}

Matrix: AI Air Filter Bq / filter

$\begin{array}{llrrrrrr}1 & \text { CO60 } & 8.813 & 0.228 & 9.160 & 0.580 & 0.962 & \mathrm{~A} \\ 1 & \text { CS137 } & 21.074 & 0.848 & 22.470 & 1.030 & 0.938 & \mathrm{~A} \\ 1 & \text { GROSS ALPHA } & 1.620 & 0.133 & 1.650 & 0.160 & 0.982 & \mathrm{~A} \\ 1 & \text { GROSS BETA } & 2.133 & 0.126 & 2.160 & 0.070 & 0.987 & \mathrm{~A} \\ 1 & \text { MN54 } & 4.866 & 0.247 & 4.920 & 0.400 & 0.989 & \mathrm{~A} \\ 1 & \text { SB125 } & 7.239 & 0.290 & 8.890 & 0.550 & 0.814 & \text { W }\end{array}$

Matrix: WA Water $\mathrm{Bq} / \mathrm{L}$

$\begin{array}{llllllll}1 & \text { CO60 } & 49.586 & 1.775 & 49.400 & 1.200 & 1.004 & \mathrm{~A} \\ 1 & \text { CS137 } & 47.630 & 2.489 & 50.000 & 1.700 & 0.953 & \mathrm{~A} \\ 1 & \text { MN54 } & 34.701 & 2.083 & 32.400 & 1.400 & 1.071 & \mathrm{~A}\end{array}$

Values for elemental uranium are reported in $\mu \mathrm{g} /$ filter, $\mathrm{g}$, or $\mathrm{mL}$.

$\mathrm{pCi} / \mathrm{g}$ or $\mathrm{mL}=\mathrm{Bq} \times 0.027$

Evaluation: $A=$ Acceptable, $W=$ Acceptable with Warning, $N=$ Not Acceptable

If the evaluation system is not appropriate for the types of analyses performed in your lab, apply a site specific evaluation. 


\section{QAP 49 Results by Laboratory}

Lab: NL Fluor Daniel Fernald, Inc., Ohio

\begin{tabular}{|c|c|c|c|c|c|c|c|}
\hline $\begin{array}{l}0 . \\
\text { est }\end{array}$ & Radionuclide & $\begin{array}{c}\text { Reported } \\
\text { Value }\end{array}$ & $\begin{array}{c}\text { Reported } \\
\text { Error }\end{array}$ & $\begin{array}{l}\text { EML } \\
\text { Value }\end{array}$ & $\begin{array}{l}\text { EML } \\
\text { Error }\end{array}$ & $\frac{\text { Reported }}{\text { EML }}$ & $\begin{array}{c}\text { QAP 48 } \\
\text { Evaluation }\end{array}$ \\
\hline
\end{tabular}

Matrix: AI Air Filter Bq / filter

$\begin{array}{llrrrrrrr}1 & \text { Bq U } & 0.538 & 0.063 & 0.530 & 0.020 & 1.015 & \mathrm{~A} & \\ 1 & \text { CO60 } & 9.300 & 1.560 & 9.160 & 0.580 & 1.015 & \mathrm{~A} & \mathrm{~A} \\ 1 & \text { CS137 } & 24.000 & 5.900 & 22.470 & 1.030 & 1.068 & \mathrm{~A} & \mathrm{~A} \\ 1 & \text { MN54 } & 5.060 & 1.610 & 4.920 & 0.400 & 1.028 & \mathrm{~A} & \mathrm{~A} \\ 1 & \text { PU238 } & 0.472 & 0.108 & 0.460 & 0.005 & 1.026 & \mathrm{~A} & \mathrm{~W} \\ 1 & \text { PU239 } & 0.447 & 0.101 & 0.420 & 0.006 & 1.064 & \mathrm{~A} & \mathrm{~A} \\ 1 & \text { SB125 } & 10.600 & 1.400 & 8.890 & 0.550 & 1.192 & \mathrm{~W} & \mathrm{~A} \\ 1 & \text { U234 } & 0.266 & 0.063 & 0.260 & 0.010 & 1.023 & \mathrm{~A} & \mathrm{~A} \\ 1 & \text { U238 } & 0.272 & 0.064 & 0.260 & 0.010 & 1.046 & \mathrm{~A} & \mathrm{~W}\end{array}$

Matrix: SO Soil $\mathrm{Bq} / \mathrm{kg}$

$\begin{array}{ll}1 & \text { AC228 } \\ 1 & \text { BI212 } \\ 1 & \text { BI214 } \\ 1 & \text { CS137 } \\ 1 & \text { K40 } \\ 1 & \text { PB212 } \\ 1 & \text { PB214 } \\ 1 & \text { PU239 } \\ 1 & \text { TH228 } \\ 1 & \text { TH234 } \\ 1 & \text { TL208 } \\ 1 & \text { U234 } \\ 1 & \text { U238 } \\ 1 & \text { ug U }\end{array}$

$\begin{array}{rrr}56.400 & 10.500 & 52.600 \\ 55.200 & 9.500 & 58.300 \\ 31.100 & 7.100 & 28.800 \\ 1010.000 & 181.000 & 954.000 \\ 332.000 & 52.000 & 314.000 \\ 55.200 & 9.500 & 52.800 \\ 31.500 & 7.200 & 29.100 \\ 13.560 & 3.160 & 13.090 \\ 55.200 & 9.500 & 52.700 \\ 95.600 & 37.200 & 114.000 \\ 53.600 & 10.800 & 18.300 \\ 113.000 & 27.000 & 113.000 \\ 117.000 & 28.000 & 120.000 \\ 9.370 & 2.220 & 9.700\end{array}$

2.900
5.900
0.500
38.000
13.000
3.700
1.200
0.570
4.000
6.000
1.100
6.000
9.000
0.700

1.072

0.947

1.080

1.059

1.057

1.045

1.082

1.036

1.047

0.839

2.929

1.000

0.975

0.966

$\begin{array}{ll}\text { A } & \\ \text { A } & \\ \text { A } & \\ \text { A } & \text { A } \\ \text { A } & \text { A } \\ \text { A } & \\ \text { A } & \\ \text { A } & \text { W } \\ \text { A } & \\ \text { A } & \\ \text { A } & \\ \text { A } & \text { A } \\ \text { A } & \text { A } \\ \text { A } & \end{array}$

Matrix: WA Water $\mathrm{Bq} / \mathrm{L}$

$\begin{array}{llrrrrrrr}1 & \text { Bq U } & 1.210 & 0.120 & 1.050 & 0.080 & 1.152 & \mathrm{~A} & \\ 1 & \text { CO60 } & 50.100 & 6.100 & 49.400 & 1.200 & 1.014 & \mathrm{~A} & \mathrm{~W} \\ 1 & \text { CS137 } & 50.600 & 8.500 & 50.000 & 1.700 & 1.012 & \mathrm{~A} & \mathrm{~W} \\ 1 & \text { MN54 } & 36.000 & 5.300 & 32.400 & 1.400 & 1.111 & \mathrm{~A} & \mathrm{~W} \\ 1 & \text { PU238 } & 1.160 & 0.260 & 1.100 & 0.010 & 1.055 & \mathrm{~A} & \mathrm{~W} \\ 1 & \text { PU239 } & 1.540 & 0.350 & 1.410 & 0.040 & 1.092 & \mathrm{~A} & \mathrm{~A} \\ 1 & \text { U234 } & 0.502 & 0.120 & 0.510 & 0.030 & 0.984 & \mathrm{~A} & \mathrm{~A} \\ 1 & \text { U238 } & 0.521 & 0.120 & 0.520 & 0.050 & 1.002 & \mathrm{~A} & \mathrm{~A}\end{array}$

\section{Values for elemental uranium are reported in $\mu \mathrm{g} /$ filter, $\mathrm{g}$, or $\mathrm{mL}$. \\ $\mathrm{pCi} / \mathrm{g}$ or $\mathrm{mL}=\mathrm{Bq} \times 0.027$}

Evaluation: $A=A c c e p t a b l e, W=A c c e p t a b l e$ with Warning, $N=$ Not Acceptable

If the evaluation system is not appropriate for the types of analyses performed in your lab, apply a site specific evaluation. 


\section{QAP 49 Results by Laboratory}

Lab: NM Environmental Evaluation Group, Carlsbad, NM

\begin{tabular}{|c|c|c|c|c|c|c|c|}
\hline est & Radionuclide & $\begin{array}{l}\text { Reported } \\
\text { Value }\end{array}$ & $\begin{array}{c}\text { Reported } \\
\text { Error }\end{array}$ & $\begin{array}{l}\text { EML } \\
\text { Value }\end{array}$ & $\begin{array}{l}\text { EML } \\
\text { Error }\end{array}$ & $\frac{\text { Reported }}{\text { EML }}$ & $\begin{array}{cc}\text { QAP 48 } \\
\text { Evaluation } \\
\text { Evaluation }\end{array}$ \\
\hline
\end{tabular}

Matrix: AI Air Filter Bq/filter

$\begin{array}{llrrrrrrr}1 & \text { AM241 } & 0.478 & 0.013 & 0.510 & 0.008 & 0.937 & \text { A } & \text { A } \\ 1 & \text { CS137 } & 17.510 & 1.300 & 22.470 & 1.030 & 0.779 & \text { W } & \text { A } \\ 1 & \text { PU238 } & 0.482 & 0.010 & 0.460 & 0.005 & 1.048 & \text { A } & \text { A } \\ 1 & \text { PU239 } & 0.433 & 0.009 & 0.420 & 0.006 & 1.031 & \text { A } & \text { A } \\ 1 & \text { SR90 } & 1.004 & 0.140 & 1.120 & 0.050 & 0.896 & \text { A } & \end{array}$

Matrix: SO Soil $\mathrm{Bq} / \mathrm{kg}$

$\begin{array}{ll}2 & \text { AM241 } \\ 3 & \text { AM241 } \\ 1 & \text { AM241 } \\ 1 & \text { CS137 } \\ 2 & \text { PU239 } \\ 1 & \text { PU239 } \\ 3 & \text { PU239 }\end{array}$

7.840
9.120
7.010
903.000
13.300
10.300
12.900

$$
\begin{array}{r}
0.380 \\
0.410 \\
0.350 \\
46.000 \\
0.400 \\
0.300 \\
0.400
\end{array}
$$

0.410
0.410
0.410
38.000
0.570
0.570
0.570

1.050

1.221

0.938

0.947

1.016

0.787

13.090

0.985

$\begin{array}{ll}\text { A } & \text { A } \\ \text { A } & \text { A } \\ \text { A } & \text { A } \\ \text { A } & \text { A } \\ \text { A } & \text { A } \\ \text { W } & \text { A } \\ \text { A } & \text { A }\end{array}$

Matrix: WA Water $\mathrm{Bq} / \mathrm{L}$

$\begin{array}{llrrrrrrr}1 & \text { AM241 } & 1.250 & 0.033 & 1.250 & 0.080 & 1.000 & \mathrm{~A} & \mathrm{~A} \\ 1 & \text { CS137 } & 54.460 & 2.800 & 50.000 & 1.700 & 1.089 & \mathrm{~A} & \mathrm{~W} \\ 1 & \text { PU238 } & 1.111 & 0.026 & 1.100 & 0.010 & 1.010 & \mathrm{~A} & \mathrm{~A} \\ 1 & \text { PU239 } & 1.458 & 0.033 & 1.410 & 0.040 & 1.034 & \mathrm{~A} & \mathrm{~A} \\ 1 & \text { SR90 } & 1.759 & 0.254 & 2.110 & 0.180 & 0.834 & \text { W } & \end{array}$

\footnotetext{
Values for elemental uranium are reported in $\mu \mathrm{g} / \mathrm{filter}, \mathrm{g}$, or $\mathrm{mL}$.
}

$\mathrm{pCi} / \mathrm{g}$ or $\mathrm{mL}=\mathrm{Bq} \times 0.027$

Evaluation: $A=A c c e p t a b l e, W=A c c e p t a b l e$ with Warning, $N=$ Not Acceptable

If the evaluation system is not appropriate for the types of analyses performed in your lab, apply a site specific evaluation. 


\section{QAP 49 Results by Laboratory}

Lab: NP JAF Environmental Laboratory, New York Power Authority

\begin{tabular}{|c|c|c|c|c|c|c|c|}
\hline $\begin{array}{l}\text { No. } \\
\text { Test }\end{array}$ & adionuclide & $\begin{array}{l}\text { Reported } \\
\text { Value }\end{array}$ & $\begin{array}{l}\text { Reported } \\
\text { Error }\end{array}$ & $\begin{array}{l}\text { EML } \\
\text { Value }\end{array}$ & $\begin{array}{l}\text { EML } \\
\text { Error }\end{array}$ & $\frac{\text { Reporte }}{\text { EML }}$ & 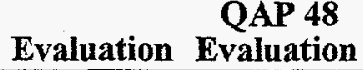 \\
\hline
\end{tabular}

Matrix: $\mathrm{AI}$ Air Filter $\mathrm{Bq} /$ filter

$\begin{array}{llrrrrrrr}1 & \text { CO60 } & 9.070 & 0.070 & 9.160 & 0.580 & 0.990 & \mathrm{~A} & \mathrm{~A} \\ 1 & \text { CS137 } & 22.000 & 0.100 & 22.470 & 1.030 & 0.979 & \mathrm{~A} & \mathrm{~A} \\ 1 & \text { GROSS BETA } & 1.990 & 0.020 & 2.160 & 0.070 & 0.921 & \mathrm{~A} & \mathrm{~A} \\ 1 & \text { MN54 } & 5.370 & 0.070 & 4.920 & 0.400 & 1.091 & \mathrm{~A} & \text { W } \\ 1 & \text { SB125 } & 9.300 & 0.100 & 8.890 & 0.550 & 1.046 & \mathrm{~A} & \mathrm{~A}\end{array}$

Matrix: SO Soil $\mathrm{Bq} / \mathrm{kg}$

$\begin{array}{lllllllll}1 & \text { CS137 } & 653.000 & 1.000 & 954.000 & 38.000 & 0.684 & \text { N } & \text { W } \\ 1 & \mathrm{~K} 40 & 259.000 & 4.000 & 314.000 & 13.000 & 0.825 & \text { W } & \end{array}$

Matrix: VE Vegetation $\mathrm{Bq} / \mathrm{kg}$

$\begin{array}{llrrrrrrr}1 & \text { CO60 } & 17.500 & 0.600 & 20.000 & 1.000 & 0.875 & \mathrm{~A} & \mathrm{~A} \\ 1 & \mathrm{CS} 137 & 334.000 & 2.000 & 390.000 & 20.000 & 0.856 & \mathrm{~W} & \mathrm{~W} \\ 1 & \mathrm{~K} 40 & 462.000 & 12.000 & 460.000 & 20.000 & 1.004 & \mathrm{~A} & \end{array}$

Matrix: WA Water $\mathrm{Bq} / \mathrm{L}$

$\begin{array}{llrrrrrrr}1 & \text { CO60 } & 52.200 & 1.000 & 49.400 & 1.200 & 1.057 & \mathrm{~A} & \mathrm{~W} \\ 1 & \text { CS137 } & 48.800 & 1.100 & 50.000 & 1.700 & 0.976 & \mathrm{~A} & \mathrm{~A} \\ 1 & \text { GROSS BETA } & 1225.000 & 5.000 & 1420.000 & 60.000 & 0.863 & \mathrm{~A} & \mathrm{~A} \\ 1 & \text { H3 } & 444.000 & 37.000 & 76.200 & 2.900 & 5.827 & \mathrm{~N} & \mathrm{~W} \\ 1 & \text { MN54 } & 32.900 & 1.000 & 32.400 & 1.400 & 1.015 & \mathrm{~A} & \mathrm{~A}\end{array}$

Values for elemental uranium are reported in $\mu \mathrm{g} /$ filter, $\mathrm{g}$, or $\mathrm{mL}$.

$\mathrm{pCi} / \mathrm{g}$ or $\mathrm{mL}=\mathrm{Bq} \times 0.027$

Evaluation: A=Acceptable, $W=$ Acceptable with Warming, $N=$ Not Acceptable

If the evaluation system is not appropriate for the types of analyses performed in your lab, apply a site specific evaluation. 


\section{QAP 49 Results by Laboratory}

Lab: NQ New Mexico Department of Health, Albuquerque

\begin{tabular}{|c|c|c|}
\hline $\begin{array}{l}\text { No. } \\
\text { Test Radionuclide }\end{array}$ & $\begin{array}{c}\text { Reported } \\
\text { Value }\end{array}$ & $\begin{array}{c}\text { Reported } \\
\text { Error }\end{array}$ \\
\hline
\end{tabular}

Matrix: $\mathrm{AI}$ Air Filter $\mathrm{Bq} /$ filter

$\begin{array}{llllllll}1 & \text { AM241 } & 0.444 & 0.027 & 0.510 & 0.008 & 0.871 & \text { W } \\ 1 & \text { GROSS ALPHA } & 1.440 & 0.220 & 1.650 & 0.160 & 0.873 & \mathrm{~A} \\ 1 & \text { GROSS BETA } & 1.860 & 0.280 & 2.160 & 0.070 & 0.861 & \text { W } \\ 1 & \text { PU238 } & 0.405 & 0.024 & 0.460 & 0.005 & 0.880 & \text { W } \\ 1 & \text { PU239 } & 0.380 & 0.022 & 0.420 & 0.006 & 0.905 & \mathrm{~A} \\ 1 & \text { U234 } & 0.261 & 0.015 & 0.260 & 0.010 & 1.004 & \mathrm{~A} \\ 1 & \text { U238 } & 0.259 & 0.015 & 0.260 & 0.010 & 0.996 & \mathrm{~A}\end{array}$

Matrix: SO Soil Bq/ $\mathrm{kg}$

$\begin{array}{ll}1 & \text { AC228 } \\ 1 & \text { AM241 } \\ 1 & \text { BI212 } \\ 1 & \text { BI214 } \\ 1 & \text { CS137 } \\ 1 & \text { K40 } \\ 1 & \text { PB212 } \\ 1 & \text { PB214 } \\ 1 & \text { PU239 } \\ 1 & \text { RA226 } \\ 1 & \text { TH234 } \\ 1 & \text { TL208 } \\ 1 & \text { U234 } \\ 1 & \text { U238 }\end{array}$

63.300
8.400
68.500
33.000
1190.000
410.000
68.100
37.800
11.300
33.900
125.000
20.700
110.700
111.900

$\begin{array}{rr}6.700 & 52.600 \\ 1.500 & 7.470 \\ 9.600 & 58.300 \\ 3.700 & 28.800 \\ 140.000 & 954.000 \\ 47.000 & 314.000 \\ 7.800 & 52.800 \\ 4.800 & 29.100 \\ 1.300 & 13.090 \\ 1.400 & 29.000 \\ 16.000 & 114.000 \\ 2.600 & 18.300 \\ 6.400 & 113.000 \\ 6.400 & 120.000\end{array}$

\subsection{0}

0.410

5.900

0.500

38.000

13.000

3.700

1.200

0.570

1.000

6.000

1.100

6.000

9.000

$\begin{array}{ll}1.203 & \mathrm{~A} \\ 1.124 & \mathrm{~A} \\ 1.175 & \mathrm{~A} \\ 1.146 & \mathrm{~A} \\ 1.247 & \mathrm{~W} \\ 1.306 & \mathrm{~W} \\ 1.290 & \mathrm{~A} \\ 1.299 & \mathrm{~A} \\ 0.863 & \mathrm{~W} \\ 1.169 & \mathrm{~A} \\ 1.096 & \mathrm{~A} \\ 1.131 & \mathrm{~A} \\ 0.980 & \mathrm{~A} \\ 0.933 & \mathrm{~A}\end{array}$

Matrix: WA Water $\mathrm{Bq} / \mathrm{L}$

$\begin{array}{llr}1 & \text { AM241 } & 1.100 \\ 1 & \text { CO60 } & 60.400 \\ 1 & \text { CS137 } & 61.000 \\ 1 & \text { GROSS ALPHA } & 1273.000 \\ 1 & \text { GROSS BETA } & 1357.000 \\ 1 & \text { MN54 } & 41.900 \\ 1 & \text { PU238 } & 1.120 \\ 1 & \text { PU239 } & 1.430 \\ 1 & \text { U234 } & 0.552 \\ 1 & \text { U238 } & 0.539\end{array}$

$$
\begin{array}{r}
0.090 \\
6.600 \\
6.900 \\
116.000 \\
115.000 \\
4.900 \\
0.070 \\
0.090 \\
0.036 \\
0.035
\end{array}
$$

1.250

49.400

50.000

1080.000

1420.000

32.400

1.100
0.080
1.200
1.700
60.000
60.000
1.400
0.010
0.040
0.030
0.050

1.410

0.510

$\begin{array}{ll}0.880 & \mathrm{~W} \\ 1.223 & \mathrm{~N} \\ 1.220 & \mathrm{~W} \\ 1.179 & \mathrm{~W} \\ 0.956 & \mathrm{~A} \\ 1.293 & \mathrm{~N} \\ 1.018 & \mathrm{~A} \\ 1.014 & \mathrm{~A} \\ 1.082 & \mathrm{~A} \\ 1.037 & \mathrm{~A}\end{array}$




\section{QAP 49 Results by Laboratory}

Lab: NR Naval Reactors Facility Chemistry, Scoville, ID

No.

Reported Reported

EML

EML

Reported

QAP 48

Test Radionuclide Value

Error

Value

Error

EML

Evaluation Evaluation

Matrix: SO Soil $\mathrm{Bq} / \mathrm{kg}$

$\begin{array}{llrrrrrrr}1 & \text { CS137 } & 1003.000 & 201.000 & 954.000 & 38.000 & 1.051 & \mathrm{~A} & \mathrm{~A} \\ 1 & \mathrm{~K} 40 & 317.000 & 63.000 & 314.000 & 13.000 & 1.010 & \mathrm{~A} & \mathrm{~A}\end{array}$

Matrix: $\mathrm{VE}$ Vegetation $\mathrm{Bq} / \mathrm{kg}$

$\begin{array}{llrrrrrrr}1 & \text { CO60 } & 19.800 & 4.000 & 20.000 & 1.000 & 0.990 & \text { A } & \text { A } \\ 1 & \text { CS137 } & 414.000 & 83.000 & 390.000 & 20.000 & 1.062 & \text { A } & \text { A } \\ 1 & \text { K40 } & 448.000 & 90.000 & 460.000 & 20.000 & 0.974 & \text { A } & \text { A }\end{array}$

Values for elemental uranium are reported in $\mu \mathrm{g} /$ filter, $\mathrm{g}$, or $\mathrm{mL}$.

$\mathrm{pCi} / \mathrm{g}$ or $\mathrm{mL}=\mathrm{Bq} \times 0.027$

Evaluation: A=Acceptable, $W=$ Acceptable with Warning, $N=$ Not Acceptable

If the evaluation system is not appropriate for the types of analyses performed in your lab, apply a site specific evaluation. 


\section{QAP 49 Results by Laboratory}

Lab: NS State Lab of Public Health, North Carolina

\begin{tabular}{|c|c|c|c|c|c|c|c|}
\hline $\begin{array}{l}0, \\
\text { est }\end{array}$ & Radionuclide & $\begin{array}{l}\text { Reported } \\
\text { Value }\end{array}$ & $\begin{array}{l}\text { Reported } \\
\text { Error }\end{array}$ & $\begin{array}{l}\text { EML } \\
\text { Value }\end{array}$ & $\begin{array}{l}\text { EML } \\
\text { Error }\end{array}$ & $\frac{\text { Reported }}{\text { EML }}$ & $\begin{array}{cc}\text { QAP 48 } \\
\text { Evaluation } & \text { Evaluation }\end{array}$ \\
\hline
\end{tabular}

Matrix: AI Air Filter Bq / filter

$1 \mathrm{CO} 60$

$1 \quad \operatorname{CS} 137$

1 GROSS ALPHA

1 GROSS BETA

I MN54

1 SB125
9.136

22.358

3.066

2.237

5.444

8.963
0.100

0.107

0.162

0.085

0.067

0.126
9.160

22.470

1.650

2.160

4.920

8.890
0.580

1.030

0.160

0.070

0.400

0.550
0.997

0.995

1.858

1.036

1.107

1.008 $\begin{array}{ll}\text { A } & \text { W } \\ \text { A } & \text { A } \\ \text { N } & \text { W }\end{array}$

A A

A $A$

A $\quad$ A

Matrix: WA Water $\mathrm{Bq} / \mathrm{L}$

$1 \mathrm{~Bq} \mathrm{U}$

$1 \quad \mathrm{CO} 60$

1 CS137

1 GROSS ALPHA

1 GROSS BETA

$1 \mathrm{H} 3$

1 MN54
0.767

53.469

53.333

1081.317

1330.600

80.918

37.741
0.227

0.593

0.518

57.279

45,863

6.412

0.481
1.050

49.400

50.000

1080.000

1420.000

76.200

32.400
0.080
1.200
1.700
60.000
60.000
2.900
1.400

0.730

1.082

1.067

1.001

0.937

1.062

$* * * * * * * *$
W
A
A
A
A
A
N

W

A

A

A

\section{Values for elemental uranium are reported in $\mu \mathrm{g} /$ filter, $g$, or $\mathrm{mL}$.}

$\mathrm{pCi} / \mathrm{g}$ or $\mathrm{mL}=\mathrm{Bq} \times 0.027$

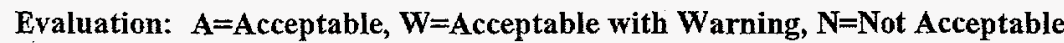

If the evaluation system is not appropriate for the types of analyses performed in your lab, apply a site specific evaluation. 


\section{QAP 49 Results by Laboratory}

Lab: NZ National Radiation Laboratory, New Zealand

\begin{tabular}{|c|c|c|c|c|c|c|c|}
\hline $\begin{array}{l}\text { o. } \\
\text { est }\end{array}$ & Radiomuclide & $\begin{array}{l}\text { Reported } \\
\text { Value }\end{array}$ & $\begin{array}{l}\text { Reported } \\
\text { Error }\end{array}$ & $\begin{array}{l}\text { EML } \\
\text { Value }\end{array}$ & $\begin{array}{l}\text { EML } \\
\text { Error }\end{array}$ & $\frac{\text { Reported }}{\text { EML }}$ & $\begin{array}{c}\text { QAP 48 } \\
\text { Evaluation } \\
\text { Evaluation }\end{array}$ \\
\hline
\end{tabular}

Matrix: AI Air Filter $\mathrm{Bq} /$ filter

\begin{tabular}{|c|c|c|c|c|c|}
\hline $\mathrm{CO} 60$ & 9.100 & 0.500 & 9.160 & 0.580 & 0.993 \\
\hline $\mathrm{CO} 60$ & 9.100 & 0.500 & 9.160 & 0.580 & 0.993 \\
\hline $\operatorname{CS} 137$ & 24.100 & 1.200 & 22.470 & 1.030 & 1.073 \\
\hline $\mathrm{CS} 137$ & 24.300 & 1.200 & 22.470 & 1.030 & 1.081 \\
\hline GROSS ALPHA & 1.400 & 0.100 & 1.650 & 0.160 & 0.848 \\
\hline GROSS ALPHA & 1.500 & 0.100 & 1.650 & 0.160 & 0.909 \\
\hline GROSS BETA & 1.900 & 0.100 & 2.160 & 0.070 & 0.880 \\
\hline GROSS BETA & 1.900 & 0.100 & 2.160 & 0.070 & 0.880 \\
\hline MN54 & 5.600 & 0.300 & 4.920 & 0.400 & 1.138 \\
\hline MN54 & 5.700 & 0.300 & 4.920 & 0.400 & 1.159 \\
\hline PU238 & 0.610 & 0.020 & 0.460 & 0.005 & 1.326 \\
\hline PU238 & 0.590 & 0.020 & 0.460 & 0.005 & 1.283 \\
\hline PU239 & 0.570 & 0.020 & 0.420 & 0.006 & 1.357 \\
\hline PU239 & 0.550 & 0.020 & 0.420 & 0.006 & 1.310 \\
\hline SB125 & 10.200 & 0.500 & 8.890 & 0.550 & 1.147 \\
\hline SB125 & 10.300 & 0.500 & 8.890 & 0.550 & 1.159 \\
\hline SR90 & 0.400 & 0.030 & 1.120 & 0.050 & 0.357 \\
\hline SR90 & 0.500 & 0.040 & 1.120 & 0.050 & 0.446 \\
\hline U234 & 0.260 & 0.020 & 0.260 & 0.010 & 1.000 \\
\hline U234 & 0.280 & 0.050 & 0.260 & 0.010 & 1.077 \\
\hline $\mathrm{U} 238$ & 0.330 & 0.050 & 0.260 & 0.010 & 1.269 \\
\hline $\mathrm{U} 238$ & 0.280 & 0.020 & 0.260 & 0.010 & 1.077 \\
\hline
\end{tabular}

Matrix: SO Soil $\mathrm{Bq} / \mathrm{kg}$

$\begin{array}{llrrrrrr}1 & \text { CS137 } & 1046.000 & 54.000 & 954.000 & 38.000 & 1.096 & \mathrm{~A} \\ 2 & \text { CS137 } & 1024.000 & 53.000 & 954.000 & 38.000 & 1.073 & \mathrm{~A} \\ 2 & \text { PU239 } & 12.700 & 1.100 & 13.090 & 0.570 & 0.970 & \mathrm{~A} \\ 1 & \text { PU239 } & 14.400 & 1.400 & 13.090 & 0.570 & 1.100 & \mathrm{~A} \\ 2 & \text { SR90 } & 40.900 & 3.500 & 39.630 & 0.003 & 1.032 & \mathrm{~A} \\ 1 & \text { SR90 } & 41.500 & 3.600 & 39.630 & 0.003 & 1.047 & \mathrm{~A} \\ 1 & \text { U234 } & 106.000 & 4.000 & 113.000 & 6.000 & 0.938 & \mathrm{~A} \\ 2 & \text { U234 } & 108.000 & 3.000 & 113.000 & 6.000 & 0.956 & \mathrm{~A} \\ 1 & \text { U238 } & 115.000 & 4.000 & 120.000 & 9.000 & 0.958 & \mathrm{~A} \\ 2 & \text { U238 } & 115.000 & 3.000 & 120.000 & 9.000 & 0.958 & \mathrm{~A}\end{array}$

Matrix: VE Vegetation $\mathrm{Bq} / \mathrm{kg}$

$\begin{array}{llrrrrrr}1 & \text { CO60 } & 20.800 & 1.200 & 20.000 & 1.000 & 1.040 & \mathrm{~A} \\ 1 & \text { CS137 } & 426.000 & 22.000 & 390.000 & 20.000 & 1.092 & \mathrm{~A} \\ 2 & \text { PU239 } & 4.300 & 0.300 & 3.720 & 0.270 & 1.156 & \mathrm{~A} \\ 1 & \text { PU239 } & 3.900 & 0.300 & 3.720 & 0.270 & 1.048 & \mathrm{~A} \\ 1 & \text { SR90 } & 577.000 & 30.000 & 606.000 & 40.000 & 0.952 & \mathrm{~A} \\ 2 & \text { SR90 } & 487.000 & 24.000 & 606.000 & 40.000 & 0.804 & \mathrm{~A}\end{array}$

Values for elemental uranium are reported in $\mu \mathrm{g} /$ filter, $\mathrm{g}$, or $\mathrm{mL} . \quad \mathrm{pCi} / \mathrm{g}$ or $\mathrm{mL}=\mathrm{Bq} \times 0.027$

Evaluation: $A=$ Acceptable, $W=$ Acceptable with Warning, $N=$ Not Acceptable

If the evaluation system is not appropriate for the types of analyses performed in your lab, apply a site specific evaluation. 


\section{QAP 49 Results by Laboratory}

Lab: NZ National Radiation Laboratory, New Zealand

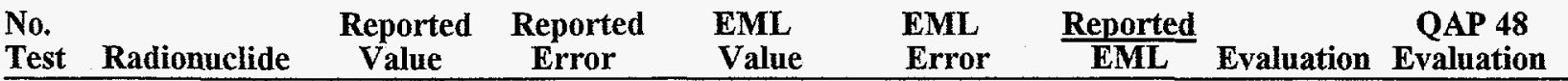

Matrix: WA Water $\mathrm{Bq} / \mathrm{L}$

$\begin{array}{llrrrrrr}1 & \text { CO60 } & 50.000 & 3.000 & 49.400 & 1.200 & 1.012 & \mathrm{~A} \\ 2 & \text { CO60 } & 48.000 & 3.000 & 49.400 & 1.200 & 0.972 & \mathrm{~A} \\ 2 & \text { CS137 } & 50.000 & 3.000 & 50.000 & 1.700 & 1.000 & \mathrm{~A} \\ 1 & \text { CS137 } & 53.000 & 3.000 & 50.000 & 1.700 & 1.060 & \mathrm{~A} \\ 1 & \text { GROSS ALPHA } & 684.000 & 39.000 & 1080.000 & 60.000 & 0.633 & \mathrm{~W} \\ 2 & \text { GROSS ALPHA } & 693.000 & 39.000 & 1080.000 & 60.000 & 0.642 & \mathrm{~W} \\ 1 & \text { GROSS BETA } & 1029.000 & 40.000 & 1420.000 & 60.000 & 0.725 & \mathrm{~A} \\ 2 & \text { GROSS BETA } & 1050.000 & 41.000 & 1420.000 & 60.000 & 0.739 & \mathrm{~A} \\ 1 & \text { MN54 } & 36.000 & 2.000 & 32.400 & 1.400 & 1.111 & \mathrm{~A} \\ 2 & \text { MN54 } & 35.000 & 2.000 & 32.400 & 1.400 & 1.080 & \mathrm{~A} \\ 1 & \text { PU238 } & 1.890 & 0.080 & 1.100 & 0.010 & 1.718 & \mathrm{~N} \\ 2 & \text { PU238 } & 1.110 & 0.040 & 1.100 & 0.010 & 1.009 & \mathrm{~A} \\ 1 & \text { PU239 } & 2.530 & 0.100 & 1.410 & 0.040 & 1.794 & \mathrm{~N} \\ 2 & \text { PU239 } & 1.510 & 0.050 & 1.410 & 0.040 & 1.071 & \mathrm{~A} \\ 1 & \text { SR90 } & 3.400 & 0.100 & 2.110 & 0.180 & 1.611 & \mathrm{~N} \\ 2 & \text { SR90 } & 4.000 & 0.100 & 2.110 & 0.180 & 1.896 & \mathrm{~N} \\ 1 & \text { U234 } & 0.570 & 0.050 & 0.510 & 0.030 & 1.118 & \mathrm{~A} \\ 2 & \text { U234 } & 0.460 & 0.040 & 0.510 & 0.030 & 0.902 & \mathrm{~A} \\ 2 & \text { U238 } & 0.520 & 0.040 & 0.520 & 0.050 & 1.000 & \mathrm{~A} \\ 1 & \text { U238 } & 0.560 & 0.050 & 0.520 & 0.050 & 1.077 & \mathrm{~A}\end{array}$

\section{Values for elemental uranium are reported in $\mu \mathrm{g} /$ filter, $\mathrm{g}$, or $\mathrm{mL} . \quad \mathrm{pCi} / \mathrm{g}$ or $\mathrm{mL}=\mathrm{Bq} \times 0.027$}

Evaluation: $A=$ Acceptable, $W=$ Acceptable with Warning, $N=$ Not Acceptable

If the evaluation system is not appropriate for the types of analyses performed in your lab, apply a site specific evaluation. 


\section{QAP 49 Results by Laboratory}

Lab: OB OBG Laboratories, East Syracuse, NY

\begin{tabular}{|c|c|c|c|c|c|c|c|}
\hline $\begin{array}{l}0 . \\
\text { est }\end{array}$ & Radionuclide & $\begin{array}{l}\text { Reported } \\
\text { Value }\end{array}$ & $\begin{array}{l}\text { Reported } \\
\text { Error }\end{array}$ & $\begin{array}{l}\text { EML } \\
\text { Value }\end{array}$ & $\begin{array}{l}\text { EML } \\
\text { Error }\end{array}$ & $\frac{\text { Reported }}{\text { EML }}$ & $\begin{array}{cc}\text { QAP 48 } \\
\text { Evaluation } \\
\text { Evaluation }\end{array}$ \\
\hline
\end{tabular}

Matrix: AI Air Filter Bq / filter

$\begin{array}{llrrrrrrr}1 & \text { CO60 } & 15.300 & 2.710 & 9.160 & 0.580 & 1.670 & \mathrm{~N} & \\ 1 & \text { CS137 } & 35.700 & 6.800 & 22.470 & 1.030 & 1.589 & \mathrm{~N} & \\ 1 & \text { GROSS ALPHA } & 1.830 & 0.183 & 1.650 & 0.160 & 1.109 & \mathrm{~A} & \mathrm{~A} \\ 1 & \text { GROSS BETA } & 1.910 & 0.190 & 2.160 & 0.070 & 0.884 & \mathrm{~W} & \mathrm{~A} \\ 1 & \text { MN54 } & 9.400 & 1.900 & 4.920 & 0.400 & 1.911 & \mathrm{~N} & \\ 1 & \text { SB125 } & 15.800 & 3.110 & 8.890 & 0.550 & 1.777 & \mathrm{~N} & \end{array}$

Matrix: SO Soil $\mathrm{Bq} / \mathrm{kg}$

$\begin{array}{ll}1 & \text { AC228 } \\ 1 & \text { BI212 } \\ 1 & \text { BI214 } \\ 1 & \text { CS137 } \\ 1 & \text { K40 } \\ 1 & \text { PB210 } \\ 1 & \text { PB212 } \\ 1 & \text { PB214 } \\ 1 & \text { RA226 }\end{array}$

75.400
63.300
34.300
1370.000
492.000
26.500
107.000
6.750
41.800
25.900

22.000

43.300

13.700

259.000

113.000

139.000

23.400

19.000

12.400

8.020

52.600

58.300

28.800

954.000

314.000

32.000

52.800

29.100

29.000

18.300
1.433

1.086

1.191

1.436

1.567

0.828

2.027

0.232

1.441

1.415
A

A

Matrix: $\mathrm{VE}$ Vegetation $\mathrm{Bq} / \mathrm{kg}$

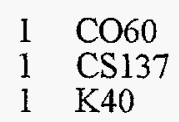

38.900
632.000
856.000

9.140

120.000

20.000

390.000

1.000
20.000
20.000

1.945

1.621

460.000

1.861

A
A
A
N
N
A
A
A
A
A

Matrix: WA Water $\mathrm{Bq} / \mathrm{L}$

$\begin{array}{llr}1 & \text { CO60 } & 51.400 \\ 1 & \text { CS137 } & 58.000 \\ 1 & \text { GROSS ALPHA } & 1110.000 \\ 1 & \text { GROSS BETA } & 1320.000 \\ 1 & \text { MN54 } & 43.500\end{array}$

8.880

11.200

7.290

49.400

50.000

8.730

1080.000

8.100

1420.000

32.400

1.200
1.700
60.000
60.000
1.400

1.040

1.160

1.028

0.930

1.343

$\begin{array}{ll}\text { A } & \\ \text { A } & \\ \text { A } & \text { W } \\ \text { A } & \text { A } \\ \text { N } & \end{array}$

\footnotetext{
Values for elemental uranium are reported in $\mu \mathrm{g} /$ filter, $\mathrm{g}$, or $\mathrm{mL}$.

$\mathrm{pCi} / \mathrm{g}$ or $\mathrm{mL}=\mathrm{Bq} \times 0.027$

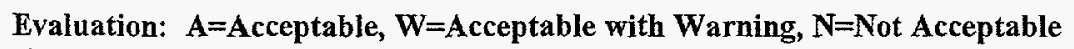

If the evaluation system is not appropriate for the types of analyses performed in your lab, apply a site specific evaluation.
} 


\section{QAP 49 Results by Laboratory}

Lab: OC Radiation Protection Service Laboratory, Ontario, Canada

\begin{tabular}{|c|c|c|c|c|c|c|c|}
\hline $\begin{array}{l}\text { No. } \\
\text { Test }\end{array}$ & Radionuclide & $\begin{array}{l}\text { Reported } \\
\text { Value }\end{array}$ & $\begin{array}{l}\text { Reported } \\
\text { Error }\end{array}$ & $\begin{array}{l}\text { EML } \\
\text { Value }\end{array}$ & $\begin{array}{l}\text { EML } \\
\text { Error }\end{array}$ & $\frac{\text { Reported }}{\text { EML }}$ & $\begin{array}{cc}\text { QAP 48 } \\
\text { Evaluation } & \text { Evaluation }\end{array}$ \\
\hline
\end{tabular}

Matrix: AI Air Filter $\mathrm{Bq} /$ filter

$\begin{array}{llrrrrrl}3 & \text { CO60 } & 10.000 & 3.000 & 9.160 & 0.580 & 1.092 & \mathrm{~A} \\ 1 & \text { CO60 } & 10.000 & 3.000 & 9.160 & 0.580 & 1.092 & \mathrm{~A} \\ 2 & \text { CO60 } & 10.000 & 3.000 & 9.160 & 0.580 & 1.092 & \mathrm{~A} \\ 3 & \text { CS137 } & 27.000 & 5.000 & 22.470 & 1.030 & 1.202 & \mathrm{~W} \\ 2 & \text { CS137 } & 28.000 & 5.000 & 22.470 & 1.030 & 1.246 & \mathrm{~W} \\ 1 & \text { CS137 } & 28.000 & 5.000 & 22.470 & 1.030 & 1.246 & \mathrm{~W} \\ 1 & \text { GROSS ALPHA } & 1.400 & 0.200 & 1.650 & 0.160 & 0.848 & \mathrm{~A} \\ 2 & \text { GROSS ALPHA } & 1.400 & 0.200 & 1.650 & 0.160 & 0.848 & \mathrm{~A} \\ 3 & \text { GROSS ALPHA } & 1.400 & 0.200 & 1.650 & 0.160 & 0.848 & \mathrm{~A} \\ 1 & \text { GROSS BETA } & 1.900 & 0.200 & 2.160 & 0.070 & 0.880 & \mathrm{~W} \\ 2 & \text { GROSS BETA } & 1.900 & 0.200 & 2.160 & 0.070 & 0.880 & \mathrm{~W} \\ 3 & \text { GROSS BETA } & 1.900 & 0.200 & 2.160 & 0.070 & 0.880 & \mathrm{~W} \\ 3 & \text { MN54 } & 6.000 & 2.000 & 4.920 & 0.400 & 1.220 & \mathrm{~W} \\ 2 & \text { MN54 } & 6.000 & 2.000 & 4.920 & 0.400 & 1.220 & \mathrm{~W} \\ 1 & \text { MN54 } & 6.000 & 2.000 & 4.920 & 0.400 & 1.220 & \mathrm{~W} \\ 1 & \text { SB125 } & 10.000 & 3.000 & 8.890 & 0.550 & 1.125 & \mathrm{~A} \\ 3 & \text { SB125 } & 11.000 & 3.000 & 8.890 & 0.550 & 1.237 & \mathrm{~W} \\ 2 & \text { SB125 } & 11.000 & 3.000 & 8.890 & 0.550 & 1.237 & \mathrm{~W}\end{array}$

Matrix: SO Soil $\mathrm{Bq} / \mathrm{kg}$

$\begin{array}{llrrrrrr}2 & \text { AC222 } & 39.000 & 18.000 & 52.600 & 2.900 & 0.741 & \mathrm{~A} \\ 1 & \text { AC228 } & 41.000 & 18.000 & 52.600 & 2.900 & 0.779 & \mathrm{~A} \\ 3 & \text { AC228 } & 48.000 & 18.000 & 52.600 & 2.900 & 0.913 & \mathrm{~A} \\ 1 & \text { BI212 } & 23.000 & 15.000 & 58.300 & 5.900 & 0.395 & \mathrm{~A} \\ 2 & \text { BI212 } & 25.000 & 15.000 & 58.300 & 5.900 & 0.429 & \mathrm{~A} \\ 3 & \text { BI212 } & 24.000 & 15.000 & 58.300 & 5.900 & 0.412 & \mathrm{~A} \\ 3 & \text { BI214 } & 21.000 & 10.000 & 28.800 & 0.500 & 0.729 & \mathrm{~A} \\ 2 & \text { BI214 } & 28.000 & 10.000 & 28.800 & 0.500 & 0.972 & \mathrm{~A} \\ 1 & \text { BI214 } & 25.000 & 10.000 & 28.800 & 0.500 & 0.868 & \mathrm{~A} \\ 1 & \text { CS137 } & 850.000 & 300.000 & 954.000 & 38.000 & 0.891 & \mathrm{~W} \\ 2 & \text { CS137 } & 840.000 & 300.000 & 954.000 & 38.000 & 0.881 & \mathrm{~W} \\ 3 & \text { CS137 } & 830.000 & 300.000 & 954.000 & 38.000 & 0.870 & \text { W } \\ 1 & \text { K40 } & 400.000 & 200.000 & 314.000 & 13.000 & 1.274 & \text { W } \\ 3 & \text { K40 } & 400.000 & 200.000 & 314.000 & 13.000 & 1.274 & \text { W } \\ 2 & \text { K40 } & 380.000 & 200.000 & 314.000 & 13.000 & 1.210 & \mathrm{~A} \\ 1 & \text { PB212 } & 58.000 & 10.000 & 52.800 & 3.700 & 1.098 & \mathrm{~A} \\ 2 & \text { PB212 } & 63.000 & 10.000 & 52.800 & 3.700 & 1.193 & \mathrm{~A} \\ 3 & \text { PB212 } & 54.000 & 10.000 & 52.800 & 3.700 & 1.023 & \mathrm{~A} \\ 2 & \text { PB214 } & 31.000 & 10.000 & 29.100 & 1.200 & 1.065 & \mathrm{~A} \\ 1 & \text { PB214 } & 28.000 & 10.000 & 29.100 & 1.200 & 0.962 & \mathrm{~A} \\ 3 & \text { PB214 } & 27.000 & 10.000 & 29.100 & 1.200 & 0.928 & \mathrm{~A} \\ 1 & \text { RA226 } & 25.000 & 10.000 & 29.000 & 1.000 & 0.862 & \mathrm{~A} \\ 2 & \text { RA226 } & 28.000 & 10.000 & 29.000 & 1.000 & 0.966 & \mathrm{~A} \\ 3 & \text { RA226 } & 21.000 & 10.000 & 29.000 & 1.000 & 0.724 & \mathrm{~A} \\ 3 & \text { TH228 } & 42.000 & 15.000 & 52.700 & 4.000 & 0.797 & \mathrm{~A} \\ 2 & \text { TH228 } & 37.000 & 15.000 & 52.700 & 4.000 & 0.702 & \mathrm{~A}\end{array}$

Values for elemental uranium are reported in $\mu \mathrm{g} /$ filter, $\mathrm{g}$, or $\mathrm{mL}$.

$\mathrm{pCi} / \mathrm{g}$ or $\mathrm{mL}=\mathrm{Bq} \times 0.027$

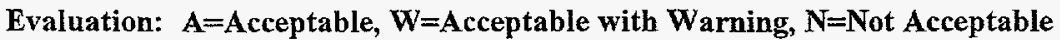

If the evaluation system is not appropriate for the types of analyses performed in your lab, apply a site specific evaluation. 


\section{QAP 49 Results by Laboratory}

Lab: OC Radiation Protection Service Laboratory, Ontario, Canada

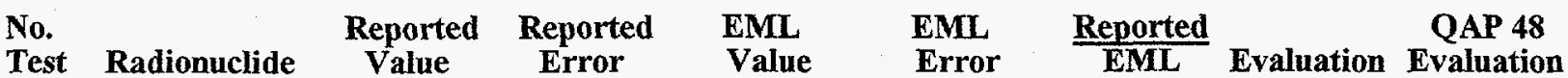

Matrix: SO Soil $\mathrm{Bq} / \mathrm{kg}$

$\begin{array}{rrrrrrrr}1 . & \text { TH228 } & 40.000 & 15.000 & 52.700 & 4.000 & 0.759 & \mathrm{~A} \\ 2 & \text { TH234 } & 140.000 & 40.000 & 114.000 & 6.000 & 1.228 & \mathrm{~A} \\ 3 & \text { TH234 } & 120.000 & 40.000 & 114.000 & 6.000 & 1.053 & \mathrm{~A} \\ 1 & \text { TH234 } & 170.000 & 40.000 & 114.000 & 6.000 & 1.491 & \mathrm{~A} \\ 1 & \text { TL208 } & 40.000 & 15.000 & 18.300 & 1.100 & 2.186 & \mathrm{~A} \\ 3 & \text { TL208 } & 42.000 & 15.000 & 18.300 & 1.100 & 2.295 & \mathrm{~A} \\ 2 & \text { TL208 } & 37.000 & 15.000 & 18.300 & 1.100 & 2.022 & \mathrm{~A}\end{array}$

Matrix: VE Vegetation $\mathrm{Bq} / \mathrm{kg}$

$\begin{array}{llrrrrrr}3 & \text { CO60 } & 16.000 & 5.000 & 20.000 & 1.000 & 0.800 & \mathrm{~W} \\ 2 & \text { CO60 } & 16.000 & 5.000 & 20.000 & 1.000 & 0.800 & \mathrm{~W} \\ 1 & \text { CO60 } & 17.000 & 5.000 & 20.000 & 1.000 & 0.850 & \mathrm{~W} \\ 1 & \text { CS137 } & 390.000 & 20.000 & 390.000 & 20.000 & 1.000 & \mathrm{~A} \\ 3 & \text { CS137 } & 380.000 & 20.000 & 390.000 & 20.000 & 0.974 & \mathrm{~A} \\ 2 & \text { CS137 } & 380.000 & 20.000 & 390.000 & 20.000 & 0.974 & \mathrm{~A} \\ 1 & \text { K40 } & 640.000 & 100.000 & 460.000 & 20.000 & 1.391 & \mathrm{~W} \\ 2 & \text { K40 } & 640.000 & 100.000 & 460.000 & 20.000 & 1.391 & \mathrm{~W} \\ 3 & \text { K40 } & 610.000 & 100.000 & 460.000 & 20.000 & 1.326 & \mathrm{~W} \\ 1 & \text { SR90 } & 510.000 & 25.000 & 606.000 & 40.000 & 0.842 & \mathrm{~A} \\ 2 & \text { SR90 } & 440.000 & 22.000 & 606.000 & 40.000 & 0.726 & \mathrm{~W} \\ 3 & \text { SR90 } & 500.000 & 25.000 & 606.000 & 40.000 & 0.825 & \mathrm{~A}\end{array}$

Matrix: WA Water $\mathrm{Bq} / \mathrm{L}$

\begin{tabular}{|c|c|c|c|c|c|}
\hline $\mathrm{CO} 60$ & 50.000 & 5.000 & 49.400 & 1.200 & 1.012 \\
\hline $\mathrm{CO60}$ & 54.000 & 5.000 & 49.400 & 1.200 & 1.093 \\
\hline $\mathrm{CO} 60$ & 53.000 & 5.000 & 49.400 & 1.200 & 1.073 \\
\hline $\mathrm{CS} 137$ & 50.000 & 5.000 & 50.000 & 1.700 & 1.000 \\
\hline $\mathrm{CS} 137$ & 50.000 & 5.000 & 50.000 & 1.700 & 1.000 \\
\hline CS 137 & 50.000 & 5.000 & 50.000 & 1.700 & 1.000 \\
\hline GROSS ALPHA & 1100.000 & 60.000 & 1080.000 & 60.000 & 1.019 \\
\hline GROSS ALPHA & 1100.000 & 60.000 & 1080.000 & 60.000 & 1.019 \\
\hline GROSS BETA & 1200.000 & 20.000 & 1420.000 & 60.000 & 0.845 \\
\hline GROSS BETA & 1200.000 & 20.000 & 1420.000 & 60.000 & 0.845 \\
\hline $\mathrm{H} 3$ & 251.000 & 10.000 & 76.200 & 2.900 & 3.294 \\
\hline $\mathrm{H3}$ & 252.000 & 10.000 & 76.200 & 2.900 & 3.307 \\
\hline $\mathrm{H} 3$ & 247.000 & 10.000 & 76.200 & 2.900 & 3.241 \\
\hline MN54 & 42.000 & 5.000 & 32.400 & 1.400 & 1.296 \\
\hline MN54 & 30.000 & 5.000 & 32.400 & 1.400 & 0.926 \\
\hline MN54 & 35.000 & 5.000 & 32.400 & 1.400 & 1.080 \\
\hline SR90 & 2.300 & 0.200 & 2.110 & 0.180 & 1.090 \\
\hline SR90 & 2.300 & 0.200 & 2.110 & 0.180 & 1.090 \\
\hline SR90 & 2.400 & 0.200 & 2.110 & 0.180 & 1.137 \\
\hline
\end{tabular}

\section{Values for elemental uranium are reported in $\mu \mathrm{g} /$ filter, $\mathrm{g}$, or $\mathrm{mL} . \quad \mathrm{pCi} / \mathrm{g}$ or $\mathrm{mL}=\mathrm{Bq} \times 0.027$}

Evaluation: $A=$ Acceptable, $W=$ Acceptable with Warning, $N=$ Not Acceptable

If the evaluation system is not appropriate for the types of analyses performed in your lab, apply a site specific evaluation. 


\section{QAP 49 Results by Laboratory}

Lab: OD ORNL, Radiobioassay Lab

\begin{tabular}{|c|c|c|c|c|c|c|c|}
\hline $\begin{array}{l}\text { o. } \\
\text { est }\end{array}$ & Radionuclide & $\begin{array}{l}\text { Reported } \\
\text { Value }\end{array}$ & $\begin{array}{l}\text { Reported } \\
\text { Error }\end{array}$ & $\begin{array}{l}\text { EML } \\
\text { Value }\end{array}$ & $\begin{array}{l}\text { EML } \\
\text { Error }\end{array}$ & $\frac{\text { Reported }}{\text { EML }}$ & $\begin{array}{cc}\text { QAP 48 } \\
\text { Evaluation } \\
\text { Evaluation }\end{array}$ \\
\hline
\end{tabular}

Matrix: AI Air Filter Bq / filter

$\begin{array}{llrlrllll}1 & \text { CO60 } & 8.996 & 0.146 & 9.160 & 0.580 & 0.982 & \mathrm{~A} & \mathrm{~A} \\ 2 & \text { CO60 } & 9.078 & 0.140 & 9.160 & 0.580 & 0.991 & \mathrm{~A} & \mathrm{~A} \\ 1 & \text { CO60 } & 9.000 & 0.140 & 9.160 & 0.580 & 0.983 & \mathrm{~A} & \mathrm{~A} \\ 2 & \text { CO60 } & 9.080 & 0.140 & 9.160 & 0.580 & 0.991 & \mathrm{~A} & \mathrm{~A} \\ 1 & \text { CS137 } & 22.780 & 1.010 & 22.470 & 1.030 & 1.014 & \mathrm{~A} & \mathrm{~A} \\ 2 & \text { CS137 } & 22.620 & 0.995 & 22.470 & 1.030 & 1.007 & \mathrm{~A} & \mathrm{~A} \\ 1 & \text { CS137 } & 22.780 & 1.010 & 22.470 & 1.030 & 1.014 & \mathrm{~A} & \mathrm{~A} \\ 2 & \text { CS137 } & 22.620 & 1.000 & 22.470 & 1.030 & 1.007 & \mathrm{~A} & \mathrm{~A} \\ 1 & \text { GROSS ALPHA } & 1.600 & 0.060 & 1.650 & 0.160 & 0.970 & \mathrm{~A} & \mathrm{~A} \\ 1 & \text { GROSS ALPHA } & 1.600 & 0.060 & 1.650 & 0.160 & 0.970 & \mathrm{~A} & \mathrm{~A} \\ 1 & \text { GROSS BETA } & 2.520 & 0.080 & 2.160 & 0.070 & 1.167 & \mathrm{~A} & \mathrm{~A} \\ 1 & \text { GROSS BETA } & 2.520 & 0.080 & 2.160 & 0.070 & 1.167 & \mathrm{~A} & \mathrm{~A} \\ 2 & \text { MN54 } & 5.030 & 0.321 & 4.920 & 0.400 & 1.022 & \mathrm{~A} & \mathrm{~A} \\ 1 & \text { MN54 } & 5.084 & 0.327 & 4.920 & 0.400 & 1.033 & \mathrm{~A} & \mathrm{~A} \\ 2 & \text { MN54 } & 5.030 & 0.320 & 4.920 & 0.400 & 1.022 & \mathrm{~A} & \mathrm{~A} \\ 1 & \text { MN54 } & 5.080 & 0.330 & 4.920 & 0.400 & 1.033 & \mathrm{~A} & \mathrm{~A} \\ 2 & \text { SB125 } & 8.329 & 0.174 & 8.890 & 0.550 & 0.937 & \mathrm{~A} & \mathrm{~A} \\ 1 & \text { SB125 } & 8.449 & 0.181 & 8.890 & 0.550 & 0.950 & \mathrm{~A} & \mathrm{~A} \\ 1 & \text { SB125 } & 8.450 & 0.180 & 8.890 & 0.550 & 0.951 & \mathrm{~A} & \mathrm{~A} \\ 2 & \text { SB125 } & 8.330 & 0.170 & 8.890 & 0.550 & 0.937 & \mathrm{~A} & \mathrm{~A}\end{array}$

Matrix: WA Water $\mathrm{Bq} / \mathrm{L}$

$\begin{array}{llrllllll}1 & \text { AM241 } & 1.316 & 0.133 & 1.250 & 0.080 & 1.053 & \mathrm{~A} & \mathrm{~A} \\ 1 & \text { AM241 } & 1.320 & 0.130 & 1.250 & 0.080 & 1.056 & \mathrm{~A} & \mathrm{~A} \\ 1 & \text { CO60 } & 50.660 & 2.170 & 49.400 & 1.200 & 1.026 & \mathrm{~A} & \mathrm{~A} \\ 2 & \text { CO60 } & 51.750 & 2.510 & 49.400 & 1.200 & 1.048 & \mathrm{~A} & \mathrm{~A} \\ 2 & \text { CO60 } & 51.750 & 2.510 & 49.400 & 1.200 & 1.048 & \mathrm{~A} & \mathrm{~A} \\ 1 & \text { CO60 } & 50.660 & 2.170 & 49.400 & 1.200 & 1.026 & \mathrm{~A} & \mathrm{~A} \\ 1 & \text { CS137 } & 51.020 & 3.370 & 50.000 & 1.700 & 1.020 & \mathrm{~A} & \mathrm{~A} \\ 2 & \text { CS137 } & 58.530 & 4.000 & 50.000 & 1.700 & 1.171 & \mathrm{~A} & \mathrm{~A} \\ 1 & \text { CS137 } & 51.020 & 3.370 & 50.000 & 1.700 & 1.020 & \mathrm{~A} & \mathrm{~A} \\ 2 & \text { CS137 } & 58.530 & 4.000 & 50.000 & 1.700 & 1.171 & \mathrm{~A} & \mathrm{~A} \\ 1 & \text { H3 } & 80.170 & 4.670 & 76.200 & 2.900 & 1.052 & \mathrm{~A} & \mathrm{~A} \\ 1 & \text { H3 } & 80.170 & 4.670 & 76.200 & 2.900 & 1.052 & \mathrm{~A} & \mathrm{~A} \\ 2 & \text { MN54 } & 34.100 & 3.190 & 32.400 & 1.400 & 1.052 & \mathrm{~A} & \mathrm{~A} \\ 1 & \text { MN54 } & 38.790 & 3.120 & 32.400 & 1.400 & 1.197 & \mathrm{~W} & \mathrm{~A} \\ 2 & \text { MN54 } & 34.100 & 3.190 & 32.400 & 1.400 & 1.052 & \mathrm{~A} & \mathrm{~A} \\ 1 & \text { MN54 } & 38.790 & 3.120 & 32.400 & 1.400 & 1.197 & \mathrm{~W} & \mathrm{~A} \\ 1 & \text { PU238 } & 1.178 & 0.121 & 1.100 & 0.010 & 1.071 & \mathrm{~A} & \mathrm{~A} \\ 1 & \text { PU238 } & 1.180 & 0.120 & 1.100 & 0.010 & 1.073 & \mathrm{~A} & \mathrm{~A} \\ 1 & \text { PU239 } & 1.542 & 0.157 & 1.410 & 0.040 & 1.094 & \mathrm{~A} & \mathrm{~A} \\ 1 & \text { PU239 } & 1.540 & 0.160 & 1.410 & 0.040 & 1.092 & \mathrm{~A} & \mathrm{~A} \\ 1 & \text { SR90 } & 2.390 & 0.280 & 2.110 & 0.180 & 1.133 & \mathrm{~A} & \mathrm{~A} \\ 1 & \text { SR90 } & 2.390 & 0.280 & 2.110 & 0.180 & 1.133 & \mathrm{~A} & \mathrm{~A} \\ 1 & \text { U234 } & 0.503 & 0.052 & 0.510 & 0.030 & 0.986 & \mathrm{~A} & \mathrm{~A} \\ 1 & \text { U234 } & 0.503 & 0.052 & 0.510 & 0.030 & 0.986 & \mathrm{~A} & \mathrm{~A}\end{array}$

Values for elemental uranium are reported in $\mu \mathrm{g} /$ filter, $\mathrm{g}$, or $\mathrm{mL} . \quad \mathrm{pCi} / \mathrm{g}$ or $\mathrm{mL}=\mathrm{Bq} \times 0.027$

Evaluation: A=Acceptable, $W=$ Acceptable with Warning, $N=$ Not Acceptable

If the evaluation system is not appropriate for the types of analyses performed in your lab, apply a site specific evaluation. 


\section{QAP 49 Results by Laboratory}

Lab: OD ORNL, Radiobioassay Lab

No.

Reported Reported

EML

EML

Reported Error

Value

Error EML

Evaluation

Matrix: WA Water $\mathrm{Bq} / \mathrm{L}$

$\begin{array}{lllllllll}1 & \mathrm{U} 238 & 0.532 & 0.055 & 0.520 & 0.050 & 1.023 & \mathrm{~A} & \mathrm{~A} \\ \mathrm{l} & \mathrm{U} 238 & 0.532 & 0.055 & 0.520 & 0.050 & 1.023 & \mathrm{~A} & \mathrm{~A}\end{array}$

Values for elemental uranium are reported in $\mu \mathrm{g} /$ filter, $\mathrm{g}$, or $\mathrm{mL}$.

$\mathrm{pCi} / \mathrm{g}$ or $\mathrm{mL}=\mathrm{Bq} \times 0.027$

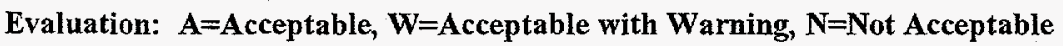

If the evaluation system is not appropriate for the types of analyses performed in your lab, apply a site specific evaluation. 


\section{QAP 49 Results by Laboratory}

Lab: OL ORNL Environmental Sciences Div.

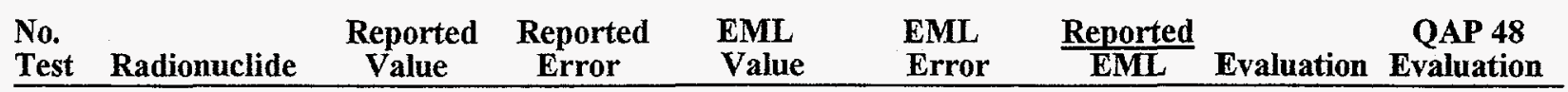

Matrix: $\mathrm{AI}$ Air Filter $\mathrm{Bq} /$ filter

$\begin{array}{llrrrrrrr}1 & \text { CO60 } & 9.790 & 0.340 & 9.160 & 0.580 & 1.069 & \mathrm{~A} & \mathrm{~A} \\ 1 & \mathrm{CS} 137 & 24.830 & 1.770 & 22.470 & 1.030 & 1.105 & \mathrm{~A} & \mathrm{~A} \\ 1 & \text { MN54 } & 5.820 & 0.360 & 4.920 & 0.400 & 1.183 & \mathrm{~W} & \mathrm{~A} \\ 1 & \mathrm{SB125} & 10.220 & 0.470 & 8.890 & 0.550 & 1.150 & \mathrm{~A} & \mathrm{~W}\end{array}$

Matrix: SO Soil $\mathrm{Bq} / \mathrm{kg}$

$1 \mathrm{CO} 60$

$1 \quad \mathrm{CS} 137$

$1 \mathrm{~K} 40$
1.320

1059.200

362.900

0.320

26.800

26.100

1.240

954.000

314.000

Matrix: VE Vegetation $\mathrm{Bq} / \mathrm{kg}$

$1 \mathrm{CO60}$

$1 \quad \operatorname{CS} 137$

$1 \mathrm{~K} 40$

20.590

439.600

527.200

1.090

6.600

20.100

20.000

390.000

460.000

1.000
20.000
20.000

1.030

1.127

1.146

0.110

38.000

13.000
1.580

1.290

$-49.400$

50.000

32.400

1.200
1.700
1.400
0.950
50.460

34.270
1 MN54
1.021
1.002
1.058

Matrix: WA Water $\mathrm{Bq} / \mathrm{L}$

$\begin{array}{ll}\mathrm{A} & \mathrm{A} \\ \mathrm{A} & \mathrm{A} \\ \mathrm{A} & \mathrm{A}\end{array}$




\section{QAP 49 Results by Laboratory}

Lab: OT ORNL Radioactive Material Analysis Lab

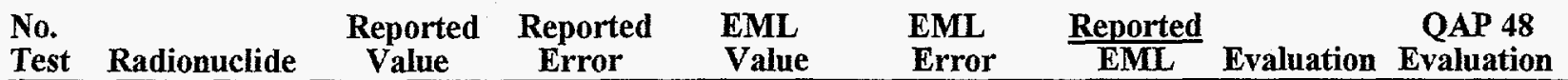

Matrix: AI Air Filter Bq/filter

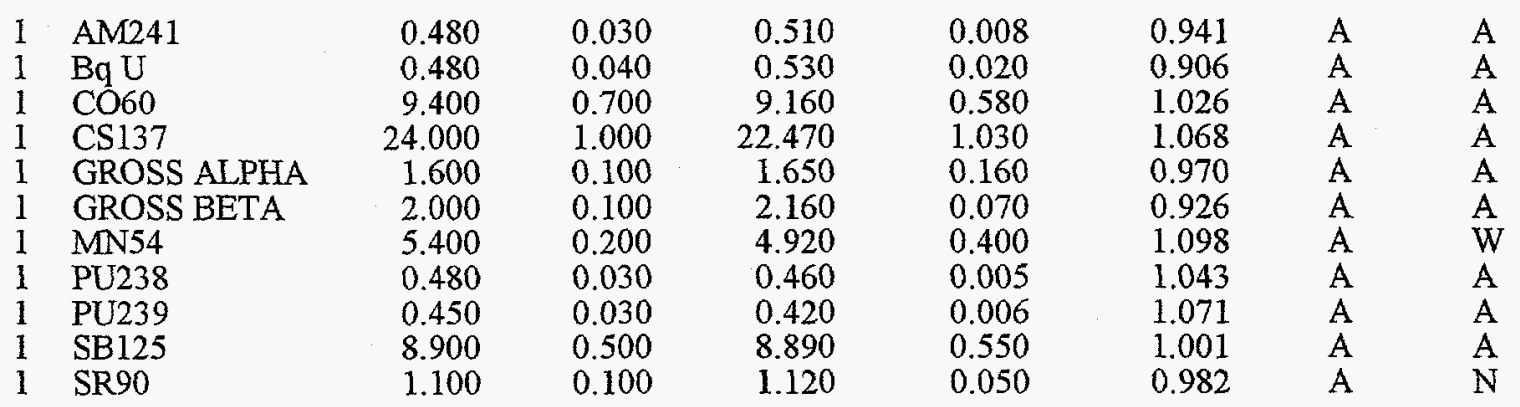

Matrix: SO Soil $\mathrm{Bq} / \mathrm{kg}$

$\begin{array}{ll}1 & \mathrm{AC} 228 \\ 1 & \mathrm{AM} 241 \\ 1 & \mathrm{BI212} \\ 1 & \mathrm{BI214} \\ 1 & \mathrm{BqU} \\ 1 & \mathrm{CS} 137 \\ 1 & \mathrm{~K} 40 \\ 1 & \mathrm{~PB} 212 \\ 1 & \mathrm{~PB} 214 \\ 1 & \mathrm{PU} 239 \\ 1 & \mathrm{RA226} \\ 1 & \mathrm{SR} 90 \\ 1 & \mathrm{TH} 228 \\ 1 & \mathrm{TH} 234 \\ 1 & \mathrm{TL} 208\end{array}$

$\begin{array}{rrr}42.000 & 10.000 & 52.600 \\ 6.500 & 1.000 & 7.470 \\ 47.000 & 11.000 & 58.300 \\ 37.000 & 14.000 & 28.800 \\ 160.000 & 20.000 & 237.000 \\ 920.000 & 10.000 & 954.000 \\ 320.000 & 40.000 & 314.000 \\ 47.000 & 11.000 & 52.800 \\ 37.000 & 14.000 & 29.100 \\ 14.000 & 2.000 & 13.090 \\ 37.000 & 14.000 & 29.000 \\ 31.000 & 5.000 & 39.630 \\ 21.000 & 1.000 & 52.700 \\ 78.000 & 6.000 & 114.000 \\ 47.000 & 11.000 & 18.300\end{array}$

Matrix: VE Vegetation $\mathrm{Bq} / \mathrm{kg}$

$\begin{array}{rrr}2.900 & 0.500 & 2.330 \\ 2.000 & 0.400 & 1.760 \\ 19.000 & 3.000 & 20.000 \\ 380.000 & 10.000 & 390.000 \\ 480.000 & 60.000 & 460.000 \\ 4.400 & 0.700 & 3.720 \\ 580.000 & 20.000 & 606.000\end{array}$

Matrix: WA Water $\mathrm{Bq} / \mathrm{L}$

\subsection{0 \\ 0.830 \\ 52.000}

53.000
0.100
0.080
2.000
2.000

2.900
0.410
5.900
0.500
16.000
38.000
13.000
3.700
1.200
0.570
1.000
0.003
4.000
6.000
1.100

0.798

0.870

0.806

1.285

0.675

0.964

1.019

0.890

1.271

1.070

1.276

0.782

0.398

0.684

2.568
A

A

A

A

A

A

$\begin{array}{ll}\text { A } & \text { A } \\ \text { A } & \text { W } \\ \text { A } & \text { A } \\ \text { A } & \text { W } \\ \text { A } & \text { A } \\ \text { A } & \text { A } \\ \text { A } & \text { A }\end{array}$

Values for elemental uranium are reported in $\mu \mathrm{g} /$ filter, $\mathrm{g}$, or $\mathrm{mL}$.

$\mathrm{pCi} / \mathrm{g}$ or $\mathrm{mL}=\mathrm{Bq} \times \mathbf{0 . 0 2 7}$

Evaluation: $A=$ Acceptable, $W=$ Acceptable with Warning, $N=$ Not Acceptable

If the evaluation system is not appropriate for the types of analyses performed in your lab, apply a site specific evaluation. 


\section{QAP 49 Results by Laboratory}

Lab: OT ORNL Radioactive Material Analysis Lab

\begin{tabular}{|c|c|c|c|c|c|c|c|}
\hline $\begin{array}{l}\text { No. } \\
\text { Test }\end{array}$ & Radionuclide & $\begin{array}{l}\text { Reported } \\
\text { Value }\end{array}$ & $\begin{array}{l}\text { Reported } \\
\text { Error }\end{array}$ & $\begin{array}{l}\text { EML } \\
\text { Value }\end{array}$ & $\begin{array}{l}\text { EML } \\
\text { Error }\end{array}$ & $\frac{\text { Reported }}{\text { EML }}$ & $\begin{array}{c}\text { QAP 48 } \\
\text { Evaluation } \\
\text { Evaluation }\end{array}$ \\
\hline
\end{tabular}

Matrix: WA Water $\mathrm{Bq} / \mathrm{L}$

$\begin{array}{llrrrrrrr}1 & \text { GROSS ALPHA } & 1200.000 & 100.000 & 1080.000 & 60.000 & 1.111 & \mathrm{~A} & \mathrm{~A} \\ 1 & \text { GROSS BETA } & 1400.000 & 100.000 & 1420.000 & 60.000 & 0.986 & \mathrm{~A} & \mathrm{~A} \\ 1 & \text { H3 } & 43.000 & 4.000 & 76.200 & 2.900 & 0.564 & \mathrm{~N} & \mathrm{~W} \\ 1 & \text { MN54 } & 36.000 & 2.000 & 32.400 & 1.400 & 1.111 & \mathrm{~A} & \mathrm{~A} \\ 1 & \text { PU238 } & 0.940 & 0.070 & 1.100 & 0.010 & 0.855 & \mathrm{~W} & \mathrm{~W} \\ 1 & \text { PU239 } & 1.400 & 0.100 & 1.410 & 0.040 & 0.993 & \mathrm{~A} & \mathrm{~A} \\ 1 & \text { SR90 } & 2.100 & 0.300 & 2.110 & 0.180 & 0.995 & \mathrm{~A} & \mathrm{~A}\end{array}$

Values for elemental uranium are reported in $\mu \mathrm{g} /$ filter, $\mathrm{g}$, or $\mathrm{mL}$.

$\mathrm{pCi} / \mathrm{g}$ or $\mathrm{mL}=\mathrm{Bq} \times \mathbf{0 . 0 2 7}$

Evaluation: $A=A c c e p t a b l e, W=$ Acceptable with Warning, $N=$ Not Acceptable

If the evaluation system is not appropriate for the types of analyses performed in your lab, apply a site specific evaluation. 


\section{QAP 49 Results by Laboratory}

Lab: OU Outreach Laboratory, Broken Arrow, OK

\begin{tabular}{|c|c|c|c|c|c|c|c|}
\hline $\begin{array}{l}\text { No. } \\
\text { Test }\end{array}$ & Radionuclide & $\begin{array}{l}\text { Reported } \\
\text { Value }\end{array}$ & $\begin{array}{c}\text { Reported } \\
\text { Error }\end{array}$ & $\begin{array}{l}\text { EML } \\
\text { Value }\end{array}$ & $\begin{array}{l}\text { EML } \\
\text { Error }\end{array}$ & $\frac{\text { Reported }}{\text { EML }}$ & $\begin{array}{cc}\text { QAP 48 } \\
\text { Evaluation } \\
\text { Evaluation }\end{array}$ \\
\hline
\end{tabular}

Matrix: AI Air Filter $\mathrm{Bq} /$ filter

\begin{tabular}{|c|c|c|c|c|c|c|c|}
\hline 1 & $\mathrm{CO} 60$ & 8.970 & 0.290 & 9.160 & 0.580 & 0.979 & A \\
\hline 1 & $\mathrm{CS} 137$ & 25.300 & 0.610 & 22.470 & 1.030 & 1.126 & A \\
\hline 1 & GROSS AIPHA & 2.190 & 0.167 & 1.650 & 0.160 & 1.327 & W \\
\hline 1 & GROSS BETA & 2.590 & 0.199 & 2.160 & 0.070 & 1.199 & A \\
\hline 1 & MN54 & 4.590 & 1.200 & 4.920 & 0.400 & 0.933 & A \\
\hline 1 & SB 125 & 8.430 & 1.800 & 8.890 & 0.550 & 0.948 & A \\
\hline
\end{tabular}

Matrix: SO Soil $\mathrm{Bq} / \mathrm{kg}$

$\begin{array}{ll}1 & \mathrm{BI} 212 \\ 1 & \mathrm{BI} 214 \\ 1 & \mathrm{~Bq} \mathrm{U} \\ 1 & \mathrm{CS} 137 \\ 1 & \mathrm{~K} 40 \\ 1 & \mathrm{~PB} 212 \\ 1 & \mathrm{~PB} 214 \\ 1 & \mathrm{SR} 90 \\ 1 & \mathrm{TH} 228 \\ 1 & \mathrm{TH} 234 \\ 1 & \mathrm{U} 234 \\ 1 & \mathrm{U} 238 \\ 1 & \mathrm{ug} \mathrm{U}\end{array}$

$\begin{array}{rrr}52.100 & 3.200 & 58.300 \\ 33.200 & 2.300 & 28.800 \\ 237.000 & 16.000 & 237.000 \\ 1110.000 & 52.100 & 954.000 \\ 326.000 & 15.200 & 314.000 \\ 49.100 & 6.200 & 52.800 \\ 31.300 & 1.700 & 29.100 \\ 37.600 & 9.200 & 39.630 \\ 121.000 & 4.000 & 52.700 \\ 115.000 & 2.400 & 114.000 \\ 115.000 & 2.340 & 113.000 \\ 93.300 & 2.160 & 120.000 \\ 9.300 & 0.400 & 9.700\end{array}$

5.900
0.500
16.000
38.000
13.000
3.700
1.200
0.003
4.000
6.000
6.000
9.000
0.700

0.894
1.153
1.000
1.164
1.038
0.930
1.076
0.949
2.296
1.009
1.018
0.778
0.959

A
A
A
A
A
A
A
A
A
A
A
A
A

W

W

A

Matrix: VE Vegetation $\mathrm{Bq} / \mathrm{kg}$

\begin{tabular}{|c|c|c|c|c|c|}
\hline CO60 & 20.100 & 1.490 & 20.000 & 1.000 & 1.005 \\
\hline CS137 & 385.000 & 62.000 & 390.000 & 20.000 & 0.987 \\
\hline $\mathrm{K} 40$ & 416.000 & 21.800 & 460.000 & 20.000 & 0.904 \\
\hline
\end{tabular}

Matrix: WA Water $\mathrm{Bq} / \mathrm{L}$

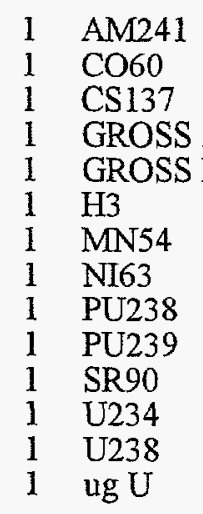

1.270
51.800
44.500
833.000
1250.000
201.000
38.800
103.000
1.070
1.540
2.010
0.399
0.402
0.033

1.250
49.400
50.000
1080.000
1420.000
76.200
32.400
95.700
1.100
1.410
2.110
0.510
0.520
0.040

1.016
1.049
0.890
0.771
0.880
2.638
1.198
1.076
0.973
1.092
0.953
0.782
0.773
0.823

$\begin{array}{ll}\text { A } & \\ \text { A } & \text { N } \\ \text { W } & \text { A } \\ \text { W } & \text { A } \\ \text { A } & \text { A } \\ \text { N } & \text { W } \\ \text { W } & \text { A } \\ \text { A } & \\ \text { A } & \\ \text { A } & \\ \text { A } & \\ \text { N } & \text { A } \\ \text { N } & \text { A } \\ \text { W } & \text { A }\end{array}$

Values for elemental uranium are reported in $\mu \mathrm{g} /$ filter, $\mathrm{g}$, or $\mathrm{mL}$.

$\mathrm{pCi} / \mathrm{g}$ or $\mathrm{mL}=\mathrm{Bq} \times 0.027$

Evaluation: $A=$ Acceptable, $W=$ Acceptable with Warning, $N=$ Not Acceptable

If the evaluation system is not appropriate for the types of analyses performed in your lab, apply a site specific evaluation. 
QAP 49 Results by Laboratory

Lab: PA Mason \& Hanger-Silas Mason Co., Inc., Battelle Pantex, Amarillo, TX

No. $\quad$ Reported Reported EML $\quad$ EML Reported $\quad$ QAP 48

Test Radionuclide Value Error Value Error $\frac{\text { EML }}{\text { Evaluation Evaluation }}$

Matrix: AI Air Filter Bq/filter

$\begin{array}{lllllllll}1 & \text { GROSS ALPHA } & 1.540 & 0.110 & 1.650 & 0.160 & 0.933 & \text { A } & \text { A } \\ 1 & \text { GROSS BETA } & 2.090 & 0.100 & 2.160 & 0.070 & 0.968 & \text { A } & \text { A }\end{array}$

Values for elemental uranium are reported in $\mu \mathrm{g} /$ filter, $\mathrm{g}$, or $\mathrm{mL}$.

$\mathrm{pCi} / \mathrm{g}$ or $\mathrm{mL}=\mathrm{Bq} \times 0.027$

Evaluation: $A=$ Acceptable, $W=$ Acceptable with Warning, $N=$ Not Acceptable

If the evaluation system is not appropriate for the types of analyses performed in your lab, apply a site specific evaluation. 


\section{QAP 49 Results by Laboratory}

Lab: PK Pakistan Institute of Nuclear Science \& Technology

\begin{tabular}{llclll}
$\begin{array}{l}\text { No. } \\
\text { Test }\end{array}$ Radionuclide & $\begin{array}{c}\text { Reported } \\
\text { Value }\end{array}$ & $\begin{array}{c}\text { Reported } \\
\text { Error }\end{array}$ & $\begin{array}{l}\text { EML } \\
\text { Value }\end{array}$ & $\begin{array}{c}\text { EML } \\
\text { Error }\end{array}$ & $\begin{array}{c}\text { Reported } \\
\text { EML }\end{array}$ Evaluation Evaluation \\
\hline
\end{tabular}

Matrix: AI Air Filter $\mathrm{Bq} /$ filter

$\begin{array}{llrlrllll}1 & \text { CO60 } & 9.035 & 0.380 & 9.160 & 0.580 & 0.986 & \text { A } & \\ 1 & \text { CS137 } & 21.190 & 0.380 & 22.470 & 1.030 & 0.943 & \text { A } & \text { N } \\ 1 & \text { MN54 } & 4.990 & 0.520 & 4.920 & 0.400 & 1.014 & \text { A } & \\ 1 & \text { SB125 } & 8.081 & 0.300 & 8.890 & 0.550 & 0.909 & \text { A } & \end{array}$

Matrix: SO Soil Bq / kg

$\begin{array}{lllllllll}1 & \mathrm{CS} 137 & 946.260 & 39.350 & 954.000 & 38.000 & 0.992 & \mathrm{~A} & \mathrm{~A}\end{array}$

Matrix: VE Vegetation $\mathrm{Bq} / \mathrm{kg}$

$\begin{array}{lllllllll}\mathrm{I} & \mathrm{CS} 137 & 430.240 & 12.230 & 390.000 & 20.000 & 1.103 & \mathrm{~A} & \mathrm{~A} \\ \mathrm{l} & \mathrm{K} 40 & 449.980 & 50.150 & 460.000 & 20.000 & 0.978 & \mathrm{~A} & \mathrm{~A}\end{array}$

Values for elemental uranium are reported in $\mu \mathrm{g} /$ filter, $\mathrm{g}$, or $\mathrm{mL}$.

$\mathrm{pCi} / \mathrm{g}$ or $\mathrm{mL}=\mathrm{Bq} \times 0.027$

Evaluation: $A=A c c e p t a b l e, ~ W=A c c e p t a b l e$ with Warning, $N=$ Not Acceptable

If the evaluation system is not appropriate for the types of analyses performed in your lab, apply a site specific evaluation. 


\section{QAP 49 Results by Laboratory}

Lab: PO Institute of Oceanology PAN, Poland

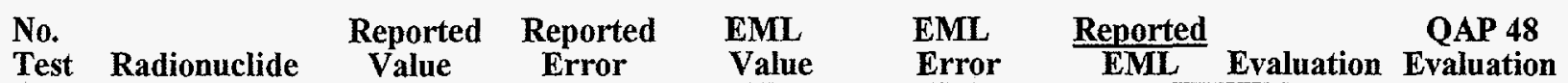

Matrix: AI Air Filter Bq/ filter

$\begin{array}{llrrrrrrr}1 & \text { AM241 } & 0.700 & 0.100 & 0.510 & 0.008 & 1.373 & \mathrm{~A} & \\ 1 & \text { CO60 } & 10.500 & 0.500 & 9.160 & 0.580 & 1.146 & \text { W } & \text { A } \\ 1 & \text { CS137 } & 26.600 & 1.300 & 22.470 & 1.030 & 1.184 & \text { W } & \text { A } \\ 1 & \text { MN54 } & 6.100 & 0.300 & 4.920 & 0.400 & 1.240 & \text { W } & \text { A } \\ 1 & \text { SB125 } & 9.900 & 0.500 & 8.890 & 0.550 & 1.114 & \text { A } & \text { A }\end{array}$

Matrix: SO Soil $\mathrm{Bq} / \mathrm{kg}$

$\begin{array}{ll}1 & \text { AM241 } \\ 1 & \text { CO60 } \\ 1 & \text { CS137 } \\ 1 & \text { K40 }\end{array}$

Matrix: VE Vegetation $\mathrm{Bq} / \mathrm{kg}$

$\begin{array}{ll}1 & \text { AM241 } \\ 1 & \text { CO60 } \\ 1 & \text { CS137 } \\ 1 & \text { K40 }\end{array}$

$\begin{array}{rr}9.000 & 0.600 \\ 1.100 & 0.400 \\ 1045.000 & 50.000 \\ 329.000 & 20.000\end{array}$

0.600

50.000

329.000

7.470
1.240
954.000
314.000

$$
\begin{array}{r}
0.410 \\
0.110 \\
38.000 \\
13.000
\end{array}
$$

$\begin{array}{rrr}2.000 & 0.300 & 2.330 \\ 19.800 & 1.200 & 20.000 \\ 412.000 & 25.000 & 390.000 \\ 496.000 & 22.000 & 460.000\end{array}$

1.205

0.887

1.095

1.048
A
A
A
A

A




\section{QAP 49 Results by Laboratory}

Lab: PR Princeton Plasma Physics Lab

\begin{tabular}{|c|c|c|c|c|c|c|}
\hline & $\begin{array}{l}\text { Reported } \\
\text { Value }\end{array}$ & $\begin{array}{l}\text { Reported } \\
\text { Error }\end{array}$ & $\begin{array}{l}\text { EML } \\
\text { Value }\end{array}$ & $\begin{array}{l}\text { EML } \\
\text { Error }\end{array}$ & $\frac{\text { Reported }}{\text { EML }}$ & $\begin{array}{c}\text { QAP 48 } \\
\text { Evaluation } \\
\text { Evaluation }\end{array}$ \\
\hline
\end{tabular}

Matrix: AI Air Filter Bq/filter

$\begin{array}{llrrrrrr}1 & \text { AM241 } & 0.629 & 0.191 & 0.510 & 0.008 & 1.234 & \mathrm{~A} \\ 1 & \text { CO60 } & 9.344 & 0.095 & 9.160 & 0.580 & 1.020 & \mathrm{~A} \\ 1 & \text { CS137 } & 21.873 & 0.290 & 22.470 & 1.030 & 0.973 & \mathrm{~A} \\ 1 & \text { MN54 } & 4.717 & 0.161 & 4.920 & 0.400 & 0.959 & \mathrm{~A} \\ 1 & \text { SB125 } & 9.289 & 0.691 & 8.890 & 0.550 & 1.045 & \mathrm{~A}\end{array}$

Matrix: WA Water $\mathrm{Bq} / \mathrm{L}$

$\begin{array}{llrrrrrrr}1 & \text { AM241 } & 1.536 & 0.004 & 1.250 & 0.080 & 1.229 & \text { A } & \\ 1 & \text { CO60 } & 53.189 & 0.308 & 49.400 & 1.200 & 1.077 & \text { A } & \\ 1 & \text { CS137 } & 52.087 & 0.284 & 50.000 & 1.700 & 1.042 & \text { A } & \text { A } \\ 1 & \text { H3 } & 81.950 & 0.850 & 76.200 & 2.900 & 1.075 & \text { A } & \text { A } \\ 1 & \text { MN54 } & 36.360 & 0.460 & 32.400 & 1.400 & 1.122 & \text { A } & \end{array}$

Values for elemental uranium are reported in $\mu \mathrm{g} / \mathrm{filter}, \mathrm{g}$, or $\mathrm{mL} . \quad \mathrm{pCi} / \mathrm{g}$ or $\mathrm{mL}=\mathrm{Bq} \times 0.027$

Evaluation: $A=$ Acceptable, $W=$ Acceptable with Warning, $N=$ Not Acceptable

If the evaluation system is not appropriate for the types of analyses performed in your lab, apply a site specific evaluation. 


\section{QAP 49 Results by Laboratory}

Lab: RA V. G. Khlopin Radium Institute, St. Petersburg, Russia

$\begin{array}{lccccc}\text { No. } & \begin{array}{c}\text { Reported } \\ \text { Test }\end{array} & \text { Radionuclide } & \begin{array}{c}\text { Reported } \\ \text { Value }\end{array} & \begin{array}{l}\text { EML } \\ \text { Error }\end{array} & \text { Value }\end{array} \quad \begin{aligned} & \text { EML } \\ & \text { Error }\end{aligned} \frac{\begin{array}{c}\text { Reported } \\ \text { EML }\end{array} \text { Evaluation Evaluation }}{\text { EAP 48 }}$

Matrix: AI Air Filter $\mathrm{Bq} /$ filter

$\begin{array}{llrrrrrrr}2 & \text { CO60 } & 9.360 & 0.220 & 9.160 & 0.580 & 1.022 & \mathrm{~A} & \mathrm{~A} \\ 1 & \text { CO60 } & 9.290 & 0.430 & 9.160 & 0.580 & 1.014 & \mathrm{~A} & \mathrm{~A} \\ 2 & \text { CS137 } & 23.300 & 0.870 & 22.470 & 1.030 & 1.037 & \mathrm{~A} & \mathrm{~A} \\ 1 & \text { CS137 } & 24.200 & 0.650 & 22.470 & 1.030 & 1.077 & \mathrm{~A} & \mathrm{~A} \\ 1 & \text { MN54 } & 5.070 & 0.070 & 4.920 & 0.400 & 1.030 & \mathrm{~A} & \mathrm{~A} \\ 2 & \text { MN54 } & 5.180 & 0.130 & 4.920 & 0.400 & 1.053 & \mathrm{~A} & \mathrm{~A} \\ 1 & \text { SB125 } & 9.750 & 0.330 & 8.890 & 0.550 & 1.097 & \mathrm{~A} & \mathrm{~A} \\ 2 & \text { SB125 } & 9.920 & 0.380 & 8.890 & 0.550 & 1.116 & \mathrm{~A} & \mathrm{~A} \\ 1 & \text { U234 } & 285.000 & 28.000 & 0.260 & 0.010 & 1,096.154 & \mathrm{~N} & \mathrm{~A} \\ 1 & \text { U238 } & 280.000 & 28.000 & 0.260 & 0.010 & 1,076.923 & \mathrm{~N} & \mathrm{~A} \\ 1 & \text { ug U } & 22.400 & 1.700 & 20.960 & 0.100 & 1.069 & \mathrm{~A} & \mathrm{~A}\end{array}$

Matrix: SO Soil $\mathrm{Bq} / \mathrm{kg}$

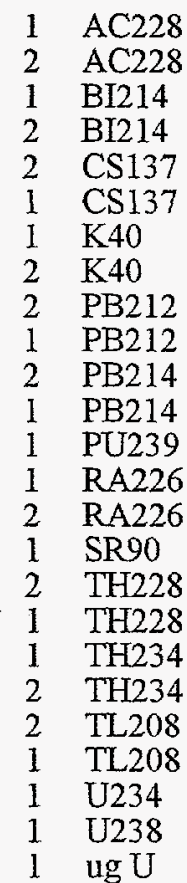

51.400
52.500
27.500
30.600
1030.000
1020.000
404.000
425.000
53.100
53.000
25.900
28.700
16.700
27.300
29.000
45.000
50.300
47.000
160.000
140.000
16.700
16.500
108.000
107.000
8.600

$$
\begin{array}{r}
52.600 \\
52.600 \\
28.800 \\
28.800 \\
954.000 \\
954.000 \\
314.000 \\
314.000 \\
52.800 \\
52.800 \\
29.100 \\
29.100 \\
13.090 \\
29.000 \\
29.000 \\
39.630 \\
52.700 \\
52.700 \\
114.000 \\
114.000 \\
18.300 \\
18.300 \\
113.000 \\
120.000 \\
9.700
\end{array}
$$

$$
\begin{array}{r}
2.900 \\
2.900 \\
0.500 \\
0.500 \\
38.000 \\
38.000 \\
13.000 \\
13.000 \\
3.700 \\
3.700 \\
1.200 \\
1.200 \\
0.570 \\
1.000 \\
1.000 \\
0.003 \\
4.000 \\
4.000 \\
6.000 \\
6.000 \\
1.100 \\
1.100 \\
6.000 \\
9.000 \\
0.700
\end{array}
$$

$\begin{array}{lll}0.977 & \mathrm{~A} & \\ 0.998 & \mathrm{~A} & \\ 0.955 & \mathrm{~A} & \\ 1.063 & \mathrm{~A} & \\ 1.080 & \mathrm{~A} & \mathrm{~W} \\ 1.069 & \mathrm{~A} & \mathrm{~W} \\ 1.287 & \mathrm{~W} & \mathrm{~W} \\ 1.354 & \mathrm{~W} & \mathrm{~W} \\ 1.006 & \mathrm{~A} & \\ 1.004 & \mathrm{~A} & \\ 0.890 & \mathrm{~A} & \\ 0.986 & \mathrm{~A} & \\ 1.276 & \mathrm{~W} & \mathrm{~W} \\ 0.941 & \mathrm{~A} & \\ 1.000 & \mathrm{~A} & \\ 1.136 & \mathrm{~A} & \mathrm{~A} \\ 0.954 & \mathrm{~A} & \\ 0.892 & \mathrm{~A} & \\ 1.404 & \mathrm{~A} & \\ 1.228 & \mathrm{~A} & \\ 0.913 & \mathrm{~A} & \\ 0.902 & \mathrm{~A} & \\ 0.956 & \mathrm{~A} & \mathrm{~A} \\ 0.892 & \mathrm{~A} & \mathrm{~A} \\ 0.887 & \mathrm{~A} & \mathrm{~A}\end{array}$

Matrix: VE Vegetation $\mathrm{Bq} / \mathrm{kg}$

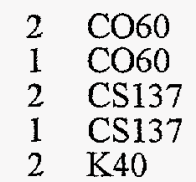

19.900
19.400
407.000
408.000
555.000

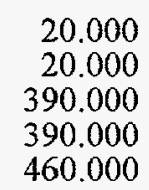

0.995
0.970
1.044
1.046
1.207

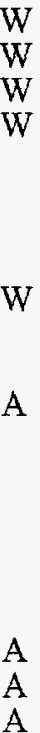

A

A

Values for elemental uranium are reported in $\mu \mathrm{g} /$ filter, $\mathrm{g}$, or $\mathrm{mL}$.

$\mathrm{pCi} / \mathrm{g}$ or $\mathrm{mL}=\mathrm{Bq} \times 0.027$

Evaluation: $A=$ Acceptable, $W=$ Acceptable with Warning, $N=$ Not Acceptable

If the evaluation system is not appropriate for the types of analyses performed in your lab, apply a site specific evaluation. 


\section{QAP 49 Results by Laboratory}

Lab: RA V. G. Khlopin Radium Institute, St. Petersburg, Russia

\begin{tabular}{|c|c|c|c|c|c|c|c|}
\hline o. & Radionuclide & $\begin{array}{l}\text { Reported } \\
\text { Value }\end{array}$ & $\begin{array}{l}\text { Reported } \\
\text { Error }\end{array}$ & $\begin{array}{l}\text { EML } \\
\text { Value }\end{array}$ & $\begin{array}{l}\text { EML } \\
\text { Error }\end{array}$ & $\frac{\text { Reported }}{\text { EML }}$ & $\begin{array}{cc}\text { QAP 48 } \\
\text { Evaluation } \\
\text { Evaluation }\end{array}$ \\
\hline
\end{tabular}

Matrix: VE Vegetation $\mathrm{Bq} / \mathrm{kg}$

$\begin{array}{llrrrrrrr}1 & \text { K40 } & 532.000 & 69.000 & 460.000 & 20.000 & 1.157 & \text { A } & \text { A } \\ 1 & \text { PU238 } & 0.300 & 0.100 & 0.310 & 0.070 & 0.968 & \text { A } & \text { A } \\ 1 & \text { PU239 } & 4.400 & 0.700 & 3.720 & 0.270 & 1.183 & \text { A } & \text { W } \\ 1 & \text { SR90 } & 590.000 & 100.000 & 606.000 & 40.000 & 0.974 & \text { A } & \text { A }\end{array}$

Values for elemental uranium are reported in $\mu \mathrm{g} /$ filter, $\mathrm{g}$, or $\mathrm{mL}$.

$\mathrm{pCi} / \mathrm{g}$ or $\mathrm{mL}=\mathrm{Bq} \times 0.027$

Evaluation: $A=A c c e p t a b l e, W=A c c e p t a b l e$ with Warning, $N=$ Not Acceptable

If the evaluation system is not appropriate for the types of analyses performed in your lab, apply a site specific evaluation. 


\section{QAP 49 Results by Laboratory}

Lab: RC US NRC Region I Laboratory, PA

\begin{tabular}{|c|c|c|c|c|c|c|c|c|}
\hline $\begin{array}{l}\text { No. } \\
\text { Test }\end{array}$ & Radionuclide & $\begin{array}{c}\text { Reported } \\
\text { Value } \\
\end{array}$ & $\begin{array}{c}\text { Reported } \\
\text { Error }\end{array}$ & $\begin{array}{l}\text { EML } \\
\text { Value } \\
\end{array}$ & $\begin{array}{l}\text { EML } \\
\text { Error } \\
\end{array}$ & $\frac{\text { Reported }}{\text { EML }}$ & Evaluation & $\begin{array}{r}\text { QA } \\
\text { Eval }\end{array}$ \\
\hline \multicolumn{9}{|c|}{ Matrix: AI Air Filter Bq / filter } \\
\hline $\begin{array}{l}1 \\
1 \\
1 \\
1 \\
1 \\
1\end{array}$ & $\begin{array}{l}\text { CO60 } \\
\text { CS137 } \\
\text { GROSS ALPHA } \\
\text { GROSS BETA } \\
\text { MN54 } \\
\text { SB125 }\end{array}$ & $\begin{array}{r}9.160 \\
22.500 \\
1.810 \\
1.960 \\
5.200 \\
9.060\end{array}$ & $\begin{array}{l}0.650 \\
1.200 \\
0.130 \\
0.140 \\
0.460 \\
0.880\end{array}$ & $\begin{array}{r}9.160 \\
22.470 \\
1.650 \\
2.160 \\
4.920 \\
8.890\end{array}$ & $\begin{array}{l}0.580 \\
1.030 \\
0.160 \\
0.070 \\
0.400 \\
0.550\end{array}$ & $\begin{array}{l}1.000 \\
1.001 \\
1.097 \\
0.907 \\
1.057 \\
1.019\end{array}$ & $\begin{array}{l}\mathrm{A} \\
\mathrm{A} \\
\mathrm{A} \\
\mathrm{A} \\
\mathrm{A} \\
\mathrm{A}\end{array}$ & $\begin{array}{l}\mathrm{A} \\
\mathrm{A} \\
\mathrm{A} \\
\mathrm{A} \\
\mathrm{A} \\
\mathrm{A}\end{array}$ \\
\hline
\end{tabular}

Matrix: $\mathrm{SO}$ Soil $\mathrm{Bq} / \mathrm{kg}$

$\begin{array}{ll}1 & \text { CO60 } \\ 1 & \text { CS137 } \\ 1 & \text { K } 40\end{array}$

1.920

980.000

352.000
0.410

67.000

19.000

\subsection{0}

954.000

314.000
0.110
38.000
13.000
1.548

1.027

1.121
QAP 48

valuation

Matrix: WA Water $\mathrm{Bq} / \mathrm{L}$

$\begin{array}{lllllllll}1 & \text { CO60 } & 48.500 & 2.500 & 49.400 & 1.200 & 0.982 & \mathrm{~A} & \mathrm{~A} \\ 1 & \text { CS137 } & 48.700 & 2.500 & 50.000 & 1.700 & 0.974 & \mathrm{~A} & \mathrm{~A} \\ 1 & \mathrm{H} 3 & 72.000 & 5.000 & 76.200 & 2.900 & 0.945 & \mathrm{~A} & \mathrm{~A} \\ 1 & \text { MN54 } & 32.800 & 1.700 & 32.400 & 1.400 & 1.012 & \mathrm{~A} & \mathrm{~A}\end{array}$

Values for elemental uranium are reported in $\mu \mathrm{g} /$ filter, $g$, or $\mathrm{mL}$.

$\mathrm{pCi} / \mathrm{g}$ or $\mathrm{mL}=\mathrm{Bq} \times 0.027$

Evaluation: $A=$ Acceptable,$W=A c c e p t a b l e$ with Warning, $N=$ Not Acceptable

If the evaluation system is not appropriate for the types of analyses performed in your lab, apply a site specific evaluation. 


\section{QAP 49 Results by Laboratory}

Lab: RE Bechtel Nevada, Mercury, NV

\begin{tabular}{|c|c|c|c|c|c|c|c|}
\hline $\begin{array}{l}\text { vo. } \\
\text { Test }\end{array}$ & adi & $\begin{array}{c}\text { Reported } \\
\text { Value }\end{array}$ & $\begin{array}{c}\text { Reported } \\
\text { Error }\end{array}$ & $\begin{array}{l}\text { EML } \\
\text { Value }\end{array}$ & $\begin{array}{l}\text { EML } \\
\text { Error }\end{array}$ & $\frac{\text { Reporte }}{\text { EML }}$ & $\begin{array}{cc}\text { QAP 48 } \\
\text { Evaluation } \\
\text { Evaluation }\end{array}$ \\
\hline
\end{tabular}

Matrix: $\mathrm{AI}$ Air Filter $\mathrm{Bq} /$ filter

$\begin{array}{llrrrrrrr}1 & \text { AM241 } & 0.515 & 0.039 & 0.510 & 0.008 & 1.010 & \mathrm{~A} & \mathrm{~A} \\ 1 & \text { CO60 } & 8.820 & 0.910 & 9.160 & 0.580 & 0.963 & \mathrm{~A} & \mathrm{~A} \\ 1 & \text { CS137 } & 21.300 & 1.900 & 22.470 & 1.030 & 0.948 & \mathrm{~A} & \mathrm{~A} \\ 1 & \text { MN54 } & 5.180 & 0.570 & 4.920 & 0.400 & 1.053 & \mathrm{~A} & \mathrm{~A} \\ 1 & \text { PU238 } & 0.403 & 0.033 & 0.460 & 0.005 & 0.876 & \mathrm{~W} & \mathrm{~A} \\ 1 & \text { PU239 } & 0.382 & 0.032 & 0.420 & 0.006 & 0.910 & \mathrm{~A} & \mathrm{~A} \\ 1 & \text { SB125 } & 8.390 & 1.010 & 8.890 & 0.550 & 0.944 & \mathrm{~A} & \mathrm{~A} \\ 1 & \text { SR90 } & 1.320 & 0.060 & 1.120 & 0.050 & 1.179 & \mathrm{~A} & \mathrm{~A} \\ 1 & \text { U234 } & 0.254 & 0.023 & 0.260 & 0.010 & 0.977 & \mathrm{~A} & \mathrm{~A} \\ 1 & \text { U238 } & 0.267 & 0.024 & 0.260 & 0.010 & 1.027 & \mathrm{~A} & \mathrm{~A}\end{array}$

Matrix: SO Soil $\mathrm{Bq} / \mathrm{kg}$

$\begin{array}{ll}1 & \text { AM241 } \\ 1 & \text { CS137 } \\ 1 & \text { K40 } \\ 1 & \text { PU239 } \\ 1 & \text { RA226 } \\ 1 & \text { SR90 } \\ 1 & \text { TH228 } \\ 1 & \text { U234 } \\ 1 & \text { U238 }\end{array}$

Matrix: VE Vegetation $\mathrm{Bq} / \mathrm{kg}$

$\begin{array}{rr}2.560 & 0.280 \\ 1.830 & 0.210 \\ 19.300 & 2.700 \\ 389.000 & 32.000 \\ 599.000 & 65.000 \\ 3.840 & 0.430 \\ 587.000 & 8.000\end{array}$

$\begin{array}{rrr}6.700 & 0.870 & 7.470 \\ 967.000 & 72.000 & 954.000 \\ 343.000 & 32.000 & 314.000 \\ 14.100 & 1.700 & 13.090 \\ 28.400 & 3.400 & 29.000 \\ 47.300 & 3.500 & 39.630 \\ 54.200 & 5.300 & 52.700 \\ 107.000 & 15.000 & 113.000 \\ 116.000 & 16.000 & 120.000\end{array}$

7.470

0.410

38.000

13.000

0.570

1.000

0.003

4.000

6.000

9.000
0.897

1.014

1.092

1.077

0.979

1.194

1.028

0.947

0.967

$\begin{array}{ll}\text { A } & \text { A } \\ \text { A } & \text { A } \\ \text { A } & \text { A } \\ \text { A } & \text { A } \\ \text { A } & \\ \text { A } & \text { A } \\ \text { A } & \\ \text { A } & \text { A } \\ \text { A } & \text { A }\end{array}$

1.099

1.040

0.965

0.997

1.302

1.032

0.969

$\begin{array}{ll}\text { A } & \text { A } \\ \text { A } & \\ \text { A } & \text { A } \\ \text { A } & \text { A } \\ \text { W } & \text { A } \\ \text { A } & \text { A } \\ \text { A } & \text { A }\end{array}$

Matrix: WA Water $\mathrm{Bq} / \mathrm{L}$

$\begin{array}{llr}1 & \text { AM241 } & 1.320 \\ 1 & \text { CO60 } & 51.600 \\ 1 & \text { CS137 } & 51.800 \\ 1 & \text { GROSS ALPHA } & 1070.000 \\ 1 & \text { GROSS BETA } & 1250.000 \\ 1 & \text { H3 } & 83.700 \\ 1 & \text { MN54 } & 37.300 \\ 1 & \text { PU238 } & 1.010 \\ 1 & \text { PU239 } & 1.410 \\ 1 & \text { SR90 } & 2.260 \\ 1 & \text { U234 } & 0.487\end{array}$

$$
\begin{array}{r}
0.120 \\
5.200 \\
5.100 \\
36.000 \\
29.000 \\
20.100 \\
3.900 \\
0.110 \\
0.140 \\
0.210 \\
0.058
\end{array}
$$

1.250
49.400
50.000
1080.000
1420.000
76.200
32.400
1.100
1.410
2.110
0.510

0.080
1.200
1.700
60.000
60.000
2.900
1.400
0.010
0.040
0.180
0.030
1.056
1.045
1.036
0.991
0.880
1.098
1.151
0.918
1.000
1.071
0.955

$\begin{array}{ll}\mathrm{A} & \mathrm{A} \\ \mathrm{A} & \mathrm{A} \\ \mathrm{A} & \mathrm{A} \\ \mathrm{A} & \mathrm{A} \\ \mathrm{A} & \mathrm{A} \\ \mathrm{A} & \mathrm{A} \\ \mathrm{A} & \mathrm{A} \\ \mathrm{A} & \mathrm{N} \\ \mathrm{A} & \mathrm{N} \\ \mathrm{A} & \mathrm{A} \\ \mathrm{A} & \mathrm{A}\end{array}$

\footnotetext{
Values for elemental uranium are reported in $\mu \mathrm{g} /$ filter, $\mathrm{g}$, or $\mathrm{mL}$.
}

$\mathrm{pCi} / \mathrm{g}$ or $\mathrm{mL}=\mathrm{Bq} \times 0.027$

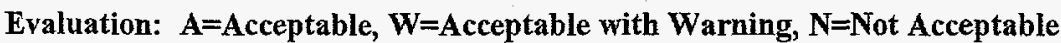

If the evaluation system is not appropriate for the types of analyses performed in your lab, apply a site specific evaluation. 


\section{QAP 49 Results by Laboratory}

Lab: RE Bechtel Nevada, Mercury, NV

No.

$\begin{array}{ccc}\text { Reported } & \text { Reported } & \text { EML } \\ \text { Value } & \text { Error } & \text { Value }\end{array}$

Value

EML

Error

Reported

EML

QAP 48

Test Radionuclide Value Error

Matrix: WA Water $\mathrm{Bq} / \mathrm{L}$

$1 \quad \mathrm{U} 238$

0.508

0.061

0.520

0.050

0.977

A

A

Values for elemental uranium are reported in $\mu \mathrm{g} /$ filter, $\mathrm{g}$, or $\mathrm{mL}$. $\mathrm{pCi} / \mathrm{g}$ or $\mathrm{mL}=\mathrm{Bq} \times 0.027$

Evaluation: $A=$ Acceptable, $W=$ Acceptable with Warning, $N=$ Not Acceptable If the evaluation system is not appropriate for the types of analyses performed in your lab, apply a site specific evaluation. 


\section{QAP 49 Results by Laboratory}

Lab: RG Thermo Nutech Rocky Flats Plant, Golden

\begin{tabular}{|c|c|c|c|c|c|c|c|}
\hline o. & Radionuclide & $\begin{array}{l}\text { Reported } \\
\text { Value }\end{array}$ & $\begin{array}{c}\text { Reported } \\
\text { Error }\end{array}$ & $\begin{array}{l}\text { EML } \\
\text { Value }\end{array}$ & $\begin{array}{l}\text { EML } \\
\text { Error }\end{array}$ & $\frac{\text { Reported }}{\text { EML }}$ & Evaluation Evalu \\
\hline
\end{tabular}

Matrix: WA Water $\mathrm{Bq} / \mathrm{L}$

$\begin{array}{lllllllll}1 & \text { GROSS ALPHA } & 1027.200 & 62.300 & 1080.000 & 60.000 & 0.951 & \text { A } & \text { A } \\ 1 & \text { GROSS BETA } & 1271.200 & 56.000 & 1420.000 & 60.000 & 0.895 & \text { A } & \text { A }\end{array}$

Values for elemental uranium are reported in $\mu \mathrm{g} /$ filter, $\mathrm{g}$, or $\mathrm{mL} . \quad \mathrm{pCi} / \mathrm{g}$ or $\mathrm{mL}=\mathrm{Bq} \times 0.027$

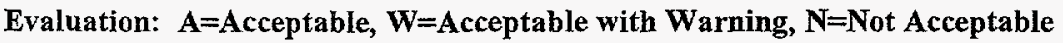

If the evaluation system is not appropriate for the types of analyses performed in your lab, apply a site specific evaluation. 


\section{QAP 49 Results by Laboratory}

Lab: RI Waste Management Services of Hanford, Inc., 222S Lab

\begin{tabular}{|c|c|c|c|c|c|c|c|}
\hline $\begin{array}{l}\text { No. } \\
\text { Test }\end{array}$ & Radionuclide & $\begin{array}{c}\text { Reported } \\
\text { Value }\end{array}$ & $\begin{array}{l}\text { Reported } \\
\text { Error }\end{array}$ & $\begin{array}{l}\text { EML } \\
\text { Value }\end{array}$ & $\begin{array}{l}\text { EML } \\
\text { Error }\end{array}$ & $\frac{\text { Reported }}{\text { EML }}$ & $\begin{array}{c}\text { QAP 48 } \\
\text { Evaluation } \\
\text { Evaluation }\end{array}$ \\
\hline
\end{tabular}

Matrix: AI Air Filter $\mathrm{Bq} /$ filter

$\begin{array}{llrrrrrrr}1 & \text { AM241 } & 0.466 & 0.032 & 0.510 & 0.008 & 0.914 & \text { A } & \text { A } \\ 1 & \text { CO60 } & 8.000 & 0.782 & 9.160 & 0.580 & 0.873 & \text { A } & \text { A } \\ 1 & \text { CS137 } & 18.300 & 1.480 & 22.470 & 1.030 & 0.814 & \text { W } & \text { A } \\ 1 & \text { MN54 } & 4.770 & 0.717 & 4.920 & 0.400 & 0.970 & \text { A } & \text { A } \\ 1 & \text { PU238 } & 0.522 & 0.038 & 0.460 & 0.005 & 1.135 & \text { A } & \text { A } \\ 1 & \text { PU239 } & 0.648 & 0.045 & 0.420 & 0.006 & 1.543 & \text { N } & \text { W } \\ 1 & \text { SB125 } & 10.300 & 1.670 & 8.890 & 0.550 & 1.159 & \text { A } & \text { A } \\ 1 & \text { SR90 } & 1.070 & 0.076 & 1.120 & 0.050 & 0.955 & \text { A } & \text { A }\end{array}$

Matrix: SO Soil $\mathrm{Bq} / \mathrm{kg}$

$\begin{array}{lllllllll}1 & \mathrm{CS} 137 & 1080.000 & 30.300 & 954.000 & 38.000 & 1.132 & \mathrm{~A} & \mathrm{~A}\end{array}$

Matrix: VE Vegetation $\mathrm{Bq} / \mathrm{kg}$

$\begin{array}{llrrrrrrr}1 & \text { CO60 } & 35.000 & 6.740 & 20.000 & 1.000 & 1.750 & \mathrm{~N} & \mathrm{~N} \\ 1 & \text { CS137 } & 459.000 & 23.600 & 390.000 & 20.000 & 1.177 & \mathrm{~A} & \mathrm{~A} \\ 1 & \text { SR90 } & 292.000 & 16.900 & 606.000 & 40.000 & 0.482 & \mathrm{~N} & \mathrm{~A}\end{array}$

Matrix: WA Water $\mathrm{Bq} / \mathrm{L}$

$\begin{array}{llrrrrrrr}1 & \text { AM241 } & 1.300 & 0.104 & 1.250 & 0.080 & 1.040 & \mathrm{~A} & \mathrm{~A} \\ 1 & \text { CO60 } & 52.100 & 3.590 & 49.400 & 1.200 & 1.055 & \mathrm{~A} & \mathrm{~N} \\ 1 & \text { CS137 } & 62.800 & 4.860 & 50.000 & 1.700 & 1.256 & \mathrm{~W} & \mathrm{~A} \\ 1 & \text { MN54 } & 33.700 & 4.250 & 32.400 & 1.400 & 1.040 & \mathrm{~A} & \mathrm{~A} \\ 1 & \text { PU238 } & 1.390 & 0.116 & 1.100 & 0.010 & 1.264 & \mathrm{~N} & \mathrm{~A} \\ 1 & \text { PU239 } & 1.630 & 0.129 & 1.410 & 0.040 & 1.156 & \text { W } & \mathrm{A} \\ 1 & \text { SR90 } & 2.410 & 0.245 & 2.110 & 0.180 & 1.142 & \mathrm{~A} & \mathrm{~A}\end{array}$

Values for elemental uranium are reported in $\mu \mathrm{g} /$ filter, $\mathrm{g}$, or $\mathrm{mL} . \quad$ pCi/g or $\mathrm{mL}=\mathrm{Bq} \times 0.027$

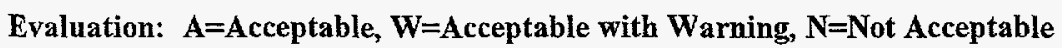

If the evaluation system is not appropriate for the types of analyses performed in your lab, apply a site specific evaluation. 


\section{QAP 49 Results by Laboratory}

Lab: RK Rock Island Arsenal, Illinois

No.

Reported Reported

EML

EML

Reported

Error EML

QAP 48

Test Radionuclide

Value

Error

Value

EML

Evaluation Evaluation

Matrix: AI Air Filter $\mathrm{Bq} /$ filter

$\begin{array}{lllllllll}1 & \text { GROSS ALPHA } & 1.300 & 0.150 & 1.650 & 0.160 & 0.788 & \text { W } & \text { W } \\ 1 & \text { GROSS BETA } & 2.190 & 0.140 & 2.160 & 0.070 & 1.014 & \text { A } & \text { A }\end{array}$

Values for elemental uranium are reported in $\mu \mathrm{g} /$ filter, $\mathrm{g}$, or $\mathrm{mL}$.

$\mathrm{pCi} / \mathrm{g}$ or $\mathrm{mL}=\mathrm{Bq} \times 0.027$

Evaluation: $A=$ Acceptable, $W=$ Acceptable with Warning, $N=$ Not Acceptable

If the evaluation system is not appropriate for the types of analyses performed in your lab, apply a site specific evaluation. 


\section{QAP 49 Results by Laboratory}

Lab: SA Sandia Labs Radioactive Sample Diag. Prog., NM

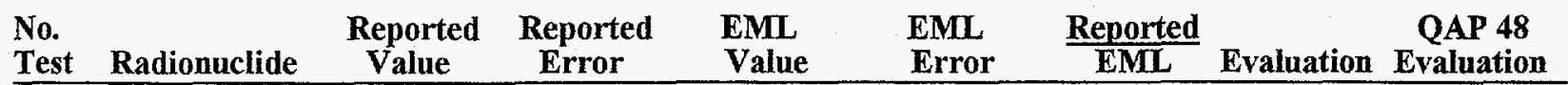

Matrix: AI Air Filter Bq / filter

\begin{tabular}{|c|c|c|c|c|c|c|}
\hline $\mathrm{CO} 60$ & 9.400 & 1.200 & 9.160 & 0.580 & 1.026 & A \\
\hline $\mathrm{CS} 137$ & 23.000 & 2.900 & 22.470 & 1.030 & 1.024 & A \\
\hline GROSS ALPHA & 1.840 & 0.240 & 1.650 & 0.160 & 1.115 & A \\
\hline GROSS BETA & 2.040 & 0.250 & 2.160 & 0.070 & 0.944 & A \\
\hline MN54 & 5.500 & 0.700 & 4.920 & 0.400 & 1.118 & A \\
\hline SB125 & 8.100 & 1.100 & 8.890 & 0.550 & 0.911 & A \\
\hline ug U & 23.300 & 1.200 & 20.960 & 0.100 & 1.112 & A \\
\hline
\end{tabular}

Matrix: SO Soil $\mathrm{Bq} / \mathrm{kg}$

$\begin{array}{lllllllll}1 & \mathrm{CS} 137 & 990.000 & 29.000 & 954.000 & 38.000 & 1.038 & \mathrm{~A} & \mathrm{~A} \\ 1 & \mathrm{~K} 40 & 353.000 & 16.000 & 314.000 & 13.000 & 1.124 & \mathrm{~A} & \mathrm{~A}\end{array}$

Matrix: WA Water $\mathrm{Bq} / \mathrm{L}$

$\begin{array}{lllllllll}1 & \text { CO60 } & 53.000 & 2.400 & 49.400 & 1.200 & 1.073 & \mathrm{~A} & \mathrm{~A} \\ 1 & \text { CS137 } & 53.000 & 2.800 & 50.000 & 1.700 & 1.060 & \mathrm{~A} & \mathrm{~A} \\ 1 & \text { MN54 } & 38.000 & 1.800 & 32.400 & 1.400 & 1.173 & \text { W } & \text { A }\end{array}$

Values for elemental uranium are reported in $\mu \mathrm{g} /$ filter, $\mathrm{g}$, or $\mathrm{mL}$.

$\mathrm{pCi} / \mathrm{g}$ or $\mathrm{mL}=\mathrm{Bq} \times 0.027$

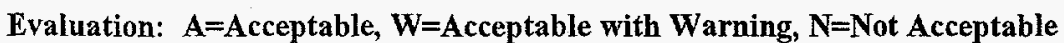

If the evaluation system is not appropriate for the types of analyses performed in your lab, apply a site specific evaluation. 


\section{QAP 49 Results by Laboratory}

Lab: SB SC Dept. of Health and Environment Control Radiological Lab

\begin{tabular}{|c|c|c|c|c|c|c|}
\hline Radionuclide & $\begin{array}{l}\text { Reported } \\
\text { Value }\end{array}$ & $\begin{array}{l}\text { Reported } \\
\text { Error }\end{array}$ & $\begin{array}{l}\text { EML } \\
\text { Value }\end{array}$ & $\begin{array}{l}\text { EML } \\
\text { Error }\end{array}$ & $\frac{\text { Reported }}{\text { EML }}$ & $\begin{array}{cc}\text { QAP 48 } \\
\text { Evaluation } \\
\text { Evaluation }\end{array}$ \\
\hline
\end{tabular}

Matrix: $\mathrm{AI}$ Air Filter $\mathrm{Bq} /$ filter

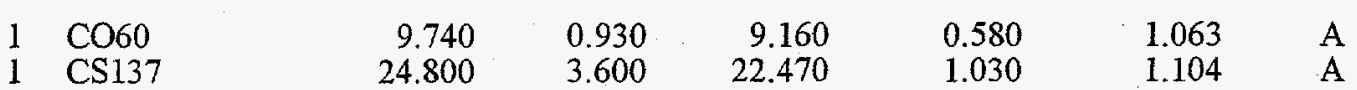

Matrix: SO Soil $\mathrm{Bq} / \mathrm{kg}$

$\begin{array}{llrrrrrr}1 & \mathrm{CS} 137 & 1031.000 & 128.000 & 954.000 & 38.000 & 1.081 & \mathrm{~A} \\ 1 & \mathrm{~K} 40 & 372.000 & 57.000 & 314.000 & 13.000 & 1.185 & \mathrm{~A}\end{array}$

Matrix: VE Vegetation $\mathrm{Bq} / \mathrm{kg}$

$\begin{array}{llrrrrrr}1 & \text { CO60 } & 21.200 & 2.810 & 20.000 & 1.000 & 1.060 & \mathrm{~A} \\ 1 & \text { CS137 } & 429.000 & 53.600 & 390.000 & 20.000 & 1.100 & \mathrm{~A} \\ 1 & \text { K40 } & 538.000 & 72.800 & 460.000 & 20.000 & 1.170 & \mathrm{~A}\end{array}$

Matrix: WA Water $\mathrm{Bq} / \mathrm{L}$

$\begin{array}{llrrrrrr}1 & \text { AM241 } & 1.390 & 0.780 & 1.250 & 0.080 & 1.112 & \text { A } \\ 1 & \text { CO60 } & 59.100 & 5.410 & 49.400 & 1.200 & 1.196 & \text { W } \\ 1 & \text { CS137 } & 61.600 & 8.890 & 50.000 & 1.700 & 1.232 & \text { W } \\ 1 & \text { H3 } & 81.500 & 6.700 & 76.200 & 2.900 & 1.070 & \text { A } \\ 1 & \text { MN54 } & 42.100 & 4.870 & 32.400 & 1.400 & 1.299 & \text { N }\end{array}$

Values for elemental uranium are reported in $\mu \mathrm{g} / \mathrm{filter}, \mathrm{g}$, or $\mathrm{mL} . \quad \mathrm{pCi} / \mathrm{g}$ or $\mathrm{mL}=\mathrm{Bq} \times 0.027$

Evaluation: $A=$ Acceptable, $W=$ Acceptable with Warning, $N=$ Not Acceptable

If the evaluation system is not appropriate for the types of analyses performed in your lab, apply a site specific evaluation. 


\section{QAP 49 Results by Laboratory}

Lab: SK Savannah River Plant

\begin{tabular}{|c|c|c|c|c|c|c|c|}
\hline $\begin{array}{l}\text { No. } \\
\text { Test }\end{array}$ & Radionuclide & $\begin{array}{l}\text { Reported } \\
\text { Value }\end{array}$ & $\begin{array}{c}\text { Reported } \\
\text { Error }\end{array}$ & $\begin{array}{l}\text { EML } \\
\text { Value }\end{array}$ & $\begin{array}{l}\text { EML } \\
\text { Error }\end{array}$ & $\frac{\text { Reporte }}{\text { EML }}$ & $\begin{array}{c}\text { QAP 48 } \\
\text { Evaluation Evaluation }\end{array}$ \\
\hline
\end{tabular}

Matrix: AI Air Filter Bq/filter

$\begin{array}{llrlrllll}1 & \text { AM241 } & 0.650 & 0.050 & 0.510 & 0.008 & 1.275 & \mathrm{~A} & \mathrm{~A} \\ 1 & \text { CO60 } & 8.600 & 0.900 & 9.160 & 0.580 & 0.939 & \mathrm{~A} & \mathrm{~A} \\ 1 & \text { CS137 } & 21.000 & 2.000 & 22.470 & 1.030 & 0.935 & \mathrm{~A} & \mathrm{~A} \\ 1 & \text { MN54 } & 4.700 & 0.400 & 4.920 & 0.400 & 0.955 & \mathrm{~A} & \mathrm{~A} \\ 1 & \text { SB125 } & 5.700 & 0.600 & 8.890 & 0.550 & 0.641 & \text { W } & \text { A }\end{array}$

Matrix: SO Soil $\mathrm{Bq} / \mathrm{kg}$

$\begin{array}{ll}1 & \text { AC228 } \\ 1 & \text { AM241 } \\ 1 & \text { B1212 } \\ 1 & \text { B1214 } \\ 1 & \text { CO60 } \\ 1 & \text { CS137 } \\ 1 & \text { K40 } \\ 1 & \text { PB212 } \\ 1 & \text { PB214 } \\ 1 & \text { RA226 } \\ 1 & \text { TH234 } \\ 1 & \text { TL208 }\end{array}$

57.000
10.200
59.000
38.000
1.740
1080.000
328.000
61.000
38.000
38.000
180.000
21.100

3.000
0.800
1.000
1.000
0.270
66.000
20.000
4.000
1.000
4.000
17.000
0.300

52.600

7.470

58.300

28.800

1.240

954.000

314.000

52.800

29.100

29.000

114.000

18.300

\subsection{0}

0.410

5.900

0.500

0.110

38.000

13.000

3.700

1.200

1.000

6.000

1.100

1.250
49.400
50.000
32.400
1.100
1.410

0.080
1.200
1.700
1.400
0.010
0.040

0.936

1.002

0.986

0.972

0.223

0.230

1.410
A
A
A
A
A

Matrix: WA Water $\mathrm{Bq} / \mathrm{L}$

$\begin{array}{ll}1 & \text { AM241 } \\ 1 & \text { CO60 } \\ 1 & \text { CS137 } \\ 1 & \text { MN54 } \\ 1 & \text { PU238 } \\ 1 & \text { PU239 }\end{array}$

$\begin{array}{rr}1.170 & 0.220 \\ 49.500 & 4.200 \\ 49.300 & 3.000 \\ 31.500 & 1.900 \\ 0.245 & 0.011 \\ 0.324 & 0.010\end{array}$

$\mathrm{A}$
$\mathrm{A}$
$\mathrm{A}$
$\mathrm{A}$
$\mathrm{N}$
$\mathrm{N}$

$\mathrm{A}$
$\mathrm{A}$
$\mathrm{A}$
$\mathrm{A}$
$\mathrm{A}$
$\mathrm{A}$

Values for elemental uranium are reported in $\mu \mathrm{g} /$ filter, $\mathrm{g}$, or $\mathrm{mL}$.

$\mathrm{pCi} / \mathrm{g}$ or $\mathrm{mL}=\mathrm{Bq} \times 0.027$

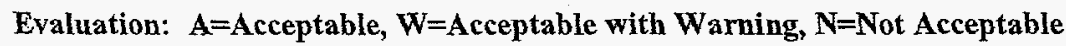

If the evaluation system is not appropriate for the types of analyses performed in your lab, apply a site specific evaluation. 


\section{QAP 49 Results by Laboratory}

Lab: SL Stanford Linear Accelerator Center

No. Reported Reported EML EML Reported

Test Radionuclide Value Error Value Error EML Evaluation Evaluation

Matrix: WA Water $\mathrm{Bq} / \mathrm{L}$

$\begin{array}{lllllllll}1 & \text { CO60 } & 49.000 & 3.000 & 49.400 & 1.200 & 0.992 & \mathrm{~A} & \mathrm{~A} \\ \mathrm{I} & \text { CS137 } & 50.000 & 3.000 & 50.000 & 1.700 & 1.000 & \mathrm{~A} & \mathrm{~A} \\ \mathrm{I} & \text { MN54 } & 34.000 & 2.000 & 32.400 & 1.400 & 1.049 & \mathrm{~A} & \mathrm{~A}\end{array}$

Values for elemental uranium are reported in $\mu \mathrm{g} /$ filter, $\mathrm{g}$, or $\mathrm{mL} . \quad \mathrm{pCi} / \mathrm{g}$ or $\mathrm{mL}=\mathrm{Bq} \times 0.027$

Evaluation: $A=$ Acceptable, $W=$ Acceptable with Warning, $N=$ Not Acceptable

If the evaluation system is not appropriate for the types of analyses performed in your lab, apply a site specific evaluation. 


\section{QAP 49 Results by Laboratory}

Lab: SN Sanford Cohen Associates, Inc., Montgomery, AL

\begin{tabular}{|c|c|c|c|c|c|c|c|}
\hline $\begin{array}{l}\text { No. } \\
\text { Test }\end{array}$ & Radionuclide & $\begin{array}{l}\text { Reported } \\
\text { Value }\end{array}$ & $\begin{array}{c}\text { Reported } \\
\text { Error }\end{array}$ & $\begin{array}{l}\text { EML } \\
\text { Value }\end{array}$ & $\begin{array}{l}\text { EML } \\
\text { Error }\end{array}$ & $\frac{\text { Reported }}{\text { EML }}$ & $\begin{array}{c}\text { QAP 48 } \\
\text { Evaluation Evaluation }\end{array}$ \\
\hline
\end{tabular}

Matrix: AI Air Filter $\mathrm{Bq} /$ filter

$\begin{array}{lllllllll}1 & \text { AM241 } & 0.590 & 0.084 & 0.510 & 0.008 & 1.157 & \text { A } & \text { A } \\ 1 & \text { PU238 } & 0.492 & 0.064 & 0.460 & 0.005 & 1.070 & \text { A } & \\ 1 & \text { PU239 } & 0.458 & 0.060 & 0.420 & 0.006 & 1.090 & \text { A } & \text { A }\end{array}$

Matrix: SO Soil $\mathrm{Bq} / \mathrm{kg}$

$\begin{array}{ll}1 & \text { AM241 } \\ 1 & \text { BI212 } \\ 1 & \text { BI214 } \\ 1 & \text { CS137 } \\ 1 & \text { K40 } \\ 1 & \text { PB212 } \\ 1 & \text { PB214 } \\ 1 & \text { PU239 } \\ 1 & \text { TL208 }\end{array}$

$\begin{array}{rr}7.736 & 3.269 \\ 37.259 & 7.767 \\ 31.988 & 4.330 \\ 1113.947 & 111.321 \\ 406.462 & 40.585 \\ 63.855 & 6.628 \\ 35.578 & 5.638 \\ 13.438 & 4.293 \\ 19.692 & 2.394\end{array}$

7.470
58.300
28.800
954.000
314.000
52.800
29.100
13.090
18.300

0.410
5.900
0.500
38.000
13.000
3.700
1.200
0.570
1.100

1.036

0.639

1.111

1.168

1.294

1.209

1.223

1.027

1.076

$\begin{array}{ll}\text { A } & \text { A } \\ \text { A } & \\ \text { A } & \\ \text { A } & \text { A } \\ \text { W } & \text { W } \\ \text { A } & \\ \text { A } & \\ \text { A } & \text { A } \\ \text { A } & \end{array}$

Matrix: VE Vegetation $\mathrm{Bq} / \mathrm{kg}$

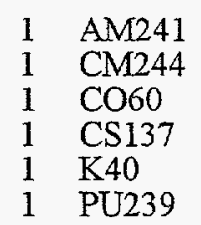

2.505
1.590
23.589
485.757
611.296
3.284

1.093
0.862
2.742
48.687
60.094
1.183

2.330
1.760
20.000
390.000
460.000
3.720

0.060
0.070
1.000
20.000
20.000
0.270
1.075
0.903
1.179
1.246
1.329
0.883

$\begin{array}{ll}\text { A } & \text { W } \\ \text { A } & \text { A } \\ \text { A } & \text { A } \\ \text { A } & \text { A } \\ \text { W } & \text { N } \\ \text { A } & \text { A }\end{array}$

Matrix: WA Water $\mathrm{Bq} / \mathrm{L}$

$\begin{array}{ll}1 & \text { AM241 } \\ 1 & \text { CO60 } \\ 1 & \text { CS137 } \\ 1 & \text { MN54 } \\ 1 & \text { PU238 } \\ 1 & \text { PU239 }\end{array}$

1.295
49.982
47.885
34.620
1.191
1.525

$\begin{array}{lr}0.202 & 1.250 \\ 4.477 & 49.400 \\ 4.280 & 50.000 \\ 3.681 & 32.400 \\ 0.189 & 1.100 \\ 0.232 & 1.410\end{array}$

0.080
1.200
1.700
1.400
0.010
0.040

1.036

1.012

0.958

1.069

1.083

1.082

$\begin{array}{ll}\mathrm{A} & \mathrm{A} \\ \mathrm{A} & \mathrm{A} \\ \mathrm{A} & \mathrm{A} \\ \mathrm{A} & \mathrm{A} \\ \mathrm{A} & \mathrm{A} \\ \mathrm{A} & \mathrm{A}\end{array}$

Values for elemental uranium are reported in $\mu \mathrm{g} /$ filter, $\mathrm{g}$, or $\mathrm{mL}$.

$\mathrm{pCi} / \mathrm{g}$ or $\mathrm{mL}=\mathrm{Bq} \times 0.027$

Evaluation: $A=A c c e p t a b l e, W=A c c e p t a b l e$ with Warning, $N=$ Not Acceptable

If the evaluation system is not appropriate for the types of analyses performed in your lab, apply a site specific evaluation. 


\section{QAP 49 Results by Laboratory}

Lab: SR Savannah River Environmental Laboratory

\begin{tabular}{llllll} 
No. & Reported \\
Test & Radionuclide & $\begin{array}{c}\text { Reported } \\
\text { Value }\end{array}$ & $\begin{array}{c}\text { EML } \\
\text { Ealue }\end{array}$ & $\begin{array}{c}\text { EML } \\
\text { Error }\end{array}$ & $\begin{array}{c}\text { Reported } \\
\text { EML }\end{array}$ \\
Evaluation Evaluation \\
\hline
\end{tabular}

Matrix: AI Air Filter $\mathrm{Bq} /$ filter

$\begin{array}{llrlrllll}1 & \text { AM241 } & 0.511 & 0.023 & 0.510 & 0.008 & 1.002 & \mathrm{~A} & \mathrm{~A} \\ 1 & \text { CO60 } & 8.760 & 0.158 & 9.160 & 0.580 & 0.956 & \mathrm{~A} & \mathrm{~A} \\ 1 & \text { CS137 } & 21.000 & 0.418 & 22.470 & 1.030 & 0.935 & \mathrm{~A} & \mathrm{~A} \\ 1 & \text { GROSS ALPHA } & 1.590 & 0.130 & 1.650 & 0.160 & 0.964 & \mathrm{~A} & \mathrm{~W} \\ 1 & \text { GROSS BETA } & 2.170 & 0.150 & 2.160 & 0.070 & 1.005 & \mathrm{~A} & \mathrm{~A} \\ 1 & \text { MN54 } & 4.870 & 0.144 & 4.920 & 0.400 & 0.990 & \mathrm{~A} & \mathrm{~A} \\ 1 & \text { PU238 } & 0.469 & 0.023 & 0.460 & 0.005 & 1.020 & \mathrm{~A} & \mathrm{~A} \\ 1 & \text { PU239 } & 0.434 & 0.024 & 0.420 & 0.006 & 1.033 & \mathrm{~A} & \mathrm{~A} \\ 1 & \text { SB125 } & 5.240 & 0.179 & 8.890 & 0.550 & 0.589 & \mathrm{~N} & \mathrm{~A} \\ 1 & \text { SR90 } & 1.240 & 0.150 & 1.120 & 0.050 & 1.107 & \mathrm{~A} & \mathrm{~W} \\ 1 & \text { U234 } & 0.262 & 0.018 & 0.260 & 0.010 & 1.008 & \mathrm{~A} & \mathrm{~A} \\ 1 & \text { U238 } & 0.264 & 0.018 & 0.260 & 0.010 & 1.015 & \mathrm{~A} & \mathrm{~A}\end{array}$

Matrix: SO Soil $\mathrm{Bq} / \mathrm{kg}$

$\begin{array}{ll}1 & \text { AC228 } \\ 1 & \text { BI212 } \\ 1 & \text { BI214 } \\ 1 & \text { CS137 } \\ 1 & \text { K40 } \\ 1 & \text { PB212 } \\ 1 & \text { PB214 } \\ 1 & \text { SR90 } \\ 1 & \text { TL208 }\end{array}$

$\begin{array}{rrr}61.400 & 1.600 & 52.600 \\ 40.800 & 3.600 & 58.300 \\ 31.400 & 1.200 & 28.800 \\ 1090.000 & 20.000 & 954.000 \\ 394.000 & 12.000 & 314.000 \\ 51.300 & 1.400 & 52.800 \\ 31.300 & 1.400 & 29.100 \\ 50.400 & 19.200 & 39.630 \\ 19.300 & 0.900 & 18.300\end{array}$

2.900
5.900
0.500
38.000
13.000
3.700
1.200
0.003
1.100

1.167

0.700

1.090

1.143

1.255

0.972

1.076

1.272

1.055

$\mathrm{A}$
$\mathrm{A}$
$\mathrm{A}$
$\mathrm{A}$
$\mathrm{W}$
$\mathrm{A}$
$\mathrm{A}$
$\mathrm{A}$
$\mathrm{A}$

Matrix: VE Vegetation $\mathrm{Bq} / \mathrm{kg}$

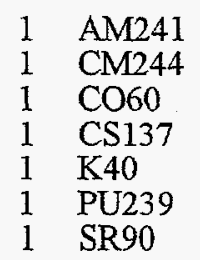

$\begin{array}{rr}3.142 & 0.525 \\ 2.200 & 0.440 \\ 23.900 & 0.700 \\ 460.000 & 8.800 \\ 563.000 & 16.600 \\ 3.986 & 0.655 \\ 754.800 & 51.800\end{array}$

$$
\begin{array}{r}
2.330 \\
1.760 \\
20.000 \\
390.000 \\
460.000 \\
3.720 \\
606.000
\end{array}
$$

0.060
0.070
1.000
20.000
20.000
0.270
40.000

$$
\begin{aligned}
& 1.348 \\
& 1.250 \\
& 1.195 \\
& 1.179 \\
& 1.224 \\
& 1.072 \\
& 1.246
\end{aligned}
$$

$\begin{array}{ll}\text { A } & \text { A } \\ \text { A } & \text { A } \\ \text { A } & \text { A } \\ \text { A } & \text { A } \\ \text { A } & \text { A } \\ \text { A } & \text { A } \\ \text { W } & \text { N }\end{array}$

Matrix: WA Water $\mathrm{Bq} / \mathrm{L}$

$\begin{array}{llr}1 & \text { AM241 } & 1.690 \\ 1 & \text { CO60 } & 50.600 \\ 1 & \text { CS137 } & 51.000 \\ 1 & \text { GROSS ALPHA } & 809.000 \\ 1 & \text { GROSS BETA } & 1816.000 \\ 1 & \text { H3 } & 77.300 \\ 1 & \text { MN54 } & 35.500 \\ 1 & \text { PU238 } & 1.310 \\ 1 & \text { PU239 } & 1.880\end{array}$

0.083
0.900
1.350
118.000
409.000
3.600
1.000
0.073
0.094

1.250
49.400
50.000
1080.000
1420.000
76.200
32.400
1.100
1.410

0.080
1.200
1.700
60.000
60.000
2.900
1.400
0.010
0.040

1.352
1.024
1.020
0.749
1.279
1.014
1.096
1.191
1.333

W
A
A
W
A
A
A
W
W

A A

Values for elemental uranium are reported in $\mu \mathrm{g} /$ filter, $\mathrm{g}$, or $\mathrm{mL}$.

$\mathrm{pCi} / \mathrm{g}$ or $\mathrm{mL}=\mathrm{Bq} \times 0.027$

Evaluation: $A=$ Acceptable, $W=$ Acceptable with Warning, $N=$ Not Acceptable

If the evaluation system is not appropriate for the types of analyses performed in your lab, apply a site specific evaluation. 


\section{QAP 49 Results by Laboratory}

Lab: SR Savannah River Environmental Laboratory

\begin{tabular}{|c|c|c|c|c|c|c|c|c|}
\hline $\begin{array}{l}\text { No. } \\
\text { Test }\end{array}$ & Radionuclide & $\begin{array}{c}\text { Reported } \\
\text { Value }\end{array}$ & $\begin{array}{c}\text { Reported } \\
\text { Error }\end{array}$ & $\begin{array}{l}\text { EML } \\
\text { Value }\end{array}$ & $\begin{array}{l}\text { EML } \\
\text { Error }\end{array}$ & $\frac{\text { Reported }}{\text { EML }}$ & Evaluation & $\begin{array}{r}\text { QA } \\
\text { Eval }\end{array}$ \\
\hline \multicolumn{9}{|c|}{ Matrix: WA Water $\mathrm{Bq} / \mathrm{L}$} \\
\hline $\begin{array}{l}1 \\
1 \\
1\end{array}$ & $\begin{array}{l}\text { SR90 } \\
\text { U234 } \\
\text { U238 }\end{array}$ & $\begin{array}{l}2.386 \\
0.547 \\
0.609\end{array}$ & $\begin{array}{l}0.721 \\
0.030 \\
0.037\end{array}$ & $\begin{array}{l}2.110 \\
0.510 \\
0.520\end{array}$ & $\begin{array}{l}0.180 \\
0.030 \\
0.050\end{array}$ & $\begin{array}{l}1.131 \\
1.073 \\
1.171\end{array}$ & $\begin{array}{l}\mathrm{A} \\
\mathrm{A} \\
\mathrm{W}\end{array}$ & $\begin{array}{l}\mathrm{A} \\
\mathrm{A} \\
\mathrm{A}\end{array}$ \\
\hline
\end{tabular}




\section{QAP 49 Results by Laboratory}

Lab: ST SC DHEC, Aiken, South Carolina

No.

Reported Reported EML Value

EML

Reported

QAP 48

Test Radionuclide Value Error Error EML Evaluation Evaluation

Matrix: WA Water $\mathrm{Bq} / \mathrm{L}$
$1 \mathrm{H} 3$
85.320
6.810
76.200
2,900
1.120
A
A

Values for elemental uranium are reported in $\mu \mathrm{g} /$ filter, $\mathrm{g}$, or $\mathrm{mL}$.

$\mathrm{pCi} / \mathrm{g}$ or $\mathrm{mL}=\mathrm{Bq} \times 0.027$

Evaluation: $A=A$ cceptable, $W=A c c e p t a b l e$ with $W$ arning, $N=$ Not Acceptable

If the evaluation system is not appropriate for the types of analyses performed in your lab, apply a site specific evaluation. 


\section{QAP 49 Results by Laboratory}

Lab: SW Southwest Research Institute, San Antonio, TX

\begin{tabular}{lccccc} 
No. & Reported \\
Test & Radionuclide & $\begin{array}{c}\text { Reported } \\
\text { Value }\end{array}$ & $\begin{array}{c}\text { EML } \\
\text { Value }\end{array}$ & $\begin{array}{c}\text { EML } \\
\text { Error }\end{array}$ & $\begin{array}{c}\text { Reported } \\
\text { EML }\end{array}$ Evaluation Evaluation \\
\hline
\end{tabular}

Matrix: AI Air Filter Bq / filter

$\begin{array}{llrrrrrrr}1 & \text { AM241 } & 0.870 & 0.050 & 0.510 & 0.008 & 1.706 & \text { W } & \text { A } \\ 1 & \text { CO60 } & 9.400 & 0.090 & 9.160 & 0.580 & 1.026 & \mathrm{~A} & \mathrm{~A} \\ 1 & \text { CS137 } & 22.740 & 0.290 & 22.470 & 1.030 & 1.012 & \mathrm{~A} & \mathrm{~A} \\ 1 & \text { GROSS ALPHA } & 1.510 & 0.060 & 1.650 & 0.160 & 0.915 & \mathrm{~A} & \mathrm{~W} \\ 1 & \text { GROSS BETA } & 1.620 & 0.060 & 2.160 & 0.070 & 0.750 & \mathrm{~W} & \mathrm{~A} \\ 1 & \text { MN54 } & 5.300 & 0.100 & 4.920 & 0.400 & 1.077 & \mathrm{~A} & \mathrm{~A} \\ 1 & \text { PU238 } & 0.760 & 0.050 & 0.460 & 0.005 & 1.652 & \mathrm{~N} & \mathrm{~N} \\ 1 & \text { PU239 } & 0.310 & 0.030 & 0.420 & 0.006 & 0.738 & \mathrm{~N} & \mathrm{~W} \\ 1 & \text { SB125 } & 9.150 & 0.300 & 8.890 & 0.550 & 1.029 & \mathrm{~A} & \mathrm{~A} \\ 1 & \text { SR90 } & 0.390 & 0.100 & 1.120 & 0.050 & 0.348 & \mathrm{~N} & \mathrm{~W} \\ 1 & \text { U238 } & 21.700 & & 0.260 & 0.010 & 83.462 & \mathrm{~N} & \end{array}$

Matrix: SO Soil $\mathrm{Bq} / \mathrm{kg}$

$\begin{array}{ll}1 & \text { AC228 } \\ 1 & \text { AM241 } \\ 1 & \text { BI212 } \\ 1 & \text { BI214 } \\ 1 & \text { CS137 } \\ 1 & \text { K40 } \\ 1 & \text { PB210 } \\ 1 & \text { PB212 } \\ 1 & \text { PB214 } \\ 1 & \text { PU239 } \\ 1 & \text { RA226 } \\ 1 & \text { SR90 } \\ 1 & \text { TH228 } \\ 1 & \text { TH234 } \\ 1 & \text { TL208 } \\ 1 & \text { ug U }\end{array}$

Matrix: VE Vegetation $\mathrm{Bq} / \mathrm{kg}$

$\begin{array}{rrr}83.140 & 13.200 & 52.600 \\ 9.410 & 1.040 & 7.470 \\ 85.470 & 5.300 & 58.300 \\ 44.550 & 2.290 & 28.800 \\ 1228.000 & 5.000 & 954.000 \\ 427.400 & 57.800 & 314.000 \\ 35.880 & 15.770 & 32.000 \\ 78.040 & 2.100 & 52.800 \\ 42.130 & 8.510 & 29.100 \\ 2.810 & 0.520 & 13.090 \\ 43.960 & 2.500 & 29.000 \\ 20.190 & 14.100 & 39.630 \\ 83.140 & 13.200 & 52.700 \\ 143.200 & 9.900 & 114.000 \\ 28.810 & 2.300 & 18.300 \\ 9.010 & & 9.700\end{array}$

$$
\begin{array}{r}
9.480 \\
1.040 \\
27.500 \\
489.950 \\
592.500 \\
2.670 \\
157.960
\end{array}
$$

2.900
0.410
5.900
0.500
38.000
13.000
3.300
3.700
1.200
0.570
1.000
0.003
4.000
6.000
1.100
0.700

1.581

1.260

1.466

1.547

1.287

1.361

1.121

1.478

1.448

0.215

1.516

0.509

1.578

1.256

1.574

0.929

$\begin{array}{ll}\text { A } & \\ \text { A } & \text { W } \\ \text { A } & \\ \text { A } & \\ \text { W } & \text { N } \\ \text { W } & \text { W } \\ \text { A } & \\ \text { A } & \\ \text { A } & \\ \text { N } & \text { N } \\ \text { A } & \\ \text { N } & \text { N } \\ \text { A } & \\ \text { A } & \\ \text { A } & \\ \text { A } & \text { A }\end{array}$




\section{QAP 49 Results by Laboratory}

Lab: SW Southwest Research Institute, San Antonio, TX

\begin{tabular}{|c|c|c|c|c|c|c|c|}
\hline $\begin{array}{l}0 . \\
\text { est }\end{array}$ & Radionuclide & $\begin{array}{l}\text { Reported } \\
\text { Value }\end{array}$ & $\begin{array}{c}\text { Reported } \\
\text { Error }\end{array}$ & $\begin{array}{l}\text { EML } \\
\text { Value }\end{array}$ & $\begin{array}{l}\text { EML } \\
\text { Error }\end{array}$ & $\frac{\text { Reported }}{\text { EML }}$ & $\begin{array}{cc}\text { QAP 48 } \\
\text { Evaluation } \\
\text { Evaluation }\end{array}$ \\
\hline
\end{tabular}

Matrix: WA Water $\mathrm{Bq} / \mathrm{L}$

$\begin{array}{llrrrrrrr}1 & \text { GROSS ALPHA } & 796.850 & 13.710 & 1080.000 & 60.000 & 0.738 & \text { W } & \text { A } \\ 1 & \text { GROSS BETA } & 1183.060 & 13.240 & 1420.000 & 60.000 & 0.833 & \mathrm{~A} & \mathrm{~A} \\ 1 & \text { H3 } & 1098.000 & 60.000 & 76.200 & 2.900 & 14.409 & \mathrm{~N} & \mathrm{~N} \\ 1 & \text { MN54 } & 37.070 & 0.040 & 32.400 & 1.400 & 1.144 & \mathrm{~A} & \mathrm{~A} \\ 1 & \text { PU238 } & 1.640 & 0.080 & 1.100 & 0.010 & 1.491 & \mathrm{~N} & \mathrm{~A} \\ 1 & \text { PU239 } & 1.180 & 0.060 & 1.410 & 0.040 & 0.837 & \mathrm{~W} & \mathrm{~N} \\ 1 & \text { SR90 } & 0.740 & 0.190 & 2.110 & 0.180 & 0.351 & \mathrm{~N} & \mathrm{~N} \\ 1 & \text { U238 } & 0.045 & & 0.520 & 0.050 & 0.086 & \mathrm{~N} & \end{array}$

Values for elemental uranium are reported in $\mu \mathrm{g} /$ filter, $\mathrm{g}$, or $\mathrm{mL} . \quad \mathrm{pCi} / \mathrm{g} \mathrm{or} \mathrm{mL}=\mathrm{Bq} \times 0.027$

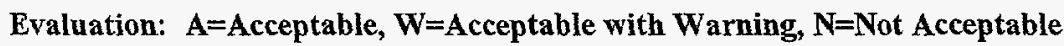

If the evaluation system is not appropriate for the types of analyses performed in your lab, apply a site specific evaluation. 


\section{QAP 49 Results by Laboratory}

Lab: TE Teledyne Isotopes Midwest Lab, Northbrook, $\mathrm{L}$

\begin{tabular}{|c|c|c|c|}
\hline $\begin{array}{l}\text { No. } \\
\text { Test Radionuclide }\end{array}$ & $\begin{array}{l}\text { Reported } \\
\text { Value }\end{array}$ & $\begin{array}{l}\text { Reported } \\
\text { Error }\end{array}$ & $\begin{array}{l}\text { EML } \\
\text { Value }\end{array}$ \\
\hline
\end{tabular}

Matrix: $\mathrm{AI}$ Air Filter $\mathrm{Bq}$ / filter

$\begin{array}{llrrrrrrr}1 & \text { CO60 } & 9.300 & 0.300 & 9.160 & 0.580 & 1.015 & \mathrm{~A} & \mathrm{~A} \\ 1 & \text { CS137 } & 22.400 & 0.500 & 22.470 & 1.030 & 0.997 & \mathrm{~A} & \mathrm{~A} \\ 1 & \text { GROSS ALPHA } & 2.200 & 0.100 & 1.650 & 0.160 & 1.333 & \mathrm{~W} & \\ 1 & \text { GROSS BETA } & 2.800 & 0.100 & 2.160 & 0.070 & 1.296 & \mathrm{~A} & \\ 1 & \text { MN54 } & 5.300 & 0.300 & 4.920 & 0.400 & 1.077 & \mathrm{~A} & \mathrm{~A} \\ 1 & \text { SB125 } & 10.000 & 0.800 & 8.890 & 0.550 & 1.125 & \mathrm{~A} & \mathrm{~A} \\ 1 & \text { SR90 } & 1.100 & 0.100 & 1.120 & 0.050 & 0.982 & \mathrm{~A} & \mathrm{~A}\end{array}$

Matrix: SO Soil $\mathrm{Bq} / \mathrm{kg}$

$\begin{array}{ll}1 & \mathrm{AC} 228 \\ 1 & \mathrm{BI212} \\ 1 & \mathrm{BI214} \\ 1 & \mathrm{CS} 137 \\ 1 & \mathrm{~K} 40 \\ 1 & \mathrm{~PB} 212 \\ 1 & \mathrm{~PB} 214 \\ 1 & \mathrm{RA226} \\ 1 & \text { SR90 } \\ 1 & \text { TL208 }\end{array}$

$\begin{array}{rrr}54.100 & 3.300 & 52.600 \\ 55.400 & 10.300 & 58.300 \\ 28.500 & 6.500 & 28.800 \\ 915.700 & 8.200 & 954.000 \\ 296.200 & 39.900 & 314.000 \\ 53.600 & 1.500 & 52.800 \\ 31.000 & 5.900 & 29.100 \\ 115.300 & 2.200 & 29.000 \\ 37.400 & 1.900 & 39.630 \\ 20.100 & 3.100 & 18.300\end{array}$

2.900
5.900
0.500
38.000
13.000
3.700
1.200
1.000
0.003
1.100

1.029

0.950

0.990

0.960

0.943

1.015

1.065

3.976

0.944

1.098

A
A
A
A
A
A
A
A
A
A

A

A

A

A

Matrix: VE Vegetation $\mathrm{Bq} / \mathrm{kg}$

$\begin{array}{llrrrrrrr}1 & \text { CO60 } & 18.100 & 1.500 & 20.000 & 1.000 & 0.905 & \mathrm{~A} & \mathrm{~A} \\ 1 & \text { CS137 } & 340.400 & 4.800 & 390.000 & 20.000 & 0.873 & \text { W } & \mathrm{A} \\ 1 & \text { K40 } & 417.500 & 28.200 & 460.000 & 20.000 & 0.908 & \mathrm{~A} & \mathrm{~A} \\ 1 & \text { SR90 } & 672.500 & 32.500 & 606.000 & 40.000 & 1.110 & \mathrm{~A} & \mathrm{~A}\end{array}$

Matrix: WA Water $\mathrm{Bq} / \mathrm{L}$

$\begin{array}{llrrrrrrr}1 & \text { CO60 } & 49.300 & 2.800 & 49.400 & 1.200 & 0.998 & \mathrm{~A} & \mathrm{~A} \\ 1 & \text { CS137 } & 50.100 & 3.200 & 50.000 & 1.700 & 1.002 & \mathrm{~A} & \mathrm{~A} \\ 1 & \text { FE55 } & 140.600 & 9.200 & 139.000 & 2.000 & 1.012 & \mathrm{~A} & \mathrm{~A} \\ 1 & \text { GROSS ALPHA } & 1178.300 & 47.200 & 1080.000 & 60.000 & 1.091 & \mathrm{~A} & \mathrm{~A} \\ 1 & \text { GROSS BETA } & 1613.600 & 171.800 & 1420.000 & 60.000 & 1.136 & \mathrm{~A} & \mathrm{~A} \\ 1 & \text { H3 } & 102.200 & 4.500 & 76.200 & 2.900 & 1.341 & \mathrm{~W} & \mathrm{~N} \\ 1 & \text { MN54 } & 35.900 & 3.400 & 32.400 & 1.400 & 1.108 & \mathrm{~A} & \mathrm{~A} \\ 1 & \text { SR90 } & 3.000 & 0.900 & 2.110 & 0.180 & 1.422 & \mathrm{~W} & \mathrm{~N}\end{array}$

Values for elemental uranium are reported in $\mu \mathrm{g} /$ filter, $\mathrm{g}$, or $\mathrm{mL}$.

$\mathrm{pCi} / \mathrm{g}$ or $\mathrm{mL}=\mathrm{Bq} \times 0.027$

Evaluation: $A=$ Acceptable, $W=$ Acceptable with Warning, $N=$ Not Acceptable

If the evaluation system is not appropriate for the types of analyses performed in your lab, apply a site specific evaluation. 


\section{QAP 49 Results by Laboratory}

Lab: TI Teledyne Brown Engineering Environmental Services, Westwood, NJ

\begin{tabular}{|c|c|c|c|c|c|c|}
\hline Radionuclide & $\begin{array}{l}\text { Reported } \\
\text { Value }\end{array}$ & $\begin{array}{l}\text { Reported } \\
\text { Error }\end{array}$ & $\begin{array}{l}\text { EML } \\
\text { Value }\end{array}$ & $\begin{array}{l}\text { EML } \\
\text { Error }\end{array}$ & $\frac{\text { Reported }}{\text { EML }}$ & $\begin{array}{c}\text { QAP 48 } \\
\text { Evaluation } \\
\text { Evaluation }\end{array}$ \\
\hline
\end{tabular}

Matrix: AI Air Filter Bq/filter

$\begin{array}{llrr}1 & \text { AM241 } & 0.540 & 0.050 \\ 1 & \text { CO60 } & 8.800 & 0.900 \\ 1 & \text { CS137 } & 22.600 & 2.300 \\ 1 & \text { GROSS ALPHA } & 2.100 & 0.100 \\ 1 & \text { GROSS BETA } & 1.700 & 0.100 \\ 1 & \text { MN54 } & 5.200 & 0.500 \\ 1 & \text { PU238 } & 0.430 & 0.050 \\ 1 & \text { PU239 } & 0.400 & 0.050 \\ 1 & \text { SB125 } & 9.000 & 0.900 \\ 1 & \text { SR90 } & 0.910 & 0.120 \\ 1 & \text { ug U } & 25.000 & 4.000\end{array}$

$\begin{array}{lrll}0.050 & 0.510 & 0.008 & 1.059 \\ 0.900 & 9.160 & 0.580 & 0.961 \\ 2.300 & 22.470 & 1.030 & 1.006 \\ 0.100 & 1.650 & 0.160 & 1.273 \\ 0.100 & 2.160 & 0.070 & 0.787 \\ 0.500 & 4.920 & 0.400 & 1.057 \\ 0.050 & 0.460 & 0.005 & 0.935 \\ 0.050 & 0.420 & 0.006 & 0.952 \\ 0.900 & 8.890 & 0.550 & 1.012 \\ 0.120 & 1.120 & 0.050 & 0.813 \\ 4.000 & 20.960 & 0.100 & 1.193\end{array}$

$\begin{array}{ll}\text { A } & \text { A } \\ \text { A } & \text { A } \\ \text { A } & \text { A } \\ \text { A } & \text { A } \\ \text { W } & \text { A } \\ \text { A } & \text { A } \\ \text { A } & \text { N } \\ \text { A } & \text { N } \\ \text { A } & \text { A } \\ \text { W } & \text { A } \\ \text { A } & \text { A }\end{array}$

Matrix: SO Soil $\mathrm{Bq} / \mathrm{kg}$

$\begin{array}{ll}1 & \text { AM241 } \\ 1 & \text { CO60 } \\ 1 & \text { CS137 } \\ 1 & \text { K40 } \\ 1 & \text { PU239 } \\ 1 & \text { SR90 } \\ 1 & \text { ug U }\end{array}$

4.300
1.850
1188.000
381.000
12.000
38.000
10.000

1.200

0.600

119.000

38.200

2.000

4.000

2.000

Matrix: VE Vegetation $\mathrm{Bq} / \mathrm{kg}$

$\begin{array}{ll}1 & \text { AM241 } \\ 1 & \text { CM244 } \\ 1 & \text { CO60 } \\ 1 & \text { CS137 } \\ 1 & \text { K40 } \\ 1 & \text { PU239 } \\ 1 & \text { SR90 }\end{array}$

2.500
1.900
21.900
496.000
523.000
3.300
520.000

0.500

0.400

2.200

49.600

52.300

0.700

10.000

2.330
1.760
20.000
390.000
460.000
3.720
606.000

0.060
0.070
1.000
20.000
20.000
0.270
40.000

1.073

1.080

1.095

1.272

1.137

0.887

0.858

$\begin{array}{ll}\mathrm{A} & \mathrm{A} \\ \mathrm{A} & \mathrm{A} \\ \mathrm{A} & \mathrm{A} \\ \mathrm{W} & \mathrm{A} \\ \mathrm{A} & \mathrm{A} \\ \mathrm{A} & \mathrm{N} \\ \mathrm{A} & \mathrm{N}\end{array}$

Matrix: WA Water $\mathrm{Bq} / \mathrm{L}$

$\begin{array}{ll}1 & \text { AM241 } \\ 1 & \text { CO60 } \\ 1 & \text { CS137 } \\ 1 & \text { FE55 } \\ 1 & \text { GROSS ALPHA } \\ 1 & \text { GROSS BETA } \\ 1 & \text { H3 } \\ 1 & \text { MN54 } \\ 1 & \text { N163 } \\ 1 & \text { PU238 } \\ 1 & \text { PU239 } \\ 1 & \text { SR90 }\end{array}$

1.300
49.000
51.100
140.000
1100.000
1100.000
450.000
33.500
110.000
1.100
1.600
2.200
0.200
4.900
5.100
20.000
100.000
100.000
50.000
3.400
10.000
0.200
0.200
0.300

$\begin{array}{rr}0.080 & 1.040 \\ 1.200 & 0.992 \\ 1.700 & 1.022 \\ 2.000 & 1.007 \\ 60.000 & 1.019 \\ 60.000 & 0.775 \\ 2.900 & 5.906 \\ 1.400 & 1.034 \\ 0.900 & 1.149 \\ 0.010 & 1.000 \\ 0.040 & 1.135 \\ 0.180 & 1.043\end{array}$

$\begin{array}{ll}\mathrm{A} & \mathrm{A} \\ \mathrm{A} & \mathrm{A} \\ \mathrm{A} & \mathrm{A} \\ \mathrm{A} & \mathrm{W} \\ \mathrm{A} & \mathrm{A} \\ \mathrm{A} & \mathrm{A} \\ \mathrm{N} & \mathrm{A} \\ \mathrm{A} & \mathrm{A} \\ \mathrm{A} & \\ \mathrm{A} & \mathrm{A} \\ \mathrm{A} & \mathrm{W} \\ \mathrm{A} & \mathrm{A}\end{array}$
A
A

A
A
A
A
A
A
N
N
A
A
A

A
A
A
A
A
A
N
N
A
A
A

\section{Values for elemental uranium are reported in $\mu \mathrm{g} /$ filter, $\mathrm{g}$, or $\mathrm{mL}$.}

$\mathrm{pCi} / \mathrm{g}$ or $\mathrm{mL}=\mathrm{Bq} \times 0.027$

Evaluation: $A=A c c e p t a b l e, W=A c c e p t a b l e$ with Warning, $N=$ Not Acceptable

If the evaluation system is not appropriate for the types of analyses performed in your lab, apply a site specific evaluation. 


\section{QAP 49 Results by Laboratory}

Lab: TI Teledyne Brown Engineering Environmental Services, Westwood, NJ

No.

Test Radionuclide

Reported Reported

EML

Value

EML

Error

Reported

EML

QAP 48

Evaluation Evaluation

Matrix: WA Water $\mathrm{Bq} / \mathrm{L}$

1 ug U

0.046

0.007

0.040

0.003

1.150

A

A

Values for elemental uranium are reported in $\mu \mathrm{g} /$ filter, $\mathrm{g}$, or $\mathrm{mL}$.

$\mathrm{pCi} / \mathrm{g}$ or $\mathrm{mL}=\mathrm{Bq} \times 0.027$

Evaluation: $A=$ Acceptable, $W=A c c e p t a b l e$ with Warning, $N=$ Not Acceptable

If the evaluation system is not appropriate for the types of analyses performed in your lab, apply a site specific evaluation. 


\section{QAP 49 Results by Laboratory}

Lab: TM Thermo NUtech Albuquerque Lab, NM

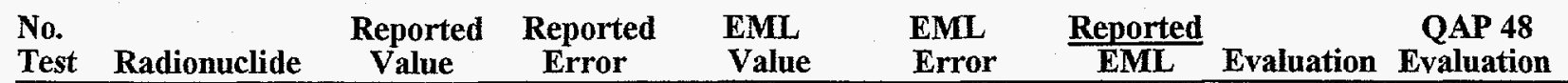

Matrix: AI Air Filter Bq / filter

$\begin{array}{llrrrrrrr}1 & \text { CO60 } & 7.940 & 0.423 & 9.160 & 0.580 & 0.867 & \mathrm{~A} & \mathrm{~A} \\ 1 & \text { CS137 } & 19.200 & 0.675 & 22.470 & 1.030 & 0.854 & \mathrm{~A} & \mathrm{~W} \\ 1 & \text { GROSS ALPHA } & 1.870 & 0.240 & 1.650 & 0.160 & 1.133 & \mathrm{~A} & \mathrm{~N} \\ 1 & \text { GROSS BETA } & 1.970 & 0.178 & 2.160 & 0.070 & 0.912 & \mathrm{~A} & \mathrm{~N} \\ 1 & \text { MN54 } & 4.540 & 0.339 & 4.920 & 0.400 & 0.923 & \mathrm{~A} & \mathrm{~A} \\ 1 & \text { PU238 } & 0.482 & 0.025 & 0.460 & 0.005 & 1.048 & \mathrm{~A} & \mathrm{~A} \\ 1 & \text { PU239 } & 0.473 & 0.025 & 0.420 & 0.006 & 1.126 & \mathrm{~A} & \mathrm{~W} \\ 1 & \text { SB125 } & 7.520 & 0.716 & 8.890 & 0.550 & 0.846 & \mathrm{~A} & \mathrm{~W} \\ 1 & \text { U234 } & 0.260 & 0.015 & 0.260 & 0.010 & 1.000 & \mathrm{~A} & \mathrm{~A} \\ 1 & \text { U238 } & 0.268 & 0.016 & 0.260 & 0.010 & 1.031 & \mathrm{~A} & \mathrm{~A} \\ 1 & \text { ug U } & 19.500 & 1.250 & 20.960 & 0.100 & 0.930 & \mathrm{~A} & \mathrm{~A}\end{array}$

Matrix: SO Soil Bq / kg

$\begin{array}{ll}1 & \text { AC228 } \\ 1 & \text { AM241 } \\ 1 & \text { BI212 } \\ 1 & \text { BI214 } \\ 1 & \text { CS137 } \\ 1 & \text { K40 } \\ 1 & \text { PB212 } \\ 1 & \text { PB214 } \\ 1 & \text { PU239 } \\ 1 & \text { RA226 } \\ 1 & \text { SR90 } \\ 1 & \text { TH228 } \\ 1 & \text { TH234 } \\ 1 & \text { TL208 } \\ 1 & \text { U234 } \\ 1 & \text { U238 } \\ 1 & \text { ug U }\end{array}$

$\begin{array}{rrr}72.500 & 7.300 & 52.600 \\ 7.930 & 2.980 & 7.470 \\ 75.500 & 20.700 & 58.300 \\ 41.000 & 3.890 & 28.800 \\ 1030.000 & 30.800 & 954.000 \\ 345.000 & 32.900 & 314.000 \\ 62.400 & 3.830 & .52 .800 \\ 35.700 & 5.060 & 29.100 \\ 14.400 & 1.420 & 13.090 \\ 31.000 & 4.110 & 29.000 \\ 42.900 & 10.200 & 39.630 \\ 65.300 & 7.260 & 52.700 \\ 92.500 & 23.700 & 114.000 \\ 60.500 & 6.730 & 18.300 \\ 115.000 & 6.440 & 113.000 \\ 123.000 & 6.810 & 120.000 \\ 8.510 & 0.438 & 9.700\end{array}$

2.900
0.410
5.900
0.500
38.000
13.000
3.700
1.200
0.570
1.000
0.003
4.000
6.000
1.100
6.000
9.000
0.700

1.378
1.062
1.295
1.424
1.080
1.099
1.182
1.227
1.100
1.069
1.083
1.239
0.811
3.306
1.018
1.025
0.877

$\begin{array}{ll}\text { A } & \\ \text { A } & \text { A } \\ \text { A } & \\ \text { A } & \\ \text { A } & \text { A } \\ \text { A } & \text { A } \\ \text { A } & \\ \text { A } & \\ \text { A } & \text { A } \\ \text { A } & \\ \text { A } & \text { W } \\ \text { A } & \\ \text { A } & \\ \text { A } & \\ \text { A } & \text { A } \\ \text { A } & \text { A } \\ \text { A } & \text { A }\end{array}$

Matrix: VE Vegetation $\mathrm{Bq} / \mathrm{kg}$

$\begin{array}{ll}1 & \text { AM241 } \\ 1 & \text { CM244 } \\ 1 & \text { CO60 } \\ 1 & \text { CS137 } \\ 1 & \text { K40 } \\ 1 & \text { PU239 } \\ 1 & \text { SR90 }\end{array}$

$$
\begin{array}{r}
2.960 \\
2.630 \\
21.500 \\
437.000 \\
574.000
\end{array}
$$$$
0.500
$$$$
2.330
$$$$
0.481
$$$$
3.750
$$$$
14.400
$$$$
85.700
$$$$
0.361
$$$$
2.350
$$

0.060
0.070
1.000
20.000
20.000
0.270
40.000

1.270

1.494

1.075

20.000

390.000

460.000

3.720

1.121

1.248

606.000

1.110

0.056

$\begin{array}{ll}\mathrm{A} & \mathrm{A} \\ \mathrm{W} & \mathrm{A} \\ \mathrm{A} & \mathrm{N} \\ \mathrm{A} & \mathrm{A} \\ \mathrm{W} & \mathrm{A} \\ \mathrm{A} & \mathrm{A} \\ \mathrm{N} & \mathrm{A}\end{array}$

Matrix: WA Water $\mathrm{Bq} / \mathrm{L}$

$\begin{array}{lllllllll}1 & \text { CO60 } & 53.400 & 1.720 & 49.400 & 1.200 & 1.081 & \text { A } & \text { W } \\ 1 & \text { CS137 } & 55.800 & 1.740 & 50.000 & 1.700 & 1.116 & \text { A } & \text { W }\end{array}$

\section{Values for elemental uranium are reported in $\mu \mathrm{g} /$ filter, $\mathrm{g}$, or $\mathrm{mL}$.}

$\mathrm{pCi} / \mathrm{g}$ or $\mathrm{mL}=\mathrm{Bq} \times 0.027$

Evaluation: $A=$ Acceptable, $W=$ Acceptable with Warning, $N=$ Nat Acceptable

If the evaluation system is not appropriate for the types of analyses performed in your lab, apply a site specific evaluation. 


\section{QAP 49 Results by Laboratory}

Lab: TM Thermo NUtech Albuquerque Lab, NM

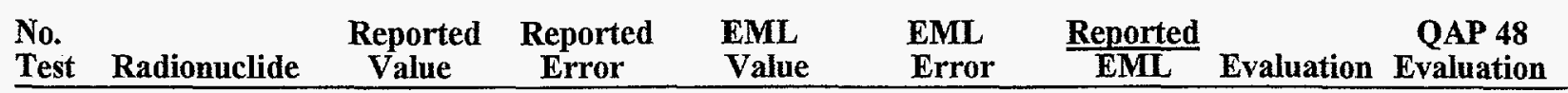

Matrix: WA Water $\mathrm{Bq} / \mathrm{L}$

$\begin{array}{llrrrrrrr}1 & \text { GROSS ALPHA } & 1480.000 & 167.000 & 1080.000 & 60.000 & 1.370 & \mathrm{~N} & \mathrm{~A} \\ 1 & \text { GROSS BETA } & 1470.000 & 110.000 & 1420.000 & 60.000 & 1.035 & \mathrm{~A} & \mathrm{~A} \\ 1 & \text { MN54 } & 38.300 & 1.280 & 32.400 & 1.400 & 1.182 & \mathrm{~W} & \mathrm{~W} \\ 1 & \text { PU238 } & 1.200 & 0.077 & 1.100 & 0.010 & 1.091 & \mathrm{~A} & \mathrm{~A} \\ 1 & \text { PU239 } & 1.520 & 0.093 & 1.410 & 0.040 & 1.078 & \mathrm{~A} & \mathrm{~A} \\ 1 & \text { SR90 } & 2.230 & 0.235 & 2.110 & 0.180 & 1.057 & \mathrm{~A} & \mathrm{~W} \\ 1 & \text { U234 } & 0.576 & 0.043 & 0.510 & 0.030 & 1.129 & \mathrm{~A} & \mathrm{~A} \\ 1 & \text { U238 } & 0.571 & 0.043 & 0.520 & 0.050 & 1.098 & \mathrm{~A} & \mathrm{~A} \\ 1 & \text { ug U } & 0.041 & 0.003 & 0.040 & 0.003 & 1.015 & \mathrm{~A} & \mathrm{~W}\end{array}$

Values for elemental uranium are reported in $\mu \mathrm{g} /$ filter, $\mathrm{g}$, or $\mathrm{mL}$.

$\mathrm{pCi} / \mathrm{g}$ or $\mathrm{mL}=\mathrm{Bq} \times 0.027$

Evaluation: $A=A c c e p t a b l e, W=A c c e p t a b l e$ with Warning, $N=$ Not Acceptable

If the evaluation system is not appropriate for the types of analyses performed in your lab, apply a site specific evaluation. 


\section{QAP 49 Results by Laboratory}

Lab: TN Thermo NUTech, Richmond, CA

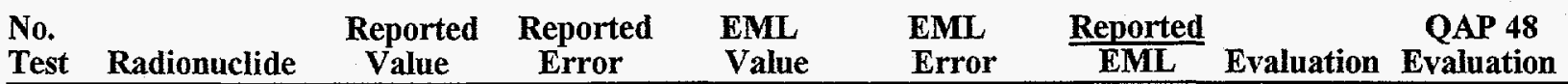

Matrix: AI Air Filter Bq / filter

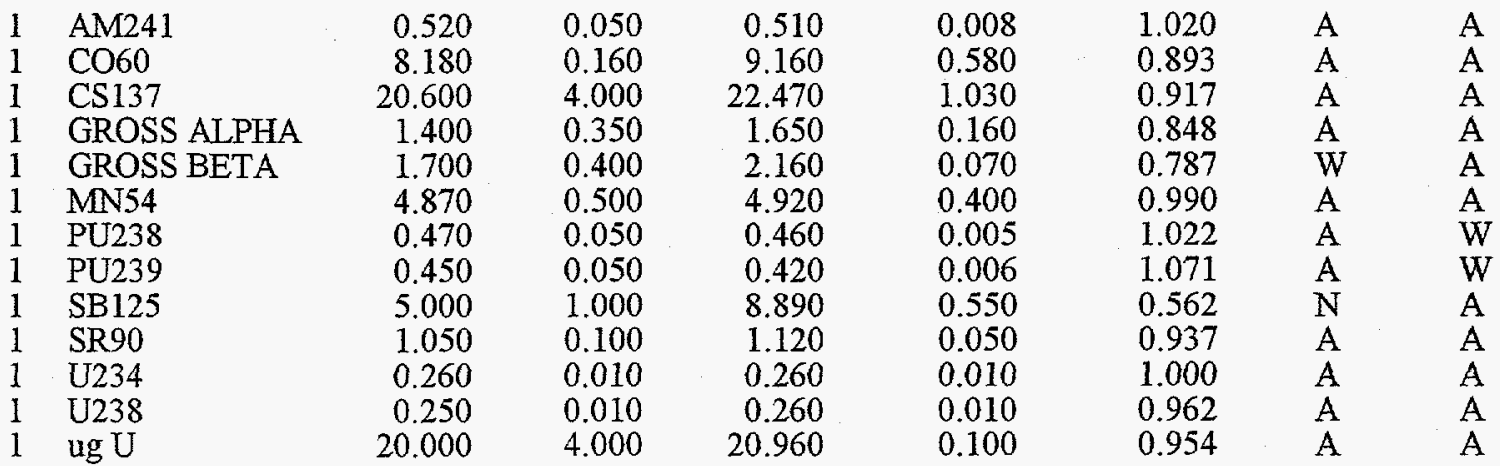

Matrix: SO Soil $\mathrm{Bq} / \mathrm{kg}$

$\begin{array}{ll}1 & \text { AM241 } \\ 1 & \text { CS137 } \\ 1 & \text { K40 } \\ 1 & \text { PU239 } \\ 1 & \text { RA226 } \\ 1 & \text { SR90 } \\ 1 & \text { TH228 } \\ 1 & \text { U234 } \\ 1 & \text { U238 } \\ 1 & \text { ug U }\end{array}$

$\begin{array}{rr}10.000 & 1.000 \\ 88.300 & 9.000 \\ 2.920 & 0.300 \\ 12.600 & 1.300 \\ 0.200 & 0.040 \\ 41.000 & 4.000 \\ 0.490 & 0.050 \\ 103.000 & 10.000 \\ 108.000 & 11.000 \\ 8.840 & 0.900\end{array}$

7.470

954.000

314.000

13.090

29.000

39.630

52.700

113.000

120.000

9.700

$$
\begin{array}{r}
0.410 \\
38.000 \\
13.000 \\
0.570 \\
1.000 \\
0.003 \\
4.000 \\
6.000 \\
9.000 \\
0.700
\end{array}
$$

Matrix: VE Vegetation $\mathrm{Bq} / \mathrm{kg}$

$$
\begin{array}{r}
2.550 \\
1.880 \\
0.220 \\
3.920 \\
4.850 \\
3.500 \\
678.000
\end{array}
$$

$$
\begin{array}{r}
0.250 \\
0.500 \\
0.030 \\
0.400 \\
1.000 \\
0.700 \\
68.000
\end{array}
$$

$$
\begin{array}{r}
2.330 \\
1.760 \\
20.000 \\
390.000 \\
460.000 \\
3.720 \\
606.000
\end{array}
$$

$$
\begin{array}{r}
0.060 \\
0.070 \\
1.000 \\
20.000 \\
20.000 \\
0.270 \\
40.000
\end{array}
$$

1.339
0.093
0.009
0.963
0.007
1.035
0.009
0.912
0.900
0.911

1.339

0.093

0.963

0.007

1.035

0.009

0.912

0.911
A

A

$\mathrm{N}$

A
A
W

Matrix: WA Water $\mathrm{Bq} / \mathrm{L}$

$\begin{array}{llr}1 & \text { AM241 } & 1.240 \\ 1 & \text { CO60 } & 0.098 \\ 1 & \text { CS137 } & 0.098 \\ 1 & \text { FE55 } & 148.000 \\ 1 & \text { GROSS ALPHA } & 820.000 \\ 1 & \text { GROSS BETA } & 1240.000 \\ 1 & \text { H3 } & 78.000\end{array}$

0.120
0.010
0.010
15.000
120.000
200.000
8.000
1.250
49.400
50.000
139.000
1080.000
1420.000
76.200

$\begin{array}{ll}1.094 & \mathrm{~A} \\ 1.068 & \mathrm{~A} \\ 0.011 & \mathrm{~N} \\ 0.010 & \mathrm{~N} \\ 0.011 & \mathrm{~N} \\ 0.941 & \mathrm{~A} \\ 1.119 & \mathrm{~A}\end{array}$

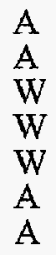

Values for elemental uranium are reported in $\mu \mathrm{g} /$ filter, $\mathrm{g}$, or $\mathrm{mL}$.

$\begin{array}{lll}0.992 & \mathrm{~A} & \mathrm{~A} \\ 0.002 & \mathrm{~N} & \mathrm{~A} \\ 0.002 & \mathrm{~N} & \mathrm{~A} \\ 1.065 & \mathrm{~A} & \mathrm{~W} \\ 0.759 & \mathrm{~W} & \mathrm{~W} \\ 0.873 & \mathrm{~A} & \mathrm{~A} \\ 1.024 & \mathrm{~A} & \mathrm{~W}\end{array}$

Evaluation: A=Acceptable, $W=$ Acceptable with Warning, $N=$ Not Acceptable

If the evaluation system is not appropriate for the types of analyses performed in your lab, apply a site specific evaluation. 


\section{QAP 49 Results by Laboratory}

Lab: TN Thermo NUTech, Richmond, CA

No.

Reported Reported

EML

EML

Reported

QAP 48

Test Radionuclide

Value

Error

Value

Error EML

Evaluation Evaluation

Matrix: WA Water $\mathrm{Bq} / \mathrm{L}$

$\begin{array}{ll}1 & \text { MN54 } \\ 1 & \text { NI63 } \\ 1 & \text { PU238 } \\ 1 & \text { PU239 } \\ 1 & \text { SR90 } \\ 1 & \text { U234 } \\ 1 & \text { U238 } \\ 1 & \text { ug U }\end{array}$

$\begin{array}{rr}0.067 & 0.007 \\ 122.000 & 12.000 \\ 1.150 & 0.110 \\ 1.480 & 0.150 \\ 2.160 & 0.210 \\ 0.530 & 0.050 \\ 0.520 & 0.050 \\ 0.043 & 0.004\end{array}$

32.400

95.700

1.100

1.410

2.110

0.510

0.520

0.040

1.400
0.900
0.010
0.040
0.180
0.030
0.050
0.003

0.002

1.275

1.045

1.050

1.024

1.039

1.000

1.075

N
A
A
A
A
A
A
A

A

$N$

$\mathrm{N}$

A

A

A

Values for elemental uranium are reported in $\mu \mathrm{g} /$ filter, $\mathrm{g}$, or $\mathrm{mL}$.

$\mathrm{pCi} / \mathrm{g}$ or $\mathrm{mL}=\mathrm{Bq} \times 0.027$

Evaluation: A=Acceptable, $W=$ Acceptable with Warning, $N=$ Not Acceptable

If the evaluation system is not appropriate for the types of analyses performed in your lab, apply a site specific evaluation. 


\section{QAP 49 Results by Laboratory}

Lab: TO Thermo NUtech Oak Ridge Laboratory

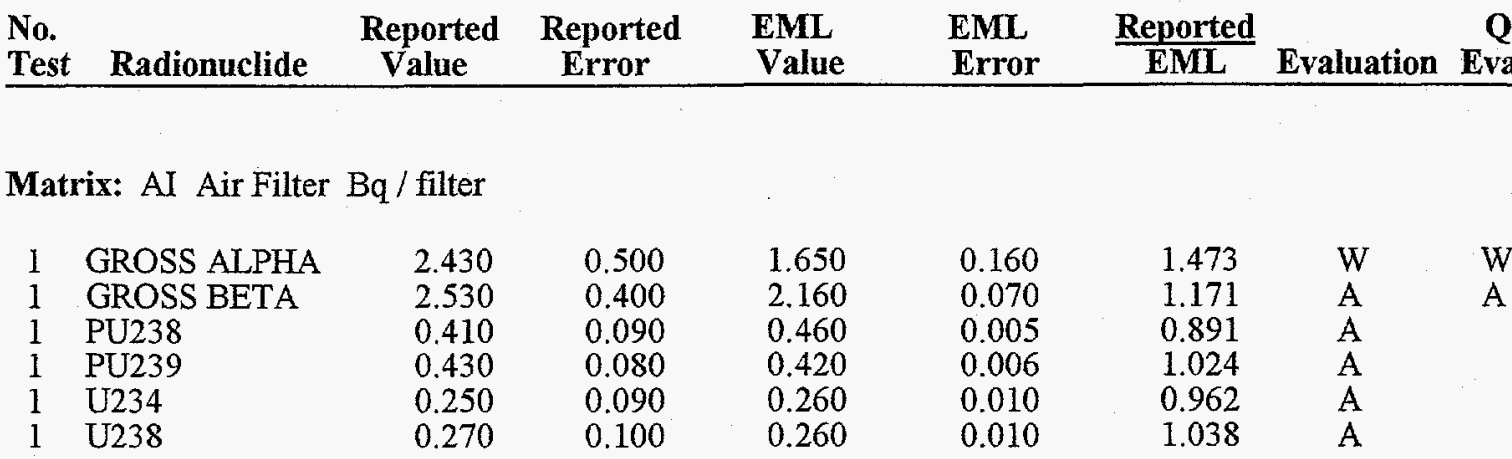

Matrix: SO Soil Bq/kg

$\begin{array}{ll}1 & \text { AC228 } \\ 1 & \text { AM241 } \\ 1 & \text { BI212 } \\ 1 & \text { BI214 } \\ 1 & \text { BI214 } \\ 1 & \text { CS137 } \\ 1 & \text { K40 } \\ 1 & \text { PB210 } \\ 1 & \text { PB212 } \\ 1 & \text { PB214 } \\ 1 & \text { PU239 } \\ 1 & \text { RA226 } \\ 1 & \text { SR90 } \\ 1 & \text { TH234 } \\ 1 & \text { TL208 } \\ 1 & \text { TL208 } \\ 1 & \text { U234 } \\ 1 & \text { U238 }\end{array}$

$\begin{array}{rrr}35.900 & 7.300 & 52.600 \\ 9.600 & 3.800 & 7.470 \\ 26.500 & 16.600 & 58.300 \\ 27.402 & 6.985 & 28.800 \\ 22.800 & 5.400 & 28.800 \\ 693.900 & 70.900 & 954.000 \\ 224.000 & 43.100 & 314.000 \\ 20.400 & 2.400 & 32.000 \\ 39.000 & 6.800 & 52.800 \\ 24.700 & 11.800 & 29.100 \\ 13.900 & 4.200 & 13.090 \\ 23.800 & 8.600 & 29.000 \\ 37.800 & 3.000 & 39.630 \\ 98.700 & 45.300 & 114.000 \\ 15.856 & 3.862 & 18.300 \\ 31.500 & 6.600 & 18.300 \\ 110.400 & 26.900 & 113.000 \\ 108.100 & 30.100 & 120.000\end{array}$

2.900
0.410
5.900
0.500
0.500
38.000
13.000
3.300
3.700
1.200
0.570
1.000
0.003
6.000
1.100
1.100
6.000
9.000

0.683

1.285

0.455

0.951

0.792

0.727

0.713

0.637

0.739

0.849

1.062

0.821

0.954

0.866

0.866

1.721

0.977

0.901

$\begin{array}{ll}\text { A } & \\ \text { A } & \text { N } \\ \text { A } & \\ \text { A } & \\ \text { A } & \\ \text { N } & \text { A } \\ \text { N } & \text { A } \\ \text { A } & \\ \text { A } & \\ \text { A } & \\ \text { A } & \text { N } \\ \text { A } & \\ \text { A } & \text { A } \\ \text { A } & \\ \text { A } & \\ \text { A } & \\ \text { A } & \text { A } \\ \text { A } & \text { A }\end{array}$

Matrix: VE Vegetation $\mathrm{Bq} / \mathrm{kg}$

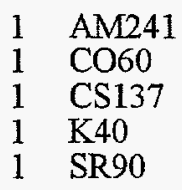

Matrix: WA Water $\mathrm{Bq} / \mathrm{L}$

$\begin{array}{ll}1 & \text { AM241 } \\ 1 & \text { CO60 } \\ 1 & \text { CS137 } \\ 1 & \text { FE55 } \\ 1 & \text { GROSS ALPHA } \\ 1 & \text { GROSS BETA } \\ 1 & \text { H3 } \\ 1 & \text { MN54 }\end{array}$

1.780
2.400
30.600
52.900
13.600

2.330

20.000

390.000

460.000

606.000

0.060
1.000
20.000
20.000
40.000

1.124

0.680

0.748

0.780

0.856
QAP 48

valuation
A

N

A

$\mathrm{A}$
$\mathrm{A}$
W

A

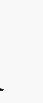

A




\section{QAP 49 Results by Laboratory}

Lab: TO Thermo NUtech Oak Ridge Laboratory

\begin{tabular}{llllll} 
No. & Reported & Reported & EML & EML & Reported \\
Test & Radionuclide & $\begin{array}{c}\text { Value } \\
\text { Error }\end{array}$ & Value & Error & $\begin{array}{c}\text { QAP 48 } \\
\text { EML }\end{array}$ Evaluation Evaluation \\
\hline
\end{tabular}

Matrix: WA Water $\mathrm{Bq} / \mathrm{L}$

$\begin{array}{llrrrrrrr}1 & \text { NI63 } & 117.700 & 1.000 & 95.700 & 0.900 & 1.230 & \mathrm{~A} & \\ 1 & \text { PU238 } & 1.240 & 0.280 & 1.100 & 0.010 & 1.127 & \text { W } & \text { A } \\ 1 & \text { PU239 } & 1.640 & 0.340 & 1.410 & 0.040 & 1.163 & \text { W } & \text { A } \\ 1 & \text { SR90 } & 2.300 & 0.100 & 2.110 & 0.180 & 1.090 & \text { A } & \text { W } \\ 1 & \text { U234 } & 0.590 & 0.220 & 0.510 & 0.030 & 1.157 & \text { A } & \text { A } \\ 1 & \text { U238 } & 0.580 & 0.230 & 0.520 & 0.050 & 1.115 & \text { A } & \text { A }\end{array}$




\section{QAP 49 Results by Laboratory}

Lab: TP Taiwan Power Company, Taipei, Taiwan

\begin{tabular}{|c|c|c|c|c|c|c|c|}
\hline $\begin{array}{l}\text { No. } \\
\text { Test }\end{array}$ & Radi & $\begin{array}{c}\text { Reported } \\
\text { Value }\end{array}$ & $\begin{array}{c}\text { Reported } \\
\text { Error }\end{array}$ & $\begin{array}{l}\text { EML } \\
\text { Value }\end{array}$ & $\begin{array}{l}\text { EML } \\
\text { Error }\end{array}$ & $\frac{\text { Reported }}{\text { EML }}$ & $\begin{array}{c}\text { QAP 48 } \\
\text { Evaluation }\end{array}$ \\
\hline
\end{tabular}

Matrix: $\mathrm{AI}$ Air Filter Bq / filter

$\begin{array}{llrrrrrrr}1 & \text { CO60 } & 9.480 & 0.050 & 9.160 & 0.580 & 1.035 & \mathrm{~A} & \mathrm{~A} \\ 1 & \text { CS137 } & 23.200 & 0.220 & 22.470 & 1.030 & 1.032 & \mathrm{~A} & \mathrm{~A} \\ 1 & \text { GROSS ALPHA } & 1.600 & 0.080 & 1.650 & 0.160 & 0.970 & \mathrm{~A} & \mathrm{~W} \\ 1 & \text { GROSS BETA } & 2.090 & 0.100 & 2.160 & 0.070 & 0.968 & \mathrm{~A} & \mathrm{~A} \\ 1 & \text { MN54 } & 4.900 & 0.100 & 4.920 & 0.400 & 0.996 & \mathrm{~A} & \mathrm{~A} \\ 1 & \text { SB125 } & 8.620 & 0.080 & 8.890 & 0.550 & 0.970 & \mathrm{~A} & \mathrm{~A} \\ 1 & \text { SR90 } & 3.270 & 0.060 & 1.120 & 0.050 & 2.920 & \mathrm{~N} & \mathrm{~A}\end{array}$

Matrix: SO Soil Bq/kg

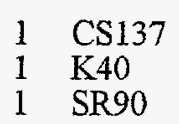

918.530
327.740
68.400

5.180
5.220
2.340

954.000

314.000

39.630

38.000

13.000

0.003

Matrix: VE Vegetation $\mathrm{Bq} / \mathrm{kg}$

$\begin{array}{ll}1 & \text { CO60 } \\ 1 & \text { CS137 } \\ 1 & \text { K40 } \\ 1 & \text { SR90 }\end{array}$

18.070
373.170
458.780
644.160

0.050

11.710

4.090

25.790

$$
1.000
$$

20.000

$\begin{array}{ll}460.000 & 20.000 \\ 606.000 & 40.000\end{array}$
Matrix: WA Water $\mathrm{Bq} / \mathrm{L}$

$\begin{array}{llr}1 & \text { CO60 } & 44.870 \\ 1 & \text { CS137 } & 47.750 \\ 1 & \text { GROSS ALPHA } & 996.700 \\ 1 & \text { GROSS BETA } & 1371.000 \\ 1 & \text { H3 } & 115.640 \\ 1 & \text { MN54 } & 31.470 \\ 1 & \text { SR90 } & 2.500\end{array}$

$$
\begin{array}{r}
49.400 \\
50.000 \\
1080.000 \\
1420.000 \\
76.200 \\
32.400 \\
2.110
\end{array}
$$

$$
\begin{array}{r}
1.200 \\
1.700 \\
60.000 \\
60.000 \\
2.900 \\
1.400 \\
0.180
\end{array}
$$

0.963

1.044

1.726

$\begin{array}{lll}0.903 & \mathrm{~A} & \mathrm{~A} \\ 0.957 & \mathrm{~A} & \mathrm{~A} \\ 0.997 & \mathrm{~A} & \mathrm{~A} \\ 1.063 & \mathrm{~A} & \mathrm{~A}\end{array}$

A




\section{QAP 49 Results by Laboratory}

Lab: TT Tracer Technologies International, Inc., Cleveland

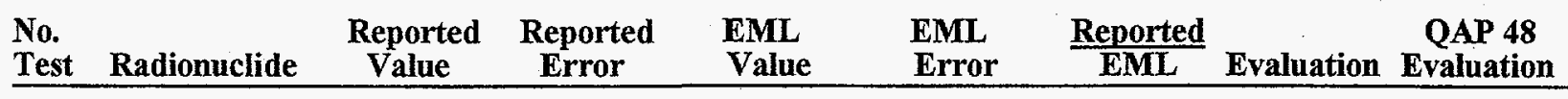

Matrix: SO Soil $\mathrm{Bq} / \mathrm{kg}$

$\begin{array}{lllllllll}1 & \text { CS137 } & 750.000 & 9.230 & 954.000 & 38.000 & 0.786 & \mathrm{~N} & \mathrm{~N} \\ 1 & \mathrm{~K} 40 & 266.000 & 5.570 & 314.000 & 13.000 & 0.847 & \mathrm{~W} & \mathrm{~W}\end{array}$

Matrix: WA Water $\mathrm{Bq} / \mathrm{L}$

$\begin{array}{lllllllll}1 & \text { CO60 } & 54.000 & 3.200 & 49.400 & 1.200 & 1.093 & \mathrm{~A} & \text { W } \\ 1 & \text { CS137 } & 55.000 & 6.820 & 50.000 & 1.700 & 1.100 & \mathrm{~A} & \mathrm{~A} \\ 1 & \text { H3 } & 69.000 & 7.090 & 76.200 & 2.900 & 0.906 & \mathrm{~A} & \mathrm{~A} \\ 1 & \text { MN54 } & 38.000 & 7.090 & 32.400 & 1.400 & 1.173 & \text { W } & \text { A }\end{array}$

Values for elemental uranium are reported in $\mu \mathrm{g} /$ filter, $\mathrm{g}$, or $\mathrm{mL}$.

$\mathrm{pCi} / \mathrm{g}$ or $\mathrm{mL}=\mathrm{Bq} \times 0.027$

Evaluation: $A=A c c e p t a b l e, W=$ Acceptable with Warning, $N=$ Not Acceptable

If the evaluation system is not appropriate for the types of analyses performed in your lab, apply a site specific evaluation. 


\section{QAP 49 Results by Laboratory}

Lab: TW Taiwan Radiation Monitoring Center

\begin{tabular}{|c|c|c|c|c|c|c|c|}
\hline $\begin{array}{l}\text { No. } \\
\text { Test }\end{array}$ & $\mathbf{R a d}$ & $\begin{array}{l}\text { Reported } \\
\text { Value }\end{array}$ & $\begin{array}{c}\text { Reported } \\
\text { Error }\end{array}$ & $\begin{array}{l}\text { EML } \\
\text { Value }\end{array}$ & $\begin{array}{l}\text { EML } \\
\text { Error }\end{array}$ & $\frac{\text { Reported }}{\text { EML }}$ & $\begin{array}{c}\text { QAP 48 } \\
\text { Evaluation } \\
\text { Evaluation }\end{array}$ \\
\hline
\end{tabular}

Matrix: AI Air Filter $\mathrm{Bq} /$ filter

$\begin{array}{llrrrrrrr}1 & \text { CO60 } & 9.400 & 0.200 & 9.160 & 0.580 & 1.026 & \mathrm{~A} & \mathrm{~A} \\ 1 & \text { CS137 } & 22.200 & 0.600 & 22.470 & 1.030 & 0.988 & \mathrm{~A} & \mathrm{~A} \\ 1 & \text { GROSS ALPHA } & 1.730 & 0.050 & 1.650 & 0.160 & 1.048 & \mathrm{~A} & \mathrm{~A} \\ 1 & \text { GROSS BETA } & 1.860 & 0.040 & 2.160 & 0.070 & 0.861 & \mathrm{~W} & \mathrm{~A} \\ 1 & \text { MN54 } & 5.000 & 0.200 & 4.920 & 0.400 & 1.016 & \mathrm{~A} & \mathrm{~A} \\ 1 & \text { SB125 } & 9.700 & 0.200 & 8.890 & 0.550 & 1.091 & \mathrm{~A} & \mathrm{~A}\end{array}$

Matrix: SO Soil $\mathrm{Bq} / \mathrm{kg}$

$\begin{array}{ll}1 & \mathrm{AC} 228 \\ 1 & \mathrm{BI} 14 \\ 1 & \mathrm{CS} 137 \\ 1 & \mathrm{~K} 40 \\ 1 & \mathrm{~PB} 212 \\ 1 & \mathrm{~PB} 214 \\ 1 & \mathrm{TL} 208\end{array}$

$\begin{array}{rrr}54.000 & 1.000 & 52.600 \\ 25.300 & 0.800 & 28.800 \\ 1040.000 & 8.000 & 954.000 \\ 371.000 & 7.000 & 314.000 \\ 52.600 & 0.900 & 52.800 \\ 29.000 & 1.000 & 29.100 \\ 16.500 & 0.500 & 18.300\end{array}$

2.900
0.500
38.000
13.000
3.700
1.200
1.100

1.027

0.878

1.090

1.182

0.996

0.997

0.902
A
A
A
A
A
A
A

A

$\mathrm{A}$
$\mathrm{A}$
$\mathrm{A}$
$\mathrm{A}$
$\mathrm{A}$
$\mathrm{A}$

Matrix: VE Vegetation $\mathrm{Bq} / \mathrm{kg}$

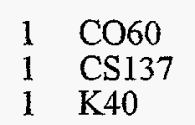

$1 \quad \operatorname{CS} 137$

$1 \mathrm{~K} 40$

\begin{abstract}
21.000
455.000

540.000
\end{abstract}

$\begin{array}{rr}20.000 & 1.000 \\ 390.000 & 20.000 \\ 460.000 & 20.000\end{array}$

20.000
1.050
1.167
1.174

$\begin{array}{ll}\mathrm{A} & \mathrm{A} \\ \mathrm{A} & \mathrm{A} \\ \mathrm{A} & \mathrm{A}\end{array}$

Matrix: WA Water $\mathrm{Bq} / \mathrm{L}$

$\begin{array}{llrrrrrrr}1 & \text { CO60 } & 49.200 & 0.700 & 49.400 & 1.200 & 0.996 & \mathrm{~A} & \mathrm{~A} \\ 1 & \text { CS137 } & 51.000 & 1.000 & 50.000 & 1.700 & 1.020 & \mathrm{~A} & \mathrm{~A} \\ 1 & \text { GROSS ALPHA } & 820.000 & 30.000 & 1080.000 & 60.000 & 0.759 & \mathrm{~W} & \mathrm{~N} \\ 1 & \text { GROSS BETA } & 1192.000 & 50.000 & 1420.000 & 60.000 & 0.839 & \mathrm{~A} & \mathrm{~A} \\ 1 & \text { MN54 } & 36.200 & 0.800 & 32.400 & 1.400 & 1.117 & \mathrm{~A} & \mathrm{~A}\end{array}$

\footnotetext{
Values for elemental uranium are reported in $\mu \mathrm{g} /$ filter, $\mathrm{g}$, or $\mathrm{mL}$.

$\mathrm{pCi} / \mathrm{g}$ or $\mathrm{mL}=\mathrm{Bq} \times 0.027$
}

Evaluation: $A=$ Acceptable, $W=$ Acceptable with Warning, $N=$ Not Acceptable

If the evaluation system is not appropriate for the types of analyses performed in your lab, apply a site specific evaluation. 
Lab: TX Texas Dept. of Health/Laboratories, Austin

$\begin{array}{llllll}\text { No. } & \text { Reported } & \text { Reported } & \text { EML } & \text { EML } & \text { Reported } \\ \text { Test } & \text { Radionuclide } & \begin{array}{l}\text { Value } \\ \text { Error }\end{array} & \text { Value } & \text { Error } & \begin{array}{c}\text { QAP 48 } \\ \text { EML }\end{array} \text { Evaluation Evaluation }\end{array}$

Matrix: AI Air Filter Bq / filter

$\begin{array}{ll}1 & \text { AM241 } \\ 1 & \text { CO60 } \\ 1 & \text { CS137 } \\ 1 & \text { GROSS ALPHA } \\ 1 & \text { GROSS BETA } \\ 1 & \text { MN54 } \\ 1 & \text { PU238 } \\ 1 & \text { PU239 } \\ 1 & \text { SB125 } \\ 1 & \text { U234 } \\ 1 & \text { U238 }\end{array}$

0.491
8.250
21.900
1.770
2.090
5.060
0.525
0.460
8.810
0.254
0.268

$\begin{array}{lr}0.014 & 0.510 \\ 0.070 & 9.160 \\ 0.100 & 22.470 \\ 0.050 & 1.650 \\ 0.070 & 2.160 \\ 0.070 & 4.920 \\ 0.016 & 0.460 \\ 0.014 & 0.420 \\ 0.110 & 8.890 \\ 0.010 & 0.260 \\ 0.011 & 0.260\end{array}$

0.008

0.580

1.030

0.160

0.070

0.400

0.005

0.006

0.550

0.010

0.010
A

0.901

0.975

1.073

0.968

1.028

1.141

1.095

0.991

0.977

1.031

$\begin{array}{lll}0.992 & \mathrm{~A} & \\ 1.319 & \mathrm{~A} & \mathrm{~A} \\ 0.801 & \mathrm{~A} & \\ 0.872 & \mathrm{~A} & \\ 0.958 & \mathrm{~A} & \mathrm{~A} \\ 1.003 & \mathrm{~A} & \mathrm{~A} \\ 0.866 & \mathrm{~A} & \\ 1.007 & \mathrm{~A} & \\ 0.940 & \mathrm{~A} & \mathrm{~A} \\ 1.528 & \mathrm{~A} & \\ 1.188 & \mathrm{~A} & \mathrm{~A} \\ 1.019 & \mathrm{~A} & \\ 1.123 & \mathrm{~A} & \\ 0.918 & \mathrm{~A} & \\ 0.929 & \mathrm{~A} & \mathrm{~A} \\ 0.933 & \mathrm{~A} & \mathrm{~A}\end{array}$

Matrix: VE Vegetation $\mathrm{Bq} / \mathrm{kg}$

$\begin{array}{ll}1 & \text { AM241 } \\ 1 & \text { CO60 } \\ 1 & \text { CS137 } \\ 1 & \text { K40 } \\ 1 & \text { PU239 } \\ 1 & \text { SR90 }\end{array}$

$\begin{array}{rr}52.200 & 1.500 \\ 9.850 & 0.890 \\ 46.700 & 1.600 \\ 25.100 & 1.200 \\ 914.000 & 4.000 \\ 315.000 & 12.000 \\ 27.700 & 9.300 \\ 29.300 & 2.100 \\ 12.300 & 0.500 \\ 44.300 & 3.500 \\ 47.100 & 9.700 \\ 53.700 & 2.200 \\ 128.000 & 15.000 \\ 16.800 & 0.700 \\ 105.000 & 2.000 \\ 112.000 & 3.000\end{array}$

52.600
7.470
58.300
28.800
954.000
314.000
32.000
29.100
13.090
29.000
39.630
52.700
114.000
18.300
113.000
120.000

2.900
0.410
5.900
0.500
38.000
13.000
3.300
1.200
0.570
1.000
0.003
4.000
6.000
1.100
6.000
9.000

0.390

1.100

2.000

20.000

0.230

33.000

$\begin{array}{rr}2.330 & 0.060 \\ 20.000 & 1.000 \\ 390.000 & 20.000 \\ 460.000 & 20.000 \\ 3.720 & 0.270 \\ 606.000 & 40.000\end{array}$
0.060
0.000
40.000
1.429
1.160
1.115
1.126
1.043
1.061

$\begin{array}{ll}\mathrm{A} & \mathrm{A} \\ \mathrm{A} & \mathrm{A} \\ \mathrm{A} & \mathrm{A} \\ \mathrm{A} & \mathrm{A} \\ \mathrm{A} & \mathrm{A} \\ \mathrm{A} & \mathrm{A}\end{array}$

Matrix: WA Water $\mathrm{Bq} / \mathrm{L}$

$\begin{array}{llrrrrrrr}1 & \text { AM241 } & 1.320 & 0.060 & 1.250 & 0.080 & 1.056 & \text { A } & \text { W } \\ 1 & \text { CO60 } & 51.900 & 0.400 & 49.400 & 1.200 & 1.051 & \text { A } & \text { A } \\ 1 & \text { CS137 } & 52.000 & 0.500 & 50.000 & 1.700 & 1.040 & \text { A } & \text { A } \\ 1 & \text { GROSS ALPHA } & 1103.000 & 30.000 & 1080.000 & 60.000 & 1.021 & \text { A } & \text { W }\end{array}$

Values for elemental uranium are reported in $\mu \mathrm{g} /$ filter, $\mathrm{g}$, or $\mathrm{mL}$.

$\mathrm{pCi} / \mathrm{g}$ or $\mathrm{mL}=\mathrm{Bq} \times 0.027$

Evaluation: $A=$ Acceptable, $W=$ Acceptable with Warning, $N=$ Not Acceptable

If the evaluation system is not appropriate for the types of analyses performed in your lab, apply a site specific evaluation. 


\section{QAP 49 Results by Laboratory}

Lab: TX Texas Dept. of Health/Laboratories, Austin

\begin{tabular}{llllll} 
No. & Reported & Reported & EML & EML \\
Test & Radionuclide & $\begin{array}{c}\text { Value } \\
\text { Error }\end{array}$ & $\begin{array}{c}\text { Value } \\
\text { Error }\end{array}$ & $\begin{array}{c}\text { Reported } \\
\text { EML }\end{array}$ Evaluation Evaluation \\
\hline
\end{tabular}

Matrix: WA Water $\mathrm{Bq} / \mathrm{L}$

$\begin{array}{llrrrrrrr}1 & \text { GROSS BETA } & 1208.000 & 35.000 & 1420.000 & 60.000 & 0.851 & \mathrm{~A} & \mathrm{~A} \\ 1 & \text { H3 } & 80.900 & 5.600 & 76.200 & 2.900 & 1.062 & \mathrm{~A} & \mathrm{~A} \\ 1 & \text { MN54 } & 36.300 & 0.400 & 32.400 & 1.400 & 1.120 & \mathrm{~A} & \mathrm{~A} \\ 1 & \text { PU238 } & 1.162 & 0.047 & 1.100 & 0.010 & 1.056 & \mathrm{~A} & \mathrm{~A} \\ 1 & \text { PU239 } & 1.567 & 0.055 & 1.410 & 0.040 & 1.111 & \mathrm{~A} & \mathrm{~A} \\ 1 & \text { SR90 } & 2.720 & 0.710 & 2.110 & 0.180 & 1.289 & \mathrm{~W} & \mathrm{~A} \\ 1 & \text { U234 } & 0.533 & 0.032 & 0.510 & 0.030 & 1.045 & \mathrm{~A} & \mathrm{~A} \\ 1 & \text { U238 } & 0.527 & 0.031 & 0.520 & 0.050 & 1.013 & \mathrm{~A} & \mathrm{~W}\end{array}$

Values for elemental uranium are reported in $\mu \mathrm{g} /$ filter, $\mathrm{g}$, or $\mathrm{mL} . \quad \mathrm{pCi} / \mathrm{g}$ or $\mathrm{mL}=\mathrm{Bq} \times 0.027$

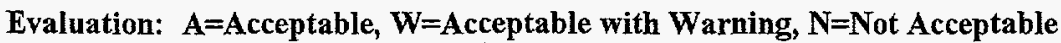

If the evaluation system is not appropriate for the types of analyses performed in your lab, apply a site specific evaluation. 


\section{QAP 49 Results by Laboratory}

Lab: TY Scientific Production Association, Russia

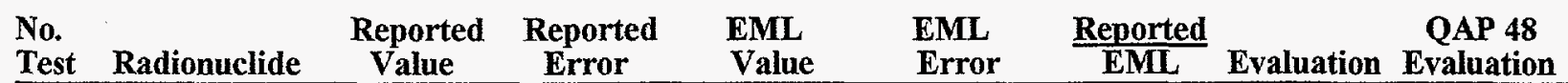

Matrix: SO Soil $\mathrm{Bq} / \mathrm{kg}$

\begin{tabular}{|c|c|c|c|c|c|}
\hline CO60 & 2.000 & 1.000 & 1.240 & 0.110 & 1.613 \\
\hline CS137 & 864.000 & 1.000 & 954.000 & 38.000 & 0.906 \\
\hline $\mathrm{K} 40$ & 336.000 & 15.000 & 314.000 & 13.000 & 1.070 \\
\hline U234 & 28.000 & 3.000 & 113.000 & 6.000 & 0.248 \\
\hline
\end{tabular}

Values for elemental uranium are reported in $\mu \mathrm{g} /$ filter, $\mathrm{g}$, or $\mathrm{mL}$.

$\mathrm{pCi} / \mathrm{g}$ or $\mathrm{mL}=\mathrm{Bq} \times 0.027$

Evaluation: A=Acceptable, $W=$ Acceptable with Warning, $N=$ Not Acceptable

If the evaluation system is not appropriate for the types of analyses performed in your lab, apply a site specific evaluation. 


\section{QAP 49 Results by Laboratory}

Lab: UC Lockheed Martin, Paducah, KY

\begin{tabular}{|c|c|c|c|c|c|c|c|}
\hline est & Radionuclide & $\begin{array}{c}\text { Reported } \\
\text { Value }\end{array}$ & $\begin{array}{c}\text { Reported } \\
\text { Error }\end{array}$ & $\begin{array}{l}\text { EML } \\
\text { Value }\end{array}$ & $\begin{array}{l}\text { EML } \\
\text { Error }\end{array}$ & $\frac{\text { Reported }}{\text { EML }}$ & $\begin{array}{c}\text { QAP 48 } \\
\text { Evaluation Evaluation }\end{array}$ \\
\hline
\end{tabular}

Matrix: AI Air Filter $\mathrm{Bq} /$ filter

$\begin{array}{llrrrrrrr}1 & \text { CO60 } & 9.117 & 1.238 & 9.160 & 0.580 & 0.995 & \mathrm{~A} & \mathrm{~A} \\ 1 & \text { CS137 } & 31.338 & 10.810 & 22.470 & 1.030 & 1.395 & \mathrm{~N} & \mathrm{~W} \\ 1 & \text { GROSS ALPHA } & 1.620 & 0.080 & 1.650 & 0.160 & 0.982 & \mathrm{~A} & \mathrm{~A} \\ 1 & \text { GROSS BETA } & 2.380 & 0.080 & 2.160 & 0.070 & 1.102 & \mathrm{~A} & \mathrm{~A} \\ 1 & \text { PU238 } & 0.107 & 0.010 & 0.460 & 0.005 & 0.233 & \mathrm{~N} & \\ 1 & \text { PU239 } & 0.100 & 0.009 & 0.420 & 0.006 & 0.238 & \mathrm{~N} & \end{array}$

Matrix: SO Soil Bq/ $\mathrm{kg}$

$\begin{array}{llrrrrrrr}1 & \mathrm{CS} 137 & 1160.320 & 98.420 & 954.000 & 38.000 & 1.216 & \mathrm{~W} & \mathrm{~W} \\ \mathrm{I} & \mathrm{K} 40 & 380.240 & 16.290 & 314.000 & 13.000 & 1.211 & \mathrm{~A} & \mathrm{~A}\end{array}$

Matrix: VE Vegetation $\mathrm{Bq} / \mathrm{kg}$

$\begin{array}{llrrrrrrr}1 & \text { CO60 } & 2.230 & 0.190 & 20.000 & 1.000 & 0.112 & \mathrm{~N} & \mathrm{~A} \\ 1 & \text { CS137 } & 44.900 & 4.620 & 390.000 & 20.000 & 0.115 & \mathrm{~N} & \mathrm{~A} \\ 1 & \mathrm{~K} 40 & 53.110 & 4.820 & 460.000 & 20.000 & 0.115 & \mathrm{~N} & \mathrm{~A}\end{array}$

Matrix: WA Water $\mathrm{Bq} / \mathrm{L}$

\begin{tabular}{|c|c|c|c|c|c|c|c|}
\hline CO60 & 52.250 & 5.280 & 49.400 & 1.200 & 1.058 & A & A \\
\hline CS137 & 53.290 & 6.200 & 50.000 & 1.700 & 1.066 & A & A \\
\hline GROSS ALPHA & 993.230 & 66.710 & 1080.000 & 60.000 & 0.920 & A & A \\
\hline GROSS BETA & 1224.570 & 50.490 & 1420.000 & 60.000 & 0.862 & A & \\
\hline ug U & 0.042 & & 0.040 & 0.003 & 1.050 & A & \\
\hline
\end{tabular}

Values for elemental uranium are reported in $\mu \mathrm{g} /$ filter, $\mathrm{g}$, or $\mathrm{mL} . \quad$ pCi/g or $\mathrm{mL}=\mathrm{Bq} \times 0.027$

Evaluation: $A=$ Acceptable, $W=$ Acceptable with Waming, $N=$ Not Acceptable

If the evaluation system is not appropriate for the types of analyses performed in your lab, apply a site specific evaluation. 


\section{QAP 49 Results by Laboratory}

Lab: UP Lockheed Martin Energy Systems, Y-12 Plant, Oak Ridge

\begin{tabular}{llllll} 
No. & Reported & Reported & EML & EML & Reported \\
Test & Radionuclide & $\begin{array}{c}\text { Value } \\
\text { Error }\end{array}$ & $\begin{array}{c}\text { Value } \\
\text { Error }\end{array}$ & $\frac{1}{\text { EML }}$ Evaluation EAP 48 \\
\hline
\end{tabular}

Matrix: AI Air Filter $\mathrm{Bq} /$ filter

$\begin{array}{llrrrrrrl}1 & \text { AM241 } & 0.557 & 0.068 & 0.510 & 0.008 & 1.092 & \mathrm{~A} & \\ 1 & \text { Bq U } & 0.678 & 0.083 & 0.530 & 0.020 & 1.279 & \mathrm{~A} & \\ 1 & \text { CO60 } & 9.580 & 0.467 & 9.160 & 0.580 & 1.046 & \mathrm{~A} & \mathrm{~W} \\ 1 & \text { CS137 } & 23.800 & 1.420 & 22.470 & 1.030 & 1.059 & \mathrm{~A} & \mathrm{~W} \\ 1 & \text { GROSS ALPHA } & 1.640 & 0.081 & 1.650 & 0.160 & 0.994 & \mathrm{~A} & \mathrm{~A} \\ 1 & \text { GROSS BETA } & 1.900 & 0.066 & 2.160 & 0.070 & 0.880 & \mathrm{~W} & \mathrm{~A} \\ 1 & \text { MN54 } & 5.410 & 0.528 & 4.920 & 0.400 & 1.100 & \mathrm{~A} & \mathrm{~W} \\ 1 & \text { PU238 } & 0.488 & 0.066 & 0.460 & 0.005 & 1.061 & \mathrm{~A} & \\ 1 & \text { PU239 } & 0.471 & 0.064 & 0.420 & 0.006 & 1.121 & \mathrm{~A} & \\ 1 & \text { SB125 } & 3.410 & 0.813 & 8.890 & 0.550 & 0.384 & \mathrm{~N} & \mathrm{~N} \\ 1 & \text { SR90 } & 1.040 & 0.223 & 1.120 & 0.050 & 0.929 & \mathrm{~A} & \\ 1 & \text { ug U } & 21.390 & 2.000 & 20.960 & 0.100 & 1.021 & \mathrm{~A} & \mathrm{~A}\end{array}$

Matrix: SO Soil $\mathrm{Bq} / \mathrm{kg}$

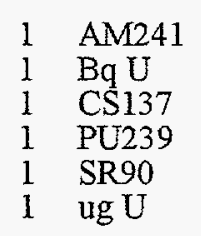

Matrix: WA Water $\mathrm{Bq} / \mathrm{L}$

$\begin{array}{ll}1 & \text { AM241 } \\ 1 & \text { Bq U } \\ 1 & \text { CO60 } \\ 1 & \text { CS137 } \\ 1 & \text { GROSS ALPHA } \\ 1 & \text { GROSS BETA } \\ 1 & \text { H3 } \\ 1 & \text { MN54 } \\ 1 & \text { PU238 } \\ 1 & \text { PU239 } \\ 1 & \text { SR90 } \\ 1 & \text { ug U }\end{array}$

9.390
234.000
1101.000
13.900
38.850
9.180

2.020

18.800

88.000

4.030

10.730

0.500

7.470
237.000
954.000
13.090
39.630
9.700

0.410
16.000
38.000
0.570
0.003
0.700

0.410
16.000

38.000

0.003

0.700

\subsection{7 \\ 0.987 \\ 1.154 \\ 1.062 \\ 0.980 \\ 0.946}

A
A
A
A
A
A

A

A

A

A

Matrix: AI Air Filter Bq / filter

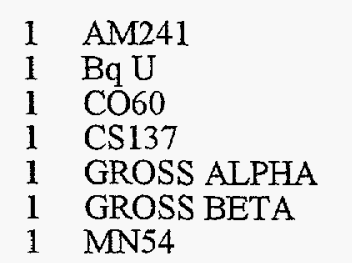

0.522

0.554

9.260

23.100

1.320

1.810

5.080

$\begin{array}{rr}0.192 & 1.250 \\ 0.212 & 1.050 \\ 1.590 & 49.400 \\ 3.220 & 50.000 \\ 105.000 & 1080.000 \\ 97.000 & 1420.000 \\ 23.600 & 76.200 \\ 3.020 & 32.400 \\ 0.166 & 1.100 \\ 0.204 & 1.410 \\ 0.606 & 2.110 \\ 0.004 & 0.040\end{array}$

0.073

0.064

1.100

1.800

0.120

0.085

0.700

$$
\begin{array}{r}
0.080 \\
0.080 \\
1.200 \\
1.700 \\
60.000 \\
60.000 \\
2.900 \\
1.400 \\
0.010 \\
0.040 \\
0.180 \\
0.003
\end{array}
$$

1.120
1.381
1.095
1.092
0.974
1.048
1.102
1.127
1.000
1.071
1.191
1.075

A
W
A
A
A
A
A
A
A
A
A
A

N

A

A

A

A

A

A

$W$
$W$

A

Values for elemental uranium are reported in $\mu \mathrm{g} /$ filter, $\mathrm{g}$, or $\mathrm{mL}$.

$\begin{array}{ll}0.008 & 1.024 \\ 0.020 & 1.045 \\ 0.580 & 1.011 \\ 1.030 & 1.028 \\ 0.160 & 0.800 \\ 0.070 & 0.838 \\ 0.400 & 1.033\end{array}$

$\begin{array}{ll}\text { A } & \text { A } \\ \text { A } & \text { A } \\ \text { A } & \text { A } \\ \text { A } & \text { A } \\ \text { W } & \text { A } \\ \text { W } & \text { A } \\ \text { A } & \text { A }\end{array}$

Evaluation: A=Acceptable, W=Acceptable with Warning, N=Not Acceptable

If the evaluation system is not appropriate for the types of analyses performed in your lab, apply a site specific evaluation. 


\section{QAP 49 Results by Laboratory}

Lab: UY Lockheed Martin Energy Systems, Y-12 Plant, Oak Ridge

\begin{tabular}{|c|c|c|c|c|c|c|c|}
\hline $\begin{array}{l}\text { No. } \\
\text { Test }\end{array}$ & Radionuclide & $\begin{array}{c}\text { Reported } \\
\text { Value }\end{array}$ & $\begin{array}{l}\text { Reported } \\
\text { Error }\end{array}$ & $\begin{array}{l}\text { EML } \\
\text { Value }\end{array}$ & $\begin{array}{l}\text { EML } \\
\text { Error }\end{array}$ & $\frac{\text { Reported }}{\text { EML }}$ & 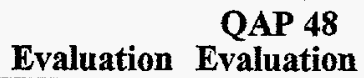 \\
\hline
\end{tabular}

Matrix: AI Air Filter Bq / filter

$\begin{array}{lllllllll}1 & \text { PU238 } & 0.465 & 0.051 & 0.460 & 0.005 & 1.011 & \mathrm{~A} & \mathrm{~A} \\ 1 & \text { PU239 } & 0.441 & 0.050 & 0.420 & 0.006 & 1.050 & \mathrm{~A} & \mathrm{~A} \\ 1 & \text { SB125 } & 8.470 & 0.800 & 8.890 & 0.550 & 0.953 & \mathrm{~A} & \mathrm{~A} \\ 1 & \text { SR90 } & 1.150 & 0.110 & 1.120 & 0.050 & 1.027 & \mathrm{~A} & \text { W }\end{array}$

Matrix: SO Soil $\mathrm{Bq} / \mathrm{kg}$

$\begin{array}{ll}1 & \text { AC228 } \\ 1 & \text { AM241 } \\ 1 & \text { BI212 } \\ 1 & \text { BI214 } \\ 1 & \text { BqU } \\ 1 & \text { CS137 } \\ 1 & \text { K40 } \\ 1 & \text { PB212 } \\ 1 & \text { PB214 } \\ 1 & \text { PU239 } \\ 1 & \text { SR90 } \\ 1 & \text { TH228 } \\ 1 & \text { TH234 } \\ 1 & \text { TL208 }\end{array}$

$\begin{array}{rr}71.000 & 32.000 \\ 7.530 & 1.800 \\ 60.000 & 30.000 \\ 33.000 & 15.000 \\ 216.000 & 22.000 \\ 1100.000 & 100.000 \\ 415.000 & 120.000 \\ 58.000 & 30.000 \\ 34.000 & 15.000 \\ 12.700 & 1.400 \\ 43.600 & 3.500 \\ 50.000 & 6.000 \\ 108.000 & 11.000 \\ 21.000 & 12.000\end{array}$

52.600
7.470
58.300
28.800
237.000
954.000
314.000
52.800
29.100
13.090
39.630
52.700
114.000
18.300

2.900
0.410
5.900
0.500
16.000
38.000
13.000
3.700
1.200
0.570
0.003
4.000
6.000
1.100

1.350

1.008

1.029

1.146

0.911

1.153

1.322

1.098

1.168

0.970

1.100

0.949

0.947

1.148

$\begin{array}{ll}\text { A } & \\ \text { A } & \text { A } \\ \text { A } & \\ \text { A } & \\ \text { A } & \text { A } \\ \text { A } & \text { A } \\ \text { W } & \text { A } \\ \text { A } & \\ \text { A } & \\ \text { A } & \text { A } \\ \text { A } & \text { A } \\ \text { A } & \\ \text { A } & \\ \text { A } & \end{array}$

Matrix: VE Vegetation $\mathrm{Bq} / \mathrm{kg}$

$\begin{array}{ll}1 & \text { AM241 } \\ 1 & \text { CM244 } \\ 1 & \text { CO60 } \\ 1 & \text { CS137 } \\ 1 & \text { K40 } \\ 1 & \text { PU239 } \\ 1 & \text { SR90 }\end{array}$

$\begin{array}{rr}2.540 & 0.450 \\ 0.811 & 0.220 \\ 28.000 & 6.000 \\ 472.000 & 33.000 \\ 595.000 & 126.000 \\ 4.060 & 0.500 \\ 541.000 & 10.000\end{array}$

2.330
1.760
20.000
390.000
460.000
3.720
606.000

0.060
0.070
1.000
20.000
20.000
0.270
40.000

1.090
0.460
1.400
1.210
1.293
1.090
0.893

$A$
W
W
A
W
A
A

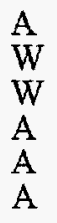

Matrix: WA Water $\mathrm{Bq} / \mathrm{L}$

$\begin{array}{llr}1 & \text { AM241 } & 1.260 \\ 1 & \text { Bq U } & 1.120 \\ 1 & \text { CO60 } & 50.200 \\ 1 & \text { CS137 } & 52.700 \\ 1 & \text { GROSS ALPHA } & 942.000 \\ 1 & \text { GROSS BETA } & 1220.000 \\ 1 & \text { H3 } & 74.800 \\ 1 & \text { MN54 } & 35.600 \\ 1 & \text { PU238 } & 1.130 \\ 1 & \text { PU239 } & 1.520 \\ 1 & \text { SR90 } & 2.000\end{array}$

$$
\begin{array}{r}
0.150 \\
0.150 \\
4.000 \\
7.000 \\
49.000 \\
45.000 \\
18.000 \\
4.000 \\
0.130 \\
0.170 \\
0.300
\end{array}
$$

1.250
1.050
49.400
50.000
1080.000
1420.000
76.200
32.400
1.100
1.410
2.110
0.080
0.080
1.200
1.700
60.000
60.000
2.900
1.400
0.010
0.040
0.180

$\begin{array}{ll}1.008 & \mathrm{~A} \\ 1.067 & \mathrm{~A} \\ 1.016 & \mathrm{~A} \\ 1.054 & \mathrm{~A} \\ 0.872 & \mathrm{~A} \\ 0.859 & \mathrm{~A} \\ 0.982 & \mathrm{~A} \\ 1.099 & \mathrm{~A} \\ 1.027 & \mathrm{~A} \\ 1.078 & \mathrm{~A} \\ 0.948 & \mathrm{~A}\end{array}$

A
A
A
A
A
A
A
A
A
A
A

Values for elemental uranium are reported in $\mu \mathrm{g} /$ filter, $\mathrm{g}$, or $\mathrm{mL}$.

$\mathrm{pCi} / \mathrm{g}$ or $\mathrm{mL}=\mathrm{Bq} \times 0.027$

Evaluation: A=Acceptable, $W=A c c e p t a b l e$ with Warning, $N=$ Not Acceptable

If the evaluation system is not appropriate for the types of analyses performed in your lab, apply a site specific evaluation. 


\section{QAP 49 Results by Laboratory}

Lab: WA Environmental Radiation Lab, Off. of Public Health Labs. Seattle

\begin{tabular}{|c|c|c|c|c|c|c|}
\hline Radionuclide & $\begin{array}{l}\text { Reported } \\
\text { Value }\end{array}$ & $\begin{array}{l}\text { Reported } \\
\text { Error }\end{array}$ & $\begin{array}{l}\text { EML } \\
\text { Value }\end{array}$ & $\begin{array}{l}\text { EML } \\
\text { Error }\end{array}$ & $\frac{\text { Reported }}{\text { EML }}$ & $\begin{array}{c}\text { QAP 48 } \\
\text { Evaluation Evaluation }\end{array}$ \\
\hline
\end{tabular}

Matrix: AI Air Filter $\mathrm{Bq} /$ filter

$\begin{array}{llrlrllll}1 & \text { AM241 } & 0.440 & 0.040 & 0.510 & 0.008 & 0.863 & \text { W } & \text { A } \\ 1 & \text { Bq U } & 0.470 & 0.060 & 0.530 & 0.020 & 0.887 & \text { W } & \text { A } \\ 1 & \text { CO60 } & 8.700 & 0.200 & 9.160 & 0.580 & 0.950 & \text { A } & \text { A } \\ 1 & \text { CS137 } & 21.400 & 0.600 & 22.470 & 1.030 & 0.952 & \text { A } & \text { A } \\ 1 & \text { GROSS ALPHA } & 1.910 & 0.070 & 1.650 & 0.160 & 1.158 & \text { A } & \text { A } \\ 1 & \text { GROSS BETA } & 2.230 & 0.060 & 2.160 & 0.070 & 1.032 & \text { A } & \text { A } \\ 1 & \text { MN54 } & 5.200 & 0.300 & 4.920 & 0.400 & 1.057 & \text { A } & \text { W } \\ 1 & \text { PU238 } & 0.510 & 0.050 & 0.460 & 0.005 & 1.109 & \text { A } & \text { A } \\ 1 & \text { PU239 } & 0.500 & 0.040 & 0.420 & 0.006 & 1.190 & \text { W } & \text { W } \\ 1 & \text { SB125 } & 9.300 & 0.500 & 8.890 & 0.550 & 1.046 & \text { A } & \text { W } \\ 1 & \text { SR90 } & 1.340 & 0.230 & 1.120 & 0.050 & 1.196 & \text { A } & \text { A } \\ 1 & \text { U234 } & 0.240 & 0.040 & 0.260 & 0.010 & 0.923 & \text { A } & \text { A } \\ 1 & \text { U238 } & 0.230 & 0.040 & 0.260 & 0.010 & 0.885 & \text { W } & \text { A }\end{array}$

Matrix: SO Soil $\mathrm{Bq} / \mathrm{kg}$

$\begin{array}{ll}1 & \mathrm{AC} 228 \\ 1 & \mathrm{AM} 241 \\ 1 & \text { BI212 } \\ 1 & \text { BI214 } \\ 1 & \text { Bq U } \\ 1 & \text { CS137 } \\ 1 & \text { K40 } \\ 1 & \text { PB212 } \\ 1 & \text { PB214 } \\ 1 & \text { PU238 } \\ 1 & \text { PU239 } \\ 1 & \text { RA226 } \\ 1 & \text { SR90 } \\ 1 & \text { TH228 } \\ 1 & \text { TH234 } \\ 1 & \text { TL208 } \\ 1 & \text { U234 } \\ 1 & \text { U238 }\end{array}$

$\begin{array}{rrr}54.700 & 2.600 & 52.600 \\ 7.160 & 0.640 & 7.470 \\ 61.400 & 8.300 & 58.300 \\ 28.700 & 1.900 & 28.800 \\ 247.000 & 9.000 & 237.000 \\ 1080.000 & 30.000 & 954.000 \\ 399.000 & 15.000 & 314.000 \\ 57.500 & 2.600 & 52.800 \\ 30.200 & 2.200 & 29.100 \\ 0.550 & 0.350 & 0.530 \\ 13.500 & 1.000 & 13.090 \\ 135.000 & 17.000 & 29.000 \\ 46.100 & 3.900 & 39.630 \\ 190.000 & 33.000 & 52.700 \\ 101.000 & 11.000 & 114.000 \\ 18.600 & 1.000 & 18.300 \\ 116.000 & 7.000 & 113.000 \\ 123.000 & 7.000 & 120.000\end{array}$

2.900
0.410
5.900
0.500
16.000
38.000
13.000
3.700
1.200
0.270
0.570
1.000
0.003
4.000
6.000
1.100
6.000
9.000

1.040

0.959

1.053

0.997

1.042

1.132

1.271

1.089

1.038

1.038

1.031

4.655

1.163

3.605

0.886

1.016

1.027

1.025

$\begin{array}{ll}\text { A } & \\ \text { A } & \text { A } \\ \text { A } & \\ \text { A } & \\ \text { A } & \text { A } \\ \text { A } & \text { A } \\ \text { W } & \text { A } \\ \text { A } & \\ \text { A } & \\ \text { A } & \\ \text { A } & \text { A } \\ \text { A } & \\ \text { A } & \text { A } \\ \text { A } & \\ \text { A } & \\ \text { A } & \\ \text { A } & \text { A } \\ \text { A } & \text { A }\end{array}$

Matrix: VE Vegetation $\mathrm{Bq} / \mathrm{kg}$

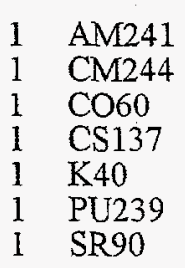

1.720
2.640
21.000
409.000
470.000
4.370
709.000

2.330
1.760
20.000
390.000
460.000
3.720
606.000

0.738
1.500
1.050
1.049
1.022
1.175
1.170

$\begin{array}{ll}\text { W } & \text { W } \\ \text { W } & \\ \text { A } & \text { A } \\ \text { A } & \text { A } \\ \text { A } & \text { A } \\ \text { A } & \text { A } \\ \text { W } & \text { A }\end{array}$

\footnotetext{
Values for elemental uranium are reported in $\mu \mathrm{g} /$ filter, $g$, or $\mathrm{mL}$.
}

$\mathrm{pCi} / \mathrm{g}$ or $\mathrm{mL}=\mathrm{Bq} \times 0.027$

Evaluation: $A=$ Acceptable, $W=$ Acceptable with Warning, $N=$ Not Acceptable

If the evaluation system is not appropriate for the types of analyses performed in your lab, apply a site specific evaluation. 


\section{QAP 49 Results by Laboratory}

Lab: WA Environmental Radiation Lab, Off. of Public Health Labs. Seattle

\begin{tabular}{|c|c|c|c|c|c|c|c|}
\hline $\begin{array}{l}\text { No. } \\
\text { Test }\end{array}$ & adionuc & $\begin{array}{c}\text { Reported } \\
\text { Value }\end{array}$ & $\begin{array}{l}\text { Reported } \\
\text { Error }\end{array}$ & $\begin{array}{l}\text { EML } \\
\text { Value }\end{array}$ & $\begin{array}{l}\text { EML } \\
\text { Error }\end{array}$ & $\frac{\text { Reported }}{\text { EML }}$ & $\begin{array}{c}\text { QAP 48 } \\
\text { Evaluation Evaluation }\end{array}$ \\
\hline
\end{tabular}

Matrix: WA Water $\mathrm{Bq} / \mathrm{L}$

$\begin{array}{llrrrrrrr}1 & \text { AM241 } & 1.090 & 0.110 & 1.250 & 0.080 & 0.872 & \text { W } & \mathrm{A} \\ 1 & \text { Bq U } & 1.130 & 0.100 & 1.050 & 0.080 & 1.076 & \mathrm{~A} & \mathrm{~A} \\ 1 & \text { CO60 } & 50.100 & 1.500 & 49.400 & 1.200 & 1.014 & \mathrm{~A} & \mathrm{~A} \\ 1 & \text { CS137 } & 50.000 & 2.200 & 50.000 & 1.700 & 1.000 & \mathrm{~A} & \mathrm{~A} \\ 1 & \text { GROSS ALPHA } & 949.000 & 83.000 & 1080.000 & 60.000 & 0.879 & \mathrm{~A} & \mathrm{~A} \\ 1 & \text { GROSS BETA } & 1370.000 & 80.000 & 1420.000 & 60.000 & 0.965 & \mathrm{~A} & \mathrm{~A} \\ 1 & \text { H3 } & 93.000 & 4.000 & 76.200 & 2.900 & 1.220 & \mathrm{~A} & \mathrm{~A} \\ 1 & \text { MN54 } & 35.400 & 2.300 & 32.400 & 1.400 & 1.093 & \mathrm{~A} & \mathrm{~A} \\ 1 & \text { N163 } & 119.000 & 4.000 & 95.700 & 0.900 & 1.243 & \mathrm{~A} & \\ 1 & \text { PU238 } & 1.180 & 0.090 & 1.100 & 0.010 & 1.073 & \mathrm{~A} & \mathrm{~A} \\ 1 & \text { PU239 } & 1.650 & 0.100 & 1.410 & 0.040 & 1.170 & \mathrm{~W} & \mathrm{~A} \\ 1 & \text { SR90 } & 2.130 & 0.440 & 2.110 & 0.180 & 1.009 & \mathrm{~A} & \mathrm{~A} \\ 1 & \text { U234 } & 0.500 & 0.070 & 0.510 & 0.030 & 0.980 & \mathrm{~A} & \mathrm{~A} \\ 1 & \text { U238 } & 0.560 & 0.070 & 0.520 & 0.050 & 1.077 & \mathrm{~A} & \mathrm{~A}\end{array}$

Values for elemental uranium are reported in $\mu \mathrm{g} /$ filter, $\mathrm{g}$, or $\mathrm{mL} . \quad \mathrm{pCi} / \mathrm{g}$ or $\mathrm{mL}=\mathrm{Bq} \times 0.027$

Evaluation: $A=A c c e p t a b l e, W=$ Acceptable with Warning, $N=$ Not Acceptable

If the evaluation system is not appropriate for the types of analyses performed in your lab, apply a site specific evaluation. 


\section{QAP 49 Results by Laboratory}

Lab: WC Waste Management Federal Services of Hanford

\begin{tabular}{|c|c|c|c|c|c|c|c|}
\hline $\begin{array}{l}\text { No. } \\
\text { Test }\end{array}$ & Radionuclide & $\begin{array}{l}\text { Reported } \\
\text { Value }\end{array}$ & $\begin{array}{c}\text { Reported } \\
\text { Error }\end{array}$ & $\begin{array}{l}\text { EML } \\
\text { Value }\end{array}$ & $\begin{array}{l}\text { EML } \\
\text { Error }\end{array}$ & $\frac{\text { Reported }}{\text { EML }}$ & $\begin{array}{cc}\text { QAP 48 } \\
\text { Evaluation } \\
\text { Evaluation }\end{array}$ \\
\hline
\end{tabular}

Matrix: $\mathrm{AI}$ Air Filter $\mathrm{Bq} /$ filter

$\begin{array}{llrrrrrrr}1 & \text { AM241 } & 0.610 & 0.100 & 0.510 & 0.008 & 1.196 & \mathrm{~A} & \mathrm{~W} \\ 1 & \text { CO60 } & 9.200 & 0.800 & 9.160 & 0.580 & 1.004 & \mathrm{~A} & \mathrm{~A} \\ 1 & \text { CS137 } & 22.000 & 3.400 & 22.470 & 1.030 & 0.979 & \mathrm{~A} & \mathrm{~A} \\ 1 & \text { GROSS ALPHA } & 1.500 & 0.200 & 1.650 & 0.160 & 0.909 & \mathrm{~A} & \mathrm{~A} \\ 1 & \text { GROSS BETA } & 2.300 & 0.200 & 2.160 & 0.070 & 1.065 & \mathrm{~A} & \mathrm{~A} \\ 1 & \text { MN54 } & 5.000 & 0.700 & 4.920 & 0.400 & 1.016 & \mathrm{~A} & \mathrm{~A} \\ 1 & \text { PU238 } & 0.500 & 0.080 & 0.460 & 0.005 & 1.087 & \mathrm{~A} & \mathrm{~W} \\ 1 & \text { PU239 } & 0.470 & 0.080 & 0.420 & 0.006 & 1.119 & \mathrm{~A} & \mathrm{~A} \\ 1 & \text { SB125 } & 9.800 & 0.800 & 8.890 & 0.550 & 1.102 & \mathrm{~A} & \mathrm{~N} \\ 1 & \text { SR90 } & 1.300 & 0.200 & 1.120 & 0.050 & 1.161 & \mathrm{~A} & \mathrm{~A} \\ 1 & \text { U234 } & 0.280 & 0.050 & 0.260 & 0.010 & 1.077 & \mathrm{~A} & \mathrm{~A} \\ 1 & \text { U238 } & 0.270 & 0.050 & 0.260 & 0.010 & 1.038 & \mathrm{~A} & \mathrm{~A}\end{array}$

Matrix: SO Soil $\mathrm{Bq} / \mathrm{kg}$

$\begin{array}{ll}1 & \text { AM241 } \\ 1 & \text { CS137 } \\ 1 & \text { K40 } \\ 1 & \text { PU239 } \\ 1 & \text { SR90 }\end{array}$

8.400
1081.000
413.000
13.000
61.000

$\begin{array}{rr}7.470 & 0.410 \\ 954.000 & 38.000 \\ 314.000 & 13.000 \\ 13.090 & 0.570 \\ 39.630 & 0.003\end{array}$

Matrix: VE Vegetation $\mathrm{Bq} / \mathrm{kg}$

$\begin{array}{ll}1 & \text { AM241 } \\ 1 & \text { CM244 } \\ 1 & \text { CO60 } \\ 1 & \text { CS137 } \\ 1 & \text { K40 } \\ 1 & \text { PU239 } \\ 1 & \text { SR90 }\end{array}$

Matrix: WA Water $\mathrm{Bq} / \mathrm{L}$.

4.800
1.700
23.000
460.000
589.000
4.000
667.000

1.300
0.700
0.200
69.000
73.000
0.400
86.000

0.060
0.070
1.000
20.000
20.000
0.270
40.000

2.060
0.966
1.150
1.179
1.280
1.075
1.101

1.124 1.133

1.315

0.993

1.539

$\begin{array}{llr}1 & \text { AM241 } & 1.400 \\ 1 & \text { CO60 } & 59.000 \\ 1 & \text { CS137 } & 62.000 \\ 1 & \text { GROSS ALPHA } & 1056.000 \\ 1 & \text { GROSS BETA } & 1341.000 \\ 1 & \text { H3 } & 80.000 \\ 1 & \text { MN54 } & 42.000 \\ 1 & \text { PU238 } & 1.100 \\ 1 & \text { PU239 } & 1.600 \\ 1 & \text { SR90 } & 2.300 \\ 1 & \text { U234 } & 0.570 \\ 1 & \text { U238 } & 0.560\end{array}$

$\begin{array}{rr}0.230 & 1.250 \\ 4.600 & 49.400 \\ 8.600 & 50.000 \\ 106.000 & 1080.000 \\ 134.000 & 1420.000 \\ 9.300 & 76.200 \\ 6.000 & 32.400 \\ 0.180 & 1.100 \\ 0.230 & 1.410 \\ 0.320 & 2.110 \\ 0.100 & 0.510 \\ 0.100 & 0.520\end{array}$

$$
\begin{array}{r}
0.080 \\
1.200 \\
1.700 \\
60.000 \\
60.000 \\
2.900 \\
1.400 \\
0.010 \\
0.040 \\
0.180 \\
0.030 \\
0.050
\end{array}
$$

$\begin{array}{ll}\text { W } & \text { A } \\ \text { A } & \text { A } \\ \text { A } & \text { A } \\ \text { A } & \text { A } \\ \text { W } & \text { A } \\ \text { A } & \text { A } \\ \text { A } & \text { A }\end{array}$

$\begin{array}{lll}1.120 & \mathrm{~A} & \mathrm{~A} \\ 1.194 & \mathrm{~W} & \mathrm{~A} \\ 1.240 & \mathrm{~W} & \mathrm{~A} \\ 0.978 & \mathrm{~A} & \mathrm{~A} \\ 0.944 & \mathrm{~A} & \mathrm{~A} \\ 1.050 & \mathrm{~A} & \mathrm{~A} \\ 1.296 & \mathrm{~N} & \mathrm{~A} \\ 1.000 & \mathrm{~A} & \mathrm{~A} \\ 1.135 & \mathrm{~A} & \mathrm{~A} \\ 1.090 & \mathrm{~A} & \mathrm{~A} \\ 1.118 & \mathrm{~A} & \mathrm{~A} \\ 1.077 & \mathrm{~A} & \mathrm{~A}\end{array}$

\section{Values for elemental uranium are reported in $\mu \mathrm{g} /$ filter, $\mathrm{g}$, or $\mathrm{mL}$.}

$\mathrm{pCi} / \mathrm{g}$ or $\mathrm{mL}=\mathrm{Bq} \times 0.027$

Evaluation: $A=A c c e p t a b l e, W=A c c e p t a b l e$ with Warning, $N=$ Not Acceptable

If the evaluation system is not appropriate for the types of analyses performed in your lab, apply a site specific evaluation. 


\section{QAP 49 Results by Laboratory}

Lab: WE Westinghouse Electric Corp., Madison, PA

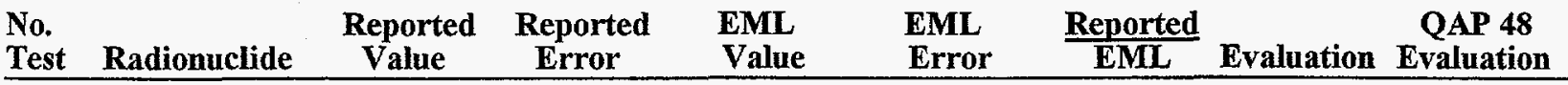

Matrix: AI Air Filter $\mathrm{Bq} /$ filter

$\begin{array}{llrrrrrr}1 & \text { CO60 } & 9.990 & 0.230 & 9.160 & 0.580 & 1.091 & \mathrm{~A} \\ 1 & \text { CS137 } & 26.900 & 4.900 & 22.470 & 1.030 & 1.197 & \mathrm{~W} \\ 1 & \text { MN54 } & 6.130 & 0.630 & 4.920 & 0.400 & 1.246 & \text { W } \\ 1 & \text { SB125 } & 10.900 & 0.590 & 8.890 & 0.550 & 1.226 & \text { W } \\ 1 & \text { SR90 } & 9.210 & 0.830 & 1.120 & 0.050 & 8.223 & \text { N }\end{array}$

Matrix: $\mathrm{SO}$ Soil $\mathrm{Bq} / \mathrm{kg}$

$\begin{array}{ll}1 & \text { AC228 } \\ 1 & \text { BI212 } \\ 1 & \text { BI214 } \\ 1 & \text { CS137 } \\ 1 & \text { K40 } \\ 1 & \text { PB212 } \\ 1 & \text { PB214 } \\ 1 & \text { RA226 } \\ 1 & \text { SR90 } \\ 1 & \text { TH228 } \\ 1 & \text { TH234 } \\ 1 & \text { TL208 }\end{array}$

37.500
37.200
33.900
1130.000
366.000
53.400
39.400
34.400
40.200
37.200
52.800
17.400

$\begin{array}{rrr}13.000 & 52.600 & 2.900 \\ 22.000 & 58.300 & 5.900 \\ 17.000 & 28.800 & 0.500 \\ 290.000 & 954.000 & 38.000 \\ 27.000 & 314.000 & 13.000 \\ 7.900 & 52.800 & 3.700 \\ 10.000 & 29.100 & 1.200 \\ 17.000 & 29.000 & 1.000 \\ 30.000 & 39.630 & 0.003 \\ 22.000 & 52.700 & 4.000 \\ 40.000 & 114.000 & 6.000 \\ 10.000 & 18.300 & 1.100\end{array}$

0.713

0.638

1.177

1.184

1.166

1.011

1.354

1.186

1.014

0.706

0.463

0.951

Matrix: VE Vegetation $\mathrm{Bq} / \mathrm{kg}$

$\begin{array}{llrrrrrr}1 & \text { CO60 } & 21.900 & 2.700 & 20.000 & 1.000 & 1.095 & \text { A } \\ 1 & \text { CS137 } & 469.000 & 120.000 & 390.000 & 20.000 & 1.203 & \text { A } \\ 1 & \text { K40 } & 522.000 & 39.000 & 460.000 & 20.000 & 1.135 & \text { A } \\ 1 & \text { SR90 } & 316.000 & 32.000 & 606.000 & 40.000 & 0.521 & \text { W }\end{array}$

Matrix: WA Water $\mathrm{Bq} / \mathrm{L}$

$\begin{array}{llrrrrrr}1 & \text { CO60 } & 53.400 & 2.600 & 49.400 & 1.200 & 1.081 & \mathrm{~A} \\ 1 & \text { CS137 } & 58.200 & 6.800 & 50.000 & 1.700 & 1.164 & \mathrm{~A} \\ 1 & \text { MN54 } & 40.500 & 2.700 & 32.400 & 1.400 & 1.250 & \mathrm{~N} \\ 1 & \text { SR90 } & 12.900 & 0.880 & 2.110 & 0.180 & 6.114 & \mathrm{~N}\end{array}$

Values for elemental uranium are reported in $\mu \mathrm{g} /$ filter, g, or $\mathrm{mL} . \quad \mathrm{pCi} / \mathrm{g}$ or $\mathrm{mL}=\mathrm{Bq} \times \mathbf{0 . 0 2 7}$

Evaluation: $A=$ Acceptable, $W=$ Acceptable with Warning, $N=$ Not Acceptable

If the evaluation system is not appropriate for the types of analyses performed in your lab, apply a site specific evaluation. 


\section{QAP 49 Results by Laboratory}

Lab: WI WIPP Site, Westinghouse Electric Corp.

No.

Reported Reported

EML

EML

Reported

QAP 48

Test Radionuclide Value Error Value Error EML Evaluation Evaluation

Matrix: WA Water $\mathrm{Bq} / \mathrm{L}$

$\begin{array}{ll}1 & \text { CO60 } \\ 3 & \text { CO60 } \\ 2 & \text { CO60 } \\ 3 & \text { CS137 } \\ 2 & \text { CS137 } \\ 1 & \text { CS137 } \\ 2 & \text { MN54 } \\ 3 & \text { MN54 } \\ 1 & \text { MN54 }\end{array}$

55.980

55.720

55.850

56.620

55.960

56.260

39.490

39.010

38.340

4.243
4.223
4.229
7.027
6.945
6.983
3.990
3.946
3.878

49.400

49.400

49.400

50.000

50.000

50.000

32.400

32.400

32.400
1.200

1.200

1.200

1.700

1.700

1.700

1.400

1.400

1.400
1.133

1.128

1.131

1.132

1.119

1.125

1.219

1.204

1.183

$\begin{array}{ll}\text { A } & \text { W } \\ \text { A } & \text { W } \\ \text { A } & \text { W } \\ \text { A } & \text { A } \\ \text { A } & \text { A } \\ \text { A } & \text { A } \\ \text { W } & \text { A } \\ \text { W } & \text { A } \\ \text { W } & \text { A }\end{array}$

Values for elemental uranium are reported in $\mu \mathrm{g} /$ filter, $g$, or $\mathrm{mL}$.

$\mathrm{pCi} / \mathrm{g}$ or $\mathrm{mL}=\mathrm{Bq} \times \mathbf{0 . 0 2 7}$

Evaluation: $A=$ Acceptable, $W=$ Acceptable with Warning, $N=$ Not Acceptable

If the evaluation system is not appropriate for the types of analyses performed in your lab, apply a site specific evaluation. 


\section{QAP 49 Results by Laboratory}

Lab: WS Weldon Springs Site, St Charles, MO

No.

Reported Reported EML

EML

Reported

QAP 48

Test Radionuclide Value Error

Value

Error EML

Evaluation Evaluation

Matrix: AI Air Filter $\mathrm{Bq}$ / filter

1 GROSS ALPHA $\quad 1.695$

$0.040 \quad 1.650$

0.160

1.027

A

A

Matrix: SO Soil Bq/kg

$1 \quad \mathrm{AM} 241$

$1 \mathrm{CS} 137$

$1 \mathrm{~K} 40$

$1 \mathrm{U} 238$
13.100

1077.100

363.710

122.800
2.410

36.000

18.900

15.700
7.470

954.000

314.000

120.000
0.410

38.000

13.000

9.000
1.754

1.129

1.158

1.023
W
A
A
A

A

A

A

Values for elemental uranium are reported in $\mu \mathrm{g} /$ filter, $g$, or $\mathrm{mL}$.

pCi/g or $\mathrm{mL}=\mathrm{Bq} \times 0.027$

Evaluation: $A=$ Acceptable,$W=$ Acceptable with Warning, $N=$ Not Acceptable

If the evaluation system is not appropriate for the types of analyses performed in your lab, apply a site specific evaluation. 


\section{QAP 49 Results by Laboratory}

Lab: WV West Valley Nuclear Services Co, Inc, NY

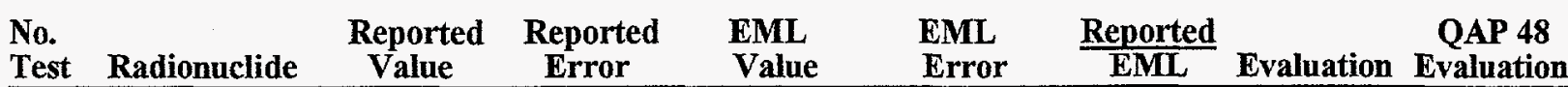

Matrix: AI Air Filter Bq/filter

1 GROSS ALPHA $\quad 1.530$

1 GROSS BETA
0.031

0.036

A

W

Matrix: WA Water $\mathrm{Bq} / \mathrm{L}$

$1 \mathrm{CO} 60$

1 CS137

1 GROSS ALPHA

1 GROSS BETA

$1 \mathrm{H} 3$

$1 \mathrm{MN} 54$

1 SR90
52.060

50.620

848.410

1279.460

80.920

36.320

2.430

$\begin{array}{rr}0.540 & 49.400 \\ 0.440 & 50.000 \\ 59.830 & 1080.000 \\ 59.760 & 1420.000 \\ 4.630 & 76.200 \\ 0.480 & 32.400 \\ 0.240 & 2.110\end{array}$

0.240

1.200
1.700
60.000
60.000
2.900
1.400
0.180

1.054

1.012

0.786

0.901

1.062

1.121

1.152

$\begin{array}{ll}\mathrm{A} & \mathrm{A} \\ \mathrm{A} & \mathrm{A} \\ \mathrm{W} & \mathrm{A} \\ \mathrm{A} & \mathrm{A} \\ \mathrm{A} & \mathrm{A} \\ \mathrm{A} & \mathrm{A} \\ \mathrm{A} & \mathrm{A}\end{array}$

Values for elemental uranium are reported in $\mu \mathrm{g} /$ filter, $\mathrm{g}$, or $\mathrm{mL}$.

$\mathrm{pCi} / \mathrm{g}$ or $\mathrm{mL}=\mathrm{Bq} \times 0.027$

Evaluation: A=Acceptable, $W=$ Acceptable with Warning, $N=$ Not Acceptable

If the evaluation system is not appropriate for the types of analyses performed in your lab, apply a site specific evaluation. 


\section{QAP 49 Results by Laboratory}

Lab: YA Duke Engineering Environmental Lab, Westboro, MA

\begin{tabular}{|c|c|c|c|c|c|c|c|}
\hline est & Radionuclide & $\begin{array}{c}\text { Reported } \\
\text { Value }\end{array}$ & $\begin{array}{l}\text { Reported } \\
\text { Error }\end{array}$ & $\begin{array}{l}\text { EML } \\
\text { Value }\end{array}$ & $\begin{array}{l}\text { EML } \\
\text { Error }\end{array}$ & $\frac{\text { Reported }}{\text { EML }}$ & $\begin{array}{c}\text { QAP 48 } \\
\text { Evaluation Evaluation }\end{array}$ \\
\hline
\end{tabular}

Matrix: AI Air Filter $\mathrm{Bq} /$ filter

$\begin{array}{llrrrrrrr}1 & \text { AM241 } & 0.524 & 0.018 & 0.510 & 0.008 & 1.027 & \mathrm{~A} & \mathrm{~A} \\ 1 & \text { BqU } & 0.567 & 0.013 & 0.530 & 0.020 & 1.070 & \mathrm{~A} & \\ 1 & \text { CO60 } & 8.845 & 0.237 & 9.160 & 0.580 & 0.966 & \mathrm{~A} & \mathrm{~A} \\ 1 & \text { CS137 } & 22.292 & 0.370 & 22.470 & 1.030 & 0.992 & \mathrm{~A} & \mathrm{~A} \\ 1 & \text { GROSS ALPHA } & 1.555 & 0.017 & 1.650 & 0.160 & 0.943 & \mathrm{~A} & \mathrm{~A} \\ 1 & \text { GROSS BETA } & 1.906 & 0.021 & 2.160 & 0.070 & 0.882 & \mathrm{~W} & \mathrm{~A} \\ 1 & \text { MN54 } & 5.223 & 0.257 & 4.920 & 0.400 & 1.062 & \mathrm{~A} & \mathrm{~A} \\ 1 & \text { PU238 } & 0.499 & 0.008 & 0.460 & 0.005 & 1.085 & \mathrm{~A} & \mathrm{~A} \\ 1 & \text { PU239 } & 0.462 & 0.008 & 0.420 & 0.006 & 1.099 & \mathrm{~A} & \mathrm{~A} \\ 1 & \text { SR90 } & 1.221 & 0.081 & 1.120 & 0.050 & 1.090 & \mathrm{~A} & \mathrm{~A}\end{array}$

Matrix: SO Soil $\mathrm{Bq} / \mathrm{kg}$

$\begin{array}{ll}1 & \text { AM241 } \\ 1 & \text { Bq U } \\ 1 & \text { CS137 } \\ 1 & \text { K40 } \\ 1 & \text { PU239 } \\ 1 & \text { SR90 } \\ 1 & U 234 \\ 1 & \text { U238 }\end{array}$

$\begin{array}{rr}6.736 & 0.241 \\ 225.805 & 6.418 \\ 1176.415 & 3.330 \\ 384.060 & 8.010 \\ 13.858 & 0.433 \\ 51.603 & 3.083 \\ 107.238 & 2.985 \\ 113.800 & 3.157\end{array}$

7.470
237.000
954.000
314.000
13.090
39.630
113.000
120.000

0.410

16.000

38.000

13.000

0.570

0.003

6.000

9.000

0.902
0.953
1.233
1.223
1.059
1.302
0.949
0.948

$\begin{array}{ll}\text { A } & \text { A } \\ \text { A } & \\ \text { W } & \text { A } \\ \text { A } & \text { A } \\ \text { A } & \text { A } \\ \text { A } & \text { W } \\ \text { A } & \text { A } \\ \text { A } & \text { A }\end{array}$

Matrix: VE Vegetation $\mathrm{Bq} / \mathrm{kg}$

$\begin{array}{ll}1 & \text { AM241 } \\ 1 & \text { CM244 } \\ 1 & \text { CO60 } \\ 1 & \text { CS137 } \\ 1 & \text { K40 } \\ 1 & \text { PU239 } \\ 1 & \text { SR90 }\end{array}$

$\begin{array}{rr}2.533 & 0.122 \\ 1.376 & 0.088 \\ 20.590 & 0.647 \\ 415.880 & 2.720 \\ 511.340 & 13.505 \\ 3.881 & 0.099 \\ 615.803 & 31.943\end{array}$

2.330
1.760
20.000
390.000
460.000
3.720
606.000

0.060
0.070
1.000
20.000
20.000
0.270
40.000

1.087

0.782

1.030

1.066

1.112

1.043

1.016

$\begin{array}{ll}\mathrm{A} & \mathrm{A} \\ \mathrm{W} & \mathrm{A} \\ \mathrm{A} & \mathrm{A} \\ \mathrm{A} & \mathrm{A} \\ \mathrm{A} & \mathrm{A} \\ \mathrm{A} & \mathrm{A} \\ \mathrm{A} & \mathrm{A}\end{array}$

Matrix: WA Water $\mathrm{Bq} / \mathrm{L}$

$\begin{array}{llr}1 & \text { AM241 } & 1.240 \\ 1 & \text { CO60 } & 49.013 \\ 1 & \text { CS137 } & 48.902 \\ 1 & \text { FE55 } & 136.543 \\ 1 & \text { GROSS ALPHA } & 720.020 \\ 1 & \text { GROSS BETA } & 1194.977 \\ 1 & \text { H3 } & 81.079 \\ 1 & \text { MN54 } & 33.781 \\ 1 & \text { NI63 } & 124.073 \\ 1 & \text { PU238 } & 1.195 \\ 1 & \text { PU239 } & 1.534 \\ 1 & \text { SR90 } & 1.449\end{array}$

0.040
0.752
0.913
11.347
16.773
16.403
2.504
0.802
9.990
0.038
0.046
0.315

1.250
49.400
50.000
139.000
1080.000
1420.000
76.200
32.400
95.700
1.100
1.410
2.110

0.080
1.200
1.700
2.000
60.000
60.000
2.900
1.400
0.900
0.010
0.040
0.180
0.992
0.992
0.978
0.982
0.667
0.842
1.064
1.043
1.296
1.086
1.088
0.687

$\begin{array}{ll}\text { A } & \text { A } \\ \text { A } & \text { A } \\ \text { A } & \text { A } \\ \text { A } & \text { W } \\ \text { W } & \text { A } \\ \text { A } & \text { A } \\ \text { A } & \text { A } \\ \text { A } & \text { A } \\ \text { A } & \\ \text { A } & \text { A } \\ \text { A } & \text { A } \\ \text { N } & \text { A }\end{array}$

Values for elemental uranium are reported in $\mu \mathrm{g} /$ filter, $\mathrm{g}$, or $\mathrm{mL}$.

$\mathrm{pCi} / \mathrm{g}$ or $\mathrm{mL}=\mathrm{Bq} \times 0.027$

Evaluation: $A=$ Acceptable, $W=$ Acceptable with Warning, $N=N o t$ Acceptable

If the evaluation system is not appropriate for the types of analyses performed in your lab, apply a site specific evaluation. 


\section{QAP 49 Results by Laboratory}

Lab: YA Duke Engineering Environmental Lab, Westboro, MA

Reported Reported

EML

EML

Reported

QAP 48

Test Radionuclide

Value

Error

Value

Error EML

Evaluation Evaluation

Matrix: WA Water $\mathrm{Bq} / \mathrm{L}$
1 ug U
0.040
0.001
0.040
0.003
0.990
A
W

Values for elemental uranium are reported in $\mu \mathrm{g} /$ filter, $\mathrm{g}$, or $\mathrm{mL}$.

$\mathrm{pCi} / \mathrm{g}$ or $\mathrm{mL}=\mathrm{Bq} \times 0.027$

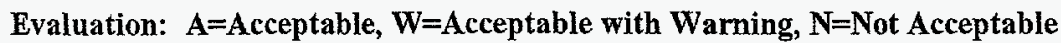

If the evaluation system is not appropriate for the types of analyses performed in your lab, apply a site specific evaluation. 


\section{QAP 49 Results by Laboratory}

Lab: YP US Army Proving Ground, Yuma, AZ

No.

Reported Reported

EML

EML

Reported

QAP 48

Test Radionuclide Value Error Value Error EML

Evaluation

Evaluation

Matrix: AI Air Filter $\mathrm{Bq}$ / filter

$0.940 \quad 20.960$

0.100

0.997

A

A

Matrix: SO Soil $\mathrm{Bq} / \mathrm{kg}$

1 ug $\mathrm{U}$

8.240

1.510

9.700

0.700

0.849

A

A

Matrix: WA Water $\mathrm{Bq} / \mathrm{L}$

1 ug U

0.040

0.002

0.040

0.003

1.000

A

A

Values for elemental uranium are reported in $\mu \mathrm{g} /$ filter, $\mathrm{g}$, or $\mathrm{mL}$.

$\mathrm{pCi} / \mathrm{g}$ or $\mathrm{mL}=\mathrm{Bq} \times 0.027$

Evaluation: A=Acceptable, $W=A c c e p t a b l e$ with Warning, $N=$ Not Acceptable

If the evaluation system is not appropriate for the types of analyses performed in your lab, apply a site specific evaluation. 


\section{QAP 49 Results by Laboratory}

Lab: YU Institute of Occupational and Radiological Health, Yugoslovia

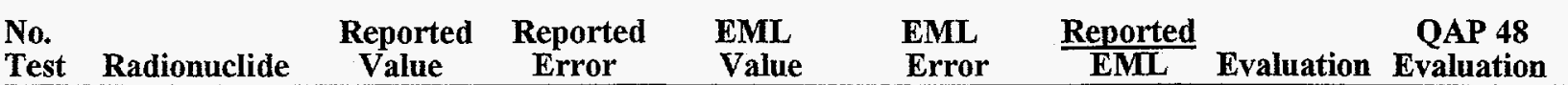

Matrix: AI Air Filter $\mathrm{Bq} /$ filter

$\begin{array}{llrrrrrrr}1 & \text { CO60 } & 8.100 & 0.500 & 9.160 & 0.580 & 0.884 & \text { A } & \text { W } \\ 1 & \text { CS137 } & 19.300 & 0.700 & 22.470 & 1.030 & 0.859 & \text { A } & \text { W } \\ 1 & \text { MN54 } & 4.300 & 0.400 & 4.920 & 0.400 & 0.874 & \text { A } & \text { W } \\ 1 & \text { SB125 } & 7.000 & 0.200 & 8.890 & 0.550 & 0.787 & \text { W } & \text { W }\end{array}$

Matrix: SO Soil $\mathrm{Bq} / \mathrm{kg}$

$\begin{array}{lllllllll}1 & \operatorname{CS} 137 & 951.000 & 20.000 & 954.000 & 38.000 & 0.997 & \mathrm{~A} & \mathrm{~A} \\ 1 & \mathrm{~K} 40 & 315.000 & 10.000 & 314.000 & 13.000 & 1.003 & \mathrm{~A} & \mathrm{~A}\end{array}$

Matrix: VE Vegetation $\mathrm{Bq} / \mathrm{kg}$

$\begin{array}{llrrrrrrr}1 & \text { CO60 } & 17.800 & 1.200 & 20.000 & 1.000 & 0.890 & \text { A } & \text { W } \\ 1 & \text { CS137 } & 367.000 & 20.000 & 390.000 & 20.000 & 0.941 & \text { A } & \text { W } \\ 1 & \text { K40 } & 535.000 & 20.000 & 460.000 & 20.000 & 1.163 & \text { A } & \text { W }\end{array}$

Matrix: WA Water $\mathrm{Bq} / \mathrm{L}$

$\begin{array}{lllllllll}1 & \text { CO60 } & 48.300 & 1.500 & 49.400 & 1.200 & 0.978 & \text { A } & \text { A } \\ 1 & \text { CS137 } & 48.200 & 1.500 & 50.000 & 1.700 & 0.964 & \text { A } & \text { A } \\ 1 & \text { MN54 } & 33.000 & 1.100 & 32.400 & 1.400 & 1.019 & \text { A } & \text { A }\end{array}$

Values for elemental uranium are reported in $\mu \mathrm{g} /$ filter, $\mathrm{g}$, or $\mathrm{mL} . \quad \mathrm{pCi} / \mathrm{g}$ or $\mathrm{mL}=\mathrm{Bq} \times 0.027$

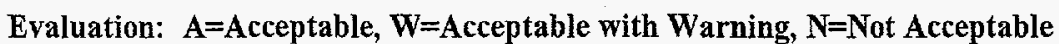

If the evaluation system is not appropriate for the types of analyses performed in your lab, apply a site specific evaluation. 


\section{QAP 49 Results by Nuclide}

\begin{tabular}{lc}
\hline Matrix: AI & Air Filter Bq / filter \\
Radionuclide: & AM241 \\
\hline EML Value: & 0.510 \\
EML Error: & 0.008 \\
\hline
\end{tabular}

\begin{tabular}{|c|c|c|c|c|c|c|}
\hline Labcode & Test \# & $\begin{array}{l}\text { Reported } \\
\text { Value }\end{array}$ & $\begin{array}{c}\text { Reported } \\
\text { Error }\end{array}$ & $\frac{\text { Reported }}{E M L}$ & $\begin{array}{c}\text { QAP 48 } \\
\text { Evaluation }\end{array}$ & Evaluation \\
\hline $\mathrm{AF}$ & 1 & 0.1400 & 0.0200 & 0.275 & W & $N$ \\
\hline $\mathrm{AG}$ & 1 & 0.2260 & 0.0300 & 0.443 & W & $\mathrm{N}$ \\
\hline AM & 1 & 0.4800 & 0.0500 & 0.941 & $\mathrm{~N}$ & A \\
\hline $\mathrm{AN}$ & 1 & 0.5300 & 0.0200 & 1.039 & A & A \\
\hline $\mathrm{AU}$ & 1 & 0.8530 & 0.0640 & 1.673 & $\mathrm{~N}$ & W \\
\hline $\mathrm{BE}$ & 1 & 0.5600 & 0.0500 & 1.098 & A & $\mathrm{A}$ \\
\hline $\mathrm{BL}$ & 1 & 0.5990 & 0.0410 & 1.175 & $\mathrm{~W}$ & A \\
\hline $\mathrm{BL}$ & 2 & 0.5520 & 0.0350 & 1.082 & W & $\mathrm{A}$ \\
\hline $\mathrm{BM}$ & 1 & 0.4600 & 0.0600 & 0.902 & A & A \\
\hline BP & 1 & 0.5750 & 0.0190 & 1.127 & A & A \\
\hline $\mathrm{BU}$ & 1 & 0.6000 & 0.0300 & 1.176 & A & A \\
\hline BX & 1 & 0.5000 & 0.0400 & 0.980 & A & A \\
\hline $\mathrm{CB}$ & 1 & 0.6000 & 0.0700 & 1.176 & & A \\
\hline $\mathrm{CB}$ & 2 & 0.4200 & 0.0900 & 0.824 & & W \\
\hline $\mathrm{CB}$ & 3 & 0.5000 & 0.0300 & 0.980 & & A \\
\hline $\mathrm{CH}$ & 1 & 0.5350 & 0.0270 & 1.049 & A & A \\
\hline $\mathrm{CL}$ & 1 & 0.5100 & 0.1000 & 1.000 & A & A \\
\hline$\overline{\mathrm{CN}}$ & 1 & 0.9100 & 0.1300 & 1.784 & & $\mathrm{~W}$ \\
\hline $\mathrm{CS}$ & 1 & 0.5198 & 0.0387 & 1.019 & W & A \\
\hline EG & 1 & 0.5680 & 0.0440 & 1.114 & A & A \\
\hline $\mathrm{EP}$ & 1 & 0.5470 & 0.0504 & 1.073 & A & A \\
\hline FJ & 1 & 0.4600 & 0.0900 & 0.902 & A & A \\
\hline FM & 1 & 0.5700 & 0.0300 & 1.118 & & A \\
\hline GA & 1 & 0.5600 & 0.0180 & 1.098 & A & A \\
\hline GE & 1 & 0.5494 & 0.1421 & 1.077 & W & A \\
\hline GP & 1 & 0.5000 & 0.0500 & 0.980 & A & A \\
\hline GT & 1 & 0.5400 & 0.1000 & 1.059 & $\mathrm{~A}$ & A \\
\hline HU & 1 & 0.3400 & 0.1900 & 0.667 & & $\mathrm{~N}$ \\
\hline IN & 1 & 0.5400 & 0.0500 & 1.059 & & $\mathrm{~A}$ \\
\hline IS & 1 & 0.3967 & 0.1841 & 0.778 & $\mathrm{~N}$ & W \\
\hline IT & 1 & 0.4600 & 0.0700 & 0.902 & A & A \\
\hline LA & 1 & 0.5430 & 0.0170 & 1.065 & A & A \\
\hline LA & 2 & 0.5600 & 0.0170 & 1.098 & A & A \\
\hline LA & 3 & 0.5630 & 0.0170 & 1.104 & A & A \\
\hline LL & 1 & 0.5670 & 0.0307 & 1.112 & A & $\mathrm{A}$ \\
\hline LN & 1 & 0.3700 & 0.0700 & 0.725 & & $\mathrm{~N}$ \\
\hline LV & 1 & 0.8940 & 0.0510 & 1.753 & $\mathrm{~N}$ & W \\
\hline ME & 1 & 0.7600 & 0.0600 & 1.490 & $\mathrm{~N}$ & W \\
\hline MS & 1 & 0.4500 & 0.0500 & 0.882 & A & A \\
\hline $\mathrm{NM}$ & 1 & 0.4780 & 0.0130 & 0.937 & A & A \\
\hline NQ & 1 & 0.4440 & 0.0270 & 0.871 & & W \\
\hline OT & 1 & 0.4800 & 0.0300 & 0.941 & A & A \\
\hline
\end{tabular}

Values for elemental uranium are reported in $\mu \mathrm{g} /$ filter, $\mathrm{g}$ or $\mathrm{mL}$.

$\mathrm{pCi} / \mathrm{g}$ or $\mathrm{mL}=\mathrm{Bq} \times \mathbf{0 . 0 2 7}$

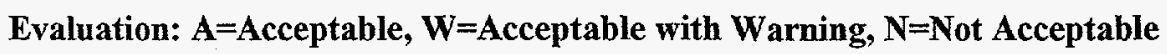

If the evaluation system is not appropriate for the types of analyses performed in your lab, apply site specific evaluation. 


\section{QAP 49 Results by Nuclide}

\begin{tabular}{|c|c|c|c|c|c|c|}
\hline \multicolumn{3}{|c|}{$\begin{array}{l}\text { Matrix: AI } \\
\text { Radionuclide: }\end{array}$} & \multirow[b]{3}{*}{$\begin{array}{l}\text { Reported } \\
\text { Error }\end{array}$} & \multirow[b]{3}{*}{$\frac{\text { Reported }}{E M L}$} & \multirow[b]{3}{*}{$\begin{array}{l}\text { QAP } 48 \\
\text { Evaluation }\end{array}$} & \multirow[b]{3}{*}{ Evaluation } \\
\hline $\begin{array}{l}\text { EML Value: } \\
\text { EML Error: }\end{array}$ & \multicolumn{2}{|c|}{$\begin{array}{l}0.510 \\
0.008\end{array}$} & & & & \\
\hline Labcode & Test \# & $\begin{array}{l}\text { Reported } \\
\text { Value }\end{array}$ & & & & \\
\hline PO & 1 & 0.7000 & 0.1000 & 1.373 & & A \\
\hline PR & 1 & 0.6291 & 0.1915 & 1.234 & & A \\
\hline RE & 1 & 0.5150 & 0.0390 & 1.010 & A & A \\
\hline RI & 1 & 0.4660 & 0.0322 & 0.914 & A & A \\
\hline SK & 1 & 0.6500 & 0.0500 & 1.275 & A & A \\
\hline $\mathrm{SN}$ & 1 & 0.5900 & 0.0840 & 1.157 & $\mathrm{~A}$ & A \\
\hline SR & 1 & 0.5110 & 0.0230 & 1.002 & $\mathrm{~A}$ & A \\
\hline SW & 1 & 0.8700 & 0.0500 & 1.706 & A & $\mathrm{W}$ \\
\hline TI & 1 & 0.5400 & 0.0500 & 1.059 & A & A \\
\hline TN & 1 & 0.5200 & 0.0500 & 1.020 & A & A \\
\hline TX & 1 & 0.4910 & 0.0140 & 0.963 & $\mathrm{~A}$ & A \\
\hline UP & 1 & 0.5570 & 0.0675 & 1.092 & & A \\
\hline UY & 1 & 0.5220 & 0.0730 & 1.024 & A & A \\
\hline WA & 1 & 0.4400 & 0.0400 & 0.863 & A & W \\
\hline WC & 1 & 0.6100 & 0.1000 & 1.196 & W & A \\
\hline YA & 1 & 0.5239 & 0.0181 & 1.027 & A & A \\
\hline
\end{tabular}

Total Number Reported: 


\section{QAP 49 Results by Nuclide}

\begin{tabular}{|c|c|c|c|c|c|c|}
\hline \multicolumn{3}{|c|}{ Matrix: AI Air Filter $\mathrm{Bq}$ / filter } & \multirow[b]{3}{*}{$\begin{array}{c}\text { Reported } \\
\text { Error }\end{array}$} & \multirow[b]{3}{*}{$\frac{\text { Reported }}{E M L}$} & \multirow[b]{3}{*}{$\begin{array}{c}\text { QAP 48 } \\
\text { Evaluation }\end{array}$} & \multirow[b]{3}{*}{ Evalu } \\
\hline \multirow{2}{*}{$\begin{array}{l}\text { EML Value: } \\
\text { EML Error: } \\
\text { Labcode }\end{array}$} & \multicolumn{2}{|c|}{$\begin{array}{l}0.530 \\
0.020 \\
\end{array}$} & & & & \\
\hline & Test \# & $\begin{array}{l}\text { Reported } \\
\text { Value }\end{array}$ & & & & \\
\hline $\mathrm{AF}$ & 1 & 0.5100 & 0.0600 & 0.962 & & A \\
\hline $\mathrm{AG}$ & 1 & 0.5260 & 0.0450 & 0.992 & A & A \\
\hline AM & 1 & 0.9400 & 0.0200 & 1.774 & W & W \\
\hline $\mathrm{BL}$ & 1 & 0.5280 & 0.0080 & 0.996 & $A$ & A \\
\hline $\mathrm{BP}$ & 1 & 0.5420 & 0.0140 & 1.023 & & A \\
\hline $\mathrm{BU}$ & 1 & 0.4900 & 0.0300 & 0.925 & A & A \\
\hline $\mathrm{CH}$ & 1 & 0.5100 & 0.0250 & 0.962 & A & A \\
\hline $\mathrm{CL}$ & 1 & 0.5500 & 0.1000 & 1.038 & A & A \\
\hline GP & 1 & 0.4200 & & 0.792 & A & $\mathrm{N}$ \\
\hline IT & 1 & 0.4900 & 0.0200 & 0.925 & & A \\
\hline NL & 1 & 0.5380 & 0.0630 & 1.015 & & A \\
\hline OT & 1 & 0.4800 & 0.0400 & 0.906 & A & A \\
\hline UP & 1 & 0.6780 & 0.0830 & 1.279 & & A \\
\hline UY & 1 & 0.5540 & 0.0640 & 1.045 & A & A \\
\hline WA & 1 & 0.4700 & 0.0600 & 0.887 & A & W \\
\hline YA & 1 & 0.5673 & 0.0128 & 1.070 & & A \\
\hline
\end{tabular}

Total Number Reported: 


\section{QAP 49 Results by Nuclide}

Matrix: AI Air Filter $\mathrm{Bq} /$ filter

Radionuclide: $\quad \mathrm{CO} 60$

\begin{tabular}{ll} 
EML Value: & 9.160 \\
EML Error: & 0.580 \\
\hline
\end{tabular}

Labcode Test \# $\begin{gathered}\text { Reported } \\ \text { Value }\end{gathered}$

Reported
Error $\frac{\text { Reported }}{\text { EML }} \quad \begin{gathered}\text { QAP 48 } \\ \text { Evaluation }\end{gathered}$

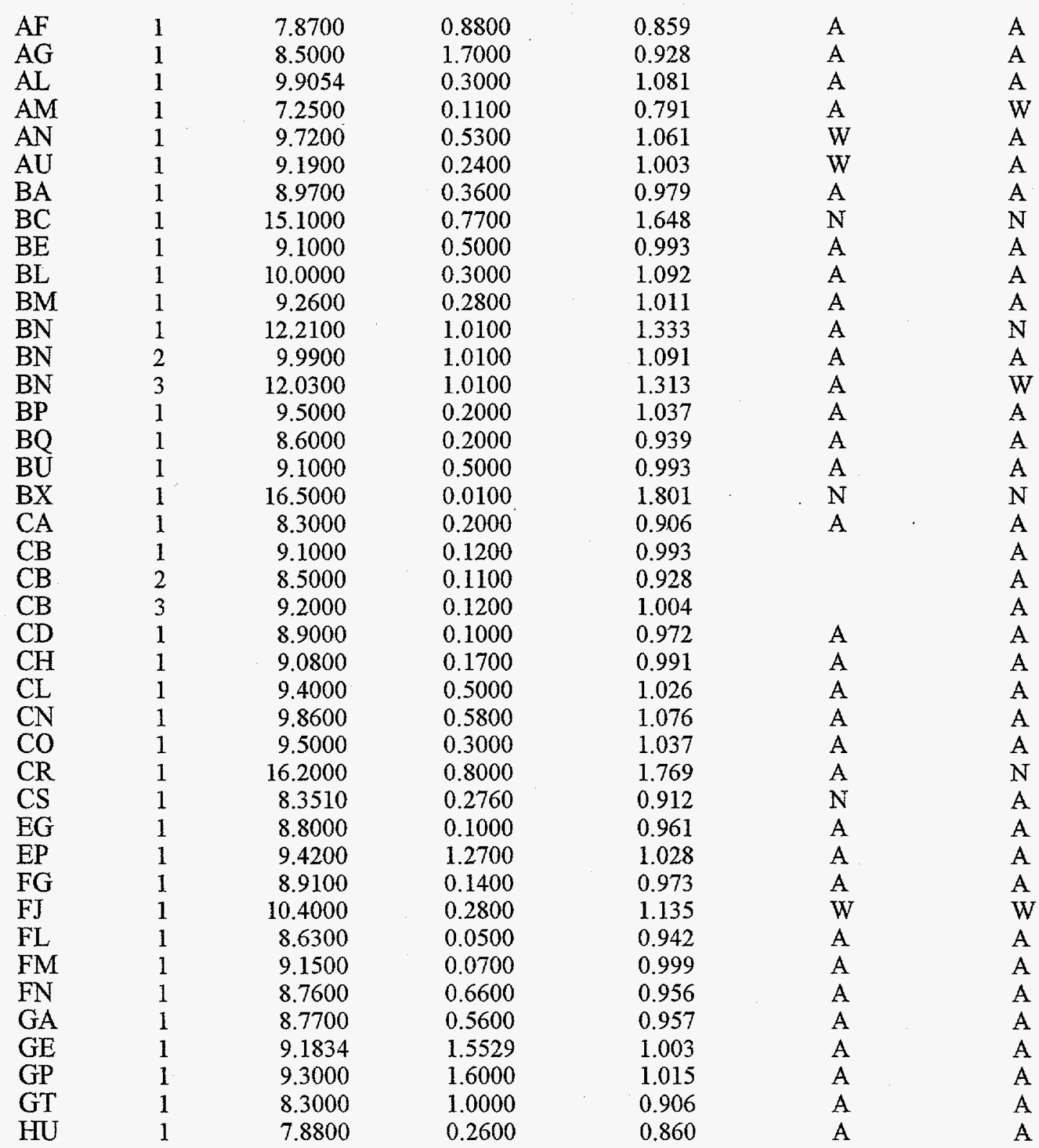

Values for elemental uranium are reported in $\mu \mathrm{g} / \mathrm{filter}, \mathrm{g}$ or $\mathrm{mL}$.

$\mathrm{pCi} / \mathrm{g}$ or $\mathrm{mL}=\mathrm{Bq} \times 0.027$

Evaluation: $A=$ Acceptable, $W=$ Acceptable with Warning, $N=$ Not Acceptable

If the evaluation system is not appropriate for the types of analyses performed in your lab, apply site specific evaluation. 


\section{QAP 49 Results by Nuclide}

Matrix: AI Air Filter Bq/filter

Radionuclide: $\quad$ CO60

\begin{tabular}{ll}
\hline EML Value: & 9.160 \\
EML Error: & 0.580 \\
\hline
\end{tabular}

Labcode Test \# $\begin{gathered}\text { Reported } \\ \text { Value }\end{gathered}$

Reported Reported QAP 48

Error Evaluation

Evaluation

\begin{tabular}{|c|c|c|c|c|c|c|}
\hline LA & 1 & 8.5300 & 0.0900 & 0.931 & W & A \\
\hline IA & 2 & 8.3100 & 0.0900 & 0.907 & W & A \\
\hline IA & 3 & 8.2300 & 0.0900 & 0.898 & W & A \\
\hline ID & 1 & 9.7000 & 0.5920 & 1.059 & W & A \\
\hline IL & 1 & 8.9000 & 0.1000 & 0.972 & A & A \\
\hline $\mathrm{IN}$ & 1 & 8.5000 & 0.3000 & 0.928 & A & A \\
\hline IS & 1 & 9.5000 & 1.1700 & 1.037 & A & A \\
\hline IT & 1 & 8.8200 & 0.3300 & 0.963 & A & A \\
\hline KR & 1 & 8.8000 & 0.9000 & 0.961 & & A \\
\hline LA & 1 & 9.1900 & 0.7200 & 1.003 & A & A \\
\hline LA & 2 & 9.1200 & 0.7400 & 0.996 & A & A \\
\hline LA & 3 & 9.0700 & 0.7300 & 0.990 & A & A \\
\hline LB & 1 & 10.4000 & 1.1000 & 1.135 & & W \\
\hline LL & 1 & 9.0100 & 0.2520 & 0.984 & A & A \\
\hline LN & 1 & 8.1000 & 0.2800 & 0.884 & A & $\mathrm{A}$ \\
\hline LV & 1 & 8.4000 & 0.1600 & 0.917 & A & A \\
\hline MA & 1 & 9398.0000 & 1295.0000 & 1025.983 & & $\mathrm{~N}$ \\
\hline $\mathrm{ME}$ & 1 & 10.2000 & 0.3100 & 1.114 & W & W \\
\hline $\mathrm{MH}$ & 1 & 9.8400 & 0.2600 & 1.074 & W & $\mathrm{A}$ \\
\hline MS & 1 & 8.9600 & 0.9000 & 0.978 & A & A \\
\hline $\mathrm{NA}$ & 1 & 8.7100 & 0.0800 & 0.951 & A & A \\
\hline ND & 1 & 8.8130 & 0.2280 & 0.962 & & A \\
\hline NL & 1 & 9.3000 & 1.5600 & 1.015 & A & A \\
\hline NP & 1 & 9.0700 & 0.0700 & 0.990 & A & A \\
\hline NS & 1 & 9.1360 & 0.1000 & 0.997 & W & A \\
\hline $\mathrm{NZ}$ & 1 & 9.1000 & 0.5000 & 0.993 & & A \\
\hline $\mathrm{NZ}$ & 2 & 9.1000 & 0.5000 & 0.993 & & A \\
\hline OB & 1 & 15.3000 & 2.7100 & 1.670 & & $\mathrm{~N}$ \\
\hline OC & 1 & 10.0000 & 3.0000 & 1.092 & & A \\
\hline $\mathrm{OC}$ & 2 & 10.0000 & 3.0000 & 1.092 & & A \\
\hline $\mathrm{OC}$ & 3 & 10.0000 & 3.0000 & 1.092 & & $\mathrm{~A}$ \\
\hline OD & 1 & 8.9960 & 0.1460 & 0.982 & A & A \\
\hline OD & 1 & 9.0000 & 0.1400 & 0.983 & A & A \\
\hline OD & 2 & 9.0780 & 0.1400 & 0.991 & A & A \\
\hline OD & 2 & 9.0800 & 0.1400 & 0.991 & A & A \\
\hline OL & 1 & 9.7900 & 0.3400 & 1.069 & A & A \\
\hline OT & 1 & 9.4000 & 0.7000 & 1.026 & A & A \\
\hline $\mathrm{OU}$ & 1 & 8.9700 & 0.2900 & 0.979 & A & A \\
\hline PK & 1 & 9.0350 & 0.3800 & 0.986 & & A \\
\hline $\mathrm{PO}$ & 1 & 10.5000 & 0.5000 & 1.146 & A & $\mathrm{W}$ \\
\hline PR & 1 & 9.3443 & 0.0945 & 1.020 & & A \\
\hline RA & 1 & 9.2900 & 0.4300 & 1.014 & A & A \\
\hline
\end{tabular}

Values for elemental uranium are reported in $\mu \mathrm{g} /$ filter, $\mathrm{g}$ or $\mathrm{mL}$.

pCi/g or $\mathrm{mL}=\mathrm{Bq} \times 0.027$

Evaluation: $A=$ Acceptable, $W=$ Acceptable with Warning, $N=$ Not Acceptable

If the evaluation system is not appropriate for the types of analyses performed in your lab, apply site specific evaluation. 
Matrix: AI Air Filter $\mathrm{Bq} /$ filter

Radionuclide:

$\mathrm{CO} 60$

\begin{tabular}{ll} 
EML Value: & 9.160 \\
EML Error: & 0.580 \\
\hline
\end{tabular}

\begin{tabular}{ccccccc} 
Labcode & Test \# & $\begin{array}{c}\text { Reported } \\
\text { Value }\end{array}$ & $\begin{array}{c}\text { Reported } \\
\text { Error }\end{array}$ & $\begin{array}{c}\text { Reported } \\
\text { EML }\end{array}$ & $\begin{array}{c}\text { QAP 48 } \\
\text { Evaluation }\end{array}$ & Evaluation \\
\hline RA & 2 & 9.3600 & 0.2200 & 1.022 & $\mathrm{~A}$ & $\mathrm{~A}$ \\
RC & 1 & 9.1600 & 0.6500 & 1.000 & $\mathrm{~A}$ & $\mathrm{~A}$ \\
RE & 1 & 8.8200 & 0.9100 & 0.963 & $\mathrm{~A}$ & $\mathrm{~A}$ \\
RI & 1 & 8.0000 & 0.7820 & 0.873 & $\mathrm{~A}$ & $\mathrm{~A}$ \\
SA & 1 & 9.4000 & 1.2000 & 1.026 & $\mathrm{~A}$ & $\mathrm{~A}$ \\
SB & 1 & 9.7400 & 0.9300 & 1.063 & & $\mathrm{~A}$ \\
SK & 1 & 8.6000 & 0.9000 & 0.939 & $\mathrm{~A}$ & $\mathrm{~A}$ \\
SR & 1 & 8.7600 & 0.1580 & 0.956 & $\mathrm{~A}$ & $\mathrm{~A}$ \\
SW & 1 & 9.4000 & 0.0900 & 1.026 & $\mathrm{~A}$ & $\mathrm{~A}$ \\
TE & 1 & 9.3000 & 0.3000 & 1.015 & $\mathrm{~A}$ & $\mathrm{~A}$ \\
TI & 1 & 8.8000 & 0.9000 & 0.961 & $\mathrm{~A}$ & $\mathrm{~A}$ \\
TM & 1 & 7.9400 & 0.4230 & 0.867 & $\mathrm{~A}$ & $\mathrm{~A}$ \\
TN & 1 & 8.1800 & 0.1600 & 0.893 & $\mathrm{~A}$ & $\mathrm{~A}$ \\
TP & 1 & 9.4800 & 0.0500 & 1.035 & $\mathrm{~A}$ & $\mathrm{~A}$ \\
TW & 1 & 9.4000 & 0.2000 & 1.026 & $\mathrm{~A}$ & $\mathrm{~A}$ \\
TX & 1 & 8.2500 & 0.0700 & 0.901 & $\mathrm{~A}$ & $\mathrm{~A}$ \\
UC & 1 & 9.1170 & 1.2380 & 0.995 & $\mathrm{~A}$ & $\mathrm{~A}$ \\
UP & 1 & 9.5800 & 0.4670 & 1.046 & $\mathrm{~W}$ & $\mathrm{~A}$ \\
UY & 1 & 9.2600 & 1.1000 & 1.011 & $\mathrm{~A}$ & $\mathrm{~A}$ \\
WA & 1 & 8.7000 & 0.2000 & 0.950 & $\mathrm{~A}$ & $\mathrm{~A}$ \\
WC & 1 & 9.2000 & 0.8000 & 1.004 & $\mathrm{~A}$ & $\mathrm{~A}$ \\
WE & 1 & 9.9900 & 0.2300 & 1.091 & & $\mathrm{~A}$ \\
YA & 1 & 8.8449 & 0.2368 & 0.966 & $\mathrm{~W}$ & \\
YU & 1 & 8.1000 & 0.5000 & 0.884 & & \\
& & & & & &
\end{tabular}

Total Number Reported: $\quad 107$ 


\section{QAP 49 Results by Nuclide}

Matrix: $\mathrm{AI}$ Air Filter $\mathrm{Bq} /$ filter
Radionuclide: $\quad \operatorname{CS} 137$

\begin{tabular}{lr}
\hline EML Value: & 22.470 \\
EML Error: & 1.030 \\
\hline
\end{tabular}

Labcode Test \# $\begin{gathered}\text { Reported } \\ \text { Value }\end{gathered}$

$\begin{array}{ccc}\text { Reported } & \text { Reported } & \text { QAP 48 } \\ \text { Error } & \text { EvLluation } & \text { Evaluation }\end{array}$

\begin{tabular}{|c|c|c|c|c|c|}
\hline $\mathrm{AF}$ & 1 & 19.5800 & 2.1400 & 0.871 & A \\
\hline$A G$ & 1 & 22.3000 & 4.1000 & 0.992 & A \\
\hline AL & 1 & 22.5560 & 0.1000 & 1.004 & W \\
\hline AM & 1 & 17.9400 & 0.1800 & 0.798 & A \\
\hline AN & 1 & 22.6000 & 0.7000 & 1.006 & A \\
\hline $\mathrm{AU}$ & 1 & 22.3500 & 1.0300 & 0.995 & $\mathrm{~N}$ \\
\hline BA & 1 & 23.6000 & 1.7000 & 1.050 & A \\
\hline $\mathrm{BC}$ & 1 & 44.2000 & 3.4000 & 1.967 & $\mathrm{~N}$ \\
\hline $\mathrm{BE}$ & 1 & 23.1000 & 2.0000 & 1.028 & A \\
\hline $\mathrm{BL}$ & 1 & 26.7000 & 0.8000 & 1.188 & A \\
\hline $\mathrm{BM}$ & 1 & 22.2000 & 0.3300 & 0.988 & A \\
\hline $\mathrm{BN}$ & 1 & 36.7000 & 1.9900 & 1.633 & W \\
\hline $\mathrm{BN}$ & 2 & 33.6700 & 1.9900 & 1.498 & $\mathrm{~W}$ \\
\hline $\mathrm{BN}$ & 3 & 38.4800 & 1.9900 & 1.713 & W \\
\hline $\mathrm{BP}$ & 1 & 22.9000 & 0.5000 & 1.019 & A \\
\hline$B Q$ & 1 & 24.4000 & 0.2000 & 1.086 & A \\
\hline $\mathrm{BU}$ & 1 & 23.0000 & 1.2000 & 1.024 & A \\
\hline BX & 1 & 46.9000 & 3.1100 & 2.087 & $\mathrm{~N}$ \\
\hline $\mathrm{CA}$ & 1 & 22.5000 & 2.3000 & 1.001 & A \\
\hline CB & 1 & 22.8000 & 0.6700 & 1.015 & \\
\hline CB & 2 & 23.1000 & 0.6400 & 1.028 & \\
\hline CB & 3 & 23.3000 & 0.5400 & 1.037 & \\
\hline $\mathrm{CD}$ & 1 & 20.7000 & 0.2000 & 0.921 & W \\
\hline $\mathrm{CH}$ & 1 & 21.4000 & 0.1800 & 0.952 & A \\
\hline CL & 1 & 22.0000 & 0.9000 & 0.979 & A \\
\hline $\mathrm{CN}$ & 1 & 23.0200 & 1.1900 & 1.024 & A \\
\hline $\mathrm{CO}$ & 1 & 22.8000 & 0.6000 & 1.015 & A \\
\hline CR & 1 & 38.5000 & 1.5000 & 1.713 & W \\
\hline CS & 1 & 19.9600 & 0.8800 & 0.888 & $\mathrm{~N}$ \\
\hline EG & 1 & 21.8000 & 0.2000 & 0.970 & A \\
\hline EP & 1 & 22.3100 & 2.8700 & 0.993 & A \\
\hline FG & 1 & 23.1800 & 0.1400 & 1.032 & A \\
\hline FJ & 1 & 23.9000 & 0.2200 & 1.064 & A \\
\hline $\mathrm{FL}$ & 1 & 23.8300 & 0.1000 & 1.061 & A \\
\hline FM & 1 & 24.4000 & 0.1600 & 1.086 & A \\
\hline FN & 1 & 21.1000 & 2.1000 & 0.939 & A \\
\hline GA & 1 & 21.5000 & 0.9000 & 0.957 & A \\
\hline GE & 1 & 22.5885 & 3.0559 & 1.005 & A \\
\hline GP & 1 & 22.0000 & 3.0000 & 0.979 & $\mathrm{~N}$ \\
\hline $\mathrm{GT}$ & 1 & 21.0000 & 4.0000 & 0.935 & A \\
\hline HU & 1 & 25.9000 & 0.7700 & 1.153 & A \\
\hline
\end{tabular}

Values for elemental uranium are reported in $\mu \mathrm{g} /$ filter, $\mathrm{g}$ or $\mathrm{mL}$.

$\mathrm{pCi} / \mathrm{g}$ or $\mathrm{mL}=\mathrm{Bq} \times 0.027$

Evaluation: $A=A c c e p t a b l e, ~ W=A c c e p t a b l e$ with Warning, $N=$ Not Acceptable

If the evaluation system is not appropriate for the types of analyses performed in your lab, apply site specific evaluation. 
QAP 49 Results by Nuclide

Matrix: AI Air Filter $\mathrm{Bq} /$ filter

Radionuclide: CS137

\begin{tabular}{lr} 
EML Value: & 22.470 \\
EML Error: & 1.030 \\
\hline
\end{tabular}

\begin{tabular}{|c|c|c|c|c|c|c|}
\hline Labcode & Test \# & $\begin{array}{l}\text { Reported } \\
\text { Value }\end{array}$ & $\begin{array}{l}\text { Reported } \\
\text { Error }\end{array}$ & $\frac{\text { Reported }}{\text { EML }}$ & $\begin{array}{c}\text { QAP } 48 \\
\text { Evaluation }\end{array}$ & Evaluation \\
\hline IA & 1 & 21.1200 & 0.1100 & 0.940 & A & $\mathrm{A}$ \\
\hline IA & 2 & 20.7100 & 0.1100 & 0.922 & A & A \\
\hline IA & 3 & 21.1900 & 0.1100 & 0.943 & A & A \\
\hline ID & 1 & 22.8200 & 1.2300 & 1.016 & W & A \\
\hline IL & 1 & 21.3000 & 0.3000 & 0.948 & A & A \\
\hline IN & 1 & 21.6000 & 0.8000 & 0.961 & A & A \\
\hline IS & 1 & 22.4000 & 2.4400 & 0.997 & A & A \\
\hline $\mathrm{IT}$ & 1 & 21.8000 & 0.3000 & 0.970 & A & A \\
\hline $\mathrm{KR}$ & 1 & 21.5000 & 2.3000 & 0.957 & & A \\
\hline LA & 1 & 22.0000 & 1.7000 & 0.979 & A & A \\
\hline LA & 2 & 22.1000 & 1.7000 & 0.984 & A & A \\
\hline $\mathrm{LA}$ & 3 & 22.1000 & 1.7000 & 0.984 & A & A \\
\hline LB & 1 & 26.0000 & 2.0000 & 1.157 & & W \\
\hline $\mathrm{LL}$ & 1 & 22.4000 & 0.4930 & 0.997 & $\mathrm{~N}$ & $\mathrm{~A}$ \\
\hline LN & 1 & 18.1000 & 0.2900 & 0.806 & A & $\mathrm{W}$ \\
\hline LV & 1 & 20.5000 & 0.2000 & 0.912 & $\mathrm{~A}$ & $\mathrm{~A}$ \\
\hline $\mathrm{MA}$ & 1 & $* * * * * * * *$ & 3663.0000 & 983.044 & & $\mathrm{~N}$ \\
\hline $\mathrm{ME}$ & 1 & 25.6000 & 1.2300 & 1.139 & W & $\mathrm{W}$ \\
\hline MH & $i$ & 25.8600 & 0.9700 . & 1.151 & W & W \\
\hline MS & 1 & 21.1000 & 1.1000 & 0.939 & A & A \\
\hline NA & 1 & 23.2800 & 0.1600 & 1.036 & A & A \\
\hline ND & 1 & 21.0740 & 0.8480 & 0.938 & & A \\
\hline NL & 1 & 24.0000 & 5.9000 & 1.068 & A & A \\
\hline NM & 1 & 17.5100 & 1.3000 & 0.779 & $\mathrm{~A}$ & $\mathrm{~W}$ \\
\hline NP & 1 & 22.0000 & 0.1000 & 0.979 & A & A \\
\hline NS & 1 & 22.3580 & 0.1070 & 0.995 & A & A \\
\hline NZ & 1 & 24.3000 & 1.2000 & 1.081 & & A \\
\hline NZ & 2 & 24.1000 & 1.2000 & 1.073 & & A \\
\hline $\mathrm{OB}$ & 1 & 35.7000 & 6.8000 & 1.589 & & $\mathrm{~N}$ \\
\hline $\mathrm{OC}$ & 1 & 28.0000 & 5.0000 & 1.246 & & W \\
\hline $\mathrm{OC}$ & 2 & 28.0000 & 5.0000 & 1.246 & & W \\
\hline OC & 3 & 27.0000 & 5.0000 & 1.202 & & W \\
\hline OD & 1 & 22.7800 & 1.0100 & 1.014 & A & A \\
\hline OD & 1 & 22.7800 & 1.0100 & 1.014 & A & A \\
\hline OD & 2 & 22.6200 & 0.9950 & 1.007 & A & A \\
\hline OD & 2 & 22.6200 & 1.0000 & 1.007 & A & A \\
\hline $\mathrm{OL}$ & 1 & 24.8300 & 1.7700 & 1.105 & A & $A$ \\
\hline OT & 1 & 24.0000 & 1.0000 & 1.068 & A & A \\
\hline OU & 1 & 25.3000 & 0.6100 & 1.126 & W & A \\
\hline PK & 1 & 21.1900 & 0.3800 & 0.943 & $\mathrm{~N}$ & A \\
\hline $\mathrm{PO}$ & 1 & 26.6000 & 1.3000 & 1.184 & A & W \\
\hline PR & 1 & 21.8733 & 0.2903 & 0.973 & & A \\
\hline
\end{tabular}

Values for elemental uranium are reported in $\mu \mathrm{g} /$ filter, $\mathrm{g}$ or $\mathrm{mL}$.

$\mathrm{pCi} / \mathrm{g}$ or $\mathrm{mL}=\mathrm{Bq} \times 0.027$

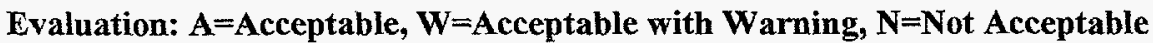

If the evaluation system is not appropriate for the types of analyses performed in your lab, apply site specific evaluation. 


\section{QAP 49 Results by Nuclide}

\begin{tabular}{|c|c|c|c|c|c|c|}
\hline $\begin{array}{l}\text { Matrix: A } \\
\text { Radionuclide }\end{array}$ & II Air Fi & $\begin{array}{l}\text { ter } \mathrm{Bq} / \text { filter } \\
\text { S137 }\end{array}$ & & & & \\
\hline $\begin{array}{l}\text { EML Value: } \\
\text { EML Error: }\end{array}$ & $\begin{array}{r}22 . \\
1 .\end{array}$ & & & & & \\
\hline Labcode & Test \# & $\begin{array}{l}\text { Reported } \\
\text { Value }\end{array}$ & $\begin{array}{c}\text { Reported } \\
\text { Error }\end{array}$ & $\frac{\text { Reported }}{E M L}$ & $\begin{array}{c}\text { QAP 48 } \\
\text { Evaluation }\end{array}$ & Evaluation \\
\hline $\mathrm{RA}$ & 1 & 24.2000 & 0.6500 & 1.077 & A & A \\
\hline $\mathrm{RA}$ & 2 & 23.3000 & 0.8700 & 1.037 & A & $\mathrm{A}$ \\
\hline $\mathrm{RC}$ & 1 & 22.5000 & 1.2000 & 1.001 & A & $\mathrm{A}$ \\
\hline $\mathrm{RE}$ & 1 & 21.3000 & 1.9000 & 0.948 & A & A \\
\hline $\mathrm{RI}$ & 1 & 18.3000 & 1.4800 & 0.814 & A & W \\
\hline SA & 1 & 23.0000 & 2.9000 & 1.024 & A & A \\
\hline SB & 1 & 24.8000 & 3.6000 & 1.104 & & $\mathrm{~A}$ \\
\hline SK & 1 & 21.0000 & 2.0000 & 0.935 & A & $\mathrm{A}$ \\
\hline $\mathrm{SR}$ & 1 & 21.0000 & 0.4180 & 0.935 & A & $\mathrm{A}$ \\
\hline SW & 1 & 22.7400 & 0.2900 & 1.012 & A & A \\
\hline TE & 1 & 22.4000 & 0.5000 & 0.997 & A & $\mathrm{A}$ \\
\hline $\mathrm{TI}$ & 1 & 22.6000 & 2.3000 & 1.006 & A & $\mathrm{A}$ \\
\hline TM & 1 & 19.2000 & 0.6750 & 0.854 & W & $\mathrm{A}$ \\
\hline TN & 1 & 20.6000 & 4.0000 & 0.917 & A & A \\
\hline $\mathrm{TP}$ & 1 & 23.2000 & 0.2200 & 1.032 & $\mathrm{~A}$ & $\mathrm{~A}$ \\
\hline TW & 1 & 22.2000 & 0.6000 & 0.988 & A & $\mathrm{A}$ \\
\hline $\mathrm{TX}$ & 1 & 21.9000 & 0.1000 & 0.975 & $\mathrm{~A}$ & $\mathrm{~A}$ \\
\hline UC & 1 & 31.3380 & 10.8100 & 1.395 & W & $N$ \\
\hline UP & 1 & 23.8000 & 1.4200 & 1.059 & W & A \\
\hline$U Y$ & 1 & 23.1000 & 1.8000 & 1.028 & $\mathrm{~A}$ & $\mathrm{~A}$ \\
\hline WA & 1 & 21.4000 & 0.6000 & 0.952 & A & A \\
\hline WC & 1 & 22.0000 & 3.4000 & 0.979 & $\mathrm{~A}$ & A \\
\hline WE & 1 & 26.9000 & 4.9000 & 1.197 & & W \\
\hline YA & 1 & 22.2925 & 0.3700 & 0.992 & A & A \\
\hline YU & 1 & 19.3000 & 0.7000 & 0.859 & $\mathrm{~W}$ & A \\
\hline
\end{tabular}

Total Number Reported: $\quad 108$

Values for elemental uranium are reported in $\mu \mathrm{g}$ /filter, $\mathrm{g}$ or $\mathrm{mL}$.

$\mathrm{pCi} / \mathrm{g}$ or $\mathrm{mL}=\mathrm{Bq} \times 0.027$

Evaluation: $A=A c c e p t a b l e, W=$ Acceptable with Warning, $N=$ Not Acceptable

If the evaluation system is not appropriate for the types of analyses performed in your lab, apply site specific evaluation. 


\section{QAP 49 Results by Nuclide}

\begin{tabular}{lc}
\hline $\begin{array}{l}\text { Matrix: AI } \\
\text { Radionuclide: }\end{array}$ & $\begin{array}{c}\text { Air Filter Bq / filter } \\
\text { GROSS ALPHA }\end{array}$ \\
\hline EML Value: & 1.650 \\
EML Error: & 0.160 \\
\hline & $\begin{array}{c}\text { Reported } \\
\text { Value }\end{array}$
\end{tabular}

Reported Error

\section{Reported EML}

QAP 48 Evaluation
Evaluation

\begin{tabular}{|c|c|c|c|c|c|c|}
\hline AI & 1 & 1.2800 & 0.0509 & 0.776 & & W \\
\hline $\mathrm{Al}$ & 1 & 1.6830 & 0.4000 & 1.020 & A & A \\
\hline $\mathrm{AM}$ & 1 & 2.4700 & 0.0200 & 1.497 & W & W \\
\hline AP & 1 & 2.0600 & 0.2400 & 1.248 & & A \\
\hline $\mathrm{AU}$ & 1 & 1.8900 & 0.0400 & 1.145 & A & $\mathrm{A}$ \\
\hline $\mathrm{BC}$ & 1 & 2.4000 & 0.0500 & 1.455 & A & W \\
\hline $\mathrm{BE}$ & 1 & 1.5800 & 0.0900 & 0.958 & A & A \\
\hline BL & 1 & 1.5600 & 0.0300 & 0.945 & A & $\mathrm{A}$ \\
\hline $\mathrm{BN}$ & 1 & 1.4600 & 0.0200 & 0.885 & W & A \\
\hline $\mathrm{BN}$ & 2 & 1.4900 & 0.0200 & 0.903 & W & A \\
\hline $\mathrm{BN}$ & 3 & 1.4500 & 0.0200 & 0.879 & W & A \\
\hline BP & 1 & 1.6500 & 0.1100 & 1.000 & $\mathrm{~A}$ & A \\
\hline $\mathrm{BQ}$ & 1 & 3.0900 & 0.0800 & 1.873 & & $N$ \\
\hline $\mathrm{BU}$ & 1 & 1.5000 & 0.1000 & 0.909 & A & A \\
\hline BX & 1 & 2.3600 & 0.0500 & 1.430 & A & W \\
\hline $\mathrm{CA}$ & 1 & 1.8000 & 0.2000 & 1.091 & A & A \\
\hline $\mathrm{CD}$ & 1 & 1.5000 & 0.1000 & 0.909 & W & A \\
\hline $\mathrm{CH}$ & 1 & 1.8000 & 0.0460 & 1.091 & A & A \\
\hline $\mathrm{CS}$ & 1 & 1.7800 & 0.0800 & 1.079 & A & A \\
\hline DH & 1 & 1.5000 & 0.2200 & 0.909 & A & A \\
\hline $\mathrm{FG}$ & 1 & 1.6020 & 0.1180 & 0.971 & A & A \\
\hline FL & 1 & 1.4600 & 0.0700 & 0.885 & W & A \\
\hline GC & 1 & 1.5500 & 0.0030 & 0.939 & W & A \\
\hline $\mathrm{GE}$ & 1 & 1.6317 & 0.0074 & 0.989 & A & A \\
\hline GP & 1 & 1.7000 & 0.2000 & 1.030 & A & A \\
\hline GT & 1 & 1.8000 & 0.1000 & 1.091 & A & A \\
\hline $\mathrm{HC}$ & 1 & 1.4970 & 0.1500 & 0.907 & $\mathrm{~W}$ & A \\
\hline ID & 1 & 1.3970 & 0.1030 & 0.847 & W & A \\
\hline$\overline{\mathrm{IL}}$ & 1 & 1.9900 & 0.0300 & 1.206 & & A \\
\hline IS & 1 & 2.0100 & 0.2000 & 1.218 & A & A \\
\hline $\mathrm{IT}$ & 1 & 1.9600 & 0.0300 & 1.188 & A & A \\
\hline $\mathrm{JE}$ & 1 & 1.8110 & 0.2000 & 1.098 & A & A \\
\hline$\pi$ & 1 & 3.0100 & 0.0700 & 1.824 & & $\mathrm{~N}$ \\
\hline $\mathrm{KA}$ & 1 & 1.7000 & 0.0600 & 1.030 & A & A \\
\hline $\mathrm{KR}$ & 1 & 1.6000 & 0.0300 & 0.970 & A & A \\
\hline LL & 2 & 1.5200 & 0.0101 & 0.921 & A & A \\
\hline LN & 1 & 1.7000 & 0.2000 & 1.030 & A & A \\
\hline LV & 1 & 1.6000 & 0.2000 & 0.970 & W & A \\
\hline ME & 1 & 2.2200 & 0.0800 & 1.345 & W & W \\
\hline $\mathrm{MH}$ & 1 & 1.6500 & 0.0600 & 1.000 & & A \\
\hline MS & 1 & 1.9800 & 0.2000 & 1.200 & & A \\
\hline
\end{tabular}

Values for elemental uranium are reported in $\mu \mathrm{g} /$ filter, $\mathrm{g}$ or $\mathrm{mL}$.

$\mathrm{pCi} / \mathrm{g}$ or $\mathrm{mL}=\mathrm{Bq} \times 0.027$

Evaluation: $A=A c c e p t a b l e, ~ W=A c c e p t a b l e$ with Warning, $N=$ Not Acceptable

If the evaluation system is not appropriate for the types of analyses performed in your lab, apply site specific evaluation. 


\section{QAP 49 Results by Nuclide}

December, 1998

Matrix: AI Air Filter $\mathrm{Bq} /$ filter

Radionuclide: $\quad$ GROSS ALPHA

\begin{tabular}{ll} 
EML Value: & 1.650 \\
EML Error: & 0.160 \\
\hline
\end{tabular}

\begin{tabular}{|c|c|c|c|c|c|c|}
\hline Labcode & Test \# & $\begin{array}{l}\text { Reported } \\
\text { Value }\end{array}$ & $\begin{array}{c}\text { Reported } \\
\text { Error }\end{array}$ & $\frac{\text { Reported }}{E M L}$ & $\begin{array}{c}\text { QAP 48 } \\
\text { Evaluation }\end{array}$ & Evaluation \\
\hline ND & 1 & 1.6200 & 0.1330 & 0.982 & & A \\
\hline NQ & 1 & 1.4400 & 0.2200 & 0.873 & & A \\
\hline NS & 1 & 3.0660 & 0.1620 & 1.858 & W & $\mathrm{N}$ \\
\hline $\mathrm{NZ}$ & 1 & 1.4000 & 0.1000 & 0.848 & & $\mathrm{~A}$ \\
\hline NZ & 2 & 1.5000 & 0.1000 & 0.909 & & A \\
\hline $\mathrm{OB}$ & 1 & 1.8300 & 0.1830 & 1.109 & A & A \\
\hline $\mathrm{OC}$ & 1 & 1.4000 & 0.2000 & 0.848 & & A \\
\hline $\mathrm{OC}$ & 2 & 1.4000 & 0.2000 & 0.848 & & A \\
\hline OC & 3 & 1.4000 & 0.2000 & 0.848 & & A \\
\hline OD & 1 & 1.6000 & 0.0600 & 0.970 & A & A \\
\hline OD & 1 & 1.6000 & 0.0600 & 0.970 & A & A \\
\hline OT & 1 & 1.6000 & 0.1000 & 0.970 & A & A \\
\hline OU & 1 & 2.1900 & 0.1670 & 1.327 & $\mathrm{~N}$ & W \\
\hline PA & 1 & 1.5400 & 0.1100 & 0.933 & A & A \\
\hline $\mathrm{RC}$ & 1 & 1.8100 & 0.1300 & 1.097 & A & A \\
\hline RK & 1 & 1.3000 & 0.1500 & 0.788 & W & W \\
\hline $\mathrm{SA}$ & 1 & 1.8400 & 0.2400 & 1.115 & A & A \\
\hline SR & 1 & 1.5900 & 0.1300 & 0.964 & W & A \\
\hline SW & 1 & 1.5100 & 0.0600 & 0.915 & W & A \\
\hline TE & 1 & 2.2000 & 0.1000 & 1.333 & & W \\
\hline $\mathrm{TI}$ & 1 & 2.1000 & 0.1000 & 1.273 & A & A \\
\hline $\mathrm{TM}$ & 1 & 1.8700 & 0.2400 & 1.133 & $\mathbf{N}$ & A \\
\hline TN & 1 & 1.4000 & 0.3500 & 0.848 & A & A \\
\hline TO & 1 & 2.4300 & 0.5000 & 1.473 & $\mathrm{~W}$ & $\mathrm{~W}$ \\
\hline TP & 1 & 1.6000 & 0.0800 & 0.970 & W & A \\
\hline TW & 1 & 1.7300 & 0.0500 & 1.048 & A & A \\
\hline $\mathrm{TX}$ & 1 & 1.7700 & 0.0500 & 1.073 & A & $\mathrm{A}$ \\
\hline $\mathrm{UC}$ & 1 & 1.6200 & 0.0800 & 0.982 & $\mathrm{~A}$ & $\mathrm{~A}$ \\
\hline UP & 1 & 1.6400 & 0.0810 & 0.994 & A & A \\
\hline UY & 1 & 1.3200 & 0.1200 & 0.800 & A & W \\
\hline WA & 1 & 1.9100 & 0.0700 & 1.158 & A & A \\
\hline WC & 1 & 1.5000 & 0.2000 & 0.909 & A & A \\
\hline WS & 1 & 1.6950 & 0.0400 & 1.027 & A & A \\
\hline WV & 1 & 1.5300 & 0.0310 & 0.927 & W & A \\
\hline YA & 1 & 1.5552 & 0.0170 & 0.943 & A & A \\
\hline
\end{tabular}

Total Number Reported:

76

Values for elemental uranium are reported in $\mu \mathrm{g} /$ filter, $\mathrm{g}$ or $\mathrm{mL}$.

$\mathrm{pCi} / \mathrm{g}$ or $\mathrm{mL}=\mathrm{Bq} \times 0.027$

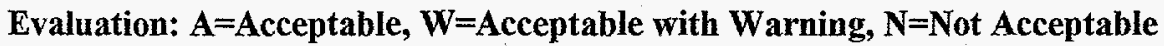

If the evaluation system is not appropriate for the types of analyses performed in your lab, apply site specific evaluation. 
QAP 49 Results by Nuclide

Matrix: AI Air Filter $\mathrm{Bq} /$ filter

Radionuclide: GROSS BETA

$\begin{array}{ll}\text { EML Value: } & 2.160 \\ \text { EML Error: } & 0.070\end{array}$

EML Error: $\quad 0.070$

\begin{tabular}{|c|c|c|c|c|c|c|}
\hline Labcode & Test \# & $\begin{array}{l}\text { Reported } \\
\text { Value }\end{array}$ & $\begin{array}{c}\text { Reported } \\
\text { Error }\end{array}$ & $\frac{\text { Reported }}{\text { EML }}$ & $\begin{array}{c}\text { QAP } 48 \\
\text { Evaluation }\end{array}$ & Evaluation \\
\hline $\mathrm{AI}$ & 1 & 1.7000 & 0.0496 & 0.787 & & W \\
\hline $\mathrm{AL}$ & 1 & 1.8660 & 0.3000 & 0.864 & $W$ & $W$ \\
\hline AM & 1 & 2.9800 & 0.0300 & 1.380 & W & A \\
\hline $\mathrm{AP}$ & 1 & 2.9300 & 0.2600 & 1.356 & & $A$ \\
\hline $\mathrm{AU}$ & 1 & 2.0400 & 0.0400 & 0.944 & A & A \\
\hline $\mathrm{BC}$ & 1 & 2.0800 & 0.0400 & 0.963 & $A$ & A \\
\hline $\mathrm{BE}$ & 1 & 1.7500 & 0.0700 & 0.810 & A & W \\
\hline BL & 1 & 1.7000 & 0.0300 & 0.787 & A & W \\
\hline $\mathrm{BN}$ & 1 & 1.6900 & 0.0400 & 0.782 & $\mathrm{~A}$ & W \\
\hline $\mathrm{BN}$ & 2 & 1.7700 & 0.0400 & 0.819 & A & W \\
\hline $\mathrm{BN}$ & 3 & 1.7600 & 0.0400 & 0.815 & A & $W$ \\
\hline BP & 1 & 1.9500 & 0.0600 & 0.903 & A & A \\
\hline $\mathrm{BQ}$ & 1 & 2.1600 & 0.0400 & 1.000 & & A \\
\hline $\mathrm{BU}$ & 1 & 1.8000 & 0.1000 & 0.833 & A & W \\
\hline $\mathrm{BX}$ & 1 & 2.1800 & 0.0400 & 1.009 & A & A \\
\hline CA & 1 & 1.7000 & 0.2000 & 0.787 & A & W \\
\hline $\mathrm{CD}$ & 1 & 3.0000 & 0.2000 & 1.389 & $\mathrm{~N}$ & A \\
\hline $\mathrm{CH}$ & 1 & 2.0600 & 0.0390 & 0.954 & A & A \\
\hline $\mathrm{CS}$ & 1 & 2.9400 & $0.2300^{\circ}$ & 1.361 & W & A \\
\hline $\mathrm{DH}$ & 1 & 1.7500 & 0.2000 & 0.810 & A & W \\
\hline FG & 1 & 1.6240 & 0.1220 & 0.752 & A & W \\
\hline FL & 1 & 2.0800 & 0.0700 & 0.963 & A & A \\
\hline GC & 1 & 1.6900 & 0.0200 & 0.782 & A & W \\
\hline GE & 1 & 1.7797 & 0.0037 & 0.824 & A & W \\
\hline GP & 1 & 2.0000 & 0.2000 & 0.926 & A & A \\
\hline GT & 1 & 2.0000 & 0.1000 & 0.926 & A & A \\
\hline $\mathrm{HC}$ & 1 & 1.6030 & 0.1600 & 0.742 & W & W \\
\hline ID & 1 & 2.2570 & 0.1160 & 1.045 & A & A \\
\hline IL & 1 & 2.2200 & 0.0200 & 1.028 & & A \\
\hline IS & 1 & 2.0100 & 0.2000 & 0.931 & A & A \\
\hline IT & 1 & 2.2000 & 0.0700 & 1.019 & A & A \\
\hline$J E$ & 1 & 2.2500 & 0.2000 & 1.042 & A & A \\
\hline JL & 1 & 0.4800 & 0.0400 & 0.222 & & $\mathrm{~N}$ \\
\hline $\mathrm{KA}$ & 1 & 2.0600 & 0.0500 & 0.954 & A & A \\
\hline KR & 1 & 1.9300 & 0.0300 & 0.894 & A & A \\
\hline LL & 2 & 2.3300 & 0.0110 & 1.079 & A & A \\
\hline $\mathrm{LN}$ & 1 & 1.6500 & 0.1600 & 0.764 & A & W \\
\hline LV & 1 & 1.7800 & 0.1000 & 0.824 & $\mathrm{~N}$ & W \\
\hline $\mathrm{ME}$ & 1 & 2.4000 & 0.0700 & 1.111 & A & A \\
\hline $\mathrm{MH}$ & 1 & 1.8500 & 0.0200 & 0.856 & & W \\
\hline MS & 1 & 2,0800 & 0.2100 & 0.963 & & $A$ \\
\hline
\end{tabular}

Values for elemental uranium are reported in $\mu \mathrm{g} /$ filter, $\mathrm{g}$ or $\mathrm{mL}$.

$\mathrm{pCi} / \mathrm{g}$ or $\mathrm{mL}=\mathrm{Bq} \times 0.027$

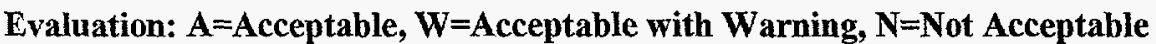

If the evaluation system is not appropriate for the types of analyses performed in your lab, apply site specific evaluation. 


\section{QAP 49 Results by Nuclide}

Matrix: AI Air Filter $\mathrm{Bq} /$ filter

Radionuclide:

GROSS BETA

\begin{tabular}{ll}
\hline EML Value: & 2.160 \\
EML Error: & 0.070 \\
\hline
\end{tabular}

Labcode Test \# $\begin{gathered}\text { Reported } \\ \text { Value }\end{gathered}$

Reported Reported QAP 48

\begin{tabular}{|c|c|c|c|c|c|c|}
\hline ND & 1 & 2.1330 & 0.1260 & 0.987 & & A \\
\hline NP & 1 & 1.9900 & 0.0200 & 0.921 & A & A \\
\hline NQ & 1 & 1.8600 & 0.2800 & 0.861 & & W \\
\hline NS & 1 & 2.2370 & 0.0850 & 1.036 & A & A \\
\hline$N Z$ & 1 & 1.9000 & 0.1000 & 0.880 & & W \\
\hline NZ & 2 & 1.9000 & 0.1000 & 0.880 & & W \\
\hline OB & 1 & 1.9100 & 0.1900 & 0.884 & A & W \\
\hline $\mathrm{OC}$ & 1 & 1.9000 & 0.2000 & 0.880 & & W \\
\hline$O C$ & 2 & 1.9000 & 0.2000 & 0.880 & & W \\
\hline $\mathrm{OC}$ & 3 & 1.9000 & 0.2000 & 0.880 & & W \\
\hline OD & 1 & 2.5200 & 0.0800 & 1.167 & A & A \\
\hline OD & 1 & 2.5200 & 0.0800 & 1.167 & A & A \\
\hline OT & 1 & 2.0000 & 0.1000 & 0.926 & A & A \\
\hline OU & 1 & 2.5900 & 0.1990 & 1.199 & A & A \\
\hline PA & 1 & 2.0900 & 0.1000 & 0.968 & A & A \\
\hline $\mathrm{RC}$ & 1 & 1.9600 & 0.1400 & 0.907 & A & A \\
\hline RK & 1 & 2.1900 & 0.1400 & 1.014 & A & A \\
\hline SA & 1 & 2.0400 & 0.2500 & 0.944 & A & A \\
\hline SR & 1 & 2.1700 & 0.1500 & 1.005 & A & A \\
\hline SW & 1 & 1.6200 & 0.0600 & 0.750 & A & W \\
\hline TE & 1 & 2.8000 & 0.1000 & 1.296 & & A \\
\hline TI & 1 & 1.7000 & 0.1000 & 0.787 & A & W \\
\hline TM & 1 & 1.9700 & 0.1780 & 0.912 & $\mathrm{~N}$ & A \\
\hline $\mathrm{TN}$ & 1 & 1.7000 & 0.4000 & 0.787 & A & W \\
\hline TO & 1 & 2.5300 & 0.4000 & 1.171 & A & A \\
\hline $\mathrm{TP}$ & 1 & 2.0900 & 0.1000 & 0.968 & A & A \\
\hline TW & 1 & 1.8600 & 0.0400 & 0.861 & A & W \\
\hline TX & 1 & 2.0900 & 0.0700 & 0.968 & A & A \\
\hline UC & 1 & 2.3800 & 0.0800 & 1.102 & A & A \\
\hline UP & 1 & 1.9000 & 0.0660 & 0.880 & A & W \\
\hline UY & 1 & 1.8100 & 0.0850 & 0.838 & $\mathrm{~A}$ & W \\
\hline WA & 1 & 2.2300 & 0.0600 & 1.032 & A & A \\
\hline WC & 1 & 2.3000 & 0.2000 & 1.065 & A & A \\
\hline WV & 1 & 2.1700 & 0.0360 & 1.005 & A & A \\
\hline YA & 1 & 1.9060 & 0.0207 & 0.882 & A & W \\
\hline
\end{tabular}

Total Number Reported:

76

Values for elemental uranium are reported in $\mu \mathrm{g} /$ filter, $\mathrm{g}$ or $\mathrm{mL}$.

$\mathrm{pCi} / \mathrm{g}$ or $\mathrm{mL}=\mathrm{Bq} \times 0.027$

Evaluation: $A=$ Acceptable, $W=$ Acceptable with Warning, $N=$ Not Acceptable

If the evaluation system is not appropriate for the types of analyses performed in your lab, apply site specific evaluation. 


\section{QAP 49 Results by Nuclide}

Matrix: $\mathrm{AI}$ Air Filter $\mathrm{Bq}$ / filter

Radionuclide: $\quad$ MN54

\begin{tabular}{ll} 
EML Value: & 4.920 \\
EML Error: & 0.400 \\
\hline
\end{tabular}

Labcode Test \# Rarted

Reported $\quad$ Reported $\quad$ QAP 48

\begin{tabular}{|c|c|c|c|c|c|c|}
\hline $\mathrm{AF}$ & 1 & 4.5000 & 0.6000 & 0.915 & A & A \\
\hline $\mathrm{AG}$ & 1 & 5.0000 & 1.3000 & 1.016 & A & A \\
\hline $\mathrm{AL}$ & 1 & 5.0532 & 0.5000 & 1.027 & W & A \\
\hline AM & 1 & 4.1500 & 0.1100 & 0.843 & A & A \\
\hline AN & 1 & 5.3000 & 0.3500 & 1.077 & A & A \\
\hline $\mathrm{AU}$ & 1 & 5.5700 & 0.4000 & 1.132 & W & A \\
\hline BA & 1 & 5.6300 & 0.4400 & 1.144 & W & A \\
\hline $\mathrm{BC}$ & 1 & 9.0700 & 0.5400 & 1.843 & $\mathrm{~N}$ & $\mathrm{~N}$ \\
\hline $\mathrm{BE}$ & 1 & 5.6000 & 0.7000 & 1.138 & A & A \\
\hline BL & 1 & 5.9800 & 0.2200 & 1.215 & A & W \\
\hline $\mathrm{BN}$ & 1 & 8.3600 & 0.6600 & 1.699 & W & $\mathrm{N}$ \\
\hline $\mathrm{BN}$ & 2 & 7.2900 & 0.6600 & 1.482 & W & $\mathrm{N}$ \\
\hline $\mathrm{BN}$ & 3 & 8.8800 & 0.6600 & 1.805 & W & $\mathrm{N}$ \\
\hline BP & 1 & 5.2000 & 0.1000 & 1.057 & A & A \\
\hline$B Q$ & 1 & 4.6000 & 0.1000 & 0.935 & A & A \\
\hline $\mathrm{BU}$ & 1 & 4.7000 & 0.8000 & 0.955 & A & A \\
\hline BX & 1 & 10.3000 & 0.0100 & 2.093 & $\mathrm{~N}$ & $\mathrm{~N}$ \\
\hline CA & 1 & 4.5000 & 0.3000 & 0.915 & A & A \\
\hline $\mathrm{CB}$ & 1 & 5.1000 & 0.1400 & 1.037 & & A \\
\hline $\mathrm{CB}$ & 2 & 5.2000 & 0.1300 & 1.057 & & A \\
\hline CB & 3 & 5.2000 & 0.1300 & 1.057 & & A \\
\hline $\mathrm{CD}$ & 1 & 5.2000 & 0.1000 & 1.057 & A & A \\
\hline $\mathrm{CH}$ & 1 & 4.8000 & 0.1300 & 0.976 & A & A \\
\hline $\mathrm{CL}$ & 1 & 5.3000 & 0.6000 & 1.077 & A & A \\
\hline $\mathrm{CN}$ & 1 & 5.0300 & 0.2900 & 1.022 & A & A \\
\hline $\mathrm{CO}$ & 1 & 5.3000 & 0.2000 & 1.077 & A & A \\
\hline CR & 1 & 8.3000 & 0.4000 & 1.687 & A & $\mathrm{N}$ \\
\hline $\mathrm{CS}$ & 1 & 4.4420 & 0.2220 & 0.903 & $\mathrm{~N}$ & A \\
\hline EG & 1 & 5.0000 & 0.1000 & 1.016 & A & A \\
\hline $\mathrm{EP}$ & 1 & 5.1300 & 0.7900 & 1.043 & A & A \\
\hline $\mathrm{FG}$ & 1 & 5.0780 & 0.1400 & 1.032 & A & A \\
\hline FJ & 1 & 7.5900 & 0.4900 & 1.543 & $\mathrm{~N}$ & $\mathrm{~N}$ \\
\hline FL & 1 & 5.2500 & 0.0500 & 1.067 & A & A \\
\hline FM & 1 & 5.6000 & 0.1600 & 1.138 & A & A \\
\hline FN & 1 & 4.6400 & 0.5100 & 0.943 & A & A \\
\hline GA & 1 & 5.2200 & 0.6400 & 1.061 & A & A \\
\hline GE & 1 & 5.3003 & 1.0216 & 1.077 & A & A \\
\hline GP & 1 & 5.0000 & 0.9000 & 1.016 & A & A \\
\hline GT & 1 & 4.8000 & 1.0000 & 0.976 & A & A \\
\hline $\mathrm{HU}$ & 1 & 5.7600 & 0.2000 & 1.171 & A & A \\
\hline IA & 1 & 4.4000 & 0.0600 & 0.894 & W & A \\
\hline
\end{tabular}

Values for elemental uranium are reported in $\mu \mathrm{g} /$ filter, $\mathrm{g}$ or $\mathrm{mL}$.

pCi/g or $\mathrm{mL}=\mathrm{Bq} \times 0.027$

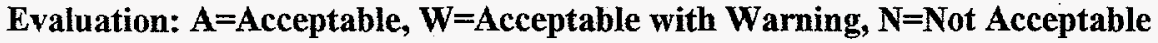

If the evaluation system is not appropriate for the types of analyses performed in your lab, apply site specific evaluation. 


\section{QAP 49 Results by Nuclide}

Matrix: AI Air Filter $\mathrm{Bq} /$ filter

Radionuclide: $\quad$ MN54

\begin{tabular}{ll}
\hline EML Value: & 4.920 \\
EML Error: & 0.400 \\
\hline
\end{tabular}

Labcode Test \# $\begin{gathered}\text { Reported } \\ \text { Value }\end{gathered} \quad \begin{gathered}\text { Reported } \\ \text { Error }\end{gathered} \frac{\text { Reported }}{\text { EML }} \quad \begin{gathered}\text { QAP 48 } \\ \text { Evaluation }\end{gathered}$ Evaluation

\begin{tabular}{|c|c|c|c|c|c|c|}
\hline IA & 2 & 4.4800 & 0.0600 & 0.911 & W & A \\
\hline IA & 3 & 4.3200 & 0.0600 & 0.878 & W & A \\
\hline ID & 1 & 5.2500 & 0.3150 & 1.067 & $W$ & A \\
\hline IL & 1 & 4.9000 & 0.1000 & 0.996 & A & A \\
\hline IN & 1 & 4.8900 & 0.5000 & 0.994 & A & A \\
\hline IS & 1 & 5.3400 & 0.6900 & 1.085 & A & A \\
\hline IT & 1 & 5.2200 & 0.1700 & 1.061 & A & A \\
\hline KR & 1 & 4.6000 & 0.5000 & 0.935 & & A \\
\hline LA & 1 & 5.1600 & 0.4300 & 1.049 & A & A \\
\hline LA & 2 & 5.0600 & 0.4200 & 1.028 & A & A \\
\hline LA & 3 & 5.1500 & 0.4300 & 1.047 & A & A \\
\hline LB & 1 & 5.9000 & 0.8000 & 1.199 & & W \\
\hline LL & 1 & 5.1600 & 0.2690 & 1.049 & A & A \\
\hline LN & 1 & 4.3000 & 0.2200 & 0.874 & A & A \\
\hline LV & 1 & 4.2800 & 0.2000 & 0.870 & A & A \\
\hline MA & 1 & 4440.0000 & 1443.0000 & 902.439 & & $\mathrm{~N}$ \\
\hline ME & 1 & 6.1000 & 0.3500 & 1.240 & W & W \\
\hline MH & 1 & 5.8400 & 0.2300 & 1.187 & W & W \\
\hline MS & 1 & 4.6200 & 0.4600 & 0.939 & A & A \\
\hline NA & 1 & 5.0100 & 0.0800 & 1.018 & A & A \\
\hline ND & 1 & 4.8660 & 0.2470 & 0.989 & & A \\
\hline NL & 1 & 5.0600 & 1.6100 & 1.028 & A & A \\
\hline NP & 1 & 5.3700 & 0.0700 & 1.091 & W & A \\
\hline NS & 1 & 5.4440 & 0.0670 & 1.107 & A & A \\
\hline NZ & 1 & 5.7000 & 0.3000 & 1.159 & & A \\
\hline $\mathrm{NZ}$ & 2 & 5.6000 & 0.3000 & 1.138 & & A \\
\hline $\mathrm{OB}$ & 1 & 9.4000 & 1.9000 & 1.911 & & $\mathrm{~N}$ \\
\hline $\mathrm{OC}$ & 1 & 6.0000 & 2.0000 & 1.220 & & W \\
\hline $\mathrm{OC}$ & 2 & 6.0000 & 2.0000 & 1.220 & & W \\
\hline $\mathrm{OC}$ & 3 & 6.0000 & 2.0000 & 1.220 & & W \\
\hline OD & 1 & 5.0840 & 0.3270 & 1.033 & A & A \\
\hline$O D$ & 1 & 5.0800 & 0.3300 & 1.033 & A & A \\
\hline OD & 2 & 5.0300 & 0.3210 & 1.022 & A & A \\
\hline $\mathrm{OD}$ & 2 & 5.0300 & 0.3200 & 1.022 & A & A \\
\hline OL & 1 & 5.8200 & 0.3600 & 1.183 & A & W \\
\hline OT & 1 & 5.4000 & 0.2000 & 1.098 & W & A \\
\hline OU & 1 & 4.5900 & 1.2000 & 0.933 & A & A \\
\hline PK & 1 & 4.9900 & 0.5200 & 1.014 & & A \\
\hline PO & 1 & 6.1000 & 0.3000 & 1.240 & A & W \\
\hline PR & 1 & 4.7166 & 0.1613 & 0.959 & & A \\
\hline RA & 1 & 5.0700 & 0.0700 & 1.030 & A & A \\
\hline $\mathrm{RA}$ & 2 & 5.1800 & 0.1300 & 1.053 & A & A \\
\hline
\end{tabular}

Values for elemental uranium are reported in $\mu \mathrm{g} /$ filter, $\mathrm{g}$ or $\mathrm{mL}$.

$\mathrm{pCi} / \mathrm{g}$ or $\mathrm{mL}=\mathrm{Bq} \times 0.027$

Evaluation: $A=A c c e p t a b l e, ~ W=A c c e p t a b l e$ with Warning, $N=$ Not Acceptable

If the evaluation system is not appropriate for the types of analyses performed in your lab, apply site specific evaluation. 


\section{QAP 49 Results by Nuclide}

\begin{tabular}{|c|c|c|c|c|c|c|}
\hline $\begin{array}{l}\text { Matrix: A } \\
\text { Radionuclid }\end{array}$ & II Air Fi & $\begin{array}{l}\text { ter Bq / filter } \\
\mathbb{N} 54\end{array}$ & & & & \\
\hline $\begin{array}{l}\text { EML Value: } \\
\text { EML Error: }\end{array}$ & & $\begin{array}{l}20 \\
00\end{array}$ & & & & \\
\hline Labcode & Test \# & $\begin{array}{l}\text { Reported } \\
\text { Value }\end{array}$ & $\begin{array}{c}\text { Reported } \\
\text { Error }\end{array}$ & $\frac{\text { Reported }}{E M L}$ & $\begin{array}{c}\text { QAP 48 } \\
\text { Evaluation }\end{array}$ & Evaluation \\
\hline $\mathrm{RC}$ & 1 & 5.2000 & 0.4600 & 1.057 & A & A \\
\hline $\mathrm{RE}$ & 1 & 5.1800 & 0.5700 & 1.053 & A & A \\
\hline RI & 1 & 4.7700 & 0.7170 & 0.970 & A & A \\
\hline SA & 1 & 5.5000 & 0.7000 & 1.118 & A & A \\
\hline SK & 1 & 4.7000 & 0.4000 & 0.955 & A & A \\
\hline SR & 1 & 4.8700 & 0.1440 & 0.990 & A & A \\
\hline SW & 1 & 5.3000 & 0.1000 & 1.077 & A & A \\
\hline $\mathrm{TE}$ & 1 & 5.3000 & 0.3000 & 1.077 & A & A \\
\hline TI & 1 & 5.2000 & 0.5000 & 1.057 & A & A \\
\hline $\mathrm{TM}$ & 1 & 4.5400 & 0.3390 & 0.923 & A & A \\
\hline TN & 1 & 4.8700 & 0.5000 & 0.990 & A & A \\
\hline $\mathrm{TP}$ & 1 & 4.9000 & 0.1000 & 0.996 & A & A \\
\hline TW & 1 & 5.0000 & 0.2000 & 1.016 & A & A \\
\hline $\mathrm{TX}$ & 1 & 5.0600 & 0.0700 & 1.028 & A & A \\
\hline UP & 1 & 5.4100 & 0.5280 & 1.100 & W & A \\
\hline UY & 1 & 5.0800 & 0.7000 & 1.033 & $\mathrm{~A}$ & A \\
\hline WA & 1 & 5.2000 & 0.3000 & 1.057 & W & A \\
\hline WC & 1 & 5.0000 & 0.7000 & 1.016 & A & A \\
\hline WE & 1 & 6.1300 & 0.6300 & 1.246 & & W \\
\hline YA & 1 & 5.2226 & 0.2572 & 1.062 & A & A \\
\hline YU & 1 & 4.3000 & 0.4000 & 0.874 & W & A \\
\hline
\end{tabular}

Total Number Reported: $\quad 104$

Values for elemental uranium are reported in $\mu \mathrm{g} /$ filter, $\mathrm{g}$ or $\mathrm{mL}$.

$\mathrm{pCi} / \mathrm{g}$ or $\mathrm{mL}=\mathrm{Bq} \times 0.027$

Evaluation: $A=A c c e p t a b l e, W=$ Acceptable with Warning, $N=$ Not Acceptable

If the evaluation system is not appropriate for the types of analyses performed in your lab, apply site specific evaluation. 


\section{QAP 49 Results by Nuclide}

December, 1998

Matrix: AI Air Filter $\mathrm{Bq} /$ filter

Radionuclide:

PU238

\begin{tabular}{ll}
\hline EML Value: & 0.460 \\
EML Error: & 0.005 \\
\hline
\end{tabular}

Reported

Labcode Test \# Value Reported
Error

0.0200

0.1073

0.3100

0.2700

0.5200

0.8190

0.5000

0.5220

0.5180

0.4970

0.5190

0.5100

0.5000

0.4840

0.0300

0.5020

0.0750

0.4760

0.5500

0.5088

0.4800

0.5200

0.4970

0.3916

0.5000

0.4670

0.4900

0.4460

0.4790

0.2510

0.4800

0.4720

0.4820

0.4050

0.6100

0.5900

0.4800

0.4030

0.5220

0.4920

0.4690

0.7600

\subsection{0}

0.0100

0.0200

0.0730

0.0400

0.0390

0.0410

0.0700

0.0100

0.0200

0.0200

0.0260

0.0100

0.0350

0.0030

0.0359

0.0210 .

0.1169

0.0500

0.1000

0.0710

0.1120

0.0300

0.0170

0.0170

0.0150

0.0453

0.0260

0.0280

0.1080

0.0100

0.0240

0.0200

0.0200

0.0300

0.0330

0.0381

0.0640

0.0230

0.0500
0.233

0.674

0.587

1.130

1.780

1.087

1.135

1.126

1.080

1.128

1.109

1.087

1.052

0.065

1.091

0.163

1.035

1.196

1.106

1.043

1.130

1.080

0.851

1.087

1.015

1.065

0.970

1.041

0.546

1.043

1.026

1.048

0.880

1.326

1.283

1.043

0.876

1.135

1.070

1.020

1.652
QAP 48

Evaluation

Evaluation

Values for elemental uranium are reported in $\mu \mathrm{g} /$ filter, $\mathrm{g}$ or $\mathrm{mL}$. $\mathrm{pCi} / \mathrm{g}$ or $\mathrm{mL}=\mathrm{Bq} \times 0.027$

Evaluation: $A=$ Acceptable, $W=$ Acceptable with Warning, $N=$ Not Acceptable

If the evaluation system is not appropriate for the types of analyses performed in your lab, apply site specific evaluation. 


\section{QAP 49 Results by Nuclide}

\begin{tabular}{|c|c|c|c|c|c|c|}
\hline \multicolumn{3}{|c|}{$\begin{array}{l}\text { Matrix: AI Air Filter } \mathrm{Bq} \text { / filter } \\
\text { Radionuclide: }\end{array}$} & \multirow[b]{3}{*}{$\begin{array}{l}\text { Reported } \\
\text { Error }\end{array}$} & \multirow[b]{3}{*}{$\frac{\text { Reported }}{E M L}$} & \multirow[b]{3}{*}{$\begin{array}{l}\text { QAP 48 } \\
\text { Evaluation }\end{array}$} & \multirow[b]{3}{*}{ Evaluation } \\
\hline $\begin{array}{l}\text { EML Value: } \\
\text { EML Error: }\end{array}$ & \multicolumn{2}{|c|}{$\begin{array}{l}0.460 \\
0.005\end{array}$} & & & & \\
\hline Labcode & Test \# & $\begin{array}{l}\text { Reported } \\
\text { Value }\end{array}$ & & & & \\
\hline TI & 1 & 0.4300 & 0.0500 & 0.935 & $\mathrm{~N}$ & A \\
\hline TM & 1 & 0.4820 & 0.0250 & 1.048 & A & A \\
\hline TN & 1 & 0.4700 & 0.0500 & 1.022 & W & $\mathrm{A}$ \\
\hline TO & 1 & 0.4100 & 0.0900 & 0.891 & & A \\
\hline $\mathrm{TX}$ & 1 & 0.5250 & 0.0160 & 1.141 & $\mathrm{~A}$ & $\mathrm{~A}$ \\
\hline UC & 1 & 0.1070 & 0.0099 & 0.233 & & $\mathrm{~N}$ \\
\hline UP & 1 & 0.4880 & 0.0661 & 1.061 & & A \\
\hline UY & 1 & 0.4650 & 0.0510 & 1.011 & A & A \\
\hline WA & 1 & 0.5100 & 0.0500 & 1.109 & A & A \\
\hline WC & 1 & 0.5000 & 0.0800 & 1.087 & W & A \\
\hline YA & 1 & 0.4989 & 0.0083 & 1.085 & A & A \\
\hline
\end{tabular}

Total Number Reported:

Values for elemental uranium are reported in $\mu \mathrm{g} /$ filter, $g$ or $\mathbf{~ m L}$.

$\mathrm{pCi} / \mathrm{g}$ or $\mathrm{mL}=\mathrm{Bq} \times 0.027$ Evaluation: $A=$ Acceptable, $W=$ Acceptable with Warning, $N=$ Not Acceptable

If the evaluation system is not appropriate for the types of analyses performed in your lab, apply site specific evaluation. 


\section{QAP 49 Results by Nuclide}

\begin{tabular}{lc}
\hline $\begin{array}{l}\text { Matrix: AI } \\
\text { Radionuclide: }\end{array}$ & $\begin{array}{c}\text { Fir Filer } \mathrm{Bq} / \text { filter } \\
\text { PU239 }\end{array}$ \\
\hline EML Value: & 0.420 \\
EML Error: & 0.006 \\
\hline
\end{tabular}

Labcode Test \# $\begin{gathered}\text { Reported } \\ \text { Value }\end{gathered}$

\section{Reported Reported QAP 48 Error EML Evaluation \\ Evaluation}

\begin{tabular}{|c|c|c|c|c|c|c|}
\hline $\mathrm{AF}$ & 1 & 0.1200 & 0.0200 & 0.286 & W & $\mathrm{N}$ \\
\hline $\mathrm{AG}$ & 1 & 0.3030 & 0.0400 & 0.721 & A & $\mathrm{N}$ \\
\hline AM & 1 & 0.3400 & 0.0100 & 0.810 & & W \\
\hline $\mathrm{AN}$ & 1 & 0.4700 & 0.0200 & 1.119 & A & A \\
\hline $\mathrm{AU}$ & 1 & 0.7350 & 0.0660 & 1.750 & $\mathrm{~N}$ & $N$ \\
\hline $\mathrm{BE}$ & 1 & 0.4500 & 0.0400 & 1.071 & A & A \\
\hline BL & 1 & 0.4760 & 0.0370 & 1.133 & A & A \\
\hline BL & 2 & 0.4440 & 0.0370 & 1.057 & A & A \\
\hline BM & 1 & 0.4720 & 0.0640 & 1.124 & A & A \\
\hline BP & 1 & 0.4890 & 0.0100 & 1.164 & A & A \\
\hline $\mathrm{BU}$ & 1 & 0.4600 & 0.0200 & 1.095 & A & A \\
\hline BX & 1 & 0.4200 & 0.0200 & 1.000 & A & A \\
\hline $\mathrm{CH}$ & 1 & 0.4410 & 0.0240 & 1.050 & A & A \\
\hline CL & 1 & 0.4800 & 0.1000 & 1.143 & A & A \\
\hline $\mathrm{EG}$ & 1 & 0.4620 & 0.0330 & 1.100 & A & A \\
\hline EM & 1 & 0.0720 & 0.0030 & 0.171 & & $\mathrm{~N}$ \\
\hline EP & 1 & 0.4460 & 0.0336 & 1.062 & A & A \\
\hline GA & 1 & 0.4800 & 0.0200 & 1.143 & W & A \\
\hline $\mathrm{GE}$ & 1 & 0.4606 & 0.1073 & 1.097 & A & A \\
\hline GP & 1 & 0.4400 & 0.0400 & 1.048 & A & A \\
\hline $\mathrm{GT}$ & 1 & 0.4900 & 0.1000 & 1.167 & A & A \\
\hline ID & 1 & 0.4630 & 0.0390 & 1.102 & $\mathrm{~N}$ & A \\
\hline IS & 1 & 0.3777 & 0.1080 & 0.899 & & W \\
\hline $\mathrm{IT}$ & 1 & 0.4800 & 0.0200 & 1.143 & A & A \\
\hline LA & 1 & 0.4710 & 0.0170 & 1.121 & A & A \\
\hline LA & 2 & 0.4660 & 0.0160 & 1.110 & A & A \\
\hline LA & 3 & 0.4370 & 0.0150 & 1.040 & A & A \\
\hline LL & 1 & 0.4630 & 0.0439 & 1.102 & A & A \\
\hline LV & 1 & 0.2480 & 0.0070 & 0.590 & & $\mathrm{~N}$ \\
\hline NA & 1 & 0.4390 & 0.0260 & 1.045 & & A \\
\hline NL & 1 & 0.4470 & 0.1010 & 1.064 & A & A \\
\hline NM & 1. & 0.4330 & 0.0090 & 1.031 & A & A \\
\hline NQ & 1 & 0.3800 & 0.0220 & 0.905 & & A \\
\hline $\mathrm{NZ}$ & 1 & 0.5700 & 0.0200 & 1.357 & & W \\
\hline $\mathrm{NZ}$ & 2 & 0.5500 & 0.0200 & 1.310 & & W \\
\hline OT & 1 & 0.4500 & 0.0300 & 1.071 & A & A \\
\hline RE & 1 & 0.3820 & 0.0320 & 0.910 & A & A \\
\hline RI & 1 & 0.6480 & 0.0447 & 1.543 & W & $\mathrm{N}$ \\
\hline SN & 1 & 0.4580 & 0.0600 & 1.090 & A & A \\
\hline SR & 1 & 0.4340 & 0.0240 & 1.033 & A & A \\
\hline SW & 1 & 0.3100 & 0.0300 & 0.738 & W & $\mathrm{N}$ \\
\hline
\end{tabular}

Values for elemental uranium are reported in $\mu \mathrm{g}$ ffilter, $\mathrm{g}$ or $\mathrm{mL}$.

$\mathrm{pCi} / \mathrm{g}$ or $\mathrm{mL}=\mathrm{Bq} \times 0.027$

Evaluation: $A=A c c e p t a b l e, W=A c c e p t a b l e$ with Warning, $N=$ Not Acceptable

If the evaluation system is not appropriate for the types of analyses performed in your lab, apply site specific evaluation. 


\section{QAP 49 Results by Nuclide}

\begin{tabular}{|c|c|c|c|c|c|c|}
\hline $\begin{array}{l}\text { Matrix: A } \\
\text { Radionuclide }\end{array}$ & II Air Fi & $\begin{array}{l}\text { U2 Bq / filter } \\
\text { U239 }\end{array}$ & & & & \\
\hline $\begin{array}{l}\text { EML Value: } \\
\text { EML Error: }\end{array}$ & & $\begin{array}{l}20 \\
06\end{array}$ & & & & \\
\hline Labcode & Test \# & $\begin{array}{l}\text { Reported } \\
\text { Value }\end{array}$ & $\begin{array}{c}\text { Reported } \\
\text { Error }\end{array}$ & $\frac{\text { Reported }}{E M L}$ & $\begin{array}{c}\text { QAP } 48 \\
\text { Evaluation }\end{array}$ & Evaluation \\
\hline TI & 1 & 0.4000 & 0.0500 & 0.952 & $\mathrm{~N}$ & A \\
\hline $\mathrm{TM}$ & 1 & 0.4730 & 0.0246 & 1.126 & W & A \\
\hline $\mathrm{TN}$ & 1 & 0.4500 & 0.0500 & 1.071 & W & A \\
\hline TO & 1 & 0.4300 & 0.0800 & 1.024 & & A \\
\hline TX & 1 & 0.4600 & 0.0140 & 1.095 & A & A \\
\hline UC & 1 & 0.1000 & 0.0093 & 0.238 & & $\mathbf{N}$ \\
\hline UP & 1 & 0.4710 & 0.0645 & 1.121 & & A \\
\hline UY & 1 & 0.4410 & 0.0500 & 1.050 & A & $\mathrm{A}$ \\
\hline WA & 1 & 0.5000 & 0.0400 & 1.190 & W & W \\
\hline WC & 1 & 0.4700 & 0.0800 & 1.119 & A & A \\
\hline YA & 1 & 0.4616 & 0.0078 & 1.099 & A & A \\
\hline
\end{tabular}

Total Number Reported:

52

Values for elemental uranium are reported in $\mu \mathrm{g} /$ filter, $\mathrm{g}$ or $\mathrm{mL}$.

$\mathrm{pCi} / \mathrm{g}$ or $\mathrm{mL}=\mathrm{Bq} \times 0.027$

Evaluation: $A=A c c e p t a b l e, W=A c c e p t a b l e$ with Warning, $N=$ Not Acceptable

If the evaluation system is not appropriate for the types of analyses performed in your lab, apply site specific evaluation. 


\section{QAP 49 Results by Nuclide}

Matrix: AI Air Filter $\mathrm{Bq} /$ filter

Radionuclide: $\quad$ SB125

\begin{tabular}{ll}
\hline EML Value: & 8.890 \\
EML Error: & 0.550 \\
\hline
\end{tabular}

Labcode Test \# $\begin{gathered}\text { Reported } \\ \text { Value }\end{gathered} \begin{gathered}\text { Reported } \\ \text { Error }\end{gathered} \frac{\text { Reported }}{E M L} \quad \begin{gathered}\text { QAP } 48 \\ \text { Evaluation }\end{gathered}$ Evaluation

\begin{tabular}{|c|c|c|c|c|c|c|}
\hline $\mathrm{AF}$ & 1 & 8.1200 & 1.2200 & 0.913 & $A$ & A \\
\hline $\mathrm{AG}$ & 1 & 9.6000 & 2.2000 & 1.080 & A & A \\
\hline $\mathrm{AL}$ & 1 & 6.1847 & 0.6000 & 0.696 & $\mathrm{~N}$ & W \\
\hline AM & 1 & 5.7200 & 0.2800 & 0.643 & W & W \\
\hline AN & 1 & 10.5900 & 0.9300 & 1.191 & A & A \\
\hline $\mathrm{AU}$ & 1 & 8.3200 & 0.7300 & 0.936 & W & A \\
\hline BA & 1 & 6.8500 & 0.3200 & 0.771 & A & W \\
\hline $\mathrm{BC}$ & 1 & 18.6000 & 0.9700 & 2.092 & $\mathrm{~N}$ & $\mathrm{~N}$ \\
\hline $\mathrm{BE}$ & 1 & 8.3000 & 0.5000 & 0.934 & A & A \\
\hline BL & 1 & 10.2000 & 0.5000 & 1.147 & A & A \\
\hline $\mathrm{BN}$ & 1 & 14.2100 & 0.3600 & 1.598 & W & $\mathrm{N}$ \\
\hline $\mathrm{BN}$ & 2 & 13.9900 & 0.3600 & 1.574 & W & $\mathrm{N}$ \\
\hline $\mathrm{BN}$ & 3 & 14.8400 & 0.3600 & 1.669 & W & $\mathrm{N}$ \\
\hline BP & 1 & 7.9000 & 0.2000 & 0.889 & A & A \\
\hline$B Q$ & 1 & 9.0000 & 0.3000 & 1.012 & $\mathrm{~N}$ & A \\
\hline $\mathrm{BU}$ & 1 & 8.5000 & 0.8000 & 0.956 & A & A \\
\hline $\mathrm{BX}$ & 1 & 19.6000 & 1.0800 & 2.205 & $N$ & $\mathrm{~N}$ \\
\hline $\mathrm{CA}$ & 1 & 8.2000 & 0.3000 . & 0.922 & A & A \\
\hline $\mathrm{CB}$ & 1 & 9.7000 & 0.1900 & 1.091 & & A \\
\hline CB & 2 & 9.4000 & 0.1700 & 1.057 & & A \\
\hline $\mathrm{CB}$ & 3 & 9.0000 & 0.1600 & 1.012 & & A \\
\hline $\mathrm{CD}$ & 1 & 8.6000 & 0.2000 & 0.967 & A & A \\
\hline $\mathrm{CH}$ & 1 & 9.6100 & 0.3100 & 1.081 & A & A \\
\hline $\mathrm{CL}$ & 1 & 8.5000 & 0.6000 & 0.956 & A & A \\
\hline $\mathrm{CN}$ & 1 & 9.3500 & 0.5200 & 1.052 & A & A \\
\hline $\mathrm{CO}$ & 1 & 8.8000 & 0.6000 & 0.990 & A & A \\
\hline CR & 1 & 16.4000 & 0.2000 & 1.845 & W & $\mathrm{N}$ \\
\hline $\mathrm{CS}$ & 1 & 8.5860 & 0.3170 & 0.966 & $\mathrm{~N}$ & A \\
\hline EG & 1 & 8.8300 & 0.1300 & 0.993 & A & A \\
\hline EP & 1 & 8.7700 & 1.5300 & 0.987 & A & A \\
\hline $\mathrm{FG}$ & 1 & 10.7700 & 0.6000 & 1.211 & A & W \\
\hline FJ & 1 & 9.5100 & 0.2100 & 1.070 & W & A \\
\hline FL & 1 & 7.3000 & 0.2000 & 0.821 & A & W \\
\hline FM & 1 & 9.7800 & 0.0900 & 1.100 & A & A \\
\hline FN & 1 & 10.0000 & 0.6000 & 1.125 & A & A \\
\hline GA & 1 & 9.2300 & 1.4300 & 1.038 & A & A \\
\hline GE & 1 & 2.7217 & 1.0818 & 0.306 & A & $\mathrm{N}$ \\
\hline GP & 1 & 13.0000 & 2.0000 & 1.462 & W & $\mathrm{N}$ \\
\hline GT & 1 & 9.6000 & 1.0000 & 1.080 & W & A \\
\hline $\mathrm{HU}$ & 1 & 10.2300 & 0.2000 & 1.151 & A & A \\
\hline IA & 1 & 8.3300 & 0.2300 & 0.937 & A & A \\
\hline
\end{tabular}

Values for elemental uranium are reported in $\mu \mathrm{g} /$ filter, $\mathrm{g}$ or $\mathrm{mL}$.

$\mathrm{pCi} / \mathrm{g}$ or $\mathrm{mL}=\mathrm{Bq} \times 0.027$

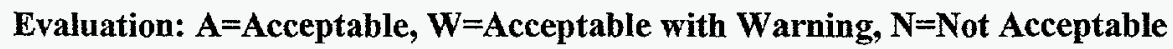

If the evaluation system is not appropriate for the types of analyses performed in your lab, apply site specific evaluation. 


\section{QAP 49 Results by Nuclide}

\begin{tabular}{lc}
\hline Matrix: AI & Air Filter $\mathrm{Bq} /$ filter \\
Radionuclide: & $\mathrm{SB} 125$ \\
\hline EML Value: & 8.890 \\
EML Error: & 0.550 \\
\hline
\end{tabular}

\begin{tabular}{|c|c|c|c|c|c|c|}
\hline Labcode & Test \# & $\begin{array}{l}\text { Reported } \\
\text { Value }\end{array}$ & $\begin{array}{c}\text { Reported } \\
\text { Error }\end{array}$ & $\frac{\text { Reported }}{E M L}$ & $\begin{array}{c}\text { QAP } 48 \\
\text { Evaluation }\end{array}$ & Evaluation \\
\hline$\overline{\mathrm{IA}}$ & 2 & 8.6400 & 0.2400 & 0.972 & $\mathrm{~A}$ & A \\
\hline IA & 3 & 7.9400 & 0.2300 & 0.893 & $\mathrm{~A}$ & A \\
\hline $\mathrm{ID}$ & 1 & 9.6000 & 0.6570 & 1.080 & W & A \\
\hline IL & 1 & 9.2000 & 0.1000 & 1.035 & A & A \\
\hline $\mathrm{N}$ & 1 & 8.9000 & 0.9000 & 1.001 & $\mathrm{~A}$ & A \\
\hline IS & 1 & 9.7100 & 1.2800 & 1.092 & $\mathrm{~A}$ & A \\
\hline IT & 1 & 9.1000 & 0.5000 & 1.024 & A & A \\
\hline $\mathrm{KR}$ & 1 & 7.7000 & 0.9000 & 0.866 & & $\mathrm{~A}$ \\
\hline $\mathrm{LA}$ & 1 & 8.9100 & 0.7500 & 1.002 & A & A \\
\hline $\mathrm{LA}$ & 2 & 9.1100 & 0.7700 & 1.025 & A & $\mathrm{A}$ \\
\hline LA & 3 & 9.1500 & 0.7700 & 1.029 & A & A \\
\hline LB & 1 & 9.6000 & 0.7000 & 1.080 & & A \\
\hline LL & 1 & 9.6700 & 0.7740 & 1.088 & W & A \\
\hline $\mathrm{LN}$ & 1 & 5.7000 & 0.3500 & 0.641 & $\mathrm{~A}$ & W \\
\hline LV & 1 & 4.4200 & 0.2500 & 0.497 & A & $\mathbf{N}$ \\
\hline MA & 1 & 8695.0000 & 3293.0000 & 978.065 & & $\mathrm{~N}$ \\
\hline $\mathrm{ME}$ & 1 & 9.7000 & 0.3900 & 1.091 & A & A \\
\hline $\mathrm{MH}$ & 1 & 6.1700 & 0.2200 & 0.694 & $\mathrm{~W}$ & W \\
\hline MS & 1 & 9.0500 & 0.4500 & 1.018 & A & A \\
\hline NA & 1 & 1.4000 & 0.1100 & 0.157 & A & $\mathrm{N}$ \\
\hline ND & 1 & 7.2390 & 0.2900 & 0.814 & & W \\
\hline NL & 1 & 10.6000 & 1.4000 & 1.192 & A & W \\
\hline NP & 1 & 9.3000 & 0.1000 & 1.046 & A & A \\
\hline NS & 1 & 8.9630 & 0.1260 & 1.008 & A & A \\
\hline $\mathrm{NZ}$ & 1 & 10.3000 & 0.5000 & 1.159 & & $\mathrm{~A}$ \\
\hline NZ & 2 & 10.2000 & 0.5000 & 1.147 & & A \\
\hline $\mathrm{OB}$ & $\overline{1}$ & 15.8000 & 3.1100 & 1.777 & & $\mathrm{~N}$ \\
\hline $\mathrm{OC}$ & 1 & 10.0000 & 3.0000 & 1.125 & & A \\
\hline $\mathrm{OC}$ & 2 & 11.0000 & 3.0000 & 1.237 & & W \\
\hline $\mathrm{OC}$ & 3 & 11.0000 & 3.0000 & 1.237 & & W \\
\hline OD & 1 & 8.4490 & 0.1810 & 0.950 & $\mathrm{~A}$ & $\mathrm{~A}$ \\
\hline OD & 1 & 8.4500 & 0.1800 & 0.951 & A & A \\
\hline OD & 2 & 8.3290 & 0.1740 & 0.937 & $\mathrm{~A}$ & $\mathrm{~A}$ \\
\hline OD & 2 & 8.3300 & 0.1700 & 0.937 & A & A \\
\hline OL & 1 & 10.2200 & 0.4700 & 1.150 & W & A \\
\hline OT & 1 & 8.9000 & 0.5000 & 1.001 & A & A \\
\hline OU & 1 & 8.4300 & 1.8000 & 0.948 & W & $\mathrm{A}$ \\
\hline PK & 1 & 8.0810 & 0.3000 & 0.909 & & A \\
\hline PO & $i$ & 9.9000 & 0.5000 & 1.114 & $\mathrm{~A}$ & A \\
\hline PR & 1 & 9.2890 & 0.6909 & 1.045 & & A \\
\hline $\mathrm{RA}$ & 1 & 9.7500 & 0.3300 & 1.097 & A & A \\
\hline RA & 2 & 9.9200 & 0.3800 & 1.116 & $\mathrm{~A}$ & A \\
\hline
\end{tabular}

Values for elemental uranium are reported in $\mu \mathrm{g} /$ filter, $\mathrm{g}$ or $\mathrm{mL}$.

$\mathrm{pCi} / \mathrm{g}$ or $\mathrm{mL}=\mathrm{Bq} \times 0.027$

Evaluation: A=Acceptable, $W=$ Acceptable with Warning, $N=$ Not Acceptable

If the evaluation system is not appropriate for the types of analyses performed in your lab, apply site specific evaluation. 


\section{QAP 49 Results by Nuclide}

Matrix: $\mathrm{AI}$ Air Filter $\mathrm{Bq} /$ filter

Radionuclide: SB 125

EML Value: $\quad 8.890$

EML Error: $\quad 0.550$

\begin{tabular}{lcrrrrr} 
Labcode & Test \# & $\begin{array}{c}\text { Reported } \\
\text { Value }\end{array}$ & $\begin{array}{c}\text { Reported } \\
\text { Error }\end{array}$ & $\begin{array}{c}\text { Reported } \\
\text { EML }\end{array}$ & $\begin{array}{c}\text { QAP 48 } \\
\text { Evaluation }\end{array}$ & Evaluation \\
\hline RC & 1 & 9.0600 & 0.8800 & 1.019 & $\mathrm{~A}$ & $\mathrm{~A}$ \\
RE & 1 & 8.3900 & 1.0100 & 0.944 & $\mathrm{~A}$ & $\mathrm{~A}$ \\
RI & 1 & 10.3000 & 1.6700 & 1.159 & $\mathrm{~A}$ & $\mathrm{~A}$ \\
SA & 1 & 8.1000 & 1.1000 & 0.911 & $\mathrm{~A}$ & $\mathrm{~A}$ \\
SK & 1 & 5.7000 & 0.6000 & 0.641 & $\mathrm{~A}$ & $\mathrm{~W}$ \\
SR & 1 & 5.2400 & 0.1790 & 0.589 & $\mathrm{~A}$ & $\mathrm{~N}$ \\
SW & 1 & 9.1500 & 0.3000 & 1.029 & $\mathrm{~A}$ & $\mathrm{~A}$ \\
TE & 1 & 10.0000 & 0.8000 & 1.125 & $\mathrm{~A}$ & $\mathrm{~A}$ \\
TI & 1 & 9.0000 & 0.9000 & 1.012 & $\mathrm{~A}$ & $\mathrm{~A}$ \\
TM & 1 & 7.5200 & 0.7160 & 0.846 & $\mathrm{~W}$ & $\mathrm{~A}$ \\
TN & 1 & 5.0000 & 1.0000 & 0.562 & $\mathrm{~A}$ & $\mathrm{~N}$ \\
TP & 1 & 8.6200 & 0.0800 & 0.970 & $\mathrm{~A}$ & $\mathrm{~A}$ \\
TW & 1 & 9.7000 & 0.2000 & 1.091 & $\mathrm{~A}$ & $\mathrm{~A}$ \\
TX & 1 & 8.8100 & 0.1100 & 0.991 & $\mathrm{~W}$ & $\mathrm{~N}$ \\
UP & 1 & 3.4100 & 0.8130 & 0.384 & $\mathrm{~N}$ & $\mathrm{~A}$ \\
UY & 1 & 8.4700 & 0.8000 & 0.953 & $\mathrm{~A}$ & $\mathrm{~A}$ \\
WA & 1 & 9.3000 & 0.5000 & 1.046 & $\mathrm{~W}$ & $\mathrm{~A}$ \\
WC & 1 & 9.8000 & 0.8000 & 1.102 & $\mathrm{~N}$ & $\mathrm{~W}$ \\
WE & 1 & 10.9000 & 0.5900 & 1.226 & & W \\
YU & 1 & 7.0000 & 0.2000 & 0.787 & &
\end{tabular}

Total Number Reported:

103

Values for elemental uranium are reported in $\mu \mathrm{g} /$ filter, $\mathrm{g}$ or $\mathrm{mL}$.

$\mathrm{pCi} / \mathrm{g}$ or $\mathrm{mL}=\mathrm{Bq} \times 0.027$

Evaluation: $A=$ Acceptable, $W=$ Acceptable with Warning, $N=$ Not Acceptable

If the evaluation system is not appropriate for the types of analyses performed in your lab, apply site specific evaluation. 


\section{QAP 49 Results by Nuclide}

\begin{tabular}{|c|c|c|c|c|c|c|}
\hline \multicolumn{3}{|c|}{$\begin{array}{l}\text { Matrix: AI } \\
\text { Radionuclide: } \\
\text { Air Filter Bq / filter } \\
\text { SR90 }\end{array}$} & \multirow[b]{3}{*}{$\begin{array}{l}\text { Reported } \\
\text { Error }\end{array}$} & \multirow[b]{3}{*}{$\frac{\text { Reported }}{\text { EML }}$} & \multirow[b]{3}{*}{$\begin{array}{c}\text { QAP } 48 \\
\text { Evaluation }\end{array}$} & \multirow[b]{3}{*}{ Evaluation } \\
\hline $\begin{array}{l}\text { EML Value: } \\
\text { EML Error: }\end{array}$ & \multicolumn{2}{|c|}{$\begin{array}{l}1.120 \\
0.050\end{array}$} & & & & \\
\hline Labcode & Test \# & $\begin{array}{l}\text { Reported } \\
\text { Value }\end{array}$ & & & & \\
\hline $\mathrm{AF}$ & 1 & 1.1600 & 0.1600 & 1.036 & W & A \\
\hline$A G$ & 1 & 1.0000 & 0.1800 & 0.893 & A & A \\
\hline AM & 1 & 0.8300 & 0.0800 & 0.741 & & W \\
\hline AN & 1 & 1.2200 & 0.0200 & 1.089 & A & A \\
\hline $\mathrm{BC}$ & 1 & 1.1500 & 0.1000 & 1.027 & W & A \\
\hline $\mathrm{BE}$ & 1 & 1.1100 & 0.0800 & 0.991 & A & A \\
\hline $\mathrm{BL}$ & 1 & 1.2200 & 0.2400 & 1.089 & A & A \\
\hline BL & 2 & 1.0900 & 0.3100 & 0.973 & A & A \\
\hline BM & 1 & 1.1400 & 0.0400 & 1.018 & $\mathrm{~A}$ & A \\
\hline $\mathrm{BP}$ & 1 & 1.1600 & 0.0900 & 1.036 & W & A \\
\hline $\mathrm{CH}$ & 1 & 1.1400 & 0.0770 & 1.018 & A & A \\
\hline CL & 1 & 1.2000 & 0.3000 & 1.071 & $\mathrm{~N}$ & A \\
\hline $\mathrm{EG}$ & 1 & 1.2300 & 0.0500 & 1.098 & A & A \\
\hline GA & 1 & 0.7800 & 0.0520 & 0.696 & & W \\
\hline $\mathrm{GE}$ & 1 & 1.1842 & 0.0972 & 1.057 & $\mathrm{~N}$ & $\mathrm{~A}$ \\
\hline GP & 1 & 1.0000 & 0.3000 & 0.893 & A & A \\
\hline GT & 1 & 0.9600 & 0.1000 & 0.857 & A & A \\
\hline ID & 1 & 1.0800 & 0.0880 & 0.964 & & A \\
\hline IS & 1 & 0.2557 & 0.0740 & 0.228 & A & $\mathrm{N}$ \\
\hline IT & 1 & 1.0900 & 0.1200 & 0.973 & A & A \\
\hline $\mathrm{KR}$ & 1 & 0.8600 & 0.0500 & 0.768 & & W \\
\hline $\mathrm{NA}$ & 1 & 0.8400 & 0.1700 & 0.750 & A & W \\
\hline NM & 1 & 1.0040 & 0.1400 & 0.896 & & A \\
\hline $\mathrm{NZ}$ & 1 & 0.4000 & 0.0300 & 0.357 & & $\mathrm{~N}$ \\
\hline NZ & 2 & 0.5000 & 0.0400 & 0.446 & & $\mathrm{~N}$ \\
\hline OT & 1 & 1.1000 & 0.1000 & 0.982 & $\mathrm{~N}$ & A \\
\hline RE & 1 & 1.3200 & 0.0600 & 1.179 & A & A \\
\hline RI & 1 & 1.0700 & 0.0759 & 0.955 & A & A \\
\hline SR & 1 & 1.2400 & 0.1500 & 1.107 & W & A \\
\hline SW & 1 & 0.3900 & 0.1000 & 0.348 & W & $\mathrm{N}$ \\
\hline TE & 1 & 1.1000 & 0.1000 & 0.982 & A & A \\
\hline TI & 1 & 0.9100 & 0.1200 & 0.813 & A & W \\
\hline TN & 1 & 1.0500 & 0.1000 & 0.937 & A & A \\
\hline $\mathrm{TP}$ & 1 & 3.2700 & 0.0600 & 2.920 & A & $\mathrm{N}$ \\
\hline UP & 1 & 1.0400 & 0.2230 & 0.929 & & A \\
\hline UY & 1 & 1.1500 & 0.1100 & 1.027 & W & A \\
\hline WA & 1 & 1.3400 & 0.2300 & 1.196 & A & A \\
\hline WC & 1 & 1.3000 & 0.2000 & 1.161 & A & A \\
\hline WE & 1 & 9.2100 & 0.8300 & 8.223 & & $\mathrm{~N}$ \\
\hline YA & 1 & 1.2210 & 0.0814 & 1.090 & $\mathrm{~A}$ & A \\
\hline
\end{tabular}

Values for elemental uranium are reported in $\mu \mathrm{g} /$ filter, $\mathrm{g}$ or $\mathrm{mL}$. $\mathrm{pCi} / \mathrm{g}$ or $\mathrm{mL}=\mathrm{Bq} \times 0.027$

Evaluation: $A=$ Acceptable, $W=A c c e p t a b l e$ with Warning, $N=$ Not Acceptable

If the evaluation system is not appropriate for the types of analyses performed in your lab, apply site specific evaluation. 
QAP 49 Results by Nuclide

December, 1998

Matrix: AI Air Filter Bq / filter

Radionuclide:

SR90

EML Value: $\quad 1.120$

EML Error: $\quad 0.050$

Labcode $\quad$ Test \# $\begin{gathered}\text { Reported } \\ \text { Value }\end{gathered} \quad \begin{gathered}\text { Reported } \\ \text { Error }\end{gathered} \frac{\text { Reported }}{E M L} \quad \begin{gathered}\text { QAP 48 } \\ \text { Evaluation }\end{gathered}$ Evaluation

Total Number Reported:

40

Values for elemental uranium are reported in $\mu \mathrm{g} /$ filter, $\mathrm{g}$ or $\mathrm{mL}$.

$\mathrm{pCi} / \mathrm{g}$ or $\mathrm{mL}=\mathrm{Bq} \times 0.027$

Evaluation: $A=$ Acceptable, $W=$ Acceptable with Warning, $N=$ Not Acceptable

If the evaluation system is not appropriate for the types of analyses performed in your lab, apply site specific evaluation. 


\section{QAP 49 Results by Nuclide}

\begin{tabular}{|c|c|c|c|c|c|c|}
\hline \multicolumn{3}{|c|}{ Matrix: AI Air Filter $\mathrm{Bq} /$ filter } & \multirow[b]{3}{*}{$\begin{array}{c}\text { Reported } \\
\text { Error }\end{array}$} & \multirow[b]{3}{*}{$\frac{\text { Reported }}{E M L}$} & \multirow[b]{3}{*}{$\begin{array}{c}\text { QAP } 48 \\
\text { Evaluation }\end{array}$} & \multirow[b]{3}{*}{ Evalu } \\
\hline $\begin{array}{l}\text { EML Value: } \\
\text { EML Error: }\end{array}$ & \multicolumn{2}{|c|}{$\begin{array}{l}0.260 \\
0.010\end{array}$} & & & & \\
\hline Labcode & Test \# & $\begin{array}{l}\text { Reported } \\
\text { Value }\end{array}$ & & & & \\
\hline$A G$ & 1 & 0.2500 & 0.0320 & 0.962 & W & A \\
\hline AM & 1 & 0.4800 & 0.0200 & 1.846 & $\mathrm{~N}$ & $W$ \\
\hline$A N$ & 1 & 0.2900 & 0.0100 & 1.115 & A & A \\
\hline $\mathrm{AU}$ & 1 & 0.2010 & 0.0240 & 0.773 & W & $\mathrm{N}$ \\
\hline $\mathrm{BC}$ & 1 & 0.3000 & 0.0200 & 1.154 & W & A \\
\hline $\mathrm{BE}$ & 1 & 0.2500 & 0.0200 & 0.962 & A & A \\
\hline $\mathrm{BL}$ & 1 & 0.2610 & 0.0040 & 1.004 & $\mathrm{~A}$ & A \\
\hline $\mathrm{BM}$ & 1 & 0.2600 & 0.0400 & 1.000 & A & A \\
\hline $\mathrm{BP}$ & 1 & 0.2640 & 0.0100 & 1.015 & & $\mathrm{~A}$ \\
\hline $\mathrm{BU}$ & 1 & 0.2400 & 0.0100 & 0.923 & A & A \\
\hline BX & 1 & 0.2500 & 0.0200 & 0.962 & W & A \\
\hline $\mathrm{CH}$ & 1 & 0.2390 & 0.0110 & 0.919 & A & A \\
\hline $\mathrm{CL}$ & 1 & 0.2800 & 0.0500 & 1.077 & A & A \\
\hline EG & 1 & 0.2710 & 0.0330 & 1.042 & A & A \\
\hline EM & 1 & 0.1010 & 0.0040 & 0.388 & & $\mathrm{~N}$ \\
\hline GA & 1 & 0.3400 & 0.0510 & 1.308 & W & A \\
\hline GE & 1 & 0.2423 & 0.0735 & 0.932 & A & A \\
\hline GP & 1 & 0.2000 & 0.0200 & 0.769 & A & $\mathrm{N}$ \\
\hline IT & 1 & 0.2400 & 0.0100 & 0.923 & A & A \\
\hline LL & 1 & 0.2510 & 0.0232 & 0.965 & $\mathrm{~N}$ & A \\
\hline NL & 1 & 0.2660 & 0.0630 & 1.023 & A & A \\
\hline NQ & 1 & 0.2610 & 0.0150 & 1.004 & & A \\
\hline $\mathrm{NZ}$ & 1 & 0.2800 & 0.0500 & 1.077 & & A \\
\hline $\mathrm{NZ}$ & 2 & 0.2600 & 0.0200 & 1.000 & & A \\
\hline $\mathrm{RA}$ & 1 & 285.0000 & 28.0000 & 1096.154 & A & $\mathrm{N}$ \\
\hline RE & 1 & 0.2540 & 0.0230 & 0.977 & A & A \\
\hline SR & 1 & 0.2620 & 0.0180 & 1.008 & A & A \\
\hline TM & 1 & 0.2600 & 0.0153 & 1.000 & A & A \\
\hline $\mathrm{TN}$ & 1 & 0.2600 & 0.0100 & 1.000 & A & A \\
\hline TO & 1 & 0.2500 & 0.0900 & 0.962 & & A \\
\hline TX & 1 & 0.2540 & 0.0100 & 0.977 & & A \\
\hline WA & 1 & 0.2400 & 0.0400 & 0.923 & A & A \\
\hline WC & 1 & 0.2800 & 0.0500 & 1.077 & A & A \\
\hline
\end{tabular}

Total Number Reported: 


\section{QAP 49 Results by Nuclide}

Matrix: $\mathrm{AI}$ Air Filter $\mathrm{Bq} /$ filter

Radionuclide: $\mathrm{U} 238$

\begin{tabular}{ll}
\hline EML Value: & 0.260 \\
EML Error: & 0.010 \\
\hline
\end{tabular}

\section{Reported Error \\ Reported EML}

0.0330

0.0200

0.0100

0.0240

0.0300

0.0200

0.0040

0.0400

0.0100

0.0100

0.0400

0.1100

0.0500

0.0390

0.0040

0.0500

0.0729

0.0300

0.0500

0.0100

0.0233

0.0640

0.0150

0.0500

0.0200

28.0000

0.0240

0.0178

0.0157

0.0100

0.1000

0.0110

0.0400

0.0500
QAP 48 Evaluation
Evaluation
A

1.008

1.808

1.077

0.746

1.192

0.962

0.977

1.038

1.027

0.923

1.231

0.958

1.077

1.088

0.365

1.308

0.945

0.808

0.962

0.885

0.969

1.046

0.996

1.269

1.077

1076.923

1.027

1.015

83.462

1.031

0.962

1.038

1.031

0.885

1.038
$\stackrel{A}{N}$

A

W

W

A

A

A

A

A

A

A

A

A

A

A

A

A

$\mathrm{N}$

W

A

A

A

A

A

A

A
A

W

A

$\mathrm{N}$

A

A

A

A

A

A

A

A

A

A

$\mathrm{N}$

A

A

$\mathrm{N}$

A

W

A

A

A

A

A

N

A

A

$\mathrm{N}$

A

A

A

A

W

A

Total Number Reported:

35

Values for elemental uranium are reported in $\mu \mathrm{g} /$ filter, $\mathrm{g}$ or $\mathrm{mL}$.

$\mathrm{pCi} / \mathrm{g}$ or $\mathrm{mL}=\mathrm{Bq} \times 0.027$

Evaluation: $A=$ Acceptable, $W=$ Acceptable with Warning, $N=$ Not Acceptable

If the evaluation system is not appropriate for the types of analyses performed in your lab, apply site specific evaluation. 


\section{QAP 49 Results by Nuclide}

December, 1998

\begin{tabular}{|c|c|c|c|c|c|c|}
\hline \multicolumn{3}{|c|}{$\begin{array}{l}\text { Matrix: AI Air Filter } \mathrm{Bq} / \text { filter } \\
\text { Radionuclide: } \\
\text { ug U }\end{array}$} & \multirow[b]{3}{*}{$\begin{array}{l}\text { Reported } \\
\text { Error }\end{array}$} & \multirow[b]{3}{*}{$\frac{\text { Reported }}{E M L}$} & \multirow[b]{3}{*}{$\begin{array}{c}\text { QAP } 48 \\
\text { Evaluation }\end{array}$} & \multirow[b]{3}{*}{ Evaluation } \\
\hline \multirow{2}{*}{$\begin{array}{l}\text { EML Value } \\
\text { EML Error } \\
\text { Labcode }\end{array}$} & \multicolumn{2}{|c|}{$\begin{array}{r}20.960 \\
0.100\end{array}$} & & & & \\
\hline & Test \# & $\begin{array}{l}\text { Reported } \\
\text { Value }\end{array}$ & & & & \\
\hline $\mathrm{AG}$ & 1 & 20.6000 & 3.4000 & 0.983 & A & A \\
\hline $\mathrm{BE}$ & 1 & 20.6000 & & 0.983 & $\mathrm{~A}$ & A \\
\hline $\mathrm{BL}$ & 1 & 21.0700 & 0.3300 & 1.005 & & A \\
\hline $\mathrm{BQ}$ & 1 & 26.0000 & 1.0000 & 1.240 & A & A \\
\hline BU & 1 & 18.0000 & 1.3000 & 0.859 & $\mathrm{~A}$ & W \\
\hline $\mathrm{CH}$ & 1 & 20.7000 & 2.1000 & 0.988 & $\mathrm{~A}$ & $\mathrm{~A}$ \\
\hline $\mathrm{GA}$ & 1 & 27.0000 & 3.8000 & 1.288 & & A \\
\hline GE & $i$ & 16.9400 & 0.6619 & 0.808 & A & W \\
\hline IS & 1 & 14.9000 & 2.0000 & 0.711 & W & $\mathrm{N}$ \\
\hline IT & 1 & 20.4000 & 0.5000 & 0.973 & $\mathrm{~A}$ & A \\
\hline $\mathrm{RA}$ & 1 & 22.4000 & 1.7000 & 1.069 & A & $\mathrm{A}$ \\
\hline $\mathrm{SA}$ & 1 & 23.3000 & 1.2000 & 1.112 & A & A \\
\hline TI & 1 & 25.0000 & 4.0000 & 1.193 & $\mathrm{~A}$ & $\mathrm{~A}$ \\
\hline TM & $i$ & 19.5000 & 1.2500 & 0.930 & $\mathrm{~A}$ & A \\
\hline TN & 1 & 20.0000 & 4.0000 & 0.954 & A & A \\
\hline UP & 1 & 21.3900 & 2.0000 & 1.021 & A & A \\
\hline YP & 1 & 20.9000 & 0.9400 & 0.997 & $\mathrm{~A}$ & $\mathrm{~A}$ \\
\hline
\end{tabular}

Total Number Reported:

Values for elemental uranium are reported in $\mu \mathrm{g} / \mathrm{filter}, \mathrm{g}$ or $\mathrm{mL}$.

$\mathrm{pCi} / \mathrm{g}$ or $\mathrm{mL}=\mathrm{Bq} \times 0.027$

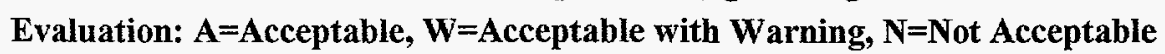

If the evaluation system is not appropriate for the types of analyses performed in your lab, apply site specific evaluation. 


\section{QAP 49 Results by Nuclide}

\begin{tabular}{lr}
\hline Matrix: SO Soil $\mathrm{Bq} / \mathrm{kg}$ \\
Radionuclide: & $\mathrm{AC} 228$ \\
\hline EML Value: & $\begin{array}{r}52.600 \\
\text { EML Error: }\end{array}$ \\
\hline
\end{tabular}

\begin{tabular}{|c|c|c|c|c|c|c|}
\hline abcode & Test \# & $\begin{array}{l}\text { Reported } \\
\text { Value }\end{array}$ & $\begin{array}{c}\text { Reported } \\
\text { Error }\end{array}$ & $\frac{\text { Reported }}{E M L}$ & $\begin{array}{c}\text { QAP 48 } \\
\text { Evaluation }\end{array}$ & Evaluation \\
\hline $\mathrm{AF}$ & 1 & 46.6200 & 8.5100 & 0.886 & & A \\
\hline $\mathrm{AG}$ & 1 & 58.0000 & 11.0000 & 1.103 & & $\mathrm{~A}$ \\
\hline AI & 1 & 51.5000 & 3.4500 & 0.979 & & $\mathrm{~A}$ \\
\hline $\mathrm{AM}$ & 1 & 51.0500 & 5.1800 & 0.971 & & A \\
\hline $\mathrm{BL}$ & 1 & 58.0000 & 5.2000 & 1.103 & & A \\
\hline $\mathrm{BN}$ & 1 & 45.0300 & 0.7500 & 0.856 & & A \\
\hline $\mathrm{BN}$ & 2 & 43.3600 & 0.7500 & 0.824 & & A \\
\hline $\mathrm{BN}$ & 3 & 43.5100 & 0.7500 & 0.827 & & A \\
\hline BP & 1 & 44.0000 & 3.0000 & 0.837 & & A \\
\hline BQ & 1 & 87.0000 & 30.0000 & 1.654 & & A \\
\hline $\mathrm{CD}$ & 1 & 55.0000 & 2.0000 & 1.046 & & $\mathrm{~A}$ \\
\hline $\mathrm{CH}$ & 1 & 57.8000 & 2.0000 & 1.099 & & A \\
\hline $\mathrm{CL}$ & 1 & 65.3000 & 11.7000 & 1.241 & & A \\
\hline $\mathrm{CR}$ & 1 & 70.0000 & 13.0000 & 1.331 & & A \\
\hline $\mathrm{CS}$ & 1 & 46.7800 & 1.0900 & 0.889 & & A \\
\hline $\mathrm{FG}$ & 1 & 60.1100 & 1.2000 & 1.143 & & A \\
\hline FS & 1 & 47.5000 & 3.6000 & 0.903 & & A \\
\hline $\mathrm{GC}$ & 1 & 54.7000 & 9.0000 & 1.040 & & $\mathrm{~A}$ \\
\hline GE & 1 & 53.5945 & 14.6050 & 1.019 & & A \\
\hline $\mathrm{HU}$ & 1 & 49.1000 & 1.2000 & 0.933 & & A \\
\hline $\mathrm{ID}$ & 1 & 60.0600 & 3.6900 & 1.142 & & A \\
\hline $\mathrm{IE}$ & 1 & 50.4100 & 7.3400 & 0.958 & & A \\
\hline II & 1 & 78.7000 & 7.4000 & 1.496 & & A \\
\hline IS & 1 & 48.5000 & 14.5000 & 0.922 & & A \\
\hline IT & 1 & 62.0000 & 1.8000 & 1.179 & & A \\
\hline $\mathrm{JE}$ & 1 & 53.2800 & 6.5900 & 1.013 & & A \\
\hline LV & 1 & 68.7000 & 3.1000 & 1.306 & & A \\
\hline MH & 1 & 51.2400 & 3.7800 & 0.974 & & A \\
\hline NL & 1 & 56.4000 & 10.5000 & 1.072 & & A \\
\hline NQ & 1 & 63.3000 & 6.7000 & 1.203 & & A \\
\hline $\mathrm{OB}$ & 1 & 75.4000 & 22.0000 & 1.433 & & A \\
\hline $\mathrm{OC}$ & 1 & 41.0000 & 18.0000 & 0.779 & & A \\
\hline $\mathrm{OC}$ & 2 & 39.0000 & 18.0000 & 0.741 & & A \\
\hline $\mathrm{OC}$ & 3 & 48.0000 & 18.0000 & 0.913 & & A \\
\hline OT & 1 & 42.0000 & 10.0000 & 0.798 & & A \\
\hline RA & 1 & 51.4000 & 6.0000 & 0.977 & & A \\
\hline $\mathrm{RA}$ & 2 & 52.5000 & 3.8000 & 0.998 & & $A$ \\
\hline SK & 1 & 57.0000 & 3.0000 & 1.084 & & A \\
\hline SR & 1 & 61.4000 & 1.6000 & 1.167 & & A \\
\hline SW & 1 & 83.1400 & 13.2000 & 1.581 & & A \\
\hline TE & 1 & 54.1000 & 3.3000 & 1.029 & & A \\
\hline
\end{tabular}

Values for elemental uranium are reported in $\mu \mathrm{g} /$ filter, g or $\mathrm{mL}$. $\mathrm{pCi} / \mathrm{g}$ or $\mathrm{mL}=\mathrm{Bq} \times 0.027$

Evaluation: $A=A c c e p t a b l e, W=A c c e p t a b l e$ with Warning, $N=$ Not Acceptable

If the evaluation system is not appropriate for the types of analyses performed in your lab, apply site specific evaluation. 


\section{QAP 49 Results by Nuclide}

\begin{tabular}{|c|c|c|c|c|c|c|}
\hline \multicolumn{3}{|c|}{$\begin{array}{lrr}\text { Matrix: } & \text { SO Soil } & \mathrm{Bq} / \mathrm{kg} \\
\text { Radionuclide: } & \text { AC228 }\end{array}$} & \multirow[b]{3}{*}{$\begin{array}{l}\text { Reported } \\
\text { Error }\end{array}$} & \multirow[b]{3}{*}{$\frac{\text { Reported }}{E M L}$} & \multirow[b]{3}{*}{$\begin{array}{c}\text { QAP } 48 \\
\text { Evaluation }\end{array}$} & \multirow[b]{3}{*}{ Evaluation } \\
\hline $\begin{array}{l}\text { EML Value: } \\
\text { EML Error: }\end{array}$ & \multicolumn{2}{|c|}{$\begin{array}{r}52.600 \\
2.900 \\
\end{array}$} & & & & \\
\hline Labcode & Test \# & $\begin{array}{l}\text { Reported } \\
\text { Value }\end{array}$ & & & & \\
\hline TM & 1 & 72.5000 & 7.3000 & 1.378 & & $\mathrm{~A}$ \\
\hline TO & 1 & 35.9000 & 7.3000 & 0.683 & & $\mathrm{~A}$ \\
\hline$T W$ & 1 & 54.0000 & 1.0000 & 1.027 & & A \\
\hline $\mathrm{TX}$ & 1 & 52.2000 & 1.5000 & 0.992 & & A \\
\hline UY & 1 & 71.0000 & 32.0000 & 1.350 & & A \\
\hline WA & 1 & 54.7000 & 2.6000 & 1.040 & & A \\
\hline WE & 1 & 37.5000 & 13.0000 & 0.713 & & A \\
\hline
\end{tabular}

Total Number Reported:

Values for elemental uranium are reported in $\mu \mathrm{g} /$ filter, $\mathrm{g}$ or $\mathrm{mL}$.

$\mathrm{pCi} / \mathrm{g}$ or $\mathrm{mL}=\mathrm{Bq} \times 0.027$

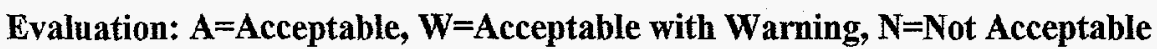

If the evaluation system is not appropriate for the types of analyses performed in your lab, apply site specific evaluation. 


\section{QAP 49 Results by Nuclide}

\begin{tabular}{lc}
\hline Matrix: SO Soil $\mathrm{Bq} / \mathrm{kg}$ \\
Radionuclide: & AM241 \\
\hline EML Value: & 7.470 \\
EML Error: & 0.410 \\
\hline
\end{tabular}

Labcode Test \# $\begin{gathered}\text { Reported } \\ \text { Value }\end{gathered}$

QAP 48

Evaluation
Evaluation

\begin{tabular}{|c|c|c|c|c|c|c|}
\hline $\mathrm{AF}$ & 1 & 7.4000 & 1.4800 & 0.991 & $\mathrm{~N}$ & A \\
\hline $\mathrm{AG}$ & 1 & 8.6000 & 2.0000 & 1.151 & A & A \\
\hline $\mathrm{AI}$ & 1 & 11.0000 & 2.0000 & 1.473 & $\mathrm{~N}$ & A \\
\hline AM & 1 & 10.3200 & 1.8500 & 1.382 & W & A \\
\hline $\mathrm{AN}$ & 1 & 7.4000 & 0.4500 & 0.991 & A & A \\
\hline AT & 1 & 7.8180 & 1.4410 & 1.047 & W & A \\
\hline $\mathrm{AU}$ & 1 & 8.3000 & 1.4000 & 1.111 & A & A \\
\hline $\mathrm{BE}$ & 1 & 7.8500 & 0.7700 & 1.051 & A & A \\
\hline BL & 1 & 3.6800 & 1.2700 & 0.493 & & $\mathrm{~N}$ \\
\hline $\mathrm{BM}$ & 1 & 7.7000 & 1.1800 & 1.031 & A & A \\
\hline $\mathrm{BU}$ & 1 & 7.6000 & 0.4000 & 1.017 & A & A \\
\hline BX & 1 & 6.5000 & 1.4000 & 0.870 & A & A \\
\hline $\mathrm{CH}$ & 1 & 8.3800 & 0.5100 & 1.122 & & A \\
\hline $\mathrm{CL}$ & 1 & 5.8000 & 1.5000 & 0.776 & A & W \\
\hline $\mathrm{CN}$ & 1 & 8.3400 & 1.1000 & 1.116 & & A \\
\hline $\mathrm{CS}$ & 1 & 7.6840 & 0.5463 & 1.029 & A & A \\
\hline DH & 1 & 5.9000 & 1.8000 & 0.790 & & W \\
\hline EG & 1 & 7.4200 & 0.7500 & 0.993 & A & A \\
\hline $\mathrm{EP}$ & 1 & 7.6200 & 0.8030 & 1.020 & A & A \\
\hline FL & 1 & 6.6000 & 0.6000 & 0.884 & A & A \\
\hline FS & 1 & 4.3700 & 0.2400 & 0.585 & A & $\mathrm{N}$ \\
\hline GA & 1 & 6.9000 & 1.2000 & 0.924 & A & A \\
\hline GE & 1 & 6.9560 & 1.5415 & 0.931 & & A \\
\hline GT & 1 & 7.4000 & 3.0000 & 0.991 & A & A \\
\hline $\mathrm{HT}$ & 1 & 41.0000 & 5.0000 & 5.489 & & $\mathrm{~N}$ \\
\hline $\mathrm{HU}$ & 1 & 8.3000 & 3.8000 & 1.111 & & A \\
\hline ID & 1 & 7.9000 & 0.7500 & 1.058 & A & A \\
\hline $\mathbb{I N}$ & 1 & 8.3000 & 1.2500 & 1.111 & A & A \\
\hline IS & 1 & 10.3400 & 5.8100 & 1.384 & A & A \\
\hline IT & 1 & 4.8800 & 0.7600 & 0.653 & A & W \\
\hline LA & 1 & 8.4700 & 0.7400 & 1.134 & $\mathrm{~A}$ & A \\
\hline LA & 2 & 7.6600 & 0.7400 & 1.025 & A & A \\
\hline LA & 3 & 7.9200 & 0.7400 & 1.060 & A & A \\
\hline LL & 1 & 7.6200 & 1.8600 & 1.020 & A & A \\
\hline LV & 1 & 13.1000 & 0.7000 & 1.754 & W & W \\
\hline LW & 1 & 9.6300 & 2.5300 & 1.289 & & A \\
\hline MA & 1 & 8.9000 & 3.0000 & 1.191 & A & A \\
\hline ME & 1 & 6.6100 & 0.7000 & 0.885 & A & A \\
\hline MH & 1 & 6.3900 & 0.6400 & 0.855 & & A \\
\hline MS & 1 & 8.7000 & 1.0000 & 1.165 & & A \\
\hline NM & 1 & 7.0100 & 0.3500 & 0.938 & $\mathrm{~A}$ & A \\
\hline
\end{tabular}

Values for elemental uranium are reported in $\mu \mathrm{g} / \mathrm{filter}, \mathrm{g}$ or $\mathrm{mL}$.

$\mathrm{pCi} / \mathrm{g}$ or $\mathrm{mL}=\mathrm{Bq} \times 0.027$

Evaluation: $A=$ Acceptable, $W=$ Acceptable with Warning, $N=$ Not Acceptable

If the evaluation system is not appropriate for the types of analyses performed in your lab, apply site specific evaluation. 


\section{QAP 49 Results by Nuclide}

\begin{tabular}{|c|c|c|c|c|c|c|}
\hline \multicolumn{3}{|c|}{$\begin{array}{lrr}\text { Matrix: SO Soil } & \mathrm{Bq} / \mathrm{kg} \\
\text { Radionuclide: } & \text { AM241 }\end{array}$} & \multirow[b]{3}{*}{$\begin{array}{l}\text { Reported } \\
\text { Error }\end{array}$} & \multirow[b]{3}{*}{$\frac{\text { Reported }}{\text { EML }}$} & \multirow[b]{3}{*}{$\begin{array}{c}\text { QAP } 48 \\
\text { Evaluation }\end{array}$} & \multirow[b]{3}{*}{ Evaluation } \\
\hline $\begin{array}{l}\text { EML Value: } \\
\text { EML Error: }\end{array}$ & \multicolumn{2}{|c|}{$\begin{array}{l}7.470 \\
0.410 \\
\end{array}$} & & & & \\
\hline Labcode & Test \# & $\begin{array}{l}\text { Reported } \\
\text { Value }\end{array}$ & & & & \\
\hline NM & 2 & 7.8400 & 0.3800 & 1.050 & A & A \\
\hline NM & 3 & 9.1200 & 0.4100 & 1.221 & A & A \\
\hline NQ & 1 & 8.4000 & 1.5000 & 1.124 & & A \\
\hline OT & 1 & 6.5000 & 1.0000 & 0.870 & A & A \\
\hline PO & 1 & 9.0000 & 0.6000 & 1.205 & & A \\
\hline $\mathrm{RE}$ & 1 & 6.7000 & 0.8700 & 0.897 & A & A \\
\hline SK & 1 & 10.2000 & 0.8000 & 1.365 & A & A \\
\hline SN & 1 & 7.7360 & 3.2690 & 1.036 & A & A \\
\hline SW & 1 & 9.4100 & 1.0400 & 1.260 & W & A \\
\hline $\mathrm{TI}$ & 1 & 4.3000 & 1.2000 & 0.576 & A & $\mathrm{N}$ \\
\hline TM & 1 & 7.9300 & 2.9800 & 1.062 & A & A \\
\hline $\mathrm{TN}$ & 1 & 10.0000 & 1.0000 & 1.339 & A & $\mathrm{A}$ \\
\hline TO & 1 & 9.6000 & 3.8000 & 1.285 & $\mathrm{~N}$ & A \\
\hline $\mathrm{TX}$ & 1 & 9.8500 & 0.8900 & 1.319 & $\mathrm{~A}$ & $\mathrm{~A}$ \\
\hline UP & 1 & 9.3900 & 2.0200 & 1.257 & $\mathrm{~A}$ & A \\
\hline UY & 1 & 7.5300 & 1.8000 & 1.008 & A & A \\
\hline WA & 1 & 7.1600 & 0.6400 & 0.959 & A & A \\
\hline WC & 1 & 8.4000 & 1.0000 & 1.124 & $\mathrm{~W}$ & $\mathrm{~A}$ \\
\hline WS & 1 & 13.1000 & 2.4100 & 1.754 & A & W \\
\hline YA & 1 & 6.7365 & 0.2405 & 0.902 & $\mathrm{~A}$ & A \\
\hline
\end{tabular}

Total Number Reported: $\quad 61$

Values for elemental uranium are reported in $\mu \mathrm{g} /$ filter, $\mathrm{g}$ or $\mathrm{mL}$.

$\mathrm{pCi} / \mathrm{g}$ or $\mathrm{mL}=\mathrm{Bq} \times 0.027$

Evaluation: $A=A c c e p t a b l e, W=$ Acceptable with Warning, $N=$ Not Acceptable

If the evaluation system is not appropriate for the types of analyses performed in your lab, apply site specific evaluation. 


\section{QAP 49 Results by Nuclide}

Matrix: SO Soil $\mathrm{Bq} / \mathrm{kg}$

Radionuclide: $\quad \mathrm{BI} 12$

\begin{tabular}{lr}
\hline EML Value: & 58.300 \\
EML Error: & 5.900 \\
\hline
\end{tabular}

Labcode Test \# $\begin{gathered}\text { Reported } \\ \text { Value }\end{gathered}$

\section{Reported Error}

QAP 48

Evaluation Evaluation

\begin{tabular}{|c|c|c|c|c|}
\hline $\mathrm{AF}$ & 1 & 37.3700 & 17.7600 & 0.641 \\
\hline$A G$ & 1 & 57.0000 & 28.0000 & 0.978 \\
\hline $\mathrm{AM}$ & 1 & 30.3300 & 7.0300 & 0.520 \\
\hline BL & 1 & 58.0000 & 2.1000 & 0.995 \\
\hline $\mathrm{BN}$ & 1 & 31.5700 & 1.5200 & 0.542 \\
\hline $\mathrm{BN}$ & 2 & 33.1500 & 1.5200 & 0.569 \\
\hline $\mathrm{BN}$ & 3 & 35.2800 & 1.5200 & 0.605 \\
\hline BP & 1 & 64.0000 & 10.0000 & 1.098 \\
\hline $\mathrm{CD}$ & 1 & 55.0000 & 1.0000 & 0.943 \\
\hline $\mathrm{CH}$ & 1 & 69.2000 & 5.0000 & 1.187 \\
\hline CR & 1 & 69.0000 & 31.0000 & 1.184 \\
\hline $\mathrm{FG}$ & 1 & 66.2800 & 6.6900 & 1.137 \\
\hline $\mathrm{GA}$ & 1 & 65.7000 & 16.6000 & 1.127 \\
\hline GE & 1 & 31.7580 & 14.9050 & 0.545 \\
\hline ID & 1 & 30.1100 & 2.3400 & 0.516 \\
\hline $\mathrm{IE}$ & 1 & 39.2200 & 8.5500 & 0.673 \\
\hline IL & 1 & 63.9000 & 3.9000 & 1.096 \\
\hline $\mathrm{IN}$ & 1 & 47.5000 & 3.0000 & 0.815 \\
\hline IS & 1 & 62.3000 & 29.0000 & 1.069 \\
\hline $\mathrm{IT}$ & 1 & 61.0000 & 3.0000 & 1.046 \\
\hline LV & 1 & 37.9000 & 2.9000 & 0.650 \\
\hline $\mathrm{MH}$ & 1 & 27.2300 & 3.6500 & 0.467 \\
\hline NA & 1 & 45.0000 & 8.0000 & 0.772 \\
\hline NL & 1 & 55.2000 & 9.5000 & 0.947 \\
\hline NQ & 1 & 68.5000 & 9.6000 & 1.175 \\
\hline $\mathrm{OB}$ & 1 & 63.3000 & 43.3000 & 1.086 \\
\hline $\mathrm{OC}$ & 1 & 23.0000 & 15.0000 & 0.395 \\
\hline $\mathrm{OC}$ & 2 & 25.0000 & 15.0000 & 0.429 \\
\hline $\mathrm{OC}$ & 3 & 24.0000 & 15.0000 & 0.412 \\
\hline OT & 1 & 47.0000 & 11.0000 & 0.806 \\
\hline $\mathrm{OU}$ & 1 & 52.1000 & 3.2000 & 0.894 \\
\hline SK & 1 & 59.0000 & 1.0000 & 1.012 \\
\hline $\mathrm{SN}$ & 1 & 37.2590 & 7.7670 & 0.639 \\
\hline SR & 1 & 40.8000 & 3.6000 & 0.700 \\
\hline SW & 1 & 85.4700 & 5.3000 & 1.466 \\
\hline TE & 1 & 55.4000 & 10.3000 & 0.950 \\
\hline TM & 1 & 75.5000 & 20.7000 & 1.295 \\
\hline TO & 1 & 26.5000 & 16.6000 & 0.455 \\
\hline TX & 1 & 46.7000 & 1.6000 & 0.801 \\
\hline UY & 1 & 60.0000 & 30.0000 & 1.029 \\
\hline WA & 1 & 61.4000 & 8.3000 & 1.053 \\
\hline
\end{tabular}

Values for elemental uranium are reported in $\mu \mathrm{g} /$ filter, $\mathrm{g}$ or $\mathrm{mL}$.

$\mathrm{pCi} / \mathrm{g}$ or $\mathrm{mL}=\mathrm{Bq} \times 0.027$

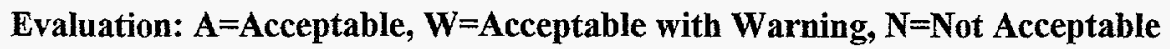

If the evaluation system is not appropriate for the types of analyses performed in your lab, apply site specific evaluation. 


\section{QAP 49 Results by Nuclide}

\begin{tabular}{lr}
\hline Matrix: SO & Soil $\mathrm{Bq} /$ \\
Radionuclide: & $\mathrm{BL} 21$ \\
\hline EML Value: & 58.300 \\
EML Error: & 5.900 \\
\hline
\end{tabular}

\begin{tabular}{ccccccc} 
Labcode & Test \# & $\begin{array}{c}\text { Reported } \\
\text { Value }\end{array}$ & $\begin{array}{c}\text { Reported } \\
\text { Error }\end{array}$ & $\frac{\text { Reported }}{\text { EML }}$ & $\begin{array}{c}\text { QAP 48 } \\
\text { Evaluation }\end{array}$ & Evaluation \\
\hline WE & 1 & 37.2000 & 22.0000 & 0.638 & $\mathrm{~A}$
\end{tabular}

Total Number Reported: 


\section{QAP 49 Results by Nuclide}

\begin{tabular}{|c|c|c|c|c|c|c|}
\hline $\begin{array}{l}\text { Matrix: S } \\
\text { Radionuclid }\end{array}$ & O Soil $\frac{\mathrm{E}}{\mathrm{E}}$ & $\mathrm{qq} / \mathrm{kg}$ & & & & \\
\hline $\begin{array}{l}\text { EML Value: } \\
\text { EML Error: }\end{array}$ & $\begin{array}{r}28.8 \\
0.5 \\
\end{array}$ & & & & & \\
\hline Labcode & Test \# & $\begin{array}{l}\text { Reported } \\
\text { Value }\end{array}$ & $\begin{array}{c}\text { Reported } \\
\text { Error }\end{array}$ & $\frac{\text { Reported }}{E M L}$ & $\begin{array}{c}\text { QAP 48 } \\
\text { Evaluation }\end{array}$ & Evaluation \\
\hline $\mathrm{AF}$ & 1 & 22.2000 & 9.6200 & 0.771 & & A \\
\hline $\mathrm{AG}$ & 1 & 32.6000 & 7.8000 & 1.132 & & A \\
\hline $\mathrm{AI}$ & 1 & 38.9000 & 2.1900 & 1.351 & & A \\
\hline AM & 1 & 40.3200 & 2.5900 & 1.400 & & A \\
\hline $\mathrm{BL}$ & 1 & 31.0000 & 2.1000 & 1.076 & & A \\
\hline $\mathrm{BN}$ & 1 & 25.8100 & 0.5800 & 0.896 & & A \\
\hline $\mathrm{BN}$ & 2 & 27.2000 & 0.5800 & 0.944 & & A \\
\hline BN & 3 & 26.2600 & 0.5800 & 0.912 & & A \\
\hline $\mathrm{BP}$ & 1 & 27.0000 & 3.0000 & 0.938 & & $A$ \\
\hline$C D$ & 1 & 32.0000 & 2.0000 & 1.111 & & A \\
\hline $\mathrm{CH}$ & 1 & 25.9000 & 1.3000 & 0.899 & & A \\
\hline $\mathrm{CL}$ & 1 & 41.0000 & 7.4000 & 1.424 & & A \\
\hline $\mathrm{CR}$ & 1 & 40.0000 & 6.0000 & 1.389 & & $\mathrm{~A}$ \\
\hline $\mathrm{CS}$ & 1 & 27.9500 & 0.7267 & 0.970 & & A \\
\hline FG & 1 & 47.8000 & 8.0000 & 1.660 & & A \\
\hline FS & 1 & 29.4000 & 2.0000 & 1.021 & & A \\
\hline GA & 1 & 31.3000 & 7.7000 & 1.087 & & A \\
\hline GC & 1 & 34.0000 & 1.8000 & 1.181 & & A \\
\hline $\mathrm{HU}$ & 1 & 25.6000 & 1.0000 & 0.889 & & $\mathrm{~A}$ \\
\hline ID & 1 & 29.8500 & 1.6500 & 1.036 & & A \\
\hline$\Pi$ & 1 & 49.1000 & 6.5000 & 1.705 & & A \\
\hline $\mathrm{IN}$ & 1 & 34.0000 & 8.0000 & 1.181 & & A \\
\hline IS & 1 & 21.9000 & 5.8000 & 0.760 & & $\mathrm{~A}$ \\
\hline IT & 1 & 32.0000 & 3.0000 & 1.111 & & A \\
\hline$J E$ & 1 & 39.8000 & 5.1200 & 1.382 & & A \\
\hline LV & 1 & 36.3000 & 1.8000 & 1.260 & & A \\
\hline $\mathrm{MH}$ & 1 & 29.1200 & 2.1600 & 1.011 & & A \\
\hline $\mathrm{NA}$ & 1 & 24.0000 & 1.5000 & 0.833 & & A \\
\hline NL & 1 & 31.1000 & 7.1000 & 1.080 & & A \\
\hline NQ & 1 & 33.0000 & 3.7000 & 1.146 & & $\mathrm{~A}$ \\
\hline $\mathrm{OB}$ & 1 & 34.3000 & 13.7000 & 1.191 & & A \\
\hline $\mathrm{OC}$ & 1 & 25.0000 & 10.0000 & 0.868 & & A \\
\hline $\mathrm{OC}$ & 2 & 28.0000 & 10.0000 & 0.972 & & A \\
\hline $\mathrm{OC}$ & 3 & 21.0000 & 10.0000 & 0.729 & & A \\
\hline OT & 1 & 37.0000 & 14.0000 & 1.285 & & A \\
\hline OU & 1 & 33.2000 & 2.3000 & 1.153 & & $\mathrm{~A}$ \\
\hline RA & 1 & 27.5000 & 3.6000 & 0.955 & & $\mathrm{~A}$ \\
\hline RA & 2 & 30.6000 & 6.8000 & 1.063 & & A \\
\hline SK & 1 & 38.0000 & 1.0000 & 1.319 & & A \\
\hline SN & 1 & 31.9880 & 4.3300 & 1.111 & & $\mathrm{~A}$ \\
\hline SR & 1 & 31.4000 & 1.2000 & 1.090 & & A \\
\hline
\end{tabular}

Values for elemental uranium are reported in $\mu \mathrm{g} / \mathrm{filter}, \mathrm{g}$ or $\mathrm{mL}$.

$\mathrm{pCi} / \mathrm{g}$ or $\mathrm{mL}=\mathrm{Bq} \times 0.027$

Evaluation: $A=$ Acceptable, $W=$ Acceptable with Warning, $N=$ Not Acceptable

If the evaluation system is not appropriate for the types of analyses performed in your lab, apply site specific evaluation. 


\begin{tabular}{|c|c|c|c|c|c|c|}
\hline $\begin{array}{l}\text { Matrix: S } \\
\text { Radionuclid }\end{array}$ & O Soil & $\begin{array}{l}\mathrm{q} / \mathrm{kg} \\
\mathrm{I} 214\end{array}$ & & & & \\
\hline $\begin{array}{l}\text { EML Value: } \\
\text { EML Error: }\end{array}$ & $\begin{array}{r}28 . \\
0 .\end{array}$ & $\begin{array}{l}00 \\
00\end{array}$ & & & & \\
\hline Labcode & Test \# & $\begin{array}{l}\text { Reported } \\
\text { Value }\end{array}$ & $\begin{array}{c}\text { Reported } \\
\text { Error }\end{array}$ & $\frac{\text { Reported }}{E M L}$ & $\begin{array}{c}\text { QAP 48 } \\
\text { Evaluation }\end{array}$ & Evaluation \\
\hline SW & 1 & 44.5500 & 2.2900 & 1.547 & & A \\
\hline $\mathrm{TE}$ & 1 & 28.5000 & 6.5000 & 0.990 & & A \\
\hline TM & 1 & 41.0000 & 3.8900 & 1.424 & & A \\
\hline TO & 1 & 22.8000 & 5.4000 & 0.792 & & A \\
\hline TO & 1 & 27.4022 & 6.9850 & 0.951 & & A \\
\hline TW & 1 & 25.3000 & 0.8000 & 0.878 & & A \\
\hline $\mathrm{TX}$ & 1 & 25.1000 & 1.2000 & 0.872 & & A \\
\hline UY & 1 & 33.0000 & 15.0000 & 1.146 & & A \\
\hline WA & 1 & 28.7000 & 1.9000 & 0.997 & & A \\
\hline WE & 1 & 33.9000 & 17.0000 & 1.177 & & A \\
\hline
\end{tabular}

Total Number Reported: 


\section{QAP 49 Results by Nuclide}

\begin{tabular}{lr}
\hline $\begin{array}{l}\text { Matrix: SO } \\
\text { Radionuclide: }\end{array}$ & $\begin{array}{c}\text { Soil } \mathrm{Bq} / \mathrm{kg} \\
\mathrm{Bq} \mathrm{U}\end{array}$ \\
\hline EML Value: & $\begin{array}{r}237.000 \\
16.000\end{array}$ \\
\hline
\end{tabular}

$\begin{array}{lcrrr}\text { Labcode } & \text { Test \# } \begin{array}{c}\text { Reported } \\ \text { Value }\end{array} & \begin{array}{c}\text { Reported } \\ \text { Error }\end{array} & \begin{array}{c}\text { Reported } \\ \text { EML }\end{array} \\ & & & & \\ \text { AF } & 1 & 121.7300 & 11.8700 & 0.514 \\ \text { AG } & 1 & 212.0000 & 18.0000 & 0.895 \\ \text { AM } & 1 & 146.0100 & 13.3100 & 0.616 \\ \text { BL } & 1 & 223.0000 & & 0.941 \\ \text { BL } & 2 & 213.0000 & 3.0000 & 0.899 \\ \text { BP } & 1 & 226.0000 & 6.0000 & 0.954 \\ \text { BU } & 1 & 218.0000 & 10.0000 & 0.920 \\ \text { CH } & 1 & 224.0000 & 11.0000 & 0.945 \\ \text { CL } & 1 & 186.0000 & 36.0000 & 0.785 \\ \text { HT } & 1 & 470.5000 & 45.0000 & 1.985 \\ \text { HU } & 1 & 9.4000 & 0.7000 & 0.040 \\ \text { ID } & 1 & 217.7700 & 12.4700 & 0.919 \\ \text { IT } & 1 & 193.0000 & 13.0000 & 0.814 \\ \text { OT } & 1 & 160.0000 & 20.0000 & 0.675 \\ \text { OU } & 1 & 237.0000 & 16.0000 & 1.000 \\ \text { UP } & 1 & 234.0000 & 18.8000 & 0.987 \\ \text { UY } & 1 & 216.0000 & 22.0000 & 0.911 \\ \text { WA } & 1 & 247.0000 & 9.0000 & 1.042 \\ \text { YA } & 1 & 225.8048 & 6.4183 & 0.953 \\ & & & & \end{array}$

QAP 48

Evaluation Evaluation

0.514

0.616

0.899

.954

0.920

.785

985

919

0.814

600

987

0.911

0.953

\begin{tabular}{|c|}
\hline $\mathrm{A}$ \\
\hline W \\
\hline $\mathrm{A}$ \\
\hline $\mathrm{A}$ \\
\hline $\mathrm{A}$ \\
\hline \\
\hline$A$ \\
\hline \\
\hline$A$ \\
\hline $\mathrm{A}$ \\
\hline \\
\hline \\
\hline$A$ \\
\hline
\end{tabular}

Total Number Reported: 


\section{QAP 49 Results by Nuclide}

Matrix: SO Soil $\mathrm{Bq} / \mathrm{kg}$

Radionuclide: $\quad$ CO60

\begin{tabular}{ll}
\hline EML Value: & 1.240 \\
EML Error: & 0.110 \\
\hline
\end{tabular}

\begin{tabular}{|c|c|c|c|c|c|c|}
\hline Labcode & Test \# & $\begin{array}{l}\text { Reported } \\
\text { Value }\end{array}$ & $\begin{array}{c}\text { Reported } \\
\text { Error }\end{array}$ & $\frac{\text { Reported }}{E M L}$ & $\begin{array}{c}\text { QAP } 48 \\
\text { Evaluation }\end{array}$ & Evaluation \\
\hline
\end{tabular}

$\begin{array}{lrrrrr}\text { CO } & 1 & 1106.0000 & 36.0000 & 891.935 & \mathrm{~N} \\ \text { DH } & 1 & 1.3700 & 0.6300 & 1.105 & \mathrm{~A} \\ \text { GT } & 1 & 1.9000 & 1.0000 & 1.532 & \mathrm{~A} \\ \text { HU } & 1 & 0.7600 & 0.2200 & 0.613 & \mathrm{~N} \\ \text { IA } & 1 & 1.5800 & 0.2000 & 1.274 & \mathrm{~A} \\ \text { IA } & 2 & 1.2100 & 0.2000 & 0.976 & \mathrm{~A} \\ \text { IA } & 3 & 0.8500 & 0.2000 & 0.685 & \mathrm{~W} \\ \text { ME } & 1 & 1.0100 & 0.2000 & 0.815 & \mathrm{~W} \\ \text { OL } & 1 & 1.3200 & 0.3200 & 1.065 & \mathrm{~A} \\ \text { PO } & 1 & 1.1000 & 0.4000 & 0.887 & \mathrm{~A} \\ \text { RC } & 1 & 1.9200 & 0.4100 & 1.548 & \mathrm{~A} \\ \text { SK } & 1 & 1.7400 & 0.2700 & 1.403 & \mathrm{~A} \\ \text { TI } & 1 & 1.8500 & 0.6000 & 1.492 & \mathrm{~A} \\ \text { TY } & 1 & 2.0000 & 1.0000 & 1.613 & \end{array}$

Total Number Reported: $\quad 14$

Values for elemental uranium are reported in $\mu \mathrm{g} /$ filter, $\mathrm{g}$ or $\mathrm{mL}$.

$\mathrm{pCi} / \mathrm{g}$ or $\mathrm{mL}=\mathrm{Bq} \times 0.027$

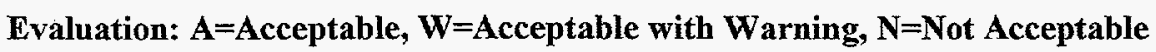

If the evaluation system is not appropriate for the types of analyses performed in your lab, apply site specific evaluation. 


\section{QAP 49 Results by Nuclide}

Matrix: SO Soil $\mathrm{Bq} / \mathrm{kg}$

Radionuclide:

CS137

EML Value: $\quad 954.000$

EML Error: $\quad 38.000$

\begin{tabular}{|c|c|c|c|c|c|c|}
\hline abcode & Test \# & $\begin{array}{l}\text { Reported } \\
\text { Value }\end{array}$ & $\begin{array}{c}\text { Reported } \\
\text { Error }\end{array}$ & $\frac{\text { Reported }}{\text { EML }}$ & $\begin{array}{c}\text { QAP 48 } \\
\text { Evaluation }\end{array}$ & Evalue \\
\hline $\mathrm{AF}$ & 1 & 723.3500 & 83.9900 & 0.758 & W & $\mathrm{N}$ \\
\hline $\mathrm{AG}$ & 1 & 1173.0000 & 200.0000 & 1.230 & A & W \\
\hline $\mathrm{AI}$ & 1 & 1010.0000 & 6.1600 & 1.059 & $\mathrm{~N}$ & A \\
\hline $\mathrm{AL}$ & 1 & 1788.3000 & 0.0700 & 1.875 & $\mathrm{~N}$ & $\mathrm{~N}$ \\
\hline $\mathrm{AM}$ & 1 & 1049.9399 & 9.9900 & 1.101 & A & A \\
\hline $\mathrm{AN}$ & l & 1092.0000 & 4.0000 & 1.145 & A & A \\
\hline $\mathrm{AT}$ & 1 & 970.5000 & 81.0000 & 1.017 & W & A \\
\hline $\mathrm{AU}$ & 1 & 1113.0000 & 58.0000 & 1.167 & A & A \\
\hline BA & 1 & 1041.0000 & 45.0000 & 1.091 & A & A \\
\hline $\mathrm{BC}$ & 1 & 1099.0000 & 76.0000 & 1.152 & A & A \\
\hline $\mathrm{BE}$ & 1 & 1069.0000 & 150.0000 & 1.121 & A & A \\
\hline $\mathrm{BL}$ & 1 & 1097.0000 & 40.0000 & 1.150 & A & $\mathrm{A}$ \\
\hline $\mathrm{BM}$ & 1 & 1041.0000 & 7.0000 & 1.091 & A & $\mathrm{A}$ \\
\hline $\mathrm{BN}$ & 1 & 959.4100 & 2.1900 & 1.006 & A & A \\
\hline $\mathrm{BN}$ & 2 & 954.9700 & 2.1900 & 1.001 & A & A \\
\hline $\mathrm{BN}$ & 3 & 959.7800 & 2.1900 & 1.006 & A & A \\
\hline BP & 1 & 902.0000 & 18.0000 & 0.945 & A & A \\
\hline $\mathrm{BQ}$ & 1 & 1070.0000 & 30.0000 & 1.122 & $\mathrm{~N}$ & A \\
\hline BÙ & 1 & 860.0000 & 90.0000 & 0.901 & W & A \\
\hline $\mathrm{BX}$ & 1 & 1094.0000 & 84.0000 & 1.147 & A & $\mathrm{A}$ \\
\hline$C D$ & 1 & 1051.0000 & 4.0000 & 1.102 & A & A \\
\hline $\mathrm{CH}$ & 1 & 1088.0000 & 2.8000 & 1.140 & & A \\
\hline CL & 1 & 950.0000 & 28.5000 & 0.996 & A & A \\
\hline $\mathrm{CM}$ & 1 & 987.0000 & 47.0000 & 1.035 & & A \\
\hline $\mathrm{CM}$ & 2 & 1010.0000 & 48.0000 & 1.059 & & A \\
\hline $\mathrm{CM}$ & 3 & 1050.0000 & 50.0000 & 1.101 & & A \\
\hline $\mathrm{CN}$ & 1 & 1046.7000 & 63.7700 & 1.097 & A & A \\
\hline $\mathrm{CO}$ & 1 & 1118.0000 & 33.0000 & 1.172 & $\mathrm{~A}$ & $\mathrm{~A}$ \\
\hline $\mathrm{CO}$ & 1 & 566.0000 & 16.0000 & 0.593 & A & $\mathrm{N}$ \\
\hline CR & 1 & 1180.0000 & 47.0000 & 1.237 & W & W \\
\hline $\mathrm{CS}$ & 1 & 950.9000 & 40.9800 & 0.997 & A & A \\
\hline $\mathrm{DH}$ & 1 & 969.0000 & 8.0000 & 1.016 & $\mathrm{~A}$ & A \\
\hline $\mathrm{EG}$ & 1 & 1110.0000 & 20.0000 & 1.164 & A & A \\
\hline $\mathrm{FG}$ & 1 & 888.3000 & 1.0000 & 0.931 & A & A \\
\hline FL & 1 & 945.0000 & 2.0000 & 0.991 & A & A \\
\hline FN & 1 & 954.0000 & 96.0000 & 1.000 & A & A \\
\hline FS & 1 & 954.0000 & 36.0000 & 1.000 & A & A \\
\hline $\mathrm{GA}$ & 1 & 1023.0000 & 30.0000 & 1.072 & A & A \\
\hline GC & 1 & 933.1000 & 35.1000 & 0.978 & A & A \\
\hline GE & 1 & 980.6850 & 140.3387 & 1.028 & A & A \\
\hline $\mathrm{GP}$ & 1 & 1000.0000 & 100.0000 & 1.048 & A & A \\
\hline
\end{tabular}

Values for elemental uranium are reported in $\mu \mathrm{g} /$ filter, $\mathrm{g}$ or $\mathrm{mL}$.

$\mathrm{pCi} / \mathrm{g}$ or $\mathrm{mL}=\mathrm{Bq} \times 0.027$

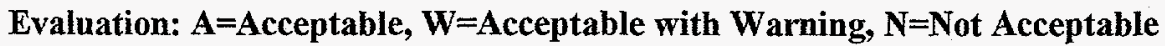

If the evaluation system is not appropriate for the types of analyses performed in your lab, apply site specific evaluation. 


\section{QAP 49 Results by Nuclide}

\begin{tabular}{|c|c|c|c|c|c|c|}
\hline \multicolumn{3}{|c|}{$\begin{array}{l}\text { Matrix: SO Soil } \mathrm{Bq} / \mathrm{kg} \\
\text { Radionuclide: }\end{array}$} & \multirow[b]{3}{*}{$\begin{array}{l}\text { Reported } \\
\text { Error }\end{array}$} & \multirow[b]{3}{*}{$\frac{\text { Reported }}{\text { EML }}$} & \multirow[b]{3}{*}{$\begin{array}{c}\text { QAP } 48 \\
\text { Evaluation }\end{array}$} & \multirow[b]{3}{*}{ Evaluation } \\
\hline $\begin{array}{l}\text { EML Value: } \\
\text { EML Error: }\end{array}$ & \multicolumn{2}{|c|}{$\begin{array}{r}954.000 \\
38.000 \\
\end{array}$} & & & & \\
\hline Labcode & Test \# & $\begin{array}{l}\text { Reported } \\
\text { Value }\end{array}$ & & & & \\
\hline $\mathrm{GT}$ & 1 & 1200.0000 & 100.0000 & 1.258 & A & $W$ \\
\hline HU & 1 & 896.0000 & 23.0000 & 0.939 & W & A \\
\hline IA & 1 & 685.0000 & 2.0000 & 0.718 & $\mathrm{~N}$ & $\mathrm{~N}$ \\
\hline $\mathrm{IA}$ & 2 & 687.0000 & 2.0000 & 0.720 & $\mathrm{~N}$ & $\mathrm{~N}$ \\
\hline IA & 3 & 683.0000 & 2.0000 & 0.716 & $\mathrm{~N}$ & $\mathrm{~N}$ \\
\hline ID & 1 & 1056.3300 & 52.9100 & 1.107 & A & A \\
\hline IE & 1 & 1125.0000 & 7.0000 & 1.179 & A & A \\
\hline IL & 1 & 994.9000 & 17.5000 & 1.043 & $N$ & A \\
\hline $\mathbb{N}$ & 1 & 1136.0000 & 175.0000 & 1.191 & A & A \\
\hline IS & 1 & 923.0000 & 116.0000 & 0.968 & A & A \\
\hline IT & 1 & 1129.0000 & 46.0000 & 1.183 & A & A \\
\hline JE & 1 & 1124.9600 & 27.6300 & 1.179 & A & A \\
\hline $\mathrm{KA}$ & 1 & 967.5000 & 108.5000 & 1.014 & A & A \\
\hline $\mathrm{KR}$ & 1 & 1043.1000 & 44.2000 & 1.093 & A & A \\
\hline LA & 1 & 628.0000 & 45.0000 & 0.658 & W & $\mathrm{N}$ \\
\hline LA & 2 & 629.0000 & 45.0000 & 0.659 & W & $\mathrm{N}$ \\
\hline $\mathrm{LA}$ & 3 & 785.0000 & 56.0000 & 0.823 & W & $\mathrm{N}$ \\
\hline LB & 1 & 1131.0000 & 124.0000 & 1.186 & & A \\
\hline $\mathrm{LL}$ & 1 & 716.0000 & 21.5000 & 0.751 & A & $\mathrm{N}$ \\
\hline LV & 1 & 1140.0000 & 4.0000 & 1.195 & W & A \\
\hline $\mathrm{MA}$ & 1 & 1114.0000 & 74.0000 & 1.168 & W & A \\
\hline $\mathrm{ME}$ & 1 & 703.0000 & 33.0000 & 0.737 & A & $\mathrm{N}$ \\
\hline MH & 1 & 969.5000 & 46.8500 & 1.016 & A & A \\
\hline MS & 1 & 1025.0000 & 103.0000 & 1.074 & & A \\
\hline NA & 1 & 923.2000 & 4.4000 & 0.968 & A & A \\
\hline NL & 1 & 1010.0000 & 181.0000 & 1.059 & A & A \\
\hline $\mathrm{NM}$ & 1 & 903.0000 & 46.0000 & 0.947 & A & A \\
\hline NP & 1 & 653.0000 & 1.0000 & 0.684 & W & $\mathrm{N}$ \\
\hline NQ & 1 & 1190.0000 & 140.0000 & 1.247 & & W \\
\hline NR & 1 & 1003.0000 & 201.0000 & 1.051 & A & A \\
\hline $\mathrm{NZ}$ & 1 & 1046.0000 & 54.0000 & 1.096 & & A \\
\hline $\mathrm{NZ}$ & 2 & 1024.0000 & 53.0000 & 1.073 & & A \\
\hline $\mathrm{OB}$ & 1 & 1370.0000 & 259.0000 & 1.436 & & $\mathrm{~N}$ \\
\hline $\mathrm{OC}$ & 1 & 850.0000 & 300.0000 & 0.891 & & W \\
\hline $\mathrm{OC}$ & 2 & 840.0000 & 300.0000 & 0.881 & & W \\
\hline $\mathrm{OC}$ & 3 & 830.0000 & 300.0000 & 0.870 & & W \\
\hline OL & 1 & 1059.2000 & 26.8000 & 1.110 & A & A \\
\hline OT & 1 & 920.0000 & 10.0000 & 0.964 & A & A \\
\hline OU & 1 & 1110.0000 & 52.1000 & 1.164 & W & A \\
\hline PK & 1 & 946.2600 & 39.3500 & 0.992 & A & A \\
\hline $\mathrm{PO}$ & 1 & 1045.0000 & 50.0000 & 1.095 & A & A \\
\hline $\mathrm{RA}$ & 1 & 1020.0000 & 40.0000 & 1.069 & $\mathrm{~W}$ & A \\
\hline
\end{tabular}

Values for elemental uranium are reported in $\mu \mathrm{g} /$ filter, $\mathrm{g}$ or $\mathrm{mL}$.

$\mathrm{pCi} / \mathrm{g}$ or $\mathrm{mL}=\mathrm{Bq} \times 0.027$

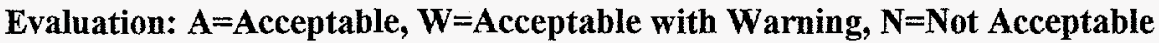

If the evaluation system is not appropriate for the types of analyses performed in your lab, apply site specific evaluation. 


\section{QAP 49 Results by Nuclide}

\begin{tabular}{|c|c|c|c|c|c|c|}
\hline \multicolumn{3}{|c|}{$\begin{array}{l}\text { Matrix: SO Soil } \mathrm{Bq} / \mathrm{kg} \\
\text { Radionuclide: }\end{array}$} & \multirow[b]{3}{*}{$\begin{array}{l}\text { Reported } \\
\text { Error }\end{array}$} & \multirow[b]{3}{*}{$\frac{\text { Reported }}{E M L}$} & \multirow[b]{3}{*}{$\begin{array}{c}\text { QAP 48 } \\
\text { Evaluation }\end{array}$} & \multirow[b]{3}{*}{ Evaluation } \\
\hline $\begin{array}{l}\text { EML Value: } \\
\text { EML Error: }\end{array}$ & \multicolumn{2}{|c|}{$\begin{array}{r}954.000 \\
38.000\end{array}$} & & & & \\
\hline Labcode & Test \# & $\begin{array}{l}\text { Reported } \\
\text { Value }\end{array}$ & & & & \\
\hline RA & 2 & 1030.0000 & 40.0000 & 1.080 & W & A \\
\hline $\mathrm{RC}$ & 1 & 980.0000 & 67.0000 & 1.027 & A & A \\
\hline $\mathrm{RE}$ & 1 & 967.0000 & 72.0000 & 1.014 & A & A \\
\hline RI & 1 & 1080.0000 & 30.3000 & 1.132 & A & A \\
\hline SA & 1 & 990.0000 & 29.0000 & 1.038 & A & A \\
\hline SB & 1 & 1031.0000 & 128.0000 & 1.081 & & A \\
\hline SK & 1 & 1080.0000 & 66.0000 & 1.132 & $W$ & A \\
\hline SN & 1 & 1113.9470 & 111.3210 & 1.168 & A & A \\
\hline SR & 1 & 1090.0000 & 20.0000 & 1.143 & A & A \\
\hline SW & 1 & 1228.0000 & 5.0000 & 1.287 & $\mathrm{~N}$ & W \\
\hline $\mathrm{TE}$ & 1 & 915.7000 & 8.2000 & 0.960 & A & A \\
\hline $\mathrm{TI}$ & 1 & 1188.0000 & 119.0000 & 1.245 & A & W \\
\hline TM & 1 & 1030.0000 & 30.8000 & 1.080 & A & A \\
\hline $\mathrm{TN}$ & 1 & 88.3000 & 9.0000 & 0.093 & & $\mathrm{~N}$ \\
\hline TO & 1 & 693.9000 & 70.9000 & 0.727 & A & $\mathrm{N}$ \\
\hline TP & 1 & 918.5300 & 5.1800 & 0.963 & A & A \\
\hline TT & 1 & 750.0000 & 9.2300 & 0.786 & $\mathrm{~N}$ & $\mathrm{~N}$ \\
\hline$T W$ & 1 & 1040.0000 & 8.0000 & 1.090 & A & A \\
\hline $\mathrm{TX}$ & 1 & 914.0000 & 4.0000 & 0.958 & A & A \\
\hline TY & 1 & 864.0000 & 1.0000 & 0.906 & & A \\
\hline UC & 1 & 1160.3199 & 98.4200 & 1.216 & W & W \\
\hline UP & 1 & 1101.0000 & 88.0000 & 1.154 & A & A \\
\hline UY & 1 & 1100.0000 & 100.0000 & 1.153 & $\mathrm{~A}$ & $\mathrm{~A}$ \\
\hline WA & 1 & 1080.0000 & 30.0000 & 1.132 & A & A \\
\hline WC & 1 & 1081.0000 & 150.0000 & 1.133 & A & A \\
\hline WE & 1 & 1130.0000 & 290.0000 & 1.184 & & A \\
\hline WS & 1 & 1077.1000 & 36.0000 & 1.129 & A & A \\
\hline YA & 1 & 1176.4150 & 3.3300 & 1.233 & A & W \\
\hline YU & 1 & 951.0000 & 20.0000 & 0.997 & A & A \\
\hline
\end{tabular}

Total Number Reported:

112

Values for elemental uranium are reported in $\mu \mathrm{g} /$ filter, $\mathrm{g}$ or $\mathrm{mL}$.

$\mathrm{pCi} / \mathrm{g}$ or $\mathrm{mL}=\mathrm{Bq} \times 0.027$

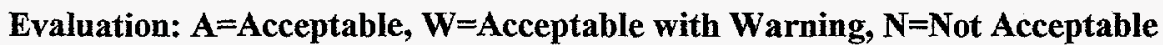

If the evaluation system is not appropriate for the types of analyses performed in your lab, apply site specific evaluation. 


\section{QAP 49 Results by Nuclide}

\begin{tabular}{lc}
\hline $\begin{array}{l}\text { Matrix: } \quad \text { SO } \\
\text { Radionuclide: }\end{array}$ & $\begin{array}{c}\text { Soil } \mathrm{Bq} / \mathrm{kg} \\
\mathrm{K} 40\end{array}$ \\
\hline EML Value: & 314.000 \\
EML Error: & 13.000 \\
\hline
\end{tabular}

Labcode Test \# $\begin{gathered}\text { Reported } \\ \text { Value }\end{gathered}$

\section{Reported Error}

\begin{tabular}{|c|c|c|c|c|}
\hline $\mathrm{AF}$ & 1 & 231.9900 & 44.7700 & 0.739 \\
\hline $\mathrm{AG}$ & 1 & 396.0000 & 74.0000 & 1.261 \\
\hline $\mathrm{AI}$ & 1 & 389.0000 & 17.7000 & 1.239 \\
\hline $\mathrm{AL}$ & 1 & 616.2400 & 0.5000 & 1.963 \\
\hline AM & 1 & 372.1800 & 38.8500 & 1.185 \\
\hline $\mathrm{AN}$ & 1 & 363.0000 & 11.0000 & 1.156 \\
\hline AT & 1 & 380.8000 & 41.1000 & 1.213 \\
\hline $\mathrm{AU}$ & 1 & 388.0000 & 24.0000 & 1.236 \\
\hline $\mathrm{BC}$ & 1 & 403.0000 & 25.2000 & 1.283 \\
\hline $\mathrm{BE}$ & 1 & 390.0000 & 80.0000 & 1.242 \\
\hline $\mathrm{BL}$ & 1 & 355.0000 & 16.0000 & 1.131 \\
\hline $\mathrm{BN}$ & 1 & 291.6700 & 3.9500 & 0.929 \\
\hline $\mathrm{BN}$ & 2 & 282.5000 & 3.9500 & 0.900 \\
\hline $\mathrm{BN}$ & 3 & 284.4600 & 3.9500 & 0.906 \\
\hline BP & 1 & 305.0000 & 7.0000 & 0.971 \\
\hline$B Q$ & 1 & 490.0000 & 5.0000 & 1.561 \\
\hline $\mathrm{BU}$ & 1 & 290.0000 & 30.0000 & 0.924 \\
\hline BX & 1 & 418.0000 & 23.7000 & 1.331 \\
\hline $\mathrm{CD}$ & 1 & 338.0000 & 10.0000 & 1.076 \\
\hline $\mathrm{CH}$ & 1 & 369.0000 & 8.0000 & 1.175 \\
\hline $\mathrm{CL}$ & 1 & 400.0000 & 36.0000 & 1.274 \\
\hline $\mathrm{CM}$ & 1 & 379.0000 & 23.0000 & 1.207 \\
\hline $\mathrm{CM}$ & 2 & 379.0000 & 23.0000 & 1.207 \\
\hline $\mathrm{CM}$ & 3 & 391.0000 & 24.0000 & 1.245 \\
\hline $\mathrm{CN}$ & 1 & 331.6000 & 31.6400 & 1.056 \\
\hline CR & 1 & 328.0000 & 58.0000 & 1.045 \\
\hline $\mathrm{CS}$ & 1 & 322.6000 & 15.1000 & 1.027 \\
\hline $\mathrm{DH}$ & 1 & 331.0000 & 27.0000 & 1.054 \\
\hline $\mathrm{EG}$ & 1 & 350.0000 & 60.0000 & 1.115 \\
\hline FG & 1 & 302.0000 & 6.4100 & 0.962 \\
\hline FL & 1 & 332.0000 & 6.0000 & 1.057 \\
\hline $\mathrm{FN}$ & 1 & 321.0000 & 34.0000 & 1.022 \\
\hline FS & 1 & 325.0000 & 20.0000 & 1.035 \\
\hline GA & 1 & 351.0000 & 59.0000 & 1.118 \\
\hline $\mathrm{GC}$ & 1 & 369.5000 & 21.2300 & 1.177 \\
\hline $\mathrm{GE}$ & 1 & 350.3160 & 63.8674 & 1.116 \\
\hline GP & 1 & 350.0000 & 30.0000 & 1.115 \\
\hline GT & 1 & 400.0000 & 80.0000 & 1.274 \\
\hline $\mathrm{HU}$ & 1 & 342.0000 & 24.0000 & 1.089 \\
\hline IA & 1 & 254.0000 & 6.0000 & 0.809 \\
\hline IA & 2 & 250.0000 & 6.0000 & 0.796 \\
\hline
\end{tabular}

QAP 48

Evaluation Evaluation

$\begin{array}{ll}A & \text { N } \\ A & \text { W }\end{array}$

W

$\mathrm{N}$

A

A

A

W

A

A

A

A

A

A

A

W

W

W

A

A

W

A

A

A

A

A

A

A

A

A

A

A

A

A

$\mathrm{N}$
$\mathrm{N}$

A

N

A

A

A

A

W

A

A

A

A

A

A

$\mathrm{N}$

A

W

A

A

W

A

A

A

A

A

A

A

A

A

A

A

A

A

A

A

A

W

A

W

Values for elemental uranium are reported in $\mu \mathrm{g} /$ filter, $\mathrm{g}$ or $\mathrm{mL}$.

$\mathrm{pCi} / \mathrm{g}$ or $\mathrm{mL}=\mathrm{Bq} \times 0.027$

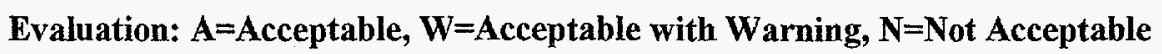

If the evaluation system is not appropriate for the types of analyses performed in your lab, apply site specific evaluation. 


\section{QAP 49 Results by Nuclide}

Matrix: SO Soil $\mathrm{Bq} / \mathrm{kg}$
Radionuclide:

\begin{tabular}{lr} 
EML Value: & 314.000 \\
EML Error: & 13.000 \\
\hline
\end{tabular}

\begin{tabular}{|c|c|c|c|c|c|c|}
\hline Labcode & Test \# & $\begin{array}{c}\text { Reported } \\
\text { Value }\end{array}$ & $\begin{array}{c}\text { Reported } \\
\text { Error }\end{array}$ & $\frac{\text { Reported }}{\text { EML }}$ & $\begin{array}{c}\text { QAP } 48 \\
\text { Evaluation }\end{array}$ & Evaluation \\
\hline$\overline{I A}$ & 3 & 246.0000 & 6.0000 & 0.783 & $\mathrm{~N}$ & W \\
\hline ID & 1 & 303.7000 & 27.1600 & 0.967 & A & $\mathrm{A}$ \\
\hline $\mathrm{IE}$ & 1 & 424.6000 & 19.2000 & 1.352 & A & $\mathrm{W}$ \\
\hline IL & 1 & 609.5000 & 90.3000 & 1.941 & $\mathrm{~N}$ & $\mathrm{~N}$ \\
\hline IN & 1 & 356.0000 & 101.0000 & 1.134 & A & A \\
\hline IS & 1 & 326.0000 & 46.9000 & 1.038 & W & A \\
\hline IT & 1 & 373.0000 & 22.0000 & 1.188 & A & A \\
\hline $\mathrm{JE}$ & 1 & 363.1100 & 58.2900 & 1.156 & A & A \\
\hline $\mathrm{KA}$ & 1 & 328.1000 & 51.3000 & 1.045 & A & A \\
\hline $\mathrm{KR}$ & 1 & 381.1000 & 49.6000 & 1.214 & A & A \\
\hline$\widetilde{\mathrm{LA}}$ & 1 & 253.0000 & 29.0000 & 0.806 & A & W \\
\hline LA & 2 & 317.0000 & 34.0000 & 1.010 & A & A \\
\hline $\mathrm{LA}$ & 3 & 333.0000 & 35.0000 & 1.061 & $\mathrm{~A}$ & A \\
\hline LB & 1 & 383.0000 & 66.0000 & 1.220 & & A \\
\hline $\mathrm{LL}$ & 1 & 242.0000 & 68.3000 & 0.771 & A & $\mathrm{N}$ \\
\hline LV & 1 & 391.0000 & 10.0000 & 1.245 & A & A \\
\hline MA & 1 & 370.0000 & 44.0000 & 1.178 & A & A \\
\hline $\mathrm{ME}$ & 1 & 280.0000 & 15.6000 & 0.892 & A & W \\
\hline $\mathrm{MH}$ & 1 & 328.4000 & 17.6600 & 1.046 & A & A \\
\hline $\mathrm{MS}$ & l & 355.0000 & 35.0000 & 1.130 & & A \\
\hline NA & 1 & 304.0000 & 12.0000 & 0.968 & A & A \\
\hline NL & 1 & 332.0000 & 52.0000 & 1.057 & A & $\mathrm{A}$ \\
\hline $\mathrm{NP}$ & 1 & 259.0000 & 4.0000 & 0.825 & & W \\
\hline NQ & 1 & 410.0000 & 47.0000 & 1.306 & & W \\
\hline NR & 1 & 317.0000 & 63.0000 & 1.010 & A & A \\
\hline $\mathrm{OB}$ & 1 & 492.0000 & 113.0000 & 1.567 & & $\mathrm{~N}$ \\
\hline $\mathrm{OC}$ & 1 & 400.0000 & 200.0000 & 1.274 & & W \\
\hline $\mathrm{OC}$ & 2 & 380.0000 & 200.0000 & 1.210 & & A \\
\hline $\mathrm{OC}$ & 3 & 400.0000 & 200.0000 & 1.274 & & W \\
\hline $\mathrm{OL}$ & 1 & 362.9000 & 26.1000 & 1.156 & A & $\mathrm{A}$ \\
\hline OT & 1 & 320.0000 & 40.0000 & 1.019 & A & A \\
\hline OU & 1 & 326.0000 & 15.2000 & 1.038 & W & A \\
\hline $\mathrm{PO}$ & 1 & 329.0000 & 20.0000 & 1.048 & A & A \\
\hline RA & 1 & 404.0000 & 42.0000 & 1.287 & W & W \\
\hline $\mathrm{RA}$ & 2 & 425.0000 & 40.0000 & 1.354 & W & W \\
\hline $\mathrm{RC}$ & 1 & 352.0000 & 19.0000 & 1.121 & A & $\mathrm{A}$ \\
\hline $\mathrm{RE}$ & 1 & 343.0000 & 32.0000 & 1.092 & A & A \\
\hline SA & 1 & 353.0000 & 16.0000 & 1.124 & A & A \\
\hline SB & 1 & 372.0000 & 57.0000 & 1.185 & & $\mathrm{~A}$ \\
\hline SK & 1 & 328.0000 & 20.0000 & 1.045 & A & A \\
\hline SN & 1 & 406.4620 & 40.5850 & 1.294 & W & W \\
\hline SR & 1 & 394.0000 & 12.0000 & 1.255 & $\mathrm{~A}$ & W \\
\hline
\end{tabular}

Values for elemental uranium are reported in $\mu \mathrm{g} /$ filter, $\mathrm{g}$ or $\mathrm{mL}$.

$\mathrm{pCi} / \mathrm{g}$ or $\mathrm{mL}=\mathrm{Bq} \times 0.027$

Evaluation: $A=A c c e p t a b l e, W=A c c e p t a b l e$ with Warning, $N=$ Not Acceptable

If the evaluation system is not appropriate for the types of analyses performed in your lab, apply site specific evaluation. 


\section{QAP 49 Results by Nuclide}

\begin{tabular}{|c|c|c|c|c|c|c|}
\hline $\begin{array}{l}\text { Matrix: SO } \\
\text { Radionuclide }\end{array}$ & $\begin{array}{l}\text { O Soil } \\
\text { e: }\end{array}$ & $\begin{array}{l}3 \mathrm{q} / \mathrm{kg} \\
40\end{array}$ & & & & \\
\hline $\begin{array}{l}\text { EML Value: } \\
\text { EML Error: }\end{array}$ & $\begin{array}{r}314 \\
13\end{array}$ & 00 & & & & \\
\hline Labcode & Test \# & $\begin{array}{l}\text { Reported } \\
\text { Value }\end{array}$ & $\begin{array}{c}\text { Reported } \\
\text { Error }\end{array}$ & $\frac{\text { Reported }}{E M L}$ & $\begin{array}{c}\text { QAP 48 } \\
\text { Evaluation }\end{array}$ & Evaluation \\
\hline SW & 1 & 427.4000 & 57.8000 & 1.361 & W & W \\
\hline TE & 1 & 296.2000 & 39.9000 & 0.943 & A & A \\
\hline $\mathrm{TI}$ & 1 & 381.0000 & 38.2000 & 1.213 & A & A \\
\hline $\mathrm{TM}$ & 1 & 345.0000 & 32.9000 & 1.099 & A & $\mathrm{A}$ \\
\hline $\mathrm{TN}$ & 1 & 2.9200 & 0.3000 & 0.009 & A & $\mathrm{N}$ \\
\hline TO & 1 & 224.0000 & 43.1000 & 0.713 & A & $\mathrm{N}$ \\
\hline$\hat{\mathrm{TP}}$ & 1 & 327.7400 & 5.2200 & 1.044 & A & A \\
\hline $\mathrm{TT}$ & 1 & 266.0000 & 5.5700 & 0.847 & W & $\mathrm{W}$ \\
\hline TW & 1 & 371.0000 & 7.0000 & 1.182 & A & A \\
\hline TX & 1 & 315.0000 & 12.0000 & 1.003 & A & A \\
\hline TY & 1 & 336.0000 & 15.0000 & 1.070 & & A \\
\hline UC & 1 & 380.2400 & 16.2900 & 1.211 & A & A \\
\hline UY & 1 & 415.0000 & 120.0000 & 1.322 & A & W \\
\hline WA & 1 & 399.0000 & 15.0000 & 1.271 & A & W \\
\hline WC & 1 & 413.0000 & 54.0000 & 1.315 & W & W \\
\hline WE & 1 & 366.0000 & 27.0000 & 1.166 & & A \\
\hline WS & 1 & 363.7100 & 18.9000 & 1.158 & A & A \\
\hline YA & 1 & 384.0600 & 8.0105 & 1.223 & A & A \\
\hline YU & 1 & 315.0000 & 10.0000 & 1.003 & A & A \\
\hline
\end{tabular}

Total Number Reported:

102

Values for elemental uranium are reported in $\mu \mathrm{g} /$ filter, $\mathrm{g}$ or $\mathrm{mL}$.

$\mathrm{pCi} / \mathrm{g}$ or $\mathrm{mL}=\mathrm{Bq} \times 0.027$

Evaluation: $A=$ Acceptable, $W=$ Acceptable with Warning, $N=$ Not Acceptable

If the evaluation system is not appropriate for the types of analyses performed in your lab, apply site specific evaluation. 


\section{QAP 49 Results by Nuclide}

\begin{tabular}{lr}
\hline Matrix: SO Soil $\mathrm{Bq} / \mathrm{k}$ \\
Radionuclide: & $\mathrm{PB} 210$ \\
\hline EML Value: & 32.000 \\
EML Error: & 3.300 \\
\hline
\end{tabular}

\begin{tabular}{lcrrrrr} 
Labcode & Test \# & $\begin{array}{c}\text { Reported } \\
\text { Value }\end{array}$ & $\begin{array}{c}\text { Reported } \\
\text { Error }\end{array}$ & $\begin{array}{c}\text { Reported } \\
\text { EML }\end{array}$ & $\begin{array}{c}\text { QAP 48 } \\
\text { Evaluation }\end{array}$ & Evaluation \\
\hline & & & & & & \\
AF & 1 & 35.1500 & 6.2300 & 1.098 & $\mathrm{~A}$ \\
AM & 1 & 72.8800 & 31.4400 & 2.277 & $\mathrm{~A}$ \\
BL & 1 & 27.7000 & 5.8000 & 0.866 & $\mathrm{~A}$ \\
BQ & 1 & 33.0000 & 10.0000 & 1.031 & $\mathrm{~A}$ \\
CS & 1 & 21.9700 & 4.6220 & 0.687 & $\mathrm{~A}$ \\
FG & 1 & 130.0000 & 58.7000 & 4.063 & $\mathrm{~A}$ \\
FS & 1 & 29.6000 & 8.4000 & 0.925 & $\mathrm{~A}$ \\
IS & 1 & 31.8000 & 32.0000 & 0.994 & $\mathrm{~A}$ \\
IT & 1 & 41.0000 & 5.5000 & 1.281 & $\mathrm{~A}$ \\
OB & 1 & 26.5000 & 139.0000 & 0.828 & $\mathrm{~A}$ \\
SW & 1 & 35.8800 & 15.7700 & 1.121 & $\mathrm{~A}$ \\
TO & 1 & 20.4000 & 2.4000 & 0.637 & 0.866 & \\
TX & 1 & 27.7000 & 9.3000 & &
\end{tabular}

Total Number Reported:

Values for elemental uranium are reported in $\mu \mathrm{g} /$ filter, $\mathrm{g}$ or $\mathrm{mL}$.

$\mathrm{pCi} / \mathrm{g}$ or $\mathrm{mL}=\mathrm{Bq} \times 0.027$

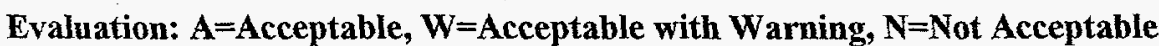

If the evaluation system is not appropriate for the types of analyses performed in your lab, apply site specific evaluation. 


\section{QAP 49 Results by Nuclide}

\begin{tabular}{|c|c|c|c|c|c|c|}
\hline \multicolumn{3}{|c|}{$\begin{array}{l}\text { Matrix: SO Soil } \underset{\mathrm{Bq} / \mathrm{kg}}{\mathrm{PB} 212} \\
\text { Radionuclide: }\end{array}$} & \multirow[b]{3}{*}{$\begin{array}{l}\text { Reported } \\
\text { Error }\end{array}$} & \multirow[b]{3}{*}{$\frac{\text { Reported }}{\text { EML }}$} & \multirow[b]{3}{*}{$\begin{array}{c}\text { QAP } 48 \\
\text { Evaluation }\end{array}$} & \multirow[b]{3}{*}{ Evalu } \\
\hline $\begin{array}{l}\text { EML Value: } \\
\text { EML Error: }\end{array}$ & \multicolumn{2}{|c|}{$\begin{array}{r}52.800 \\
3.700 \\
\end{array}$} & & & & \\
\hline Labcode & Test \# & $\begin{array}{l}\text { Reported } \\
\text { Value }\end{array}$ & & & & \\
\hline $\mathrm{AF}$ & 1 & 39.5900 & 7.4000 & 0.750 & & A \\
\hline$A G$ & 1 & 60.0000 & 11.0000 & 1.136 & & A \\
\hline $\mathrm{AI}$ & 1 & 12.6000 & 2.2600 & 0.239 & & A \\
\hline $\mathrm{AM}$ & 1 & 60.3000 & 1.4700 & 1.142 & & A \\
\hline BL & 1 & 62.1000 & 3.7000 & 1.176 & & A \\
\hline $\mathrm{BN}$ & 1 & 51.2800 & 0.6900 & 0.971 & & A \\
\hline $\mathrm{BN}$ & 2 & 49.9500 & 0.6900 & 0.946 & & A \\
\hline BN & 3 & 49.7300 & 0.6900 & 0.942 & & A \\
\hline $\mathrm{BP}$ & 1 & 50.0000 & 8.0000 & 0.947 & & A \\
\hline $\mathrm{BQ}$ & 1 & 52.0000 & 7.0000 & 0.985 & & A \\
\hline $\mathrm{CD}$ & 1 & 58.0000 & 1.0000 & 1.098 & & A \\
\hline $\mathrm{CH}$ & 1 & 55.5000 & 0.9400 & 1.051 & & A \\
\hline $\mathrm{CL}$ & 1 & 61.5000 & 3.1000 & 1.165 & & A \\
\hline CR & 1 & 63.0000 & 6.0000 & 1.193 & & A \\
\hline $\mathrm{CS}$ & 1 & 54.1700 & 2.7840 & 1.026 & & A \\
\hline $\mathrm{FG}$ & 1 & 69.0400 & 1.2000 & 1.308 & & A \\
\hline GA & 1 & 53.7000 & 7.4000 & 1.017 & & A \\
\hline $\mathrm{GC}$ & 1 & 48.1000 & 2.4000 . & 0.911 & & A \\
\hline GE & 1 & 56.5340 & 9.2900 & 1.071 & & A \\
\hline $\mathrm{HU}$ & 1 & 52.6000 & 1.5000 & 0.996 & & A \\
\hline ID & 1 & 57.2800 & 3.0700 & 1.085 & & A \\
\hline IE & 1 & 46.7500 & 1.6500 & 0.885 & & A \\
\hline$\overline{\mathrm{IL}}$ & 1 & 64.0000 & 3.9000 & 1.212 & & A \\
\hline $\mathrm{IN}$ & 1 & 56.9000 & 6.0000 & 1.078 & & A \\
\hline IS & 1 & 50.1000 & 10.0000 & 0.949 & & A \\
\hline IT & 1 & 61.0000 & 3.0000 & 1.155 & & A \\
\hline $\mathrm{JE}$ & 1 & 42.6100 & 3.3700 & 0.807 & & A \\
\hline LV & 1 & 70.3000 & 4.6000 & 1.331 & & A \\
\hline MH & 1 & 50.6800 & 3.7000 & 0.960 & & A \\
\hline NA & 1 & 49.8000 & 1.5000 & 0.943 & & A \\
\hline NL & 1 & 55.2000 & 9.5000 & 1.045 & & A \\
\hline NQ & 1 & 68.1000 & 7.8000 & 1.290 & & A \\
\hline $\mathrm{OB}$ & 1 & 107.0000 & 23.4000 & 2.027 & & A \\
\hline $\mathrm{OC}$ & 1 & 58.0000 & 10.0000 & 1.098 & & A \\
\hline $\mathrm{OC}$ & 2 & 63.0000 & 10.0000 & 1.193 & & A \\
\hline $\mathrm{OC}$ & 3 & 54.0000 & 10.0000 & 1.023 & & A \\
\hline OT & 1 & 47.0000 & 11.0000 & 0.890 & & $\mathrm{~A}$ \\
\hline OU & 1 & 49.1000 & 6.2000 & 0.930 & & A \\
\hline $\mathrm{RA}$ & 1 & 53.0000 & 2.3000 & 1.004 & & A \\
\hline $\mathrm{RA}$ & 2 & 53.1000 & 1.9000 & 1.006 & & A \\
\hline SK & 1 & 61.0000 & 4.0000 & 1.155 & & A \\
\hline
\end{tabular}

Values for elemental uranium are reported in $\mu \mathrm{g}$ filter, $\mathrm{g}$ or $\mathrm{mL}$.

$\mathrm{pCi} / \mathrm{g}$ or $\mathrm{mL}=\mathrm{Bq} \times 0.027$

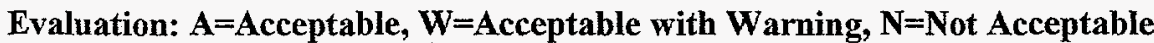

If the evaluation system is not appropriate for the types of analyses performed in your lab, apply site specific evaluation. 


\section{QAP 49 Results by Nuclide}

\begin{tabular}{lr}
\hline Matrix: $\begin{array}{l}\text { SO } \\
\text { Radionuclide: }\end{array}$ & $\begin{array}{r}\mathrm{Bq} / \\
\mathrm{PB} 2\end{array}$ \\
\hline EML Value: & 52.800 \\
EML Error: & 3.700 \\
\hline
\end{tabular}

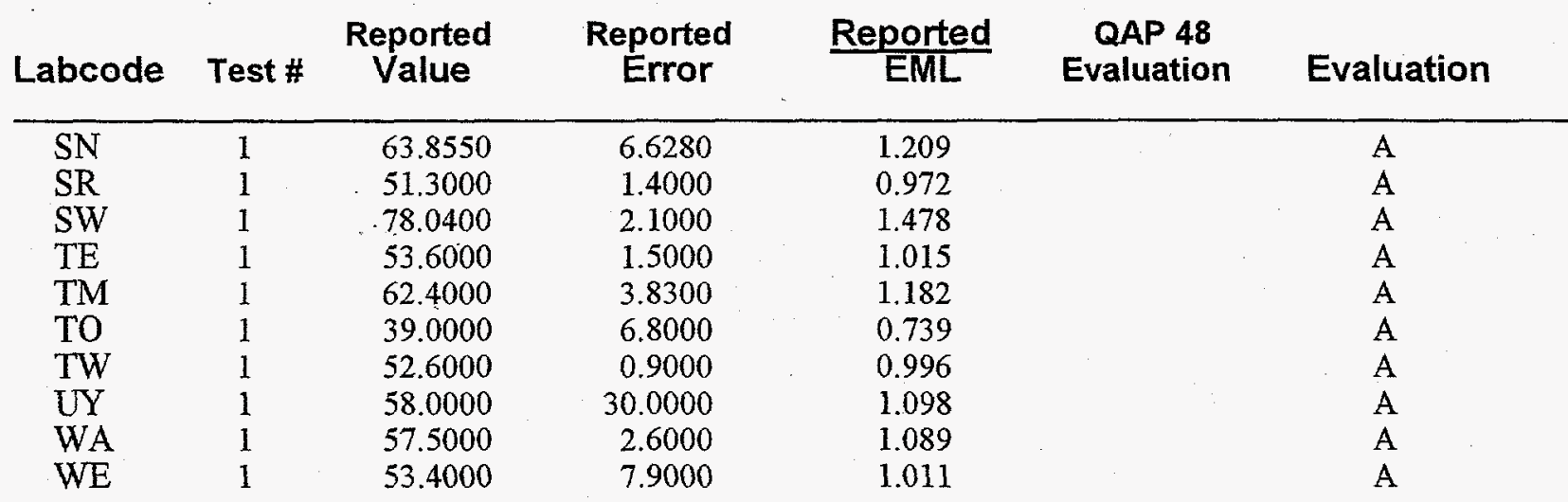

Total Number Reported:

Values for elemental uranium are reported in $\mu \mathrm{g} /$ filter, $\mathrm{g}$ or $\mathrm{mL}$.

$\mathrm{pCi} / \mathrm{g}$ or $\mathrm{mL}=\mathrm{Bq} \times 0.027$

Evaluation: $A=$ Acceptable, $W=$ Acceptable with Warning, $N=$ Not Acceptable

If the evaluation system is not appropriate for the types of analyses performed in your lab, apply site specific evaluation. 


\section{QAP 49 Results by Nuclide}

\begin{tabular}{|c|c|c|c|c|c|c|}
\hline \multicolumn{3}{|c|}{$\begin{array}{l}\text { Matrix: SO Soil } \mathrm{Bq} / \mathrm{kg} \\
\text { Radionuclide: }\end{array}$} & \multirow[b]{3}{*}{$\begin{array}{l}\text { Reported } \\
\text { Error }\end{array}$} & \multirow[b]{3}{*}{$\frac{\text { Reported }}{E M L}$} & \multirow[b]{3}{*}{$\begin{array}{c}\text { QAP } 48 \\
\text { Evaluation }\end{array}$} & \multirow[b]{3}{*}{ Evaluation } \\
\hline $\begin{array}{l}\text { EML Value: } \\
\text { EML Error: }\end{array}$ & \multicolumn{2}{|c|}{$\begin{array}{r}29.100 \\
1.200 \\
\end{array}$} & & & & \\
\hline Labcode & Test \# & $\begin{array}{l}\text { Reported } \\
\text { Value }\end{array}$ & & & & \\
\hline $\mathrm{AF}$ & 1 & 29.6000 & 6.2900 & 1.017 & & A \\
\hline $\mathrm{AG}$ & 1 & 35.0000 & 7.4000 & 1.203 & & A \\
\hline $\mathrm{AI}$ & 1 & 37.0000 & 2.6300 & 1.271 & & A \\
\hline $\mathrm{AM}$ & 1 & 36.2600 & 3.7000 & 1.246 & & A \\
\hline BL & 1 & 35.5000 & 2.3000 & 1.220 & & A \\
\hline $\mathrm{BN}$ & 1 & 26.8500 & 1.7900 & 0.923 & & A \\
\hline $\mathrm{BN}$ & 2 & 27.3800 & 1.7900 & 0.941 & & A \\
\hline $\mathrm{BN}$ & 3 & 30.9000 & 1.7900 & 1.062 & & A \\
\hline $\mathrm{BP}$ & 1 & 29.0000 & 3.0000 & 0.997 & & A \\
\hline$C D$ & 1 & 30.0000 & 2.0000 & 1.031 & & A \\
\hline $\mathrm{CH}$ & 1 & 28.8000 & 1.6000 & 0.990 & & A \\
\hline $\mathrm{CL}$ & 1 & 42.9000 & 7.3000 & 1.474 & & A \\
\hline CR & 1 & 41.0000 & 7.0000 & 1.409 & & A \\
\hline $\mathrm{CS}$ & 1 & 28.9500 & 1.0310 & 0.995 & & A \\
\hline FG & $i$ & 41.4100 & 2.0500 & 1.423 & & $\mathrm{~A}$ \\
\hline FS & 1 & 25.0000 & 4.8000 & 0.859 & & A \\
\hline GA & 1 & 36.8000 & 10.3000 & 1.265 & & A \\
\hline GE & 1 & 32.5600 & 8.9840 & 1.119 & & A \\
\hline $\mathrm{HU}$ & 1 & 31.2000 & 1.0000 & 1.072 & & A \\
\hline ID & 1 & 35.4700 & 2.5100 & 1.219 & & A \\
\hline IE & 1 & 33.2000 & 2.4300 & 1.141 & & A \\
\hline IL & 1 & 49.1000 & 6.5000 & 1.687 & & A \\
\hline $\mathrm{IN}$ & 1 & 33.4000 & 9.8000 & 1.148 & & A \\
\hline IS & 1 & 28.5000 & 7.4000 & 0.979 & & A \\
\hline IT & 1 & 35.0000 & 1.5000 & 1.203 & & A \\
\hline $\mathrm{JE}$ & 1 & 28.3900 & 5.5000 & 0.976 & & A \\
\hline LV & 1 & 43.7000 & 2.5000 & 1.502 & & A \\
\hline $\mathrm{ME}$ & 1 & 13.7000 & 1.0800 & 0.471 & & A \\
\hline $\mathrm{MH}$ & 1 & 29.0400 & 2.1700 & 0.998 & & A \\
\hline NA & 1 & 27.4000 & 1.7000 & 0.942 & & A \\
\hline NL & 1 & 31.5000 & 7.2000 & 1.082 & & A \\
\hline NQ & 1 & 37.8000 & 4.8000 & 1.299 & & A \\
\hline $\mathrm{OB}$ & 1 & 6.7500 & 19.0000 & 0.232 & & A \\
\hline $\mathrm{OC}$ & 1 & 28.0000 & 10.0000 & 0.962 & & $\mathrm{~A}$ \\
\hline $\mathrm{OC}$ & 2 & 31.0000 & 10.0000 & 1.065 & & A \\
\hline $\mathrm{OC}$ & 3 & 27.0000 & 10.0000 & 0.928 & & A \\
\hline OT & 1 & 37.0000 & 14.0000 & 1.271 & & A \\
\hline OU & 1 & 31.3000 & 1.7000 & 1.076 & & $\mathrm{~A}$ \\
\hline RA & 1 & 28.7000 & 2.9000 & 0.986 & & A \\
\hline RA & 2 & 25.9000 & 6.0000 & 0.890 & & $\mathrm{~A}$ \\
\hline SK & 1 & 38.0000 & 1.0000 & 1.306 & & A \\
\hline
\end{tabular}

Values for elemental uranium are reported in $\mu \mathrm{g} /$ filter, $g$ or $\mathbf{~ m L}$.

$\mathrm{pCi} / \mathrm{g}$ or $\mathrm{mL}=\mathrm{Bq} \times 0.027$

Evaluation: $A=$ Acceptable, $W=$ Acceptable with Warning, $N=$ Not Acceptable

If the evaluation system is not appropriate for the types of analyses performed in your lab, apply site specific evaluation. 


\section{QAP 49 Results by Nuclide}

Matrix: SO Soil $\mathrm{Bq} / \mathrm{kg}$

Radionuclide:

PB214

EML Value: $\quad 29.100$

EML Error: $\quad 1.200$

\begin{tabular}{|c|c|c|c|c|c|c|}
\hline Labcode & Test \# & $\begin{array}{l}\text { Reported } \\
\text { Value }\end{array}$ & $\begin{array}{c}\text { Reported } \\
\text { Error }\end{array}$ & $\frac{\text { Reported }}{E M L}$ & $\begin{array}{c}\text { QAP 48 } \\
\text { Evaluation }\end{array}$ & Evaluation \\
\hline SN & 1 & 35.5780 & 5.6380 & 1.223 & & A \\
\hline SR & 1 & 31.3000 & 1.4000 & 1.076 & & A \\
\hline SW & 1 & 42.1300 & 8.5100 & 1.448 & & $\mathrm{~A}$ \\
\hline TE & 1 & 31.0000 & 5.9000 & 1.065 & & A \\
\hline $\mathrm{TM}$ & 1 & 35.7000 & 5.0600 & 1.227 & & A \\
\hline TO & 1 & 24.7000 & 11.8000 & 0.849 & & A \\
\hline TW & 1 & 29.0000 & 1.0000 & 0.997 & & $\mathrm{~A}$ \\
\hline $\mathrm{TX}$ & 1 & 29.3000 & 2.1000 & 1.007 & & A \\
\hline UY & 1 & 34.0000 & 15.0000 & 1.168 & & A \\
\hline WA & 1 & 30.2000 & 2.2000 & 1.038 & & A \\
\hline WE & 1 & 39.4000 & 10.0000 & 1.354 & & A \\
\hline
\end{tabular}

Total Number Reported:

52

Values for elemental uranium are reported in $\mu \mathrm{g} /$ filter, $\mathrm{g}$ or $\mathrm{mL}$.

$\mathrm{pCi} / \mathrm{g}$ or $\mathrm{mL}=\mathrm{Bq} \times 0.027$

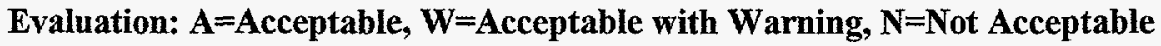

If the evaluation system is not appropriate for the types of analyses performed in your lab, apply site specific evaluation. 


\section{QAP 49 Results by Nuclide}

\begin{tabular}{lr}
\hline $\begin{array}{l}\text { Matrix: SO Soil } \mathrm{Bq} / \\
\text { Radionuclide: }\end{array}$ & PU2 \\
\hline EML Value: & 0.530 \\
EML Error: & 0.270 \\
\hline
\end{tabular}

\begin{tabular}{|c|c|c|c|c|c|c|}
\hline Labcode & Test \# & $\begin{array}{l}\text { Reported } \\
\text { Value }\end{array}$ & $\begin{array}{c}\text { Reported } \\
\text { Error }\end{array}$ & $\frac{\text { Reported }}{E M L}$ & $\begin{array}{c}\text { QAP } 48 \\
\text { Evaluation }\end{array}$ & Evalu \\
\hline $\mathrm{EG}$ & 1 & 0.4500 & 0.0800 & 0.849 & & A \\
\hline IT & 1 & 0.7700 & 0.0800 & 1.453 & & W \\
\hline WA & 1 & 0.5500 & 0.3500 & 1.038 & & \\
\hline
\end{tabular}

Total Number Reported: $\quad 3$

Values for elemental uranium are reported in $\mu \mathrm{g} /$ filter, $\mathrm{g}$ or $\mathrm{mL}$.

$\mathrm{pCi} / \mathrm{g}$ or $\mathrm{mL}=\mathrm{Bq} \times 0.027$

Evaluation: $A=A c c e p t a b l e, W=A c c e p t a b l e$ with Warning, $N=$ Not Acceptable

If the evaluation system is not appropriate for the types of analyses performed in your lab, apply site specific evaluation. 


\section{QAP 49 Results by Nuclide}

Matrix: SO Soil $\mathrm{Bq} / \mathrm{kg}$

Radionuclide:

PU239

\begin{tabular}{|c|}
\hline EML Value: \\
\hline EML Error: \\
\hline
\end{tabular}

Reported Value

Reported Error

\section{Reported} EML

0.763

2.5900

2.1000

1.8600

2.2200

0.6000

1.9000

1.3000

0.5000

0.6000

2.5000

0.3000

0.8000

0.8000

0.6600

2.7000

1.1000

0.0010

1.0300

1.3000

0.7900

2.5536

5.0000

0.6000

0.6500

2.7000

5.2260

1.2000

0.0800

0.2000

0.5000

0.5000

0.5000

1.5700

0.7400

1.5400

1.0300

2.2000

3.1600

0.3000

0.4000

0.4000
1.024

1.253

0.960

1.042

0.947

1.016

1.184

1.001

0.963

1.070

0.840

1.008

1.039

1.001

1.047

0.001

0.993

0.722

0.840

0.930

1.016

0.382

0.957

0.992

1.304

1.108

1.051

0.894

0.963

1.001

0.993

1.008

0.754

0.978

1.034

0.932

1.036

0.787

1.016

0.985
QAP 48

Evaluation Evaluation

\begin{tabular}{lll} 
NM & 1 & 10.3000 \\
\hline & 2 & 13.3000
\end{tabular}

NM $\quad 3 \quad 12.9000$

Values for elemental uranium are reported in $\mu \mathrm{g} /$ filter, $\mathrm{g}$ or $\mathrm{mL}$.

A

A

A

A

A

A

A

A

W

A

A

A.

A

A

A

A

A

A

W

A

A

A

A

A

A

A

$\mathrm{N}$

A

A

W

A

A

A
W

A

A

A

A

A

A

A

A

A

W

A

A

A

A

N

A

W

W

A

A

$\mathrm{N}$

A

A

W

A

A

A

A

A

A

A

W

A

A

A

A

A

A

Evaluation: $A=A c c e p t a b l e, ~ W=A c c e p t a b l e$ with Warning, $N=$ Not Acceptable

If the evaluation system is not appropriate for the types of analyses performed in your lab, apply site specific evaluation. 


\section{QAP 49 Results by Nuclide}

Matrix: SO Soil $\mathrm{Bq} / \mathrm{kg}$

Radionuclide:

PU239

\begin{tabular}{lr} 
EML Value: & 13.090 \\
EML Error: & 0.570 \\
\hline
\end{tabular}

\begin{tabular}{|c|c|c|c|c|c|c|}
\hline Labcode & Test \# & $\begin{array}{l}\text { Reported } \\
\text { Value }\end{array}$ & $\begin{array}{c}\text { Reported } \\
\text { Error }\end{array}$ & $\frac{\text { Reported }}{E M L}$ & $\begin{array}{c}\text { QAP 48 } \\
\text { Evaluation }\end{array}$ & Evaluation \\
\hline NQ & 1 & 11.3000 & 1.3000 & 0.863 & & W \\
\hline $\mathrm{NZ}$ & 1 & 14.4000 & 1.4000 & 1.100 & & A \\
\hline $\mathrm{NZ}$ & 2 & 12.7000 & 1.1000 & 0.970 & & A \\
\hline $\mathrm{OT}$ & 1 & 14.0000 & 2.0000 & 1.070 & A & $\mathrm{A}$ \\
\hline RA & 1 & 16.7000 & 2.5000 & 1.276 & W & W \\
\hline RE & 1 & 14.1000 & 1.7000 & 1.077 & A & A \\
\hline SN & 1 & 13.4380 & 4.2930 & 1.027 & A & A \\
\hline SW & 1 & 2.8100 & 0.5200 & 0.215 & $\mathbf{N}$ & $\mathrm{N}$ \\
\hline TI & 1 & 12.0000 & 2.0000 & 0.917 & A & A \\
\hline $\mathrm{TM}$ & 1 & 14.4000 & 1.4200 & 1.100 & A & A \\
\hline TN & 1 & 12.6000 & 1.3000 & 0.963 & W & A \\
\hline TO & $i$ & 13.9000 & 4.2000 & 1.062 & $\mathrm{~N}$ & $\mathrm{~A}$ \\
\hline TX & 1 & 12.3000 & 0.5000 & 0.940 & A & A \\
\hline UP & 1 & 13.9000 & 4.0300 & 1.062 & A & A \\
\hline UY & 1 & 12.7000 & 1.4000 & 0.970 & A & A \\
\hline WA & 1 & 13.5000 & 1.0000 & 1.031 & A & A \\
\hline WC & 1 & 13.0000 & 2.3000 & 0.993 & A & A \\
\hline YA & 1 & 13.8577 & 0.4329 & 1.059 & A & A \\
\hline
\end{tabular}

Total Number Reported:

Values for elemental uranium are reported in $\mu \mathrm{g} /$ filter, $\mathrm{g}$ or $\mathrm{mL}$.

pCi/g or $\mathrm{mL}=\mathrm{Bq} \times 0.027$

Evaluation: A=Acceptable, $W=A c c e p t a b l e$ with Warning, $N=$ Not Acceptable

If the evaluation system is not appropriate for the types of analyses performed in your lab, apply site specific evaluation. 


\section{QAP 49 Results by Nuclide}

Matrix: SO Soil $\mathrm{Bq} / \mathrm{kg}$

Radionuclide:

RA226

\begin{tabular}{lr} 
EML Value: $\quad 29.000$ \\
EML Error: $\quad 1.000$ \\
\hline
\end{tabular}

Labcode Test \# $\begin{gathered}\text { Reported } \\ \text { Value }\end{gathered}$

\section{Reported Error}

QAP 48

Evaluation
Evaluation

\begin{tabular}{|c|c|c|c|c|}
\hline $\mathrm{AF}$ & 1 & 113.2200 & 96.2000 & 3.904 \\
\hline AI & 1 & 174.0000 & 33.6000 & 6.000 \\
\hline AL & 1 & 308.3400 & 3.4000 & 10.632 \\
\hline $\mathrm{AM}$ & 1 & 54.7500 & 12.2100 & 1.888 \\
\hline BL & 1 & 44.4000 & 3.3000 & 1.531 \\
\hline$B Q$ & 1 & 39.0000 & $2: 0000$ & 1.345 \\
\hline $\mathrm{BU}$ & 1 & 34.0000 & 2.0000 & 1.172 \\
\hline $\mathrm{BX}$ & 1 & 222.0000 & 27.0000 & 7.655 \\
\hline $\mathrm{CH}$ & 1 & 25.9000 & 1.3000 & 0.893 \\
\hline CL & 1 & 331.0000 & 66.3000 & 11.414 \\
\hline $\mathrm{CM}$ & 1 & 155.0000 & 13.5000 & 5.345 \\
\hline $\mathrm{CM}$ & 2 & 156.0000 & 13.7000 & 5.379 \\
\hline $\mathrm{CM}$ & 3 & 154.0000 & 13.6000 & 5.310 \\
\hline CR & 1 & 152.0000 & 37.0000 & 5.241 \\
\hline $\mathrm{CS}$ & 1 & 69.8500 & 4.4770 & 2.409 \\
\hline FG & 1 & 204.1000 & 15.3000 & 7.038 \\
\hline GC & 1 & 212.6000 & 56.1000 & 7.331 \\
\hline GE & 1 & 29.9811 & 11.3800 & 1.034 \\
\hline IA & 1 & 21.5000 & 1.6000 & 0.741 \\
\hline IA & 2 & 23.7000 & 1.5000 & 0.817 \\
\hline IA & 3 & 21.3000 & 1.5000 & 0.734 \\
\hline IL & 1 & 62.7000 & 8.3000 & 2.162 \\
\hline IS & 1 & 103.6000 & 50.4000 & 3.572 \\
\hline IT & 1 & 32.0000 & 3.0000 & 1.103 \\
\hline LL & 1 & 26.3000 & 20.5000 & 0.907 \\
\hline NA & 1 & 35.0000 & 1.5000 & 1.207 \\
\hline NQ & 1 & 33.9000 & 1.4000 & 1.169 \\
\hline $\mathrm{OB}$ & 1 & 41.8000 & 12.4000 & 1.441 \\
\hline $\mathrm{OC}$ & 1 & 25.0000 & 10.0000 & 0.862 \\
\hline $\mathrm{OC}$ & 2 & 28.0000 & 10.0000 & 0.966 \\
\hline $\mathrm{OC}$ & 3 & 21.0000 & 10.0000 & 0.724 \\
\hline OT & 1 & 37.0000 & 14.0000 & 1.276 \\
\hline RA & 1 & 27.3000 & 3.9000 & 0.941 \\
\hline RA & 2 & 29.0000 & 4.2000 & 1.000 \\
\hline $\mathrm{RE}$ & 1 & 28.4000 & 3.4000 & 0.979 \\
\hline SK & 1 & 38.0000 & 4.0000 & 1.310 \\
\hline SW & 1 & 43.9600 & 2.5000 & 1.516 \\
\hline TE & 1 & 115.3000 & 2.2000 & 3.976 \\
\hline TM & 1 & 31.0000 & 4.1100 & 1.069 \\
\hline $\mathrm{TN}$ & 1 & 0.2000 & 0.0400 & 0.007 \\
\hline TO & 1 & 23.8000 & 8.6000 & 0.821 \\
\hline
\end{tabular}

Values for elemental uranium are reported in $\mu \mathrm{g} /$ filter, $\mathrm{g}$ or $\mathrm{mL}$. $\mathrm{pCi} / \mathrm{g}$ or $\mathrm{mL}=\mathrm{Bq} \times 0.027$ Evaluation: $A=A c c e p t a b l e, W=A c c e p t a b l e$ with $W$ arning, $N=$ Not Acceptable

If the evaluation system is not appropriate for the types of analyses performed in your lab, apply site specific evaluation. 


\section{QAP 49 Results by Nuclide}

Matrix: SO Soil $\mathrm{Bq} / \mathrm{kg}$

Radionuclide: $\quad$ RA226

EML Value: $\quad 29.000$

EML Error: $\quad 1.000$

\begin{tabular}{|c|c|c|c|c|c|c|}
\hline Labcode & Test \# & $\begin{array}{l}\text { Reported } \\
\text { Value }\end{array}$ & $\begin{array}{c}\text { Reported } \\
\text { Error }\end{array}$ & $\frac{\text { Reported }}{\text { EML }}$ & $\begin{array}{c}\text { QAP 48 } \\
\text { Evaluation }\end{array}$ & Evaluation \\
\hline $\mathrm{TX}$ & 1 & 44.3000 & 3.5000 & 1.528 & & A \\
\hline WA & 1 & 135.0000 & 17.0000 & 4.655 & & A \\
\hline WE & 1 & 34.4000 & 17.0000 & 1.186 & & $\mathrm{~A}$ \\
\hline
\end{tabular}

Total Number Reported: $\quad 44$

Values for elemental uranium are reported in $\mu \mathrm{g} /$ filter, $\mathrm{g}$ or $\mathrm{mL}$.

$\mathrm{pCi} / \mathrm{g}$ or $\mathrm{mL}=\mathrm{Bq} \times 0.027$

Evaluation: $A=$ Acceptable, $W=$ Acceptable with Warning, $N=$ Not Acceptable

If the evaluation system is not appropriate for the types of analyses performed in your lab, apply site specific evaluation. 


\section{QAP 49 Results by Nuclide}

December, 1998

\begin{tabular}{|c|c|c|c|c|c|c|}
\hline \multicolumn{3}{|c|}{$\begin{array}{lcc}\text { Matrix: SO Soil } & \mathrm{Bq} / \mathrm{kg} \\
\text { Radionuclide: } & \text { SR90 }\end{array}$} & \multirow[b]{3}{*}{$\begin{array}{l}\text { Reported } \\
\text { Error }\end{array}$} & \multirow[b]{3}{*}{$\frac{\text { Reported }}{\text { EML }}$} & \multirow[b]{3}{*}{$\begin{array}{c}\text { QAP 48 } \\
\text { Evaluation }\end{array}$} & \multirow[b]{3}{*}{ Evaluation } \\
\hline $\begin{array}{l}\text { EML Value: } \\
\text { EML Error: }\end{array}$ & \multicolumn{2}{|c|}{$\begin{array}{r}39.630 \\
0.003\end{array}$} & & & & \\
\hline Labcode & Test \# & $\begin{array}{l}\text { Reported } \\
\text { Value }\end{array}$ & & & & \\
\hline $\mathrm{AC}$ & 1 & 39.6000 & 2.6000 & 0.999 & & A \\
\hline $\mathrm{AF}$ & 1 & 48.1000 & 18.5000 & 1.214 & $N$ & $\mathrm{~A}$ \\
\hline $\mathrm{AG}$ & 1 & 45.3000 & 9.3000 & 1.143 & A & A \\
\hline $\mathrm{AM}$ & 1 & 54.7500 & 8.5100 & 1.382 & & A \\
\hline $\mathrm{AN}$ & 1 & 44.8000 & 0.9000 & 1.130 & A & $\mathrm{A}$ \\
\hline $\mathrm{AU}$ & 1 & 49.0000 & 7.0000 & 1.236 & $\mathrm{~A}$ & $\mathrm{~A}$ \\
\hline $\mathrm{BA}$ & 1 & 24.4000 & 2.3300 & 0.616 & & $\mathrm{~W}$ \\
\hline$B C$ & 1 & 27.2000 & 2.5000 & 0.686 & & W \\
\hline $\mathrm{BE}$ & 1 & 41.8000 & 4.5000 & 1.055 & A & A \\
\hline $\mathrm{BL}$ & 1 & 34.0000 & 4.4000 & 0.858 & $\mathrm{~A}$ & $\mathrm{~A}$ \\
\hline $\mathrm{BL}$ & 2 & 39.7000 & 4.9000 & 1.002 & A & $\mathrm{A}$ \\
\hline$B M$ & 1 & 40.6000 & 2.7000 & 1.024 & A & A \\
\hline $\mathrm{BP}$ & 1 & 88.0000 & 16.0000 & 2.221 & W & $\mathrm{W}$ \\
\hline $\mathrm{BX}$ & 1 & 26.5000 & 2.5000 & 0.669 & & W \\
\hline $\mathrm{CH}$ & 1 & 46.2000 & 4.0000 & 1.166 & & $\mathrm{~A}$ \\
\hline$\overline{C L}$ & 1 & 26.3000 & 12.5000 & 0.664 & W & W \\
\hline$\overline{E G}$ & 1 & 48.6000 & 2.1000 & 1.226 & $\mathrm{~A}$ & $\mathrm{~A}$ \\
\hline $\mathrm{GE}$ & 1 & 32.9300 & 4.1861 & 0.831 & A & A \\
\hline GP & 1 & 19.0000 & 7.0000 & 0.479 & & $\mathrm{~N}$ \\
\hline GT & 1 & 44.0000 & 20.0000 & 1.110 & & $\mathrm{~A}$ \\
\hline ID & 1 & 48.6300 & 4.1100 & 1.227 & A & A \\
\hline IS & 1 & 51.0600 & 17.3900 & 1.288 & A & A \\
\hline IT & 1 & 43.0000 & 2.4000 & 1.085 & A & $A$ \\
\hline $\mathrm{KA}$ & 1 & 43.3000 & 1.9000 & 1.093 & A & A \\
\hline $\mathrm{KR}$ & 1 & 35.7000 & 0.7000 & 0.901 & A & A \\
\hline $\mathrm{NZ}$ & 1 & 41.5000 & 3.6000 & 1.047 & & $\mathrm{~A}$ \\
\hline $\mathrm{NZ}$ & 2 & 40.9000 & 3.5000 & 1.032 & & A \\
\hline OT & 1 & 31.0000 & 5.0000 & 0.782 & A & $\mathrm{A}$ \\
\hline $\mathrm{OU}$ & $\mathrm{l}$ & 37.6000 & 9.2000 & 0.949 & & A \\
\hline RA & 1 & 45.0000 & 10.0000 & 1.136 & A & A \\
\hline$R E$ & 1 & 47.3000 & 3.5000 & 1.194 & A & A \\
\hline SR & 1 & 50.4000 & 19.2000 & 1.272 & & $\mathrm{~A}$ \\
\hline SW & 1 & 20.1900 & 14.1000 & 0.509 & $\mathrm{~N}$ & $\mathrm{~N}$ \\
\hline $\mathrm{TE}$ & $i$ & 37.4000 & 1.9000 & 0.944 & A & A \\
\hline $\mathrm{TI}$ & 1 & 38.0000 & 4.0000 & 0.959 & A & A \\
\hline $\mathrm{TM}$ & 1 & 42.9000 & 10.2000 & 1.083 & $\mathrm{~W}$ & $\mathrm{~A}$ \\
\hline $\mathrm{TN}$ & 1 & 41.0000 & 4.0000 & 1.035 & $\mathrm{~N}$ & A \\
\hline TO & 1 & 37.8000 & 3.0000 & 0.954 & A & A \\
\hline $\mathrm{TP}$ & 1 & 68.4000 & 2.3400 & 1.726 & A & W \\
\hline TX & 1 & 47.1000 & 9.7000 & 1.188 & A & $\mathrm{A}$ \\
\hline UP & 1 & 38.8500 & 10.7300 & 0.980 & & A \\
\hline
\end{tabular}

Values for elemental uranium are reported in $\mu \mathrm{g}$ /filter, $\mathrm{g}$ or $\mathrm{mL}$.

$\mathrm{pCi} / \mathrm{g}$ or $\mathrm{mL}=\mathrm{Bq} \times 0.027$

Evaluation: $A=A c c e p t a b l e, W=A c c e p t a b l e$ with Warning, $N=$ Not Acceptable

If the evaluation system is not appropriate for the types of analyses performed in your lab, apply site specific evaluation. 


\section{QAP 49 Results by Nuclide}

Matrix: SO Soil $\mathrm{Bq} / \mathrm{kg}$

Radionuclide: $\quad$ SR90

EML Value: $\quad 39.630$
EML Error: $\quad 0.003$

\begin{tabular}{ccccccc} 
Labcode & Test \# & $\begin{array}{c}\text { Reported } \\
\text { Value }\end{array}$ & $\begin{array}{c}\text { Reported } \\
\text { Error }\end{array}$ & $\begin{array}{c}\text { Reported } \\
\text { EML }\end{array}$ & $\begin{array}{c}\text { QAP 48 } \\
\text { Evaluation }\end{array}$ & Evaluation \\
\hline UY & 1 & 43.6000 & 3.5000 & 1.100 & $\mathrm{~A}$ & $\mathrm{~A}$ \\
WA & 1 & 46.1000 & 3.9000 & 1.163 & $\mathrm{~A}$ & $\mathrm{~A}$ \\
WC & 1 & 61.0000 & 10.0000 & 1.539 & $\mathrm{~N}$ & $\mathrm{~A}$ \\
WE & 1 & 40.2000 & 30.0000 & 1.014 & & $\mathrm{~A}$ \\
YA & 1 & 51.6027 & 3.0833 & 1.302 & W & $\mathrm{A}$
\end{tabular}

Total Number Reported: $\quad 46$

Values for elemental uranium are reported in $\mu \mathrm{g} /$ filter, $\mathrm{g}$ or $\mathrm{mL}$.

$\mathrm{pCi} / \mathrm{g}$ or $\mathrm{mL}=\mathrm{Bq} \times 0.027$

Evaluation: $A=A c c e p t a b l e, W=A c c e p t a b l e$ with Warning, $N=$ Not Acceptable

If the evaluation system is not appropriate for the types of analyses performed in your lab, apply site specific evaluation. 


\section{QAP 49 Results by Nuclide}

Matrix: SO Soil $\mathrm{Bq} / \mathrm{kg}$

Radionuclide:

TH228

EML Value: $\quad 52.700$

EML Error: $\quad 4.000$

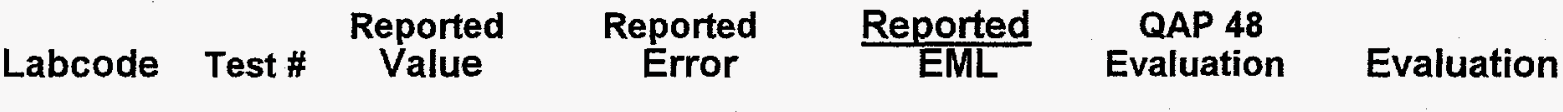

\begin{tabular}{|c|c|c|c|c|}
\hline $\mathrm{AF}$ & 1 & 103.2300 & 49.5800 & 1.959 \\
\hline $\mathrm{AG}$ & 1 & 59.0000 & 32.0000 & 1.120 \\
\hline $\mathrm{AI}$ & 1 & 49.7000 & 1.8300 & 0.943 \\
\hline $\mathrm{AM}$ & 1 & 29.5100 & 2.6000 & 0.560 \\
\hline $\mathrm{AN}$ & 1 & 55.8000 & 4.0000 & 1.059 \\
\hline BL & 1 & 60.3000 & 2.2000 & 1.144 \\
\hline$B Q$ & 1 & 53.0000 & 3.0000 & 1.006 \\
\hline $\mathrm{CH}$ & 1 & 54.2000 & 3.4000 & 1.028 \\
\hline $\mathrm{CL}$ & 1 & 58.2000 & 5.8000 & 1.104 \\
\hline FG & 1 & 32.6400 & 5.6000 & 0.619 \\
\hline GE & 1 & 53.6000 & 14.6000 & 1.017 \\
\hline IS & 1 & 57.8000 & 20.2000 & 1.097 \\
\hline IS & 2 & 65.4000 & 23.4000 & 1.241 \\
\hline IS & 3 & 66.3000 & 22.3000 & 1.258 \\
\hline IT & 1 & 57.0000 & 7.0000 & 1.082 \\
\hline LL & 1 & 39.9000 & 6.5400 & 0.757 \\
\hline ME & 1 & 115.0000 & 18.4000 & 2.182 \\
\hline $\mathrm{NA}$ & 1 & 55.0000 & 8.0000 & 1.044 \\
\hline NL & 1 & 55.2000 & 9.5000 & 1.047 \\
\hline OC & 1 & 40.0000 & 15.0000 & 0.759 \\
\hline OC & 2 & 37.0000 & 15.0000 & 0.702 \\
\hline $\mathrm{OC}$ & 3 & 42.0000 & 15.0000 & 0.797 \\
\hline OT & 1 & 21.0000 & 1.0000 & 0.398 \\
\hline OU & 1 & 121.0000 & 4.0000 & 2.296 \\
\hline $\mathrm{RA}$ & 1 & 47.0000 & 7.1000 & 0.892 \\
\hline RA & 2 & 50.3000 & 6.2000 & 0.954 \\
\hline RE & 1 & 54.2000 & 5.3000 & 1.028 \\
\hline SW & 1 & 83.1400 & 13.2000 & 1.578 \\
\hline $\mathrm{TM}$ & 1 & 65.3000 & 7.2600 & 1.239 \\
\hline $\mathrm{TN}$ & 1 & 0.4900 & 0.0500 & 0.009 \\
\hline TX & 1 & 53.7000 & 2.2000 & 1.019 \\
\hline UY & 1 & 50.0000 & 6.0000 & 0.949 \\
\hline WA & 1 & 190.0000 & 33.0000 & 3.605 \\
\hline WE & 1 & 37.2000 & 22.0000 & 0.706 \\
\hline
\end{tabular}

Total Number Reported:

A

A

A

A

A

A

A

A

A

A

A

A

A

A

A

A

A

A

A

A

A

A

A

A

A

A

A

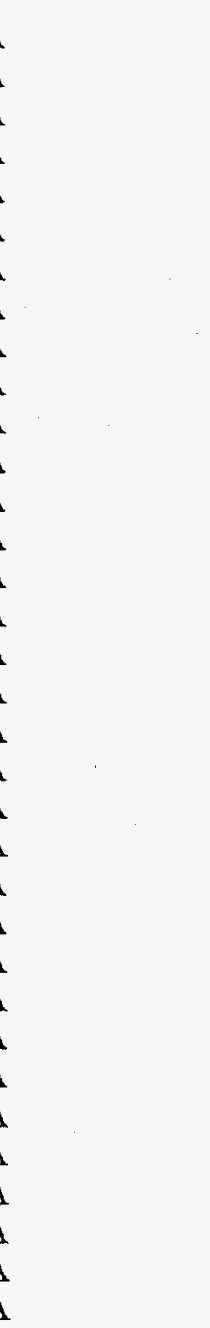




\section{QAP 49 Results by Nuclide}

Matrix: SO Soil $\mathrm{Bq} / \mathrm{kg}$

Radionuclide:

$\mathrm{TH} 234$

$\begin{array}{lr}\text { EML Value: } \quad 114.000 \\ \text { EML Error: } & 6.000\end{array}$

Labcode $\quad$ Test \# $\begin{gathered}\text { Reported } \\ \text { Value }\end{gathered} \quad \begin{gathered}\text { Reported } \\ \text { Error }\end{gathered} \frac{\begin{array}{c}\text { Reported } \\ \text { EML }\end{array}}{\begin{array}{c}\text { QAP 48 } \\ \text { Evaluation }\end{array} \text { Evaluation }}$

$\begin{array}{lrrrrr}\text { AF } & 1 & 67.3400 & 54.0200 & 0.591 & \mathrm{~A} \\ \text { AG } & 1 & 110.0000 & 31.0000 & 0.965 & \mathrm{~A} \\ \text { AI } & 1 & 103.0000 & 17.7000 & 0.904 & \mathrm{~A} \\ \text { AM } & 1 & 139.1100 & 31.4500 & 1.220 & \mathrm{~A} \\ \text { BL } & 1 & 141.0000 & 10.0000 & 1.237 & \mathrm{~A} \\ \text { BQ } & 1 & 120.0000 & 40.0000 & 1.053 & \mathrm{~A} \\ \text { CS } & 1 & 97.6600 & 6.6900 & 0.857 & \mathrm{~A} \\ \text { FG } & 1 & 66.3000 & 6.1000 & 0.582 & \mathrm{~A} \\ \text { FS } & 1 & 96.2000 & 17.8000 & 0.844 & \mathrm{~A} \\ \text { GA } & 1 & 108.0000 & 22.0000 & 0.947 & \mathrm{~A} \\ \text { GE } & 1 & 109.2795 & 84.1470 & 0.959 & \mathrm{~A} \\ \text { IE } & 1 & 226.7000 & 17.7000 & 1.989 & \mathrm{~A} \\ \text { IN } & 1 & 114.5000 & 5.0000 & 1.004 & \mathrm{~A} \\ \text { IS } & 1 & 110.0000 & 31.0000 & 0.965 & \mathrm{~A} \\ \text { IT } & 1 & 236.0000 & 13.0000 & 2.070 & \mathrm{~A} \\ \text { LV } & 1 & 90.1000 & 5.1000 & 0.790 & \mathrm{~A} \\ \text { ME } & 1 & 209.0000 & 9.8700 & 1.833 & \mathrm{~A} \\ \text { NA } & 1 & 98.0000 & 10.0000 & 0.860 & \mathrm{~A} \\ \text { NL } & 1 & 95.6000 & 37.2000 & 0.839 & \mathrm{~A} \\ \text { NQ } & 1 & 125.0000 & 16.0000 & 1.096 & \mathrm{~A} \\ \text { OC } & 1 & 170.0000 & 40.0000 & 1.491 & \mathrm{~A} \\ \text { OC } & 2 & 140.0000 & 40.0000 & 1.228 & \mathrm{~A} \\ \text { OC } & 3 & 120.0000 & 40.0000 & 1.053 & \mathrm{~A} \\ \text { OT } & 1 & 78.0000 & 6.0000 & 0.684 & \mathrm{~A} \\ \text { OU } & 1 & 115.0000 & 2.4000 & 1.009 & \mathrm{~A} \\ \text { RA } & 1 & 160.0000 & 50.0000 & 1.404 & \mathrm{~A} \\ \text { RA } & 2 & 140.0000 & 40.0000 & 1.228 & \mathrm{~A} \\ \text { SK } & 1 & 180.0000 & 17.0000 & 1.579 & \mathrm{~A} \\ \text { SW } & 1 & 143.2000 & 9.9000 & 1.256 & \mathrm{~A} \\ \text { TM } & 1 & 92.5000 & 23.7000 & 0.811 & \mathrm{~A} \\ \text { TO } & 1 & 98.7000 & 45.3000 & 0.866 & \mathrm{~A} \\ \text { TX } & 1 & 128.0000 & 15.0000 & 1.123 & \mathrm{~A} \\ \text { UY } & 1 & 108.0000 & 11.0000 & 0.947 & \mathrm{~A} \\ \text { WA } & 1 & 101.0000 & 11.0000 & 0.886 & \mathrm{~A} \\ \text { WE } & 1 & 52.8000 & 40.0000 & 0.463 & \end{array}$

Total Number Reported:

Values for elemental uranium are reported in $\mu \mathrm{g} /$ filter, $\mathrm{g}$ or $\mathrm{mL}$.

$\mathrm{pCi} / \mathrm{g}$ or $\mathrm{mL}=\mathrm{Bq} \times 0.027$

Evaluation: $A=A c c e p t a b l e, ~ W=A c c e p t a b l e$ with $W$ arning, $N=$ Not Acceptable

If the evaluation system is not appropriate for the types of analyses performed in your lab, apply site specific evaluation. 


\section{QAP 49 Results by Nuclide}

Matrix: SO Soil $\mathrm{Bq} / \mathrm{kg}$

Radionuclide: TL208

\begin{tabular}{lr}
\hline EML Value: & 18.300 \\
EML Error: & 1.100 \\
\hline
\end{tabular}

Labcode Test \# $\begin{gathered}\text { Reported } \\ \text { Value }\end{gathered} \begin{gathered}\text { Reported } \\ \text { Error }\end{gathered} \frac{\text { Reported }}{E M L} \quad \begin{gathered}\text { QAP 48 } \\ \text { Evaluation }\end{gathered} \quad$ Evaluation

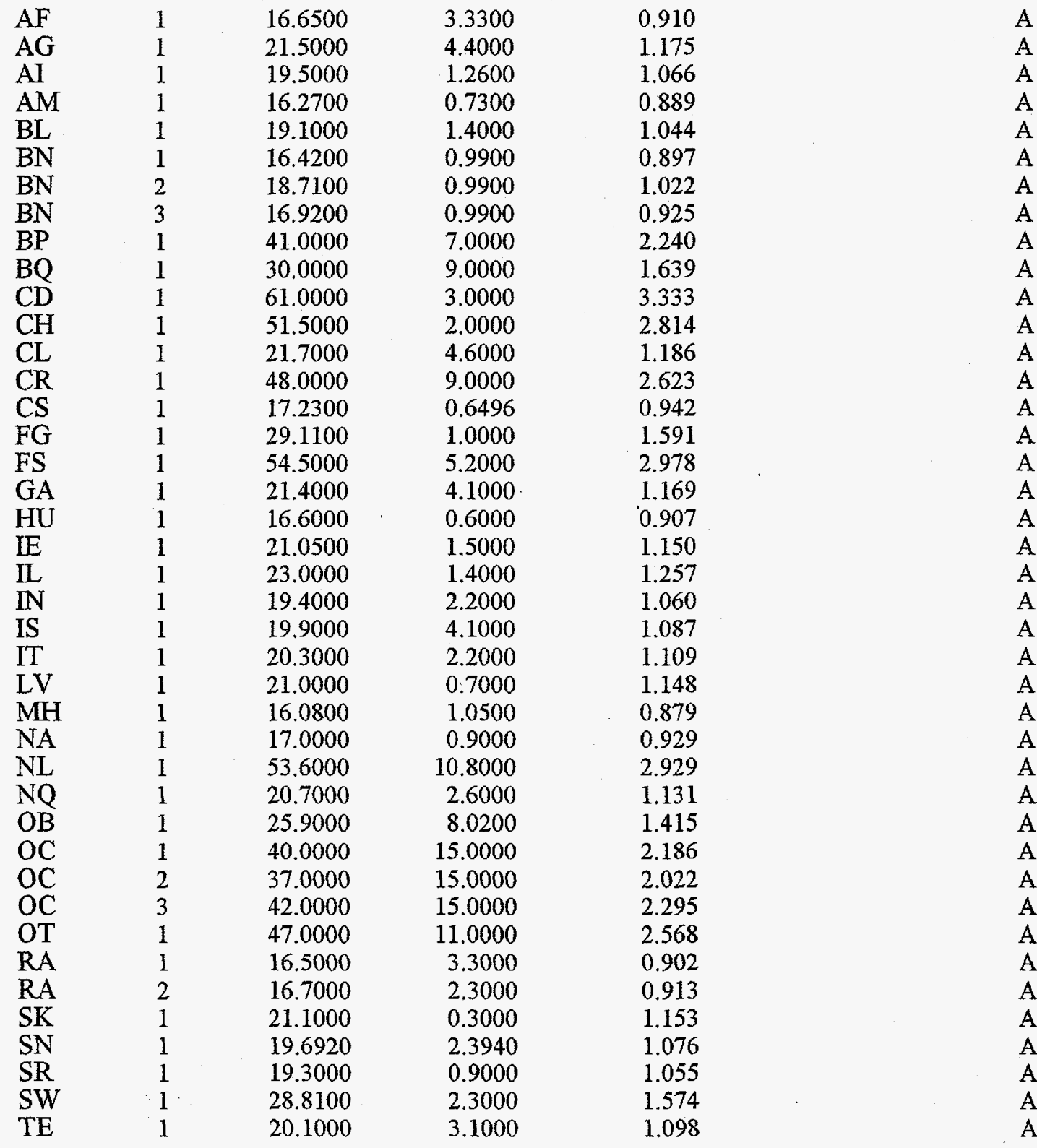

A

A

A

A

A

A

A

A

A

A

A

A

A

A

A

A

A

A

A

A

A

A

A

A

A

A

A

A

A

A

A

A

A

A

Values for elemental uranium are reported in $\mu \mathrm{g} /$ filter, $\mathrm{g}$ or $\mathrm{mL}$.

$\mathrm{pCi} / \mathrm{g}$ or $\mathrm{mL}=\mathrm{Bq} \times 0.027$

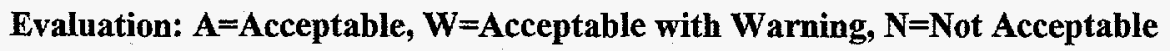

If the evaluation system is not appropriate for the types of analyses performed in your lab, apply site specific evaluation. 


\section{QAP 49 Results by Nuclide}

Matrix: SO Soil $\mathrm{Bq} / \mathrm{kg}$

Radionuclide: TL208

EML Value: $\quad 18.300$

EML Error: $\quad 1.100$

\begin{tabular}{lccccc} 
Labcode & Test \# $\begin{array}{c}\text { Reported } \\
\text { Value }\end{array}$ & $\begin{array}{c}\text { Reported } \\
\text { Error }\end{array}$ & $\begin{array}{c}\text { Reported } \\
\text { EML }\end{array}$ & $\begin{array}{c}\text { QAP 48 } \\
\text { Evaluation }\end{array}$ & Evaluation \\
\hline TM & 1 & 60.5000 & 6.7300 & 3.306 & $\mathrm{~A}$ \\
TO & 1 & 15.8560 & 3.8620 & 0.866 & $\mathrm{~A}$ \\
TO & 1 & 31.5000 & 6.6000 & 1.721 & $\mathrm{~A}$ \\
TW & 1 & 16.5000 & 0.5000 & 0.902 & $\mathrm{~A}$ \\
TX & 1 & 16.8000 & 0.7000 & 0.918 & $\mathrm{~A}$ \\
UY & 1 & 21.0000 & 12.0000 & 1.148 & $\mathrm{~A}$ \\
WA & 1 & 18.6000 & 1.0000 & 1.016 & $\mathrm{~A}$
\end{tabular}

Total Number Reported:

49

Values for elemental uranium are reported in $\mu \mathrm{g}$ /filter, $\mathrm{g}$ or $\mathbf{m L}$.

$\mathrm{pCi} / \mathrm{g}$ or $\mathrm{mL}=\mathrm{Bq} \times 0.027$

Evaluation: $A=$ Acceptable, $W=$ Acceptable with $W$ arning, $N=N$ ot Acceptable

If the evaluation system is not appropriate for the types of analyses performed in your lab, apply site specific evaluation. 


\section{QAP 49 Results by Nuclide}

\begin{tabular}{lr}
\hline $\begin{array}{l}\text { Matrix: } \\
\text { Radionuclide: }\end{array}$ & $\begin{array}{r}\text { Soil } \mathrm{Bq} / \mathrm{kg} \\
\mathrm{U} 234\end{array}$ \\
\hline EML Value: & 113.000 \\
EML Error: & 6.000 \\
\hline
\end{tabular}

\begin{tabular}{|c|c|c|c|}
\hline abcode & Test \# & $\begin{array}{l}\text { Reported } \\
\text { Value }\end{array}$ & $\begin{array}{l}\text { orted } \\
\text { rror }\end{array}$ \\
\hline
\end{tabular}

\begin{tabular}{|c|c|c|c|c|c|c|}
\hline $\mathrm{AG}$ & 1 & 102.0000 & 13.0000 & 0.903 & A & A \\
\hline $\mathrm{AM}$ & 1 & 68.4700 & 18.2000 & 0.606 & W & W \\
\hline AN & 1 & 174.0000 & 19.0000 & 1.540 & A & $\mathrm{N}$ \\
\hline $\mathrm{AU}$ & 1 & 102.0000 & 12.0000 & 0.903 & A & A \\
\hline $\mathrm{BC}$ & 1 & 165.0000 & 8.9000 & 1.460 & A & $\mathrm{N}$ \\
\hline $\mathrm{BE}$ & 1 & 105.1000 & 7.9000 & 0.930 & $\mathrm{~A}$ & A \\
\hline $\mathrm{BL}$ & 1 & 110.0000 & & 0.973 & A & A \\
\hline BL & 2 & 105.0000 & 2.0000 & 0.929 & A & A \\
\hline $\mathrm{BM}$ & 1 & 110.0000 & 14.6000 & 0.973 & A & A \\
\hline BP & 1 & 109.0000 & 4.0000 & 0.965 & & A \\
\hline $\mathrm{BU}$ & 1 & 105.0000 & 8.0000 & 0.929 & A & A \\
\hline BX & 1 & 146.2000 & 7.4000 & 1.294 & A & W \\
\hline $\mathrm{CH}$ & 1 & 109.0000 & 5.1000 & 0.965 & & A \\
\hline CL & 1 & 101.0000 & 15.7000 & 0.894 & A & A \\
\hline $\mathrm{EG}$ & 1 & 106.0000 & 11.0000 & 0.938 & $\mathrm{~A}$ & A \\
\hline EM & 1 & 0.1180 & 0.0070 & 0.001 & & $\mathrm{~N}$ \\
\hline $\mathrm{FG}$ & 1 & 110.4000 & 8.9900 & 0.977 & & A \\
\hline GA & 1 & 110.0000 & 9.4000 . & 0.973 & W & A \\
\hline GE & 1 & 103.0450 & 21.0090 & 0.912 & A & A \\
\hline HT & 1 & 220.0000 & 25.0000 & 1.947 & & $N$ \\
\hline $\mathrm{IN}$ & 1 & 116.0000 & 17.0000 & 1.027 & W & A \\
\hline IT & 1 & 92.0000 & 4.0000 & 0.814 & A & A \\
\hline LL & 1 & 98.9000 & 8.5400 & 0.875 & A & A \\
\hline $\mathrm{LW}$ & 1 & 88.9000 & 20.7000 & 0.787 & & A \\
\hline ML & 1 & 99.0900 & 7.5600 & 0.877 & A & A \\
\hline NA & 1 & 96.0000 & 12.0000 & 0.850 & A & A \\
\hline NL & 1 & 113.0000 & 27.0000 & 1.000 & A & A \\
\hline NQ & 1 & 110.7000 & 6.4000 & 0.980 & & A \\
\hline $\mathrm{NZ}$ & 1 & 106.0000 & 4.0000 & 0.938 & & A \\
\hline NZ & 2 & 108.0000 & 3.0000 & 0.956 & & A \\
\hline OU & 1 & 115.0000 & 2.3400 & 1.018 & & A \\
\hline RA & 1 & 108.0000 & 11.0000 & 0.956 & A & $\mathrm{A}$ \\
\hline RE & 1 & 107.0000 & 15.0000 & 0.947 & A & A \\
\hline $\mathrm{TM}$ & 1 & 115.0000 & 6.4400 & 1.018 & A & A \\
\hline TN & 1 & 103.0000 & 10.0000 & 0.912 & A & A \\
\hline TO & 1 & 110.4000 & 26.9000 & 0.977 & A & A \\
\hline TX & 1 & 105.0000 & 2.0000 & 0.929 & A & A \\
\hline TY & 1 & 28.0000 & 3.0000 & 0.248 & & $\mathrm{~N}$ \\
\hline WA & 1 & 116.0000 & 7.0000 & 1.027 & A & A \\
\hline YA & I & 107.2383 & 2.9847 & 0.949 & A & A \\
\hline
\end{tabular}

Values for elemental uranium are reported in $\mu \mathrm{g} /$ filter, $\mathrm{g}$ or $\mathrm{mL}$.

$\mathrm{pCi} / \mathrm{g}$ or $\mathrm{mL}=\mathrm{Bq} \times 0.027$

Evaluation: $A=A c c e p t a b l e, W=A c c e p t a b l e$ with $W$ arning, $N=$ Not Acceptable

If the evaluation system is not appropriate for the types of analyses performed in your lab, apply site specific evaluation. 


\section{QAP 49 Results by Nuclide}

Matrix: SO Soil $\mathrm{Bq} / \mathrm{kg}$
Radionuclide:

EML Value: $\quad 113.000$

EML Error: $\quad 6.000$

Labcode $\quad$ Test \# $\begin{gathered}\text { Reported } \\ \text { Value }\end{gathered} \quad \begin{gathered}\text { Reported } \\ \text { Error }\end{gathered} \frac{\text { Reported }}{E M L} \quad \begin{gathered}\text { QAP 48 } \\ \text { Evaluation }\end{gathered} \quad$ Evaluation

Total Number Reported:

Values for elemental uranium are reported in $\mu$ gffilter, $g$ or $\mathrm{mL}$.

$\mathrm{pCi} / \mathrm{g}$ or $\mathrm{mL}=\mathrm{Bq} \times 0.027$

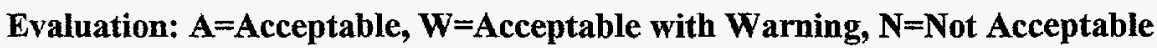

If the evaluation system is not appropriate for the types of analyses performed in your lab, apply site specific evaluation. 
QAP 49 Results by Nuclide

\begin{tabular}{|c|c|c|c|c|c|c|}
\hline $\begin{array}{l}\text { Matrix: S } \\
\text { Radionuclide }\end{array}$ & $\begin{array}{l}\text { O Soil } \\
\text { e: }\end{array}$ & $\begin{array}{l}3 \mathrm{q} / \mathrm{kg} \\
\mathrm{J} 238\end{array}$ & & & & \\
\hline $\begin{array}{l}\text { EML Value: } \\
\text { EML Error: }\end{array}$ & $\begin{array}{r}120 \\
9 \\
\end{array}$ & 00 & & & & \\
\hline Labcode & Test \# & $\begin{array}{l}\text { Reported } \\
\text { Value }\end{array}$ & $\begin{array}{c}\text { Reported } \\
\text { Error }\end{array}$ & $\frac{\text { Reported }}{E M L}$ & $\begin{array}{c}\text { QAP } 48 \\
\text { Evaluation }\end{array}$ & Evaluation \\
\hline $\mathrm{AG}$ & 1 & 105.0000 & 13.0000 & 0.875 & A & A \\
\hline AM & 1 & 72.5400 & 18.6000 & 0.604 & W & W \\
\hline $\mathrm{AN}$ & 1 & 186.0000 & 22.0000 & 1.550 & A & $\mathbf{N}$ \\
\hline $\mathrm{AU}$ & 1 & 105.0000 & 14.0000 & 0.875 & A & A \\
\hline $\mathrm{BC}$ & 1 & 95.8000 & 6.3000 & 0.798 & $\mathrm{~A}$ & $\mathrm{~A}$ \\
\hline $\mathrm{BE}$ & 1 & 108.3000 & 8.0000 & 0.903 & A & A \\
\hline $\mathrm{BL}$ & 1 & 108.0000 & & 0.900 & A & $\mathrm{A}$ \\
\hline BL & 2 & 103.0000 & 2.0000 & 0.858 & A & A \\
\hline $\mathrm{BM}$ & 1 & 114.4000 & 15.2000 & 0.953 & A & A \\
\hline $\mathrm{BP}$ & 1 & 112.0000 & 4.0000 & 0.933 & & A \\
\hline $\mathrm{BU}$ & 1 & 108.0000 & 6.0000 & 0.900 & A & $\mathrm{A}$ \\
\hline BX & 1 & 105.5000 & 5.9000 & 0.879 & $\mathrm{~A}$ & A \\
\hline $\mathrm{CH}$ & 1 & 110.0000 & 5.2000 & 0.917 & & A \\
\hline $\mathrm{CL}$ & 1 & 86.1000 & 14.9000 & 0.717 & A & A \\
\hline $\mathrm{CS}$ & 1 & 100.5000 & 21.9400 & 0.837 & $\mathrm{~N}$ & A \\
\hline EG & 1 & 111.0000 & 13.0000 & 0.925 & A & A \\
\hline $\mathrm{EM}$ & 1 & 0.1080 & 0.0060 & 0.001 & & $\mathrm{~N}$ \\
\hline $\mathrm{FG}$ & 1 & 97.3700 & 4.6600 . & 0.811 & & A \\
\hline FL & 1 & 82.0000 & 4.0000 & 0.683 & A & W \\
\hline $\mathrm{GA}$ & 1 & 113.0000 & 4.2000 & 0.942 & W & A \\
\hline GE & 1 & 117.2900 & 23.7846 & 0.977 & A & A \\
\hline GT & 1 & 102.0000 & 20.0000 & 0.850 & A & A \\
\hline $\mathrm{HT}$ & 1 & 223.5000 & 25.0000 & 1.862 & & $\mathrm{~N}$ \\
\hline ID & 1 & 112.1700 & 6.4100 & 0.935 & & A \\
\hline IE & 1 & 226.7000 & 17.7000 & 1.889 & A & $\mathrm{N}$ \\
\hline $\mathrm{IN}$ & 1 & 115.0000 & 17.0000 & 0.958 & W & A \\
\hline IT & 1 & 98.0000 & 7.0000 & 0.817 & A & A \\
\hline $\mathrm{LL}$ & 1 & 105.0000 & 9.0100 & 0.875 & $\mathrm{~N}$ & A \\
\hline LW & 1 & 94.0000 & 21.5000 & 0.783 & & A \\
\hline $\mathrm{ML}$ & 1 & 109.8000 & 8.2800 & 0.915 & A & $\mathrm{A}$ \\
\hline NA & 1 & 100.0000 & 11.0000 & 0.833 & A & A \\
\hline NL & 1 & 117.0000 & 28.0000 & 0.975 & A & A \\
\hline NQ & 1 & 111.9000 & 6.4000 & 0.933 & & A \\
\hline $\mathrm{NZ}$ & i & 115.0000 & 4.0000 & 0.958 & & A \\
\hline $\mathrm{NZ}$ & 2 & 115.0000 & 3.0000 & 0.958 & & A \\
\hline $\mathrm{OU}$ & 1 & 93.3000 & 2.1600 & 0.778 & & A \\
\hline RA & 1 & 107.0000 & 11.0000 & 0.892 & A & A \\
\hline $\mathrm{RE}$ & 1 & 116.0000 & 16.0000 & 0.967 & A & A \\
\hline $\mathrm{TM}$ & 1 & 123.0000 & 6.8100 & 1.025 & A & A \\
\hline $\mathrm{TN}$ & 1 & 108.0000 & 11.0000 & 0.900 & A & A \\
\hline TO & 1 & 108.1000 & 30.1000 & 0.901 & A & A \\
\hline
\end{tabular}

Values for elemental uranium are reported in $\mu \mathrm{g} /$ filter, $\mathrm{g}$ or $\mathrm{mL}$.

$\mathrm{pCi} / \mathrm{g}$ or $\mathrm{mL}=\mathrm{Bq} \times 0.027$

Evaluation: $A=$ Acceptable, $W=$ Acceptable with Warning, $N=$ Not Acceptable

If the evaluation system is not appropriate for the types of analyses performed in your lab, apply site specific evaluation. 


\section{QAP 49 Results by Nuclide}

\begin{tabular}{lr}
\hline Matrix: SO & Soil $\mathrm{Bq} /$ \\
Radionuclide: & U238 \\
\hline EML Value: & 120.000 \\
EML Error: & 9.000 \\
\hline
\end{tabular}

\begin{tabular}{ccccccc} 
Labcode & Test \# & $\begin{array}{c}\text { Reported } \\
\text { Value }\end{array}$ & $\begin{array}{c}\text { Reported } \\
\text { Error }\end{array}$ & $\begin{array}{c}\text { Reported } \\
\text { EML }\end{array}$ & $\begin{array}{c}\text { QAP 48 } \\
\text { Evaluation }\end{array}$ & Evaluation \\
\hline TX & 1 & 112.0000 & 3.0000 & 0.933 & $\mathrm{~A}$ & $\mathrm{~A}$ \\
WA & 1 & 123.0000 & 7.0000 & 1.025 & $\mathrm{~A}$ & $\mathrm{~A}$ \\
WS & 1 & 122.8000 & 15.7000 & 1.023 & $\mathrm{~A}$ & $\mathrm{~A}$ \\
YA & 1 & 113.7997 & 3.1573 & 0.948 & $\mathrm{~A}$ & $\mathrm{~A}$
\end{tabular}

Total Number Reported:

Values for elemental uranium are reported in $\mu \mathrm{g} /$ filter, $\mathrm{g}$ or $\mathrm{mL}$.

$\mathrm{pCi} / \mathrm{g}$ or $\mathrm{mL}=\mathrm{Bq} \times 0.027$

Evaluation: $A=$ Acceptable, $W=$ Acceptable with Warning, $N=$ Not Acceptable

If the evaluation system is not appropriate for the types of analyses performed in your lab, apply site specific evaluation. 


\section{QAP 49 Results by Nuclide}

\begin{tabular}{|c|c|c|c|c|c|c|}
\hline $\begin{array}{l}\text { Matrix: S } \\
\text { Radionuclid }\end{array}$ & $\begin{array}{ll}0 & \text { Soil } \\
\text { : } & \end{array}$ & $\begin{array}{l}\mathrm{Bq} / \mathrm{kg} \\
\mathrm{gU}\end{array}$ & & & & \\
\hline $\begin{array}{l}\text { EML Value: } \\
\text { EML Error: }\end{array}$ & & $\begin{array}{l}00 \\
00\end{array}$ & & & & \\
\hline Labcode & Test \# & $\begin{array}{l}\text { Reported } \\
\text { Value }\end{array}$ & $\begin{array}{c}\text { Reported } \\
\text { Error }\end{array}$ & $\frac{\text { Reported }}{\text { EML }}$ & $\begin{array}{c}\text { QAP } 48 \\
\text { Evaluation }\end{array}$ & Evaluation \\
\hline $\mathrm{AC}$ & 1 & 8.7800 & 0.8800 & 0.905 & & A \\
\hline $\mathrm{AG}$ & 1 & 8.9000 & 1.5000 & 0.918 & A & A \\
\hline $\mathrm{BE}$ & 1 & 8.9000 & & 0.918 & A & A \\
\hline BL & 1 & 8.9100 & & 0.919 & & A \\
\hline $\mathrm{BL}$ & 2 & 8.5100 & 0.1400 & 0.877 & & A \\
\hline $\mathrm{BQ}$ & 1 & 11.3000 & 0.3000 & 1.165 & A & W \\
\hline $\mathrm{BU}$ & 1 & 8.4000 & 0.6000 & 0.866 & A & A \\
\hline $\mathrm{CA}$ & 1 & 9.4000 & 0.9000 & 0.969 & A & $\mathrm{A}$ \\
\hline $\mathrm{CH}$ & 1 & 9.9900 & 1.0000 & 1.030 & & A \\
\hline $\mathrm{GA}$ & 1 & 8.5600 & & 0.882 & A & A \\
\hline $\mathrm{HT}$ & 1 & 18.1000 & 1.5000 & 1.866 & & $\mathrm{~N}$ \\
\hline IS & 1 & 2.7000 & 0.3900 & 0.278 & $\mathrm{~N}$ & $\mathrm{~N}$ \\
\hline IS & 2 & 2.6300 & 0.3800 & 0.271 & $\mathrm{~N}$ & $\mathrm{~N}$ \\
\hline IS & 3 & 2.5600 & 0.3700 & 0.264 & $\mathrm{~N}$ & $\mathrm{~N}$ \\
\hline $\mathrm{IT}$ & 1 & 10.2000 & 0.5000 & 1.052 & $\mathrm{~A}$ & $\mathrm{~A}$ \\
\hline NL & 1 & 9.3700 & 2.2200 & 0.966 & & A \\
\hline OU & 1 & 9.3000 & 0.4000 & 0.959 & A & $\mathrm{A}$ \\
\hline $\mathrm{RA}$ & 1 & 8.6000 & 0.5000 & 0.887 & A & A \\
\hline SW & 1 & 9.0100 & & 0.929 & $\mathrm{~A}$ & $\mathrm{~A}$ \\
\hline TI & 1 & 10.0000 & 2.0000 & 1.031 & A & A \\
\hline TM & 1 & 8.5100 & 0.4380 & 0.877 & A & A \\
\hline TN & 1 & 8.8400 & 0.9000 & 0.911 & W & A \\
\hline UP & 1 & 9.1800 & 0.5000 & 0.946 & A & $\mathrm{A}$ \\
\hline YP & 1 & 8.2400 & 1.5100 & 0.849 & A & A \\
\hline
\end{tabular}

Total Number Reported:

Values for elemental uranium are reported in $\mu \mathrm{g} /$ filter, $g$ or $\mathrm{mL}$.

$\mathrm{pCi} / \mathrm{g}$ or $\mathrm{mL}=\mathrm{Bq} \times 0.027$

Evaluation: A=Acceptable, $W=$ Acceptable with Warning, $N=$ Not Acceptable

If the evaluation system is not appropriate for the types of analyses performed in your lab, apply site specific evaluation. 


\section{QAP 49 Results by Nuclide}

\begin{tabular}{|c|c|c|c|c|c|c|}
\hline \multicolumn{3}{|c|}{$\begin{array}{lcc}\text { Matrix: } & \text { VE Vegetation } \mathrm{Bq} / \mathrm{kg} \\
\text { Radionuclide: } & \text { AM } 241\end{array}$} & \multirow[b]{3}{*}{$\begin{array}{l}\text { Reported } \\
\text { Error }\end{array}$} & \multirow[b]{3}{*}{$\frac{\text { Reported }}{\text { EML }}$} & \multirow[b]{3}{*}{$\begin{array}{c}\text { QAP } 48 \\
\text { Evaluation }\end{array}$} & \multirow[b]{3}{*}{ Evaluation } \\
\hline $\begin{array}{l}\text { EML Value: } \\
\text { EML Error: }\end{array}$ & \multicolumn{2}{|c|}{$\begin{array}{l}2.330 \\
0.060 \\
\end{array}$} & & & & \\
\hline Labcode & Test \# & $\begin{array}{l}\text { Reported } \\
\text { Value }\end{array}$ & & & & \\
\hline $\mathrm{AF}$ & 1 & 6.2900 & 1.1100 & 2.700 & W & $\mathrm{W}$ \\
\hline $\mathrm{AG}$ & 1 & 2.2300 & 0.4600 & 0.957 & A & A \\
\hline $\mathrm{AI}$ & 1 & 2.0100 & 0.4400 & 0.863 & A & W \\
\hline $\mathrm{AM}$ & 1 & 3.8400 & 1.8500 & 1.648 & A & W \\
\hline $\mathrm{AT}$ & 1 & 2.7680 & 0.9630 & 1.188 & & A \\
\hline $\mathrm{AU}$ & 1 & 2.1600 & 0.3000 & 0.927 & A & $\mathrm{A}$ \\
\hline $\mathrm{BE}$ & 1 & 2.5400 & 0.3600 & 1.090 & A & A \\
\hline BL & 1 & 2.9300 & 0.5100 & 1.258 & $\mathrm{~N}$ & A \\
\hline $\mathrm{BM}$ & 1 & 2.3600 & 0.6000 & 1.013 & $\mathrm{~A}$ & A \\
\hline BP & 1 & 2.6700 & 0.1700 & 1.146 & A & A \\
\hline $\mathrm{BU}$ & 1 & 2.9000 & 0.2000 & 1.245 & $\mathrm{~A}$ & $\mathrm{~A}$ \\
\hline $\mathrm{BX}$ & 1 & 3.0000 & 0.9000 & 1.288 & W & A \\
\hline $\mathrm{CH}$ & 1 & 2.5300 & 0.1300 & 1.086 & A & A \\
\hline $\mathrm{CL}$ & 1 & 3.8000 & 1.5000 & 1.631 & $\mathrm{~N}$ & W \\
\hline $\mathrm{CN}$ & 1 & 3.7000 & 0.2300 & 1.588 & & A \\
\hline CS & 1 & 2.4760 & 0.6348 & 1.063 & $\mathrm{~N}$ & A \\
\hline $\mathrm{CS}$ & 1 & 2.4760 & 0.6348 & 1.063 & $\mathrm{~N}$ & $\mathrm{~A}$ \\
\hline $\mathrm{EG}$ & 1 & 2.5100 & 0.2300 & 1.077 & A & $\mathrm{A}$ \\
\hline $\mathrm{EP}$ & 1 & 2.6300 & 0.3050 & 1.129 & $\mathrm{~A}$ & $\mathrm{~A}$ \\
\hline $\mathrm{FL}$ & 1 & 2.6000 & 0.5000 & 1.116 & W & A \\
\hline GA & 1 & 2.9000 & 0.2000 & 1.245 & A & A \\
\hline GE & 1 & 2.6899 & 0.8245 & 1.154 & A & A \\
\hline GP & 1 & 2.8000 & 0.8000 & 1.202 & & $\mathrm{~A}$ \\
\hline GT & 1 & 1.9000 & 0.5000 & 0.815 & A & W \\
\hline HU & 1 & 2.7000 & 1.2000 & 1.159 & & A \\
\hline IS & 1 & 3.3540 & 1.6750 & 1.439 & $\mathrm{~N}$ & A \\
\hline IT & 1 & 2.2000 & 0.2400 & 0.944 & $\mathrm{~A}$ & $\mathrm{~A}$ \\
\hline LL & 1 & 2.8100 & 0.6380 & 1.206 & A & A \\
\hline $\mathrm{LV}$ & $i$ & 3.7900 & 1.4400 & 1.627 & & W \\
\hline MA & 1 & 0.3700 & 0.3700 & 0.159 & W & $\mathrm{N}$ \\
\hline $\mathrm{ME}$ & 1 & 2.0700 & 0.4700 & 0.888 & & W \\
\hline $\mathrm{MH}$ & 1 & 1.9700 & 0.4100 & 0.845 & & W \\
\hline OT & 1 & 2.9000 & 0.5000 & 1.245 & A & $\mathrm{A}$ \\
\hline $\mathrm{PO}$ & 1 & 2.0000 & 0.3000 & 0.858 & & W \\
\hline RE & 1 & 2.5600 & 0.2800 & 1.099 & A & A \\
\hline SN & 1 & 2.5050 & 1.0930 & 1.075 & W & $\mathrm{A}$ \\
\hline SR & 1 & 3.1420 & 0.5250 & 1.348 & A & A \\
\hline SW & 1 & 9.4800 & 0.9600 & 4.069 & W & $\mathrm{N}$ \\
\hline TI & 1 & 2.5000 & 0.5000 & 1.073 & $\ddot{A}$ & A \\
\hline $\mathrm{TM}$ & 1 & 2.9600 & 0.5000 & 1.270 & A & A \\
\hline $\mathrm{TN}$ & 1 & 2.5500 & 0.2500 & 1.094 & A & A \\
\hline
\end{tabular}

Values for elemental uranium are reported in $\mu \mathrm{g} /$ filter, $\mathrm{g}$ or $\mathrm{mL}$.

$\mathrm{pCi} / \mathrm{g}$ or $\mathrm{mL}=\mathrm{Bq} \times 0.027$

Evaluation: $A=A c c e p t a b l e, ~ W=A c c e p t a b l e$ with Warning, $N=$ Not Acceptable

If the evaluation system is not appropriate for the types of analyses performed in your lab, apply site specific evaluation. 


\section{QAP 49 Results by Nuclide}

Matrix: VE Vegetation $\mathrm{Bq} / \mathrm{kg}$

Radionuclide: $\quad$ AM241

$\begin{array}{ll}\text { EML Value: } & 2.330 \\ \text { EML Error: } & 0.060\end{array}$

\begin{tabular}{lcccccc} 
Labcode & Test \# & $\begin{array}{c}\text { Reported } \\
\text { Value }\end{array}$ & $\begin{array}{c}\text { Reported } \\
\text { Error }\end{array}$ & $\begin{array}{c}\text { Reported } \\
\text { EML }\end{array}$ & $\begin{array}{c}\text { QAP 48 } \\
\text { Evaluation }\end{array}$ & Evaluation \\
\hline TO & 1 & 2.6200 & 1.7800 & 1.124 & $\mathrm{~A}$ & $\mathrm{~A}$ \\
TX & 1 & 3.3300 & 0.3900 & 1.429 & $\mathrm{~A}$ & $\mathrm{~A}$ \\
WA & 1 & 1.7200 & 0.2500 & 0.738 & $\mathrm{~W}$ & $\mathrm{~W}$ \\
WC & $\mathrm{I}$ & 4.8000 & 1.3000 & 2.060 & $\mathrm{~A}$ & $\mathrm{~W}$ \\
YA & 1 & 2.5333 & 0.1221 & 1.087 & $\mathrm{~A}$ & $\mathrm{~A}$
\end{tabular}

Total Number Reported:

46

Values for elemental uranium are reported in $\mu$ gfilter, $\mathrm{g}$ or $\mathrm{mL}$.

$\mathrm{pCi} / \mathrm{g}$ or $\mathrm{mL}=\mathrm{Bq} \times 0.027$

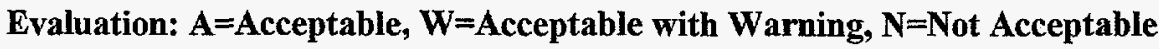

If the evaluation system is not appropriate for the types of analyses performed in your lab, apply site specific evaluation. 


\section{QAP 49 Results by Nuclide}

\begin{tabular}{lc}
\hline Matrix: VE & $\begin{array}{r}\text { Vegetation } \\
\text { Radionuclide: }\end{array}$ \\
\hline EM24
\end{tabular}

\begin{tabular}{|c|c|c|c|c|c|c|}
\hline Labcode & Test \# & $\begin{array}{l}\text { Reported } \\
\text { Value }\end{array}$ & $\begin{array}{c}\text { Reported } \\
\text { Error }\end{array}$ & $\frac{\text { Reported }}{E M L}$ & $\begin{array}{c}\text { QAP 48 } \\
\text { Evaluation }\end{array}$ & Evaluation \\
\hline $\mathrm{AG}$ & 1 & 2.0900 & 0.4400 & 1.188 & A & A \\
\hline AI & 1 & 2.6200 & 0.7300 & 1.489 & A & W \\
\hline $\mathrm{BE}$ & 1 & 2.0700 & 0.2900 & 1.176 & A & A \\
\hline $\mathrm{BL}$ & 1 & 1.3300 & 0.2500 & 0.756 & $\mathrm{~N}$ & W \\
\hline $\mathrm{BP}$ & 1 & 1.6200 & 0.1600 & 0.920 & A & A \\
\hline $\mathrm{BU}$ & 1 & 1.8000 & 0.1000 & 1.023 & A & A \\
\hline $\mathrm{BX}$ & 1 & 1.6000 & 0.6000 & 0.909 & $\mathrm{~W}$ & A \\
\hline $\mathrm{CH}$ & 1 & 1.8100 & 0.1200 & 1.028 & A & A \\
\hline $\mathrm{CL}$ & 1 & 1.2000 & 1.1000 & 0.682 & $\mathrm{~N}$ & W \\
\hline $\mathrm{EG}$ & 1 & 1.8900 & 0.1800 & 1.074 & A & A \\
\hline$\overline{\mathrm{EP}}$ & 1 & 1.8800 & 0.1900 & 1.068 & A & $\mathrm{A}$ \\
\hline GA & 1 & 1.9000 & 0.1600 & 1.080 & A & A \\
\hline $\mathrm{GE}$ & 1 & 2.0350 & 0.6725 & 1.156 & A & A \\
\hline $\mathrm{GP}$ & 1 & 1.5000 & 0.6000 & 0.852 & & A \\
\hline $\mathrm{IT}$ & 1 & 1.9400 & 0.1100 & 1.102 & W & $\mathrm{A}$ \\
\hline LL & 1 & 3.5800 & 0.8060 & 2.034 & A & $\mathrm{N}$ \\
\hline $\mathrm{OT}$ & 1 & 2.0000 & 0.4000 & 1.136 & W & $\mathrm{A}$ \\
\hline $\mathrm{RE}$ & 1 & 1.8300 & 0.2100 & 1.040 & & A \\
\hline $\mathrm{SN}$ & 1 & 1.5900 & 0.8620 & 0.903 & A & $\mathrm{A}$ \\
\hline SR & 1 & 2.2000 & 0.4400 & 1.250 & A & A \\
\hline SW & 1 & 1.0400 & 0.5200 & 0.591 & & W \\
\hline $\mathrm{TI}$ & 1 & 1.9000 & 0.4000 & 1.080 & A & A \\
\hline $\mathrm{TM}$ & 1 & 2.6300 & 0.4810 & 1.494 & A & W \\
\hline $\mathrm{TN}$ & 1 & 1.8800 & 0.5000 & 1.068 & A & A \\
\hline WA & 1 & 2.6400 & 0.3000 & 1.500 & & W \\
\hline WC & 1 & 1.7000 & 0.7000 & 0.966 & A & A \\
\hline YA & 1 & 1.3764 & 0.0876 & 0.782 & $\mathrm{~A}$ & W \\
\hline
\end{tabular}

Total Number Reported:

If the evaluation system is not appropriate for the types of analyses performed in your lab, apply site specific evaluation. 


\section{QAP 49 Results by Nuclide}

Matrix: VE Vegetation $\mathrm{Bq} / \mathrm{kg}$

Radionuclide: $\quad \mathrm{CO} 60$

\begin{tabular}{lr} 
EML Value: & 20.000 \\
EML Error: & 1.000 \\
\hline
\end{tabular}

Labcode Test \# $\begin{gathered}\text { Reported } \\ \text { Value }\end{gathered}$

Reported Reported QAP 48

\begin{tabular}{|c|c|c|c|c|c|c|}
\hline $\mathrm{AF}$ & 1 & 31.4500 & 27.0100 & 1.572 & A & $\mathrm{N}$ \\
\hline $\mathrm{AG}$ & 1 & 21.6000 & 4.1000 & 1.080 & A & A \\
\hline $\mathrm{AI}$ & 1 & 21.3000 & 1.4700 & 1.065 & A & A \\
\hline $\mathrm{AL}$ & 1 & 36.3730 & 0.8000 & 1.819 & $\mathrm{~N}$ & $\mathrm{~N}$ \\
\hline $\mathrm{AM}$ & 1 & 23.3100 & 2.9600 & 1.165 & A & A \\
\hline AT & 1 & 19.5500 & 2.6800 & 0.977 & & A \\
\hline $\mathrm{AU}$ & 1 & 23.0000 & 3.0000 & 1.150 & A & A \\
\hline BA & 1 & 24.7000 & 3.9000 & 1.235 & & A \\
\hline $\mathrm{BC}$ & 1 & 21.1000 & 1.5000 & 1.055 & A & A \\
\hline $\mathrm{BE}$ & 1 & 22.0000 & 3.0000 & 1.100 & A & A \\
\hline $\mathrm{BL}$ & 1 & 20.0000 & 1.4000 & 1.000 & A & A \\
\hline BM & 1 & 20.5000 & 1.6000 & 1.025 & A & A \\
\hline $\mathrm{BN}$ & 1 & 18.6300 & 0.3800 & 0.931 & A & A \\
\hline $\mathrm{BN}$ & 2 & 18.1600 & 0.3800 & 0.908 & A & A \\
\hline $\mathrm{BN}$ & 3 & 17.7000 & 0.3800 & 0.885 & A & A \\
\hline BP & 1 & 18.0000 & 0.4000 & 0.900 & A & A \\
\hline $\mathrm{BQ}$ & 1 & 68.0000 & 20.0000 & 3.400 & & $\mathrm{~N}$ \\
\hline BÙ & 1 & 21.0000 & 3.0000 & 1.050 & A & A \\
\hline BX & 1 & 24.6000 & 1.9000 & 1.230 & A & A \\
\hline$C D$ & 1 & 21.0000 & 1.0000 & 1.050 & A & A \\
\hline $\mathrm{CH}$ & 1 & 23.2000 & 0.9000 & 1.160 & $\mathrm{~A}$ & A \\
\hline CL & 1 & 23.3000 & 2.6000 & 1.165 & W & A \\
\hline $\mathrm{CN}$ & 1 & 19.9400 & 1.2000 & 0.997 & A & A \\
\hline $\mathrm{CO}$ & 1 & 23.0000 & 3.0000 & 1.150 & A & A \\
\hline CR & 1 & 25.0000 & 3.0000 & 1.250 & W & W \\
\hline $\mathrm{CS}$ & 1 & 18.2400 & 0.6676 & 0.912 & A & A \\
\hline EG & 1 & 18.9000 & 2.3000 & 0.945 & A & A \\
\hline FL & 1 & 20.2000 & 0.5000 & 1.010 & A & A \\
\hline $\mathrm{FN}$ & 1 & 20.7000 & 1.7000 & 1.035 & W & A \\
\hline GA & 1 & 26.6000 & 4.3000 & 1.330 & A & W \\
\hline $\mathrm{GC}$ & 1 & 21.2000 & 2.5000 & 1.060 & A & A \\
\hline GE & 1 & 19.3491 & 4.4226 & 0.967 & A & A \\
\hline GP & 1 & 21.0000 & 3.0000 & 1.050 & $\mathrm{~A}$ & A \\
\hline GT & 1 & 25.0000 & 5.0000 & 1.250 & A & W \\
\hline $\mathrm{HU}$ & 1 & 21.5000 & 0.5000 & 1.075 & A & A \\
\hline IA & 1 & 15.7000 & 0.7000 & 0.785 & W & W \\
\hline IA & 2 & 16.7000 & 0.7000 & 0.835 & W & W \\
\hline IA & 3 & 16.6000 & 0.7000 & 0.830 & W & W \\
\hline ID & 1 & 17.9600 & 0.9400 & 0.898 & A & A \\
\hline IE & 1 & 27.2000 & 1.6700 & 1.360 & A & W \\
\hline IL & 1 & 18.8000 & 2.5000 & 0.940 & W & A \\
\hline
\end{tabular}

Values for elemental uranium are reported in $\mu \mathrm{g} /$ filter, $\mathrm{g}$ or $\mathrm{mL}$.

pCi/g or $\mathrm{mL}=\mathrm{Bq} \times 0.027$

Evaluation: $A=$ Acceptable, $W=$ Acceptable with Warning, $N=$ Not Acceptable

If the evaluation system is not appropriate for the types of analyses performed in your lab, apply site specific evaluation. 


\section{QAP 49 Results by Nuclide}

\begin{tabular}{|c|c|c|c|c|c|c|}
\hline \multicolumn{3}{|c|}{$\begin{array}{l}\text { Matrix: VE Vegetation } \mathrm{Bq} / \mathrm{kg} \\
\text { Radionuclide: } \\
\text { CO60 }\end{array}$} & \multirow[b]{3}{*}{$\begin{array}{l}\text { Reported } \\
\text { Error }\end{array}$} & \multirow[b]{3}{*}{$\frac{\text { Reported }}{\text { EML }}$} & \multirow[b]{3}{*}{$\begin{array}{c}\text { QAP } 48 \\
\text { Evaluation }\end{array}$} & \multirow[b]{3}{*}{ Evaluation } \\
\hline $\begin{array}{l}\text { EML Value: } \\
\text { EML Error: }\end{array}$ & \multicolumn{2}{|c|}{$\begin{array}{r}20.000 \\
1.000\end{array}$} & & & & \\
\hline Labcode & Test \# & $\begin{array}{l}\text { Reported } \\
\text { Value }\end{array}$ & & & & \\
\hline IN & 1 & 21.2000 & 4.0000 & 1.060 & $\mathrm{~A}$ & A \\
\hline IS & 1 & 23.2000 & 5.5000 & 1.160 & W & $\mathrm{A}$ \\
\hline $\mathrm{IT}$ & 1 & 24.7000 & 2.4000 & 1.235 & W & $\mathrm{A}$ \\
\hline $\mathrm{KR}$ & 1 & 20.4000 & 1.4000 & 1.020 & A & A \\
\hline $\mathrm{LA}$ & 1 & 25.8000 & 3.1000 & 1.290 & A & $\mathrm{W}$ \\
\hline $\mathrm{LA}$ & 2 & 26.8000 & 3.2000 & 1.340 & $\mathrm{~A}$ & $\mathrm{~W}$ \\
\hline LA & 3 & 25.0000 & 2.7000 & 1.250 & A & W \\
\hline $\mathrm{LB}$ & 1 & 34.0000 & 4.0000 & 1.700 & & $N$ \\
\hline LL & 1 & 22.3000 & 3.5200 & 1.115 & A & A \\
\hline LV & 1 & 20.0000 & 0.7000 & 1.000 & A & A \\
\hline MA & 1 & 24.0000 & 3.3000 & 1.200 & W & A \\
\hline $\mathrm{ME}$ & 1 & 21.5000 & 0.7900 & 1.075 & W & A \\
\hline $\mathrm{MH}$ & 1 & 20.7100 & 0.8100 & 1.035 & $\mathrm{~A}$ & A \\
\hline $\mathrm{NA}$ & 1 & 22.2000 & 0.7000 & 1.110 & W & A \\
\hline $\mathrm{NP}$ & 1 & 17.5000 & 0.6000 & 0.875 & A & $\mathrm{A}$ \\
\hline NR & 1 & 19.8000 & 4.0000 & 0.990 & A & A \\
\hline $\mathrm{NZ}$ & 1 & 20.8000 & 1.2000 & 1.040 & & $\mathrm{~A}$ \\
\hline $\mathrm{OB}$ & 1 & 38.9000 & 9.1400 & 1.945 & & $\mathrm{~N}$ \\
\hline$\widehat{O C}$ & 1 & 17.0000 & 5.0000 & 0.850 & & W \\
\hline $\mathrm{OC}$ & 2 & 16.0000 & 5.0000 & 0.800 & & W \\
\hline $\mathrm{OC}$ & 3 & 16.0000 & 5.0000 & 0.800 & & W \\
\hline OL & 1 & 20.5900 & 1.0900 & 1.030 & $\mathrm{~A}$ & A \\
\hline OT & 1 & 19.0000 & 3.0000 & 0.950 & A & A \\
\hline OU & 1 & 20.1000 & 1.4900 & 1.005 & W & A \\
\hline PO & 1 & 19.8000 & 1.2000 & 0.990 & A & A \\
\hline RA & 1 & 19.4000 & 1.2000 & 0.970 & A & A \\
\hline RA & 2 & 19.9000 & 1.2000 & 0.995 & A & A \\
\hline $\mathrm{RE}$ & 1 & 19.3000 & 2.7000 & 0.965 & A & A \\
\hline RI & 1 & 35.0000 & 6.7400 & 1.750 & $\mathrm{~N}$ & $\mathrm{~N}$ \\
\hline $\mathrm{SB}$ & 1 & 21.2000 & 2.8100 & 1.060 & & A \\
\hline SN & 1 & 23.5890 & 2.7420 & 1.179 & A & $\mathrm{A}$ \\
\hline SR & 1 & 23.9000 & 0.7000 & 1.195 & A & $\mathrm{A}$ \\
\hline SW & 1 & 27.5000 & 1.4000 & 1.375 & $\mathrm{~N}$ & W \\
\hline $\mathrm{TE}$ & 1 & 18.1000 & 1.5000 & 0.905 & A & A \\
\hline TI & 1 & 21.9000 & 2.2000 & 1.095 & $\mathrm{~A}$ & A \\
\hline TM & 1 & 21.5000 & 3.7500 & 1.075 & $\mathrm{~N}$ & A \\
\hline $\mathrm{TN}$ & 1 & 0.2200 & 0.0300 & 0.011 & W & $\mathrm{N}$ \\
\hline TO & 1 & 13.6000 & 2.4000 & 0.680 & A & $\mathrm{N}$ \\
\hline $\mathrm{TP}$ & 1 & 18.0700 & 0.0500 & 0.903 & A & A \\
\hline TW & 1 & 21.0000 & 0.6000 & 1.050 & $\mathrm{~A}$ & A \\
\hline $\mathrm{TX}$ & 1 & 23.2000 & 1.1000 & 1.160 & A & A \\
\hline UC & 1 & 2.2300 & 0.1900 & 0.112 & A & $\mathrm{N}$ \\
\hline
\end{tabular}

Values for elemental uranium are reported in $\mu \mathrm{g} /$ filter, $\mathrm{g}$ or $\mathrm{mL}$.

$\mathrm{pCi} / \mathrm{g}$ or $\mathrm{mL}=\mathrm{Bq} \times 0.027$

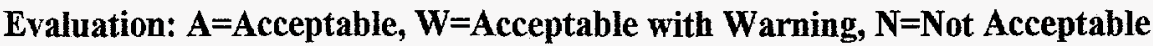

If the evaluation system is not appropriate for the types of analyses performed in your lab, apply site specific evaluation. 


\section{QAP 49 Results by Nuclide}

\begin{tabular}{lr}
\hline Matrix: VE & $\begin{array}{r}\text { Vegetatio } \\
\text { Radionuclide: }\end{array}$ \\
\hline COML Value: & 20.000 \\
EML Error: & 1.000 \\
\hline
\end{tabular}

\begin{tabular}{ccccccc} 
Labcode & Test \# & $\begin{array}{c}\text { Reported } \\
\text { Value }\end{array}$ & $\begin{array}{c}\text { Reported } \\
\text { Error }\end{array}$ & $\begin{array}{c}\text { Reported } \\
\text { EML }\end{array}$ & $\begin{array}{c}\text { QAP 48 } \\
\text { Evaluation }\end{array}$ & Evaluation \\
\hline WA & 1 & 21.0000 & 1.5000 & 1.050 & $\mathrm{~A}$ & $\mathrm{~A}$ \\
WC & 1 & 23.0000 & 0.2000 & 1.150 & $\mathrm{~A}$ & $\mathrm{~A}$ \\
WE & 1 & 21.9000 & 2.7000 & 1.095 & $\mathrm{~A}$ & $\mathrm{~A}$ \\
YA & 1 & 20.5905 & 0.6475 & 1.030 & $\mathrm{~A}$ & $\mathrm{~A}$ \\
YU & 1 & 17.8000 & 1.2000 & 0.890 & $\mathrm{~W}$
\end{tabular}

Total Number Reported:

88

Values for elemental uranium are reported in $\mu \mathrm{g} /$ filter, $\mathrm{g}$ or $\mathrm{mL}$.

$\mathrm{pCi} / \mathrm{g}$ or $\mathrm{mL}=\mathrm{Bq} \times 0.027$

Evaluation: $A=$ Acceptable, $W=$ Acceptable with Warning, $N=$ Not Acceptable

If the evaluation system is not appropriate for the types of analyses performed in your lab, apply site specific evaluation. 


\section{QAP 49 Results by Nuclide}

\begin{tabular}{l} 
Matrix: VE \\
Radionuclide: \\
\hline
\end{tabular}

EML Value: $\quad 390.000$

EML Error: $\quad 20.000$

\begin{tabular}{|c|c|c|c|c|c|c|}
\hline Labcode & Test \# & $\begin{array}{l}\text { Reported } \\
\text { Value }\end{array}$ & $\begin{array}{c}\text { Reported } \\
\text { Error }\end{array}$ & $\frac{\text { Reported }}{E M L}$ & $\begin{array}{c}\text { QAP } 48 \\
\text { Evaluation }\end{array}$ & Evaluation \\
\hline $\mathrm{AF}$ & 1 & 444.0000 & 58.8300 & 1.138 & W & $\mathrm{A}$ \\
\hline $\mathrm{AG}$ & 1 & 480.0000 & 81.0000 & 1.231 & A & A \\
\hline $\mathrm{AI}$ & 1 & 459.0000 & 5.2900 & 1.177 & $\mathrm{~N}$ & A \\
\hline $\overrightarrow{\mathrm{AL}}$ & 1 & 763.8000 & 0.1000 & 1.958 & $\mathrm{~N}$ & $\mathrm{~N}$ \\
\hline AM & 1 & 432.8500 & 6.2800 & 1.110 & A & A \\
\hline $\mathrm{AT}$ & 1 & 396.6000 & 33.2000 & 1.017 & & $\mathrm{~A}$ \\
\hline $\mathrm{AU}$ & 1 & 446.0000 & 25.0000 & 1.144 & A & A \\
\hline BA & 1 & 420.0000 & 20.0000 & 1.077 & A & A \\
\hline$B C$ & 1 & 447.0000 & 29.6000 & 1.146 & A & A \\
\hline $\mathrm{BE}$ & 1 & 447.0000 & 50.0000 & 1.146 & A & A \\
\hline $\mathrm{BL}$ & 1 & 438.0000 & 16.0000 & 1.123 & A & A \\
\hline $\mathrm{BM}$ & 1 & 431.0000 & 4.0000 & 1.105 & A & A \\
\hline $\mathrm{BN}$ & 1 & 432.5300 & 1.4900 & 1.109 & A & A \\
\hline $\mathrm{BN}$ & 2 & 435.8600 & 1.4900 & 1.118 & A & A \\
\hline $\mathrm{BN}$ & 3 & 432.9000 & 1.4900 & 1.110 & A & A \\
\hline $\mathrm{BP}$ & 1 & 374.0000 & 8.0000 & 0.959 & A & A \\
\hline $\mathrm{BQ}$ & 1 & 434.0000 & 17.0000 & 1.113 & $\mathrm{~A}$ & A \\
\hline BÙ & 1 & 430.0000 & 25.0000 & 1.103 & W & A \\
\hline BX & 1 & 480.0000 & 33.3000 & 1.231 & A & A \\
\hline$C D$ & 1 & 419.0000 & 3.0000 & 1.074 & A & A \\
\hline $\mathrm{CH}$ & 1 & 457.0000 & 2.3000 & 1.172 & A & $\mathrm{A}$ \\
\hline $\mathrm{CL}$ & 1 & 366.0000 & 14.6000 & 0.938 & W & A \\
\hline $\mathrm{CN}$ & 1 & 437.5000 & 27.8500 & 1.122 & A & A \\
\hline $\mathrm{CO}$ & 1 & 434.0000 & 8.0000 & 1.113 & A & A \\
\hline CR & 1 & 464.0000 & 19.0000 & 1.190 & A & A \\
\hline $\mathrm{CS}$ & 1 & 355.8000 & 15.4100 & 0.912 & A & A \\
\hline $\mathrm{EG}$ & 1 & 397.0000 & 9.0000 & 1.018 & A & A \\
\hline FL & 1 & 402.0000 & 2.0000 & 1.031 & A & A \\
\hline FN & 1 & 388.0000 & 39.0000 & 0.995 & W & A \\
\hline GA & 1 & 432.0000 & 15.0000 & 1.108 & A & A \\
\hline $\mathrm{GC}$ & 1 & 399.2000 & 5.3000 & 1.024 & A & $\mathrm{A}$ \\
\hline GE & 1 & 377.9550 & 54.5604 & 0.969 & A & A \\
\hline GP & 1 & 410.0000 & 40.0000 & 1.051 & A & A \\
\hline GT & 1 & 490.0000 & 100.0000 & 1.256 & A & W \\
\hline $\mathrm{HU}$ & 1 & 430.0000 & 11.0000 & 1.103 & A & A \\
\hline IA & 1 & 370.0000 & 2.0000 & 0.949 & $\mathrm{~N}$ & $\mathrm{~A}$ \\
\hline IA & 2 & 359.0000 & 2.0000 & 0.921 & $\mathrm{~N}$ & A \\
\hline IA & 3 & 361.0000 & 2.0000 & 0.926 & $\mathbf{N}$ & A \\
\hline ID & 1 & 367.2700 & 18.5400 & 0.942 & A & A \\
\hline IE & 1 & 565.7000 & 6.4000 & 1.451 & $\mathrm{~A}$ & $\mathrm{~N}$ \\
\hline IL & 1 & 437.8000 & 10.3000 & 1.123 & $\mathrm{~W}$ & A \\
\hline
\end{tabular}

Values for elemental uranium are reported in $\mu \mathrm{g} /$ filter, $\mathrm{g}$ or $\mathrm{mL}$.

$\mathrm{pCi} / \mathrm{g}$ or $\mathrm{mL}=\mathrm{Bq} \times 0.027$

Evaluation: $A=$ Acceptable, $W=$ Acceptable with Warning, $N=$ Not Acceptable

If the evaluation system is not appropriate for the types of analyses performed in your lab, apply site specific evaluation. 


\section{QAP 49 Results by Nuclide}

\begin{tabular}{lc}
\hline Matrix: VE & $\begin{array}{c}\text { Vegetation Bq } / \mathrm{kg} \\
\text { Radionuclide: }\end{array}$ \\
\hline CS137
\end{tabular}

Labcode Test \# $\begin{gathered}\text { Reported } \\ \text { Value }\end{gathered}$

Reported
Error $\frac{\text { Reported }}{E M L} \quad \begin{gathered}\text { QAP 48 } \\ \text { Evaluation }\end{gathered}$ Evaluation

\begin{tabular}{|c|c|c|c|c|c|c|}
\hline $\mathrm{IN}$ & 1 & 455.8000 & 18.4000 & 1.169 & A & A \\
\hline IS & 1 & 459.2000 & 54.2000 & 1.177 & A & A \\
\hline IT & 1 & 486.0000 & 20.0000 & 1.246 & A & A \\
\hline KR & 1 & 397.4000 & 10.2000 & 1.019 & A & A \\
\hline LA & 1 & 443.0000 & 36.0000 & 1.136 & A & A \\
\hline LA & 2 & 462.0000 & 37.0000 & 1.185 & A & A \\
\hline LA & 3 & 456.0000 & 37.0000 & 1.169 & A & A \\
\hline LB & 1 & 648.0000 & 52.0000 & 1.662 & & $\mathrm{~N}$ \\
\hline $\mathrm{LL}$ & 1 & 454.0000 & 14.5000 & 1.164 & A & A \\
\hline LV & 1 & 415.0000 & 6.0000 & 1.064 & A & A \\
\hline MA & 1 & 477.0000 & 33.0000 & 1.223 & W & $\mathrm{A}$ \\
\hline ME & 1 & 437.0000 & 20.0000 & 1.121 & $\mathrm{~N}$ & A \\
\hline $\mathrm{MH}$ & 1 & 421.6900 & 20.4400 & 1.081 & A & A \\
\hline NA & 1 & 486.6000 & 3.5000 & 1.248 & A & A \\
\hline $\mathrm{NP}$ & 1 & 334.0000 & 2.0000 & 0.856 & W & W \\
\hline NR & 1 & 414.0000 & 83.0000 & 1.062 & A & A \\
\hline NZ & 1 & 426.0000 & 22.0000 & 1.092 & & A \\
\hline OB & 1 & 632.0000 & 120.0000 & 1.621 & & $\mathrm{~N}$ \\
\hline $\mathrm{OC}$ & 1 & 390.0000 & 20.0000 & 1.000 & & A \\
\hline $\mathrm{OC}$ & 2 & 380.0000 & 20.0000 & 0.974 & & A \\
\hline $\mathrm{OC}$ & 3 & 380.0000 & 20.0000 & 0.974 & & A \\
\hline OL & 1 & 439.6000 & 6.6000 & 1.127 & A & A \\
\hline OT & 1 & 380.0000 & 10.0000 & 0.974 & W & A \\
\hline OU & 1 & 385.0000 & 62.0000 & 0.987 & $\mathrm{~N}$ & A \\
\hline PK & 1 & 430.2400 & 12.2300 & 1.103 & A & A \\
\hline $\mathrm{PO}$ & 1 & 412.0000 & 25.0000 & 1.056 & A & A \\
\hline RA & 1 & 408.0000 & 18.0000 & 1.046 & A & A \\
\hline RA & 2 & 407.0000 & 20.0000 & 1.044 & A & A \\
\hline RE & 1 & 389.0000 & 32.0000 & 0.997 & A & A \\
\hline RI & 1 & 459.0000 & 23.6000 & 1.177 & A & A \\
\hline SB & 1 & 429.0000 & 53.6000 & 1.100 & & A \\
\hline SN & 1 & 485.7570 & 48.6870 & 1.246 & A & A \\
\hline SR & 1 & 460.0000 & 8.8000 & 1.179 & A & A \\
\hline SW & 1 & 489.9500 & 3.3000 & 1.256 & $\mathrm{~N}$ & W \\
\hline TE & 1 & 340.4000 & 4.8000 & 0.873 & A & W \\
\hline TI & 1 & 496.0000 & 49.6000 & 1.272 & A & W \\
\hline TM & 1 & 437.0000 & 14.4000 & 1.121 & A & A \\
\hline TN & 1 & 3.9200 & 0.4000 & 0.010 & W & $\mathrm{N}$ \\
\hline TO & 1 & 291.6000 & 30.6000 & 0.748 & & $\mathrm{~N}$ \\
\hline $\mathrm{TP}$ & 1 . & 373.1700 & 11.7100 & 0.957 & A & A \\
\hline TW & 1 & 455.0000 & 4.0000 & 1.167 & A & A \\
\hline $\mathrm{TX}$ & 1 & 435.0000 & 2.0000 & 1.115 & A & A \\
\hline
\end{tabular}

Values for elemental uranium are reported in $\mu \mathrm{g} /$ filter, $\mathrm{g}$ or $\mathrm{mL}$.

$\mathrm{pCi} / \mathrm{g}$ or $\mathrm{mL}=\mathrm{Bq} \times 0.027$

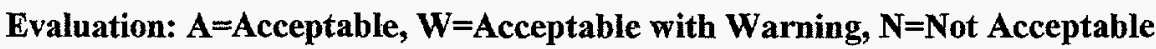

If the evaluation system is not appropriate for the types of analyses performed in your lab, apply site specific evaluation. 


\section{QAP 49 Results by Nuclide}

\begin{tabular}{lr}
\hline Matrix: VE & $\begin{array}{r}\text { Vegetation } \\
\text { Radionuclide: }\end{array}$ \\
\hline CS137 \\
EML Value: & 390.000 \\
EML Error: & 20.000 \\
\hline
\end{tabular}

\begin{tabular}{lcrrrrr} 
Labcode & Test \# & $\begin{array}{c}\text { Reported } \\
\text { Value }\end{array}$ & $\begin{array}{c}\text { Reported } \\
\text { Error }\end{array}$ & $\begin{array}{c}\text { Reported } \\
\text { EML }\end{array}$ & $\begin{array}{c}\text { QAP 48 } \\
\text { Evaluation }\end{array}$ & Evaluation \\
\hline UC & 1 & 44.9000 & 4.6200 & 0.115 & $\mathrm{~A}$ & $\mathrm{~N}$ \\
WA & 1 & 409.0000 & 30.0000 & 1.049 & $\mathrm{~A}$ & $\mathrm{~A}$ \\
WC & 1 & 460.0000 & 69.0000 & 1.179 & $\mathrm{~A}$ & $\mathrm{~A}$ \\
WE & 1 & 469.0000 & 120.0000 & 1.203 & & $\mathrm{~A}$ \\
YA & 1 & 415.8800 & 2.7195 & 1.066 & $\mathrm{~A}$ & $\mathrm{~A}$ \\
YU & 1 & 367.0000 & 20.0000 & 0.941 & $\mathrm{~W}$ & $\mathrm{~A}$
\end{tabular}

Total Number Reported: 


\section{QAP 49 Results by Nuclide}

Matrix: VE Vegetation $\mathrm{Bq} / \mathrm{kg}$ Radionuclide: $\quad$ K40

\begin{tabular}{lr}
\hline EML Value: & 460.000 \\
EML Error: & 20.000 \\
\hline
\end{tabular}

Labcode Test \# $\begin{gathered}\text { Reported } \\ \text { Value }\end{gathered}$

\section{Reported Error \\ Reported EML}

QAP 48

Evaluation Evaluation

\begin{tabular}{|c|c|c|c|c|}
\hline $\mathrm{AF}$ & 1 & 1400.4500 & 407.3700 & 3.044 \\
\hline $\mathrm{AG}$ & 1 & 516.0000 & 93.0000 & 1.122 \\
\hline $\mathrm{AI}$ & 1 & 646.0000 & 28.3000 & 1.404 \\
\hline $\mathrm{AL}$ & 1 & 870.1400 & 0.7000 & 1.892 \\
\hline $\mathrm{AM}$ & 1 & 547.5400 & 35.5200 & 1.190 \\
\hline $\mathrm{AT}$ & 1 & 514.5000 & 56.3000 & 1.118 \\
\hline $\mathrm{AU}$ & 1 & 559.0000 & 48.0000 & 1.215 \\
\hline $\mathrm{BC}$ & 1 & 559.0000 & 31.5000 & 1.215 \\
\hline $\mathrm{BE}$ & 1 & 601.0000 & 140.0000 & 1.307 \\
\hline $\mathrm{BL}$ & 1 & 483.0000 & 25.0000 & 1.050 \\
\hline $\mathrm{BN}$ & 1 & 451.0300 & 8.9700 & 0.980 \\
\hline $\mathrm{BN}$ & 2 & 429.5700 & 8.9700 & 0.934 \\
\hline $\mathrm{BN}$ & 3 & 444.3700 & 8.9700 & 0.966 \\
\hline BP & 1 & 451.0000 & 9.0000 & 0.980 \\
\hline $\mathrm{BQ}$ & 1 & 721.0000 & 7.0000 & 1.567 \\
\hline $\mathrm{BU}$ & 1 & 530.0000 & 60.0000 & 1.152 \\
\hline BX & 1 & 596.0000 & 34.0000 & 1.296 \\
\hline $\mathrm{CD}$ & 1 & 481.0000 & 1.0000 & 1.046 \\
\hline $\mathrm{CH}$ & 1 & 594.0000 & 13.0000 & 1.291 \\
\hline $\mathrm{CL}$ & 1 & 500.0000 & 45.0000 & 1.087 \\
\hline $\mathrm{CN}$ & 1 & 469.9000 & 40.1200 & 1.022 \\
\hline CR & 1 & 546.0000 & 74.0000 & 1.187 \\
\hline $\mathrm{CS}$ & 1 & 433.8000 & 20.4300 & 0.943 \\
\hline EG & 1 & 483.0000 & 68.0000 & 1.050 \\
\hline $\mathrm{FL}$ & 1 & 499.0000 & 9.0000 & 1.085 \\
\hline $\mathrm{FN}$ & 1 & 452.0000 & 47.0000 & 0.983 \\
\hline GA & 1 & 581.0000 & 79.0000 & 1.263 \\
\hline GC & 1 & 494.0000 & 47.4000 & 1.074 \\
\hline GE & 1 & 468.4200 & 81.0729 & 1.018 \\
\hline GP & 1 & 520.0000 & 50.0000 & 1.130 \\
\hline GT & 1 & 580.0000 & 50.0000 & 1.261 \\
\hline $\mathrm{HU}$ & 1 & 588.0000 & 40.0000 & 1.278 \\
\hline IA & 1 & 397.0000 & 11.0000 & 0.863 \\
\hline LA & 2 & 424.0000 & 11.0000 & 0.922 \\
\hline IA & 3 & 437.0000 & 11.0000 & 0.950 \\
\hline ID & 1 & 403.5300 & 23.8800 & 0.877 \\
\hline ID & 1 & 403.5300 & 23.8800 & 0.877 \\
\hline $\mathrm{IE}$ & 1 & 672.4000 & 28.3000 & 1.462 \\
\hline IL & 1 & 580.1000 & 99.5000 & 1.261 \\
\hline $\mathrm{IN}$ & 1. & 513.6000 & 48.3000 & 1.117 \\
\hline IS & 1 & 563.5000 & 78.8000 & 1.225 \\
\hline
\end{tabular}

W

A

A

$\mathrm{N}$

A

A

A

W

A

A

A

A

A

W

A

W

A

A

A

W

A

A

A

A

A

A

A

A

A

A

W

W

W

A

A

A

$\mathrm{N}$

A

W
$\mathrm{N}$

A

W

$\mathrm{N}$

A

A

A

A

W

A

A

A

A

A

$\mathrm{N}$

A

W

A

W

A

A

A

A

A

A

A

W

A

A

A

W

W

W

A

A

W

W

$\mathrm{N}$

W

A

A

Values for elemental uranium are reported in $\mu \mathrm{g}$ /filter, $\mathrm{g}$ or $\mathrm{mL}$.

$\mathrm{pCi} / \mathrm{g}$ or $\mathrm{mL}=\mathrm{Bq} \times \mathbf{0 . 0 2 7}$

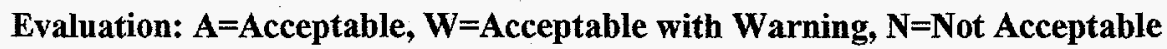

If the evaluation system is not appropriate for the types of analyses performed in your lab, apply site specific evaluation. 


\section{QAP 49 Results by Nuclide}

\begin{tabular}{lr}
\hline Matrix: $\begin{array}{l}\text { VE } \\
\text { Radionuclide: }\end{array}$ & $\begin{array}{c}\text { Kegetation } \mathrm{Bq} / \mathrm{kg} \\
\mathrm{K} 40\end{array}$ \\
\hline EML Value: & 460.000 \\
EML Error: & 20.000 \\
\hline
\end{tabular}

\begin{tabular}{|c|c|c|c|c|c|c|}
\hline Labcode & Test \# & $\begin{array}{l}\text { Reported } \\
\text { Value }\end{array}$ & $\begin{array}{c}\text { Reported } \\
\text { Error }\end{array}$ & $\frac{\text { Reported }}{\text { EML }}$ & $\begin{array}{c}\text { QAP 48 } \\
\text { Evaluation }\end{array}$ & Evaluation \\
\hline IT & 1 & 567.0000 & 8.0000 & 1.233 & A & $\mathrm{A}$ \\
\hline $\mathrm{KR}$ & 1 & 486.8000 & 42.4000 & 1.058 & A & A \\
\hline LA & 1 & 382.0000 & 43.0000 & 0.830 & W & W \\
\hline LA & 2 & 436.0000 & 48.0000 & 0.948 & W & A \\
\hline LA & 3 & 470.0000 & 51.0000 & 1.022 & W & A \\
\hline LB & 1 & 753.0000 & 90.0000 & 1.637 & & $\mathrm{~N}$ \\
\hline LL & 1 & 519.0000 & 76.8000 & 1.128 & A & A \\
\hline LV & 1 & 475.0000 & 27.0000 & 1.033 & $\mathrm{~N}$ & A \\
\hline MA & $i$ & 562.0000 & 64.0000 & 1.222 & $\mathrm{~A}$ & A \\
\hline ME & 1 & 491.0000 & 27.3000 & 1.067 & $\mathrm{~N}$ & A \\
\hline MH & 1 & 505.9900 & 26.5400 & 1.100 & A & A \\
\hline NA & 1 & 544.0000 & 17.0000 & 1.183 & A & A \\
\hline NP & 1 & 462.0000 & 12.0000 & 1.004 & & $\mathrm{~A}$ \\
\hline NR & 1 & 448.0000 & 90.0000 & 0.974 & A & A \\
\hline $\mathrm{OB}$ & 1 & 856.0000 & 188.0000 & 1.861 & & $\mathrm{~N}$ \\
\hline $\mathrm{OC}$ & 1 & 640.0000 & 100.0000 & 1.391 & & W \\
\hline $\mathrm{OC}$ & 2 & 640.0000 & 100.0000 & 1.391 & & W \\
\hline $\mathrm{OC}$ & 3 & 610.0000 & 100.0000 & 1.326 & & W \\
\hline OL & 1 & 527.2000 & 20.1000 & 1.146 & A & A \\
\hline OT & 1 & 480.0000 & 60.0000 & 1.043 & A & A \\
\hline OU & 1 & 416.0000 & 21.8000 & 0.904 & $\mathrm{~N}$ & A \\
\hline PK & 1 & 449.9800 & 50.1500 & 0.978 & A & A \\
\hline PO & 1 & 496.0000 & 22.0000 & 1.078 & A & A \\
\hline RA & 1 & 532.0000 & 69.0000 & 1.157 & A & A \\
\hline RA & 2 & 555.0000 & 60.0000 & 1.207 & $\mathrm{~A}$ & A \\
\hline $\mathrm{RE}$ & 1 & 599.0000 & 65.0000 & 1.302 & $\mathrm{~A}$ & W \\
\hline SB & 1 & 538.0000 & 72.8000 & 1.170 & & A \\
\hline SN & 1 & 611.2960 & 60.0940 & 1.329 & $N$ & W \\
\hline SR & 1 & 563.0000 & 16.6000 & 1.224 & A & A \\
\hline SW & 1 & 592.5000 & 26.8000 & 1.288 & $\mathrm{~N}$ & W \\
\hline TE & 1 & 417.5000 & 28.2000 & 0.908 & A & A \\
\hline TI & 1 & 523.0000 & 52.3000 & 1.137 & A & A \\
\hline TM & 1 & 574.0000 & 85.7000 & 1.248 & A & W \\
\hline TN & 1 & 4.8500 & 1.0000 & 0.011 & W & $\mathrm{N}$ \\
\hline TO & 1 & 358.9000 & 52.9000 & 0.780 & A & $N$ \\
\hline $\mathrm{TP}$ & 1 & 458.7800 & 4.0900 & 0.997 & A & A \\
\hline TW & 1 & 540.0000 & 20.0000 & 1.174 & A & A \\
\hline TX & 1 & 518.0000 & 20.0000 & 1.126 & A & A \\
\hline UC & 1 & 53.1100 & 4.8200 & 0.115 & A & $\mathrm{N}$ \\
\hline WA & 1 & 470.0000 & 31.0000 & 1.022 & A & A \\
\hline WC & 1 & 589.0000 & 73.0000 & 1.280 & A & W \\
\hline WE & 1 & 522.0000 & 39.0000 & 1.135 & & A \\
\hline
\end{tabular}

Values for elemental uranium are reported in $\mu \mathrm{g} /$ filter, $\mathrm{g}$ or $\mathrm{mL}$.

$\mathrm{pCi} / \mathrm{g}$ or $\mathrm{mL}=\mathrm{Bq} \times 0.027$

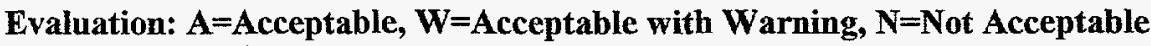

If the evaluation system is not appropriate for the types of analyses performed in your lab, apply site specific evaluation. 


\section{QAP 49 Results by Nuclide}

Matrix: VE Vegetation $\mathrm{Bq} / \mathrm{kg}$

Radionuclide:

$\mathrm{K} 40$

EML Value: $\quad 460.000$

EML Error: $\quad 20.000$

\begin{tabular}{ccccccc} 
Labcode & Test \# & $\begin{array}{c}\text { Reported } \\
\text { Value }\end{array}$ & $\begin{array}{c}\text { Reported } \\
\text { Error }\end{array}$ & $\begin{array}{c}\text { Reported } \\
\text { EML }\end{array}$ & $\begin{array}{c}\text { QAP 48 } \\
\text { Evaluation }\end{array}$ & Evaluation \\
\hline YA & 1 & 511.3400 & 13.5050 & 1.112 & $\mathrm{~A}$ & $\mathrm{~A}$ \\
YU & 1 & 535.0000 & 20.0000 & 1.163 & $\mathrm{~W}$ & $\mathrm{~A}$
\end{tabular}

Total Number Reported:

85

Values for elemental uranium are reported in $\mu \mathrm{g} /$ filter, $g$ or $\mathrm{mL}$.

$\mathrm{pCi} / \mathrm{g}$ or $\mathrm{mL}=\mathrm{Bq} \times 0.027$

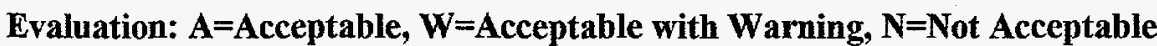

If the evaluation system is not appropriate for the types of analyses performed in your lab, apply site specific evaluation. 


\section{QAP 49 Results by Nuclide}

Matrix: VE Vegetation $\mathrm{Bq} / \mathrm{kg}$

Radionuclide: $\quad$ PU238

\begin{tabular}{ll}
\hline EML Value: & 0.310 \\
EML Error: & 0.070 \\
\hline
\end{tabular}

Reported

Reported Reported

QAP 48

Labcode Test \# Value

Error

EML

Evaluation

Evaluation

$\begin{array}{lll}\text { EG } & 1 & 0.3000 \\ \text { GP } & 1 & 0.2600 \\ \text { NA } & 1 & 0.5100 \\ \text { RA } & 1 & 0.3000\end{array}$

0.0400

0.968

$0.1500 \quad 0.839$

1.645

0.1000

0.968

A

A

0.1000

A

A

A

A

Total Number Reported:

Values for elemental uranium are reported in $\mu \mathrm{g} /$ filter, $\mathrm{g}$ or $\mathrm{mL}$.

$\mathrm{pCi} / \mathrm{g}$ or $\mathrm{mL}=\mathrm{Bq} \times 0.027$

Evaluation: $A=A c c e p t a b l e, ~ W=A c c e p t a b l e$ with Warning, $N=$ Not Acceptable

If the evaluation system is not appropriate for the types of analyses performed in your lab, apply site specific evaluation. 


\section{QAP 49 Results by Nuclide}

\begin{tabular}{lc}
\hline Matrix: VE & $\begin{array}{r}\text { Vegetation } \\
\text { Radionuclide: }\end{array}$ \\
\hline PU239 \\
EML Value: & 3.720 \\
EML Error: & 0.270 \\
\hline
\end{tabular}

Labcode Test \# $\begin{gathered}\text { Reported } \\ \text { Value }\end{gathered}$

Reported
Error

0.7400

0.5600

0.2700

0.6300

0.4600

0.4600

0.2400

0.3700

0.7300

0.4000

0.2500

0.0100

0.1900

1.9000

0.4200

0.3550

0.3500

1.0910

0.6000

0.5000

0.5050

1.6190

0.1700

0.6200

0.4000

0.3300

0.3000

0.3000

0.7000

0.7000

0.4300

1.1830

0.6550

0.3700

0.7000

0.3610

0.7000

0.2300

0.4000

0.4000

0.0987
1.094

1.056

0.927

0.586

1.000

1.210

1.175

1.062

0.987

1.102

1.156

0.134

1.040

1.102

1.167

1.097

1.022

1.353

0.914

1.156

0.960

1.672

1.056

0.919

1.169

1.239

1.048

1.156

1.183

1.183

1.032

0.883

1.072

0.718

0.887

1.110

0.941

1.043

1.175

1.075

1.043
QAP 48

Evaluation Evaluation

WC

4.0000

3.8813

1.043

$\mathrm{N}$

A

A

A

A

A

A

A

A

A

W

A

W

A

A

A

W

A

A

W

A

A

A

A

A

W

A

A

A

$\mathrm{N}$

$\mathrm{N}$

A

A

A

A

A

A
A

A

A

$\mathrm{N}$

A

A

A

A

A

A

A

$\mathrm{N}$

A

A

A

A

A

W

A

A

A

$\mathrm{N}$

A

A

A

W

A

A

A

A

A

A

A

W

A

A

A.

A

A

A

A

Values for elemental uranium are reported in $\mu \mathrm{g} /$ filter, $\mathrm{g}$ or $\mathrm{mL}$.

$\mathrm{pCi} / \mathrm{g}$ or $\mathrm{mL}=\mathrm{Bq} \times 0.027$

Evaluation: $A=A c c e p t a b l e, W=A c c e p t a b l e$ with Warning, $N=$ Not Acceptable

If the evaluation system is not appropriate for the types of analyses performed in your lab, apply site specific evaluation. 


\section{QAP 49 Results by Nuclide}

Matrix: VE Vegetation $\mathrm{Bq} / \mathrm{kg}$

Radionuclide: PU239

EML Value: $\quad 3.720$

EML Error: $\quad 0.270$

Labcode Test \# $\begin{gathered}\text { Reported } \\ \text { Value }\end{gathered} \quad \begin{gathered}\text { Reported } \\ \text { Error }\end{gathered} \frac{\text { Reported }}{\text { EML }} \quad \begin{gathered}\text { QAP 48 } \\ \text { Evaluation }\end{gathered} \quad$ Evaluation

Total Number Reported:

Values for elemental uranium are reported in $\mu \mathrm{g} /$ filter, $\mathrm{g}$ or $\mathrm{mL}$.

$\mathrm{pCi} / \mathrm{g}$ or $\mathrm{mL}=\mathrm{Bq} \times 0.027$

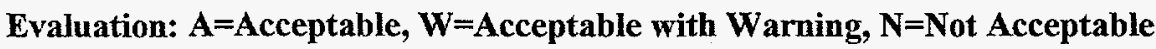

If the evaluation system is not appropriate for the types of analyses performed in your lab, apply site specific evaluation. 


\section{QAP 49 Results by Nuclide}

Matrix: VE Vegetation $\mathrm{Bq} / \mathrm{kg}$

Radionuclide: $\quad$ SR90

EML Value: $\quad 606.000$

EML Error: $\quad 40.000$

Labcode Test \# $\begin{gathered}\text { Reported } \\ \text { Value }\end{gathered}$

Reported Error
Reported EML
QAP 48 Evaluation
Evaluation

\begin{tabular}{|c|c|c|c|c|c|}
\hline $\mathrm{AF}$ & 1 & 551.3000 & 22.2000 & 0.910 & $\mathrm{~N}$ \\
\hline $\mathrm{AG}$ & 1 & 644.0000 & 115.0000 & 1.063 & A \\
\hline AI & 1 & 4.6100 & 0.0341 & 0.008 & \\
\hline AM & 1 & 406.2100 & 73.9900 & 0.670 & \\
\hline $\mathrm{AU}$ & 1 & 624.0000 & 16.0000 & 1.030 & A \\
\hline $\mathrm{BC}$ & 1 & 337.8000 & 22.7000 & 0.557 & W \\
\hline $\mathrm{BE}$ & 1 & 635.0000 & 39.0000 & 1,048 & A \\
\hline BL & 1 & 417.0000 & 18.0000 & 0.688 & A \\
\hline BM & 1 & 663.0000 & 12.0000 & 1.094 & A \\
\hline BP & 1 & 651.0000 & 88.0000 & 1.074 & A \\
\hline $\mathrm{BU}$ & 1 & 678.0000 & 34.0000 & 1.119 & A \\
\hline BX & 1 & 370.0000 & 27.7000 & 0.611 & W \\
\hline $\mathrm{CH}$ & 1 & 613.0000 & 7.9000 & 1.012 & A \\
\hline CL & 1 & 610.0000 & 30.0000 & 1.007 & $\mathrm{~N}$ \\
\hline EG & 1 & 718.0000 & 21.0000 & 1.185 & A \\
\hline GE & 1 & 588.2390 & 7.9497 & 0.971 & A \\
\hline GP & 1 & 580.0000 & 50.0000 & 0.957 & A \\
\hline GT & 1 & 440.0000 & 10.0000 & 0.726 & A \\
\hline ID & 1 & 586.3300 & 29.5300 & 0.968 & A \\
\hline IS & 1 & 416.2500 & 83.2500 & 0.687 & W \\
\hline $\mathrm{KR}$ & 1 & 714.4000 & 7.5000 & 1.179 & A \\
\hline LA & 1 & 620.0000 & 39.4000 & 1.023 & \\
\hline LA & 2 & 671.0000 & 41.7000 & 1.107 & \\
\hline LA & 3 & 622.4000 & 39.2000 & 1.027 & \\
\hline NA & 1 & 627.0000 & 12.0000 & 1.035 & A \\
\hline $\mathrm{NZ}$ & 1 & 577.0000 & 30.0000 & 0.952 & \\
\hline NZ & 2 & 487.0000 & 24.0000 & 0.804 & \\
\hline $\mathrm{OC}$ & 1 & 510.0000 & 25.0000 & 0.842 & \\
\hline $\mathrm{OC}$ & 2 & 440.0000 & 22.0000 & 0.726 & \\
\hline $\mathrm{OC}$ & 3 & 500.0000 & 25.0000 & 0.825 & \\
\hline OT & 1 & 580.0000 & 20.0000 & 0.957 & A \\
\hline RA & 1 & 590.0000 & 100.0000 & 0.974 & A \\
\hline $\mathrm{RE}$ & 1 & 587.0000 & 8.0000 & 0.969 & A \\
\hline RI & 1 & 292.0000 & 16.9000 & 0.482 & A \\
\hline SR & 1 & 754.8000 & 51.8000 & 1.246 & $\mathrm{~N}$ \\
\hline SW & 1 & 157,9600 & 25.0000 & 0.261 & $\mathrm{~N}$ \\
\hline TE & 1 & 672.5000 & 32.5000 & 1.110 & A \\
\hline TI & 1 & 520.0000 & 10.0000 & 0.858 & $\mathrm{~N}$ \\
\hline $\mathrm{TM}$ & 1 & 33.8000 & 2.3500 & 0.056 & A \\
\hline TN & 1 & 678.0000 & 68.0000 & 1.119 & $\mathrm{~A}$ \\
\hline TO & 1 & 519.0000 & 13.6000 & 0.856 & $\mathrm{~N}$ \\
\hline
\end{tabular}

Values for elemental uranium are reported in $\mu \mathrm{g} /$ filter, $\mathrm{g}$ or $\mathbf{~ m L}$.

$\mathrm{pCi} / \mathrm{g}$ or $\mathrm{mL}=\mathrm{Bq} \times 0.027$

Evaluation: $A=A c c e p t a b l e, W=A c c e p t a b l e$ with Warning, $N=$ Not Acceptable

If the evaluation system is not appropriate for the types of analyses performed in your lab, apply site specific evaluation. 


\section{QAP 49 Results by Nuclide}

Matrix: VE Vegetation $\mathrm{Bq} / \mathrm{kg}$

Radionuclide: $\quad$ SR90

EML Value: $\quad 606.000$

EML Error: $\quad 40.000$

\begin{tabular}{lcccccc} 
Labcode & Test \# & $\begin{array}{c}\text { Reported } \\
\text { Value }\end{array}$ & $\begin{array}{c}\text { Reported } \\
\text { Error }\end{array}$ & $\begin{array}{c}\text { Reported } \\
\text { EML }\end{array}$ & $\begin{array}{c}\text { QAP 48 } \\
\text { Evaluation }\end{array}$ & Evaluation \\
\hline TP & 1 & 644.1600 & 25.7900 & 1.063 & $\mathrm{~A}$ & $\mathrm{~A}$ \\
TX & 1 & 643.0000 & 33.0000 & 1.061 & $\mathrm{~A}$ & $\mathrm{~A}$ \\
WA & 1 & 709.0000 & 22.0000 & 1.170 & $\mathrm{~A}$ & $\mathrm{~W}$ \\
WC & 1 & 667.0000 & 86.0000 & 1.101 & $\mathrm{~A}$ & $\mathrm{~A}$ \\
WE & 1 & 316.0000 & 32.0000 & 0.521 & & $\mathrm{~W}$ \\
YA & 1 & 615.8033 & 31.9433 & 1.016 & $\mathrm{~A}$ & $\mathrm{~A}$
\end{tabular}

Total Number Reported:

Values for elemental uranium are reported in $\mu \mathrm{g} /$ filter, $\mathrm{g}$ or $\mathrm{mL}$.

$\mathrm{pCi} / \mathrm{g}$ or $\mathrm{mL}=\mathrm{Bq} \times 0.027$

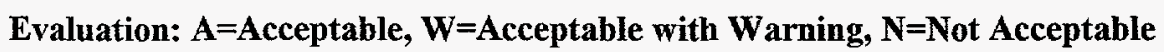

If the evaluation system is not appropriate for the types of analyses performed in your lab, apply site specific evaluation. 
QAP 49 Results by Nuclide

Matrix: WA Water $\mathrm{Bq} / \mathrm{L}$

Radionuclide: $\quad$ AM241

\begin{tabular}{ll}
\hline EML Value: & 1.250 \\
EML Error: & 0.080 \\
\hline
\end{tabular}

Reported Value

Reported Error
Reported EML
QAP 48

Evaluation
Evaluation

\begin{tabular}{|c|c|c|c|c|c|c|}
\hline $\mathrm{AF}$ & 1 & 1.0500 & 0.1700 & 0.840 & A & W \\
\hline$A G$ & 1 & 1.2800 & 0.1600 & 1.024 & A & A \\
\hline $\mathrm{AI}$ & 1 & 0.9600 & 0.0130 & 0.768 & & W \\
\hline AM & 1 & 2.1800 & 0.5300 & 1.744 & A & $\mathrm{N}$ \\
\hline AN & 1 & 1.2500 & 0.0300 & 1.000 & A & A \\
\hline $\mathrm{AT}$ & 1 & 1.3000 & 0.2210 & 1.040 & A & A \\
\hline $\mathrm{AU}$ & 1 & 1.3500 & 0.1200 & 1.080 & W & A \\
\hline $\mathrm{BE}$ & 1 & 1.4000 & 0.1400 & 1.120 & A & A \\
\hline BL & 1 & 1.5100 & 0.2300 & 1.208 & A & A \\
\hline BL & 2 & 1.2700 & 0.2200 & 1.016 & A & A \\
\hline BM & 1 & 1.2200 & 0.1800 & 0.976 & A & A \\
\hline BP & 1 & 1.4300 & 0.0400 & 1.144 & A & A \\
\hline BU & 1 & 1.3900 & 0.0900 & 1,112 & A & A \\
\hline BX & 1 & 1.2700 & 0.1400 & 1.016 & A & A \\
\hline $\mathrm{CH}$ & 1 & 1.3100 & 0.0250 & 1.048 & & A \\
\hline CL & 1 & 1.3000 & 0.2000 & 1.040 & W & A \\
\hline $\mathrm{CS}$ & 1 & 1.2750 & 0.1082 & 1.020 & A & A \\
\hline EG & 1 & 1.2400 & 0.1000 & 0.992 & A & A \\
\hline EP & 1 & 1.3200 & 0.1200 & 1.056 & A & A \\
\hline FG & 1 & 1.6380 & 0.0630 & 1.310 & $\mathrm{~N}$ & W \\
\hline FL & 1 & 1.6000 & 0.4000 & 1.280 & W & W \\
\hline$F M$ & 1 & 1.3000 & 0.1100 & 1.040 & & A \\
\hline GA & 1 & 1.4000 & 0.2100 & 1.120 & A & A \\
\hline $\mathrm{GE}$ & 1 & 1.2303 & 0.2394 & 0.984 & A & A \\
\hline GP & 1 & 1.4000 & 0.3000 & 1.120 & A & A \\
\hline GT & 1 & 1.2000 & 0.4000 & 0.960 & A & A \\
\hline HT & 1 & 2.6000 & 0.2000 & 2.080 & & $\mathrm{~N}$ \\
\hline IE & 1 & 1.9100 & 1.3800 & 1.528 & A & $\mathrm{N}$ \\
\hline $\mathrm{IN}$ & 1 & 1.2600 & 0.1200 & 1.008 & A & A \\
\hline IS & 1 & 1.0320 & 0.2490 & 0.826 & W & W \\
\hline $\mathrm{IT}$ & 1 & 1.4100 & 0.0800 & 1.128 & A & A \\
\hline JL & 1 & 0.7500 & 0.3600 & 0.600 & W & $\mathrm{N}$ \\
\hline LA & 1 & 1.3000 & 0.0400 & 1.040 & A & A \\
\hline LA & 2 & 1.1800 & 0.0400 & 0.944 & A & A \\
\hline LA & 3 & 1.3400 & 0.0400 & 1.072 & A & A \\
\hline LL & 1 & 1.3500 & 0.1190 & 1.080 & A & A \\
\hline LV & 1 & 1.4700 & 0.2400 & 1.176 & W & A \\
\hline $\mathrm{ME}$ & 1 & 1.2500 & 0.3200 & 1.000 & A & A \\
\hline MS & 1 & 1.5900 & 0.2000 & 1.272 & A & A \\
\hline NM & 1 & 1.2500 & 0.0330 & 1.000 & A & A \\
\hline NQ & 1 & 1.1000 & 0.0900 & 0.880 & & W \\
\hline
\end{tabular}

Values for elemental uranium are reported in $\mu$ gffilter, $g$ or $\mathrm{mL}$.

$\mathrm{pCi} / \mathrm{g}$ or $\mathrm{mL}=\mathrm{Bq} \times 0.027$

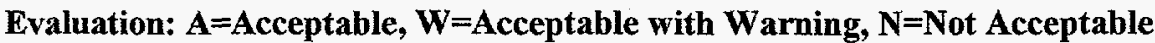

If the evaluation system is not appropriate for the types of analyses performed in your lab, apply site specific evaluation. 


\section{QAP 49 Results by Nuclide}

\begin{tabular}{|c|c|c|c|c|c|c|}
\hline $\begin{array}{l}\text { Matrix: V } \\
\text { Radionuclide }\end{array}$ & VA Wate & $\begin{array}{l}\mathrm{Bq} / \mathrm{L} \\
\mathrm{M} 241\end{array}$ & & & & \\
\hline $\begin{array}{l}\text { EML Value: } \\
\text { EML Error: }\end{array}$ & & $\begin{array}{l}50 \\
80\end{array}$ & & & & \\
\hline Labcode & Test \# & $\begin{array}{l}\text { Reported } \\
\text { Value }\end{array}$ & $\begin{array}{c}\text { Reported } \\
\text { Error }\end{array}$ & $\frac{\text { Reported }}{E M L}$ & $\begin{array}{c}\text { QAP 48 } \\
\text { Evaluation }\end{array}$ & Evaluation \\
\hline OD & 1 & 1.3160 & 0.1330 & 1.053 & A & A \\
\hline $\mathrm{OD}$ & 1 & 1.3200 & 0.1300 & 1.056 & A & A \\
\hline OT & 1 & 1.2000 & 0.1000 & 0.960 & W & A \\
\hline OU & 1 & 1.2700 & 0.1900 & 1.016 & & A \\
\hline PR & 1 & 1.5365 & 0.0038 & 1.229 & & $\mathrm{~A}$ \\
\hline $\mathrm{RE}$ & 1 & 1.3200 & 0.1200 & 1.056 & A & A \\
\hline RI & 1 & 1.3000 & 0.1040 & 1.040 & A & A \\
\hline SB & 1 & 1.3900 & 0.7800 & 1.112 & & A \\
\hline SK & 1 & 1.1700 & 0.2200 & 0.936 & A & $\mathrm{A}$ \\
\hline SN & 1 & 1.2950 & 0.2020 & 1.036 & A & A \\
\hline SR & 1 & 1.6900 & 0.0830 & 1.352 & & W \\
\hline SW & 1 & 1.6300 & 0.0800 & 1.304 & A & W \\
\hline $\mathrm{TI}$ & 1 & 1.3000 & 0.2000 & 1.040 & A & A \\
\hline $\mathrm{TN}$ & 1 & 1.2400 & 0.1200 & 0.992 & A & A \\
\hline TO & 1 & 1.4000 & 0.3000 & 1.120 & $\mathrm{~A}$ & A \\
\hline TX & 1 & 1.3200 & 0.0600 & 1.056 & W & A \\
\hline UP & 1 & 1.4000 & 0.1920 & 1.120 & $\mathrm{~N}$ & A \\
\hline UY & 1 & 1.2600 & 0.1500 & 1.008 & A & A \\
\hline WA & 1 & 1.0900 & 0.1100 & 0.872 & A & W \\
\hline WC & 1 & 1.4000 & 0.2300 & 1.120 & A & A \\
\hline YA & 1 & 1.2395 & 0.0395 & 0.992 & A & A \\
\hline
\end{tabular}

Total Number Reported:

Values for elemental uranium are reported in $\mu \mathrm{g} /$ filter, $\mathrm{g}$ or $\mathrm{mL}$.

$\mathrm{pCi} / \mathrm{g}$ or $\mathrm{mL}=\mathrm{Bq} \times 0.027$

Evaluation: $A=$ Acceptable, $W=A c c e p t a b l e$ with Warning, $N=$ Not Acceptable

If the evaluation system is not appropriate for the types of analyses performed in your lab, apply site specific evaluation. 


\section{QAP 49 Results by Nuclide}

December, 1998

\begin{tabular}{lr}
\hline Matrix: WA & $\begin{array}{r}\text { Water } \\
\text { Radionuclide: }\end{array}$ \\
\hline $\mathrm{Bq}$
\end{tabular}

\begin{tabular}{|c|c|c|c|c|c|c|}
\hline Labcode & Test \# & $\begin{array}{l}\text { Reported } \\
\text { Value }\end{array}$ & $\begin{array}{c}\text { Reported } \\
\text { Error }\end{array}$ & $\frac{\text { Reported }}{E M L}$ & $\begin{array}{c}\text { QAP } 48 \\
\text { Evaluation }\end{array}$ & Evalua \\
\hline $\mathrm{AF}$ & 1 & 1.1700 & 0.1100 & 1.114 & & A \\
\hline $\mathrm{AG}$ & 1 & 1.0800 & 0.1000 & 1.029 & A & $\mathrm{A}$ \\
\hline AI & 1 & 0.8600 & 0.0430 & 0.819 & $\mathrm{~A}$ & W \\
\hline $\mathrm{AM}$ & 1 & 1.0600 & 0.2200 & 1.010 & A & A \\
\hline $\mathrm{BP}$ & 1 & 1.1300 & 0.0300 & 1.076 & & A \\
\hline $\mathrm{BU}$ & 1 & 1.1600 & 0.0600 & 1.105 & A & A \\
\hline $\mathrm{CH}$ & 1 & 1.0900 & 0.0390 & 1.038 & & A \\
\hline $\mathrm{CL}$ & 1 & 1.0000 & 0.2000 & 0.952 & W & A \\
\hline $\mathrm{FG}$ & 1 & 1.0580 & 0.3700 & 1.008 & W & A \\
\hline $\mathrm{GP}$ & 1 & 0.9700 & & 0.924 & $\mathrm{~A}$ & A \\
\hline HT & 1 & 1.6700 & 0.1670 & 1.590 & & $\mathrm{~N}$ \\
\hline ID & 1 & 1.1300 & 0.0680 & 1.076 & A & A \\
\hline $\mathrm{IT}$ & 1 & 1.0500 & 0.1000 & 1.000 & & A \\
\hline LA & 1 & 1.0250 & 0.2500 & 0.976 & & A \\
\hline LA & 2 & 1.0250 & 0.2500 & 0.976 & & A \\
\hline LA & 3 & 1.0500 & 0.2500 & 1.000 & & A \\
\hline NL & 1 & 1.2100 & 0.1200 & 1.152 & & A \\
\hline NS & 1 & 0.7670 & 0.2270 & 0.730 & & $\mathrm{~W}$ \\
\hline OT & 1 & 0.8300 & 0.0800 & 0.790 & A & W \\
\hline UP & 1 & 1.4500 & 0.2120 & 1.381 & & W \\
\hline UY & 1 & 1.1200 & 0.1500 & 1.067 & A & $\mathrm{A}$ \\
\hline WA & 1 & 1.1300 & 0.1000 & 1.076 & $\mathrm{~A}$ & A \\
\hline
\end{tabular}

Total Number Reported:

Values for elemental uranium are reported in $\mu \mathrm{g} /$ filter, $\mathrm{g}$ or $\mathrm{mL}$.

$\mathrm{pCi} / \mathrm{g}$ or $\mathrm{mL}=\mathrm{Bq} \times 0.027$

Evaluation: $A=$ Acceptable, $W=$ Acceptable with Warning, $N=$ Not Acceptable

If the evaluation system is not appropriate for the types of analyses performed in your lab, apply site specific evaluation. 


\section{QAP 49 Results by Nuclide}

\begin{tabular}{|c|c|c|c|c|c|c|}
\hline $\begin{array}{l}\text { Matrix: } \\
\text { Radionuclid }\end{array}$ & $\begin{array}{l}\text { VA Wate } \\
\text { e: }\end{array}$ & $\begin{array}{l}\mathrm{Bq} / \mathrm{L} \\
060\end{array}$ & & & & \\
\hline $\begin{array}{l}\text { EML Value: } \\
\text { EML Error: }\end{array}$ & $\begin{array}{r}49 . \\
1.2\end{array}$ & & & & & \\
\hline Labcode & Test \# & $\begin{array}{l}\text { Reported } \\
\text { Value }\end{array}$ & $\begin{array}{c}\text { Reported } \\
\text { Error }\end{array}$ & $\frac{\text { Reported }}{\text { EML }}$ & $\begin{array}{c}\text { QAP 48 } \\
\text { Evaluation }\end{array}$ & Evaluation \\
\hline $\mathrm{AF}$ & 1 & 0.2100 & 0.0200 & 0.004 & $\mathrm{~N}$ & $\mathrm{~N}$ \\
\hline $\mathrm{AG}$ & 1 & 53.4000 & 8.5000 & 1.081 & A & $\mathrm{A}$ \\
\hline $\mathrm{AI}$ & 1 & 54.5000 & 0.4430 & 1.103 & A & A \\
\hline $\mathrm{AL}$ & 1 & 71.4700 & 0.2000 & 1.447 & W & $\mathrm{N}$ \\
\hline $\mathrm{AM}$ & 1 & 49.5700 & 0.6300 & 1.003 & W & A \\
\hline AN & 1 & 50.6000 & 2.1000 & 1.024 & A & A \\
\hline $\mathrm{AT}$ & 1 & 48.9800 & 4.4600 & 0.991 & W & A \\
\hline $\mathrm{AU}$ & 1 & 52.0000 & 1.5000 & 1.053 & W & A \\
\hline $\mathrm{BA}$ & 1 & 50.3000 & 3.3000 & 1.018 & A & A \\
\hline $\mathrm{BC}$ & 1 & 51.5000 & 2.6000 & 1.043 & A & A \\
\hline $\mathrm{BE}$ & 1 & 52.2000 & 4.0000 & 1.057 & $\mathrm{~N}$ & A \\
\hline BL & 1 & 51.0000 & 2.1000 & 1.032 & A & A \\
\hline $\mathrm{BM}$ & 1 & 46.5000 & 3.5000 & 0.941 & A & A \\
\hline $\mathrm{BN}$ & 1 & 51.5000 & 1.0900 & 1.043 & A & $\mathrm{A}$ \\
\hline $\mathrm{BN}$ & 2 & 52.8400 & 1.0900 & 1.070 & A & A \\
\hline $\mathrm{BN}$ & 3 & 54.1700 & 1.0900 & 1.097 & A & A \\
\hline BP & 1 & 50.3000 & 0.1000 & 1.018 & A & A \\
\hline$B Q$ & 1 & 49.0000 & 1.0000 & 0.992 & A & A \\
\hline $\mathrm{BU}$ & 1 & 57.0000 & 3.0000 & 1.154 & A & W \\
\hline BX & 1 & 50.5000 & 2.8000 & 1.022 & W & A \\
\hline $\mathrm{CA}$ & 1 & 50.8000 & 5.1000 & 1.028 & A & A \\
\hline $\mathrm{CD}$ & 1 & 51.0000 & 0.5000 & 1.032 & A & A \\
\hline $\mathrm{CH}$ & 1 & 52.7000 & 0.7400 & 1.067 & & A \\
\hline CL & 1 & 49.7000 & 2.0000 & 1.006 & A & A \\
\hline $\mathrm{CM}$ & 1 & 47.9000 & 1.5000 & 0.970 & & A \\
\hline $\mathrm{CM}$ & 2 & 48.8000 & 1.6000 & 0.988 & & A \\
\hline $\mathrm{CM}$ & 3 & 48.5000 & 1.6000 & 0.982 & & A \\
\hline $\mathrm{CS}$ & 1 & 50.6600 & 1.6060 & 1.026 & A & A \\
\hline CS & 1 & 50.6600 & 1.6060 & 1.026 & A & A \\
\hline $\mathrm{DH}$ & 1 & 49.1000 & 2.1000 & 0.994 & $\mathrm{~N}$ & A \\
\hline EG & 1 & 50.5000 & 1.1000 & 1.022 & A & A \\
\hline EP & 1 & 50.5700 & 6.6000 & 1.024 & W & A \\
\hline FG & 1 & 50.7600 & 0.4000 & 1.028 & A & A \\
\hline FL & 1 & 51.3000 & 0.4000 & 1.038 & A & A \\
\hline FM & 1 & 49.7000 & 0.3200 & 1.006 & $\mathrm{~A}$ & A \\
\hline FN & 1 & 47.5000 & 3.5000 & 0.962 & A & A \\
\hline GA & 1 & 46.8000 & 4.6000 & 0.947 & W & $\mathrm{A}$ \\
\hline $\mathrm{GC}$ & 1 & 51.2000 & 2.5300 & 1.036 & A & A \\
\hline GE & 1 & 53.5020 & 7.4932 & 1.083 & A & A \\
\hline GP & 1 & 51.0000 & 5.0000 & 1.032 & $N$ & A \\
\hline GT & 1 & 50.0000 & 6.0000 & 1.012 & $\mathrm{~A}$ & $\mathrm{~A}$ \\
\hline
\end{tabular}

Values for elemental uranium are reported in $\mu \mathrm{g} /$ filter, $\mathrm{g}$ or $\mathrm{mL}$.

$\mathrm{pCi} / \mathrm{g}$ or $\mathrm{mL}=\mathrm{Bq} \times 0.027$

Evaluation: A=Acceptable, $W=$ Acceptable with Warning, $N=$ Not Acceptable

If the evaluation system is not appropriate for the types of analyses performed in your lab, apply site specific evaluation. 


\section{QAP 49 Results by Nuclide}

\begin{tabular}{|c|c|c|c|c|c|c|}
\hline \multicolumn{3}{|c|}{ Matrix: WA Water $\mathrm{Bq} / \mathrm{L}$} & \multirow[b]{3}{*}{$\begin{array}{l}\text { Reported } \\
\text { Error }\end{array}$} & \multirow[b]{3}{*}{$\frac{\text { Reported }}{E M L}$} & \multirow[b]{3}{*}{$\begin{array}{c}\text { QAP 48 } \\
\text { Evaluation }\end{array}$} & \multirow[b]{3}{*}{ Evaluation } \\
\hline $\begin{array}{l}\text { EML Value: } \\
\text { EML Error: }\end{array}$ & $\begin{array}{r}49 . \\
1.2\end{array}$ & 200 & & & & \\
\hline Labcode & Test \# & $\begin{array}{l}\text { Reported } \\
\text { Value }\end{array}$ & & & & \\
\hline ID & 1 & 51.5400 & 2.7500 & 1.043 & A & A \\
\hline IE & 1 & 51.9800 & 1.2000 & 1.052 & $\mathrm{~A}$ & $\mathrm{~A}$ \\
\hline IL & 1 & 52.2000 & 0.6000 & 1.057 & A & A \\
\hline $\mathrm{IN}$ & 1 & 51.2000 & 3.4000 & 1.036 & $\mathrm{~W}$ & A \\
\hline IS & 1 & 55.1000 & 5.4000 & 1.115 & W & A \\
\hline IT & 1 & 53.4000 & 3.0000 & 1.081 & $\mathrm{~W}$ & A \\
\hline $\mathrm{JE}$ & 1 & 91.6700 & 2.7100 & 1.856 & $\mathrm{~N}$ & $\mathrm{~N}$ \\
\hline $\mathrm{JL}$ & 1 & 50.9000 & 2.5000 & 1.030 & $\mathrm{~N}$ & A \\
\hline $\mathrm{KA}$ & 1 & 47.9900 & 1.6900 & 0.971 & A & A \\
\hline $\mathrm{LA}$ & 1 & 56.9000 & 6.1000 & 1.152 & $\mathrm{~W}$ & $\mathrm{~W}$ \\
\hline LA & 2 & 54.3000 & 5.8000 & 1.099 & W & A \\
\hline LA & 3 & 52.1000 & 5.7000 & 1.055 & W & A \\
\hline LB & 1 & 51.0000 & 9.0000 & 1.032 & & A \\
\hline LL & 1 & 49.1000 & 3.2400 & 0.994 & A & A \\
\hline $\mathrm{LN}$ & 1 & 50.3000 & 2.8900 & 1.018 & $\mathrm{~N}$ & A \\
\hline LV & 1 & 50.1000 & 1.1000 & 1.014 & W & $\mathrm{A}$ \\
\hline $\mathrm{ME}$ & 1 & 53.0000 & 1.5000 & 1.073 & A & A \\
\hline $\mathrm{MH}$ & 1 & 50.0300 & 1.5400 & 1.013 & $\mathrm{~W}$ & A \\
\hline MS & 1 & 52.5000 & 5.3000 & 1.063 & A & A \\
\hline NA & 1 & 49.8000 & 0.6000 & 1.008 & A & A \\
\hline ND & 1 & 49.5860 & 1.7750 & 1.004 & & $\mathrm{~A}$ \\
\hline NL & 1 & 50.1000 & 6.1000 & 1.014 & W & A \\
\hline NP & 1 & 52.2000 & 1.0000 & 1.057 & W & A \\
\hline NQ & 1 & 60.4000 & 6.6000 & 1.223 & & $\mathrm{~N}$ \\
\hline NS & 1 & 53.4690 & 0.5930 & 1.082 & W & A \\
\hline $\mathrm{NZ}$ & 1 & 50.0000 & 3.0000 & 1.012 & & A \\
\hline $\mathrm{NZ}$ & 2 & 48.0000 & 3.0000 & 0.972 & & A \\
\hline $\mathrm{OB}$ & 1 & 51.4000 & 8.8800 & 1.040 & & A \\
\hline $\mathrm{OC}$ & 1 & 54.0000 & 5.0000 & 1.093 & & A \\
\hline $\mathrm{OC}$ & 2 & 53.0000 & 5.0000 & 1.073 & & A \\
\hline $\mathrm{OC}$ & 3 & 50.0000 & 5.0000 & 1.012 & & $\mathrm{~A}$ \\
\hline$O D$ & 1 & 50.6600 & 2.1700 & 1.026 & A & A \\
\hline OD & 1 & 50.6600 & 2.1700 & 1.026 & A & A \\
\hline$O D$ & 2 & 51.7500 & 2.5100 & 1.048 & A & A \\
\hline OD & 2 & 51.7500 & 2.5100 & 1.048 & $\mathrm{~A}$ & $\mathrm{~A}$ \\
\hline $\mathrm{OL}$ & 1 & 50.4600 & 1.5800 & 1.021 & A & A \\
\hline$\overline{\mathrm{OT}}$ & 1 & 52.0000 & 2.0000 & 1.053 & $\mathrm{~A}$ & $\mathrm{~A}$ \\
\hline OU & 1 & 51.8000 & 1.6000 & 1.049 & $\mathrm{~N}$ & A \\
\hline PR & 1 & 53.1893 & 0.3080 & 1.077 & & A \\
\hline $\mathrm{RC}$ & 1 & 48.5000 & 2.5000 & 0.982 & A & A \\
\hline $\mathrm{RE}$ & 1. & 51.6000 & 5.2000 & 1.045 & A & A \\
\hline RI & 1 & 52.1000 & 3.5900 & 1.055 & $\mathrm{~N}$ & A \\
\hline
\end{tabular}

Values for elemental uranium are reported in $\mu \mathrm{g}$ /filter, $\mathrm{g}$ or $\mathrm{mL}$.

$\mathrm{pCi} / \mathrm{g}$ or $\mathrm{mL}=\mathrm{Bq} \times 0.027$

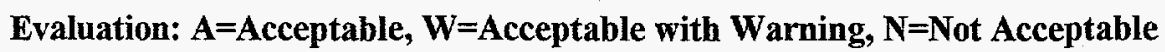

If the evaluation system is not appropriate for the types of analyses performed in your lab, apply site specific evaluation. 


\section{QAP 49 Results by Nuclide}

\begin{tabular}{|c|c|c|c|c|c|c|}
\hline \multicolumn{3}{|c|}{ Matrix: WA Water $\mathrm{Bq} / \mathrm{L}$} & \multirow[b]{3}{*}{$\begin{array}{c}\text { Reported } \\
\text { Error }\end{array}$} & \multirow[b]{3}{*}{$\frac{\text { Reported }}{E M L}$} & \multirow[b]{3}{*}{$\begin{array}{c}\text { QAP } 48 \\
\text { Evaluation }\end{array}$} & \multirow[b]{3}{*}{ Evaluation } \\
\hline $\begin{array}{l}\text { EML Value: } \\
\text { EML Error: }\end{array}$ & \multicolumn{2}{|c|}{$\begin{array}{r}49.400 \\
1.200\end{array}$} & & & & \\
\hline Labcode & Test \# & $\begin{array}{l}\text { Reported } \\
\text { Value }\end{array}$ & & & & \\
\hline $\mathrm{SA}$ & 1 & 53.0000 & 2.4000 & 1.073 & A & $\mathrm{A}$ \\
\hline SB & 1 & 59.1000 & 5.4100 & 1.196 & & W \\
\hline SK & 1 & 49.5000 & 4.2000 & 1.002 & A & $\mathrm{A}$ \\
\hline SL & 1 & 49.0000 & 3.0000 & 0.992 & A & A \\
\hline SN & 1 & 49.9820 & 4.4770 & 1.012 & A & A \\
\hline SR & 1 & 50.6000 & 0.9000 & 1.024 & A & A \\
\hline SW & 1 & 52.3400 & 0.0300 & 1.060 & A & A \\
\hline $\mathrm{TE}$ & 1 & 49.3000 & 2.8000 & 0.998 & A & A \\
\hline TI & 1 & 49.0000 & 4.9000 & 0.992 & A & A \\
\hline $\mathrm{TM}$ & 1 & 53.4000 & 1.7200 & 1.081 & W & A \\
\hline $\mathrm{TN}$ & 1 & 0.0980 & 0.0100 & 0.002 & $\mathrm{~A}$ & $\mathrm{~N}$ \\
\hline TO & 1 & 50.1000 & 3.7000 & 1.014 & A & A \\
\hline $\mathrm{TP}$ & 1 & 44.8700 & 0.4000 & 0.908 & A & A \\
\hline TT & 1 & 54.0000 & 3.2000 & 1.093 & W & A \\
\hline TW & 1 & 49.2000 & 0.7000 & 0.996 & $\mathrm{~A}$ & $\mathrm{~A}$ \\
\hline TX & 1 & 51.9000 & 0.4000 & 1.051 & A & A \\
\hline UC & 1 & 52.2500 & 5.2800 & 1.058 & A & A \\
\hline UP & 1 & 54.1000 & 1.5900 & 1.095 & A & A \\
\hline UY & 1 & 50.2000 & 4.0000 & 1.016 & $\mathrm{~A}$ & A \\
\hline WA & 1 & 50.1000 & 1.5000 & 1.014 & $\mathrm{~A}$ & A \\
\hline WC & 1 & 59.0000 & 4.6000 & 1.194 & A & W \\
\hline WE & 1 & 53.4000 & 2.6000 & 1.081 & & $\mathrm{~A}$ \\
\hline WI & 1 & 55.9800 & 4.2430 & 1.133 & W & A \\
\hline WI & 2 & 55.8500 & 4.2290 & 1.131 & W & A \\
\hline WI & 3 & 55.7200 & 4.2230 & 1.128 & W & A \\
\hline WV & 1 & 52.0600 & 0.5400 & 1.054 & $\mathrm{~A}$ & A \\
\hline YA & 1 & 49.0127 & 0.7523 & 0.992 & A & A \\
\hline YU & 1 & 48.3000 & 1.5000 & 0.978 & $\mathrm{~A}$ & A \\
\hline
\end{tabular}

Total Number Reported:

Values for elemental uranium are reported in $\mu \mathrm{g} /$ filter, $\mathrm{g}$ or $\mathrm{mL}$.

pCi/g or $\mathrm{mL}=\mathrm{Bq} \times 0.027$

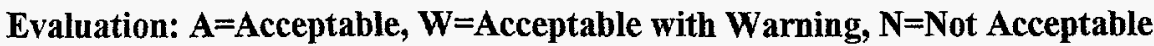

If the evaluation system is not appropriate for the types of analyses performed in your lab, apply site specific evaluation. 


\section{QAP 49 Results by Nuclide}

\begin{tabular}{lc}
\hline Matrix: WA & Water $\mathrm{Bq} /$ \\
Radionuclide: & CS 137 \\
\hline EML Value: & 50.000 \\
EML Error: & 1.700 \\
\hline
\end{tabular}

Labcode Test \# $\begin{gathered}\text { Reported } \\ \text { Value }\end{gathered}$

Reported
Error

0.2000

51.6000

50.2000

79.0250

53.3900

50.8000

47.8200

52.0000

53.3000

55.3000

53.1000

52.0000

49.3000

61.2400

61.6800

61.3100

52.1000

45.0000

47.0000

54.2000

51.0000

51.0000

53.4000

50.7000

48.3000

49.4000

49.6000

50.7500

49.3000

51.3000

50.1900

46.1600

52.7000

51.2000

49.6000

51.0000

54.0000

52.5585

52.0000

52.0000

51.3900
0.0300

8.5000

4.7400

0.2000

0.7500

0.8000

4.2200

2.6000

7.0000

4.2000

4.0000

2.1000

3.3000

0.1900

0.1900

0.1900

1.0000

1.0000

3.0000

3.9000

5.1000

0.5000

0.6400

1.5000

1.7000

1.7000

1.6000

2.1970

1.9000

1.1000

6.5000

0.8500

0.6000

0.3600

5.1000

4.0000

3.1400

7.2378

5.0000

8.0000

2.6300

\section{Reported EML}

QAP 48

Evaluation Evaluation

GT

ID

1

Values for elemental uranium are reported in $\mu \mathrm{g} / \mathrm{filter}, \mathrm{g}$ or $\mathrm{mL}$.

0.004

1.032

1.004

1.581

1.068

1.016

0.956

1.040

1.066

1.106

1.062

1.040

0.986

1.225

1.234

1.226

1.042

0.900

0.940

1.084

1.020

1.020

1.068

1.014

0.966

0.988

0.992

1.015

0.986

1.026

1.004

0.923

1.054

1.024

0.992

1.020

1.080

1.051

1.040

1.040

1.028
A

A

A

W

W

A

A

A

A

A

A

A

$\mathrm{N}$

$\mathrm{N}$

A

A

A

A

A

A

A

A

A

A

A

A

A

A

A

A

A

A

A

A

A
$\mathrm{N}$

A

A

$\mathrm{N}$

A

A

A

A

A

A

A

A

A

W

W

W

A

A

A

A

A

A

A

A

A

A

A

A

A

A

A

A

A

A

A

A

A

A

A

A

A

Evaluation: $A=A c c e p t a b l e, W=A c c e p t a b l e$ with Warning, $N=$ Not Acceptable

If the evaluation system is not appropriate for the types of analyses performed in your lab, apply site specific evaluation. 


\section{QAP 49 Results by Nuclide}

\begin{tabular}{|c|c|c|c|c|c|c|}
\hline \multicolumn{3}{|c|}{ Matrix: WA Water $\mathrm{Bq} / \mathrm{L}$} & \multirow[b]{3}{*}{$\begin{array}{c}\text { Reported } \\
\text { Error }\end{array}$} & \multirow[b]{3}{*}{$\frac{\text { Reported }}{\text { EML }}$} & \multirow[b]{3}{*}{$\begin{array}{c}\text { QAP 48 } \\
\text { Evaluation }\end{array}$} & \multirow[b]{3}{*}{ Evaluation } \\
\hline $\begin{array}{l}\text { EML Value: } \\
\text { EML Error: }\end{array}$ & \multicolumn{2}{|c|}{$\begin{array}{r}50.000 \\
1.700 \\
\end{array}$} & & & & \\
\hline Labcode & Test \# & $\begin{array}{l}\text { Reported } \\
\text { Value }\end{array}$ & & & & \\
\hline IE & 1 & 54.7700 & 1.5300 & 1.095 & A & $\mathrm{A}$ \\
\hline$\overline{\mathrm{IL}}$ & 1 & 52.3000 & 0.8000 & 1.046 & A & A \\
\hline $\mathbb{N}$ & 1 & 52.0000 & 3.2000 & 1.040 & A & A \\
\hline IS & 1 & 55.9000 & 5.7000 & 1.118 & W & A \\
\hline IT & 1 & 51.8000 & 2.5000 & 1.036 & A & A \\
\hline $\mathrm{JE}$ & 1 & 92.4500 & 3.5100 & 1.849 & A & $\mathrm{N}$ \\
\hline $\mathrm{JL}$ & 1 & 50.6000 & 3.5000 & 1.012 & A & A \\
\hline $\mathrm{KA}$ & 1 & 48.4200 & 3.3100 & 0.968 & A & A \\
\hline LA & 1 & 60.1000 & 6.3000 & 1.202 & W & W \\
\hline $\mathrm{LA}$ & 2 & 58.8000 & 6.2000 & 1.176 & W & A \\
\hline LA & 3 & 58.8000 & 6.2000 & 1.176 & W & A \\
\hline LB & 1 & 52.0000 & 7.0000 & 1.040 & & A \\
\hline $\mathrm{LL}$ & 1 & 49.4000 & 3.5600 & 0.988 & A & $A$ \\
\hline $\mathrm{LN}$ & 1 & 61.6000 & 8.9300 & 1.232 & $\mathrm{~N}$ & W \\
\hline LV & 1 & 51.3000 & 1.2000 & 1.026 & A & A \\
\hline $\mathrm{ME}$ & 1 & 55.2000 & 2.5300 & 1.104 & A & A \\
\hline MH & 1 & 50.7300 & 2.5300 & 1.015 & A & A \\
\hline MS & 1 & 52.1000 & 5.2000 & 1.042 & A & A \\
\hline NA & 1 & 52.6000 & 0.8000 & 1.052 & A & A \\
\hline ND & 1 & 47.6300 & 2.4890 & 0.953 & & A \\
\hline NL & 1 & 50.6000 & 8.5000 & 1.012 & W & A \\
\hline NM & 1 & 54.4600 & 2.8000 & 1.089 & W & A \\
\hline NP & 1 & 48.8000 & 1.1000 & 0.976 & A & A \\
\hline NQ & 1 & 61.0000 & 6.9000 & 1,220 & & W \\
\hline NS & 1 & 53.3330 & 0.5180 & 1.067 & A & A \\
\hline NZ & 1 & 53.0000 & 3.0000 & 1.060 & & A \\
\hline $\mathrm{NZ}$ & 2 & 50.0000 & 3.0000 & 1.000 & & A \\
\hline $\mathrm{OB}$ & 1 & 58.0000 & 11.2000 & 1.160 & & A \\
\hline $\mathrm{OC}$ & 1 & 50.0000 & 5.0000 & 1.000 & & A \\
\hline $\mathrm{OC}$ & 2 & 50.0000 & 5.0000 & 1.000 & & A \\
\hline $\mathrm{OC}$ & 3 & 50.0000 & 5.0000 & 1.000 & & A \\
\hline OD & 1 & 51.0200 & 3.3700 & 1.020 & A & A \\
\hline OD & 1 & 51.0200 & 3.3700 & 1.020 & A & A \\
\hline$O D$ & 2 & 58.5300 & 4.0000 & 1.171 & A & A \\
\hline OD & 2 & 58.5300 & 4.0000 & 1.171 & A & A \\
\hline $\mathrm{OL}$ & 1 & 50.1200 & 1.2900 & 1.002 & A & A \\
\hline OT & 1 & 53.0000 & 2.0000 & 1.060 & A & A \\
\hline $\mathrm{OU}$ & 1 & 44.5000 & 1.1000 & 0.890 & A & W \\
\hline PR & 1 & 52.0870 & 0.2837 & 1.042 & & A \\
\hline $\mathrm{RC}$ & 1 & 48.7000 & 2.5000 & 0.974 & A & A \\
\hline $\mathrm{RE}$ & 1 & 51.8000 & 5.1000 & 1.036 & A & A \\
\hline RI & 1 & 62.8000 & 4.8600 & 1.256 & $\mathrm{~A}$ & W \\
\hline
\end{tabular}

Values for elemental uranium are reported in $\mu \mathrm{g} /$ filter, $\mathrm{g}$ or $\mathrm{mL}$.

$\mathrm{pCi} / \mathrm{g}$ or $\mathrm{mL}=\mathrm{Bq} \times 0.027$

Evaluation: $A=$ Acceptable, $W=$ Acceptable with Warning, $N=$ Not Acceptable

If the evaluation system is not appropriate for the types of analyses performed in your lab, apply site specific evaluation. 


\section{QAP 49 Results by Nuclide}

Matrix: WA Water $\mathrm{Bq} / \mathrm{L}$ Radionuclide: $\quad \operatorname{CS} 137$

\begin{tabular}{|c|c|c|c|c|c|c|}
\hline \multirow{2}{*}{$\begin{array}{l}\text { EML Value: } \\
\text { EML Error: } \\
\text { Labcode }\end{array}$} & \multicolumn{2}{|c|}{$\begin{array}{r}50.000 \\
1.700\end{array}$} & \multirow[b]{2}{*}{$\begin{array}{c}\text { Reported } \\
\text { Error }\end{array}$} & \multirow[b]{2}{*}{$\frac{\text { Reported }}{\text { EML }}$} & \multirow[b]{2}{*}{$\begin{array}{c}\text { QAP } 48 \\
\text { Evaluation }\end{array}$} & \multirow[b]{2}{*}{ Evaluation } \\
\hline & Test \# & $\begin{array}{c}\text { Reported } \\
\text { Value }\end{array}$ & & & & \\
\hline $\mathrm{SA}$ & 1 & 53.0000 & 2.8000 & 1.060 & A & $\mathrm{A}$ \\
\hline SB & 1 & 61.6000 & 8.8900 & 1.232 & & $W$ \\
\hline SK & 1 & 49.3000 & 3.0000 & 0.986 & A & A \\
\hline $\mathrm{SL}$ & 1 & 50.0000 & 3.0000 & 1.000 & A & A \\
\hline SN & 1 & 47.8850 & 4.2800 & 0.958 & $\mathrm{~A}$ & A \\
\hline SR & 1 & 51.0000 & 1.3500 & 1.020 & A & A \\
\hline SW & 1 & 54.7600 & 0.0400 & 1.095 & A & A \\
\hline $\mathrm{TE}$ & 1 & 50.1000 & 3.2000 & 1.002 & A & A \\
\hline$\overline{\mathrm{TI}}$ & 1 & 51.1000 & 5.1000 & 1.022 & A & A \\
\hline TM & 1 & 55.8000 & 1.7400 & 1.116 & $W$ & A \\
\hline $\mathrm{TN}$ & 1 & 0.0980 & 0.0100 & 0.002 & $\mathrm{~A}$ & $\mathrm{~N}$ \\
\hline TO & 1 & 49.7000 & 4.9000 & 0.994 & A & A \\
\hline TP & 1 & 47.7500 & 1.1000 & 0.955 & A & $\mathrm{A}$ \\
\hline $\mathrm{TT}$ & 1 & 55.0000 & 6.8200 & 1.100 & A & $\mathrm{A}$ \\
\hline $\mathrm{TW}$ & 1 & 51.0000 & 1.0000 & 1.020 & A & A \\
\hline $\mathrm{TX}$ & 1 & 52.0000 & 0.5000 & 1.040 & A & A \\
\hline $\mathrm{UC}$ & 1 & 53.2900 & 6.2000 & 1.066 & A & A \\
\hline UP & 1 & 54.6000 & 3.2200 & 1.092 & A & A \\
\hline UY & 1 & 52.7000 & 7.0000 & 1.054 & A & A \\
\hline WA & 1 & 50.0000 & 2.2000 & 1.000 & A & $\mathrm{A}$ \\
\hline WC & 1 & 62.0000 & 8.6000 & 1.240 & A & $W$ \\
\hline WE & 1 & 58.2000 & 6.8000 & 1.164 & & $\mathrm{~A}$ \\
\hline WI & 1 & 56.2600 & 6.9830 & 1.125 & A & A \\
\hline WI & 2 & 55.9600 & 6.9450 & 1.119 & A & A \\
\hline WI & 3 & 56.6200 & 7.0270 & 1.132 & A & A \\
\hline WV & 1 & 50.6200 & 0.4400 & 1.012 & A & A \\
\hline $\mathrm{YA}$ & 1 & 48.9017 & 0.9127 & 0.978 & A & A \\
\hline YU & 1 & 48.2000 & 1.5000 & 0.964 & A & A \\
\hline
\end{tabular}

Total Number Reported:

Values for elemental uranium are reported in $\mu \mathrm{g} /$ filter, $\mathrm{g}$ or $\mathrm{mL}$.

$\mathrm{pCi} / \mathrm{g}$ or $\mathrm{mL}=\mathrm{Bq} \times 0.027$

Evaluation: $A=$ Acceptable, $W=$ Acceptable with Warning, $N=$ Not Acceptable

If the evaluation system is not appropriate for the types of analyses performed in your lab, apply site specific evaluation. 


\section{QAP 49 Results by Nuclide}

\begin{tabular}{|c|c|c|c|c|c|c|}
\hline \multicolumn{3}{|c|}{$\begin{array}{lcc}\text { Matrix: WA } & \text { Water } \mathrm{Bq} / \mathrm{L} \\
\text { Radionuclide: } & \text { FE55 }\end{array}$} & \multirow[b]{3}{*}{$\begin{array}{l}\text { Reported } \\
\text { Error }\end{array}$} & \multirow[b]{3}{*}{$\frac{\text { Reported }}{\text { EML }}$} & \multirow[b]{3}{*}{$\begin{array}{c}\text { QAP } 48 \\
\text { Evaluation }\end{array}$} & \multirow[b]{3}{*}{ Evalu } \\
\hline $\begin{array}{l}\text { EML Value: } \\
\text { EML Error: }\end{array}$ & \multicolumn{2}{|c|}{$\begin{array}{r}139.000 \\
2.000\end{array}$} & & & & \\
\hline Labcode & Test \# & $\begin{array}{l}\text { Reported } \\
\text { Value }\end{array}$ & & & & \\
\hline $\mathrm{BE}$ & 1 & 142.2000 & 6.8000 & 1.023 & & A \\
\hline $\mathrm{BL}$ & 1 & 148.0000 & 5.0000 & 1.065 & W & A \\
\hline BP & 1 & 145.0000 & 10.0000 & 1.043 & A & A \\
\hline $\mathrm{BX}$ & 1 & 116.2000 & 9.7700 & 0.836 & A & A \\
\hline $\mathrm{CH}$ & 1 & 142.0000 & 1.9000 & 1.022 & & A \\
\hline $\mathrm{CL}$ & 1 & 136.0000 & 8.0000 & 0.978 & A & A \\
\hline EG & 1 & 114.0000 & 48.0000 & 0.820 & A & A \\
\hline $\mathrm{GC}$ & 1 & 141.0000 & 3.7000 & 1.014 & A & A \\
\hline GE & 1 & 116.9709 & 21.3281 & 0.842 & A & A \\
\hline GP & 1 & 160.0000 & 20.0000 & 1.151 & $\mathrm{~N}$ & A \\
\hline $\mathrm{KA}$ & 1 & 149.0000 & 6.0000 & 1.072 & W & A \\
\hline $\mathrm{TE}$ & 1 & 140.6000 & 9.2000 & 1.012 & A & A \\
\hline$\overline{T I}$ & 1 & 140.0000 & 20.0000 & 1.007 & W & A \\
\hline $\mathrm{TN}$ & 1 & 148.0000 & 15.0000 & 1.065 & W & \\
\hline To & 1 & 118.2000 & 43.8000 & 0.850 & W & A \\
\hline YA & 1 & 136.5433 & 11.3467 & 0.982 & W & A \\
\hline
\end{tabular}

Total Number Reported: $\quad 16$

Values for elemental uranium are reported in $\mu$ g/filter, $g$ or $\mathrm{mL}$.

$\mathrm{pCi} / \mathrm{g}$ or $\mathrm{mL}=\mathrm{Bq} \times 0.027$

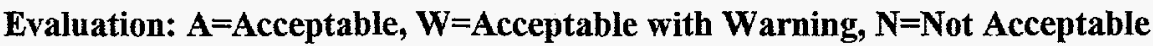

If the evaluation system is not appropriate for the types of analyses performed in your lab, apply site specific evaluation. 


\section{QAP 49 Results by Nuclide}

Matrix: WA Water $\mathrm{Bq} / \mathrm{L}$

Radionuclide:

\begin{tabular}{lr}
\hline EML Value: & 1080.000 \\
EML Error: & 60.000 \\
\hline
\end{tabular}

Reported

Labcode Test \# Value

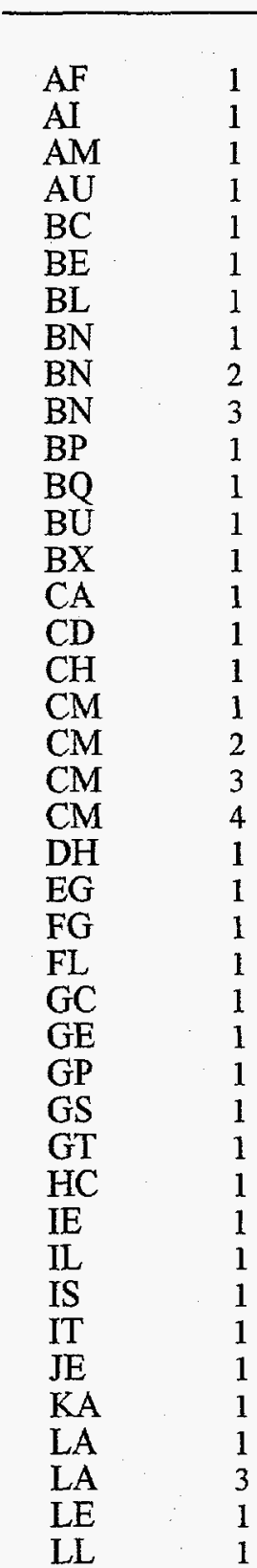

Reported
Error

\begin{abstract}
1110.0000
\end{abstract}
915.0000

1139.3700

991.0000

1113.7000

1045.0000

956.0000

8.4100

8.7200

9.8200

1066.0000

1140.0000

1140.0000

980.5000

180.0000

770.0000

885.0000

977.0000

1121.0000

1069.0000

1073.0000

867.0000

1160.0000

1153.0000

1207.3800

504.0000

1124.8000

980.0000

1208.7000

1000.0000

1012.0000

932.6000

1034.9000

1188.0000

933.0000

967.7500

1052.0000

884.3000

1110.0000

545.0000

819.0000
59.2000

70.1000

7.7400

40.0000

25.6000

71.0000

14.0000

0.6000

0.6000

0.6000

26.0000

40.0000

75.0000

23.8300

20.0000

40.0000

117.0000

26.0000

42.0000

40.0000

40.0000

120.0000

70.0000

53.0000

19.5100

53.2000

46.9900

100.0000

81.0000

50.0000

58.0000

19.9000

29.2000

122.0000

4.0000

97.8000

61.0000

156.8800

193.5100

13.4000 $\frac{\text { Reported }}{\text { EML }}$

1.028

0.847

1.055

0.918

1.031

0.968

0.885

0.008

0.008

0.009

0.987

1.056

1.056

0.908

0.167

0.713

0.819

0.905

1.038

0.990

0.994

0.803

1.074

1.068

1.118

0.467

1.041

0.907

1.119

0.926

0.937

0.864

0.958

1.100

0.864

0.896

0.974

0.819

1.028

0.505

0.758
QAP 48

Evaluation Evaluation

Values for elemental uranium are reported in $\mu \mathrm{g} /$ filter, $\mathrm{g}$ or $\mathrm{mL}$.

A

W

A

W

A

W

A

A

A

A

W

W

W

$\mathrm{N}$

W

W

$\mathrm{N}$

A

W

A

N

A

A

A

A

A

A

A

A

A
A

A

A

A

A

A

A

$\mathrm{N}$

$\mathrm{N}$

$\mathrm{N}$

A

A

A

A

$\mathrm{N}$

W

W

A

A

A

A

W

A

A

A

$\mathrm{N}$

A

A

A

A

A

A

A

A

A

A

A

W

A

$\mathrm{N}$

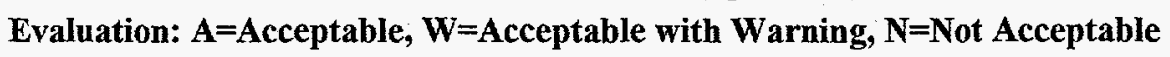

If the evaluation system is not appropriate for the types of analyses performed in your lab, apply site specific evaluation.

$\mathrm{pCi} / \mathrm{g}$ or $\mathrm{mL}=\mathrm{Bq} \times \mathbf{0 . 0 2 7}$ 


\section{QAP 49 Results by Nuclide}

\begin{tabular}{|c|c|c|c|c|c|c|}
\hline $\begin{array}{l}\text { Matrix: Y } \\
\text { Radionuclide }\end{array}$ & $\begin{array}{l}\text { VA Wat } \\
\text { e: }\end{array}$ & $\begin{array}{l}\mathrm{Bq} / \mathrm{L} \\
\mathrm{ROSS} \text { ALPHA }\end{array}$ & & & & \\
\hline $\begin{array}{l}\text { EML Value: } \\
\text { EML Error: }\end{array}$ & $\begin{array}{r}1080 . \\
60 .\end{array}$ & & & & & \\
\hline Labcode & Test \# & $\begin{array}{c}\text { Reported } \\
\text { Value }\end{array}$ & $\begin{array}{c}\text { Reported } \\
\text { Error }\end{array}$ & $\frac{\text { Reported }}{E M L}$ & $\begin{array}{c}\text { QAP 48 } \\
\text { Evaluation }\end{array}$ & Evaluation \\
\hline LV & 1 & 1240.0000 & 25.0000 & 1.148 & $\mathrm{~N}$ & A \\
\hline LW & 1 & 1048.6000 & 6.9000 & 0.971 & & A \\
\hline MH & 1 & 1229.9301 & 6.2100 & 1.139 & W & A \\
\hline MS & 1 & 1025.0000 & 100.0000 & 0.949 & & A \\
\hline NQ & 1 & 1273.0000 & 116.0000 & 1.179 & & W \\
\hline NS & 1 & 1081.3170 & 57.2790 & 1.001 & & $\mathrm{~A}$ \\
\hline NZ & 1 & 684.0000 & 39.0000 & 0.633 & & W \\
\hline NZ & 2 & 693.0000 & 39.0000 & 0.642 & & W \\
\hline $\mathrm{OB}$ & 1 & 1110.0000 & 7.2900 & 1.028 & W & A \\
\hline $\mathrm{OC}$ & 1 & 1100.0000 & 60.0000 & 1.019 & & A \\
\hline $\mathrm{OC}$ & 2 & 1100.0000 & 60.0000 & 1.019 & & A \\
\hline OT & 1 & 1200.0000 & 100.0000 & 1.111 & A & A \\
\hline OU & 1 & 833.0000 & 104.0000 & 0.771 & A & W \\
\hline RE & 1 & 1070.0000 & 36.0000 & 0.991 & A & $\mathrm{A}$ \\
\hline$\widehat{R G}$ & 1 & 1027.2000 & 62.3000 & 0.951 & A & A \\
\hline SR & 1 & 809.0000 & 118.0000 & 0.749 & A & W \\
\hline SW & 1 & 796.8500 & 13.7100 & 0.738 & A & W \\
\hline TE & 1 & 1178.3000 & 47.2000 & 1.091 & A & A \\
\hline $\mathrm{TI}$ & 1 & 1100.0000 & 100.0000 & 1.019 & A & A \\
\hline TM & 1 & 1480.0000 & 167.0000 & 1.370 & A & $\mathrm{N}$ \\
\hline $\mathrm{TN}$ & 1 & 820.0000 & 120.0000 & 0.759 & W & W \\
\hline TO & 1 & 936.0000 & 26.0000 & 0.867 & W & A \\
\hline $\mathrm{TP}$ & 1 & 996.7000 & 98.0000 & 0.923 & & A \\
\hline TW & 1 & 820.0000 & 30.0000 & 0.759 & $\mathrm{~N}$ & W \\
\hline $\mathrm{TX}$ & 1 & 1103.0000 & 30.0000 & 1.021 & W & A \\
\hline UC & 1 & 993.2300 & 66.7100 & 0.920 & A & A \\
\hline UP & 1 & 1052.0000 & 105.0000 & 0.974 & A & A \\
\hline UY & 1 & 942.0000 & 49.0000 & 0.872 & A & A \\
\hline WA & 1 & 949.0000 & 83.0000 & 0.879 & A & $\mathrm{A}$ \\
\hline WC & 1 & 1056.0000 & 106.0000 & 0.978 & A & A \\
\hline WV & 1 & 848.4100 & 59.8300 & 0.786 & A & W \\
\hline YA & 1 & 720.0200 & 16.7733 & 0.667 & A & W \\
\hline
\end{tabular}

Total Number Reported:

Values for elemental uranium are reported in $\mu \mathrm{g} /$ filter, $\mathrm{g}$ or $\mathrm{mL}$.

$\mathrm{pCi} / \mathrm{g}$ or $\mathbf{m L}=\mathrm{Bq} \times 0.027$

Evaluation: $A=$ Acceptable, $W=$ Acceptable with Warning, $N=$ Not Acceptable

If the evaluation system is not appropriate for the types of analyses performed in your lab, apply site specific evaluation. 


\section{QAP 49 Results by Nuclide}

Matrix: WA Water $\mathrm{Bq} / \mathrm{L}$
Radionuclide: $\quad$ GROSS BETA

EML Value: $\quad \begin{aligned} 1420.000 \\ \text { EML Error: }\end{aligned} \quad 60.000$

Reported

Labcode Test \# Value

Reported Reported QAP 48

Error EML Evaluation Evaluation

\begin{tabular}{|c|c|c|c|c|c|}
\hline $\mathrm{AF}$ & 1 & 1235.8000 & 44.4000 & 0.870 & A \\
\hline AI & 1 & 1230.0000 & 56,9000 & 0.866 & \\
\hline AM & 1 & 1801.1500 & 8.1700 & 1.268 & A \\
\hline $\mathrm{AU}$ & 1 & 1303.0000 & 37.0000 & 0.918 & A \\
\hline $\mathrm{BC}$ & 1 & 1198.0000 & 20.0000 & 0.844 & A \\
\hline $\mathrm{BE}$ & 1 & 1122.0000 & 53.0000 & 0.790 & A \\
\hline BL & 1 & 1055.0000 & 14.0000 & 0.743 & A \\
\hline BN & 1 & 12.4300 & 0.2700 & 0.009 & A \\
\hline BN & 2 & 12.0900 & 0.2700 & 0.009 & A \\
\hline $\mathrm{BN}$ & 3 & 12.7700 & 0.2700 & 0.009 & A \\
\hline BP & 1 & 1376.0000 & 30.0000 & 0.969 & A \\
\hline$B Q$ & 1 & 1130.0000 & 20.0000 & 0.796 & \\
\hline $\mathrm{BU}$ & 1 & 1090.0000 & 50.0000 & 0.768 & \\
\hline $\mathrm{BX}$ & 1 & 1206.0000 & 20.0000 & 0.849 & A \\
\hline $\mathrm{CA}$ & 1 & 950.0000 & 90.0000 & 0.669 & \\
\hline $\mathrm{CD}$ & 1 & 1170.0000 & 60.0000 & 0.824 & A \\
\hline $\mathrm{CH}$ & 1 & 1176.0000 & 86.0000 & 0.828 & \\
\hline CM & 1 & 1413.0000 & 33.0000 & 0.995 & \\
\hline $\mathrm{CM}$ & 2 & 1439.0000 & 33.0000 & 1.013 & \\
\hline $\mathrm{CM}$ & 3 & 1439.0000 & 33.0000 & 1.013 & \\
\hline $\mathrm{CM}$ & 4 & 1398.0000 & 32.0000 & 0.985 & \\
\hline $\mathrm{DH}$ & 1 & 1388.0000 & 103.0000 & 0.977 & $\mathrm{~N}$ \\
\hline $\mathrm{EG}$ & 1 & 1630.0000 & 50.0000 & 1.148 & \\
\hline FG & 1 & 1231.0000 & 63.0000 & 0.867 & A \\
\hline FL & 1 & 1405.5000 & 13.9400 & 0.990 & A \\
\hline $\mathrm{GC}$ & 1 & 1218.0000 & 25.2000 & 0.858 & W \\
\hline GE & 1 & 1228.4000 & 39.2200 & 0.865 & A \\
\hline GP & 1 & 1300.0000 & 100.0000 & 0.915 & A \\
\hline GS & 1 & 1363.5000 & 65.5000 & 0.960 & A \\
\hline GT & 1 & 1300.0000 & 50.0000 & 0.915 & A \\
\hline $\mathrm{HC}$ & 1 & 1347.0000 & 57.0000 & 0.949 & A \\
\hline IE & 1 & 962.1000 & 15.5000 & 0.678 & A \\
\hline IL & 1 & 1060.0000 & 25.5000 & 0.746 & \\
\hline IS & 1 & 1243.0000 & 124.0000 & 0.875 & A \\
\hline IT & 1 & 1182.0000 & 2.0000 & 0.832 & A \\
\hline $\mathrm{JE}$ & 1 & 1477.5000 & 95.6000 & 1.040 & A \\
\hline KA & 1 & 1389.0000 & 55.0000 & 0.978 & A \\
\hline LA & 1 & 1102.6000 & 62.1600 & 0.776 & A \\
\hline LA & 3 & 1158.1000 & 65.1200 & 0.816 & A \\
\hline LE & 1 & 1630.0000 & & 1.148 & \\
\hline LL & 1 & 1330.0000 & 14.4000 & 0.937 & A \\
\hline
\end{tabular}

Values for elemental uranium are reported in $\mu$ g/filter, $g$ or $\mathrm{mL}$.

$\mathrm{pCi} / \mathrm{g}$ or $\mathrm{mL}=\mathrm{Bq} \times 0.027$

Evaluation: $A=A c c e p t a b l e, W=A c c e p t a b l e$ with $W$ arning, $N=$ Not Acceptable

If the evaluation system is not appropriate for the types of analyses performed in your lab, apply site specific evaluation. 


\section{QAP 49 Results by Nuclide}

\begin{tabular}{|c|c|c|c|c|c|c|}
\hline \multicolumn{3}{|c|}{$\begin{array}{l}\text { Matrix: WA Water Bq / L } \\
\text { Radionuclide: } \quad \text { GROSS BETA }\end{array}$} & \multirow[b]{3}{*}{$\begin{array}{c}\text { Reported } \\
\text { Error }\end{array}$} & \multirow[b]{3}{*}{$\frac{\text { Reported }}{\text { EML }}$} & \multirow[b]{3}{*}{$\begin{array}{c}\text { QAP } 48 \\
\text { Evaluation }\end{array}$} & \multirow[b]{3}{*}{ Evaluation } \\
\hline $\begin{array}{l}\text { EML Value: } \\
\text { EML Error: }\end{array}$ & \multicolumn{2}{|c|}{$\begin{array}{r}1420.000 \\
60.000\end{array}$} & & & & \\
\hline Labcode & Test \# & $\begin{array}{l}\text { Reported } \\
\text { Value }\end{array}$ & & & & \\
\hline $\mathrm{LV}$ & 1 & 1030.0000 & 120.0000 & 0.725 & $\mathrm{~N}$ & A \\
\hline LW & 1 & 1292.4000 & 4.8000 & 0.910 & & $\mathrm{~A}$ \\
\hline MH & 1 & 1551.5699 & 10.1700 & 1.093 & A & $\mathrm{A}$ \\
\hline $\mathrm{MS}$ & 1 & 1300.0000 & 130.0000 & 0.915 & & A \\
\hline $\mathrm{NP}$ & 1 & 1225.0000 & 5.0000 & 0.863 & A & $\mathrm{A}$ \\
\hline $\mathrm{NQ}$ & 1 & 1357.0000 & 115.0000 & 0.956 & & A \\
\hline NS & 1 & 1330.6000 & 45.8630 & 0.937 & & A \\
\hline NZ & 1 & 1029.0000 & 40.0000 & 0.725 & & A \\
\hline $\mathrm{NZ}$ & 2 & 1050.0000 & 41.0000 & 0.739 & & $\mathrm{~A}$ \\
\hline $\mathrm{OB}$ & 1 & 1320.0000 & 8.7300 & 0.930 & A & A \\
\hline $\mathrm{OC}$ & 1 & 1200.0000 & 20.0000 & 0.845 & & A \\
\hline $\mathrm{OC}$ & 2 & 1200.0000 & 20.0000 & 0.845 & & A \\
\hline OT & 1 & 1400.0000 & 100.0000 & 0.986 & A & A \\
\hline $\mathrm{OU}$ & 1 & 1250.0000 & 144.0000 & 0.880 & A & A \\
\hline $\mathrm{RE}$ & 1 & 1250.0000 & 29.0000 & 0.880 & $\mathrm{~A}$ & A \\
\hline $\mathrm{RG}$ & 1 & 1271.2000 & 56.0000 & 0.895 & A & A \\
\hline SR & 1 & 1816.0000 & 409.0000 & 1.279 & A & A \\
\hline SW & 1 & 1183.0601 & 13.2400 & 0.833 & $\mathrm{~A}$ & A \\
\hline $\mathrm{TE}$ & 1 & 1613.6000 & 171.8000 & 1.136 & A & A \\
\hline $\mathrm{TI}$ & 1 & 1100.0000 & 100.0000 & 0.775 & A & A \\
\hline TM & 1 & 1470.0000 & 110.0000 & 1.035 & A & $\mathrm{A}$ \\
\hline $\mathrm{TN}$ & 1 & 1240.0000 & 200.0000 & 0.873 & A & A \\
\hline TO & 1 & 1248.0000 & 20.0000 & 0.879 & $\mathrm{~A}$ & $\mathrm{~A}$ \\
\hline TP & 1 & 1371.0000 & 105.0000 & 0.965 & A & A \\
\hline TW & 1 & 1192.0000 & 50.0000 & 0.839 & A & $\mathrm{A}$ \\
\hline $\mathrm{TX}$ & 1 & 1208.0000 & 35.0000 & 0.851 & A & $\mathrm{A}$ \\
\hline UC & $\hat{i}$ & 1224.5699 & 50.4900 & 0.862 & & A \\
\hline UP & 1 & 1488.0000 & 97.0000 & 1.048 & A & A \\
\hline UY & 1 & 1220.0000 & 45.0000 & 0.859 & A & $\mathrm{A}$ \\
\hline WA & 1 & 1370.0000 & 80.0000 & 0.965 & A & A \\
\hline WC & 1 & 1341.0000 & 134.0000 & 0.944 & A & $A$ \\
\hline WV & 1 & 1279.4600 & 59.7600 & 0.901 & A & $\mathrm{A}$ \\
\hline $\mathrm{YA}$ & 1 & 1194.9767 & 16.4033 & 0.842 & A & $\mathrm{A}$ \\
\hline
\end{tabular}

Total Number Reported:

Values for elemental uranium are reported in $\mu \mathrm{g} /$ filter, $\mathrm{g}$ or $\mathrm{mL}$.

$\mathrm{pCi} / \mathrm{g}$ or $\mathrm{mL}=\mathrm{Bq} \times 0.027$

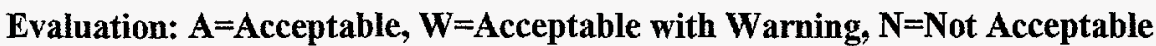

If the evaluation system is not appropriate for the types of analyses performed in your lab, apply site specific evaluation. 


\section{QAP 49 Results by Nuclide}

\begin{tabular}{|c|c|c|c|c|c|c|}
\hline \multicolumn{3}{|c|}{ Matrix: WA Water $\mathrm{Bq} / \mathrm{L}$} & \multirow[b]{3}{*}{$\begin{array}{c}\text { Reported } \\
\text { Error }\end{array}$} & \multirow[b]{3}{*}{$\frac{\text { Reported }}{\text { EML }}$} & \multirow[b]{3}{*}{$\begin{array}{c}\text { QAP } 48 \\
\text { Evaluation }\end{array}$} & \multirow[b]{3}{*}{ Evaluation } \\
\hline $\begin{array}{l}\text { EML Value: } \\
\text { EML Error: }\end{array}$ & \multicolumn{2}{|c|}{$\begin{array}{r}76.200 \\
2.900 \\
\end{array}$} & & & & \\
\hline Labcode & Test \# & $\begin{array}{l}\text { Reported } \\
\text { Value }\end{array}$ & & & & \\
\hline $\mathrm{AF}$ & 1 & 1498.5000 & 3.7000 & 19.665 & $\mathrm{~N}$ & $\mathrm{~N}$ \\
\hline$A G$ & 1 & 79.0000 & 21.0000 & 1.037 & A & A \\
\hline $\mathrm{AI}$ & 1 & 634.0000 & 86.0000 & 8.320 & $\mathrm{~N}$ & $\mathrm{~N}$ \\
\hline $\mathrm{AM}$ & 1 & 323.1000 & 15.0000 & 4.240 & & $\mathrm{~N}$ \\
\hline $\mathrm{AN}$ & 1 & 79.4000 & 3.0000 & 1.042 & A & A \\
\hline $\mathrm{AU}$ & 1 & 61.0000 & 15.0000 & 0.801 & A & W \\
\hline $\mathrm{BE}$ & 1 & 93.0000 & 7.4000 & 1.220 & $\mathrm{~A}$ & A \\
\hline BL & 1 & 81.8000 & 4.1000 & 1.073 & W & A \\
\hline $\mathrm{BN}$ & 1 & 50.4100 & 3.0200 & 0.662 & A & $\mathrm{N}$ \\
\hline $\mathrm{BN}$ & 2 & 56.1800 & 3.0200 & 0.737 & $\mathrm{~A}$ & W \\
\hline $\mathrm{BN}$ & 3 & 57.3100 & 3.0200 & 0.752 & $\mathrm{~A}$ & W \\
\hline BP & 1 & 80.8000 & 4.3000 & 1.060 & A & A \\
\hline $\mathrm{BU}$ & 1 & 76.9000 & 1.5000 & 1.009 & $\mathrm{~A}$ & A \\
\hline BX & 1 & 116.5000 & 10.7000 & 1.529 & $\mathrm{~A}$ & W \\
\hline $\mathrm{CH}$ & 1 & 80.4000 & 4.4000 & 1.055 & & A \\
\hline CL & 1 & 108.0000 & 7.6000 & 1.417 & A & W \\
\hline $\mathrm{CM}$ & 1 & 83.9000 & 2.3000 & 1.101 & & $A$ \\
\hline $\mathrm{CM}$ & 2 & 79.7000 & 2.3000 & 1.046 & & A \\
\hline $\mathrm{CM}$ & 3 & 80.8000 & 2.3000 & 1.060 & & A \\
\hline EG & 1 & 75.0000 & 6.0000 & 0.984 & & A \\
\hline $\mathrm{EP}$ & 1 & 74.6600 & 5.8900 & 0.980 & A & A \\
\hline FG & 1 & 3220.0000 & 340.0000 & 42.257 & A & $\mathrm{N}$ \\
\hline FL & 1 & 84.0800 & 3.1000 & 1.103 & A & A \\
\hline $\mathrm{FN}$ & 1 & 79.8000 & 8.7000 & 1.047 & A & A \\
\hline $\mathrm{GC}$ & 1 & 79.0000 & 1.3100 & 1.037 & A & A \\
\hline GE & 1 & 91.8654 & 17.1094 & 1.206 & A & A \\
\hline GT & 1 & 82.0000 & 10.0000 & 1.076 & A & A \\
\hline $\mathrm{HC}$ & 1 & 78.1000 & 14.4000 & 1.025 & A & A \\
\hline ID & 1 & 118.3300 & 11.0400 & 1.553 & A & W \\
\hline IE & 1 & 65.5500 & 8.0100 & 0.860 & A & $\mathrm{A}$ \\
\hline $\mathbb{N}$ & 1 & 53.0000 & 14.0000 & 0.696 & & $\mathrm{~N}$ \\
\hline IS & 1 & 118.4000 & 12.1000 & 1.554 & A & W \\
\hline IT & 1 & 79.0000 & 5.0000 & 1.037 & A & A \\
\hline $\mathrm{KA}$ & 1 & 99.0000 & 9.0000 & 1.299 & $\mathrm{~A}$ & W \\
\hline $\mathrm{LA}$ & 1 & 115.0000 & 31.0000 & 1.509 & A & $\mathrm{W}$ \\
\hline $\mathrm{LA}$ & 2 & 104.0000 & 30.0000 & 1.365 & $\mathrm{~A}$ & W \\
\hline LA & 3 & 106.0000 & 30.0000 & 1.391 & A & W \\
\hline LL & 1 & 91.5000 & 4.0300 & 1.201 & $\mathrm{~A}$ & A \\
\hline LN & 1 & 370.0000 & 13.6900 & 4.856 & W & $\mathrm{N}$ \\
\hline LV & 1 & 89.0000 & 7.1000 & 1.168 & A & A \\
\hline LW & 1 & 78.4400 & 20.0000 & 1.029 & A & A \\
\hline
\end{tabular}

Values for elemental uranium are reported in $\mu \mathrm{g} /$ filter, $\mathrm{g}$ or $\mathrm{mL}$.

$\mathrm{pCi} / \mathrm{g}$ or $\mathrm{mL}=\mathrm{Bq} \times 0.027$

Evaluation: $A=$ Acceptable, $W=$ Acceptable with Warning, $N=$ Not Acceptable

If the evaluation system is not appropriate for the types of analyses performed in your lab, apply site specific evaluation. 


\section{QAP 49 Results by Nuclide}

\begin{tabular}{|c|c|c|c|c|c|c|}
\hline $\begin{array}{l}\text { Matrix: Y } \\
\text { Radionuclide }\end{array}$ & $\begin{array}{l}\text { VA Wat } \\
\text { e: }\end{array}$ & $\mathrm{r} \mathrm{Bq} / \mathrm{L}$ & & & & \\
\hline $\begin{array}{l}\text { EML Value: } \\
\text { EML Error: }\end{array}$ & $\begin{array}{r}76 \\
2\end{array}$ & 100 & & & & \\
\hline Labcode & Test \# & $\begin{array}{l}\text { Reported } \\
\text { Value }\end{array}$ & $\begin{array}{c}\text { Reported } \\
\text { Error }\end{array}$ & $\frac{\text { Reported }}{E M L}$ & $\begin{array}{c}\text { QAP } 48 \\
\text { Evaluation }\end{array}$ & Evaluation \\
\hline $\mathrm{ME}$ & 1 & 109.0000 & 4.5000 & 1.430 & $W$ & $\mathrm{~W}$ \\
\hline $\mathrm{MH}$ & 1 & 78.3600 & 5.2200 & 1.028 & A & $\mathrm{A}$ \\
\hline $\mathrm{ML}$ & 1 & 81.0300 & 17.3900 & 1.063 & A & $\mathrm{A}$ \\
\hline MS & 1 & 74.0000 & 3.7000 & 1.015 & $\mathrm{~N}$ & $\mathrm{~A}$ \\
\hline NA & 1 & 79.2000 & 3.0000 & 1.039 & W & $\mathrm{A}$ \\
\hline NP & 1 & 444.0000 & 37.0000 & 5.827 & W & $\mathrm{N}$ \\
\hline NS & 1 & 80.9180 & 6.4120 & 1.062 & $\mathrm{~A}$ & A \\
\hline $\mathrm{OC}$ & 1 & 247.0000 & 10.0000 & 3.241 & & $\mathrm{~N}$ \\
\hline $\mathrm{OC}$ & 2 & 252.0000 & 10.0000 & 3.307 & & $\mathrm{~N}$ \\
\hline $\mathrm{OC}$ & 3 & 251.0000 & 10.0000 & 3.294 & & $\mathrm{~N}$ \\
\hline $\mathrm{OD}$ & 1 & 80.1700 & 4.6700 & 1.052 & A & A \\
\hline OD & 1 & 80.1700 & 4.6700 & 1.052 & A & A \\
\hline OT & 1 & 43.0000 & 4.0000 & 0.564 & W & $\mathrm{N}$ \\
\hline $\mathrm{OU}$ & 1 & 201.0000 & 12.9000 & 2.638 & W & $\mathrm{N}$ \\
\hline PR & 1 & 81.9500 & 0.8500 & 1.075 & A & A \\
\hline $\mathrm{RC}$ & $\hat{1}$ & 72.0000 & 5.0000 & 0.945 & A & $\mathrm{A}$ \\
\hline $\mathrm{RE}$ & 1 & 83.7000 & 20.1000 & 1.098 & A & A \\
\hline SB & 1 & 81.5000 & 6.7000 & 1.070 & & A \\
\hline SR & 1 & 77.3000 & 3.6000 & 1.014 & A & A \\
\hline ST & 1 & 85.3200 & 6.8100 & 1.120 & $\mathrm{~A}$ & A \\
\hline SW & 1 & 1098.0000 & 60.0000 & 14.409 & $\mathrm{~N}$ & $\mathrm{~N}$ \\
\hline $\mathrm{TE}$ & 1 & 102.2000 & 4.5000 & 1.341 & $\mathrm{~N}$ & W \\
\hline $\mathrm{TI}$ & 1 & 450.0000 & 50.0000 & 5.906 & A & $\mathrm{N}$ \\
\hline TN & 1 & 78.0000 & 8.0000 & 1.024 & W & A \\
\hline TO & 1 & 85.5000 & 26.0000 & 1.122 & W & $\mathrm{A}$ \\
\hline $\mathrm{TP}$ & 1 & 115.6400 & 9.9900 & 1.518 & A & W \\
\hline TT & 1 & 69.0000 & 7.0900 & 0.906 & A & A \\
\hline TX & 1 & 80.9000 & 5.6000 & 1.062 & A & $\mathrm{A}$ \\
\hline UP & 1 & 84.0000 & 23.6000 & 1.102 & A & A \\
\hline$U Y$ & 1 & 74.8000 & 18.0000 & 0.982 & A & A \\
\hline WA & 1 & 93.0000 & 4.0000 & 1.220 & A & A \\
\hline WC & 1 & 80.0000 & 9.3000 & 1.050 & A & A \\
\hline WV & 1 & 80.9200 & 4.6300 & 1.062 & A & A \\
\hline $\mathrm{YA}$ & 1 & 81.0793 & 2.5037 & 1.064 & A & A \\
\hline
\end{tabular}

Total Number Reported: 


\section{QAP 49 Results by Nuclide}

Matrix: WA Water $\mathrm{Bq} / \mathrm{L}$

Radionuclide: MN54

\begin{tabular}{lr}
\hline EML Value: & 32.400 \\
EML Error: $\quad 1.400$ \\
\hline
\end{tabular}

Labcode Test \# $\begin{gathered}\text { Reported } \\ \text { Value }\end{gathered}$

\section{Reported} Error

\subsection{0}

5.9000

0.4730

0.3000

3.0300

0.7000

3.6000

2.3000

5.3000

2.4000

3.0000

1.5000

1.3600

1.3600

1.3600

0.7000

0.9000

2.1000

3.4000

0.5000

0.5800

1.4000

1.1000

1.1000

1.1000

1.8980

1.8980

1.7000

1.0000

4.7700

0.9000

0.5000

0.3200

3.5000

5.3000

2.1800

5.7875

3.0000

6.0000

1.9200

1.2800
Reported EML
QAP 48

Evaluation
Evaluation

$\begin{array}{ll}0.004 & \mathrm{~A} \\ 1.086 & \mathrm{~A} \\ 1.080 & \mathrm{~A} \\ 1.405 & \mathrm{~A} \\ 0.933 & \mathrm{~A} \\ 1.074 & \mathrm{~A} \\ 1.002 & \mathrm{~A} \\ 1.136 & \mathrm{~A} \\ 1.185 & \mathrm{~A} \\ 1.148 & \mathrm{~A} \\ 1.188 & \mathrm{~W} \\ 1.083 & \mathrm{~A} \\ 1.216 & \mathrm{~A} \\ 1.209 & \mathrm{~A} \\ 1.302 & \mathrm{~A} \\ 1.083 & \mathrm{~A} \\ 0.944 & \mathrm{~A} \\ 1.102 & \mathrm{~A} \\ 1.062 & \mathrm{~A} \\ 1.080 & \mathrm{~A} \\ 1.108 & \\ 1.090 & \mathrm{~A}\end{array}$

$\mathrm{N}$

A

A

$\mathrm{N}$

A

A

A

W

A

W

A

W

N

A

A

A

A

A

A

A

A

A

A

W

W

A

A

A

A

A

A

A

W

A

A

A

A

A

A

Values for elemental uranium are reported in $\mu \mathrm{g}$ filter, $\mathrm{g}$ or $\mathrm{mL}$.

$\mathrm{pCi} / \mathrm{g}$ or $\mathrm{mL}=\mathrm{Bq} \times 0.027$

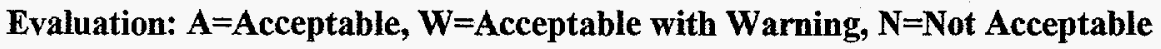

If the evaluation system is not appropriate for the types of analyses performed in your lab, apply site specific evaluation. 


\section{QAP 49 Results by Nuclide}

Matrix: WA Water $\mathrm{Bq} / \mathrm{L}$

Radionuclide: MN54

EML Value: $\quad 32.400$

EML Error: $\quad 1.400$

\begin{tabular}{|c|c|c|c|c|c|c|}
\hline Labcode & Test \# & $\begin{array}{l}\text { Reported } \\
\text { Value }\end{array}$ & $\begin{array}{l}\text { Reported } \\
\text { Error }\end{array}$ & $\frac{\text { Reported }}{E M L}$ & $\begin{array}{c}\text { QAP } 48 \\
\text { Evaluation }\end{array}$ & Evaluation \\
\hline $\mathrm{IL}$ & 1 & 36.0000 & 0.5000 & 1.111 & A & A \\
\hline IN & 1 & 35.8000 & 2.1000 & 1.105 & W & A \\
\hline IS & 1 & 39.0000 & 3.9000 & 1.204 & W & W \\
\hline IT & 1 & 35.8000 & 1.5000 & 1.105 & A & A \\
\hline $\mathrm{JE}$ & 1 & 68.3100 & 3.2500 & 2.108 & W & $\mathrm{N}$ \\
\hline $\mathrm{JL}$ & 1 & 35.2000 & 2.7000 & 1.086 & A & A \\
\hline $\mathrm{KA}$ & 1 & 31.0100 & 2.2700 & 0.957 & A & $\mathrm{A}$ \\
\hline $\mathrm{LA}$ & $i$ & 40.6000 & 4.6000 & 1.253 & W & $\mathrm{N}$ \\
\hline LA & 2 & 37.1000 & 4.2000 & 1.145 & W & A \\
\hline LA & 3 & 37.2000 & 4.3000 & 1.148 & W & A \\
\hline LB & 1 & 34.0000 & 5.0000 & 1.049 & & A \\
\hline LL & 1 & 33.6000 & 3.4300 & 1.037 & A & A \\
\hline LN & 1 & 48.7000 & 8.9700 & 1.503 & A & $\mathrm{N}$ \\
\hline LV & 1 & 37.4000 & 3.0000 & 1.154 & W & A \\
\hline $\mathrm{ME}$ & 1 & 34.2000 & 1.2800 & 1.056 & A & A \\
\hline MH & 1 & 34.2700 & 1.4200 & 1.058 & A & A \\
\hline MS & 1 & 35.6000 & 3.6000 & 1.099 & A & A \\
\hline NA & 1 & 35.3000 & 0.7000 & 1.090 & A & A \\
\hline ND & 1 & 34.7010 & 2.0830 & 1.071 & & A \\
\hline NL & 1 & 36.0000 & 5.3000 & 1.111 & W & A \\
\hline NP & 1 & 32.9000 & 1.0000 & 1.015 & A & A \\
\hline NQ & 1 & 41.9000 & 4.9000 & 1.293 & & $\mathrm{~N}$ \\
\hline NS & 1 & 37.7410 & 0.4810 & $* * * * * * * *$ & A & $\mathrm{N}$ \\
\hline $\mathrm{NZ}$ & 1 & 36.0000 & 2.0000 & 1.111 & & A \\
\hline $\mathrm{NZ}$ & 2 & 35.0000 & 2.0000 & 1.080 & & A \\
\hline $\mathrm{OB}$ & 1 & 43.5000 & 8.1000 & 1.343 & & $\mathrm{~N}$ \\
\hline $\mathrm{OC}$ & 1 & 42.0000 & 5.0000 & 1.296 & & $\mathrm{~N}$ \\
\hline $\mathrm{OC}$ & 2 & 30.0000 & 5.0000 & 0.926 & & A \\
\hline OC & 3 & 35.0000 & 5.0000 & 1.080 & & A \\
\hline $\mathrm{OD}$ & 1 & 38.7900 & 3.1200 & 1.197 & A & W \\
\hline$O D$ & 1 & 38.7900 & 3.1200 & 1.197 & A & W \\
\hline OD & 2 & 34.1000 & 3.1900 & 1.052 & A & A \\
\hline OD & 2 & 34.1000 & 3.1900 & 1.052 & A & A \\
\hline $\mathrm{OL}$ & 1 & 34.2700 & 0.9500 & 1.058 & A & A \\
\hline $\mathrm{OT}$ & 1 & 36.0000 & 2.0000 & 1.111 & A & A \\
\hline OU & 1 & 38.8000 & 1.1000 & 1.198 & A & $\mathrm{W}$ \\
\hline PR & 1 & 36.3599 & 0.4595 & 1.122 & & A \\
\hline $\mathrm{RC}$ & 1 & 32.8000 & 1.7000 & 1.012 & A & A \\
\hline RE & 1 & 37.3000 & 3.9000 & 1.151 & A & A \\
\hline $\mathrm{RI}$ & 1 & 33.7000 & 4.2500 & 1.040 & $\mathrm{~A}$ & A \\
\hline SA & 1 & 38.0000 & 1.8000 & 1.173 & $\mathrm{~A}$ & W \\
\hline $\mathrm{SB}$ & 1 & 42.1000 & 4.8700 & 1.299 & & $\mathrm{~N}$ \\
\hline
\end{tabular}

Values for elemental uranium are reported in $\mu \mathrm{g} /$ filter, $\mathrm{g}$ or $\mathrm{mL}$.

$\mathrm{pCi} / \mathrm{g}$ or $\mathrm{mL}=\mathrm{Bq} \times 0.027$

Evaluation: $A=$ Acceptable, $W=$ Acceptable with Warning, $N=$ Not Acceptable

If the evaluation system is not appropriate for the types of analyses performed in your lab, apply site specific evaluation. 


\section{QAP 49 Results by Nuclide}

\begin{tabular}{|c|c|c|c|c|c|c|}
\hline \multicolumn{3}{|c|}{ Matrix: WA Water $\mathrm{Bq} / \mathrm{L}$} & \multirow[b]{3}{*}{$\begin{array}{c}\text { Reported } \\
\text { Error }\end{array}$} & \multirow[b]{3}{*}{$\frac{\text { Reported }}{\text { EML }}$} & \multirow[b]{3}{*}{$\begin{array}{c}\text { QAP 48 } \\
\text { Evaluation }\end{array}$} & \multirow[b]{3}{*}{ Evaluation } \\
\hline $\begin{array}{l}\text { EML Value: } \\
\text { EML Error: }\end{array}$ & \multicolumn{2}{|c|}{$\begin{array}{r}32.400 \\
1.400\end{array}$} & & & & \\
\hline Labcode & Test \# & $\begin{array}{l}\text { Reported } \\
\text { Value }\end{array}$ & & & & \\
\hline SK & 1 & 31.5000 & 1.9000 & 0.972 & A & A \\
\hline SL & 1 & 34.0000 & 2.0000 & 1.049 & A & A \\
\hline SN & 1 & 34.6200 & 3.6810 & 1.069 & A & A \\
\hline SR & 1 & 35.5000 & 1.0000 & 1.096 & A & $\mathrm{A}$ \\
\hline SW & 1 & 37.0700 & 0.0400 & 1.144 & A & A \\
\hline $\mathrm{TE}$ & 1 & 35.9000 & 3.4000 & 1.108 & A & A \\
\hline $\mathrm{TI}$ & 1 & 33.5000 & 3.4000 & 1.034 & A & A \\
\hline TM & 1 & 38.3000 & 1.2800 & 1.182 & W & W \\
\hline TN & 1 & 0.0670 & 0.0070 & 0.002 & A & $\mathrm{N}$ \\
\hline TO & 1 & 34.6000 & 16.9000 & 1.068 & A & A \\
\hline $\mathrm{TP}$ & 1 & 31.4700 & 0.7500 & 0.971 & A & A \\
\hline TT & 1 & 38.0000 & 7.0900 & 1.173 & A & W \\
\hline TW & 1 & 36.2000 & 0.8000 & 1.117 & A & A \\
\hline $\mathrm{TX}$ & 1 & 36.3000 & 0.4000 & 1.120 & A & A \\
\hline UP & 1 & 36.5000 & 3.0200 & 1.127 & $\mathrm{~A}$ & A \\
\hline UY & 1 & 35.6000 & 4.0000 & 1.099 & A & A \\
\hline WA & 1 & 35.4000 & 2.3000 & 1.093 & A & A \\
\hline WC & 1 & 42.0000 & 6.0000 & 1.296 & A & $\mathrm{N}$ \\
\hline WE & 1 & 40.5000 & 2.7000 & 1.250 & & $\mathrm{~N}$ \\
\hline WI & 1 & 38.3400 & 3.8780 & 1.183 & A & W \\
\hline WI & 2 & 39.4900 & 3.9900 & 1.219 & A & W \\
\hline WI & 3 & 39.0100 & 3.9460 & 1.204 & A & W \\
\hline WV & 1 & 36.3200 & 0.4800 & 1.121 & $\mathrm{~A}$ & A \\
\hline YA & 1 & 33.7810 & 0.8017 & 1.043 & A & A \\
\hline $\mathrm{YU}$ & 1 & 33.0000 & 1.1000 & 1.019 & A & A \\
\hline
\end{tabular}

Total Number Reported:

108

Values for elemental uranium are reported in $\mu \mathrm{g} /$ filter, $\mathrm{g}$ or $\mathrm{mL}$.

$\mathrm{pCi} / \mathrm{g}$ or $\mathrm{mL}=\mathrm{Bq} \times 0.027$

Evaluation: $A=$ Acceptable, $W=$ Acceptable with Warning, $N=$ Not Acceptable

If the evaluation system is not appropriate for the types of analyses performed in your lab, apply site specific evaluation. 


\section{QAP 49 Results by Nuclide}

Matrix: WA Water $\mathrm{Bq} / \mathrm{L}$

Radionuclide: NI63

EML Value: $\quad 95.700$

EML Error: $\quad 0.900$

\begin{tabular}{ccrrrrr} 
Labcode & Test \# & $\begin{array}{c}\text { Reported } \\
\text { Value }\end{array}$ & $\begin{array}{c}\text { Reported } \\
\text { Error }\end{array}$ & $\begin{array}{c}\text { Reported } \\
\text { EML }\end{array}$ & $\begin{array}{c}\text { QAP 48 } \\
\text { Evaluation }\end{array}$ & Evaluat \\
\hline & & & & & & \\
BE & 1 & 122.2000 & 1.9000 & 1.277 & $\mathrm{~A}$ \\
$\mathrm{BL}$ & 1 & 103.0000 & 3.0000 & 1.076 & $\mathrm{~A}$ \\
$\mathrm{BP}$ & 1 & 86.0000 & 10.0000 & 0.899 & $\mathrm{~A}$ \\
$\mathrm{BX}$ & 1 & 93.6000 & 7.8000 & 0.978 & $\mathrm{~A}$ \\
$\mathrm{CL}$ & 1 & 136.0000 & 5.0000 & 1.421 & $\mathrm{~A}$ \\
EG & 1 & 78.0000 & 7.0000 & 0.815 & $\mathrm{~A}$ \\
FL & 1 & 95.2300 & 0.6700 & 0.995 & $\mathrm{~A}$ \\
GE & 1 & 55.4450 & 6.6600 & 0.579 & $\mathrm{~A}$ \\
OU & 1 & 103.0000 & 15.2000 & 1.076 & $\mathrm{~A}$ \\
TI & 1 & 110.0000 & 10.0000 & 1.149 & $\mathrm{~A}$ \\
TN & 1 & 122.0000 & 12.0000 & 1.275 & $\mathrm{~A}$ \\
TO & 1 & 117.7000 & 1.0000 & 1.230 & $\mathrm{~A}$ \\
WA & 1 & 119.0000 & 4.0000 & 1.243 & $\mathrm{~A}$ \\
YA & 1 & 124.0733 & 9.9900 & 1.296 &
\end{tabular}

Total Number Reported:

A

A

A

A

A

A

A

A

A

A

A

Values for elemental uranium are reported in $\mu \mathrm{g} /$ filter, $\mathrm{g}$ or $\mathrm{mL}$.

$\mathrm{pCi} / \mathrm{g}$ or $\mathrm{mL}=\mathrm{Bq} \times 0.027$

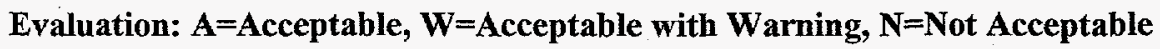

If the evaluation system is not appropriate for the types of analyses performed in your lab, apply site specific evaluation. 


\section{QAP 49 Results by Nuclide}

\begin{tabular}{lc}
\hline Matrix: WA & Water $\mathrm{Bq} / \mathrm{L}$ \\
Radionuclide: & PU238 \\
\hline EML Value: & 1.100 \\
EML Error: & 0.010 \\
\hline
\end{tabular}

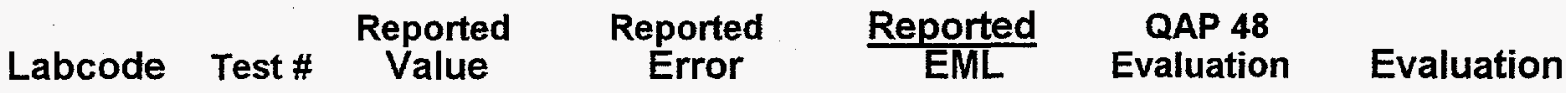

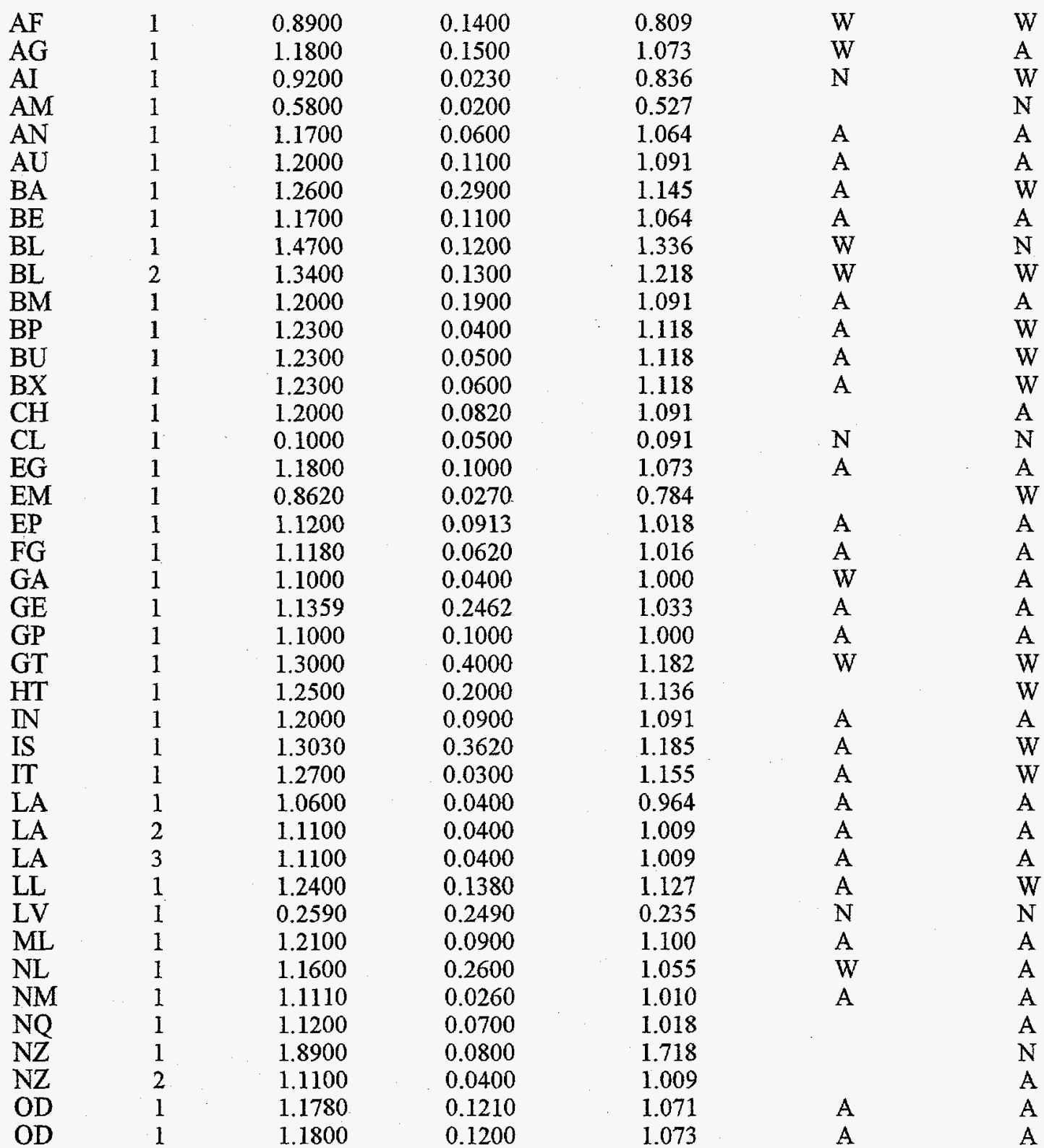

Values for elemental uranium are reported in $\mu \mathrm{g}$ /filter, $\mathrm{g}$ or $\mathrm{mL}$. $\mathrm{pCi} / \mathrm{g}$ or $\mathrm{mL}=\mathrm{Bq} \times 0.027$ Evaluation: $A=$ Acceptable, $W=$ Acceptable with Warning, $N=$ Not Acceptable If the evaluation system is not appropriate for the types of analyses performed in your lab, apply site specific evaluation. 


\section{QAP 49 Results by Nuclide}

Matrix: WA Water $\mathrm{Bq} / \mathrm{L}$

Radionuclide: $\quad$ PU238

$\begin{array}{lr}\text { EML Value: } & 1.100 \\ \text { EML Error: } & 0.010\end{array}$

EML Error: $\quad 0.010$

\begin{tabular}{|c|c|c|c|c|c|c|}
\hline Labcode & Test \# & $\begin{array}{c}\text { Reported } \\
\text { Value }\end{array}$ & $\begin{array}{c}\text { Reported } \\
\text { Error }\end{array}$ & $\frac{\text { Reported }}{E M L}$ & $\begin{array}{c}\text { QAP } 48 \\
\text { Evaluation }\end{array}$ & Evaluation \\
\hline OT & 1 & 0.9400 & 0.0700 & 0.855 & W & W \\
\hline OU & 1 & 1.0700 & 0.1400 & 0.973 & & A \\
\hline RE & 1 & 1.0100 & 0.1100 & 0.918 & $\mathrm{~N}$ & A \\
\hline RI & 1 & 1.3900 & 0.1160 & 1.264 & A & $\mathrm{N}$ \\
\hline SK & 1 & 0.2450 & 0.0110 & 0.223 & A & $\mathrm{N}$ \\
\hline SN & 1 & 1.1910 & 0.1890 & 1.083 & A & A \\
\hline SR & 1 & 1.3100 & 0.0730 & 1.191 & A & W \\
\hline SW & 1 & 1.6400 & 0.0800 & 1.491 & A & $N$ \\
\hline TI & 1 & 1.1000 & 0.2000 & 1.000 & A & A \\
\hline $\mathrm{TM}$ & 1 & 1.2000 & 0.0772 & 1.091 & A & A \\
\hline $\mathrm{TN}$ & 1 & 1.1500 & 0.1100 & 1.045 & $\mathrm{~N}$ & A \\
\hline TO & 1 & 1.2400 & 0.2800 & 1.127 & A & W \\
\hline TX & 1 & 1.1620 & 0.0470 & 1.056 & A & A \\
\hline UP & 1 & 1.1000 & 0.1660 & 1.000 & W & A \\
\hline UY & 1 & 1.1300 & 0.1300 & 1.027 & $\mathrm{~A}$ & A \\
\hline WA & 1 & 1.1800 & 0.0900 & 1.073 & A & A \\
\hline WC & 1 & 1.1000 & 0.1800 & 1.000 & A & A \\
\hline YA & 1 & 1.1951 & 0.0382 & 1.086 & $\mathrm{~A}$ & $\mathrm{~A}$ \\
\hline
\end{tabular}

Total Number Reported:

Values for elemental uranium are reported in $\mu \mathrm{g} /$ filter, $\mathrm{g}$ or $\mathrm{mL}$.

$\mathrm{pCi} / \mathrm{g}$ or $\mathrm{mL}=\mathrm{Bq} \times 0.027$

Evaluation: $A=$ Acceptable, $W=$ Acceptable with $W$ arning, $N=$ Not Acceptable

If the evaluation system is not appropriate for the types of analyses performed in your lab, apply site specific evaluation. 


\section{QAP 49 Results by Nuclide}

Matrix: WA Water $\mathrm{Bq} / \mathrm{L}$

Radionuclide: $\quad$ PU239

\begin{tabular}{ll} 
EML Value: & 1.410 \\
EML Error: & 0.040 \\
\hline
\end{tabular}

Labcode $\quad$ Test \# $\begin{gathered}\text { Reported } \\ \text { Value }\end{gathered} \quad \begin{gathered}\text { Reported } \\ \text { Error }\end{gathered} \frac{\text { Reported }}{E M L} \quad \begin{gathered}\text { QAP 48 } \\ \text { Evaluation }\end{gathered}$ Evaluation

\begin{tabular}{|c|c|c|c|c|c|}
\hline $\mathrm{AF}$ & 1 & 1.4400 & 0.2000 & 1.021 & A \\
\hline $\mathrm{AG}$ & 1 & 1.4300 & 0.1800 & 1.014 & A \\
\hline $\mathrm{AI}$ & 1 & 1.2100 & 0.0290 & 0.858 & $\mathrm{~N}$ \\
\hline AM & 1 & 0.8200 & 0.0200 & 0.582 & \\
\hline AN & 1 & 1.5700 & 0.0800 & 1.113 & A \\
\hline $\mathrm{AU}$ & 1 & 1.5000 & 0.1300 & 1.064 & W \\
\hline BA & 1 & 1.7000 & 0.3700 & 1.206 & A \\
\hline $\mathrm{BE}$ & 1 & 1.5900 & 0.1500 & 1.128 & A \\
\hline BL & 1 & 1.6800 & 0.1300 & 1.191 & A \\
\hline BL & 2 & 1.7900 & 0.1400 & 1.270 & A \\
\hline BM & 1 & 1.5800 & 0.2400 & 1.121 & A \\
\hline BP & 1 & 1.6400 & 0.0300 & 1.163 & A \\
\hline BU & 1 & 1.4500 & 0.0600 & 1.028 & A \\
\hline BX & 1 & 1.5300 & 0.0800 & 1.085 & A \\
\hline $\mathrm{CH}$ & 1 & 1.5300 & 0.0950 & 1.085 & \\
\hline $\mathrm{CL}$ & 1 & 1.4000 & 0.2000 & 0.993 & $N$ \\
\hline EG & 1 & 1.5400 & 0.1300 & 1.092 & A \\
\hline EM & I & 1.1490 & 0.0320 & 0.815 & \\
\hline EP & 1 & 1.4700 & 0.1160 & 1.043 & A \\
\hline $\mathrm{FG}$ & 1 & 1.4140 & 0.0708 & 1.003 & A \\
\hline GA & 1 & 1.6000 & 0.0500 & 1.135 & A \\
\hline GE & 1 & 1.4615 & 0.3080 & 1.037 & A \\
\hline GP & 1 & 1.5000 & 0.2000 & 1.064 & A \\
\hline GT & 1 & 1.8000 & 0.4000 & 1.277 & W \\
\hline HT & 1 & 1.1000 & 0.1500 & 0.780 & \\
\hline ID & 1 & 1.1000 & 0.3500 & 0.780 & A \\
\hline IN & 1 & 1.4400 & 0.1000 & 1.021 & A \\
\hline IS & 1 & 1.7330 & 0.4700 & 1.229 & W \\
\hline $\mathrm{IT}$ & 1 & 1.5800 & 0.1200 & 1.121 & A \\
\hline $\mathrm{KA}$ & 1 & 1.6820 & 0.0080 & 1.193 & A \\
\hline LA & 1 & 1.4600 & 0.0500 & 1.035 & A \\
\hline LA & 2 & 1.4800 & 0.0500 & 1.050 & A \\
\hline LA & 3 & 1.4500 & 0.0500 & 1.028 & A \\
\hline LL & 1 & 1.5300 & 0.1650 & 1.085 & A \\
\hline LV & 1 & 0.3940 & 0.0120 & 0.279 & $\mathrm{~N}$ \\
\hline ML & 1 & 1.6000 & 0.1200 & 1.135 & A \\
\hline NL & 1 & 1.5400 & 0.3500 & 1.092 & A \\
\hline NM & 1 & 1.4580 & 0.0330 & 1.034 & A \\
\hline NQ & 1 & 1.4300 & 0.0900 & 1.014 & \\
\hline $\mathrm{NZ}$ & 1 & 2.5300 & 0.1000 & 1.794 & \\
\hline NZ & 2 & 1.5100 & 0.0500 & 1.071 & \\
\hline
\end{tabular}

Values for elemental uranium are reported in $\mu \mathrm{g} /$ filter, $\mathrm{g}$ or $\mathrm{mL}$.

$\mathrm{pCi} / \mathrm{g}$ or $\mathrm{mL}=\mathrm{Bq} \times 0.027$

Evaluation: $A=$ Acceptable, $W=$ Acceptable with Warning, $N=$ Not Acceptable

If the evaluation system is not appropriate for the types of analyses performed in your lab, apply site specific evaluation. 


\section{QAP 49 Results by Nuclide}

\begin{tabular}{|c|c|c|c|c|c|c|}
\hline $\begin{array}{l}\text { Matrix: W } \\
\text { Radionuclide }\end{array}$ & $\begin{array}{l}\text { VA Wate } \\
\text { e: } \quad I\end{array}$ & $\underset{U}{\mathrm{U} 23 \mathrm{~Bq} / \mathrm{L}}$ & & & & \\
\hline $\begin{array}{l}\text { EML Value: } \\
\text { EML Error: }\end{array}$ & & $\begin{array}{l}10 \\
40\end{array}$ & & & & \\
\hline Labcode & Test \# & $\begin{array}{c}\text { Reported } \\
\text { Value }\end{array}$ & $\begin{array}{c}\text { Reported } \\
\text { Error }\end{array}$ & $\frac{\text { Reported }}{E M L}$ & $\begin{array}{c}\text { QAP 48 } \\
\text { Evaluation }\end{array}$ & Evaluation \\
\hline OD & 1 & 1.5420 & 0.1570 & 1.094 & A & A \\
\hline $\mathrm{OD}$ & 1 & 1.5400 & 0.1600 & 1.092 & A & A \\
\hline OT & $\hat{1}$ & 1.4000 & 0.1000 & 0.993 & A & A \\
\hline OU & 1 & 1.5400 & 0.0900 & 1.092 & & A \\
\hline $\mathrm{RE}$ & 1 & 1.4100 & 0.1400 & 1.000 & $\mathrm{~N}$ & A \\
\hline RI & 1 & 1.6300 & 0.1290 & 1.156 & A & W \\
\hline SK & 1 & 0.3240 & 0.0100 & 0.230 & A & $\mathrm{N}$ \\
\hline SN & 1 & 1.5250 & 0.2320 & 1.082 & A & A \\
\hline SR & 1 & 1.8800 & 0.0940 & 1.333 & A & W \\
\hline SW & 1 & 1.1800 & 0.0600 & 0.837 & $\mathrm{~N}$ & $\mathrm{~W}$ \\
\hline TI & 1 & 1.6000 & 0.2000 & 1.135 & W & A \\
\hline TM & 1 & 1.5200 & 0.0925 & 1.078 & A & A \\
\hline TN & 1 & 1.4800 & 0.1500 & 1.050 & $\mathrm{~N}$ & $\mathrm{~A}$ \\
\hline TO & 1 & 1.6400 & 0.3400 & 1.163 & A & W \\
\hline TX & 1 & 1.5670 & 0.0550 & 1.111 & A & A \\
\hline UP & 1 & 1.5100 & 0.2040 & 1.071 & W & A \\
\hline UY & 1 & 1.5200 & 0.1700 & 1.078 & A & A \\
\hline WA & 1 & 1.6500 & 0.1000 & 1.170 & A & W \\
\hline WC & 1 & 1.6000 & 0.2300 & 1.135 & A & $\mathrm{A}$ \\
\hline YA & 1 & 1.5343 & 0.0456 & 1.088 & A & A \\
\hline
\end{tabular}

Total Number Reported:

61

Values for elemental uranium are reported in $\mu \mathrm{g} /$ filter, $\mathrm{g}$ or $\mathrm{mL}$.

$\mathrm{pCi} / \mathrm{g}$ or $\mathrm{mL}=\mathrm{Bq} \times 0.027$

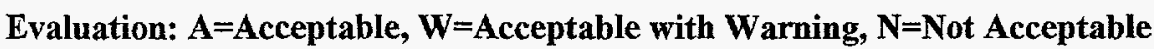

If the evaluation system is not appropriate for the types of analyses performed in your lab, apply site specific evaluation. 


\section{QAP 49 Results by Nuclide}

Matrix: WA Water $\mathrm{Bq} / \mathrm{L}$

Radionuclide: $\quad$ SR90

\begin{tabular}{ll} 
EML Value: & 2.110 \\
EML Error: & 0.180 \\
\hline
\end{tabular}

Reported

Labcode Test \# Value

Reported Error
Reported EML

1.014

1.066

0.896

0.678

0.882

1.024

0.981

1.142

2.280

1.043

1.028

1.081

1.057

3.351

3.630

1.071

1.166

1.076

1.303

1.280

1.043

1.100

1.232

1.242

0.095

0.711

0.900

1.175

1.275

0.805

1.062

1.136

1.351

1.306

1.336

0.569

0.834

1.611

1.896

1.137

1.090
QAP 48

Evaluation

Evaluation

$\mathrm{A} \quad \mathrm{A}$

A
A
A
N
W
A
A
A
N
A
A
A
A
N
N
A
A
A
W
W

A

A
A
A
N
W
A
A
A
N
A
A
A
A
N
N
A
A
A
W
W

A
A
A
N
W
A
A
A
N
A
A
A
A
N
N
A
A
A
W
W

A $A$

A $A$

A $\quad$ A

W

A

A

A

A

A

A

W

A

A

A

A

A

A

A

A

A.

W

A

A

A

A

A

A

A

A

A

$\mathrm{N}$

A

A

A

W

A

A

W

W

$\mathrm{N}$

$\mathrm{N}$

A

A

W

W

A

A

W

W

W

$\mathrm{N}$

W

$\mathrm{N}$

$\mathrm{N}$

A

A

Values for elemental uranium are reported in $\mu \mathrm{g}$ /filter, $\mathrm{g}$ or $\mathrm{mL}$. Evaluation: $A=$ Acceptable, $W=$ Acceptable with Warning, $N=$ Not Acceptable

If the evaluation system is not appropriate for the types of analyses performed in your lab, apply site specific evaluation. 


\section{QAP 49 Results by Nuclide}

\begin{tabular}{lr}
\hline $\begin{array}{l}\text { Matrix: WA } \\
\text { Radionuclide: }\end{array}$ & SR9ter B \\
\hline EML Value: & 2.110 \\
EML Error: & 0.180 \\
\hline
\end{tabular}

\begin{tabular}{|c|c|c|c|c|c|c|}
\hline Labcode & Test \# & $\begin{array}{l}\text { Reported } \\
\text { Value }\end{array}$ & $\begin{array}{c}\text { Reported } \\
\text { Error }\end{array}$ & $\frac{\text { Reported }}{E M L}$ & $\begin{array}{c}\text { QAP 48 } \\
\text { Evaluation }\end{array}$ & Evaluation \\
\hline $\mathrm{OC}$ & 3 & 2.3000 & 0.2000 & 1.090 & & A \\
\hline $\mathrm{OD}$ & 1 & 2.3900 & 0.2800 & 1.133 & A & A \\
\hline OD & 1 & 2.3900 & 0.2800 & 1.133 & A & A \\
\hline OT & 1 & 2.1000 & 0.3000 & 0.995 & A & A \\
\hline OU & 1 & 2.0100 & 0.2700 & 0.953 & & A \\
\hline RE & 1 & 2.2600 & 0.2100 & 1.071 & A & A \\
\hline RI & 1 & 2.4100 & 0.2450 & 1.142 & A & A \\
\hline SR & 1 & 2.3860 & 0.7210 & 1.131 & $\mathrm{~A}$ & A \\
\hline SW & 1 & 0.7400 & 0.1900 & 0.351 & $\mathrm{~N}$ & $\mathrm{~N}$ \\
\hline $\mathrm{TE}$ & 1 & 3.0000 & 0.9000 & 1.422 & $\mathrm{~N}$ & $\mathrm{~W}$ \\
\hline TI & 1 & 2.2000 & 0.3000 & 1.043 & A & A \\
\hline $\mathrm{TM}$ & $i$ & 2.2300 & 0.2350 & 1.057 & W & A \\
\hline TN & 1 & 2.1600 & 0.2100 & 1.024 & $\mathrm{~A}$ & A \\
\hline TO & 1 & 2.3000 & 0.1000 & 1.090 & $\mathrm{~W}$ & A \\
\hline $\mathrm{TP}$ & 1 & 2.5000 & 0.2300 & 1.185 & A & A \\
\hline TX & 1 & 2.7200 & 0.7100 & 1.289 & $\mathrm{~A}$ & W \\
\hline UP & 1 & 2.5130 & 0.6060 & 1.191 & A & A \\
\hline UY & 1 & 2.0000 & 0.3000 & 0.948 & $\mathrm{~A}$ & A \\
\hline WA & 1 & 2.1300 & 0.4400 & 1.009 & A & A \\
\hline WC & 1 & 2.3000 & 0.3200 & 1.090 & A & A \\
\hline WE & 1 & 12.9000 & 0.8800 & 6.114 & & $\mathrm{~N}$ \\
\hline WV & 1 & 2.4300 & 0.2400 & 1.152 & A & A \\
\hline YA & 1 & 1.4492 & 0.3145 & 0.687 & A & $\mathrm{N}$ \\
\hline
\end{tabular}

Total Number Reported:

64

Values for elemental uranium are reported in $\mu \mathrm{g} /$ filter, $\mathrm{g}$ or $\mathrm{mL}$.

$\mathrm{pCi} / \mathrm{g}$ or $\mathrm{mL}=\mathrm{Bq} \times 0.027$

Evaluation: $A=A c c e p t a b l e, W=A c c e p t a b l e$ with Warning, $N=$ Not Acceptable

If the evaluation system is not appropriate for the types of analyses performed in your lab, apply site specific evaluation. 


\section{QAP 49 Results by Nuclide}

December, 1998

Matrix: WA Water $\mathrm{Bq} / \mathrm{L}$

Radionuclide: $\quad$ U234

\begin{tabular}{ll}
\hline EML Value: & 0.510 \\
EML Error: & 0.030 \\
\hline
\end{tabular}

Labcode $\quad$ Test \# $\begin{gathered}\text { Reported } \\ \text { Value }\end{gathered} \begin{gathered}\text { Reported } \\ \text { Error }\end{gathered} \frac{\text { Reported }}{\text { EML }} \quad \begin{gathered}\text { QAP 48 } \\ \text { Evaluation }\end{gathered}$ Evaluation

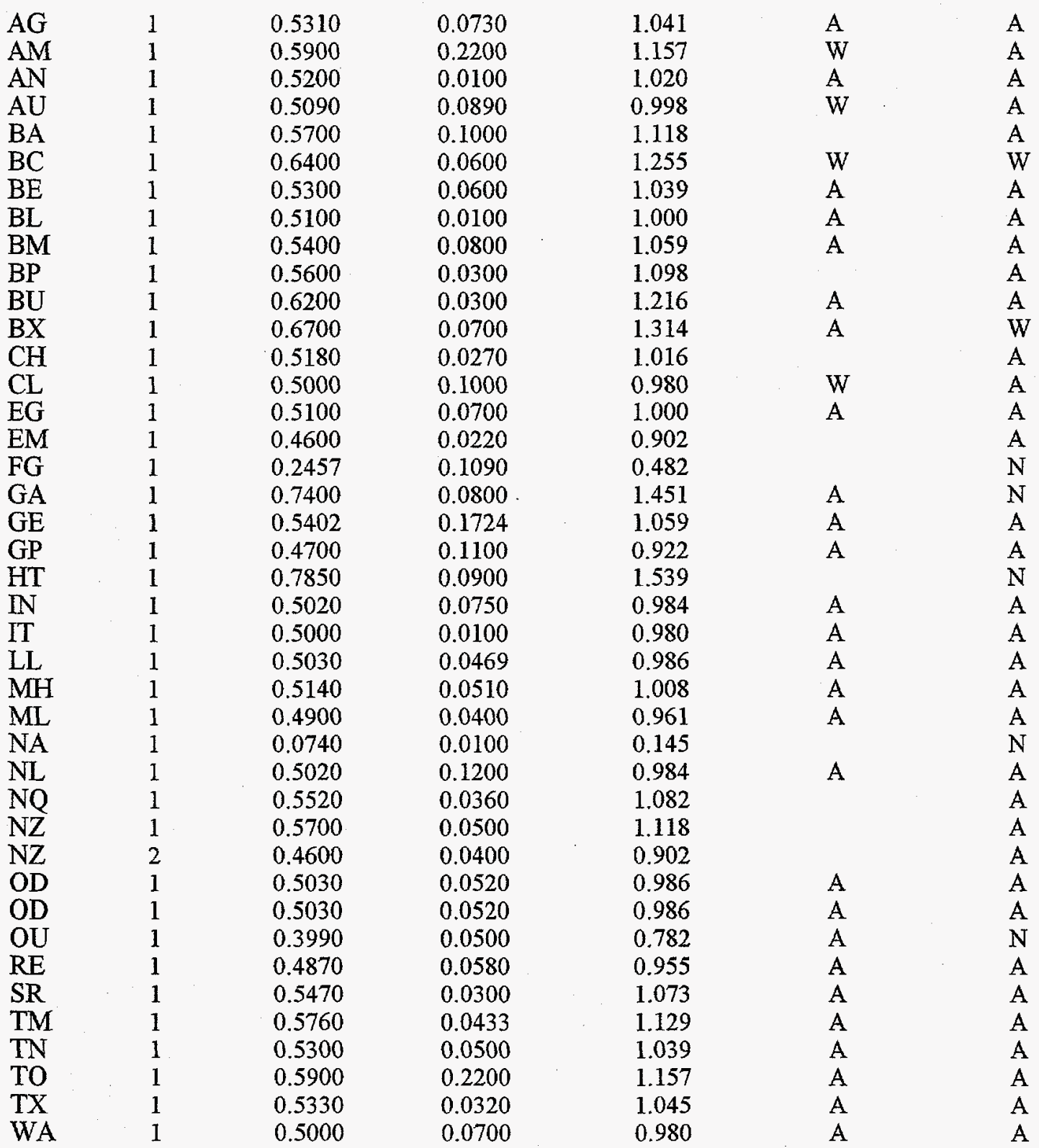

Values for elemental uranium are reported in $\mu \mathrm{g} /$ filter, $\mathrm{g}$ or $\mathrm{mL}$.

$\mathrm{pCi} / \mathrm{g}$ or $\mathrm{mL}=\mathrm{Bq} \times 0.027$

Evaluation: $A=A c c e p t a b l e, W=A c c e p t a b l e$ with Warning, $N=$ Not Acceptable

If the evaluation system is not appropriate for the types of analyses performed in your lab, apply site specific evaluation. 


\section{QAP 49 Results by Nuclide}

Matrix: WA Water $\mathrm{Bq} / \mathrm{L}$

Radionuclide: $\quad$ U234

EML Value: $\quad 0.510$

EML Error: $\quad 0.030$

\begin{tabular}{ccccccc} 
Labcode & Test \# & $\begin{array}{c}\text { Reported } \\
\text { Value }\end{array}$ & $\begin{array}{c}\text { Reported } \\
\text { Error }\end{array}$ & $\begin{array}{c}\text { Reported } \\
\text { EML }\end{array}$ & $\begin{array}{c}\text { QAP 48 } \\
\text { Evaluation }\end{array}$ & Evaluation \\
\hline WC & 1 & 0.5700 & 0.1000 & 1.118 & $\mathrm{~A}$ & $\mathrm{~A}$
\end{tabular}

Total Number Reported:

42

Values for elemental uranium are reported in $\mu \mathrm{g} /$ filter, $\mathrm{g}$ or $\mathrm{mL}$.

$\mathrm{pCi} / \mathrm{g}$ or $\mathrm{mL}=\mathrm{Bq} \times 0.027$

Evaluation: $A=$ Acceptable, $W=$ Acceptable with Warning, $N=$ Not Acceptable

If the evaluation system is not appropriate for the types of analyses performed in your lab, apply site specific evaluation. 
QAP 49 Results by Nuclide

December, 1998

Matrix: WA Water $\mathrm{Bq} / \mathrm{L}$

Radionuclide: $\quad \mathrm{U} 238$

\begin{tabular}{ll}
\hline EML Value: & 0.520 \\
EML Error: & 0.050 \\
\hline
\end{tabular}

\begin{tabular}{|c|c|c|c|}
\hline Labcode & Test \# & $\begin{array}{c}\text { Reported } \\
\text { Value }\end{array}$ & $\begin{array}{c}\text { Reported } \\
\text { Error }\end{array}$ \\
\hline
\end{tabular}

\begin{tabular}{|c|c|c|c|c|c|c|}
\hline $\mathrm{AG}$ & 1 & 0.5230 & 0.0720 & 1.006 & A & A \\
\hline AM & 1 & 0.4900 & 0.2200 & 0.942 & A & A \\
\hline $\mathrm{AN}$ & 1 & 0.5100 & 0.0100 & 0.981 & A & A \\
\hline $\mathrm{AU}$ & 1 & 0.5140 & 0.0890 & 0.988 & $\mathrm{~N}$ & A \\
\hline $\mathrm{BA}$ & 1 & 0.5400 & 0.1000 & 1.038 & & A \\
\hline $\mathrm{BC}$ & 1 & 0.5500 & 0.0700 & 1.058 & $\mathrm{~W}$ & A \\
\hline $\mathrm{BE}$ & 1 & 0.5300 & 0.0600 & 1.019 & A & A \\
\hline BL & 1 & 0.4950 & 0.0100 & 0.952 & W & A \\
\hline BM & 1 & 0.5400 & 0.0800 & 1.038 & A & A \\
\hline BP & 1 & 0.5500 & 0.0100 & 1.058 & A & A \\
\hline $\mathrm{BU}$ & 1 & 0.5500 & 0.0300 & 1.058 & A & A \\
\hline $\mathrm{BX}$ & 1 & 0.6800 & 0.0900 & 1.308 & A & $\mathrm{N}$ \\
\hline $\mathrm{CH}$ & 1 & 0.5510 & 0.0280 & 1.060 & & A \\
\hline CL & 1 & 0.6000 & 0.2000 & 1.154 & A & A \\
\hline $\mathrm{EG}$ & 1 & 0.4900 & 0.0800 & 0.942 & A & A \\
\hline EM & 1 & 0.4890 & 0.0230 & 0.940 & & A \\
\hline $\mathrm{FG}$ & 1 & 0.1902 & 0.0600 & 0.366 & & $\mathrm{~N}$ \\
\hline GA & 1 & 0.7400 & 0.0800 & 1.423 & A & $\mathrm{N}$ \\
\hline GE & 1 & 0.5180 & 0.1660 & 0.996 & A & A \\
\hline GP & 1 & 0.5000 & 0.1200 & 0.962 & A & A \\
\hline GT & 1 & 0.6900 & 0.1500 & 1.327 & A & $\mathrm{N}$ \\
\hline $\mathrm{HT}$ & 1 & 0.8000 & 0.0900 & 1.538 & & $\mathrm{~N}$ \\
\hline ID & 1 & 0.5770 & 0.0360 & 1.110 & A & A \\
\hline $\mathrm{IN}$ & 1 & 0.5000 & 0.0800 & 0.962 & A & A \\
\hline $\mathrm{IT}$ & 1 & 0.5300 & 0.0800 & 1.019 & A & A \\
\hline LL & 1 & 0.5190 & 0.0481 & 0.998 & A & A \\
\hline MH & 1 & 0.5290 & 0.0510 & 1.017 & A & A \\
\hline ML & 1 & 0.5000 & 0.0400 & 0.962 & A & A \\
\hline NA & 1 & 0.0600 & 0.0090 & 0.115 & & $\mathrm{~N}$ \\
\hline NL & 1 & 0.5210 & 0.1200 & 1.002 & A & A \\
\hline $\mathrm{NQ}$ & 1 & 0.5390 & 0.0350 & 1.037 & & A \\
\hline NZ & 1 & 0.5600 & 0.0500 & 1.077 & & A \\
\hline $\mathrm{NZ}$ & 2 & 0.5200 & 0.0400 & 1.000 & & A \\
\hline OD & 1 & 0.5320 & 0.0550 & 1.023 & A & A \\
\hline OD & 1 & 0.5320 & 0.0550 & 1.023 & A & A \\
\hline OU & 1 & 0.4020 & 0.0500 & 0.773 & A & $\mathrm{N}$ \\
\hline RE & 1 & 0.5080 & 0.0610 & 0.977 & A & A \\
\hline SR & 1 & 0.6090 & 0.0370 & 1.171 & A & W \\
\hline SW & 1 & 0.0445 & & 0.086 & & $\mathrm{~N}$ \\
\hline TM & 1 & 0.5710 & 0.0429 & 1.098 & A & A \\
\hline $\mathrm{TN}$ & 1 & 0.5200 & 0.0500 & 1.000 & A & A \\
\hline
\end{tabular}

Values for elemental uranium are reported in $\mu \mathrm{g} /$ filter, $\mathrm{g}$ or $\mathrm{mL}$.

$\mathrm{pCi} / \mathrm{g}$ or $\mathrm{mL}=\mathrm{Bq} \times 0.027$

Evaluation: $A=$ Acceptable, $W=$ Acceptable with Warning, $N=$ Not Acceptable

If the evaluation system is not appropriate for the types of analyses performed in your lab, apply site specific evaluation. 


\section{QAP 49 Results by Nuclide}

Matrix: WA Water $\mathrm{Bq} / \mathrm{L}$

Radionuclide: U238

\begin{tabular}{ll}
\hline EML Value: & 0.520 \\
EML Error: & 0.050 \\
\hline
\end{tabular}

\begin{tabular}{ccccccc} 
Labcode & Test \# & $\begin{array}{c}\text { Reported } \\
\text { Value }\end{array}$ & $\begin{array}{c}\text { Reported } \\
\text { Error }\end{array}$ & $\begin{array}{c}\text { Reported } \\
\text { EML }\end{array}$ & $\begin{array}{c}\text { QAP 48 } \\
\text { Evaluation }\end{array}$ & Evaluation \\
\hline TO & 1 & 0.5800 & 0.2300 & 1.115 & $\mathrm{~A}$ & $\mathrm{~A}$ \\
TX & 1 & 0.5270 & 0.0310 & 1.013 & $\mathrm{~W}$ & $\mathrm{~A}$ \\
WA & 1 & 0.5600 & 0.0700 & 1.077 & $\mathrm{~A}$ & $\mathrm{~A}$ \\
WC & 1 & 0.5600 & 0.1000 & 1.077 & $\mathrm{~A}$ & $\mathrm{~A}$
\end{tabular}

Total Number Reported: 


\section{QAP 49 Results by Nuclide}

Matrix: WA Water $\mathrm{Bq} / \mathrm{L}$

Radionuclide: $\quad$ ug U

$\begin{array}{ll}\text { EML Value: } & 0.040 \\ \text { EML Error: } & 0.003\end{array}$

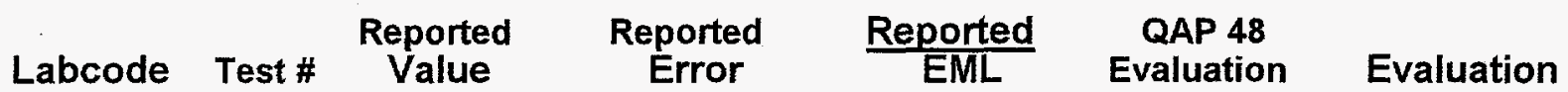

\begin{tabular}{|c|c|c|c|c|c|c|}
\hline \multirow{2}{*}{\multicolumn{7}{|c|}{$\begin{array}{l}0.0044 \\
0.0071\end{array}$}} \\
\hline $\mathrm{AG}$ & 1 & 0.0431 & 0.0071 & 1.077 & A & \\
\hline $\mathrm{BE}$ & 1 & 0.0424 & & 1.060 & A & A \\
\hline BL & 1 & 1.0300 & 0.0200 & 25.750 & & $\mathrm{~N}$ \\
\hline BL & 2 & 0.0410 & 0.0007 & 1.025 & & \\
\hline$B Q$ & 1 & 0.0480 & 0.0020 & 1.200 & A & \\
\hline $\mathrm{BU}$ & 1 & 0.0430 & 0.0030 & 1.075 & A & $A$ \\
\hline $\mathrm{CA}$ & 1 & 0.0405 & 0.0041 & 1.013 & W & $\mathrm{A}$ \\
\hline $\mathrm{CH}$ & 1 & 0.0406 & 0.0041 & 1.015 & & \\
\hline GA & 1 & 0.0590 & 0.0070 & 1.475 & & \\
\hline GE & 1 & 0.0405 & 0.0007 & 1.013 & A & \\
\hline GS & 1 & 0.0400 & 0.0020 & 1.000 & A & $A$ \\
\hline HT & 1 & 0.0650 & 0.0070 & 1.625 & & $\mathrm{~N}$ \\
\hline IS & 1 & 0.0403 & 0.0055 & 1.008 & & \\
\hline IT & 1 & 0.0399 & 0.0004 & 0.998 & A & \\
\hline $\mathrm{KA}$ & 1 & 0.0420 & 0.0004 & 1.050 & A & \\
\hline $\mathrm{OU}$ & 1 & 0.0329 & 0.0003 & 0.823 & A & \\
\hline $\mathrm{TI}$ & 1 & 0.0460 & 0.0070 & 1.150 & A & \\
\hline TM & 1 & 0.0406 & 0.0026 & 1.015 & W & \\
\hline $\mathrm{TN}$ & 1 & 0.0430 & 0.0040 & 1.075 & A & \\
\hline UC & 1 & 0.0420 & & 1.050 & A & \\
\hline UP & 1 & 0.0430 & 0.0040 & 1.075 & A & \\
\hline YA & 1 & 0.0396 & 0.0005 & 0.990 & W & \\
\hline YP & 1 & 0.0400 & 0.0016 & 1.000 & A & \\
\hline
\end{tabular}

Total Number Reported: $\quad 24$

Values for elemental uranium are reported in $\mu \mathrm{g} / \mathrm{filter}, \mathrm{g}$ or $\mathrm{mL}$.

$\mathrm{pCi} / \mathrm{g}$ or $\mathrm{mL}=\mathrm{Bq} \times 0.027$

Evaluation: $A=A c c e p t a b l e, W=A c c e p t a b l e$ with Warning, $N=$ Not Acceptable

If the evaluation system is not appropriate for the types of analyses performed in your lab, apply site specific evaluation. 


\section{Participating Laboratories in EML QAP49}

\section{Laboratories Reporting Data}

\section{Code Laboratory Name}

AC Analytical Chemistry Laboratory, Argonne National Lab

AF Air Force Analytical Lab, Brooks AFB

AG Paragon Analytics, Inc, Fort Collins, CO

AI Nuclear Technology Services, Inc., Roswell, GA

AL Ames Laboratory, Ames, IA

AM American Radiation Services, Inc., Baton Rouge

AN Argonne National Laboratory

AP Aberdeen Proving Ground, Aberdeen, MD

AT ATL International inc., Germantown, MD

AU ORISE EESD/ESSAP, Oak Ridge

BA Bettis Atomic Power Lab, West Mifflin, PA

BC Babcock \& Wilcox MC \#42, Lynchburg, VA

BE RUST Geotech, Grand Junction, CO

BL Barringer Laboratories Inc., Golden, CO

BM Battelle Memorial Institute, Columbus, $\mathrm{OH}$

BN Brookhaven National Laboratory, Upton, NY

BP Battelle Pacific Northwest National Laboratory

BQ Becquerel Laboratories Inc., Mississauga, Ontario, Canada

BU Autoridad Regulatoria, Buenos Aires, Argentina

BX B\&W Nuclear Envir. Services, Lynchburg, VA

CA Atomic Energy Control Board, Ottawa, Canada

CB Radiation Protection Bureau, Ontario, Canada

CD Gentilly-2 Nuclear Power Plant, Quebec Canada

CH California State Dept. Health Serv., Sanitation \& Radiation Laboratory

CL Core Laboratories, Casper, WY

CM Metropolitan Water Reclamation District of Greater Chicago, IL

CN China Institute for Radiation Protection

CO Bedford Institute of Oceanography, Dartmouth. Nova Scotia, Canada

CR Laboratorio de Fisica Nuclear Aplicada, Costa Rica

CS Boeing North American, Canoga Park, CA

DH Duke Engineering Services Hanford

EG LMITCO/INEL, Scoville

EM 3M, Empore Disks, St. Paul, MN

EP US EPA, Las Vegas

FG FGL Environmental, Santa Paula, CA

FJ The University of the South Pacific, Fiji Islands

FL Florida Dept of Health \& Rehab. Serv., Orlando

FM Florida Mobile Emergency Radiological Laboratory, Orlando

FN Fermi Lab, Batavia, IL

FS Florida State University, Tallahassee

GA Lockheed Martin, Pikton, $\mathrm{OH}$

GC Georgia Power Company Environmental Lab

GE General Engineering Labs, Charleston, SC

GP GPU Nuclear, Inc., Harrisburg, PA

GS USGS/NWQL, Arvada, CO

GT Georgia Institute of Technology

HC Lawrence Livermore Laboratory, California

HT Technical University, Budapest, Hungary

HU Water Resources Research Centre (VITUKD), Hungary

IA Bhabha Atomic Research Centre, India

ID Institute of Radiation Protection and Dosimetry, Rio de Janeiro, Brazil 


\section{Participating Laboratories in EML QAP49}

\section{Laboratories Reporting Data}

\section{Code Laboratory Name}

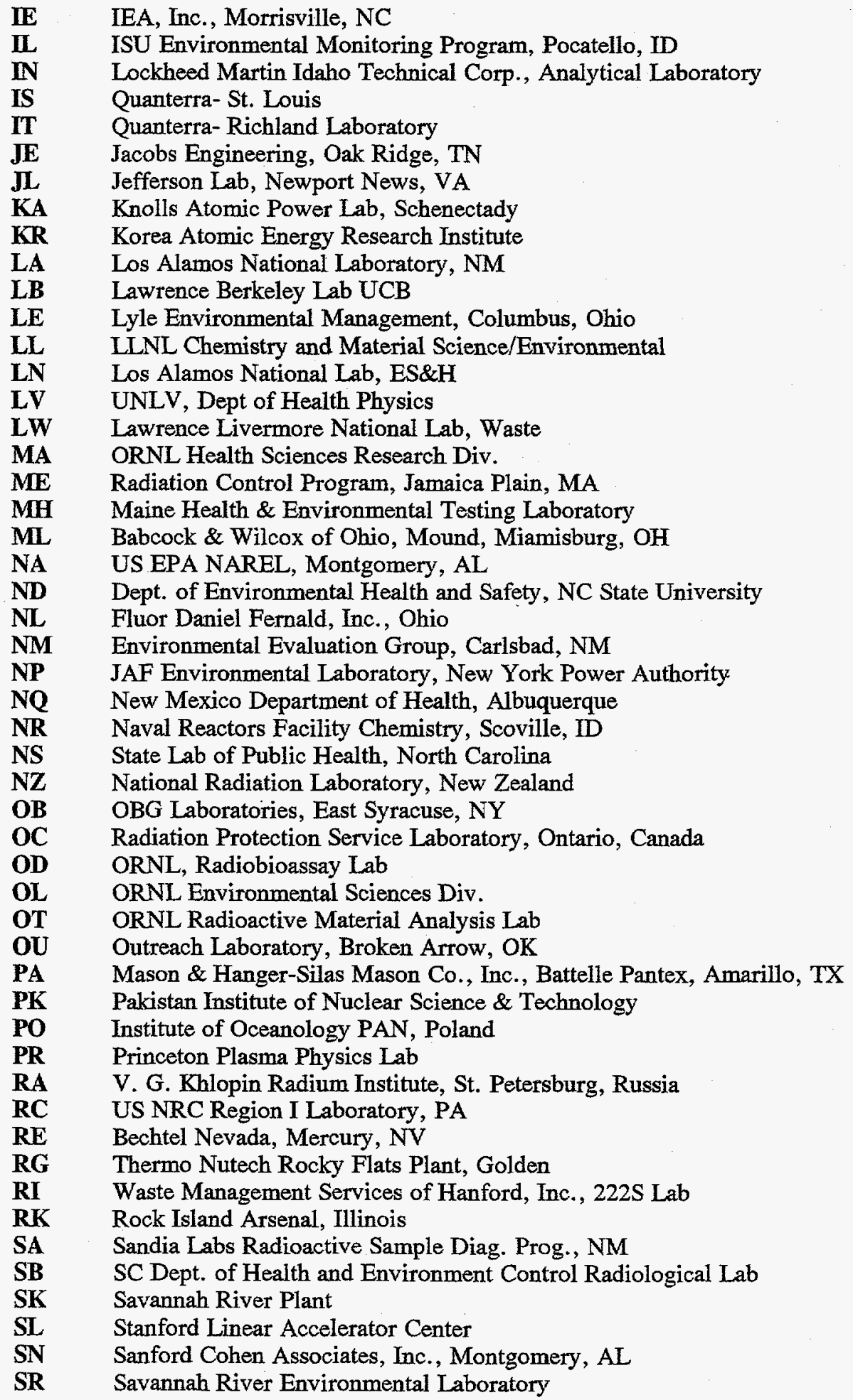




\section{Participating Laboratories in EML QAP49}

\section{Laboratories Reporting Data}

\section{Code Laboratory Name}

ST SC DHEC, Aiken, South Carolina

SW Southwest Research Institute, San Antonio, TX

TE Teledyne Isotopes Midwest Lab, Northbrook, IL

TI Teledyne Brown Engineering Environmental Services, Westwood, NJ

TM Thermo NUtech Albuquerque Lab, NM

TN Thermo NUTech, Richmond, CA

TO Thermo NUtech Oak Ridge Laboratory

TP Taiwan Power Company, Taipei, Taiwan

TT Tracer Technologies International, Inc., Cleveland

TW Taiwan Radiation Monitoring Center

TX Texas Dept. of Health/Laboratories, Austin

TY Scientific Production Association, Russia

UC Lockheed Martin, Paducah, KY

UP Lockheed Martin Energy Systems, Y-12 Plant, Oak Ridge

UY Lockheed Martin Energy Systems, Y-12 Plant, Oak Ridge

WA Environmental Radiation Lab, Off. of Public Health Labs. Seattle

WC Waste Management Federal Services of Hanford

WE Westinghouse Electric Corp., Madison, PA

WI WIPP Site, Westinghouse Electric Corp.

WS Weldon Springs Site, St Charles, MO

WV West Valley Nuclear Services Co, Inc, NY

YA Duke Engineering Environmental Lab, Westboro, MA

YP US Army Proving Ground, Yuma, AZ

YU Institute of Occupational and Radiological Health, Yugoslovia

MS Manufacturing Sciences Corporation, Oak Ridge

Total Reporting Labs: 127 


\section{Participating Laboratories in EML QAP49}

\section{Laboratories NOT Reporting Data}

\section{Code Laboratory Name}

AR Accu-Labs Research Inc., Golden, CO

AW Argonne National Laboratory, Idaho Falls

AY Analytics, Inc. Atlanta, GA

BR US Army Research Laboratory, Aberdeen Proving Ground

BS B\&W Nuclear Envir. Services, Leechburg, PA

CW Carlsbad Environmental Monitoring Research Center, NM

DC Datachem Laboratories, Salt Lake City

DP Duke Power Company, Huntersville, NC

EL Energy Laboratories, Inc., Casper, WY

FR CEA/DAM - SPR/B3

FT USACECOM-DSRM, Fort Monmouth, NJ

KO Korea Institute of Nuclear Safety

LM Los Alamos National Lab, Mercury, NV

MI Massachusetts Institute of Technology

NF Nuclear Fuel Services, Erwin, TN

NW Naval Research Lab, Washington, DC

OK Southwest Laboratory of Oklahoma

OS Oregon Health Division Radiation Controis Section, Portland

RL Thermo Hanford

SE Shealy Environmental Services Inc., Cayce, SC

SH Savannah River Ecology Lab

TR University of Istanbul, Turkey

TU Texas A\&M University, Dept of Nuclear Engineering

UK Lockheed Martin Energy Systems, Oak Ridge

WP Washington Public Power Supply System, Richland

Total Non-Reporting Labs: 25 